\title{
Wide Angle and Out-of-Plane
}

Correlations in ${ }^{7} \mathrm{Li}$ Fragmentation

\section{Thesis}

Submitted by

John Yorkston, B.Sc.

for the degree of

\section{Doctor of Philosophy}

Department of Physics

Edinburgh University 1988

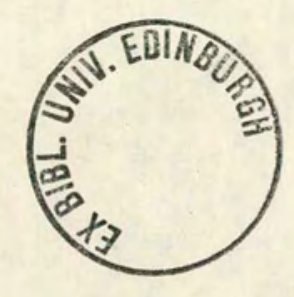


I would like to dedicate this thesis to my parents who gave me their love and support through all my decisions........even the bad ones ! 


\begin{abstract}
$\underline{\text { Abstract }}$
The reaction mechanisms producing wide angle $\alpha+\mathrm{t}$ coincidence events in the interaction of $70 \mathrm{MeV}{ }^{7} \mathrm{Li}$ with ${ }^{12} \mathrm{C},{ }^{120} \mathrm{Sn}$ and ${ }^{208} \mathrm{~Pb}$ have been investigated. The use of large area Ion Implanted Silicon Strip Detectors allowed the out-of-plane coincidence distributions to be measured simultaneously with the in-plane data. Angular distributions for the inclusive and exclusive $\alpha-\mathrm{t}$ reaction channel, in and out of the reaction plane, have been measured. The quasi-elastic alpha particle projected energy spectra are presented and compared with the results of a Monte Carlo simulation modelling sequential break-up from the excited states of ${ }^{7} \mathrm{Li}$. At larger angular separations between the outgoing fragments the data are inconsistent with this process. A simple plane wave Born approximation calculation was found to give a reasonable fit to the forward angled coincidence events and to reproduce the out-of-plane variation with changing detection angle. An attempt was made to factorise the quasi-elastic angular distributions into the product of the fragment inclusive yields. Whilst this was partly successful for the in-plane data, the out-of-plane data exhibited a significant in-plane enhancement, strongly dependant on the fragment detection angles. This behaviour was predicted from a semi-classical three body kinematic calculation where the forces on the fragments were explicitly calculated at all points along their trajectory.
\end{abstract}




\section{Acknowledgements}

I would like to thank my supervisors Dr. Alan Shotter and Dr. Derek Branford for their enthusiasm and encouragement throughout the work for this thesis. $\mathrm{My}$ grateful thanks are also extended to the other members of the Edinburgh Nuclear Physics Group both past and present whose help, company and friendship have helped me along the path to finishing this work. Special mention must be given to Dr. Euan Macdonald for his help with the Monte Carlo simulation programme and the numerous graphics packages he produced just when they were needed. I would like to thank my industrial supervisor Dr. Bryan Syme for his help with the experiments at Harwell and Gordon Turnbull for his expertise in producing the strip detector mounts and numerous other bits and bobs essential for a successful experimental run. Thanks should also be given to the staff of the N.S.F. without whom this thesis would never have been possible. I acknowledge the S.E.R.C. for the C.A.S.E studentship in conjunction with the U.K.A.E.A. and the D.H.S.S for their further research grant which enabled the completion of this work. I would also like to thank Professors Wallace and Cowley for the continuing use of the facilities of the Edinburgh University Physics Department and Dr. Dougal for his understanding viewpoint concerning overrun theses. Finally I would like to thank Christine for typing this thesis and for putting up with all the hassle for so long. 


\section{Contents}

1 Introduction 1

1.1 Terminology .................... 3

1.2 Experimental Review . . . . . . . . . . . . . 5

1.2 .1 Light Ions .................. 5

1.2.2 Light-Heavy Ions ............... 8

1.3 Theoretical Review . . . . . . . . . . . . . . 16

1.3.1 Serber Model . . . . . . . . . . . . 16

1.3.2 Coulomb Excitation ............... 18

1.3.3 Born Approximation Techniques ........... 19

1.3.4 High Energy Adiabatic Method . . . . . . . . . . 23

1.3.5 Continuum Discretised Coupled Channels . . . . . . . 24

1.4 Kinematics .......................... 25

1.5 Angular Definitions . . . . . . . . . . . . . . . . 29

1.6 Objectives and Outline of Thesis .............. 30

2 Strip Detectors $\quad 32$

2.1 Introduction .................... 32

2.2 Construction and Manufacture .............. 34

2.3 General Behaviour and Properties ... . . . . . . . 36 
2.3.1 Full Depletion Voltage ............... 37

2.3.2 Energy Resolution ................ 40

2.3.3 Continuity of Strip Output and Detection Efficiency . . . 42

2.3.4 Stability of Leakage Current . . . . . . . . . . . . 43

2.4 Charge Sharing Effects . . . . . . . . . . . . . 47

2.5 Anomalous Polarity Pulses ............... 51

2.6 Further development .................. 56

3 Experimental Apparatus and Method 60

3.1 Accelerator and beam-line ............... 60

3.2 The Detector System . . . . . . . . . . . . . . . 64

3.2.1 Detectors and Mounts ............. 66

3.2.2 Strip Groupings and Nomenclature .......... 68

3.2.3 Energy Calibration ................ 69

3.2 .4 Summary ....................... 70

3.3 Experimental Set-up .................. 70

3.3 .1 Overview .......................... 70

3.3 .2 The Event Manager .............. 71

3.3.3 Review of Computer Hardware ............ 72

3.3.4 Analogue Signal Processing ... . . . . . . . . 73

3.3.5 Logic Signal Processing . . . . . . . . . . . 78

3.3.6 Data Collection Programme (DCP) ......... 79

3.3.7 Dead-time Calculations .............. 86

3.3.8 Cross-section Calculations ............. 87

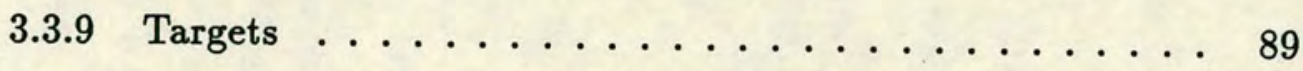


4.1 Inclusive Data . . . . . . . . . . . . . 91

4.2 Exclusive Data . . . . . . . . . . . . . 105

4.2 .1 In-Plane Data . . . . . . . . . . . . . . . 112

4.2 .2 Out-of-plane Data ................ 145

4.3 Integrated Cross-Sections $\ldots \ldots \ldots \ldots$

5 Discussion and Conclusions $\quad 164$

5.1 Sequential Decay and Partial Fusion . . . . . . . . . 167

5.1.1 Monte Carlo Simulations ............. 168

5.1 .2 Sequential Decay ................... 170

5.2 P.W.B.A Calculations . . . . . . . . . . . . . 213

5.3 Factorisation and Uncorrelated Processes . . . . . . . . . 219

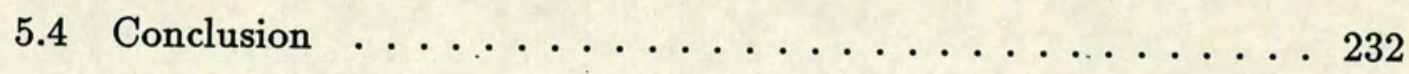

Appendix A. Strip Detector Solid Angles 236

Appendix B. Out-of-Plane Data Presentation. 237

Appendix C. Strip Detector Paper 268

$\begin{array}{ll}\text { References } & 269\end{array}$ 


\section{Chapter 1}

\section{Introduction}

This thesis is an investigation into the mechanisms and reactions leading to coincident alpha and triton fragments at large angular separations from the interaction of $10 \mathrm{MeV} / \mathrm{A}{ }^{7} \mathrm{Li}$ with ${ }^{12} \mathrm{C},{ }^{120} \mathrm{Sn}$ and ${ }^{208} \mathrm{~Pb}$.

Ever since the earliest days of experimentation with artificially accelerated nuclear particles, the concept of projectile break-up has been proposed to explain the presence of outgoing fragments detected at beam velocity $[\mathrm{Op} 35][\mathrm{Op} 35 \mathrm{~b}]$ [He47] [La35].

Only in more recent years has attention again been given to this type of interpretation. This has mainly been motivated by the increased cross-section attributed to these fragmentation processes as the energy of interaction has increased and more ion species have become available for acceleration [Br61].

Fig.1.1 is an example of the energy spectra for outgoing particles from the interaction of $70 \mathrm{MeV}{ }^{7} \mathrm{Li}$ with a ${ }^{12} \mathrm{C}$ target. At high ejectile energies one can clearly see the transitions to well isolated states in the residual nucleus. At lower energies the contributions from equilibrium and pre-equilibrium compound emission are visible.

Between these two regions one can see that a large proportion of the total crosssection is contained in the broad bump commonly termed the continuum. In this region one can envisage events corresponding to excitation of the many broad 


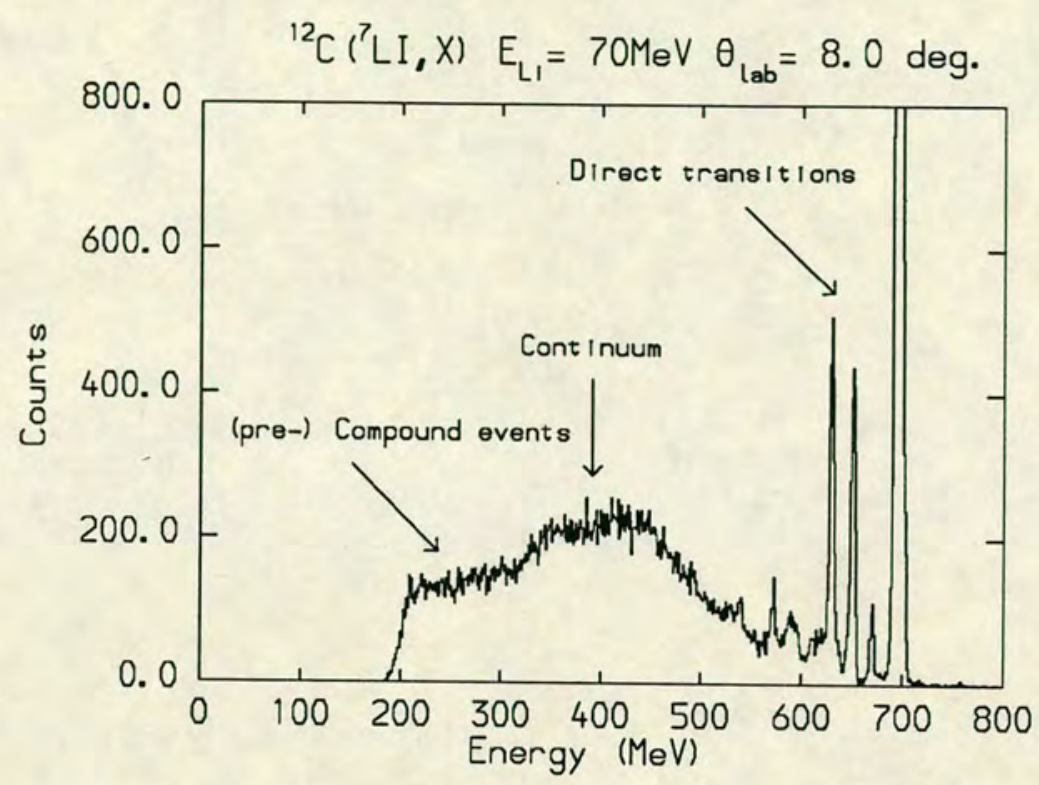

Figure 1.1: Energy spectrum for particles from $70 \mathrm{MeV}^{7} \mathrm{Li}$ incident on a ${ }^{12} \mathrm{C}$ target.

overlapping states and resonances present in the nuclear system at such high excitation energies. It is also possible for reactions with three or more particles in the final state to contribute to this region.

In recent years interest has focused on the identification and quantification of the different processes contributing to this continuum. (An excellent example of this decomposition is given in De Meijers review on He projectile reactions [Me85].) As has been pointed out, this has mainly been motivated by the increased cross-section for this region as the range of available projectile energies and masses has extended. Apart from trying to understand and identify the reaction processes themselves, detailed knowledge of their contribution to events in this energy region is essential for investigating the other components such as giant resonances and excited states well above the particle emission threshold, which also contribute to the continuum.

The elastic scattering of projectiles with strong cluster like behaviour eg. ${ }^{6} \mathrm{Li}$, ${ }^{7} \mathrm{Li},{ }^{9} \mathrm{Be}$ is also dramatically affected by the fragmentation channel. In recent calculations Sakuragi et.al. [Sa86] have managed to fit the elastic scattering angular distributions for this type of nuclei without the puzzling renormalisation 
of the interaction potential that was an anomalous feature with these projectiles. This was achieved within a C.D.C.C. approach (see section 1.3) which incorporated the effects of projectile break-up in a realistic way. The possibility of cleanly identifying the cross-section for specific types of break-up reactions eg. sequential decay, also providesa stringent testing ground for the most sophisticated theoretical codes. These require realistic representations of the internal wavefunctions of the projectile as well as detailed knowledge of the relevant interaction potentials.

It is also possible for break-up reactions to provide information on the timereversed fusion reaction that would be impossible to obtain from the fusion reaction on its own ie. very low energy fusion cross-sections. The extraction of such information is still at an early stage and it has already been pointed out that the current procedures for deducing the relevant fusion cross-section from the corresponding break-up cross-section are fraught with difficulties [Sh88].

\subsection{Terminology}

In the field of break-up studies there is unfortunately no universally accepted terminology. This short section will define the terms used in this thesis. It is not claimed that these definitions are in any way better than those of other authors but are the ones this author has become most familiar with.

\section{Sequential break-up}

This involves the excitation of the projectile to distinct states above its particle emission threshold. The excited projectile then breaks up into the constituent clusters at a distance from the target large compared to the interaction distance. The excitation and decay are pictured as two distinctly separate events.

\section{Direct break-up}

This involves the excitation of the projectile straight into the continuum without any intermediate state. This is a one-step process and occurs close to the target where final state interactions between the resulting clusters and the target are 
possible.

Coulomb break-up

This type of event is defined as a break-up initiated by the Coulomb force alone.

\section{Final state interactions}

In this thesis this term will refer to interactions between either of the fragments and the target after the projectile has broken-up. There is also the possibility of fragment-fragment final state interactions but these will not be considered here.

\section{Quasi-elastic events}

These are events where the target maintains its identity and all the outgoing particles (including the target) are in their ground state.

\section{Inelastic events}

Events which leave one of the outgoing fragments in an excited state. In this thesis this will normally be the target.

\section{Fragmentation or break-up}

These will be used to specify an event where there are more than two particles in the outgoing channel (again including the target).

\section{Fragmentation-Absorption}

This will apply to reactions where one of the fragments fuses with the target after the ${ }^{7} \mathrm{Li}$ has broken up into its constituent clusters.

\section{Partial fusion or transfer}

These both refer to a process whereby part of the projectile fuses with the target nucleus. This is normally taken to happen in a one step process. 


\subsection{Experimental Review}

\subsubsection{Light Ions}

Experiments with light ions $(A \leq 4)$ have been performed since the earliest days of nuclear physics research. The relatively simple composition of the deuteron and He nuclei limit the number of possible reaction paths. This makes the interpretation of the data easier than with heavier projectiles. Theoretical calculations are also simplified by certain approximations that lose their validity with more complex projectiles (eg. zero-range approximation and the representation of the internal wave function of the projectile).

The deuteron was first investigated by Oppenheimer and Phillips [Op35] [Op35b] where, at energies below the Coulomb barrier, they interpreted the excess of beam velocity protons over neutrons by the dissociation of the deuteron in the Coulomb field of the target. Consequently more protons survive due to their Coulomb repulsion from the target. At energies well above the Coulomb barrier Helmholtz et.al. [He 45] concluded that the beam velocity neutrons seen at forward angles originated from nuclear induced fragmentation. More recently the theoretical and experimental behaviour of deuteron break-up have been investigated by Baur et.al. [Ba72], [Ba72b], [Ba73] and by Jarczyk et.al. [Ja73]. Analysing the data taken around the Coulomb barrier with a pure Coulomb interaction Jarczyk et.al. were unable to achieve complete agreement between the data and the theoretical predictions. This was attributed to, and later confirmed to be due to neglecting the nuclear interaction between the target and the fragments. A post form DWBA calculation by Baur et.al. [Ba72b] taking into account the neutron target interaction produced much better fits to the experimental $n+p$ coincidence data over the whole angular range. This work was extensively reviewed in the paper by Baur and Trautmann [Ba76]. At energies well above the Coulomb barrier inclusive measurements of the proton angular distribution [ $\mathrm{Pa} 78],[\mathrm{Ma} 80]$ have been performed which show that the crosssection for projectile break-up is a significant contributor to the deuteron total reaction cross-section. Coincidence $n+p$ data havealso been collected at these high energies ( $56 \mathrm{MeV}$ [Ma82]) where theoretical calculations are complicated by 
the requirement for inclusion of the nuclear part of the interaction. It was found that protons were emitted predominantly on the opposite sides of the beam from the neutrons and that the correlated ejectiles arose mainly from elastic break-up of the deuteron. For smaller momentum transfer interactions where the neutron and proton were detected on opposite sides of the beam, the prior form DWBA calculation performed by Matsuoka et.al. achieved reasonable fits to the data. For larger momentum transfers, when both ejectiles were detected on the same side of the beam, the calculations overpredicted the data by factors of $\sim 2-10$. This shows that even with the simplest projectile there are still problems associated with the theoretical predictions of the experimental results when both nuclear and Coulomb components have to be included.

The extension to He projectiles of the ideas behind the break-up interpretation of the deuteron data has been extensively reviewed in the excellent paper by De Meijer and Kamermans [Me85]. The following will give a brief review of the reactions and processes outlined in that work.

Since the first experiments investigating ${ }^{3} \mathrm{He}$ [Ma78] and ${ }^{4} \mathrm{He}[\mathrm{Bu} 78]$ break-up, many authors have produced detailed works revealing numerous different reaction mechanisms. Some of these are common to both projectiles whilst others are not. The dependance of the reaction strength on the reaction $Q$ value has a marked effect on this behaviour with the large binding energy of the ${ }^{4} \mathrm{He}$ $(20 \mathrm{MeV})$ playing a major role in suppressing reaction channels seen with the more weakly bound ${ }^{3} \mathrm{He}(5.5 \mathrm{MeV})$. The absence of beam velocity enhancements in the inclusive $(\alpha, t)$ and $\left(\alpha,{ }^{3} \mathrm{He}\right)$ spectra [Me83] is attributed to this $\mathrm{Q}$ value dependance. At higher energies the inclusive yields of $t$ and ${ }^{3} \mathrm{He}$ were predominantly due to single particle transfer reactions rather than the fragmentation processes responsible for the inclusive yields seen with the ${ }^{3} \mathrm{He}$ projectiles.

In the ${ }^{3} \mathrm{He}$ experiments performed at $52 \mathrm{MeV}$ [Aa81] Aarts et.al. have identified numerous different reaction processes leading to $p+d$ coincidences. The main method for identification of these mechanisms was by projection of the relevant particle energy onto the axis of a 2 dimensional scatter plot of deuteron energy against proton energy. At all angles loci corresponding to events leaving the target nucleus in distinct excitation states (including the ground state) 
were observed. These were identified as direct break-up of the ${ }^{3} \mathrm{He}$ into its constituent clusters. In addition, when the deuteron was detected in the forward direction and the proton in the backward direction events were recorded that corresponded to a beam velocity deuteron and an evaporated proton. These were interpreted as arising from a process where the deuteron is a spectator maintaining the projectile velocity but the proton originates from compound (or pre-compound) emission from the excited target after proton fusion. This type of process has been termed absorptive break-up and is a light ion version of the reaction type called partial fusion with heavier ions. This process has been shown to be the biggest contributor to the continuum region of spectra produced by ${ }^{3} \mathrm{He}$ projectiles [Aa84]. These authors have also been able to kinematically distinguish the two similar but distinct processes of break-up transfer and transfer break-up. In the prior case nuclear transfer to one of the fragments occurs after fragmentation, in the latter, transfer takes place before fragmentation. Both are detected via the $\left({ }^{3} \mathrm{He}, \mathrm{t}+\mathrm{p}\right),\left({ }^{3} \mathrm{He},{ }^{3} \mathrm{He}+\mathrm{p}\right),\left({ }^{3} \mathrm{He}, \mathrm{d}+\mathrm{d}\right)$ reactions and break-up transfer reactions were found to be the most prominent of the two mechanisms. In events where not all the fragments from the projectile were detected eg. $\left({ }^{3} \mathrm{He}, \mathrm{p}+\mathrm{p}\right)$, events from a mechanism where both fragments have the beam velocity (two spectator events) have also been recorded along with evaporative+beam velocity coincidence events (one spectator, one particpant events).

In general these results show the range of mechanisms occuring in collisions involving He projectiles. The projectile itself may undergo any of the 'normal' nuclear reactions such as elastic scattering, nuclear pick-up or stripping before breaking up or either of the fragments may undergo similar interactions with the target after projectile fragmentation. Due to the lack of any well defined excited states in ${ }^{3} \mathrm{He}$, no sequential decay has been reported for this projectile. The alpha particle has several excited states but the large thresholds for $\mathrm{p}+\mathrm{t}$ or $d+d$ fragmentation inhibit sequential decay of this projectile and no clear identification of this decay channel has been reported. Sequential decay has however been observed from the products of transfer or stripping reactions with both projectiles eg. $\left({ }^{3} \mathrm{He},{ }^{2} \mathrm{He} \rightarrow \mathrm{p}+\mathrm{p}\right),\left(\alpha,{ }^{2} \mathrm{He} \rightarrow \mathrm{p}+\mathrm{p}\right)$ and $\left(\alpha,{ }^{7} \mathrm{Li}^{*} \rightarrow \alpha+\mathrm{t}\right)$. In addition to understanding the basic reaction mechanisms, experiments with He projectiles have supplied large amounts of spectroscopic information for many excited 
states of various nuclei as well as information on their own internal wavefunctions. This is covered in detail in [Me85].

\subsubsection{Light-Heavy Ions}

The interaction of ${ }^{6} \mathrm{Li}$ with various heavy targets $\left({ }^{118} \mathrm{Sn},{ }^{208} \mathrm{~Pb}\right)$ has been investigated by the group from Heidelberg University at energies below and around the Coulomb barrier $\mathrm{E}_{L i} \sim 22-24 \mathrm{MeV}$ [Sc77] [Os74] and $\mathrm{E}_{L i} \sim 36-48 \mathrm{MeV}$ [Ge78]. This research identified four main reaction channels contributing to the coincident charged particle cross-section. These were:

1. sequential decay of the $2.18 \mathrm{MeV}$ state in ${ }^{6} \mathrm{Li}$ into $\alpha+\mathrm{d}$ leaving the ${ }^{208} \mathrm{~Pb}$ target in its ground state.

2. a non-sequential component from direct ${ }^{6} \mathrm{Li}$ fragmentation or transfer reemission processes.

3. deuteron pick-up to the ground state of ${ }^{8} \mathrm{Be}$ followed by decay into two alphas.

4. neutron transfer to the target followed by decay of the ${ }^{5} \mathrm{Li}$ into $\alpha+\mathrm{p}$.

The sequential process (1) was found to be the strongest of these reaction channels and was proposed to be initiated via Coulomb excitation of the ${ }^{6} \mathrm{Li}$. Complementary to this work, Cunsolo et.al. [Cu78] [Cu80] have investigated the much lighter system ${ }^{6} \mathrm{Li}+{ }^{12} \mathrm{C}$ at similar projectile energies. Here the main component contributing to the quasi-elastic $\alpha+\mathrm{d}$ coincidence rate was found to be $\alpha$ transfer to excited states in ${ }^{16} \mathrm{O}$ followed by re-emission to the ground state of ${ }^{12} \mathrm{C}$. In this system the interaction energy is much higher than the Coulomb barrier so it is perhaps understandable that nuclear interactions will dominate over the Coulomb effects found to be important with the heavier targets.

Neumann and co-workers at Karlsruhe have performed various experiments aimed at interpreting the ${ }^{6} \mathrm{Li}+$ heavy target system at much higher energies $\left(\mathrm{E}_{L i}=156 \mathrm{MeV}\right)[\mathrm{Ne} 79][\mathrm{Ne} 80][\mathrm{Ne} 82]$ [P186]. In addition to the mechanisms seen 
by the Heidelberg group, they observe ${ }^{6} \mathrm{Li} \rightarrow \mathrm{t}+{ }^{3} \mathrm{He}$ dissociation. The lack of identification of this channel at lower energies was attributed to the prohibitively large $Q$ value $(Q=-15.8 \mathrm{MeV})$. In their experiments detecting the recoiling target residues, Neumann et.al. identified the fusion of ${ }^{6} \mathrm{Li}$ fragments with the target. They concluded that this reaction was identical to that of free beam velocity alpha particles interacting with the target, with the deuteron acting as a spectator.

The importance of partial fusion was also identified by Castenada et.al. [Ca80] in their excellent paper investigating the mechanisms of $Z=1$ and 2 fast particle production in ${ }^{6} \mathrm{Li}$ induced reactions at $75 \mathrm{MeV}$ on ${ }^{197} \mathrm{Au}$. This paper outlined the identification, via particle-particle and particle- $\gamma$ coincidence measurements, of partial fusion and ${ }^{6} \mathrm{Li} \rightarrow \alpha+\mathrm{d}$ sequential decay. In contrast to Neumann et.al. no ${ }^{3} \mathrm{He}+\mathrm{t}$ coincidences were seen but they did record $\alpha+\mathrm{p}$ coincidences from the sequential decay of ${ }^{5} \mathrm{Li}$ after neutron transfer to the target. Their particle- $\gamma$ coincidence yields also followed closely the free fragment + target fusion behaviour seen with alphas or deuterons incident on ${ }^{197} \mathrm{Au}$ at beam velocity. This supports the viewpoint of Neumann et.al. that at these energies the partial fusion channel arises mainly from separate interactions of the deuteron and alpha with the target after ${ }^{6} \mathrm{Li}$ dissociation. A calculation modelling the Coulomb excitation of the sequentially decaying $2.18 \mathrm{MeV}$ state agreed with the data at forward angles but it required a full DWBA calculation incorporating both Coulomb and nuclear effects to fit their data at larger scattering angles of the excited ${ }^{6} \mathrm{Li}$ ejectile. This indicates the importance of nuclear effects as the projectile energy is raised and the scattering angle approaches the grazing value.

Some early experiments with ${ }^{7} \mathrm{Li}$ around the Coulomb barrier were performed by Quebert et.al. [Qu 74] and Hausser et.al. [Ha72]. No evidence was observed for sequential decay of ${ }^{7} \mathrm{Li}$ into the $\alpha+\mathrm{t}$ channel on either ${ }^{208} \mathrm{~Pb}$ or ${ }^{197} \mathrm{Au}$ but neutron transfer followed by sequential decay via ${ }^{6} \mathrm{Li}_{2.18 \mathrm{MeV}} \rightarrow \alpha+\mathrm{d}$ was found to be the dominant coincidence channel at $32 \mathrm{MeV}$. Hausser et.al. attributed the enhanced cross-section at large scattering angles for inclusive $\alpha$ s over the inclusive tritons by the observation that a 'free' beam velocity alpha was below the Coulomb barrier whilst a 'free' beam velocity triton was above the barrier. The triton therefore, could be absorbed at the smaller impact parameters leading 
to these higher scattering angles.

A more recent experiment at similar energies [Ra82] on ${ }^{12} \mathrm{C}$ showed clear evidence for sequential decay from the $4.63 \mathrm{MeV}$ state in ${ }^{7} \mathrm{Li}$ along with sequential decay from ${ }^{6} \mathrm{Li}_{2.18 \mathrm{MeV}}$ after neutron transfer to the target. Events from ${ }^{5} \mathrm{Li} \rightarrow \alpha+\mathrm{p}$ and the decay of ${ }^{8} \mathrm{Be}_{\text {g.s. }} \rightarrow \alpha+\alpha$ following proton pick-up from the target were also seen. The observation of ${ }^{7} \mathrm{Li}$ decay in this system is presumably due to the increased influence of nuclear interactions when the centre of mass kinetic energy is above the Coulomb barrier.

At higher bombarding energies of $10 \mathrm{MeV} / \mathrm{A}$ Shotter and co-workers have identified sequential decay from this ${ }^{7} \mathrm{Li}_{4.63 \mathrm{MeV}}$ state for heavy and light target systems [Sh81] [Sh84] [Da87]. They have also recorded events due to proton and neutron pick-up and transfer processes as were seen in the reactions at lower energy [Da87]. Their detection system was specifically designed to enhance the efficiency for detecting outgoing fragments with low relative energies and allowed clear unambiguous identification of these sequential processes. For heavier targets at forward angles these workers have also identified another component contributing to the $\alpha-\mathrm{t}$ coincident cross-section at small relative energies $(0.16<\varepsilon<2.0 \mathrm{MeV})$ [Sh81] [Sh84]. These events were outwith the kinematically allowed region for sequential decay and were attributed to a direct break-up process where the ${ }^{7} \mathrm{Li}$ was excited straight into the $\alpha$-t continuum in a one step interaction with the target.

Since this break-up mode was concentrated at forward angles inside grazing it has been suggested that this direct process is initiated by the differential Coulomb force experienced by the two composite clusters due to their different charge to mass ratios. The probability of excitation via an $\mathrm{E} 1$ transition is related to the difference between these ratios, and for an $\alpha$-t cluster system at $70 \mathrm{MeV}$ this excitation mode has a sizeable probability.

The experimental measurements of these two break-up processes $\left({ }^{7} \mathrm{Li}\right.$ sequential break-up and break-up direct to the continuum) has prompted numerous theoretical calculations attempting to explain the observed strength and angular distributions. Their unambiguous identification over a large angular range pro- 
vides particularly stringent tests for the theoretical cluster wave functions used in the quantuum mechanical approaches and the interplay between nuclear and Coulomb interactions at these energies. At present no single approach has been able to incorporate both the nuclear and Coulomb components in a consistent manner and produce results which agree quantatively with the experimental data.

Coulomb calculations that use available $\alpha(t, \gamma){ }^{7} \mathrm{Li}$ fusion data succeed in predicting the magnitude of both the direct and sequential decay modes in the direction forward of grazing but their over estimation at larger angles was attributed to the increased importance of nuclear processes [Sh88] [Sh88b] [Sr88]. In the forward direction these calculations were able to reproduce the experimentally observed variation with changing target and projectile energy [Sh88c] [Sh88b].

The lack of data points in the region inside grazing (due to experimental difficulties at these small angles) makes it impossible to determine whether the data follow the decrease in cross-section for both the sequential and direct processes at forward angles predicted from these Coulomb calculations. It has been indicated [Th83] [Sh88] that at very forward angles the nuclear excitation of the direct break-up component should become dominant and keep this cross-section high in contrast to the dramatic fall predicted from pure Coulomb calculations. A recently published paper [Ut88] outlines a ${ }^{7} \mathrm{Li} \rightarrow \alpha+\mathrm{t}$ coincidence experiment using an Enge split-pole spectrometer as the particle detector. This system has none of the experimental problems associated with measurements at small angles that hindered the previous experiments and the extension of the angular distributions to small angles (even $0.0^{\circ}$ ) seems only a matter of time.

In contrast to this pure Coulomb approach a number of authors have attempted a full quantum mechanical calculation of these sequential [Sa86] and direct [Th83] [Sa86] components. Thompson and Nagarajan [Th83] managed to fit the direct component of break-up from ${ }^{208} \mathrm{~Pb}$ using a high energy adiabatic approximation for the incoming wave function. They used only the nuclear component of the cluster-target interactions and obtained quantative fits to the data over the full angular range. Inclusion of the Coulomb component of the interaction potential 
resulted in a substantial overprediction for the break-up cross-section and was attributed to the validity of the adiabatic approximation breaking down for the long range Coulomb force.

Sakuragi et.al. [Sa86] have applied the powerful technique of Continuum Discretised Coupled Channels (C.D.C.C.) to both the sequential break-up and the non-resonant direct break-up. The potential they used for calculation was obtained from fits to ${ }^{7} \mathrm{Li}$ elastic scattering and were of the M3Y form for the real part and a Woods-Saxon form for the imaginary part. Initially they only included the nuclear component of the interaction. It is interesting to note that in contrast to other coupled channels fits to ${ }^{7} \mathrm{Li}$ elastic scattering which required a renormalisation of the real and imaginary parts of the interaction potential obtained from a similar M3Y technique, their inclusion of the break-up channels in the calculation allowed excellent fits without renormalisation. Using this potential they obtained rather good quantative fits to the sequential components for targets over a wide range of masses from ${ }^{12} \mathrm{C}$ to ${ }^{208} \mathrm{~Pb}$. They also managed to fit the direct break-up component for ${ }^{120} \mathrm{Sn}$ but predicted a non-negligibledirect component for the ${ }^{12} \mathrm{C}$ target. Unfortunately no such component was seen with this target [Da87]. In their procedure, inclusion of the Coulomb excitation of direct break-up states was rather difficult but they have managed to include Coulomb excitation for break-up via the sequential channel. This improved the relative phase between the oscillations in the data and the theoretical predictions for both the ${ }^{120} \mathrm{Sn}$ and ${ }^{208} \mathrm{~Pb}$ targets but resulted in an over prediction by 3-4 in the absolute magnitude. The lack of inclusion of Coulomb excitation of direct break-up states has been suggested as a reason for this overprediction but work is still being done on this problem.

It seems that a theory capable of handling simultaneously the nuclear excitations that are dominant at large scattering angles and the Coulomb excitations dominant inside grazing has almost been constructed. This should unravel the source of the two different break-up modes and possibly explain the absence of direct break-up with lighter targets.

In addition to the reaction mechanisms outlined so far Davinson [Da87] and Utsunomiya et.al. [Ut83] have identified a strong partial fusion channel by 
particle- $\gamma$ coincidence measurements. This channel produces a substantial proportion of the beam velocity alpha yield measured in inclusive experiments. Davinson found that triton fusion is approximately twice as likely as alpha fusion. From particle- $\gamma$ and particle-fission experiments Utsunomiya concluded that spin alignment of the residual nucleus is higher for high energy $\alpha$ emission than for low energy $\alpha$ emission and that the amount of transferred angular momentum is proportional to the fraction of projectile mass captured by the target. Their measurements were also consistent with a direct reaction taking place in a peripheral region, where the total angular momentum is close to the grazing value.

As one progresses to heavier projectiles and higher projectile energies, the increase in projectile complexity is reflected by an increase in the number of possible reaction channels. Projectile fragmentation with more than three ejectiles in the outgoing channel becomes important. Identification of the exact reaction channels then requires triple coincidence experiments which normally have prohibitively small detection efficiences. The possibility of outgoing fragment excitation also complicates matters.

Recent work done at $\sim 10 \mathrm{MeV} / \mathrm{A}$ with the neutron rich ${ }^{9} \mathrm{Be}$ projectile and a ${ }^{120} \mathrm{Sn}$ target [Ma88] has identified events consistent with break-up of ${ }^{9} \mathrm{Be}_{2.43 \mathrm{MeV}}$ into ${ }^{5} \mathrm{He}+\alpha$ followed by decay of the ${ }^{5} \mathrm{He}$ into $\alpha+\mathrm{n}$. The sequential decay of the ${ }^{9} \mathrm{Be}_{1.96 \mathrm{MeV}}$ state into the ${ }^{8} \mathrm{Be}+\mathrm{n}$ channel has also been identified as well as events proposed to arise from direct break-up of the ${ }^{9} \mathrm{Be}$ into ${ }^{8} \mathrm{Be}+\mathrm{n}$. It is interesting to note that as with ${ }^{7} \mathrm{Li}$ no events corresponding to small relative energies were seen when a ${ }^{12} \mathrm{C}$ target was used. A pure Coulomb calculation for this direct component from ${ }^{120} \mathrm{Sn}$ overpredicted the observed cross-section but it will require data at more forward angles to test the applicability of this approach.

Experiments performed by Bice et.al. [Bi82] have shown that the sequential decay mechanism identified with the lighter projectiles was also present in the interaction of $10-20 \mathrm{MeV} / \mathrm{A}{ }^{12} \mathrm{Cwith}$ a ${ }^{208} \mathrm{~Pb}$ target. They identified sequential break-up from ${ }^{12} \mathrm{C}_{7.65 \mathrm{MeV}}$ and ${ }^{12} \mathrm{C}_{9.64 \mathrm{MeV}}$ into a ${ }^{8} \mathrm{Be}+\alpha$ followed by decay of the ${ }^{8} \mathrm{Be}$ into two alphas. They also showed that at the higher energies direct fragmentation from ${ }^{12} \mathrm{C} \rightarrow \alpha+\alpha+\alpha$ became important. From an investigation 
of the inclusive alpha particle cross-sectional balance, incorporating the partial fusion data of Siwek-Wilczynska et.al. [Si79] they also inferred the existence of a direct ${ }^{12} \mathrm{C} \rightarrow{ }^{8} \mathrm{Be}+\alpha$ component in their data at $187 \mathrm{MeV}$ bombarding energy. Parker et.al. $[\mathrm{Pa} 84]$ have shown that at energies $<100 \mathrm{MeV}$ with a ${ }^{51} \mathrm{~V}$ target the direct reaction ${ }^{12} \mathrm{C} \rightarrow \alpha+\alpha+\alpha$ is of minimal importance with a substantial fraction of the cross-section going into the ${ }^{8} \mathrm{Be}$ and alpha partial fusion channels forming ${ }^{59} \mathrm{Co}$ and ${ }^{55} \mathrm{Mn}$ respectively.

This energy dependance of the complete fragmentation cross-section is consistent with the limiting angular momentum model of Wilczynski [Wi73] [Wi73b]. This model balances the nuclear, Coulomb and centrifugal potentials to determine whether a colliding projectile or only a projectile fragment fuses with the target. It implicitly assumes that the fragments of the projectile carry a fraction of the total projectile angular momentum that is proportional to their mass. The model has had remarkable success, considering its simplicity, in accounting for the $\left({ }^{12} \mathrm{C}, \alpha \mathrm{xn} \gamma\right)$ and $\left({ }^{12} \mathrm{C}, 2 \alpha \mathrm{xn} \gamma\right)$ cross-sectional dependance with bombarding energy [Si79b]. It also agrees well with independantly measured values of the limiting angular momentum for fusion of the ${ }^{8} \mathrm{Be}$ or alpha fragments with the target.

Experimental evidence for the predicted localisation in angular momentum space has come from a number of experiments involving various projectiles identifying the products of partial fusion by particle- $\gamma$ ray coincidence measurements [In77] [Ge79] [Zo78] [Ba80], the lack of 'side-feeding' in the $\gamma$-ray decay chains being used as evidence for this localisation. The idea of limiting angular momentum also predicts in a natural way the increase in ${ }^{12} \mathrm{C} \rightarrow \alpha+\alpha+\alpha$ cross-section with higher projectile energies mentioned earlier.

Recent experiments have investigated the possibility of identifying the residual nucleus arising from partial fusion from its $\mathrm{X}$-ray spectra in coincidence with outgoing particles [Wi83] [Ba84b]. These have shown that, compared to the particle $\gamma$-ray method, detection of the $\mathrm{K} \mathrm{X}$-rays from the target residues is relatively straightforward although calculation of the absolute cross-sections require knowledge of the $\mathrm{X}$-ray multiplicities of each reaction channel. 
The clear identification of this partial fusion mechanism may be seen as a step in connecting the nucleon transfer and break-up behaviour seen for lighter projectiles with the deep inelastic processes more characteristic of heavier projectiles.

Bhowmik et.al. have successfully factorised the heavy ion $+\alpha$ coincidence data from ${ }^{14} \mathrm{~N}$ interactions with heavy targets into the product of the heavy ion and alpha inclusive cross-sections (see section 5.3). This behaviour is interpreted as arising from an interaction where the alpha is emitted early in the reaction and the heavy fragment undergoes further interaction with the target. These interactions tend to be rather like the deep inelastic collisions characteristic of true heavy ion processes where there is a substantial exchange of energy and mass between the participating nuclei. This further supports the picture of these intermediate mass projectiles linking the distinctive phenomenon of light and heavy ion interactions. Sequential decay has also been reported for this projectile both from excited states in ${ }^{14} \mathrm{~N}$ and excited states in fragments resulting from massive transfer to and from the target [Dr80].

As a final example of the diversity of the reaction processes possible with relatively simple systems, Siwek-Wilczynska et.al. [Si87] have clearly identified the transfer reemission of alpha particles in the quasi-elastic interaction of $157 \mathrm{MeV}$ ${ }^{20} \mathrm{Ne}$ with a ${ }^{12} \mathrm{C}$ target. They conclude that when the $\alpha$ and ${ }^{16} \mathrm{O}$ fragments are detected at large angular separations they find evidence for excitation of a $20.8 \mathrm{MeV}$ state in ${ }^{16} \mathrm{O}$ populated by an alpha transfer reaction from the ${ }^{20} \mathrm{Ne}$ to

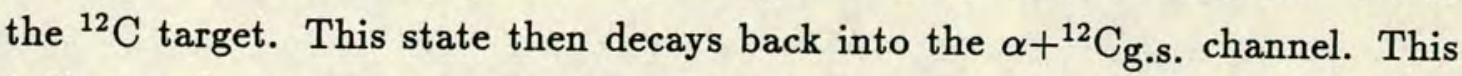
indicates the complexity of reaction mechanisms possible when light systems are involved and changed particle emission is not suppressed by the Coulomb barrier.

The experimental investigations outlined so far have in general addressed themselves to the problem of identifying the underlying mechanisms producing the outgoing particles detected in inclusive experiments. Most attention has generally been focused on reproducing their energy spectra and decomposing the inclusive cross-section into its various components. There are however other reasons for experimenting with these systems. For example, the pronounced cluster structure of many of the lighter projectiles make them ideal for investigating the 
clustering aspects of systems formed by particle transfer. In particular projectiles such as ${ }^{6} \mathrm{Li},{ }^{12} \mathrm{C}$, and even ${ }^{10} \mathrm{~B}$, have been used by various authors to populate $\alpha$ cluster states in light targets [Ar82],[Bi81],[Wo72], [Ra85].

\subsection{Theoretical Review}

At the present time the available methods for theoretically describing the large amount of experimental data now available on break-up reactions tend to be extensions of approaches used to explain 'simpler' interactions such as elastic scattering and single nucleon transfer. It is not surprising therefore that the application of these methods encountersproblems with evaluation of cross-sections arising from rather more complicated and possibly multi-step interactions. For example it is only in very recent years that models have been able to incorporate in a consistent manner the excitation of a projectile into the continuum region above the particle emission threshold [Sa86]. Even this most up to date calculation requires an averaging over fixed momentum bins to allow feasible calculation times on the most powerful computers. These most realistic approaches are not surprisingly, extremely complicated and the following review will be at a rather elementary level. Nevertheless it is hoped that the underlying physical assumptions behind them will become apparent.

\subsubsection{Serber Model}

Perhaps the simplest and most intuitively appealing approach was that of Serber [Se47] used to explain the high energy deuteron break-up data of Helmholtz et.al. [He47].

Serber proposed that the projectile be viewed as consisting of two parts. One that underwent an interaction with the target, known as the participant, and the other which carried on its inital trajectory undisturbed. This latter fragment was known as the spectator and it was assumed to be the particle detected by the inclusive measurements of the experimenter. 
The outgoing velocity of this spectator was then found from the sum of the momentum the fragment possessed due to the movement of the projectile and the intrinsic momentum the fragment had inside the projectile ie. its Fermi momentum. The theoretical cross-section was then proportional to the square of the internal wave function of the projectile in the momentum representation ie.

$$
\frac{d^{2} \sigma}{d \Omega d E} \sim\left|\phi\left(\underline{\mathbf{p}}_{\mathbf{i}}\right)\right|^{2}\left(E\left(E_{\max }-E\right)\right)^{\frac{1}{2}}
$$

where $\mathrm{E}_{\max }$ is the maximum allowed energy. This reproduced the observed beam velocity bump seen in the experimental inclusive data and explained the width of the bump as arising from the coupling to the internal momentum of the projectile.

This simple picture and its success in reproducing the observed experimental behaviour has motivated the use of the spectator participant concept in many of the more sophisticated theoretical approaches. Predictably however, this simple Serber model has a number of limitations. The model says nothing about the interaction of the participant, whether it's elastically scattered, inelastically scattered or absorbed forming an excited compound nucleus. Consequently the model only describes the inclusive channel and cannot be used to explain any exclusive measurements. Also, the predicted particle distributions do not take into account any distortion of the outgoing fragments due to the final state interactions, although recently a local Coulomb corrected wave function for the projectile has been used to improve the theoretical predictions for heavier projectiles and targets. These basically corrected the the wave functions' energy and direction for Coulomb distortion effects [Mc80]. It has been pointed out [Ba74] that these final state interactions complicate the extraction of any information on the internal wave function of the projectile by distorting the experimentally observed inclusive spectra energy widths.

In summary the Serber model is useful in describing inclusive energy spectra and introduces the concept of a participant and a spectator part of the projectile. 


\subsubsection{Coulomb Excitation}

It has been proposed [Sh84] that for certain projectiles with strong cluster like properties and low break-up thresholds it may be possible for Coulomb excitation to play an important role in inducing projectile fragmentation. This is particularly true for systems where the Sommerfeld parameter is large [Ba80] and the centre of mass of the projectile is scattered in the forward direction inside grazing.

Unlike the nuclear force the Coulomb force is well understood and accurate theories concerning excitation and de-excitation of projectiles and targets via the Coulomb field have been developed over many years [Ad56]. Coulomb calculations tend to employ a semi-classical approach which are good approximations for systems with Sommerfeld parameters $>10$.

The Coulomb field operator is expanded into an infinite series of magnetic and electric multipole operators. Since the strength of the magnetic multipoles is proportional to the speed of the projectile compared to the velocity of light, they can generally be ignored for the applications to projectile excitation in the energy range $\sim 10 \mathrm{MeV} / \mathrm{A}$. In practice it is also normally acceptable to neglect all but the first or second electric multipole operators ie. E1 or E2 transitions. The cross-section for excitation of the projectile by one of these multipoles is then dependant on two factors. One, the reduced transition probability, describes how likely it is for the projectile to be excited by a specific multipole transition to a specific excitation state and contains the nuclear structure information. The other is related to the strength of the Coulomb multipole field between the projectile and the target. This is known as the standard Coulomb orbital function.

A softer collision, with the projectile being scattered to more forward angles, is less likely to excite as high excitation states as a more abrupt collision where the projectile is scattered to larger angles. In practice Coulomb excitation is rather weak for excitation states above $\sim 4 \mathrm{MeV}$. 
As has been noted in the experimental review, Shotter et al. have applied the Coulomb excitation approach to the direct break-up mode of ${ }^{7} \mathrm{Li}$ from ${ }^{120} \mathrm{Sn}$ and ${ }^{208} \mathrm{~Pb}$ targets at $70 \mathrm{MeV}$. They use $\alpha+\mathrm{t}$ fusion data to calculate the reduced transition probability for ${ }^{7} \mathrm{Li} \rightarrow \alpha+\mathrm{t}$ along with standard Coulomb orbital functions to produce angular distributions for Coulomb excitation of the direct break-up channel. A good fit to the magnitude of the forward angle experimental data was achieved along with an excellent agreement with the shape of the fragment energy spectra arising from this reaction mode. At scattering angles beyond grazing the calculation overpredicts the experimental data and this was attributed to the increased influence of nuclear interactions. The theoretical simplicity of these calculations makes them rather appealing for explaining break-up data in the angular regions not contaminated by the less well known nuclear interaction.

\subsubsection{Born Approximation Techniques}

The Serber model provided an intuitive method for calculation of the inclusive energy distributions but to achieve a more rigorous theoretical approach with more detailed predictive powers a full quantum mechanical approach is necessary. These are normally impossible to solve exactly but certain approximations can be made to make the calculations tractable. The main idea behind the Born Approximation approach is to expand the full wave function of the system in terms of the potential causing the scattering. For a system with a hamiltonian of the form:

$$
H=H_{\circ}+V
$$

it can be shown that the full solution $\Psi$ of $H$ can be written as [Ja70]:

$$
\left|\Psi^{ \pm}>=\right| \Phi>+\frac{1}{E-H_{\circ} \pm i \epsilon} V\left|\Psi^{ \pm}\right\rangle
$$

This is known as the Lipmann-Schwinger equation of the full hamiltonian and is exactly equivalent to the Schrodinger equation of the system written in a different form. The wave function $|\Phi\rangle$ is a solution of the operator $H_{0}$. The plus and minus choice is made to satisfy the boundary conditions of outgoing or incoming solutions respectively. This can be further expanded into the Born 
Series by replacing the $\left|\Psi^{ \pm}\right\rangle$on the right hand side by the full right hand side expansion for $\left|\Psi^{ \pm}\right\rangle$. This leads to the infinite series:

$$
\begin{aligned}
\left|\Psi^{ \pm}\right\rangle=\mid \Phi> & +\frac{1}{E-H_{\circ} \pm i \epsilon} V \mid \Phi> \\
& +\frac{1}{E-H_{\circ} \pm i \epsilon} V \frac{1}{E-H_{\circ} \pm i \epsilon} V \mid \Phi>+\ldots \ldots
\end{aligned}
$$

The different Born Approximation methods vary by termination of this series to different powers of the potential operator V. The plane wave Born Approximation (P.W.B.A.) uses only the first term in this expansion with the Hamiltonian divided such that $\mathrm{H}_{\circ}$ represents the kinetic energy operator series of the free particles and $\mathrm{V}$ is the remainder of the full Hamiltonian. It can be seen from equ.1.3 that this will be valid when the other terms in the expansion are small or in other words the distortion due to the potential $\mathrm{V}$ is small. The solution of $\mathrm{H}_{\circ}$ is then a plane wave, hence the approximation's name. This method is dealt with in detail in Section 5.2 so will not be explained further here.

The distorted wave Born Approximation (D.W.B.A.) includes the effect of the second term in the series. This will be a more realistic approximation but increases the complexity of the evaluation of the corresponding wave function.

There are two different types of D.W.B.A. calculations currently being applied to explain different experimental data. They differ mainly in the way they decompose the Hamiltonian of the system. This will be briefly explained in the following example. If one considers the system of projectile a (composed of fragments $b$ and $x$ ) incident on target $A$, the full Hamiltonian of the system can be written as

$$
H=T_{A}+T_{b}+T_{x}+V_{x A}+V_{b A}+V_{b x}
$$

where $T_{i}$ is the kinetic energy operator of the $i^{t h}$ particle and $V_{i j}$ is the potential acting between particles $\mathrm{i}$ and $\mathrm{j}$ (eg. $\mathrm{V}_{b x}$ is the potential binding $\mathrm{b}$ to $\mathrm{x}$ to form $a$ in the incoming channel). One can then divide the Hamiltonian in two ways:

$$
\begin{aligned}
\mathrm{H}^{\text {prior }} & =\underbrace{T_{k i n}+V_{b x}+V_{a A}}_{H_{i}}+\underbrace{V_{x A}+V_{b A}-V_{a A}}_{V_{i}} \\
H^{\text {post }} & =\underbrace{T_{k i n}+V_{b A}+V_{x A}}_{H_{f}}+\underbrace{V_{b x}}_{V_{f}}
\end{aligned}
$$


Using these decompositions of the full Hamiltonian it is possible to write exact expressions for the corresponding $\mathrm{T}$ matrices as:

$$
\begin{aligned}
\mathrm{T}^{\text {prior }} & =<\Psi_{f}^{-}\left|V_{i}\right| \chi_{i}^{+}>=<\Psi_{f}^{-}\left|V_{x A}+V_{b A}-V_{a A}\right| \chi_{i}^{+}> \\
T^{\text {post }} & =<\chi_{f}^{-}\left|V_{f}\right| \Psi_{i}^{+}>=\left\langle\chi_{f}^{-}\left|V_{b x}\right| \Psi_{i}^{+}\right\rangle
\end{aligned}
$$

In this exact representation the $\Psi_{i, f}^{ \pm}$are the full solutions of $H$ with the correct boundary conditions. The wave functions $\mid \chi_{i}^{+}>$and $\left\langle\chi_{f}^{-}\right|$are distorted solutions of the decomposed Hamiltonians $\mathrm{H}_{i}$ and $\mathrm{H}_{f}$ respectively, ie. they are calculated to first order in the interactions $\mathrm{V}_{b x}+\mathrm{V}_{a A}$ and $\mathrm{V}_{x A}+\mathrm{V}_{b A}$ respectively, see equ.1.3. In practice these distorted wave functions are obtained from the solution of elastic scattering of $a$ on $A$ or $b$ and $x$ on $A$, eg. for the (d,np) reaction the solution $\chi_{i}$ is the wave function obtained from elastic deuteron scattering whilst $\chi_{f}$ is the product of elastic proton and elastic neutron scattering wave functions at the correct energy.

The approximation to these exact solutions is then in the choice of wave function used to represent the $\Psi_{i}$ and $\Psi_{f}$. In the post form approximation the initial wavefunction $\Psi_{i}$ is replaced by $\chi_{i}$ and in the prior form the final wave function $\Psi_{f}$ is replaced by $\chi_{f}$. It is thus seen that in the post form the approximation to the exact solution enters via the initial channel and in the prior form the approximation enters via the final channel. This gives the following for the $\mathrm{T}$ matrix elements in the different approximations.

$$
\begin{aligned}
& T_{D W B A}^{\text {prior }}=\left\langle\chi_{f}^{-}\left|V_{x A}+V_{b A}-V_{a A}\right| \chi_{i}^{+}\right\rangle \\
& T_{D W B A}^{\text {post }}=\left\langle\chi_{f}^{-}\left|V_{b x}\right| \chi_{i}^{+}\right\rangle
\end{aligned}
$$

If one replaces $\mathrm{H}_{\circ}$ and $\mathrm{V}$ in equ.1.2 by $\mathrm{H}_{i}$ and $\mathrm{V}_{i}$ or $\mathrm{H}_{f}$ and $\mathrm{V}_{f}$ it becomes clear when these different approximations are appropriate. The prior approximation ie. replacing $\Psi_{f}$ by $\chi_{f}$, is valid when there is no strong interaction between the outgoing fragments and $\mathrm{V}_{b x}$ can be ignored. This tends to be the case for direct break-up reactions where the interaction between fragments in the final channel is negligible. In the post form approximation the potential being neglected is the $V_{x A}+V_{b A}-V_{a A}$ term. This method is more appropriate when inelastic processes exciting the projectile are weak since these are proportional to the differential forces acting on the projectile clusters ie. $\mathrm{V}_{i}$. 
Once the best $\mathrm{T}$ matrix for the particular problem has been chosen there still remains the problem of calculating the solution. This generally involves a six dimensional integral. This can rarely be done analytically and normally requires further simplifying approximations. One common simplifying approximation is the zero-range approximation which replaces the integral over the internal coordinates of the projectile with a delta function reducing the problem to three dimensions. This has the problem of producing an infinitely wide momentum distribution for the relative momentum between the projectile fragments. A correction term is normally added to take account of this unphysical aspect of the zero-range approximation, but for heavier projectiles a full finite range calculation must be performed. A correction term is also included to account for the non-local nature of the actual nuclear potential since only local potentials are used in the construction of the wave functions and the potentials $V_{i}$ and $V_{f}$. The post form approximation has an added advantage over the prior form in that the interaction potential $V_{b x}$ has a relatively short range compared to $V_{x A}+V_{b A}-$ $V_{a A}$ and the numerical calculations are consequently much faster. Bauer and Trautman have applied such a post-form D.W.B.A. formation to deuteron breakup below and above the Coulomb barrier [Ba76] and have achieved good fits to the experimental data. For heavier projectiles [Ba84] these authors concluded that the zero-range approximation breaks down and full finite range calculations are necessary. The inclusion of Coulomb distortion effects also complicate the calculations.

One disadvantage of the D.W.B.A. approaches is that they cannot distinguish between different inelastic channels but calculate the total inelastic cross-section from the unitarity of the scattering matrix and the elastic scattering crosssections previously mentioned. Neumann et al [Ne82] have also applied the D.W.B.A. approach to ${ }^{6} \mathrm{Li}$ inclusive data and achieved good fits to the energy spectra but failed to fit the angular distributions. This was attributed to finite range effects and use of optical model potentials derived from elastic scattering which is strongly influenced by the break-up process itself.

Thompson and Nagarajan [Th83] have performed a prior form D.W.B.A. calculation to model the direct elastic break-up of ${ }^{7} \mathrm{Li}$ on ${ }^{208} \mathrm{~Pb}$ using a cluster model for the $\alpha+t$ internal wave function of the ${ }^{7} \mathrm{Li}$. Calculations with the nuclear 
potential only and the nuclear + Coulomb potential both over-predicted the experimental data by factors of $20-40$. This was taken as andication that the ${ }^{7} \mathrm{Li}$ projectile tends to recover from the forces trying to break it up and that a calculation involving a more realistic three body model with coupling between elastic and break-up channels was required.

\subsubsection{High Energy Adiabatic Method}

In the high energy adiabatic method first outlined by Johnson and Soper [Jo70] the total wave function of the system is expressed as the product of the projectile internal wave function $\phi(\underline{\mathbf{r}})$ and a projectile target wave function $\chi(\underline{\mathbf{R}}, \underline{\mathbf{r}})$ ie.

$$
\Psi(\underline{\mathbf{R}}, \underline{\mathbf{r}})=\phi(\underline{\mathbf{r}}) \chi(\underline{\mathbf{R}}, \underline{\mathbf{r}})
$$

with $\underline{\mathbf{R}}$ the separation between target and projectile and $\underline{\mathbf{r}}$ the internal coordinate between the fragments in the projectile. With this decompostion the potential operator describing the relative motion of the clusters within the projectile can be replaced by the corresponding eigenvalue of the internal wavefunction $\phi(\underline{\mathbf{r}})$. For different excited states of the projectile the wavefunctions $\phi$ and the eigenvalues will be different but in the high energy adiabatic approximation both are replaced by their ground state values. The eigenvalue of the potential operator is then the binding energy of the projectile. This approximation is valid in situations where the excitation of the projectile is small compared to the projectile's kinetic energy. This criterion is fulfilled with loosely bound, cluster like nuclei such as ${ }^{7} \mathrm{Li}$ and Thompson and Nagarajan [Na82] have used this approach in an attempt to explain the elastic scattering of ${ }^{7} \mathrm{Li}$ from ${ }^{40} \mathrm{Ca}$ and ${ }^{48} \mathrm{Ca}$. They point out that in their approach finite range effects are implicitly included in the calculation so it should be a more realistic model for heavier projectiles than the D.W.B.A. method. They have also extended their calculations to the direct break-up of ${ }^{7} \mathrm{Li}$ from a ${ }^{208} \mathrm{~Pb}$ target in an attempt to explain the data of Shotter et.al. [Sh81] [Th83]. They achieved a good fit to the experimental data when only the nuclear potential was used but when the Coulomb potential was included they overpredicted the cross-section by a factor of three. This was attributed to the improper representation of the Coulomb polarising force on 
the clusters which tends to distort the incoming projectile. The adiabatic approximation is equivalent to assuming that the nuclear volume of the projectile stays constant over the interaction which will not be the case if the Coulomb polarising potentials are present.

\subsubsection{Continuum Discretised Coupled Channels}

In the Continuum Discretised Couple Channels method [Ra75] [Sa86] the total wave function of the colliding system is expanded in terms of the eigenfunctions of the internal states of the projectile $\psi_{i}$ and those of the target $\phi_{j}$ as:

$$
\Psi=\sum_{i, j} \psi_{i} \phi_{j} \chi_{i j}(\underline{\mathbf{R}})
$$

where $\chi_{i j}$ describes the relative motion between the projectile in state $\mathrm{i}$ and the target in state $\mathrm{j}$. For weakly bound projectiles such as ${ }^{6} \mathrm{Li},{ }^{7} \mathrm{Li}$ and ${ }^{9} \mathrm{Be}$ the continuum states corresponding to 'free' projectile clusters are incorporated into the expression for $\psi$ along with the wave functions for the ground and bound excited states of the projectile. The inclusion of these continuum states in the internal wave function of the projectilecausesdifficulties in evaluation of the $\psi$ due to the continuous nature of the solutions to the internal Schrodinger equation. To circumvent this problem the continuum part of the projectile internal wave function is divided into bins in relative momentum space. The average of the wave function over each individual bin is then used within a framework of a coupled channels calculation to calculate the scattering cross-section for excitation of both resonance states in the continuum (sequential decay) and non-resonant solutions in the continuum (direct break-up). The scattering potential used to couple the different states of the projectile was calculated by folding an effective nucleon-nucleon interaction potential between the internal states of the colliding nuclei.

As has been mentioned in the experimental review, C.D.C.C. calculations using this method have been extremely successful in explaining the direct and sequential break-up of the ${ }^{7} \mathrm{Li}$ projectile. Inclusion of both nuclear and Coulomb components in the interaction potential with a ${ }^{120} \mathrm{Sn}$ target improved the phase of the theoretical and experimental oscillations at forward angles over that achieved 
with the nuclear potential alone. Unfortunately however, the inclusion of the Coulomb potential resulted in an overprediction for the absolute magnitude of the cross-section of $\sim \times 3$. This was attributed to the omission of coupling to the non-resonant direct break-up channel which was not included due to computational difficulties.

The C.D.C.C. method has also been applied to elastic scattering of other cluster like nuclei such as ${ }^{6} \mathrm{Li}$ and ${ }^{12} \mathrm{C}$. The results have been very successful in describing the quantative and qualitative behaviour of the elastic scattering cross-section for a range of target nuclei.

There are however some outstanding problems such as the non-negligible direct break-up component predicted for ${ }^{7} \mathrm{Li}$ projectiles on a ${ }^{12} \mathrm{C}$ target. No experimental evidence is seen for this channel. The inclusion of the Coulomb interaction coupling between the direct non-resonant states also requires investigation. Even after the discretisation procedure the amount of time required for calculation of the cross-sections on the most powerful computers is rather prohibitive. The limits on relative momentum set by this problem mean that coupling to large relative momentum via multi-step processes (or final state interactions) may be impossible to model at this stage. It may require more powerful computers or more sophisticated methods for continuum discretisation before calculations for the wide angle data measured in this thesis can be performed.

\subsection{Kinematics}

This section will briefly review some of the kinematical ideas used in the identification of different reaction mechanisms. The average velocities $(\sim 0.1-0.2 \mathrm{c})$ involved in the experiment allow the use of non-relativistic kinematics. In a reaction of the form:

$$
\mathrm{a}+\mathrm{A} \longrightarrow 1+2+3
$$

(where a and $A$ are the projectile and target respectively and 1,2 and 3 refer to the outgoing fragments) there are ten unknown quantities. These are the momenta of the outgoing ejectiles and the $Q$ value for the reaction. The three 
body ground state $Q$ value is defined as

$$
\mathrm{Q}_{3}^{g s}=\mathrm{m}_{a}+\mathrm{m}_{A}-\mathrm{m}_{1}-\mathrm{m}_{2}-\mathrm{m}_{3}
$$

Conservation of momentum and energy reduces the number of parameters required to completely specify the reaction kinematics to six. Thus, measurement of the energy and direction of two of the outgoing fragments allows exact calculation of the kinematical properties of the remaining ejectile. For a coincidence measurement at fixed angles $\theta_{1}, \phi_{1}, \theta_{2}, \phi_{2}$ and $Q$ value $Q_{3}$ it can be shown [Oh65] that the energies of the two detected fragments are related by the equation:

$$
\begin{aligned}
\frac{1}{m_{3}}( & E_{1}\left(m_{1}+m_{2}\right)+E_{2}\left(m_{2}+m_{3}\right)-2\left(m_{a} m_{1} E_{a} E_{1}\right)^{\frac{1}{2}} \cos \theta_{1} \\
& \left.-2\left(m_{a} m_{2} E_{a} E_{2}\right)^{\frac{1}{2}} \cos \theta_{2}+2\left(m_{1} m_{2} E_{1} E_{2}\right)^{\frac{1}{2}} \cos \theta_{12}\right) \\
& =\mathrm{Q}_{3}+E_{a}\left(1-\frac{m_{a}}{m_{3}}\right)
\end{aligned}
$$

where:

$$
\cos \theta_{1-2}=\cos \theta_{1} \cos \theta_{2}+\sin \theta_{1} \sin \theta_{2} \cos \left(\phi_{1}-\phi_{2}\right)
$$

This equation describes a closed curve in a two dimensional plot of $E_{1}$ versus $E_{2}$. Such a plot is extremely useful in identifying events which have different final state $Q$ values either through excitation of the fragments or more usually, excitation of the target recoil. Events of this type lie on a different locus from the quasi-elastic events where all the outgoing fragments are in their ground states. This type of two dimensional plot also allows identification of events due to contamination of the target. This is shown in fig.1.2 which shows a coincident alpha versus triton energy scatterplot from the ${ }^{12} \mathrm{C}$ target used in the experiment. Lines corresponding to equ.1.10 are shown for quasi-elastic events $\left(Q_{3}^{g . s}=-2.47 \mathrm{MeV}\right)$ and events leaving the ${ }^{12} \mathrm{C}$ in its $4.44 \mathrm{MeV}$ excited state $\left(Q_{3}=-6.91 \mathrm{MeV}\right)$. The data shown were summed together from neighbouring elements of the strip detector telescope and exhibit significant kinematic broadening on the ground state locus. The dotted line encloses the region specified by equ.1.10 for the reaction ${ }^{1} \mathrm{H}\left({ }^{7} \mathrm{Li}, \alpha+\mathrm{t}\right)^{1} \mathrm{H}$ and shows the contamination from hydrogen present on the surface of the ${ }^{12} \mathrm{C}$ target (see section 3.9) By placing windows on such a two dimensional plot it is possible to select out events with specific target excitation and discriminate against unwanted events such as target contamination. 


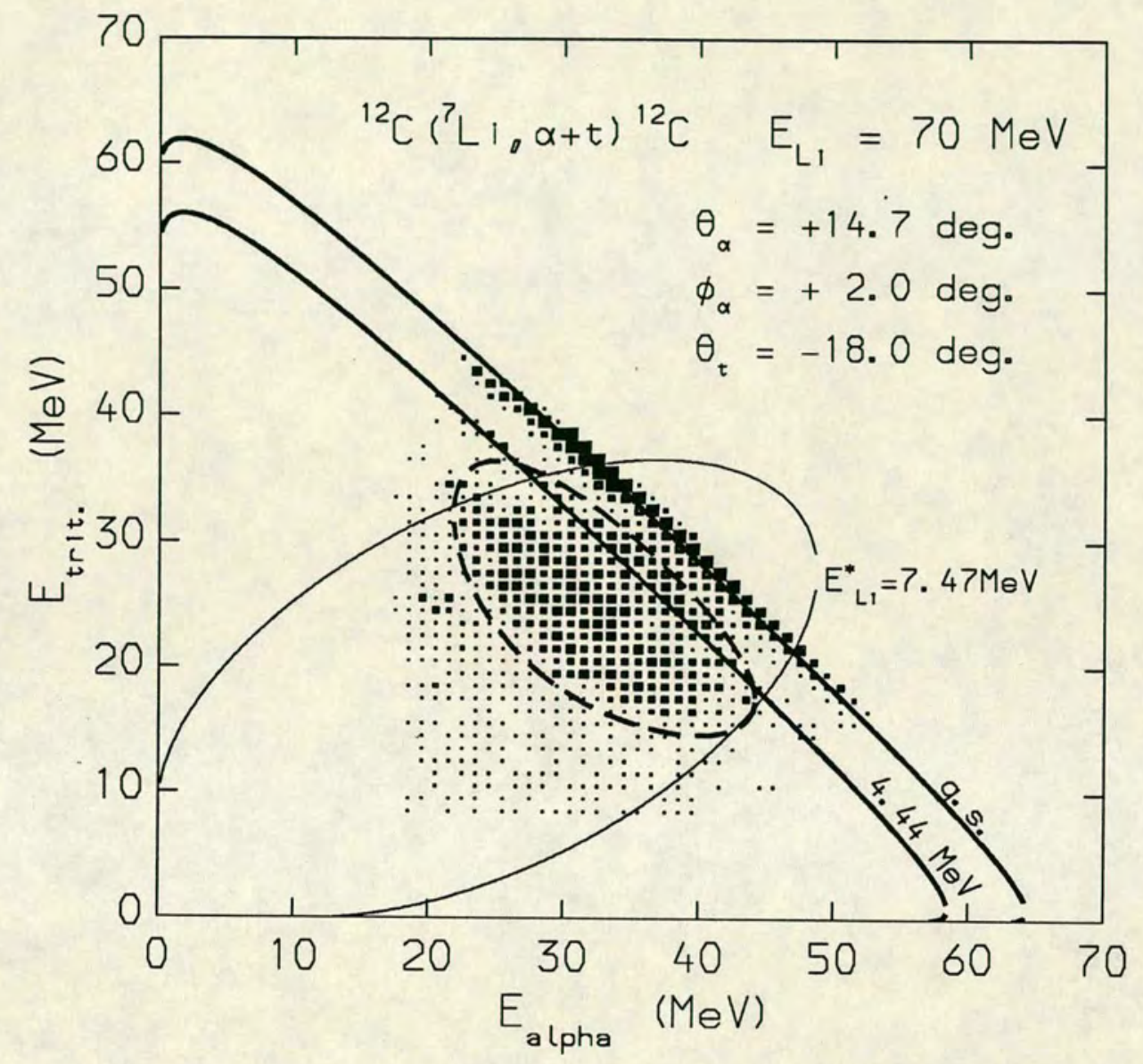

Figure 1.2: Two dimensional plot showing kinematical solutions for different $Q$ value events (see equ.1.10). Thick lines are for events leaving the ${ }^{12} \mathrm{C}$ target in its ground and first excited states. The thin elliptical line is for events with a fixed value of relative energy corresponding to break-up from the ${ }^{7} \mathrm{Li}_{7.47 \mathrm{MeV}}$ state. The dotted ellipse is the kinematical limit for break-up from the hydrogen contamination on the target surface. 


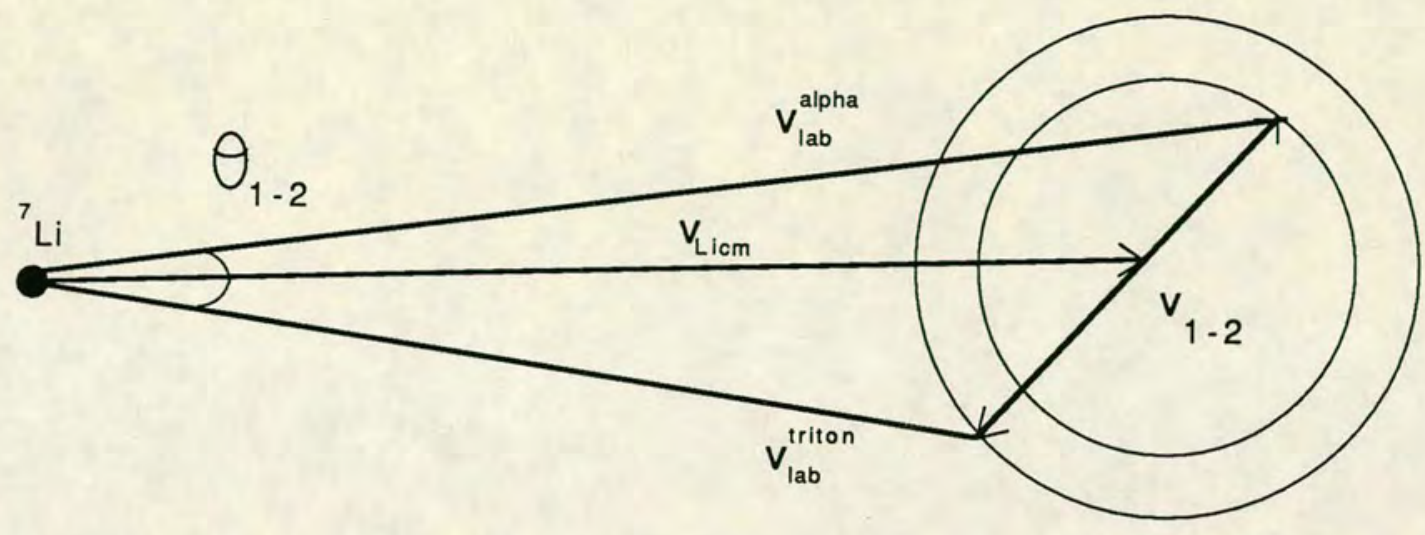

Figure 1.3: Velocity vector diagram for break-up event.

One of the most useful features of this type of data presentation is related to the identification of sequential decay. In this type of reaction mechanism the outgoing fragments have a fixed relative energy $\varepsilon$, for break-up from a specific state in the composite ejectile. This relative energy is defined as:

$$
\varepsilon_{1-2}=\frac{1}{2} \mu_{1-2} v_{1-2}^{2}=\frac{1}{m_{1}+m_{2}}\left(m_{2} E_{1}+m_{1} E_{2}-2 m_{1} m_{2} E_{1} E_{2}\right)^{\frac{1}{2}} \cos \theta_{1-2}
$$

where $\theta_{1-2}$ and $v_{1-2}$ are defined in fig.1.3. The thin elliptical locus shown in fig.1.2 plots the points of constant $\varepsilon$ for a fixed $\theta_{1-2}$ corresponding to sequential break-up from the ${ }^{7} \mathrm{Li}_{7.47 \mathrm{MeV}}$ state. This indicates that events belonging to the ground state locus, originating from sequential break-up of this state will be concentrated at the two points of intersection of the two loci. Projection of this ground state locus onto either the $\mathrm{E}_{\alpha}$ or $\mathrm{E}_{t}$ axis then allows 'easy' identification of such sequential peaks [Sh84]. Events outside these peaks are kinematically excluded from originating solely from sequential break-up of this state.

In conclusion it has been illustrated in this section how it is possible to extract information about the relevant reaction processes from two dimensional, coincident particle energy plots. 


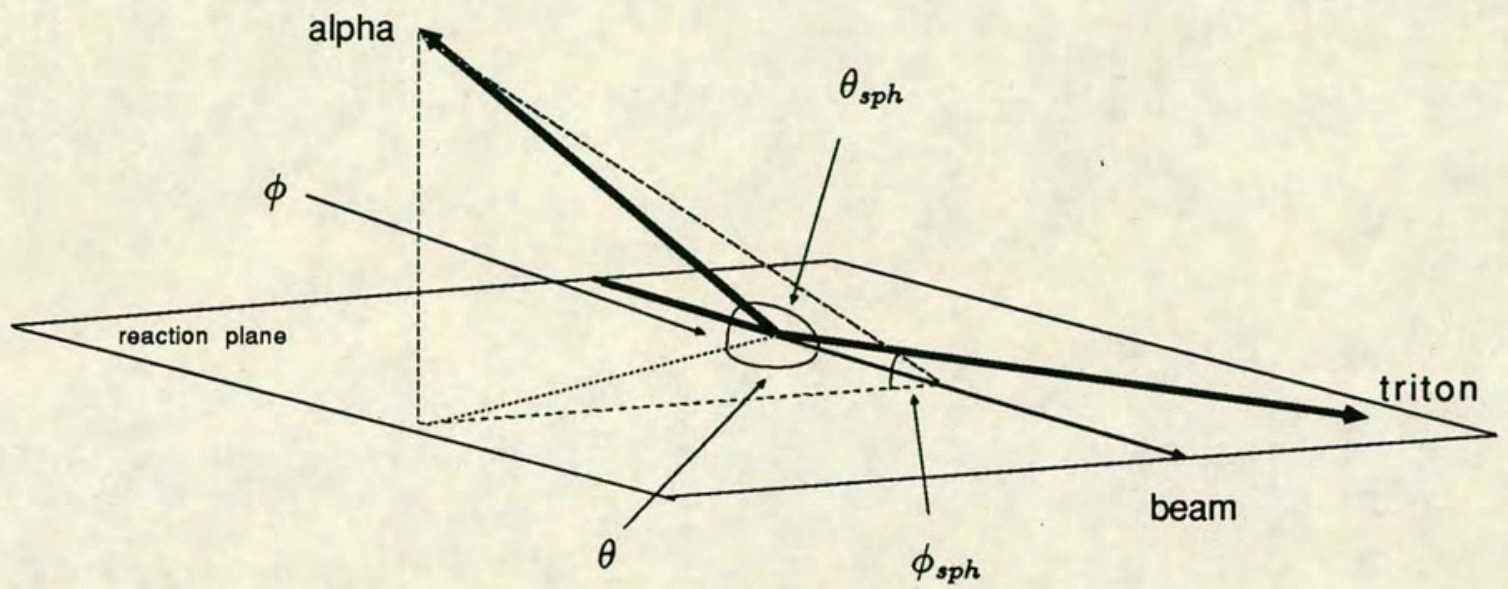

Figure 1.4: Diagram of angular definitions

\subsection{Angular Definitions}

The normal method for defining angles in nuclear reactions is to use spherical polar coordinates with the spherical azimuthal angle $\phi_{s p h}$ measured round the beam direction. The detector set-up used in this thesis however, lends itself to a different angular definition. The square detector pixels measuring the outof-plane angle are more naturally defined by polar coordinates similar to the longitude and latitude used to define positions on the earth's surface. This is shown in fig.1.4. The reaction plane is defined as the plane containing the beam and the triton detector telescopes. Unless otherwise specified, all the angles defined in this thesis will be in this system. If an angle is quoted in spherical coordinates the suffix sph. will appear against the angle symbol ie. $\theta_{s p h}$ or $\phi_{s p h}$. This is mainly used in section 4.1 and 5.3. The transformations between these two definitions are given below:

$$
\begin{aligned}
\theta_{s p h} & =\cos ^{-1}(\cos \theta \cos \phi) \\
\phi_{s p h} & =\tan ^{-1}\left(\frac{\tan \phi}{\sin \theta}\right) \\
\theta & =\tan ^{-1}\left(\tan \theta_{s p h} \cos \phi_{s p h}\right) \\
\phi & =\sin ^{-1}\left(\sin \theta_{s p h} \sin \phi_{s p h}\right)
\end{aligned}
$$




\subsection{Objectives and Outline of Thesis}

The main objective of this thesis was the measurement of the wide-angle coincidence cross-section for the $\alpha+\mathrm{t}$ break-up channel of $70 \mathrm{MeV}{ }^{7} \mathrm{Li}$ incident on the targets ${ }^{12} \mathrm{C},{ }^{120} \mathrm{Sn}$ and ${ }^{208} \mathrm{~Pb}$. The importance of the different mechanisms producing these break-up events is of intense interest at the present time. Theoretical calculations are currently being performed to model both the Coulomb interaction and the nuclear interaction between the projectile and target and the fragments and target. These hope to determine the regions where these different processes are dominant. For a more detailed understanding of the interplay between the two mechanisms it is important that experimental data is obtained over the whole scattering range. It has been proposed that final-stateinteractions (f.s.i.) between the fragments and the target are responsible for the large cross-section seen at large angular separations and it is conceivable that these may destroy any momentum correlation between the outgoing fragments. Consequently, it is important that the out-of-plane intensity distribution be measured to determine if this changes with angular separation between the fragments. The small angular separation coincidence cross-section has already been measured [ $\mathrm{Da} 87$ ] and the $\alpha+\mathrm{t}$ channel was found to be dominated by quasielastic break-up events where all the outgoing fragments were in their ground state. One objective of this thesis was to determine whether this remained true for large angular separations. One might suppose that if f.s.i. are responsible for the wide-angle cross-section, as one progresses to larger angles the inelasticity of the reaction should increase. It is even possible that these types of measurement open up the possibility of studying a smooth transition from the projectile break-up mode to the partial-fusion mode where one of the fragments is absorbed by the target.

This chapter has been a quick review of the reaction mechanisms that have been observed with similar mass projectiles and the theoretical models that are currently being applied to explain the experimental behaviour of the different break-up processes observed. Chapter 2 deals with the behaviour of a new type of charged particle detector that was used in the experiment to detect the outgoing alpha particles. These were large area silicon detectors which allowed 
out-of-plane data to be collected simultaneously with the in-plane data. Chapter 3 details the complete experimental set-up used to record the data and the procedures used to analyse it. Chapter 4 presents the inclusive and exclusive data collected during the experiment and finally chapter 5 presents a discussion of the results with a view to identifying the reaction mechanisms responsible for the wide angle coincidence data. The last section of chapter 5 is a conclusion summarising the work carried out and the results presented in the rest of the thesis. 


\section{Chapter 2}

\section{Strip Detectors}

\subsection{Introduction}

The experiment outlined in this thesis involved the use of a novel design of radiation detector, and this chapter will be a description of its construction and performance.

In recent high energy physics experiments the necessity for detecting more and more particles with better and better spatial resolution has prompted the evolution of a class of detectors which combine the advantages of solid state detectors with the improved manufacturing techniques of state of the art semi-conductor technology. These detectors have been termed STRIP DETECTORS and are essentially a set of separate detectors, usually rectangular in shape, manufactured on the same wafer of silicon. The basic design of a strip detector is the implantation of fixed regions of donor (or acceptor) atoms in a wafer of high resistivity silicon (typically $\sim 10-20 \mathrm{k} \Omega \mathrm{cm}$ ). These regions define the active area that is to be sensitive to particle irradiation. In our detectors they were rectangular strips typically $50 \mathrm{~mm} \times 2 \mathrm{~mm}$ separated by $100 \mu \mathrm{m}$. Each detector had a number of these separate strips implanted on its surface, (in our case 25 strips) with a separate output for each one.

Some of the more obvious advantages associated with strip detectors and their 
related technology are:

- improved stopping power of $\mathrm{Si}$ as compared to gas or liquid detectors.

- excellent intrinsic energy resolution.

- well controlled manufacturing process producing thin dead layers with extremely accurate position resolution $\leq 1 \mu \mathrm{m}$.

- relative ease of production of detectors of arbitrary shape and size (limited by size of available Si wafers).

- reduction of the physical space and capital cost necessary for instrumenting each of the individual strips.

- multi-hit capability.

- large surface area.

- increased counting rate over conventional detectors of comparable size.

- low leakage current 10-50 times smaller than equivalent surface barrier.

- very small spatial separation between detector elements.

These strip detectors have been used extensively [Hy83],[Hy84],[Be84],[Ho84] in a number of high energy experiments where the high spatial resolution attainable with modern semi-conductor fabrication techniques makes them especially suitable for particle tracking and vertex location.

These experiments tend to employ the strip detectors more as logic devices with their output used solely to determine the point of impact of the particle. No energy measurements are generally taken. In contrast to this application, the use of strip detectors in heavy ion experiments requires the accurate measurement of the amount of energy deposited in the detector. The novel construction of these strip detectors with closely spaced regions of active silicon, may create special problems associated with the charge collection for these energy measurements. 
To investigate this and, more generally, to become more familiar with the behaviour of these new detectors under irradiation with highly ionising particles, a series of tests were carried out.

The rest of this chapter will be a review of these tests and their results, but before this is done a review of the manufacturing processes involved in the construction of the detectors will be given in the next section.

\subsection{Construction and Manufacture}

The strip detectors used in this experiment were supplied by Micron Semiconductor Limited but tests were also performed on detectors made by Enertec Schlumberger of France.

Both types of detectors were manufactured using the 'planar process' [Ke80], [Ke82]. Fig.2.1 shows the main stages of this process. The oxide layer, grown on the high resistivity $\mathrm{n}$-type wafer, is etched to open windows for implantation of the Boron atoms used to form the $\mathrm{p}^{+}$region underneath each strip. After implantation and annealing the aluminium contacts are evaporated onto the front and the rear of the detector and the complete unit is mounted on its printed circuit board. Connections are made with $30 \mu \mathrm{m}$ thick aluminium wire, wedge bonded onto each strip. The rear of the detector is also implanted with Arsenic atoms to ensure an ohmic contact between the rear aluminium contact and the bulk of the detector. Each strip then acts as a separate detector in the same way as any conventional semiconductor detector operates under reverse bias. This Oxide Passivated Ion Implanted technology results in detectors with more repeatable characteristics, lower noise, higher interstrip resistance and fewer inactive strips.

Fig.2.2 shows four typical strip detector designs, two from Micron and two from Enertec. The detectors used in the experiment were the large area Micron detector type. The Micron design was chosen mainly because of the problems associated with the output connections to the Enertec detector. This was in 
1
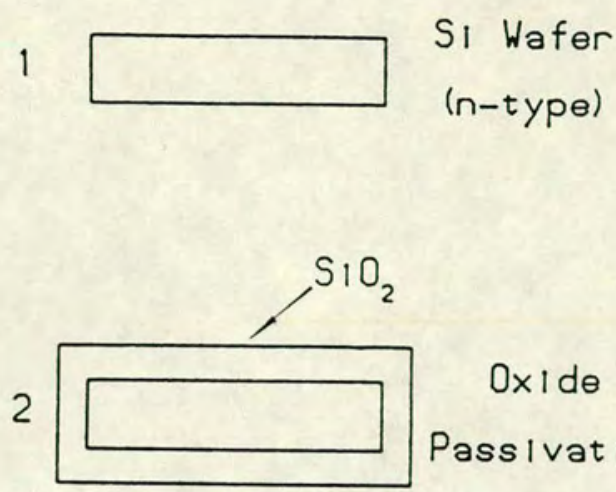

Oxide

Passivation

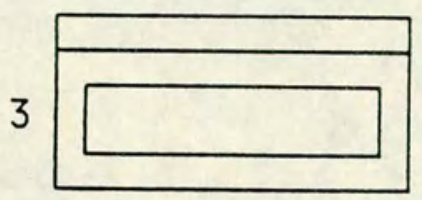

Coating with

Posltive

Photo Resist

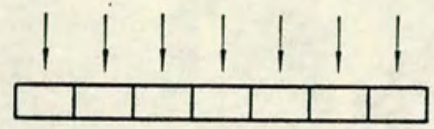

4

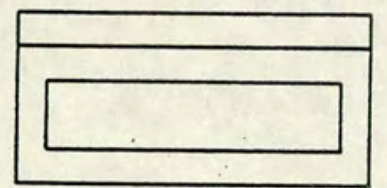

Alignment and

Exposure

5

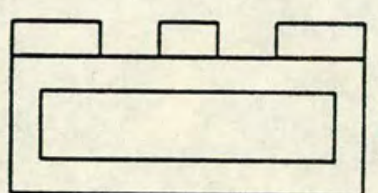

Development

11

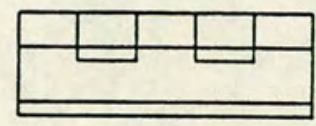

6

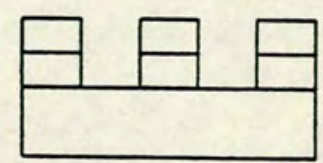

$\mathrm{SiO}_{2}$ Etching

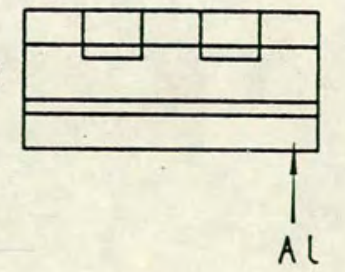

Removal of $A L$ from $0 \times 1$ de

Removal of Resist

Ion Implantation

Annealing

Al Metalization $(p-s \mid d e)$

Al Metalization (n-s|de)

Figure 2.1: Stages in the manufacture of Ion Implanted, Oxide Passivated, Silicon Strip Detectors. 


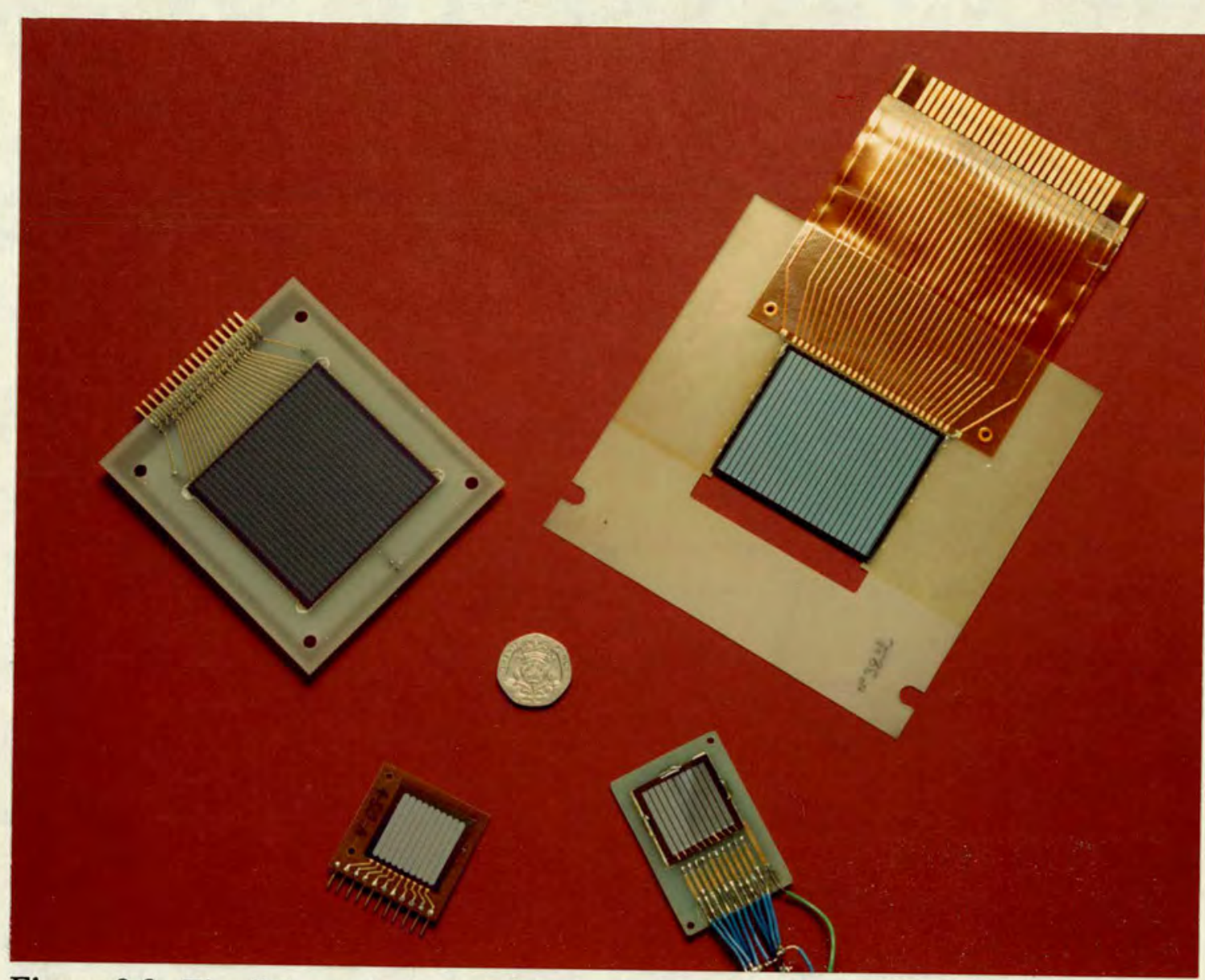

Figure 2.2: The different designs of detector tested. The large and small area detectors on the left were made by Micron, the ones on the right by Enertec

the form of a flexible plastic strip which unfortunately had a habit of becoming bent close to the detector mount. This caused fatigue in the gold output tracks which frequently resulted in failure of the strip contacts. If this problem could be overcome, by mounting the Enertec detectors on more robust mounts with better output connections, then they would be preferable to the Micron design. This judgement is made on the basis that the Enertec wafers were lapped on both sides before the detectors were made, making their thickness more uniform over the surface. This improves their particle identification properties and simplifies the energy calibration (see section 3.3).

\subsection{General Behaviour and Properties}

The tests performed on the detectors were to achieve the following aims: 
- investigate the detectors general response to highly ionising particles.

- measure the full depletion voltage of the detector.

- measure the energy resolution of each strip at this voltage.

- check that the full length of each strip was active.

- test for any charge sharing effects between adjacent strips.

- test the stability of the detectors.

To achieve these aims a beam of $5.48 \mathrm{MeV}$ alpha particles from an ${ }^{241} \mathrm{Am}$ source was collimated onto the surface of the detector being tested. In general the strip detectors behaved like any typical surface barrier or ion implanted semiconductor detector under ionising radiation, with the output signal being related to the amount of energy deposited in the depletion layer. The rest of this section will deal with how these measurements were made.

\subsubsection{Full Depletion Voltage}

To determine the full depletion voltage of each detector the peak position of the ${ }^{241} \mathrm{Am}$ alpha particles collimated onto the strip side was measured with increasing bias voltage. The alphas were then collimated onto the back of the detector and the process repeated. Fig.2.3 shows the results for a typical strip on an Enertec large area detector. It can be seen that this detector requires a voltage of $\sim-70 \mathrm{~V}$ to become completely depleted. The slight difference in peak height between front and back of $\sim 0.5 \%$ is due to the different thicknesses of the dead layers on the front and back of the detector. The Enertec detectors tended to deplete evenly over the length of a strip whereas the Micron detectors required a substantial ( $\sim 20 \%)$ overbias compared to the manufacturers recommendation, to fully deplete the strips at their centre. This is illustrated in fig.2.4 which shows the energy spectrum for alpha particles incident on the front and back of a strip on a Micron detector. Fig.2.4(b)-(d) shows the energy spectrum from alphas collimated on the edges and the centre of the back of the detector at the recommended bias voltage. It is clear that incomplete charge collection 


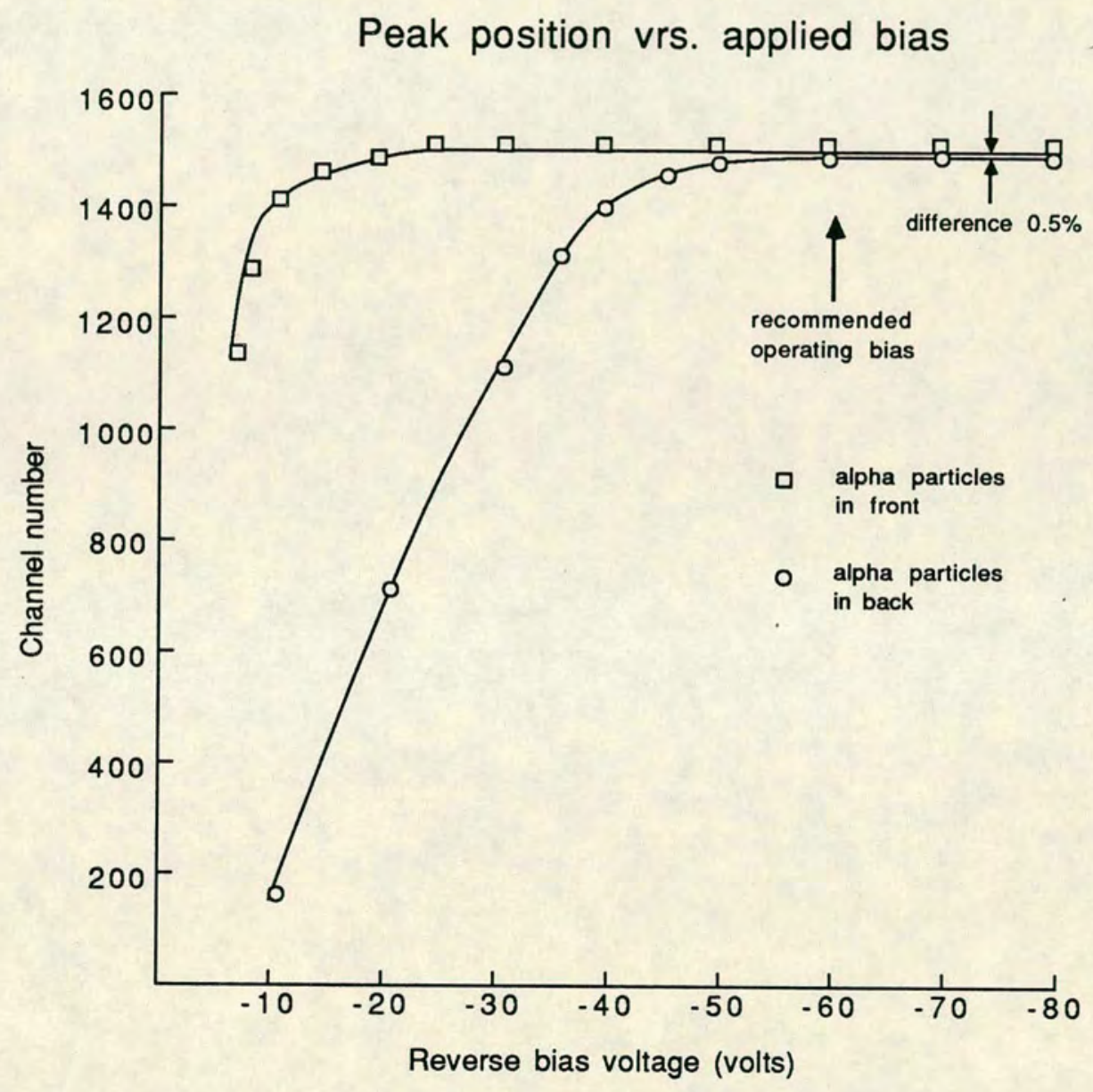

Figure 2.3: Peak position vrs. bias voltage for a typical strip with alpha particles incident on the front and back of a $300 \mu \mathrm{m}$ thick Enertec large area detector. 
(a)

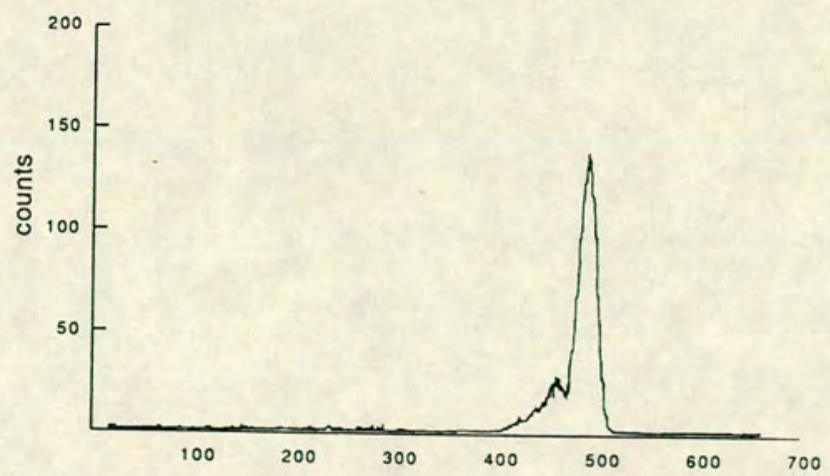

(b)

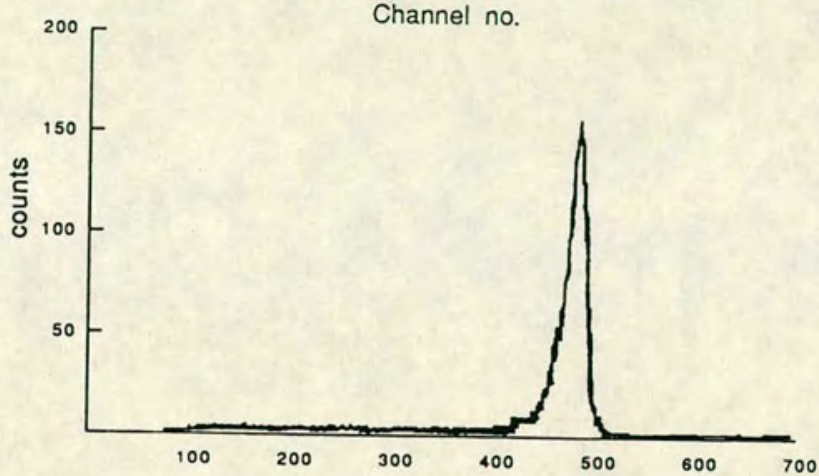

(c)

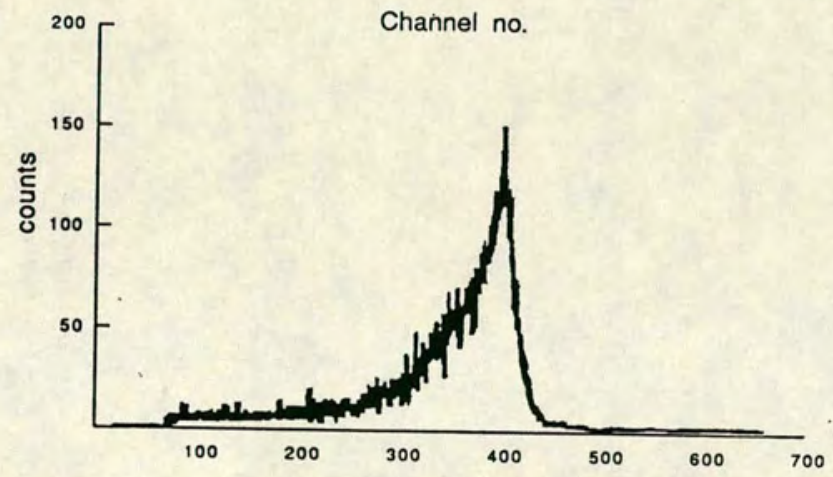

(d)

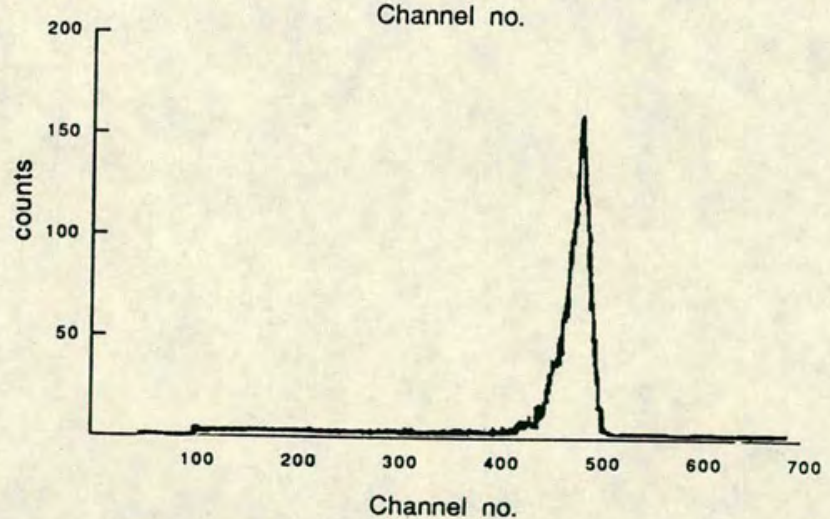

Figure 2.4: Energy spectrum from ${ }^{241} \mathrm{Am}$ alpha particles incident on a large area Micron detector.(a) alphas incident on front of strip, (b) alphas collimated onto contact edge of rear of strip, (c) alphas collimated onto centre of rear of strip, (d) alphas collimated onto bottom edge of rear of strip. All measurements taken at recommended full depletion bias voltage. 
is occuring at the centre of the strip. At higher bias voltages all the alpha particles in the back gave the same energy signal which was $\sim 2.0 \%$ lower than those in the front (again because of increased dead layer at the rear). This difference in depletion behaviour may have been due to varying thickness along the strip length but it was observed with a number of Micron detectors. This points to some other cause. Although this was of minor importance in the applications in this thesis it may become important for applications where high energy resolution is essential since an increased bias voltage results in an increase in leakage current and degradation of energy resolution.

\subsubsection{Energy Resolution}

Whilst the depletion of voltage measurements were being taken the energy resolution of the ${ }^{241} \mathrm{Am}$ alpha peak was also measured. The typical energy resolution of the $5.48 \mathrm{MeV}$ peak at full depletion was $20-25 \mathrm{keV}$ fwhm for the large area Enertec detectors and $20-40 \mathrm{keV}$ for the large area Micron detectors. Different Micron detectors showed much larger variations around this typical value than the Enertec detectors. The small area Enertec detectors had a typical resolution of 15-17 keV fwhm whilst the small area Micron detectors were slightly better with resolutions of $\sim 15 \mathrm{keV}$ fwhm. Fig. 2.5 shows a plot of the fwhm energy resolution across the face of a 'good' large area Micron detector with its strips connected in groups of two (the remaining five strips were left at a floating potential). The leakage current at $-50 \mathrm{~V}$ bias voltage for each strip group is also shown. These were measured with each group individually biased up and the rest left at a floating potential. It is clear that for this detector the energy resolution, in general, follows the leakage current distribution across the detector surface. Some of the large area Micron detectors however, exhibited a rather unfortunate energy resolution degradation with increasing bias voltage. This was present in strips 9 and 10 of the detector used in fig.2.5. Fig.2.6 shows the energy resolution versus bias voltage for this strip group. It can be seen that the energy resolution reaches an optimum at a bias of $\sim-25 \mathrm{~V}$, well below that required for full depletion $(\sim-70 \mathrm{~V})$. This degradation in the energy resolution was traced to an unusual type of low frequency break down. This was distinguished from the normal type of microplasma breakdown by its characteristic 


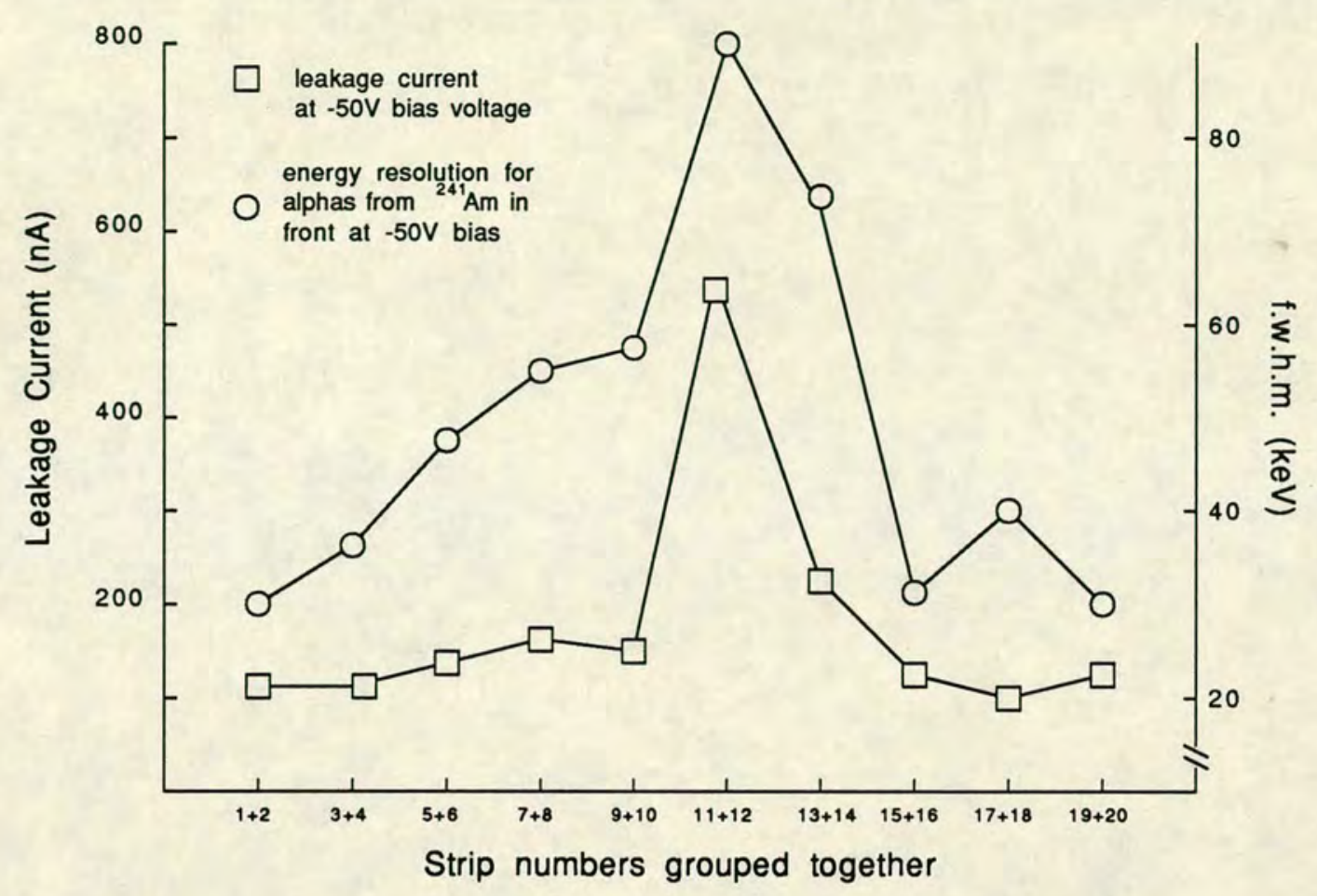

Figure 2.5: Energy resolution for ${ }^{241} \mathrm{Am}$ alphas across the surface of a large area Micron detector. The leakage current of each strip group is also shown.

time scale of milliseconds. The interstrip resistance between the strip exhibiting this behaviour and its neighbouring strips also showed an unusual diode effect with the resistance measured towards the offending strip markedly smaller than when measured with the measuring bias in the opposite direction. This property may be used as a quick test for this behaviour. More recent detectors however, seem to be less susceptible to this problem. The detectors chosen for this experiment were not affected by this behaviour to any significant degree.

Finally, during the energy resolution measurements, it was found that grouping strips together to form larger elements on the same detector degraded the energy resolution. This was expected due to the increased capacitance and leakage current from the group as a whole. This degradation had the general approximate behaviour of adding the individual resolutions in quadrature except when the low frequency breakdown was present. If any of the strips exhibited this breakdown then the resolution of the group as a whole was much worse than expected. 


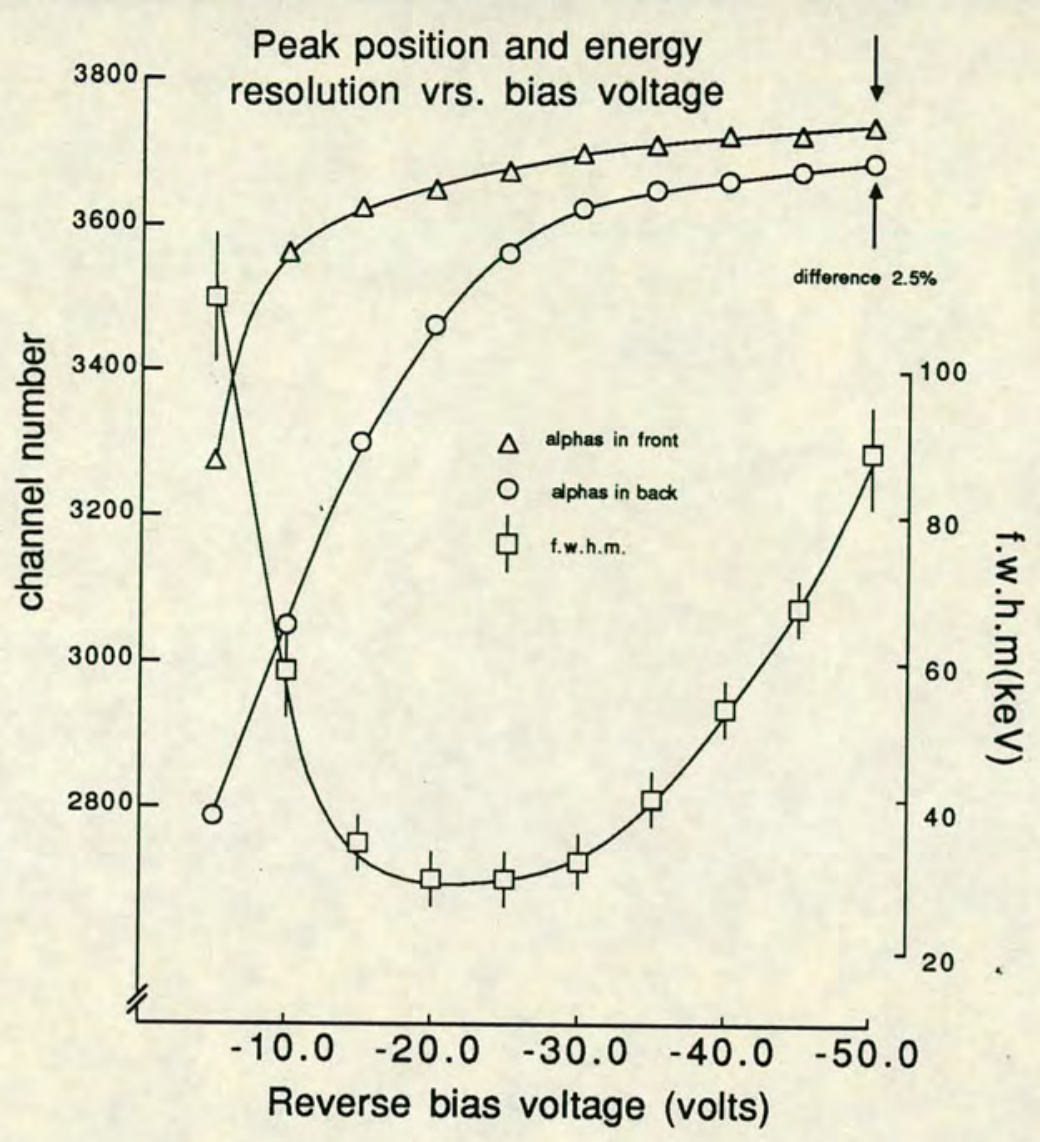

Figure 2.6: Energy resolution vrs. bias voltage for the strip group $9+10$ used in fig.2.5. This group exhibits the low frequency breakdown behaviour mentioned in the text.

\subsubsection{Continuity of Strip Output and Detection Efficiency}

Tests on similar ion-implanted detectors at CERN [He80],[Bo81] have shown that they are $100 \%$ efficient in detecting minimum ionising particles i.e. there are no dead regions between or underneath the strips. This was verified by shining an uncollimated ${ }^{241} \mathrm{Am}$ source onto the surface of the detectors and integrating the number of counts from each strip collected over a fixed time interval. The count rate across the detector face followed that which would be expected from a consideration of the geometry of this set-up. This indicated that none of the detectors had substantial dead regions underneath any of their strips. It did not however, check for any dead regions between strips and this will be discussed more in section 2.4 . 


\subsubsection{Stability of Leakage Current}

One of the main advantages of the ion-implanted planar process over the other manufacturing techniques (in particular, surface barrier techniques) is the extremely low reverse bias leakage currents achieved by the planar method. In surface barrier technology the leakage current is dominated by the surface leakage but in the planar process this component is reduced by surface passivation. Consequently the bulk generation current is the main contributor to the leakage. This means that it is important to keep a long minority carrier lifetime to ensure good charge collection, low leakage currents and optimum energy resolution. Although the planar process involves high temperatures $\left(1030^{\circ} \mathrm{C}\right.$ for oxidation and $200^{\circ} \mathrm{C}$ for annealing) the expected reduction in minority carrier lifetime and related increase in leakage current is, surprisingly, much smaller than predicted $[\mathrm{Ke} 80]$. On the contrary it has been shown that the annealing may actually increase the minority carrier lifetime over that of the starting material. This has been attributed to gettering effects reducing the number of recombination generation centres [Ke82]. The resulting detectors have leakage currents at least an order of magnitude better than equivalent surface barrier detectors. Fig.2.7 shows a typical leakage versus bias current for one strip on the Enertec large area detector at room temperature. It can be seen that the strip has a leakage current of $\sim 25 \mathrm{nA}$ at full depletion voltage. Fig.2.8 shows a leakage versus bias curve for two separate strips from a large area Micron detector, again at room temperature. Curve (a) has a similar bias dependance to the Enertec strip. Curve (b) clearly has a different behaviour to that of the Enertec detector with no evidence of the plateau region around full depletion. This difference was attributed to the presence of the low frequency breakdown component mentioned previously. The leakage current expected for the full detector could be approximately calculated by adding all the individual strips leakages together. Fig.2.9 shows the total measured leakage as a function of the number of strips connected compared to that expected from addition of the individual strip leakages. These measurements were made in air at room temperature.

The long term stability of the leakage current is also important in applications where the detector is to be used over a number of days. Fig.2.10 is the time dependance for both a large area Enertec detector and a large area Micron de- 


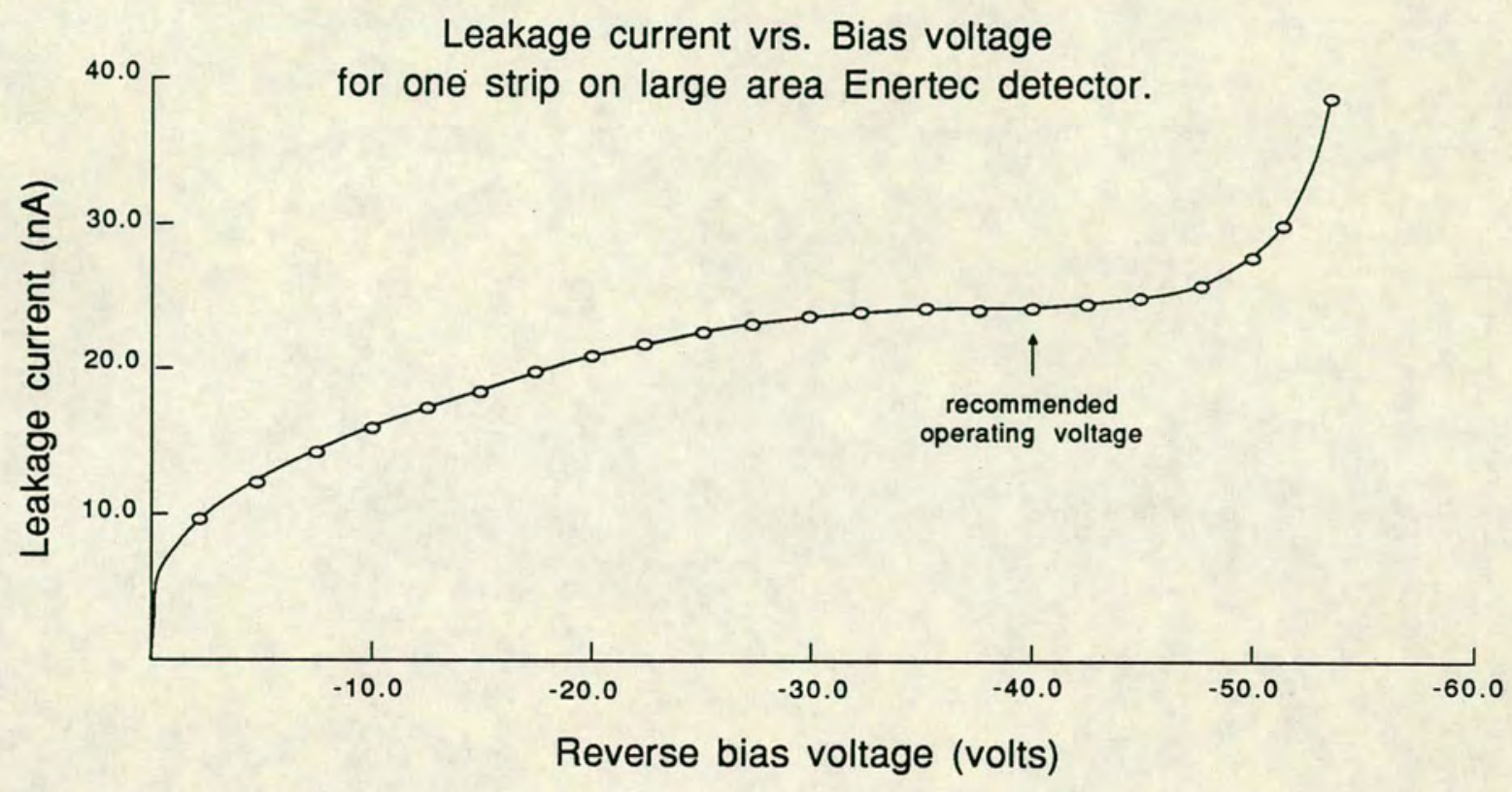

Figure 2.7: Leakage current vrs. bias for a single strip on a large area Enertec detector.

Leakage vrs. Bias voltage for a good strip and a bad strip on a

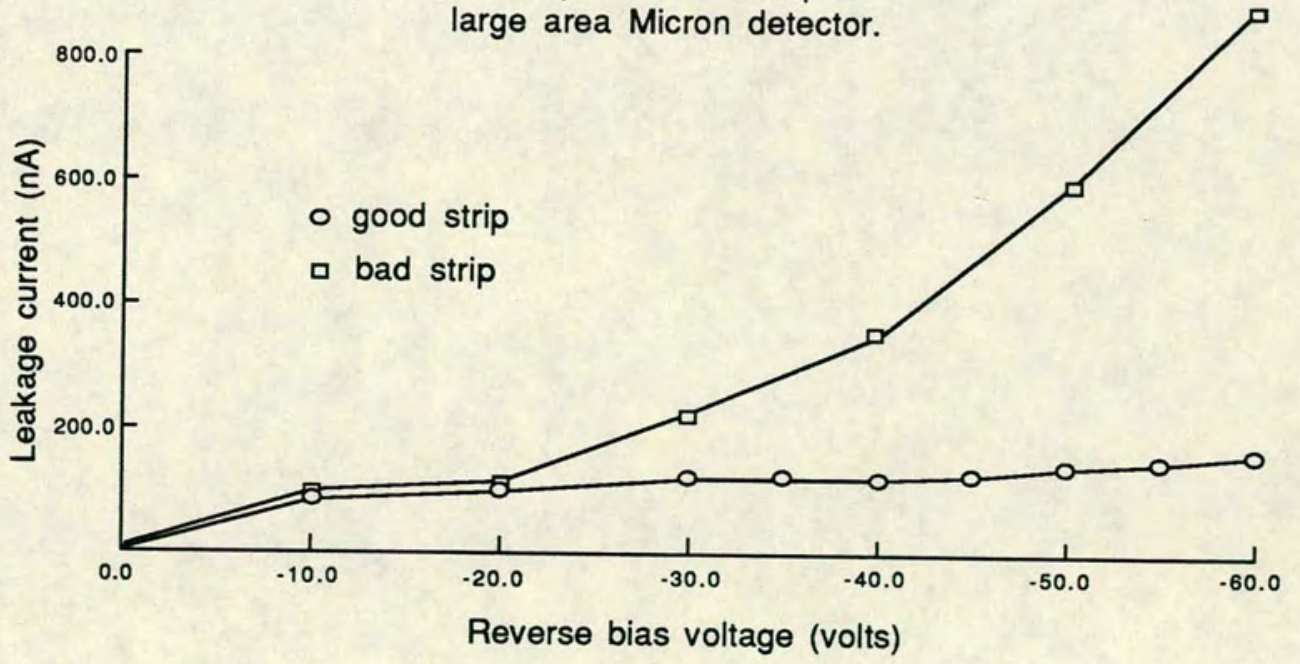

Figure 2.8: Leakage current vrs. bias for two separate strips on a large area Micron detector. (a) a normal strip. (b) a strip exhibiting low frequency breakdown. 


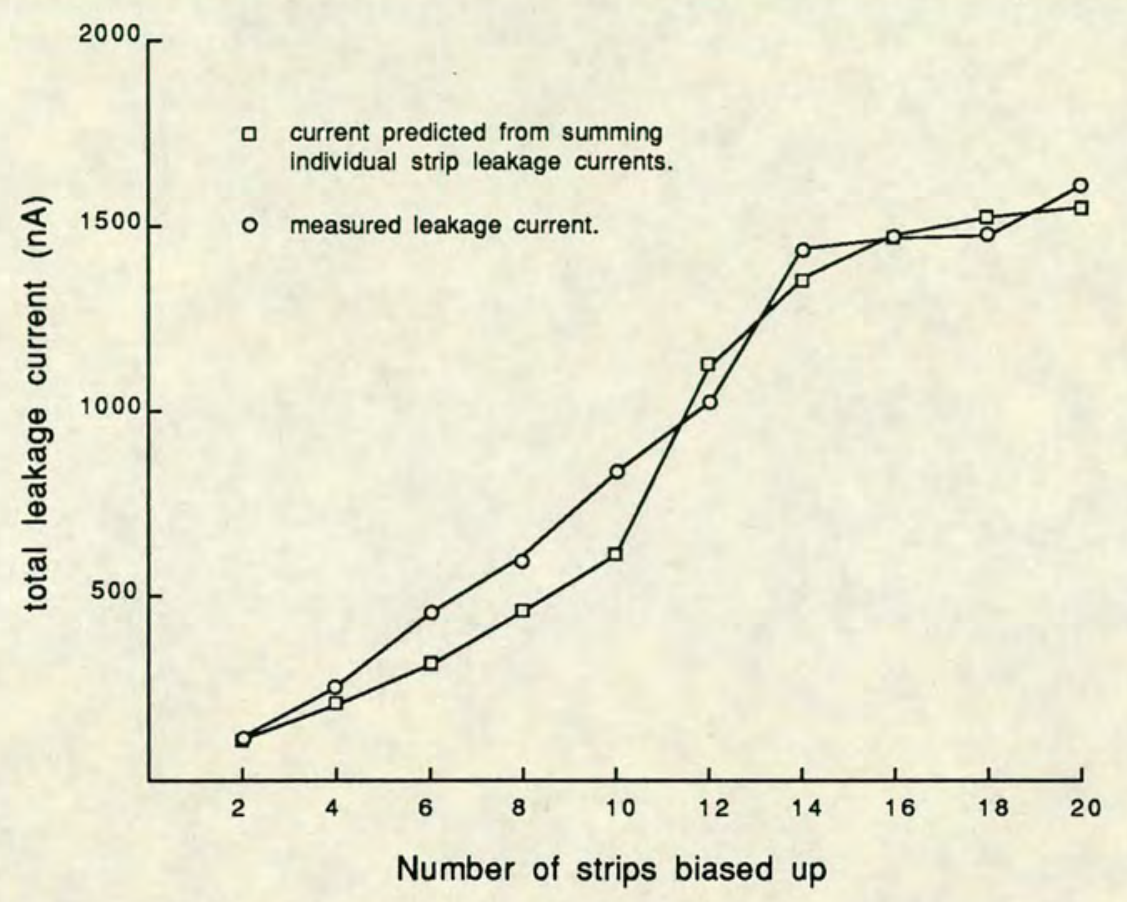

Figure 2.9: Leakage current as a function of the number of strips under bias. The square points represent the leakage expected from addition of the individual strip leakages.

tector. The abrupt change in leakage at $\sim 16$ hours is attributed to temperature variations in the lab. These measurements were again taken in air at room temperature.

One interesting property was the short term time dependance of the maximum bias voltage applicable before breakdown of the detector. When some of the detectors were initially biased, the maximum voltage that could be applied was less than the recommended full depletion voltage. However, after $\sim 3$ hours at a low bias, this upper bias limit increased to a value above the full depletion voltage. Fig.2.11 is an example of this time dependant bias limit for a Micron large area detector. This 'self-healing' effect has been observed in the tests on strip detectors [Go86] and has been attributed to trapping of electrons in the oxide on the detector surface. This trapped charge may improve the field profile at the $\mathrm{p}^{+}$junction edges resulting in higher breakdown voltages.

In summary the leakage current measurements gave a general indication of how well the detectors would perform under irradiation but certain aspects of the leakage behaviour need further study e.g. breakdown voltage time dependance 


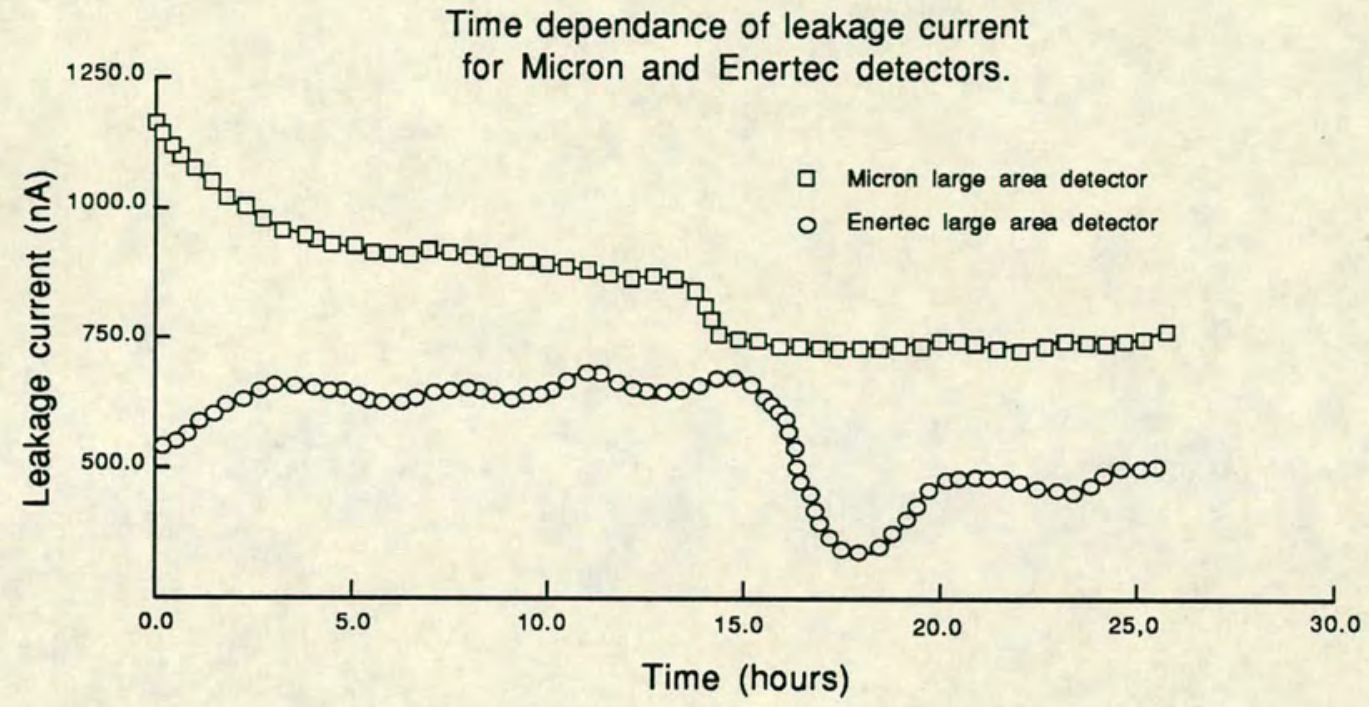

Figure 2.10: Time dependance of full leakage current for large area Micron detector and large area Enertec detector.

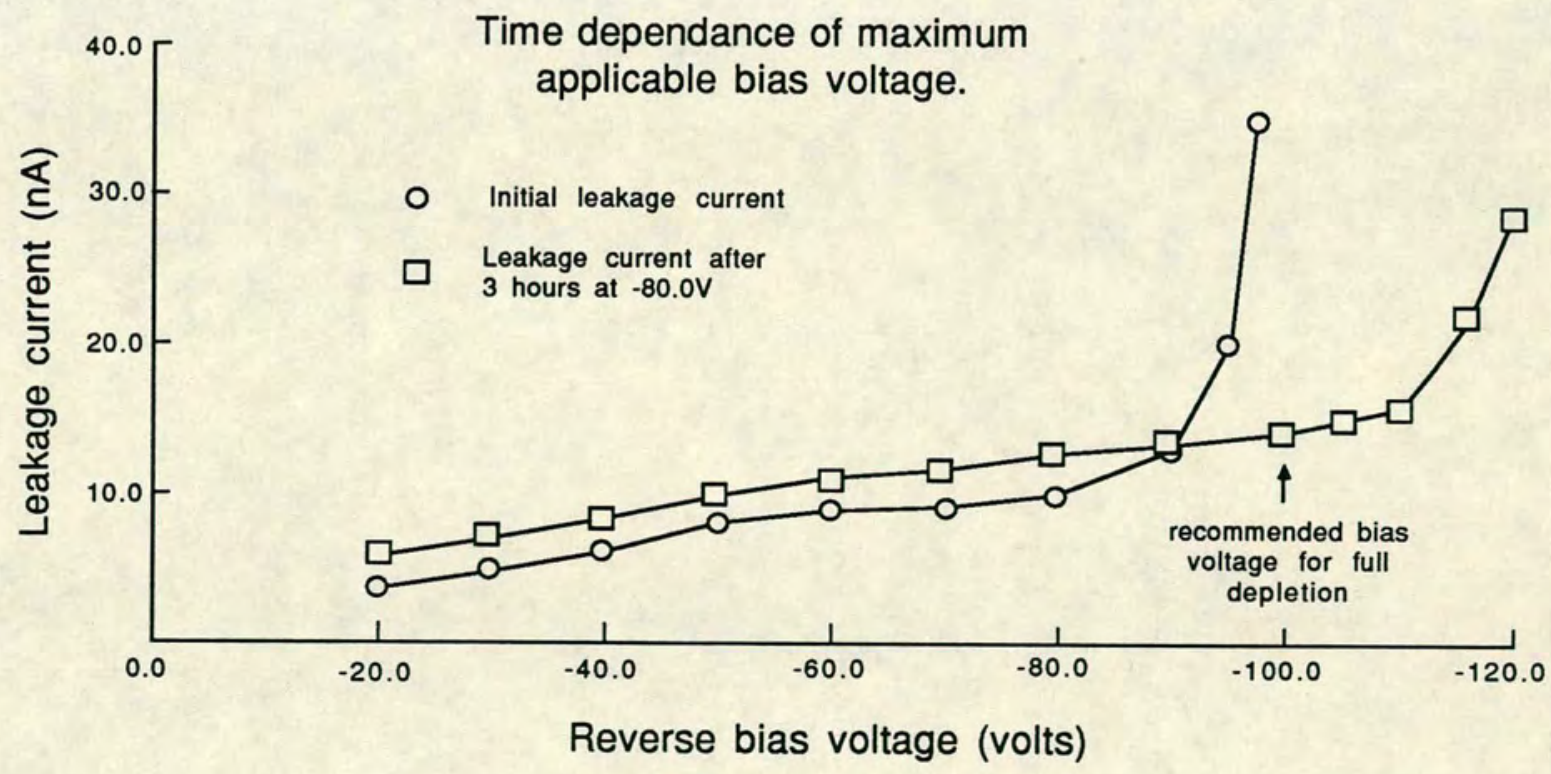

Figure 2.11: Time dependance of maximum applicable bias voltage before breakdown. 
and low frequency breakdown. More recently, detectors have become much more stable with typical leakage currents of $\sim 10 \mathrm{nA}$ per strip for a large area detector. This is most likely due to improvements in the manufacturing process. It is probable that these improvements are also responsible for the absence of the low frequency breakdown behaviour present in the earlier detectors.

\subsection{Charge Sharing Effects}

One of the main reasons for performing tests on the strip detectors was to determine any problems associated with charge sharing between adjacent strips. Such charge sharing, through capacitive or resistive coupling of neighbouring strips, has been used to improve the determination of the centre of gravity of ionisation tracks [En81], [En82] but for the intended use in this thesis it is an unwanted property.

Any charge division between strips that are not connected together in the same group, will result in an event with two elements on the same detector recording a hit. This type of event is ambiguous and is disregarded in the event analysis (see section 3.3).

Extensive tests have been performed at CERN [Bo81] to determine the extent of this charge sharing in surface barrier and ion implanted microstrip detectors. These tests concluded that for minimum ionising particles $\left(10 \mathrm{GeV} \pi^{-}\right)$the ion-implanted strip detectors showed no evidence of any charge sharing effects from neighbouring strips. In addition to this result they also concluded that the surface barrier strip detectors did exhibit charge sharing between strips and that the intensity of this was directly related to the dimensions of the interstrip gap.

In contrast to the minimum ionising particles used in these tests, highly ionising particles will deposit large amounts of energy in the active volume of the detector. This will release large amounts of charge, creating electric fields comparable to those present in the detector under its full depletion voltage. It was 
therefore necessary to verify these previously mentioned results and to quantify any charge sharing found.

To test for this charge sharing, a beam of ${ }^{241} \mathrm{Am}$ alpha particles, half the width of an individual strip, was shone onto the front of the detector. It was positioned in such a way that it straddled the interstrip gap with a part on each neighbouring strip. A normal, slow coincidence set-up was used to determine any events which occured simultaneously in both strips. The resulting coincidence rate was negligible and consistent with the rate of random coincidences expected from two independant counters. The range of $5.48 \mathrm{MeV}$ alpha particles in $\mathrm{Si}$ is $\sim 30 \mu \mathrm{m}$ so, to test the full active volume, the alphas were shone, uncollimated; into the back of the detector. In this configuration the holes have to traverse the complete thickness of the detector $(300 \mu \mathrm{m})$. Taking a hole mobility of $480 \mathrm{~cm}^{2}$ volt $^{-1}$ $\sec ^{-1}[E G+G]$ and an electric field strength of $1600 \mathrm{~V} \mathrm{~cm}^{-1}$ (50V over $\left.300 \mu \mathrm{m}\right)$ it takes a hole $\sim 40 \mathrm{~ns}$ to traverse the complete $300 \mu \mathrm{m}$ of silicon. One can then work out an approximate size for the hole cloud from the formula [Da84]:

$$
\mathrm{L}_{\mathrm{D}}=\sqrt{\mathrm{D} \tau}
$$

with $\mathrm{D}=$ diffusion constant $=\frac{k t}{e} \mu_{h}=12.3 \mathrm{~cm}^{2} \mathrm{~s}^{-1}$

$\mu_{h} \quad=$ hole mobility

and $\tau=$ the time for diffusion $=40 \mathrm{~ns}$.

This gives a diffusion distance of $\sim 10 \mu \mathrm{m}$ compared to the initial lateral extent of $\sim 0.1 \mu \mathrm{m}$ [Hs83]. A more accurate calculation using a more realistic, linearly varying electric field instead of a constant field gives results of the same order of magnitude differing only by a factor of $\sim \times 2$. This diffusion was assumed to be the cause of the increased coincidence rate when the alphas were incident on the back of the detector. This rate was still less than $0.5 \%$ of the individual strip singles rate. Why ion-implanted detectors exhibit this lack of charge sharing compared to surface barrier detectors is not clear but a possible explanation is given in the next section.

During these tests for charge sharing it was noticed there was a non-negligible rate of pulses with opposite polarity to the normal output signals. These pulses 
were only present when the front of the detector was being irradiated and in particular when the interstrip gap was being illuminated. The origin and behaviour of these anomalous polarity pulses was the subject of a paper published in Nims, a pre-print of which is contained at the end of this thesis. The next section will be a summary of the contents of this paper along with a number of other observations concerning these negative polarity pulses.

\subsection{Anomalous Polarity Pulses}

To investigate the behaviour of the anomalous polarity pulses a number of simple observations were made with a beam of ${ }^{241} \mathrm{Am}$ alpha particles collimated as in the previous section. As has been mentioned these negative pulses were only present when the alphas were incident on the side of the detector with the $\mathrm{p}^{+}$ implantation. This suggested that they arose from some surface effect and that an investigation of the interstrip surface structure may yield an explanation of their origin. Details of this interstrip region for the detector used in these tests are shown in fig.2.12. Fig.2.13 shows an example of the alpha particle energy spectrum obtained from one strip when the beam straddled the interstrip gap between it and its neighbour. It is clear that a number of anomalous features are present. The main ${ }^{241} \mathrm{Am}$ energy peak is clearly seen as the most intense peak in the spectrum but there is another peak approximately $90 \mathrm{keV}$ higher. This upper energy peak has been identified as those alpha particles entering the active volume through the $\mathrm{SiO}_{2}$ window. This is thinner than the $\mathrm{Al}$ strip and the difference in thickness is consistent with this $90 \mathrm{keV}$ energy loss for alphas entering through the strip. The lower energy satellite peak is rather more confusing. This peak was identified as arising from alpha particles entering the detector in the region between the $\mathrm{p}^{+}$implantation volumes [Yo87]. The pulses contributing to this peak were also found to occur coincidentally with an anomalous polarity pulse in the adjacent strip. The presence of these abnormally low amplitude pulses and the associated negative pulses was obviously an unwanted property of the interstrip region. Not only would they degrade the energy resolution of the detector but may, depending on the application, result in particles being misidentified by any $\Delta \mathrm{E}-\mathrm{E}$ mass algorithm as well as disabling certain types of 


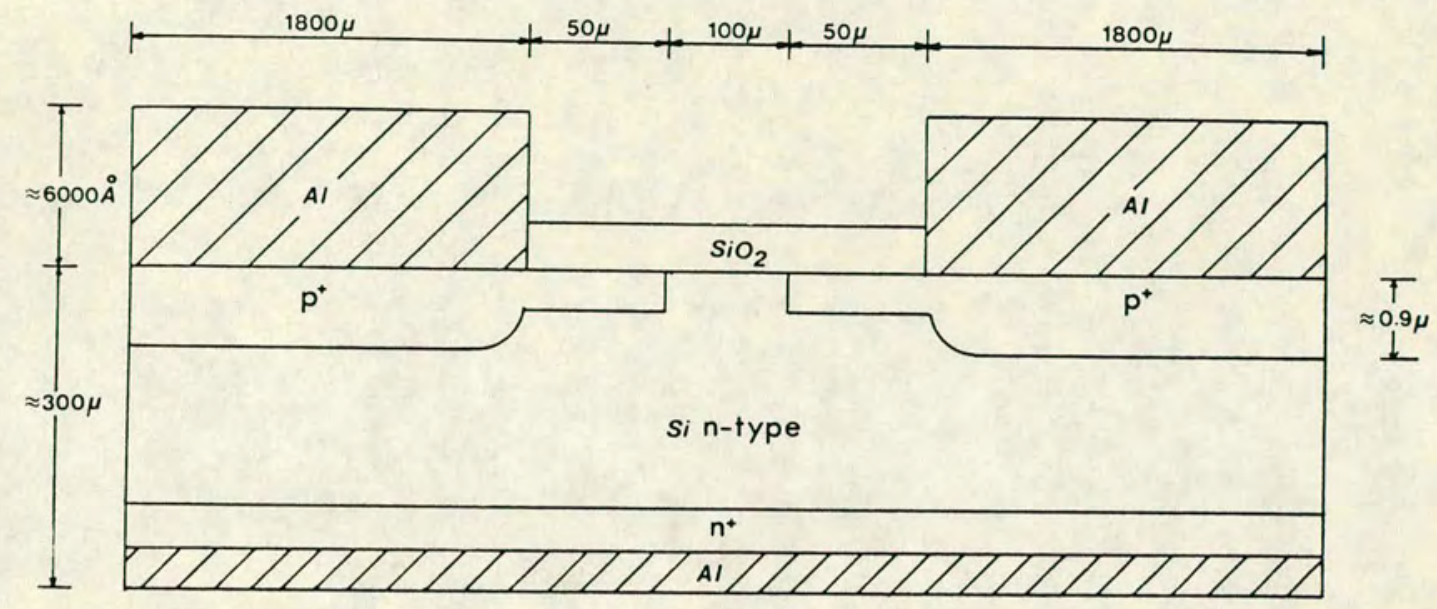

Figure 2.12: Details of the interstrip gap region.

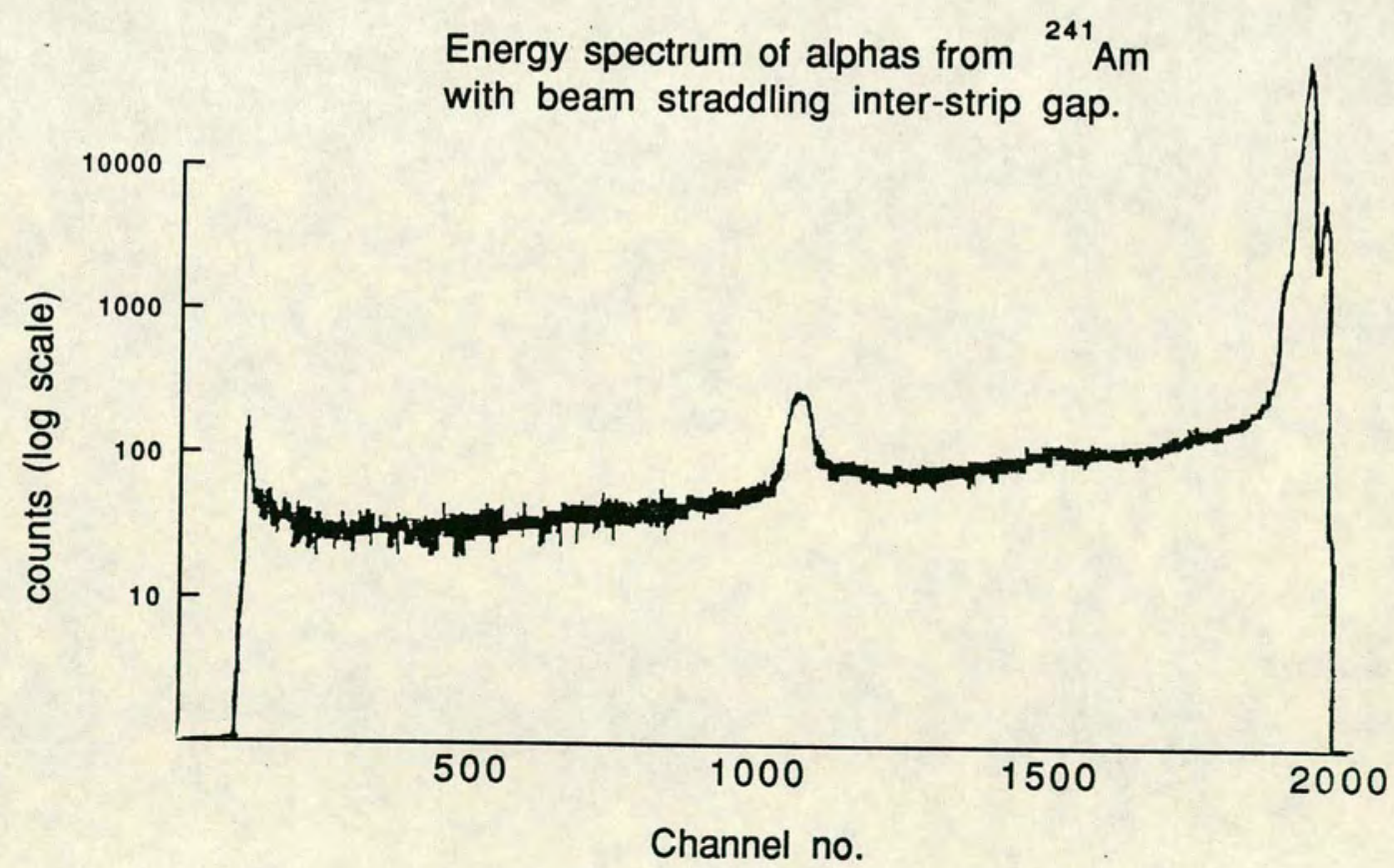

Figure 2.13: Energy spectrum of alphas from ${ }^{241} \mathrm{Am}$ with beam collimated across the interstrip gap. 
ADC unable to accept bipolar input signals.

To clarify and quantify the properties and behaviour of these anomalous pulses an experiment was performed using the Intense Bunched Ion Source (I.B.I.S.) accelerator facility at A.E.R.E. Harwell. This is a small Van-de-Graaff accelerator capable of producing a $3 \mathrm{MeV}$ alpha beam. When used in its microbeam mode the I.B.I.S. set up can produce a beam of $10 \mu \mathrm{m} \times 10 \mu \mathrm{m}$ cross-sectional area [Co79] which seemed ideal for probing the interstrip region. Unfortunately the low beam currents $\left(\sim 10^{-16} \mathrm{amps}\right)$ required to allow direct exposure onto the detector surface meant that the beam profile did not approach this value. From measurements during the experiment it became obvious that the beam was an intense spot with one sharp edge $\sim 5 \mu \mathrm{m}$ in width, but with an extended tail on the opposite edge wider than the interstrip gap. The main advantage of the experimental set-up was the detector mounting table which had a reproducible horizontal movement resolution of $\sim 1 \mu \mathrm{m}$. With this set-up, the beam was stepped from one strip towards the other, sharp edge first, in steps of $25 \mu \mathrm{m}$.

In summary, the results (given in detail in the pre-print in appendix c), verified the supposition previously outlined, that the different peaks were indeed produced by alpha particles entering the detector at distinctly different positions, the location of these positions being determined by the detector's structure. In particular the negative pulses in one strip were associated with alpha particles entering the detector between the $\mathrm{p}^{+}$regions at a distance further away than the midpoint between it and its neighbouring strip. The coincident, positive polarity pulses contributed to the energy spectrum from the adjacent strip in the region from the main peak down to and including the lower satellite peak. The sum of the moduli of the coincident negative and positive pulses was found to be a constant that was comparable to the amplitude expected for a normal full energy pulse.

Other observations about these negative pulses include that;

- they disappeared below a threshold bias voltage of $\sim-8 \mathrm{~V}$ with a detector whose full depletion voltage was $-40 \mathrm{~V}$.

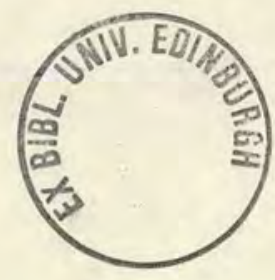


- they disappeared from one strip when both its neighbouring strips were left at a floating potential.

- they occured simultaneously (to within $\sim 2 \mathrm{~ns}$ ) with the correlated positive polarity pulse from the neighbouring strip.

- they were present with fission fragments from ${ }^{252} \mathrm{Cf}$.

- their behaviour seems to be independant of the time under bias.

- no $(-)(-)$ coincident pulses were recorded.

- they were not detectable with minimum ionising electrons from ${ }^{207} \mathrm{Bi}$.

This last observation is consistent with the tests at CERN where no such anomalous features were reported with minimum ionising particles. The effect is probably still present but not detectable above the detector noise due to the small amount of charge released in the surface region by minimum ionising particles.

In the paper published in Nims we presented a possible mechanism for these anomalous events. It was proposed that positive charge, trapped at the $\mathrm{Si}-\mathrm{SiO}_{2}$ interface, caused a local field reversal and that any electrons created near this region were swept towards the surface instead of the rear of the detector. These electrons induced a charge of an anomalous polarity on both the nearest strips. It is the addition of this induced charge onto the normal charge signal that is responsible for the reduced height $(+)$ polarity pulse and coincident $(-)$ polarity pulses.

Subsequent to the publication of this paper, computer simulations were performed [Bu88] to model the interstrip field in the case of fixed charges at the $\mathrm{Si}-\mathrm{SiO}_{2}$ interface. These produced results that were in excellent agreement with the proposed model. Fig.2.14 (a) shows the potential distribution through a $300 \mu \mathrm{m}$ detector with no trapped charge at the surface, whereas simulation (b) has a fixed charge of $1 \times 10^{11} \mathrm{q}$ (where $\mathrm{q}$ is unit of electron charge) on the oxide layer. These simulations were for $\mathrm{n}$ type substrate with $3.5 \mathrm{k} \Omega$ resistivity at a bias of $-100 \mathrm{~V}$. The formation of a potential well is clearly visible in (b).

This model explains the following experimentally observed effects: 

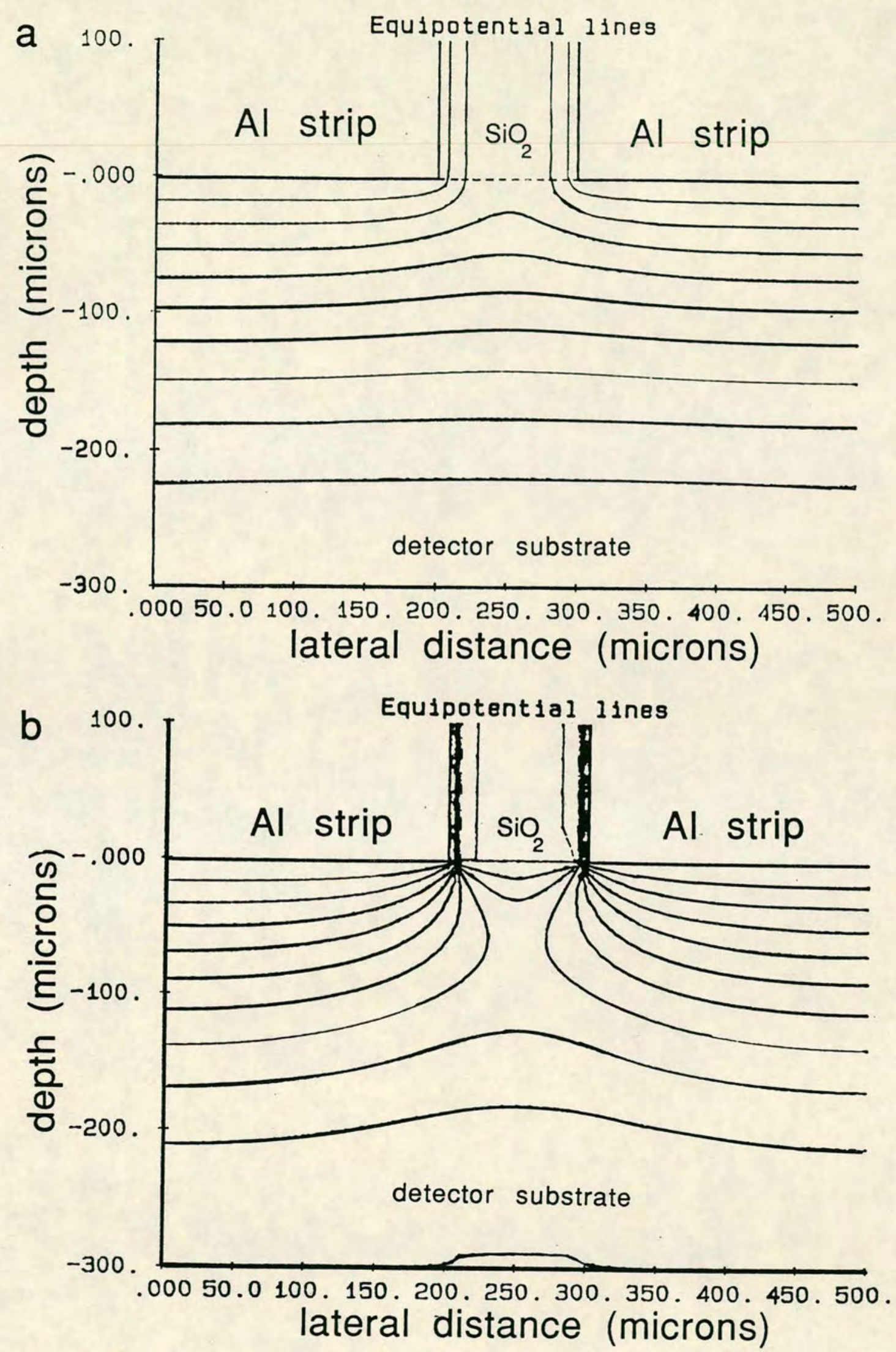

Figure 2.14: Equipotential lines from computer simulation of interstrip region with and without trapped charge at the $\mathrm{Si}_{-} \mathrm{SiO}_{2}$ interface. (a) no trapped charge. (b) trapped charge of $1 \times 10^{11} \mathrm{q}$ ( $\mathrm{q}$ is unit of electric charge). 
- sharp splitting of signals between strips due to potential hump.

- the fact that only $(+)(-)$ coincident events are seen when particles penetrate the front surface.

- the detailed form of the correlation function between simultaneous pulses from adjacent strips (see appendix C fig.5 and fig.8).

Another observation concerning the shape of the pulse height spectrum for the (-) pulses was that whilst all the Enertec detectors exhibited a distinct peak followed by a sharp cut-off at approximately half the full energy amplitude, the Micron detectors had a much less well defined shape. The spectrum for these detectors tended to be on a gradual fall off in intensity to the upper cut-off level, with no obvious peak. This difference is probably due to the different manufacturing techniques creating slightly different surface structures which in turn affects the strength of the field reversal responsible for the (-) pulses.

Once these negative pulses had been identified it was necessary to establish how much of an affect they would have on the detectors capability to record valid events. Effectively this meant finding out how much of the detector contributed to these anomalous events. This was determined by using an uncollimated ${ }^{241} \mathrm{Am}$ source and illuminating the strip side of the detector. The number of negative events compared to the number of normal events in the main peak then gave the ratio of the areas producing the different pulses. For the Micron detectors used in the experiment, each strip had an average of $\sim 80 \mu \mathrm{m}$ of the interstrip gap contributing to the negative events. This value was used in the calculation of the elemental solid angles to account for events that were corrupted due to this behaviour. It meant a decrease of the calculated solid angles by approximately $10 \%$ for each element. This correction assumes that all the particles entering this region are totally lost. This approximation will not be completely true but should only add a small amount to the overall systematic error. The error associated with this approximation was taken as producing a $2 \%$ contribution via the error in the solid angle of each element.

In an effort to reduce the effect of this anomalous behaviour, Burger has proposed the use of a field plate evaporated onto the surface of the $\mathrm{SiO}_{2}$. A bias applied 


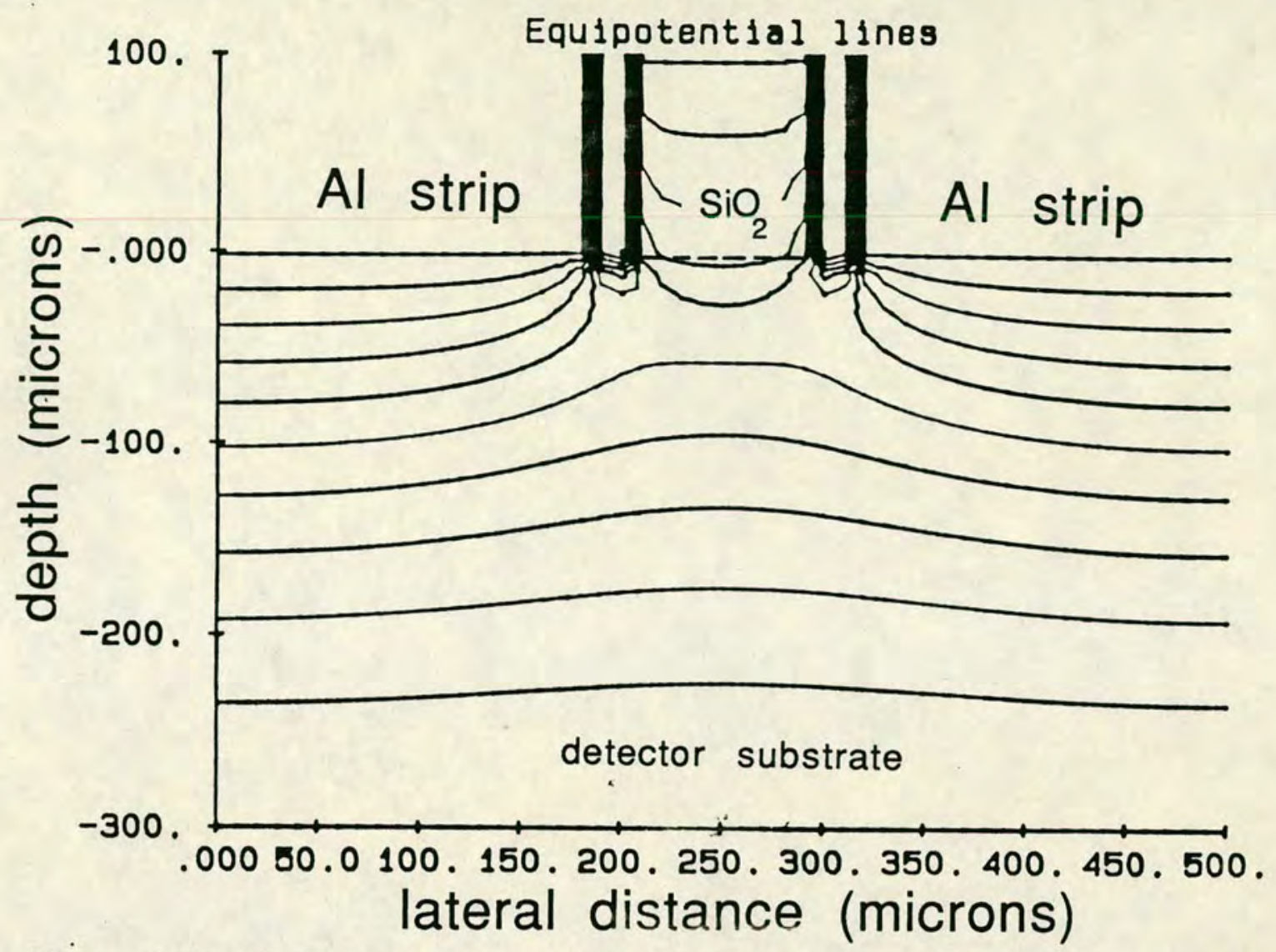

Figure 2.15: Computer simulation of field with field plate compensation. Trapped charge same as in fig.2.14(b).

to this plate could then compensate for the trapped charge and reduce the field reversed region. Fig. 2.15 shows the results of a calculation similar to that shown in fig.2.14(b) but this time with a field plate used as a compensator. It can be seen that the result is a reduction in the potential well. This method may indeed result in a reduction of the negative pulse rate but it may also result in an increase in the occurrence of $(+)(+)$ coincidences. In some applications it is conceivable that the negative pulses are of minor importance, being much out-weighed by the advantage of the sharp division of events between adjacent strips. In this situation the trapped charge has a beneficial effect. Clearly the end use of the detector will play an important role in determining whether or not such field plates are desirable. If a detector is constructed with a field plate it is always possible to leave it unconnected. This makes the detector more versatile, therefore the field plates are probably a useful design feature.

In summary, normal charge sharing between neighbouring strips was absent, but an anomalous interstrip surface effect which generated abnormal polarity output pulses was identified and investigated. This was found to originate from a 
field reversal near the detector surface caused by charge trapped at the $\mathrm{Si}-\mathrm{SiO}_{2}$ interface. The proposed field shape agreed well with computer simulations for this situation and a method of reducing its effect was proposed.

\subsection{Further development}

There are three main areas where future development is essential if strip detectors are to achieve their full potential in nuclear physics experiments. These are:

- improvement of the detectors themselves and their manufacturing techniques.

- improvement of the testing facilities.

- improvement of the output electronics.

The detectors themselves suffer from the limitation that the positional information which they supply is one dimensional. This requires the use of two detectors at $90^{\circ}$ to one another if accurate $\mathrm{X}-\mathrm{Y}$ positional resolution is required. Such a set-up was used in the experiment outlined later in this thesis. Clearly, to register in the second detector and record the full information, the particle must traverse the first detector. This results in a lower energy threshold limit for any applications of this configuration. This problem is further compounded by the limitations on the thickness of the detectors available (this being another area requiring development). A much better arrangement would be to have both the co-ordinates measured by the one detector. Two simple methods of achieving this would be to have strips on the front and back of the same detector orientated at $90^{\circ}$ to one another or to have one set of strips on the front that operate like conventional position sensitive detectors.

Unfortunately both these proposals double the number of output signals that have to be instrumented and recorded. However, recent developments in micro electronics specifically designed for nuclear physics applications will soon 
eliminate this problem [Wa84], [Ho84], [Da88]. Prototype detectors of both the double-sided and position-sensitive design are already being tested and look promising for the future.

Up until now, the behaviour of the individual detectors has been rather unpredictable with no two behaving similarly. This is attributed to the complex manufacturing techniques which, although well known, are being applied to a rather different situation from normal. It seems reasonable to expect that improvements will be made in this reproducability, with detector properties becoming more reliable. One observation that should be mentioned concerns the number of working strips on a detector. When one has a large number of strips $\sim 1000$, a surface defect which disables one or two neighbouring strips has a small effect on the percentage of strips still operating $\sim 0.1-1.0 \%$. However, when the number of strips is as low as 10-20, a similar defect is much more destructive. If this defect affects only one strip it will result in $5-10 \%$ of the detector becoming unuseable. It is clear that producing these large area strip detectors, with large surface area strips, requires a much higher quality control than is being applied to detectors which have an average of $\sim 2 \%$ of their strips (on a 512 strip detector) inoperative due to abnormally high leakage currents [Go86].

During the testing of the detectors it became clear that a number of improvements in the testing routines would have to be made if strip detectors were to be routinely used in nuclear physics experiments. To say that the testing of each individual strip for leakage, energy resolution and thickness was a rather tedious procedure would be somewhat of an understatement. The automation of this testing procedure is essential. It may be envisaged that the detectors are mounted on a CAMAC controlled X-Y table in front of a collimated $\alpha$ or $\gamma$ ray source with the output from each strip multiplexed into a single ADC. The whole energy and thickness testing procedure could then be computer controlled with an enormous saving of time and effort. Similarly, the design of a test-bed for measuring the leakage, capacitance and full depletion voltage for each detector is also desireable. Such a system has already been built [Go86] for measuring these properties for micro-strip detectors with 520 microstrips on each detector. Again an enormous saving in time and effort would result from such a system. 
As far as output electronics is concerned, one of the main obstacles to fully utilising the potential of strip detectors is the capital cost of instrumenting each strip separately. At current prices this is in excess of $£ 3000$ per channel, making it unfeasible to instrument each individual strip on a single 25 strip detector, let alone a telescope constructed from two or three such detectors. Recently there has been intense development of hybrid pre-amplifiers and associated electronics which has not only reduced the cost to $£ 100$ per channel but has also reduced the physical space required for this instrumentation. This means that it will be possible to mount the electronics much closer to the detector and reduce the stray capacitance from leads etc. to a minimum. These hybrid systems will undoubtedly have their own special problems, for example, care must be taken to ensure that a compact hybrid electronic set-up is cooled correctly, with heat sinks or heat pumps incorporated into the overall design at an early stage. This is necessary because although these condensed micro-electronic circuits use only small amounts of power, typically milliwatts, this is concentrated in a small physical space with little or no convection cooling (especially if the pre-amps are mounted close to the detector inside the vacuum chamber). Other similar problems are sure to be encountered as this technology progresses but the saving in space and money will surely outweigh them. Fig.2.16 is an impression of a system currently being developed in the Edinburgh Physics Department that all for the cost of one normal Nim unit.

One final consideration in the implementation of strip detector systems is the philosophy behind the data analysis. One option is to perform only a very basic event selection in hardware and leave the more sophisticated selection to software. This has the advantage of simplicity of hardware but a disadvantage related to the rate at which the computer interface can read the larger number of events. This may become serious when large numbers of strips are being used. The other approach is to make a much more elaborate event selection at the hardware stage resulting in a much reduced rate of event recording. This method has the disadvantge of requiring a more complex decision making logic set-up. One realistic solution may be a combination of both approaches with a logic system incorporating programmable logic chips to provide rough trigger signals to the computer which would read predefined groups of ADCs on 


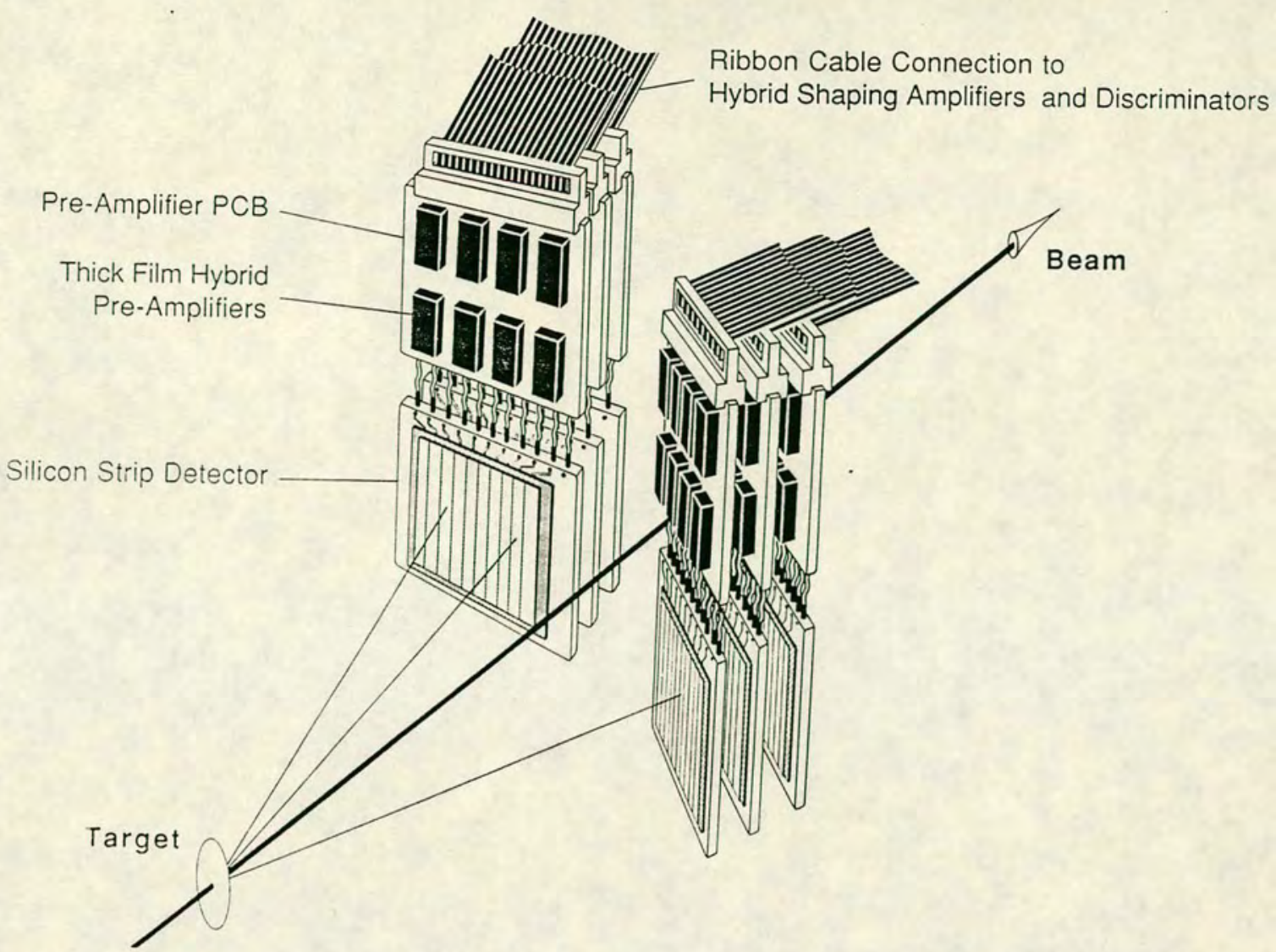

Figure 2.16: Impression of proposed strip detector and hybrid electronics system that will be realised in the near future.

receipt of these trigger signals. This has the advantage of generality of application with different detector configurations using different programmable chips incorporated into a standard logic set-up. The application of strip detectors to the increasingly complicated detector configurations used in new heavy-ion experiments will undoubtedlyresult in increased activity in all these fields. 


\section{Chapter 3}

\section{Experimental Apparatus and Method}

\subsection{Accelerator and beam-line}

All the data presented in the following sections were obtained at the Nuclear Structure Facility ( N.S.F. ), of Daresbury Laboratory. The N.S.F. is a $20 \mathrm{MV}$ tandem Van-de-Graaff accelerator with several experimental areas. Figures 3.1 and 3.2 show the general layout of the accelerator and experimental areas. All the experiments were performed in the 1 metre scattering chamber on the $79^{\circ}$ line.

A beam of ${ }^{7} \mathrm{Li}^{(-)}$ions was extracted from a Middleton source and injected into the main accelerating column. The ions were then accelerated towards the central terminal which was held accurately at a positive voltage of about $17.5 \mathrm{MV}$. On passing through the central terminal they were stripped of their electrons by thin carbon stripper foils and the required charge state selected by an offset quadrupole charge selector. Emerging from the selector the ${ }^{7} \mathrm{Li}$ ions, now $3(+)$ charged, were accelerated away from the central terminal. At the base of the accelerating column the ions, now at their full energy, were deflected into the experimental area by a $90^{\circ}$ inflector magnet with an intrinsic energy resolution of $\sim 0.01 \%$.

After injection into area $1 \mathrm{~A}$ the beam encountered a set of $\mathrm{X}-\mathrm{Y}$ collimators and then passed through a focusing quadrupole doublet and a $5^{\circ}$ bending dipole into 


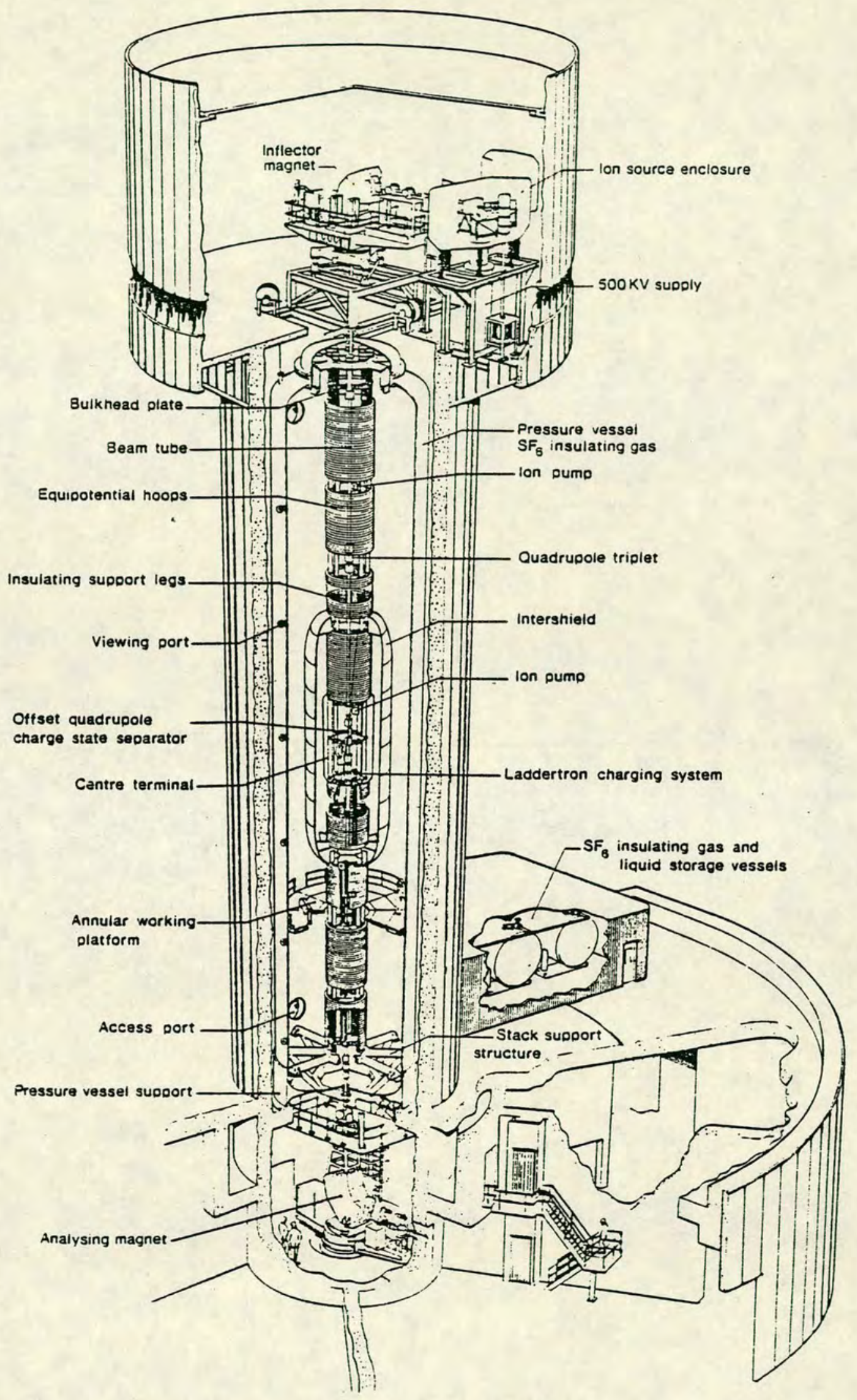

DARESBURY TANDEM ACCELERATOR

Figure 3.1: The 20MV tandem Van-De-Graaff accelerator of the Nuclear Structure Facility at Daresbury Laboratory. 


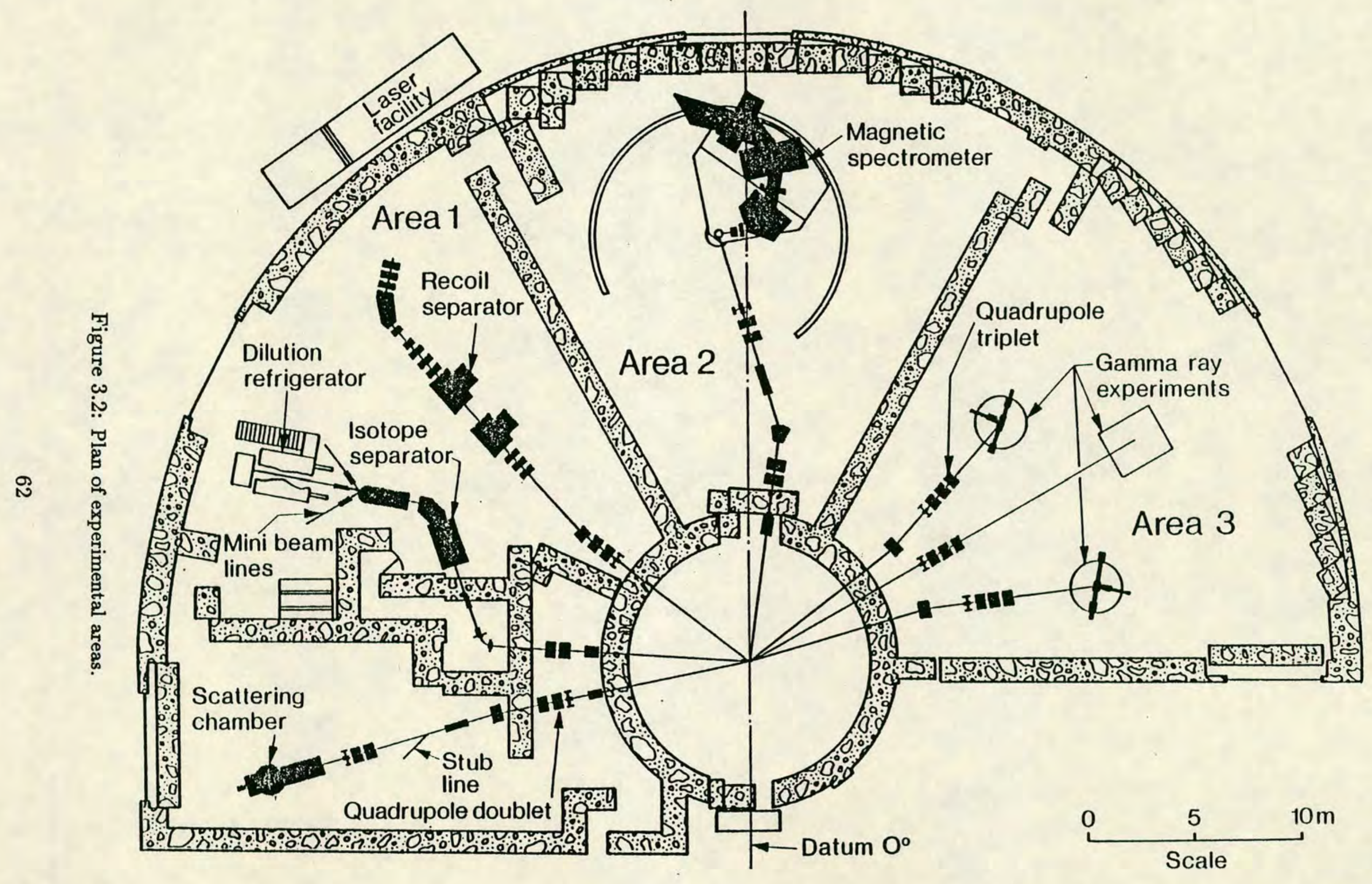


the final section of the beam line. The experimental area diagnostic section was immediately behind the $5^{\circ}$ magnet and consisted of a quartz screen viewed by a video camera, a Faraday cup and a $2 \mathrm{~mm}$ diameter circular aperture. During the experiment these were periodically checked to monitor the beam position and intensity. The final focusing quadrupole doublet was followed by two collimator turrets. In this experiment the first was left empty and the turret closest to the scattering chamber was used to mount a $2 \mathrm{~mm} \times 2 \mathrm{~mm}$ tantalum collimator at a distance of $0.75 \mathrm{~m}$ from the target. A $4 \mathrm{~mm} \times 5 \mathrm{~mm}$ anti-scatter collimator, made from tantalum, was also placed $90 \mathrm{~mm}$ from the target to remove any unwanted beam spray. The target ladder contained a $2 \mathrm{~mm}$ diameter aperture and an empty $10 \mathrm{~mm}$ diameter target holder along with the three targets used during the experiment. The beam was finally collected by a magnetically suppressed, oil cooled, Faraday cup.

The scattering chamber itself was 1 metre in diameter with a number of viewing ports and insulated cable feedouts. Fixed out-of-plane monitor detectors were mounted in the chamber walls at $\pm 15^{\circ}$ and $\pm 30^{\circ}$. The main detectors were mounted on two independently moveable arms. The angle of the arms and the height and angle of the target ladder were remotely controlled from the N.S.F control room. The chamber was pumped by three water cooled diffusion pumps and was typically at $\sim 3 \times 10^{-5}$ torr. during the experiment. The beam line was pumped by three ion pumps and close to the second quadrupole the pressure was typically $\sim 5 \times 10^{-6}$ torr.

During the beam alignment the current collected on all the relevant collimators and apertures was monitored. With the $2 \mathrm{~mm}$ diameter aperture in place in the target ladder, the beam transmission from the diagnostic box to the final Faraday cup was better than $99 \%$.

The beam current was adjusted during the experiment to keep the $\Delta \mathrm{E}$ detector rates at $\leq 15 \mathrm{KHz}$. and ranged from $<1 \mathrm{nA}$ for the ${ }^{208} \mathrm{~Pb}$ target at forward angles to almost $20 \mathrm{nA}$ for the ${ }^{12} \mathrm{C}$ target at backward angles. From past experience [Da85] it has been found that these beam intensities present no problems as far as target degradation is concerned thus the insitu monitor detectors were not required during the experiment. 


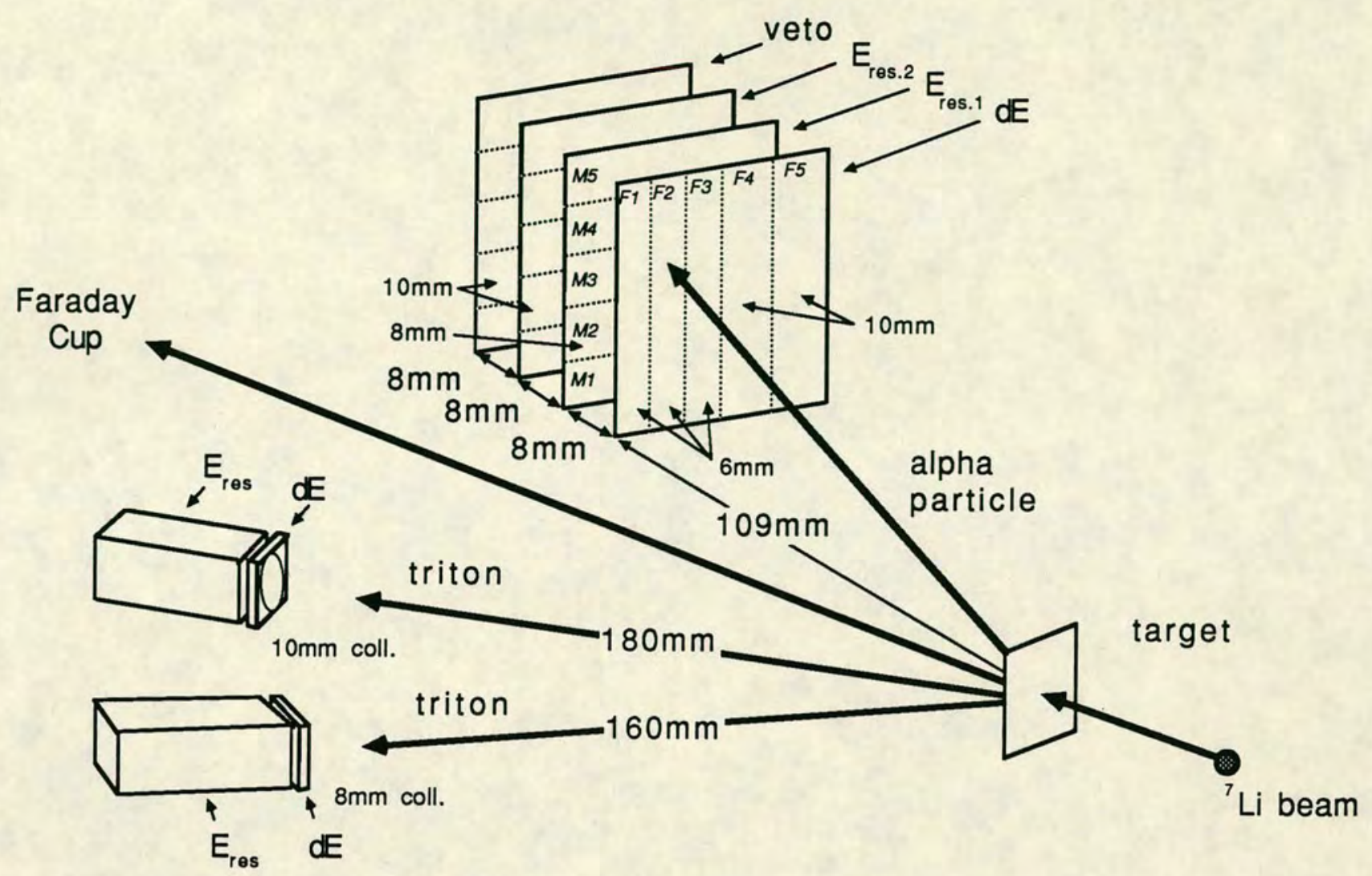

Figure 3.3: Detector set-up used during the experiment.

\subsection{The Detector System}

\subsubsection{Detectors and Mounts}

Figure 3.3 shows the details of the detection system used during the experiment. The system comprised of two $\Delta \mathrm{E}-\mathrm{E}$ silicon detector telescopes and a strip detector telescope system (constructed from large area Micron strip detectors). For more details of the strip detectors see chapter 2. The design of the telescopes was primarily governed by the requirement that they should be suitable for detecting the relevant energy triton and alpha particles from the fragmentation of the ${ }^{7} \mathrm{Li}$. The thickness, the angular resolution and the energy resolution had to lie within certain limits compatible with this objective.

Particle identification was performed by the standard $\Delta \mathrm{E}-\mathrm{E}$ technique [Ce66]. 
An ideal telescope for this purpose would have a thin $\Delta \mathrm{E}$ detector with good energy resolution and minimal dead layer followed by an $\mathrm{E}$ detector thick enough to completely stop the particles of interest. Ideally, the strip detector telescope and the silicon detector telescopes would each be able to identify the correct energy alpha and triton particles incident on them. In this experiment however, a number of compromises had to be made which limited the versatility of the set-up. The $\Delta \mathrm{E}$ detector had to be thick enough for the most energetic triton particles to deposit sufficient energy to overcome any hardware thresholds but thin enough to allow through the low energy alpha particles. Two transmission mounted surface barrier detectors of $220 \mu \mathrm{m}$ and $200 \mu \mathrm{m}$ were chosen as the $\Delta \mathrm{E}$ for telescopes 1 and 2 respectively. The E detectors used were thick, lithium drifted silicon detectors (commonly known as SiLi detectors). At $4 \mathrm{~mm}$ and $5 \mathrm{~mm}$ thick for telescopes 1 and 2 respectively they were thick enough to completely stop all the particles of interest. The strip detectors presented a bit more of a problem. The processes involved in the manufacture of these large area detectors make it difficult to produce detectors outwith the limits of $\sim 200-500 \mu \mathrm{m}$ thick. The lower limit is just on the edge of what is acceptable for the $\Delta \mathrm{E}$ detector of an alpha particle telescope suitable for this work. The upper limit is, unfortunately, too thin to stop the higher energy alpha particles of interest. The final design for the strip detector telescope was a $190 \mu \mathrm{m} \Delta \mathrm{E}$ detector and a $1000 \mu \mathrm{m} \mathrm{E}$ detector formed from two $500 \mu \mathrm{m}$ strip detectors mounted one behind the other. This was sufficient to just stop $\sim 55 \mathrm{MeV}$ alpha particles but the higher energy tritons $(\geq 40 \mathrm{MeV})$ and other $\mathrm{Z}=1$ particles passed completely through the strip detector system. The energy limits imposed by this constraint meant that the strip detector telescope was only used as an alpha particle detector. To prevent any mass identification problems, due to particles passing completely through the strip detectors, a fourth strip detector was used to veto such unwanted events.

The solid angles of telescopes 1 and 2 were $2.5 \mathrm{msr}$ and $2.1 \mathrm{msr}$ and were defined by $10 \mathrm{~mm}$ and $8 \mathrm{~mm}$ diameter lead apertures positioned at a distance of $180 \pm 1 \mathrm{~mm}$ and $160 \pm 1 \mathrm{~mm}$ from the target. The reaction plane was defined as the plane containing the centres of these detectors and the beam direction. The angular resolution of telescope 1 was $\pm 1.6^{\circ}$ and telescope 2 had an angular resolution of $\pm 1.4^{\circ}$. 


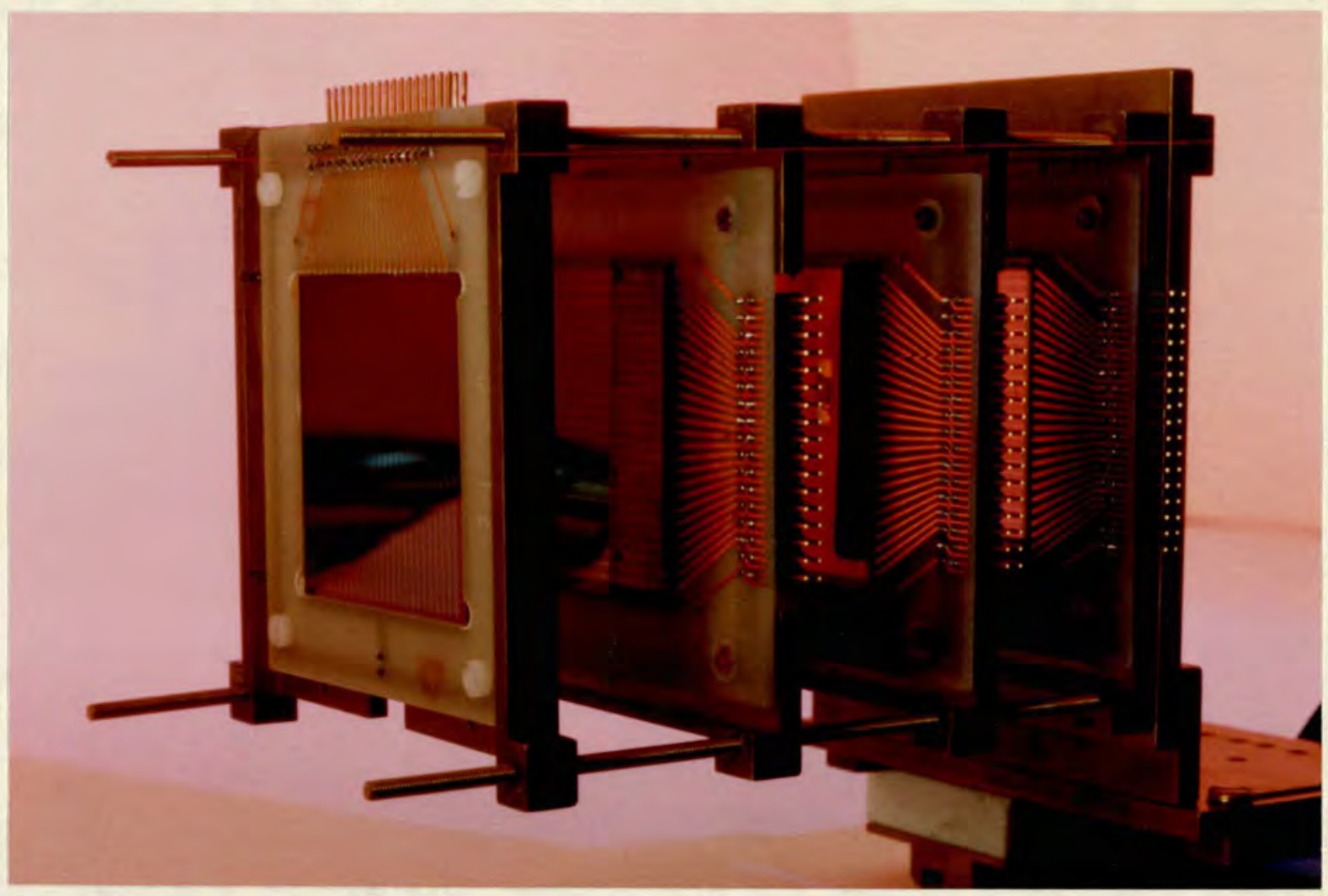

Figure 3.4: Photograph showing the strip detector mount.

The strip detector mounts were designed subject to a number of constraints imposed by the aims of the experiment. The whole mount was constructed from brass and consisted of a stand arrangement onto which the strip detectors, each on their individual holders, were mounted ( see fig.3.4). The stand was rigid enough to allow accurate, reproducible mounting of the detector holders. The strip detectors were mounted on their holders with an accuracy of $\sim 0.1 \mathrm{~mm}$ using a travelling microscope. The stand and holders were designed such that each holder could be rotated through $90^{\circ}$ to orientate the strips on the detector either horizontally or vertically. This arrangement had the advantage that any detector could be quickly replaced during the experiment without the necessity of realigning the telescope system or having problems with handedness of holders.

One aim of the experiment was to take measurements as close to the beam direction as possible. To facilitate this requirement the strip detector telescope was orientated such that the edge of the nearest strip to the beam, rather than the centre of the detector as a whole, was at $90^{\circ}$ to the defined angle direction. This is illustrated in fig. 3.5 

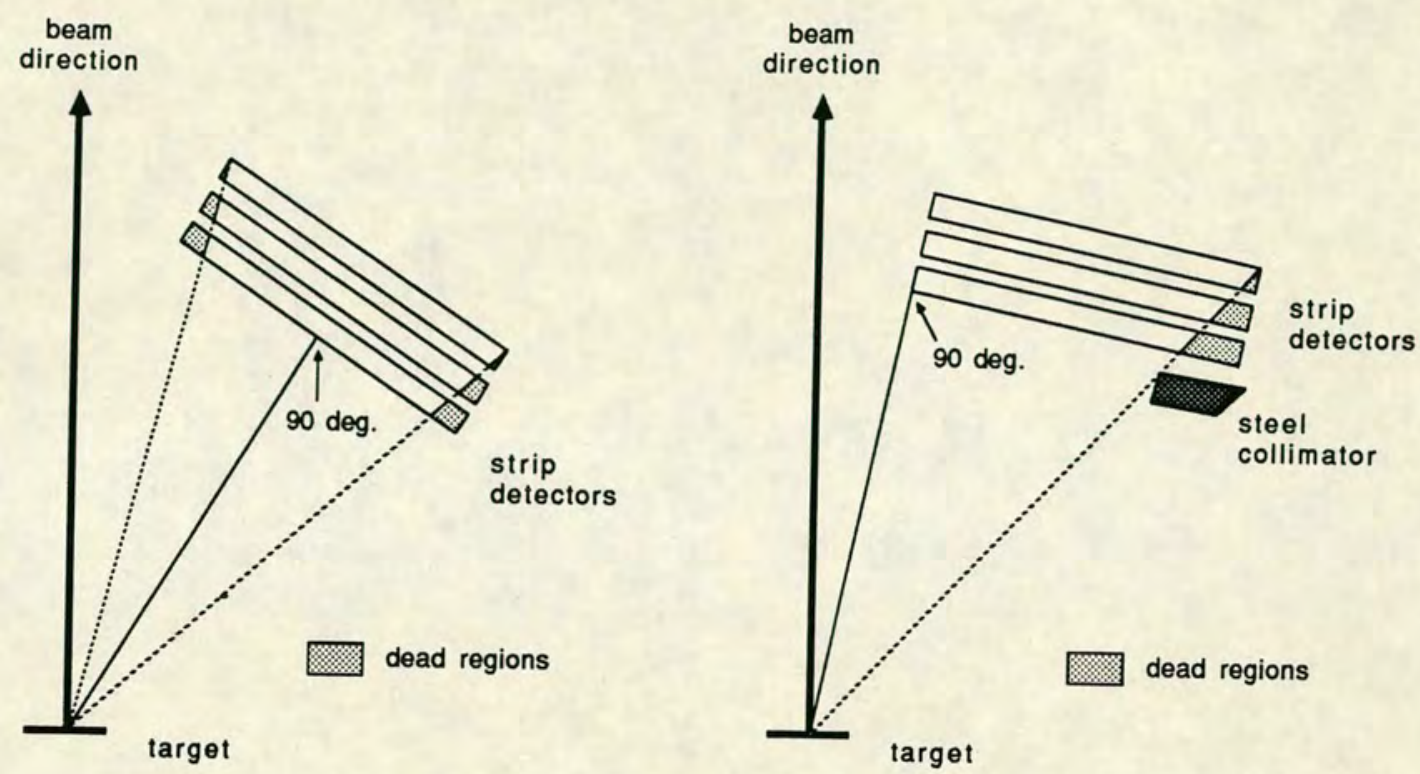

Figure 3.5: Angular orientation of strip detector telescope showing dead-regions and steel collimator

This meant that the smallest angle was defined as close as possible to the active region of the detector. Another aim was to measure coincidences as far as possible from the reaction plane. This was accomplished by mounting the strip detectors as close together as possible and mounting the stand such that the bottom edge of the lowest strip was on the reaction plane. This arrangement assumes that any reaction process involving an unpolarised beam and target will be symmetric about this plane. This orientation of the stand gave an increase of $\sim 2^{\circ}$ in the final, maximum out-of-plane angle. Clearly, the closer the strip detector telescope was placed to the target the larger would be this maximum out-of-plane angle. This was counteracted by the increase in the closest angle to the beam as the detector-target distance was decreased. A compromise was chosen that gave $\theta_{\min }=13.0^{\circ}$ and $\phi_{\max }=20^{\circ}$. This was a target to front strip detector distance of $109 \mathrm{~mm}$. At this distance there was no great improvement in one limit for a small change in the other.

A $3 \mathrm{~mm}$ thick stainless steel collimator was mounted in front of the strip detector telescope. The purpose of this was not to define the active area but to reduce 
the number of particles hitting the 'dead' regions of the detectors. These dead regions were the parts of the front and middle detectors that were not covered by the active region of the rear strip detector (see fig.3.5). A particle hitting these areas would not be able to produce a true $\Delta \mathrm{E}-\mathrm{E}$ coincidence, although they would contribute to the dead-time and pile-up of the system. The active region of the detector was defined by the edges of the horizontal and vertical strips used during the experiment and the holders were mounted as close together as possible to maximise this active area. The detectors large surface area made them particularly vulnerable to pick-up and this configuration had the added advantage of shielding the inner ones.

Magnetic supression collimators were fixed in front of the strip detector system and the SiLi detectors to prevent them being hit by stray electrons from the target. All the detectors were also electrically isolated from their mounts and from the vacuum chamber to reduce problems with pick-up.

During the experiment the front detector was orientated with its strips vertical. The three remaining detectors had their strips horizontal. The direction of the outgoing fragment was then determined from the horizontal and vertical strips that were present in the event.

\subsubsection{Strip Groupings and Nomenclature}

The number of strips on each detector that could be used was limited by the instrumentation of the output signals. It was not possible to instrument each of the 25 strips on each detector, so neighbouring strips were connected together in groups of varying width and the signal from the full group was inputted into a single pre-amplifier. Unfortunately, this led to a decrease in the angular and energy resolution but the increased solid angle improved the count rate. The set-up in this experiment was such that the front detector was connected into 3 groups of 3 strips each and 2 groups with 5 strips each. This used 19 strips out of the total of 25 on the detector. The second detector had 5 groups of 4 strips each and the remaining two detectors had 5 groups of 5 strips each. The resulting detector system was one with a $5 \times 5$ array of pixels ranging in size from 
$\sim 6 \times 8 \mathrm{~mm}$ to $\sim 10 \times 8 \mathrm{~mm}$ horizontally and vertically. The separation between the front and middle detectors meant that the effective vertical height of each pixel was slightly different from this and had to be calculated from the projection of the horizontal middle strips onto the front detector. The actual sizes, angular resolution and lab solid angles for each pixel are given in appendix A.

The nomenclature used to label these pixels was that the vertical strips on the front detector were numbered F1-F5, F1 being the closest to the beam. The horizontal strips on the middle detector were numbered M1-M5, with M1 being the strip group closest to the reaction plane. Thus, for example, pixel F1M1 was the pixel in the bottom left hand corner of the detector, looking from the target. Similarly F5-M5 was the element furthest from the beam and the reaction plane, in the top right hand corner.

\subsubsection{Energy Calibration}

During the experiment, initial and final energy calibrations were obtained for each detector from ${ }^{241} \mathrm{Am}$ alpha sources placed between the $\Delta \mathrm{E}$ and $\mathrm{E}$ detectors of the triton telescopes and between each of the strip detectors. These alphas entered the strip detectors at very oblique angles and only supplied a first approximation for the strip detector energy calibration. This was later improved using the position of the elastic ${ }^{7} \mathrm{Li}$ events. To make this calibration the thickness of the strip detector had to be measured by $\gamma$ ray attenuation. If similar strip detector set-ups are to be used in future, it would be advantageous to devise a better method for calibrating the energy. This could possibly be done by changing the energy of a light particle beam scattered from a heavy target such that the scattered particles are stepped into each of the strip detectors separately. Alternatively it may be possible to inject a fixed amount of charge from a pulser via a charge terminator into each electronic channel to obtain their relative gains. Once this is done it is much simpler to obtain an absolute calibration for each strip detector as a whole. Finally, the back-bias on each channel was measured using a conventional pulser step-through technique. 


\subsubsection{Summary}

In conclusion, the detector system was comprised of two standard, particle telescopes and a $5 \times 5$ pixel array formed from four crossed strip detectors. Each telescope had a thin surface barrier $\Delta \mathrm{E}$ and a thick $\mathrm{SiLi}, \mathrm{E}$ detector and was used to detect the tritons from the fragmentation of the ${ }^{7} \mathrm{Li}$ projectile. The alphas were detected by the strip detectors with the in-plane angle being measured by the vertical front strips and the out-of-plane angle measured by the horizontal middle strips. The third strip detector recorded the alpha's residual energy and the fourth acted as a veto.

\subsection{Experimental Set-up}

\subsubsection{Overview}

The experimental set-up can be divided into three main sections:

- signal processing and event logic.

- the computer and computer interface (the event manager EM ).

- the analysis software (the data collection programme DCP ).

Each of these different parts of the set-up had an influence on the design of the others. For example, the way in which the DCP analysed the recorded events affected the design of the event logic and the way in which the EM registered the events affected the DCP design. Consequently, each will be reviewed in turn.

The system as a whole had to be capable of performing a number of functions, the most important of which are listed below:

- amplify and shape the output signals from the individual detector elements to allow accurate determination of the energy deposited in each detector. 
- define the required logic coincidences for different events of interest.

- reject any events that are corrupted due to pile-up or incomplete energy measurement.

- record and analyse the signals from the correct detector elements.

- record the total beam current used throughout the experiment.

- allow calculation of the system dead-time for absolute normalisation of the differential cross-sections.

- monitor on-line the status of the detectors and electronics.

How each of these functions was achieved will now be explained in more detail. Since the design of the logic circuit and the DCP depended on the way the EM responded to the input signals it received, a brief description of the EM's operation, along with a short review of the overall computer architecture at the time of the data collection run will be given first. A description of the analogue and logic signal processing will then be given and finally the DCP and the deadtime calculations will be outlined.

\subsubsection{The Event Manager}

At the time of the experiment there were three EMs, each differing in the number of analogue to digital converters (ADCs) available to them. The EM is an intelligent, front end interface between the experimental set-up which provides the analogue signals to the ADCs and the DCP which analyses the converted signals. The way in which the two separate parts interface together is controlled by user defined responses to the logic TRIGGERS sent from the electronic setup. This system allows the user to define the action to be taken on receipt of any one of a number of these predefined trigger signals. For example, if an event is recorded and produces a trigger, then the user can define this as a command to the EM to read a predetermined set of ADCs participating in this event. If another type of event is recorded and produces a different trigger, this can correspond to reading another set of ADCs which may or may not overlap 
those of the first trigger. This is an example of a Direct Trigger multi-parameter event. The EM can support 16 different direct triggers and for each one a register within the EM is loaded with the pattern of the ADCs to be read for that specific trigger. When the direct trigger is sent to the EM it looks at the relevant internal register and checks that all the ADCs that are to be incorporated into the event are processing a signal. If they are all present the event is read, packaged and sent to the data collection processor for writing to tape and analysis by the DCP. If any of the ADCs are absent the event is aborted and the ADCs cleared ready for the next trigger.

These direct triggers are useful when there is only a limited number of types of event that are of interest. In this experiment however, the large number of ADCs (22 in all) and the enormous number of possible different events that could occur meant that it was infeasible to use direct triggers. Another more general type of trigger, the Indirect Trigger, was more useful in this situation. This externally defined indirect trigger, or trigger 24 for short, is formed in a slightly different way from the direct trigger. Instead of predefining the ADCs to be read for each specific trigger signal the pattern of ADCs to be read at each separate event is supplied, by the user, to an external trigger pattern unit. The logical 'AND' of this pattern and the pattern of ADCs in pre-conversion is loaded into a register and the resulting pattern treated as a direct trigger.

The method used in this experiment was to produce this externally defined pattern from the trigger 24 logic signal such that there was always an input present on all the external pattern inputs whenever there was a trigger 24. This meant that the EM read all the ADCs that were in preconversion when the trigger 24 signal arrived. This mode of operation made it easier to define the production of the trigger signal but produced difficulties in calculation of the dead-time of the circuit. This is explained in more detail in section 3.3.7.

\subsubsection{Review of Computer Hardware}

The data handling system at the NSF is based around a network of 5 GEC 4000 series computers. The main idea behind the design of the system is to separate 
the more demanding tasks onto different processors where the performance will not be degraded by less important tasks. The functions of the Data Handling System were divided amongst the five machines as shown below:

- Resources Machine ( R1 machine ): This was a GEC 4190 processor, known as NNGA, which controlled the system peripherals. These included $3 \times 80$ $\mathrm{Mb}$ bulk disc stores, 4 tape drives (1600 bpi), line printer, Benson pen plotter and versatec electrostatic plotter. It was also connected via the PAD connection to the SERCNET computer network.

- Accumulation Machines (A machines ): Each EM had its controlling A machine ( EM1 - NNGC, EM2 - NNGE and EM3 - NNGD respectively). These were GEC 4085 processors whose function was to set-up and control the EM, read out blocks of multi parameter data, generate sorted spectra and send these multi-parameter events to the R1 machine for writing to magnetic tape. This was where the data acquisition software was run.

- Control Machine ( C machine ): The C machine was a GEC 4190 processor known as NNGB linked by a CAMAC serial highway to four graphics stations. Its function was to provide an interactive environment for setting up and controlling experimental data taking, monitoring of spectra generated by the sort programme and to provide facilities for off-line analysis of data.

The data were collected and monitored online using this computer network and were later analysed more extensively offline using the Edinburgh GEC 4190 workstation.

\subsubsection{Analogue Signal Processing}

The analogue side of the signal processing will now be discussed in detail. Fig.3.6 is a circuit diagram of the full experimental logic set-up used for the data collection. 


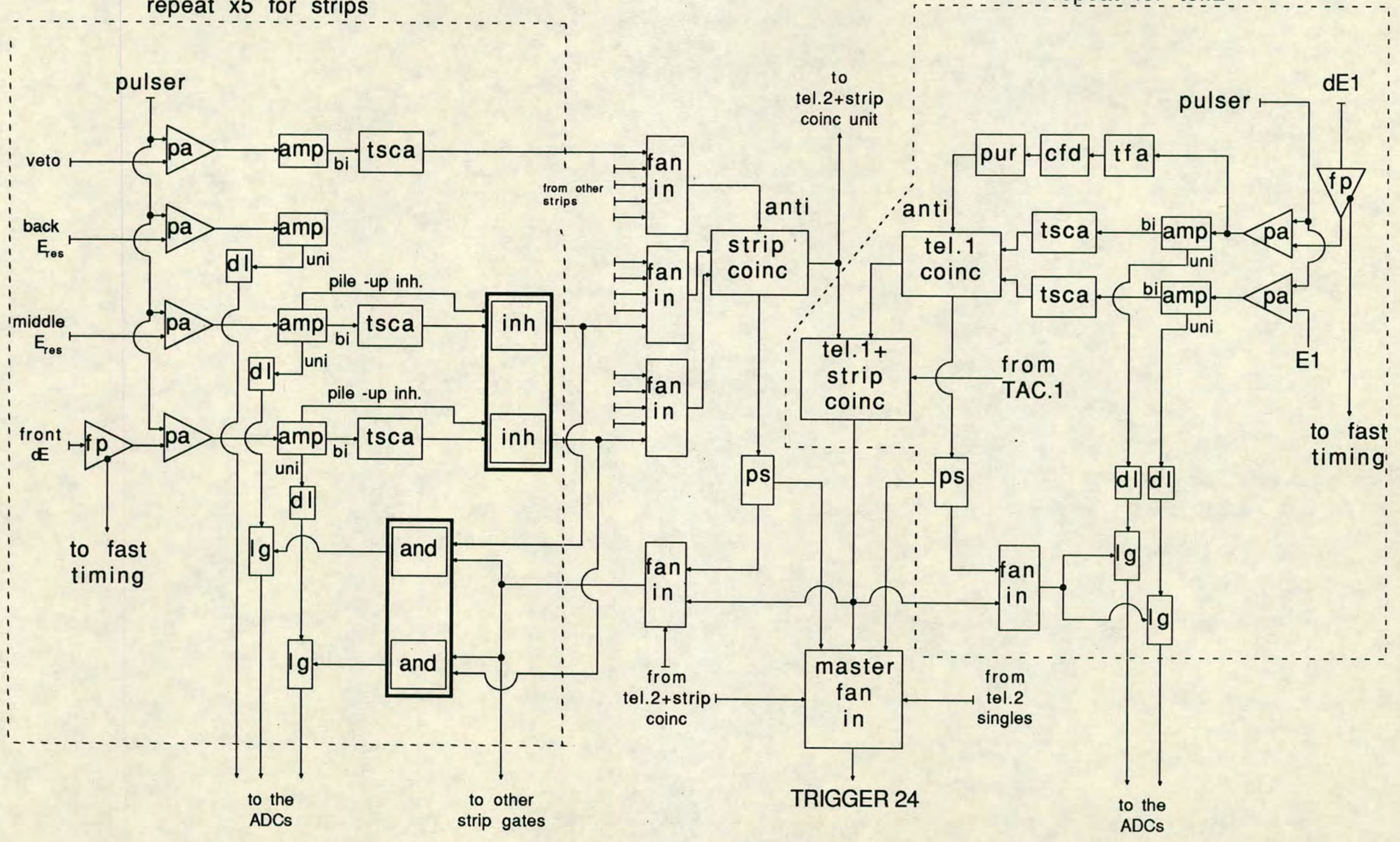

Figure 3.6: Experimental set-up of data collection circuit. 
Each strip detector had its own bias voltage supply and the voltage to each of its groups was obtained from a voltage divider unit with $10 \mathrm{M} \Omega$ bias resistors on each branch. The detectors in the triton telescopes were also biased separately.

The outputs from the detectors and strip groups were fed into Cooknell charge sensitive pre-amps (PA) with a nominal gain of $20 \mathrm{mV}$ per $\mathrm{MeV}$ of energy deposited in the detector. On the $\Delta \mathrm{E}$ channels the inputs to the pre-amps were picked off with Cooknell W2000 fast timing pre-amplifiers (FP) and $\times 10$ D.C. amplifiers to be used on the fast timing side of the logic circuit. After being transfered to the main N.S.F. control room the output of the charge sensitive pre-amps were further amplified by Ortec 572 main amplifiers. The amplification at this stage was chosen to have the dynamic range required for the $\Delta \mathrm{E}$ and $\mathrm{E}$ detectors. All the energy signals were processed with a $0.5 \mu$ sec shaping constant. The unipolar output from all the detectors, except the veto detector channels, were then routed into the relevant ADCs via Canberra 1457 delay line amplifiers (DL) and 1454 linear gates (LG). These were necessary for the dead-time calculations and will be explained later.

The bipolar outputs from the 572 amplifiers were used to produce the event logic and were fed into Canberra 2037A timing single channel analysers (TSCAs). The rest of the logic processing will be described in the next section.

Each of the triton $\Delta \mathrm{E}$ detectors and every second strip group on the front and middle detectors had a ratemeter attached to the corresponding 572 count-ratemonitor output. These allowed the beam current to be optimised for each target and detector angle combination. This was important in achieving the optimum data collection rate compatible with keeping the detector rates $\leq 10-12 \mathrm{KHz}$. It was possible to have rates somewhat higher than this without degradation of the energy signals from piled-up events by using a pile-up reject unit (PUR). For the two triton telescopes this PUR was achieved by using the output of the $\Delta \mathrm{E}$ fast timing amplifiers (TFA) into constant fraction discriminators (CFD). The CFD output was then inspected by an Ortec 404A PUR unit which supplied an anticoincidence logic signal to the relevant triton telescope $\Delta \mathrm{E}-\mathrm{E}$ coincidence unit on detection of a piled-up event. The inspect time used for this was $4 \mu$ sec with an inhibit pulse of $8 \mu \mathrm{sec}$ duration. The PUR for the strip detector groups was more 
important due to their larger surface area. PUR also had to be performed on both the front and middle groups because they were at $90^{\circ}$ to one another. The back strip detector was not inspected because, being aligned with the middle detector, any event that piles up in it would also pile-up in the middle detector and thus be vetoed anyway. The internal PUR circuit in the 572 shaping amp was used to perform this pile-up inspection for the front and middle strip groups. This part of the circuit had an inspect and inhibit time of six times the shaping constant used. For this set-up it was $6 \times 0.5=3 \mu \mathrm{sec}$ inspect and inhibit. The output pulse from the detection of a piled-up event in the strip detector was used to inhibit the output of the corresponding TSCA and hence suppress the detection of a corrupted $\Delta \mathrm{E}-\mathrm{E}$ coincidence. Scalers were attached to all these pile-up outputs to monitor the pile-up rate and to ensure this remained at a small percentage of the valid coincidence rate.

The fast timing side of the analogue signal processing, see fig.3.7, was done on the output from the W2000 fast timing pre-amps. These were on the $\Delta \mathrm{E}$ branches of the output signals from the telescopes and each group on the front strip detector. These fast timing signals were amplified with Ortec 474 fast amps and then fed into Ortec 934 CFDs. The outputs from the telescope CFDs were used to start two time to amplitude convertors (TACs), one for tel. $1+$ strip det. coincidence events and another for tel.2 + strip det. coincidence events. The five front strip constant fraction ouputs were fanned together with a Lecroy 428 linear fan in and the resulting signal, delayed by $200 \mathrm{~ns}$, was used as a stop signal for both the TACs. The analogue output from the TACs were inputted into individual ADCs and the logic outputs used to complete the coincidence requirement for a particle-particle coincidence.

During the experiment the beam current was monitored by a Brookhaven Current Integrator (BCI). This was connected to the final Faraday cup and gave out a predetermined number of pulses for every electrical nano-amp of current collected. The BCI output was used to fire a pulser whose output was injected into the pre-amplifier test inputs of all the strip detector groups (except the veto detector) and triton telescope detectors. This meant that the total integrated beam current for each experimental run could be calculated from the number of output pulses from the pulser. This number was recorded on both Camac 


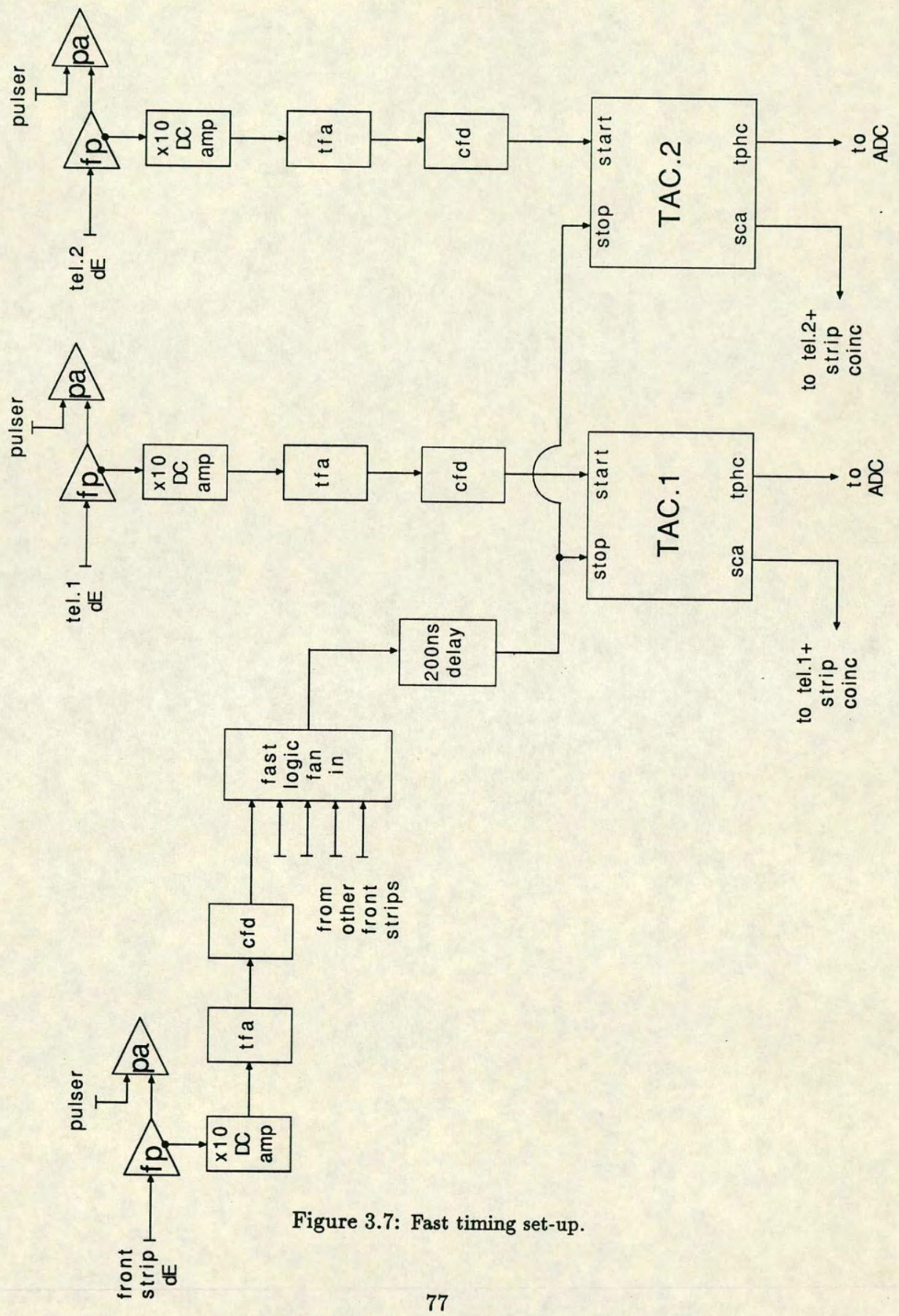


and NIM scalers. The BCI output was also inputted directly into an ADC. This meant that every pulser event injected into the system that caused a trigger 24 was 'tagged' by a conversion in this ADC and could easily be identified. This was extremely useful in the estimation of the dead-times necessary for absolute cross-section calculations.

\subsubsection{Logic Signal Processing}

The following section, on the rational behind the event logic design, should be read in close conjunction with fig.3.6.

The logic used throughout was based on the normal fast-slow coincidence technique. Slow logic, with characteristic times of the order of a few micro-seconds, was used to define a coincidence between the detectors within a telescope for $\Delta \mathrm{E}$-E identification and between telescope and strip detector for particle-particle coincidences. Fast logic, with characteristic times of the order of a few nanoseconds, was later used in software analysis to improve the time window for particle-particle coincidence events. This cut down the the number of random coincidences mistaken for real events.

The electronic logic set-up had to be capable of performing the following functions:

- allow uncorrupted inclusive data to be collected at a predetermined prescale rate by detecting a $\Delta \mathrm{E}-\mathrm{E}$ coincidence between the relevant detectors in each separate detector system.

- allow collection of uncorrupted particle-particle coincidence events by detecting a coincidence between a valid telescope event and a valid strip detector event.

- provide a measure of the total dead-time of the data collection system for absolute cross-section measurements. 
(a)

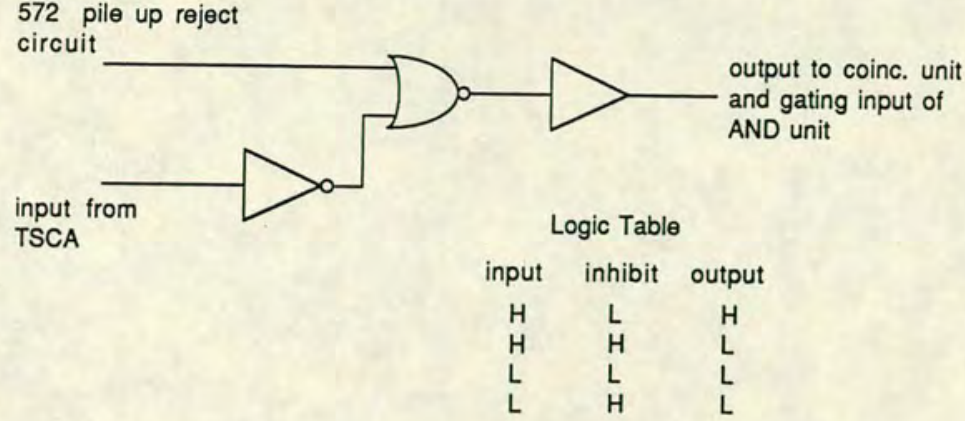

(b)

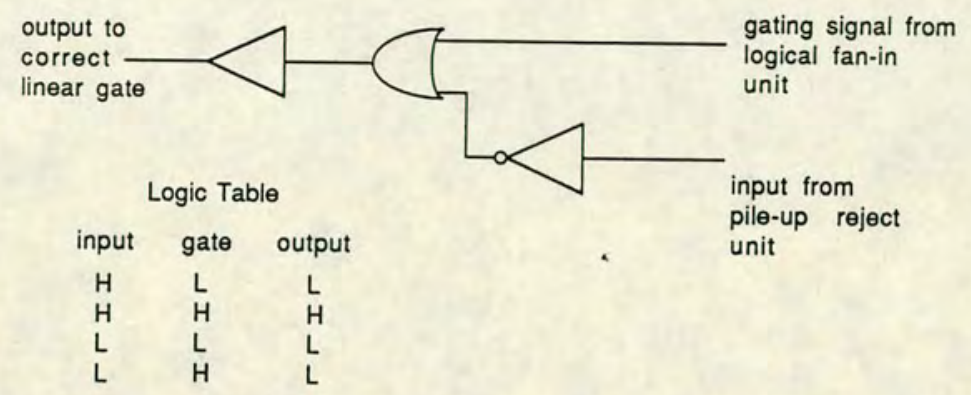

Figure 3.8: Circuit diagram of home built units. (a) pile-up inhibit unit. (b) and gating unit.

As previously mentioned, the bipolar signal from the 572 main amp was used to produce a logic signal via a TSCA operating in its crossover mode. The output from this was then inhibited if a pile-up event had been detected. This inhibit was performed at different stages for the strip detector and the telescope systems. The latter inhibit was anti-coincidenced with the $\Delta \mathrm{E}-\mathrm{E}$ signals at the telescope valid event coincidence unit. The strip detector logic signals had to be inhibited before this as the logic signals from all the front detector groups were OR'd together (as were the logic signals from the middle detector). It was important for the calculation of the individual element dead-times, that the information on which group piled-up was preserved. This inhibit was performed by a home built pile-up gate with each TSCA output being inhibited by its corresponding 572 pile-up output signal ( circuit marked 'inh' in fig.3.6). The circuit for one channel of this unit is shown in fig.3.8. The full unit had 10 such channels, one for each of the five front and five middle strip groups. Careful tests were performed with pulser signals to ensure there was no cross-talk between different channels in this unit. 
Once the strip logic signals had survived the pile-up gate, the front strips were OR'd together using an Ortec $418 \mathrm{~A}$ coincidence unit set on coincidence requirement 1 ( marked as 'fan-in' in fig.3.6). The middle strip groups were treated similarly. From this point on all the front groups and middle groups were treated as a single $\Delta \mathrm{E}$ or $\mathrm{E}$ detector respectively. To register a valid strip detector event, the next coincidence unit required a front detector and middle detector signal with no veto signal. The third strip detector, the back detector, did not participate in the event logic. If there was an ADC from the back detector in preconversion when a strip detector event caused a trigger 24 then this ADC was read as part of the event structure. If there wasn't, the strip detector event was treated as if it had only a front and a middle detector conversion.

The output from this valid strip detector coincidence event was then used to test for a tel. 1 + strip det. + TAC. 1 coincidence and a tel. 2 + strip det. + TAC. 2 coincidence. If there was a valid coincidence event the output was used to send a trigger 24 signal to the EM and to open the correct linear gates. This allowed the correct unipolar signals to reach the ADCs. All the ADCs in preconversion were then read by the EM, stored for online analysis and written, event by event, to tape for further, more detailed offline analysis.

To succeed in opening the strip detector linear gates the coincidence logic signal ( unit marked 'and' in fig.3.6) also required a signal from the output of the pileup gate unit. This ensured that only the gates which corresponded to the strip detector groups forming the coincidence were opened. This had a twofold effect in that it reduced the dead-time associated with the ADCs being run at the full uninterrupted singles rate and it allowed the calculation of each individual element dead-time. This latter point will be explained more fully later. One channel of the home built unit that performed this gating is also shown in fig.3.8. Again, the full unit had ten such channels.

The linear gates and EM were also triggered by the output of the telescope and strip detector pre-scaled singles events, allowing the collection of the inclusive data. In summary, the EM recorded an event when a trigger 24 was produced 
by one of the following types of event :

- a telescope 1 , telescope 2 , or strip detector pre-scaled singles event

- a telescope 1 and strip detector coincidence event

- a telescope 2 and strip detector coincidence event

- a pulser signal simulating any one of the above

The logic circuit that produced this trigger signal could be divided into four main parts :

1. logic to determine a valid strip detector $\Delta \mathrm{E}-\mathrm{E}$ event.

2. logic to determine a valid tel.1 or tel.2 $\Delta \mathrm{E}-\mathrm{E}$ event.

3. logic to determine a valid coincidence between (1) and (2).

4. a section for production of the correct gating and trigger logic.

This is illustrated in fig.3.9 which shows a simplified block diagram of the logic set-up.

Once the event was recorded, the event package was written to tape and the DCP inspected the ADC conversions present, to determine the type of event that had occured. How this was done is the topic of the next section.

\subsubsection{Data Collection Programme (DCP)}

The data collection programme is a fortran routine, written by the user, structured for his particular experimental application. It consists of a number of Entry Points which are accessed from the main acquisition programme during a data collection run. The main entry point initiates the analysis of the event package read from the EM into the correct mass and energy gated spectra. Other entry 


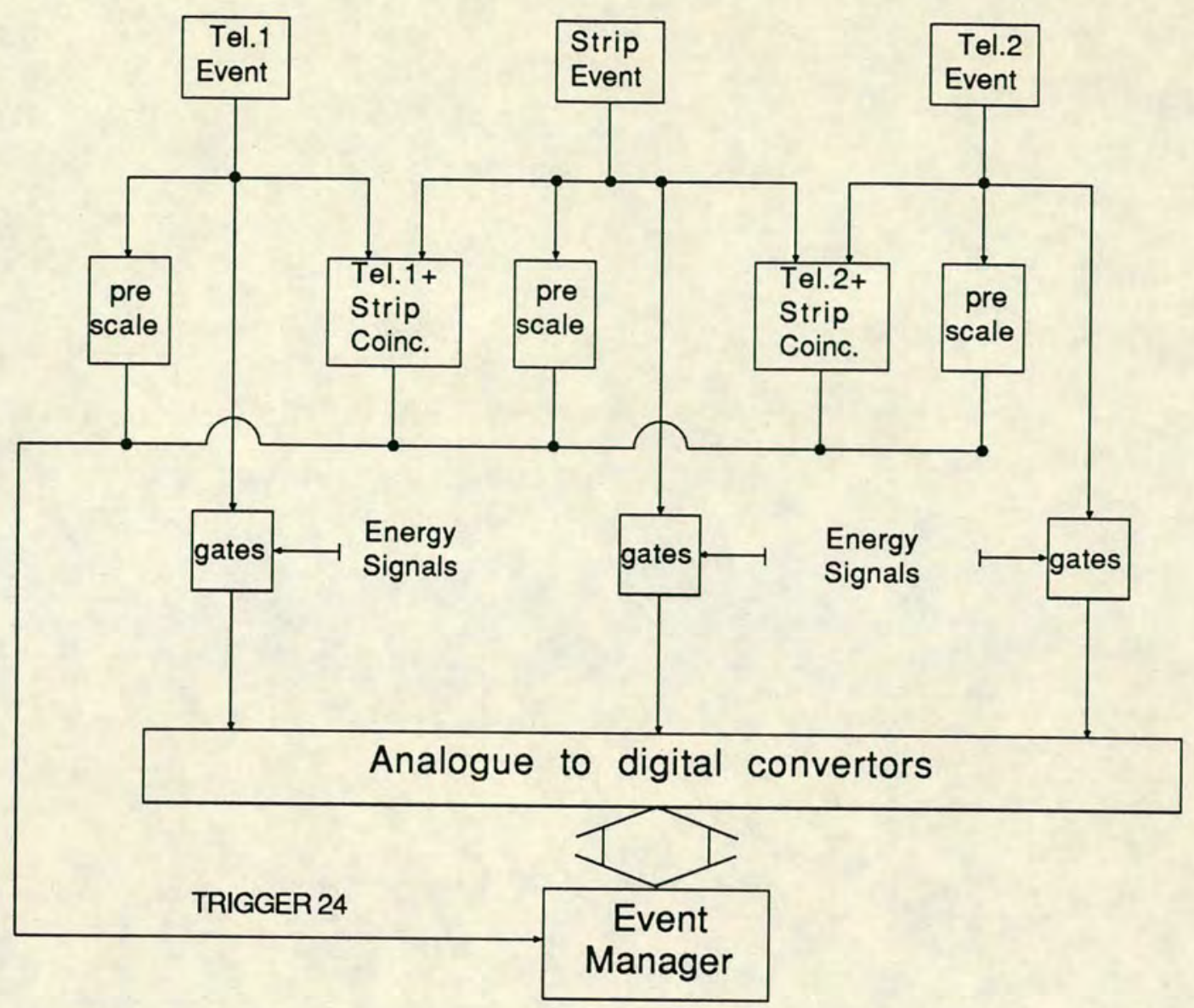

Figure 3.9: Simplified block diagram of event logic

points are defined which set-up the online spectra, acquire the energy conversion factors, the back biases, the energy and mass windows etc. These latter entry points are normally only accessed once during the experimental run whereas the event analysis entry point is called for every event package. One of the more frequently used entry points was for the dead-time calculation routine. This compared the number of events formed from pulser coincidences with the total number of pulser events detected by the EM. It gave an accurate online monitor of the dead-time for the triton telescopes and each detection element of the strip detector in both singles and coincidence mode. This was valuable because it allowed the beam current to be optimised whilst keeping the dead-time for coincidence measurements at a reasonable level $(\leq 20 \%)$. It also helped pinpoint problems with the electronic set-up by highlighting any anomalous dead-time behaviour.

Identification of events: The main entry point routine took the event supplied 
from the EM and created a bit pattern index number (BPI). This was done by assigning the value:

$\mathrm{BPI}=\sum_{n} 2^{n} \quad$ where $\mathrm{n}$ is the EM channel number of all ADCs in event.

This meant that every conceivable combination of EM channels had a unique value of this BPI. The routine then inspected this index to identify the following types of event:

1. strip det. telescope singles event i.e. only conversions from the strip detector EM channels present.

2. triton telescope singles event i.e. only conversions from one of the triton telescopes are present.

3. strip det. and tel.1 coincidence event, identified by having a TAC.1 conversion without a TAC. 2 or pulser ADC conversion present.

4. strip det. and tel.2 coincidence event, identified as in (3).

5. a pulser event, identified by the presence of the pulser ADC in the event.

The inspection was done by requiring the BPI to be equal to a certain value or less than a certain limit, determined by the type of event eg. a tel.2 singles event incorporating EM channels 16 and 17 will have a $\mathrm{BPI}=2^{16}+2^{17}=196608$ so the BPI index had to equal this if it was to be identified as a tel.2 singles event. It was impossible to test for each of the possible combinations of the strip detector groups so a strip detector singles event was identified as having a BPI less than $2^{16}$ (the strip detectors occupying the first $15 \mathrm{EM}$ channels). Similarly a strip detector + tel. 1 coincidence event had to have a TAC. 1 event present. The resulting BPI therefore had to lie within the limits $2^{18}$ and $2^{19}$ when TAC. 1 was in EM channel 18 and all higher channels were occupied by signals not involved in this type of event.

Once the event had been identified the routine then checked all the EM channels present. This was important for events identified by a limit on the BPI (e.g. the strip detector singles events) rather than an explicit value, because these events 
may still not contain the correct EM channels. As an example, a strip detector singles event must have its first conversion from the front strips, its second from the middle strips and its third, if any, from the back strips. If there was more than one conversion from a specific strip detector it was impossible to tell which was the valid conversion and the event was discarded. The event also failed if there was a conversion missing.

If the event survived all these checks on the event package construction the energies of the ADC conversions were calculated. From these the mass of the particles in each detector was calculated from the standard mass identification algorithm [Ce66].

$$
P I \propto T Z^{2} M^{n-1} \propto\left(\Delta E+E_{\text {res }}\right)^{n}-E_{\text {res }}^{n}
$$

with $\mathrm{T}$ the detectors thickness, $\mathrm{Z}$ the particles charge and $\mathrm{M}$ its mass. The parameter $\mathrm{n}$ ranges from $\sim 1.69-1.76$ and was optimised by viewing a 2 dimensional PI vrs $\left(\Delta \mathrm{E}+\mathrm{E}_{\text {res }}\right)$ plot. An example of the mass resolution obtained from the triton telescope systems is shown in fig.3.10. The $\mathrm{Z}=1$ particles are clearly resolved. Figure 3.11 shows an example of the excellent mass resolution obtained for each pixel across the face of the strip detector telescope. Again the $\mathrm{Z}=1$ and $\mathrm{Z}=2$ particles are clearly resolved. Mass, timing and enery gates were then applied and any surviving events used to increment the spectra of interest.

Unfortunately this method of selecting valid events had an intrinsic problem. This arose due to the possibility of a random event accompanying the event of interest. This random conversion would cause the event to fail by either affecting the BPI or violating the requirement of one detector, one conversion. They thus contributed to the dead-time of the system by causing a valid event to be rejected. A solution to this problem was achieved in the way the pulser events were used to define a valid event for the dead-time calculation. This will be discussed in more detail in the next section.

In summary the DCP was a user defined fortran routine which accepted event packages from the event manager, ensured they had a valid event construction, tested these valid events against predetermined energy, mass and timing gates 


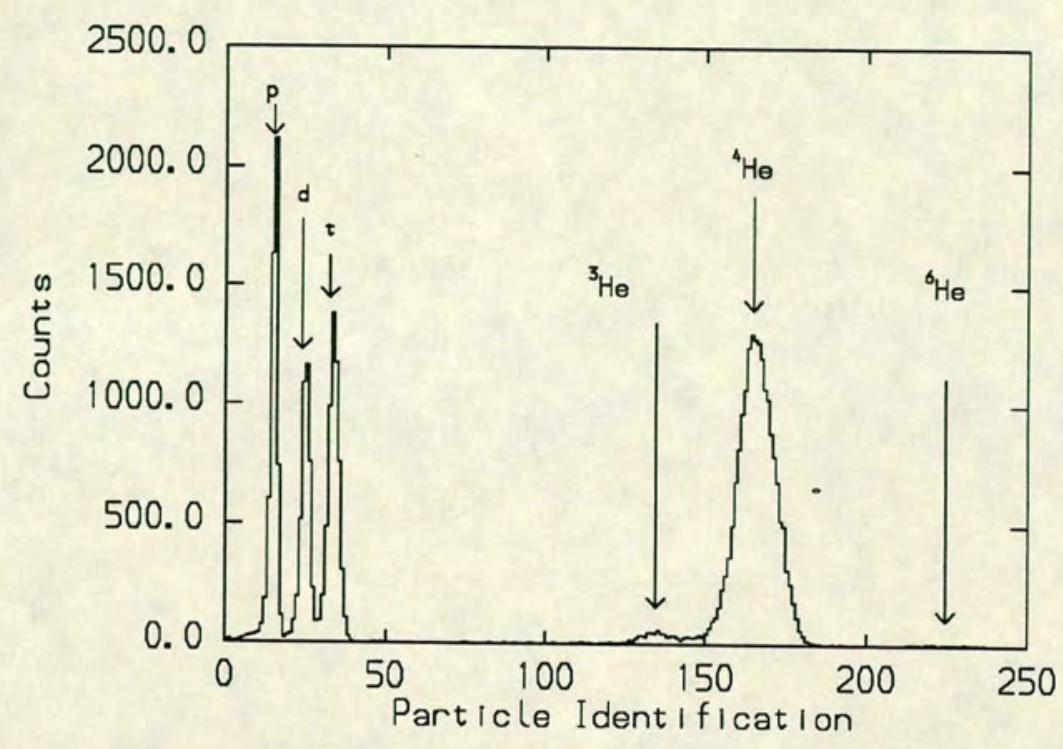

Figure 3.10: Typical mass spectrum obtained from the triton telescope systems.

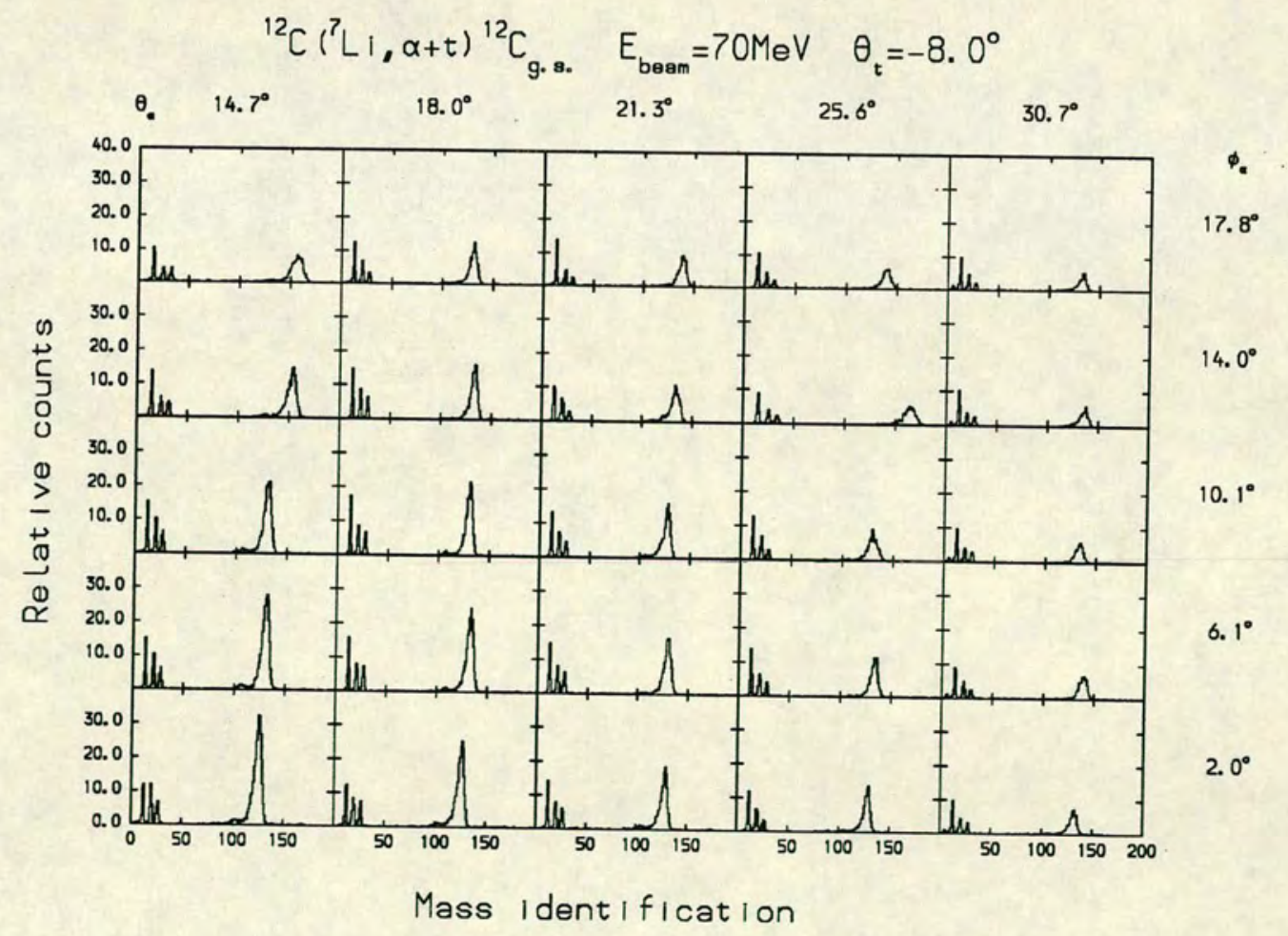

Figure 3.11: Mass spectra from each of the 25 pixels on the strip detector telescope showing the excellent mass separation. The individual peaks correspond to, from the left, protons, deuterons, tritons, ${ }^{3} \mathrm{He}$ and ${ }^{4} \mathrm{He}$. 
and incremented the correct spectra. It also allowed online calculation of detector dead-times and acted as an online monitor for detecting any problems with the electronic set-up.

\subsubsection{Dead-time Calculations}

When a pulser event was triggered into the detection system by the BCI, all the detectors (except the veto detectors) registered this event at the same time. As has been explained previously, this caused problems with identifying which groups, if any, of the strip detectors were responsible for the resulting trigger 24 sent to the EM. Unless the whole detection system was completely dead a trigger 24 was sent to the EM for every BCI output. Assuming that every pulser event was tagged by a pulser ADC conversion, it was possible to calculate the deadtime for all the possible detector configurations of interest. A comparison of the pulser ADC spectra in singles and multi-parameter mode ${ }^{1}$ showed that the system produced a trigger 24 for $99.9 \%$ of all the injected BCI pulses. On a number of occasions, deviations from this value pinpointed problems with the EM acquisition system.

In a set-up where a number of channels are fanned together, as in this case, it is necessary to calculate the individual pixel dead-times as this will vary across the face of the strip detector. This was achieved by requiring that if pixel F.n,M.m was to be registered as live, then both these ADCs had to record an event within their pulser peak windows. Similarly if this pixel was to be registered as live in coincidence with a tel.1 event, then the tel.1 detectors and the TAC.1 ADCs all had to register an event within their specified pulser windows. In this way the dead-time for each pixel and telescope system was calculated in both singles and coincidence mode.

Care must be taken with this procedure to ensure that the full dead-time is calculated for each event configuration. One possible pitfall can be seen as follows. If one of the strip detector TSCAs was to stop operating, this channel

\footnotetext{
${ }^{1}$ The multi-parameter mode spectrum was only incremented for those pulser events accompanied by a trigger 24 .
} 
would become $100 \%$ dead. However, because the other four strip groups are fanned into the strip detector coincidence unit it only requires one of them to complete a strip event. This would create a trigger 24 and the pulser conversion at the faulty channel's ADC would be recorded as a live event. A similar type of error will be recorded if any of the pulser events cause a pile-up on the strip detector, vetoing one of its TSCA outputs. What effectively happens is that all the strip groups fanned together appear to have a dead-time smaller than the smallest of the group. A solution to this problem was achieved by the use of the linear gates and the home built 'AND' unit used to gate them. This meant that only those channels whose TSCA had produced an unvetoed logic output in coincidence with a trigger 24 were opened to their ADCs.

This method of calculating the dead-time also took into account the effect of random pulses destroying the event structure of valid events. This was because when the pulser event package was inspected, if any of the EM channels had a conversion outside the relevant pulser window, this was interpreted as a random event which would destroy any valid coincidence event. In this case the pulser event was aborted and the whole system taken as dead.

So, a telescope+pixel configuration was assumed dead in one of two situations, either:

- a random event had been detected outside the pulser windows,

- or a strip group, telescope or TAC conversion was completely absent.

By this method it was possible to calculate a realistic dead-time for each of the possible detection configurations.

\subsubsection{Cross-section Calculations}

Once the data were sorted into the required energy spectra the actual differential cross-section for coincidence events was calculated from equation 3.1. 


$$
\frac{d^{2} \sigma}{d \Omega_{\alpha} d \Omega_{t}}=\frac{2.66 \times 10^{-7} N Z A}{I d \Omega_{\alpha} d \Omega_{t} f T} \mathrm{mb} / \mathrm{sr}^{2}
$$

where

- $\mathrm{N}$ - is the number of counts of interest

- $\mathrm{Z}$ - is the average charge of the beam after traversing the target (for all targets used $\mathrm{Z}=3(+)$ )

- A - is the target mass ( in A.M.U.)

- I - is the integrated beam current ( in $\mu \mathrm{C}$ )

- $\mathrm{d} \Omega_{\alpha}$ - is the solid angle of the alpha detector (in sr )

- $\mathrm{d} \Omega_{t}$ - is the solid angle of the triton detector (in sr )

- $\mathrm{f}$ - is the fractional live time

- $\mathrm{T}$ - is the target thickness ( in $\mathrm{mg} / \mathrm{cm}^{2}$ )

When sequential cross-sections are being discussed this formula was modified by replacing $d \Omega_{\alpha} d \Omega_{t}$ with $d \Omega_{\text {eff }}$. This $d \Omega_{\text {eff }}$ is the effective solid angle of the detector set-up for detection of particles from a specific sequential state. It was calculated from a Monte Carlo simulation code written to model the detector system used in this experiment. This is discussed in more detail in section 5.1.

The main source of error entering into the double differential cross-section calculations depended on the target being studied. For the heavier targets the statistical errors tend to dominate whereas for carbon these tend to be less important.

The transmission of the beam from the diagnostics box to the beam dump Faraday cup was better than $99 \%$ and so the error in the beam current on target was assumed to have an upper limit of $\sim 2 \%$ ( which includes an estimate of the error on the BCI output). The error on the solid angles of the different detectors was estimated at $\sim 2 \%$ from the error in the target to collimator distances. The 
error in $d \Omega_{\text {eff }}$ was much more sensitive to the angle between the alpha and triton detectors and depended greatly on the different targets and sequential states.

The main experimental error, outwith the statistics, was from the determination of the target thickness. This was estimated to have an upper limit of $\sim 10 \%$.

All these factors gave an upper limit of the systematic error of $\leq 12 \%$ for the measurements of the double differential cross-sections.

\subsubsection{Targets}

All the targets used in this thesis were solid, self supporting sheets of enriched target material. A number of considerations influenced the choice of targets. These were:

- availability of other data for comparison

- clean separation of ground and first excited states

- ease of manufacture of target

The targets chosen were ${ }^{12} \mathrm{C},{ }^{120} \mathrm{Sn}$ and ${ }^{208} \mathrm{~Pb}$.

Each target was mounted on its own aluminium holder and this, in turn, mounted on the target ladder in the centre of the scattering chamber. The thickness of the targets used in the experiment are shown in table 3.1.

\begin{tabular}{|c|c|}
\hline target & $\begin{array}{c}\text { thickness } \\
\left(\mathrm{mg} / \mathrm{cm}^{2}\right)\end{array}$ \\
\hline${ }^{12} \mathrm{C}$ & 0.54 \\
${ }^{120} \mathrm{Sn}$ & 2.9 \\
${ }^{208} \mathrm{~Pb}$ & 4.2 \\
\hline
\end{tabular}

Table 3.1: Target thicknesses 


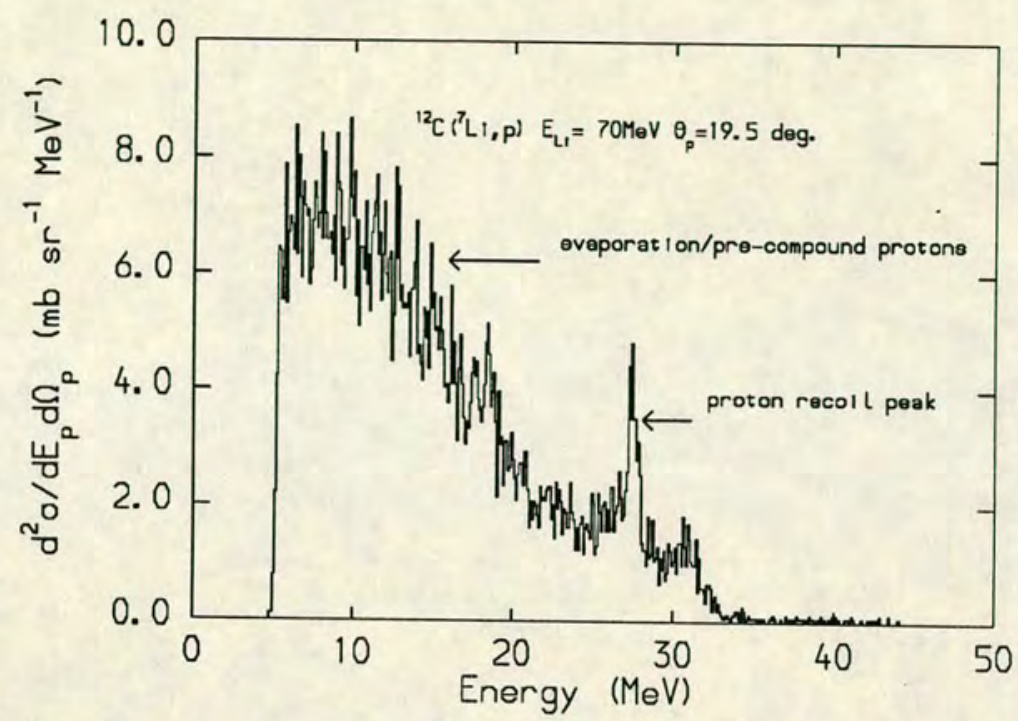

Figure 3.12: Inclusive proton spectrum from ${ }^{12} \mathrm{C}$ target showing peak from recoil protons.

These thicknesses were measured by the energy loss of $5.486 \mathrm{MeV}$ alpha particles, from ${ }^{241} \mathrm{Am}$ passing through the foils and were estimated to be $\leq 10 \%$ accurate.

No trace of any impurities (eg. ${ }^{16} \mathrm{O}$ ) were seen in the ${ }^{7} \mathrm{Li}$ elastic scattering energy spectra from any of the targets, however fig.3.12 shows the inclusive proton energy spectrum for scattering from the ${ }^{12} \mathrm{C}$ target. The obvious peak at $27.4 \mathrm{MeV}$ was identified as recoil protons from hydrogen in the target. This was further supported by the existence of events in the $\alpha$ vrs. $t$ two dimensional energy spectrum ( see fig.1.2) and the total kinetic energy spectra (eg. fig.4.10) which were consistent with break-up of ${ }^{7} \mathrm{Li}$ on hydrogen. Events of this type were also seen in similar plots from the ${ }^{120} \mathrm{Sn}$ and ${ }^{208} \mathrm{~Pb}$ targets. This was indicative of hydrocarbons from the vacuum system contaminating the surface of all the targets. For most of the angular settings used during the experiment the detectors were outwith the kinematically allowed break-up cone for break-up from hydrogen. For these settings the contamination did not affect the data. At more forward angles the unwanted events were avoided by gates placed on the TKE peaks of interest. 


\section{Chapter 4}

\section{Data Presentation}

In this chapter, the inclusive and exclusive data recorded with the ${ }^{12} \mathrm{C},{ }^{120} \mathrm{Sn}$ and ${ }^{208} \mathrm{~Pb}$ targets will be presented. The inclusive data will be presented in section 4.1 and compared to similar data collected in another experiment [Da87]. This will show the good agreement between these two data sets, and give increased confidence in the exclusive in and out-of-plane data presented in sections 4.2.1 and 4.2.2 respectively. Angular distributions for quasi-elastic events and events leaving the ${ }^{12} \mathrm{C}$ target in its first excited state $\left(4.44 \mathrm{MeV}, 2^{+}\right)$will be presented in these sections. For completeness, the angular distributions for the full $\alpha$-t coincidence channel, irrespective of the final state $Q$ value, will also be presented. Finally the angle integrated cross-sections for fixed values of $\theta_{t}$ will be presented in section 4.3.

\subsection{Inclusive Data}

In this section the inclusive data from the reaction of $70 \mathrm{MeV}{ }^{7} \mathrm{Li}$ with ${ }^{12} \mathrm{C},{ }^{120} \mathrm{Sn}$ and ${ }^{208} \mathrm{~Pb}$ will be presented. These data were collected simultaneously with the exclusive $\alpha$-t coincidence data. Similar datahave already been reported [Da 87] and the present data were collected more as a test of the experimental set-up and data analysis system than as an extensive investigation of the inclusive data in its own right. Nevertheless the material exhibits features worthy of mention. Typical energy spectra for inclusive alpha and triton particles from different 
targets are presented in figs.4.1 to 4.6 .

These data, taken at forward and backward angles, exhibit the typical features common to other investigations using light heavy ions at similar energies [Me85], [Ca80], [Ne82], [Sh84]. These features are:

- distinct, broad, structureless bumps at energies around the beam velocity ( $\left.\mathrm{E}_{\text {bump }} \sim \mathrm{E}_{\text {beam }}\left(\mathrm{m}_{\text {frag }} / \mathrm{m}_{\mathrm{Li}}\right)\right)$.

- a long exponential background extending to high energies.

- high energy structure attributed to massive transfer eg. ${ }^{12} \mathrm{C}\left({ }^{7} \mathrm{Li}, \mathrm{t}\right){ }^{16} \mathrm{O}^{*}$.

The bump is concentrated at forward angles whereas at greater deflections the exponential background becomes increasingly important. This forward focusing is taken as evidence that the particles contributing to the bump originate from direct processes, whereas the background is assumed to arise from equilibrium and pre-equilibrium emission from the excited compound nucleus. The ${ }^{12} \mathrm{C}$ background may also consist of significant contributions from target fragmentation. It can be seen that the absolute magnitude of the evaporation component is significantly reduced with the heavier targets. This is a consequence of the reduced emission probability due to the increased Coulomb barrier. At the higher energy end of the ${ }^{12} \mathrm{C}$ inclusive triton spectrum there is evidence of alpha transfer to states in ${ }^{16} \mathrm{O}$. These correspond to excitations of $\sim 21 \mathrm{MeV}$ and $\sim 17.5 \mathrm{MeV}$ where a number of resonances in the ${ }^{12} \mathrm{C}+\alpha$ system are known to exist [Aj86]. The beam velocity bump is interpreted as arising from reactions in which the projectile has fragmented into its constituent clusters under the influence of the Coulomb and/or nuclear force between it and the target. These clusters then continue on their initial trajectories affected by only the Coulomb field, or by further target fragment interactions. The former are known as spectator fragments and the latter as participant fragments. This target fragment interaction takes the form of normal nuclear interactions such as elastic scattering, inelastic scattering with the target or fragment left in an excited state, or fusion of the fragment with the target. 

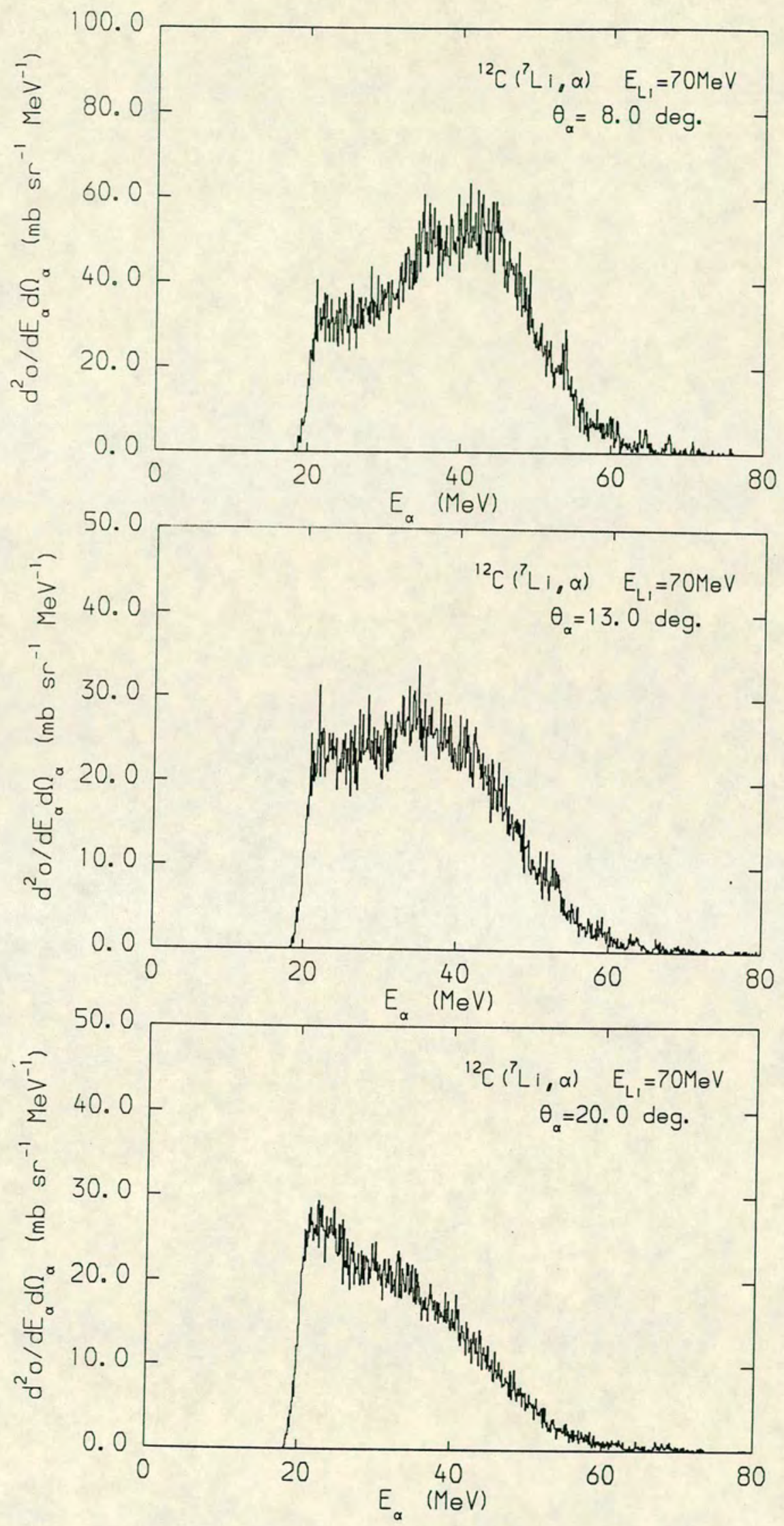

Figure 4.1: Inclusive alpha energy spectra from ${ }^{12} \mathrm{C}$ for varying $\theta_{\alpha}$. 

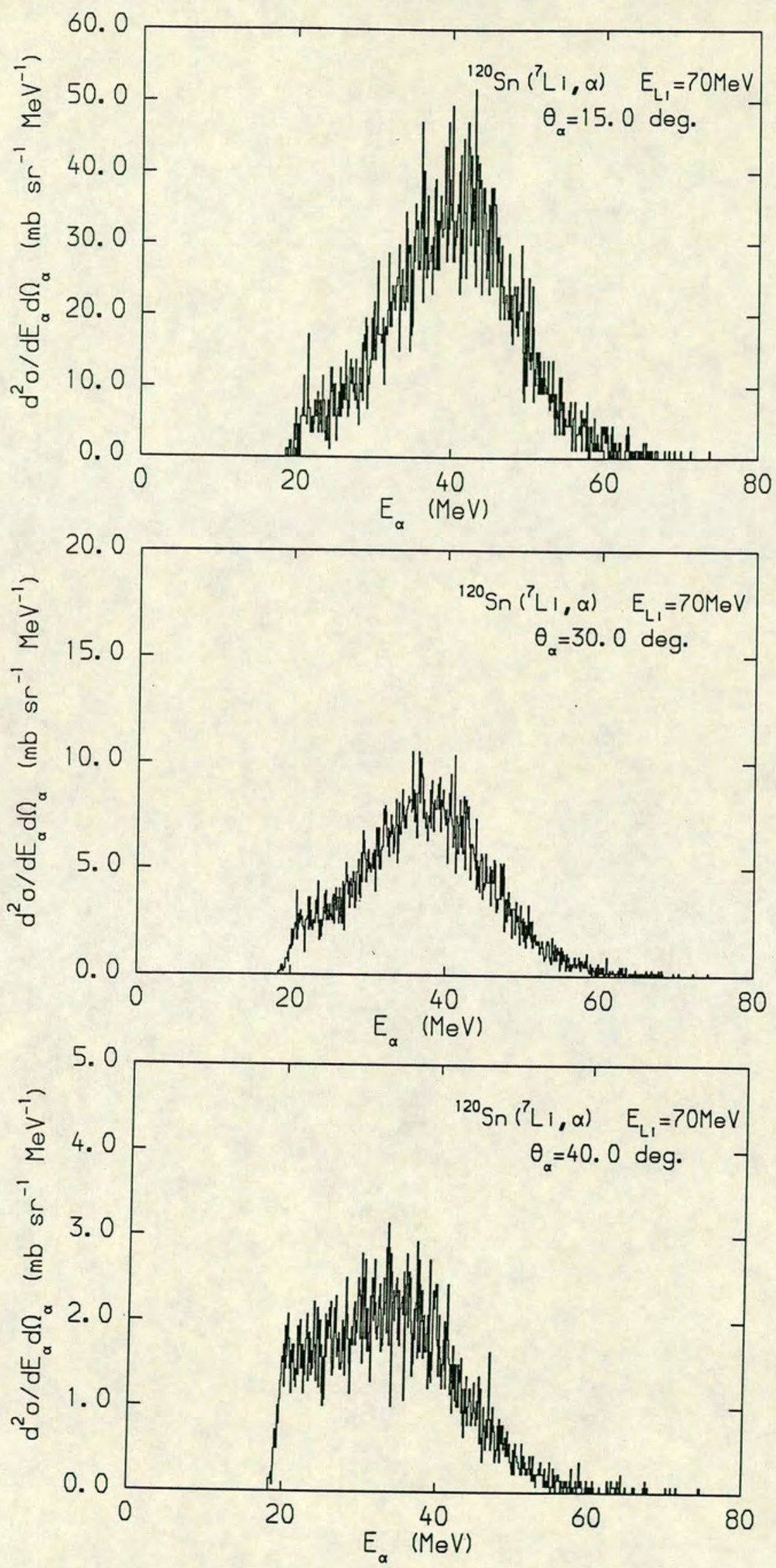

Figure 4.2: Inclusive alpha energy spectra from ${ }^{120} \mathrm{Sn}$ for varying $\theta_{\alpha}$. 

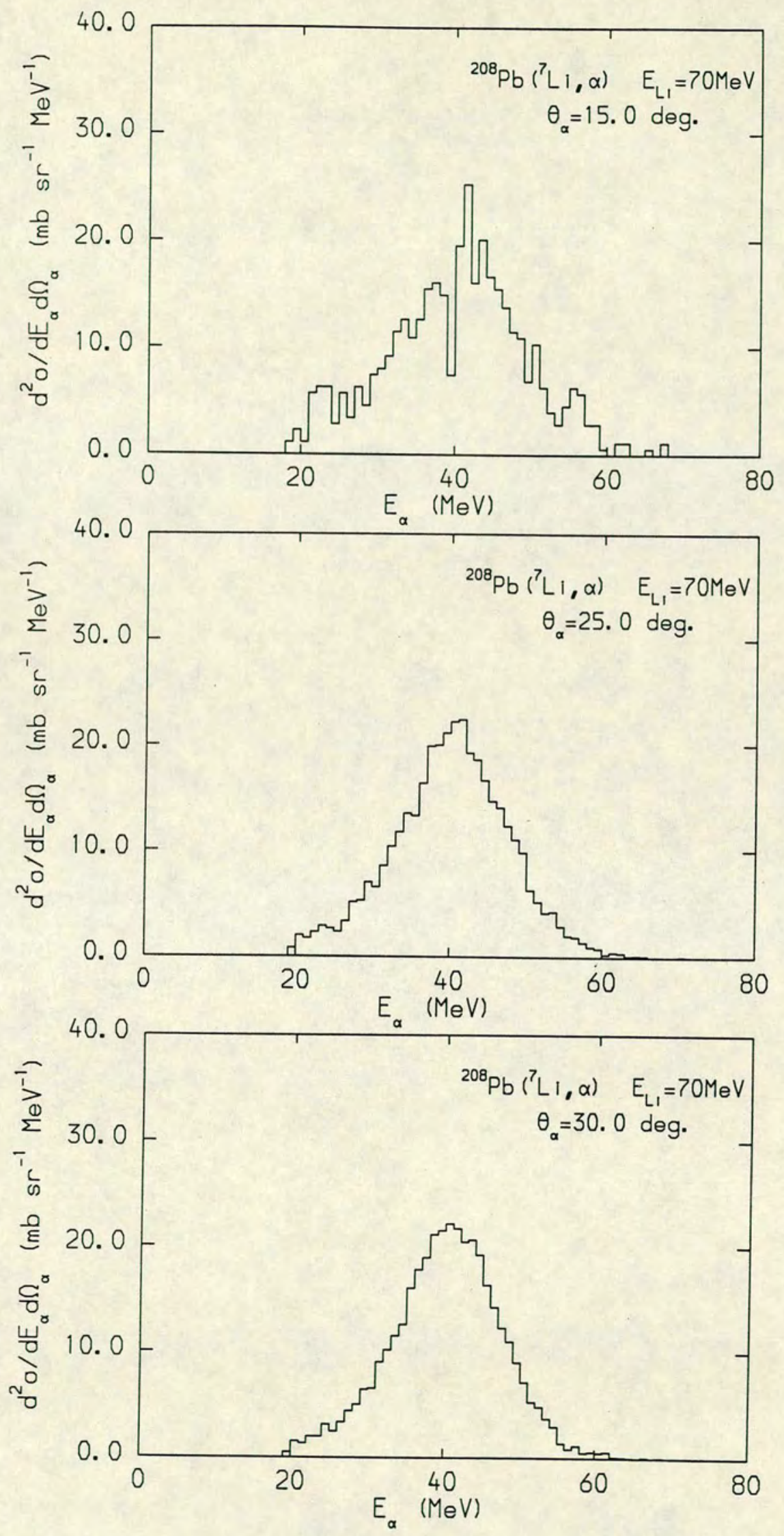

Figure 4.3: Inclusive alpha energy spectra from ${ }^{208} \mathrm{~Pb}$ for varying $\theta_{\alpha}$. 

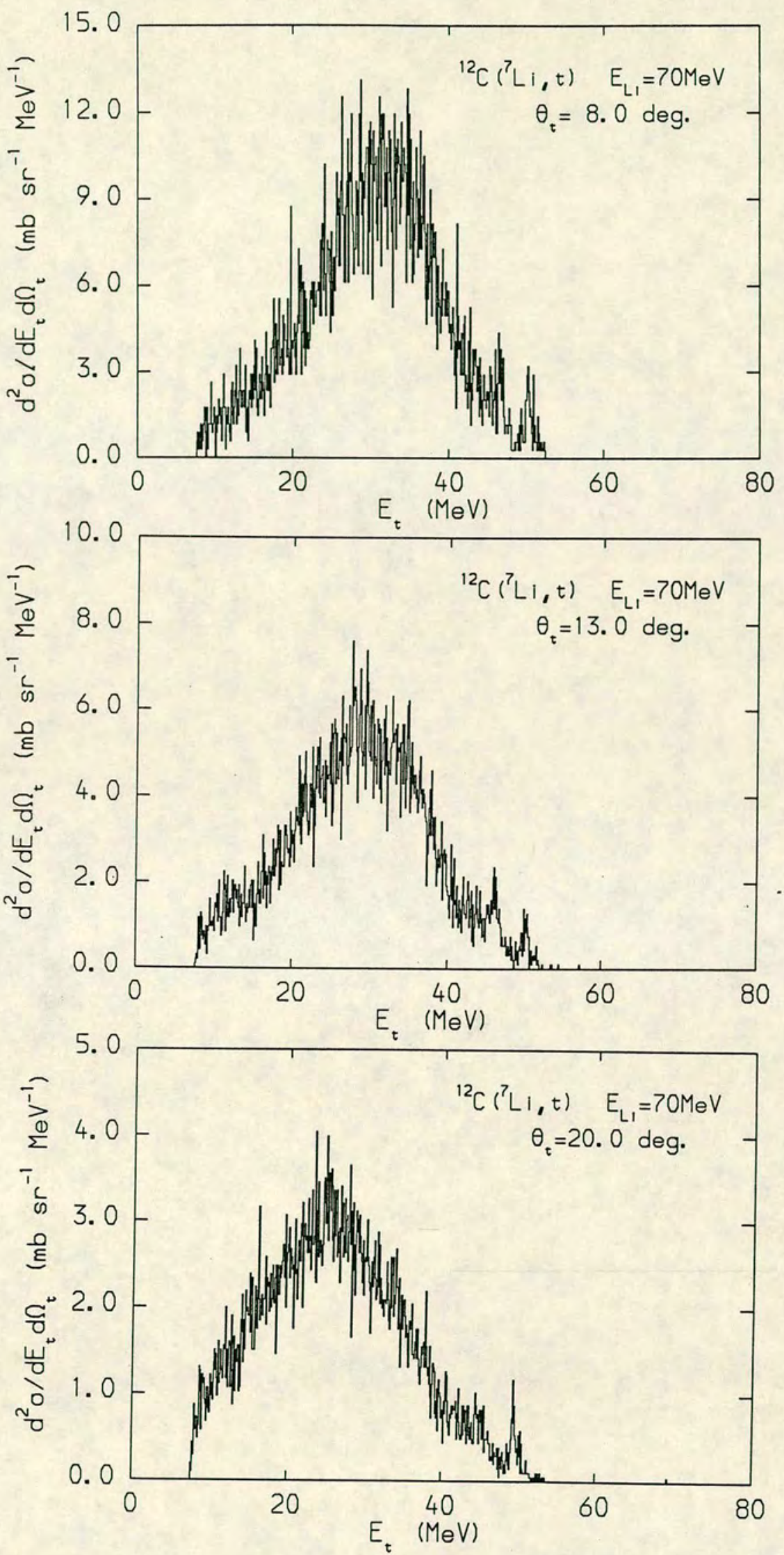

Figure 4.4: Inclusive triton energy spectra from ${ }^{12} \mathrm{C}$ for varying $\theta_{t}$. 

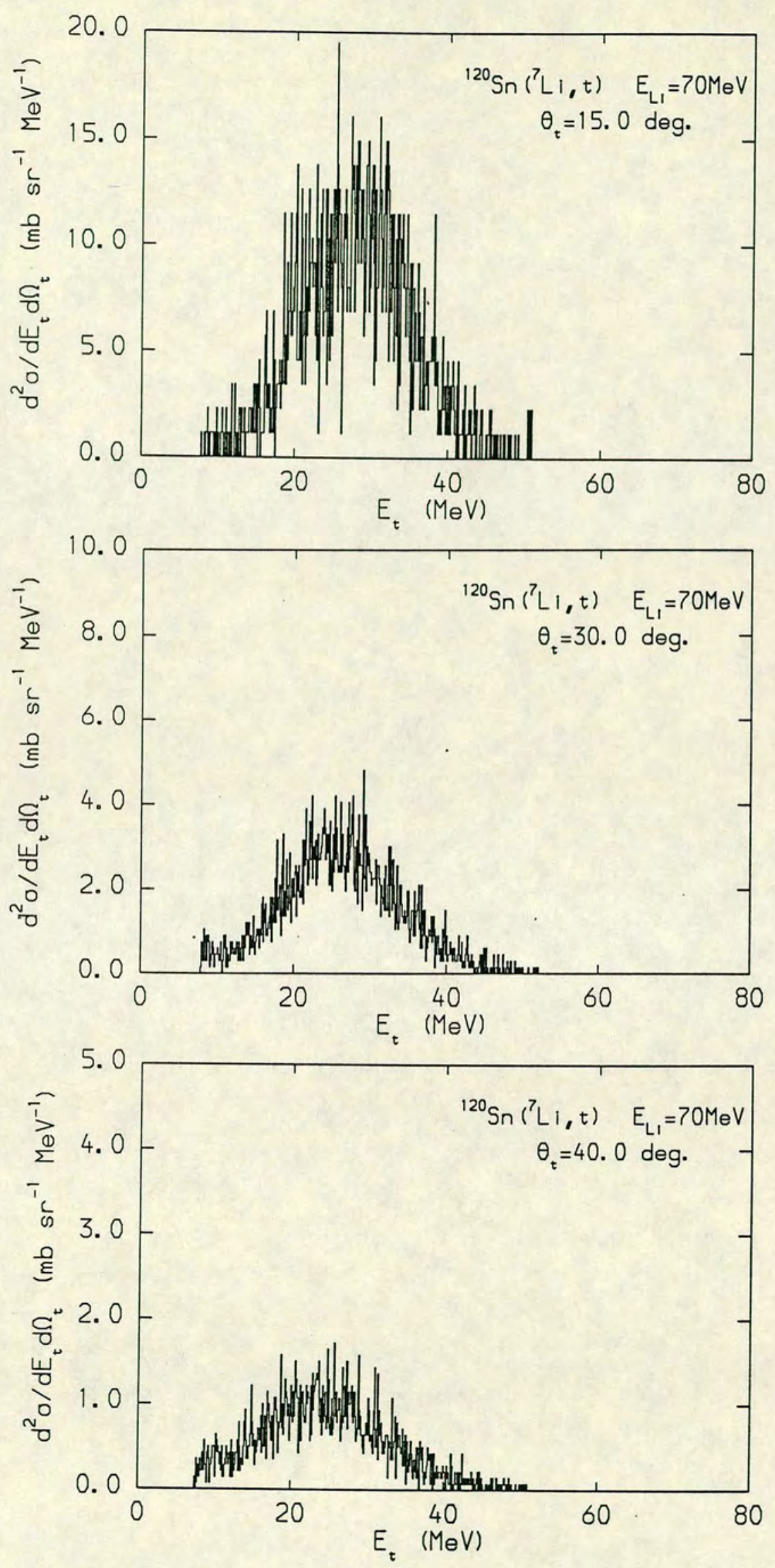

Figure 4.5: Inclusive triton energy spectra from ${ }^{120} \mathrm{Sn}$ for varying $\theta_{t}$. 

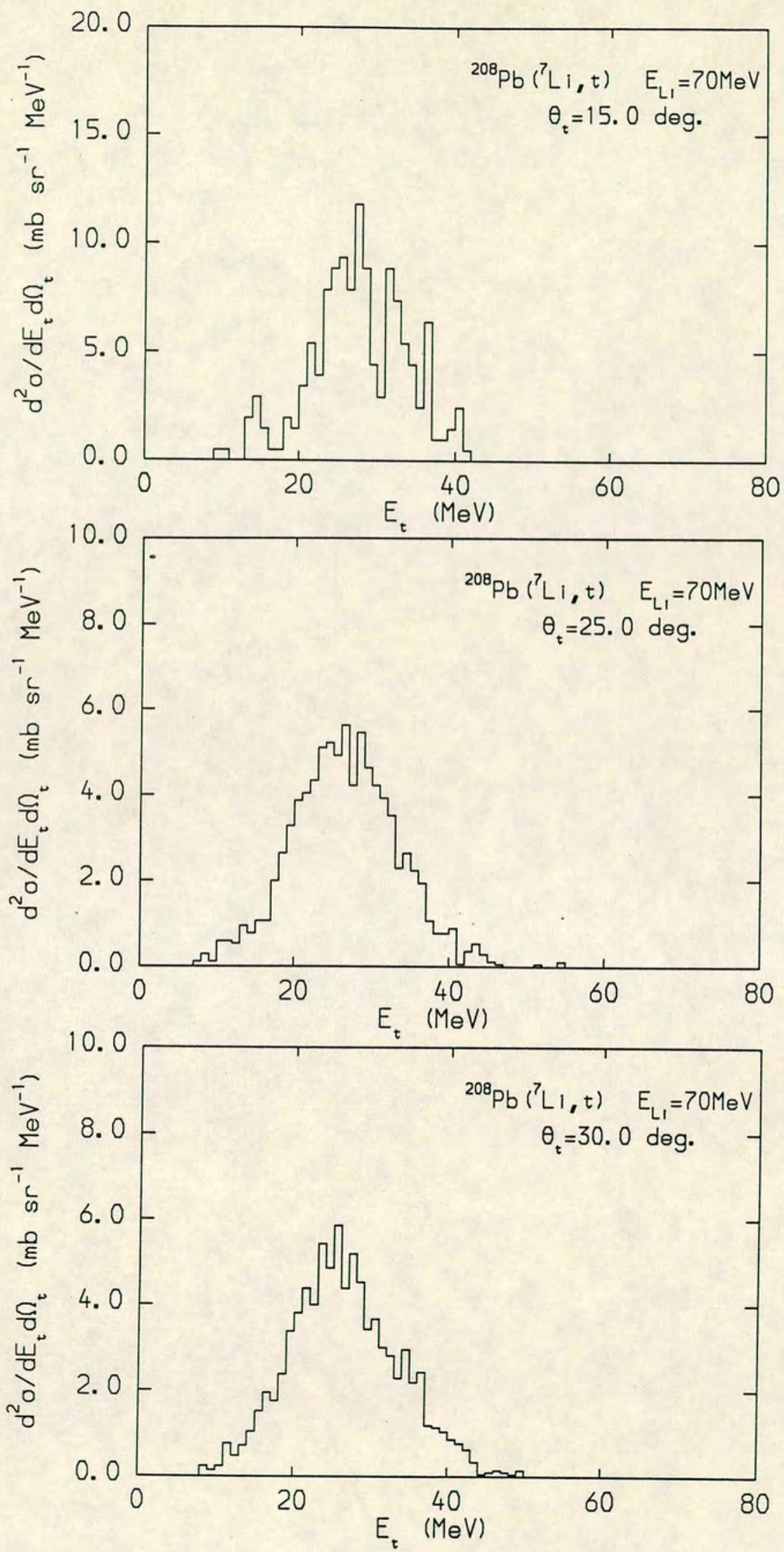

Figure 4.6: Inclusive triton energy spectra from ${ }^{208} \mathrm{~Pb}$ for varying $\theta_{t}$. 
It has been shown [Sc77] [Me84] that it is possible for other reaction mechanisms eg. incomplete fusion, sequential break-up to contribute to this bump and consequently that any conclusions about these underlying reaction mechanisms, drawn from inclusive data alone must be viewed with caution.

This spectator participant picture has been the basis for a number of simple models mentioned in chapter 1 from the original Serber model [Se47] through the plane wave analysis of Matsuoka [Ma78] to the more realistic D.W.B.A. break-up theory developed by Baur et.al. [Ba84]. These theories progress from viewing the target as a completely absorbing disc to a full calculation of the target-fragment interaction, including the distortion of the wave function of the incoming projectile and the outgoing fragments. Although they all produce fits of acceptable quality for the shape of the inclusive bump they all have difficulty reproducing the magnitude and angular dependance of the differential crosssection. As would be expected, the simpler models have the greatest trouble with these calculations, completely overestimating the intensity. Nevertheless their good fits to the shape of the energy spectrum, with the only input the internal momentum distribution of the projectile, lend further weight to the interpretation of the inclusive bump as originating from projectile fragmentation [Ma78]. Again however, it should be acknowledged that this may also be attributed to the inclusive data being rather insensitive to the reaction mechanism.

One of the main reasons for measuring the inclusive bump cross-section is its magnitude. In other reactions [P186] it has been measured to contain up to $50 \%$ of the total reaction cross-section and as such is an important reaction channel. It is also important to understand its behaviour in order to perform accurate subtraction from the energy spectrum to allow investigation of other processes contributing to this energy region e.g. giant resonances and particle emission from high excited states.

The intensity of the break-up bump was extracted by fitting the bump with a Gaussian on top of a contribution from the pre-compound processes. For the ${ }^{12} \mathrm{C}$ data this latter contribution was fitted with an exponential whereas the other targets were fitted with a linearly decreasing background normalised to the low 
energy end of the break-up bumps. The resulting angular distributions for the break-up bumps are shown in fig.4.7 to 4.9 along with similar data taken from [Da87].

The datahavebeen fitted with a weighted least squares fit to the three constants $\mathrm{A}, \mathrm{B}$ and $\mathrm{C}$ in the parameterisation.

$$
\frac{\mathrm{d}^{2} \sigma(\theta)}{\mathrm{d} \Omega \mathrm{dE}}=A e^{B \theta} e^{C \theta^{2}}
$$

As can be seen this produced an adequate fit to the data for all targets. In view of the uncertainty associated with the background extraction the agreement between the two sets of data is quite acceptable.

The disagreement at larger angles on the ${ }^{12} \mathrm{C}$ target is due to the lack of data in the region $\theta>20^{\circ}$ with the results of [Da87] being more reliable in this range. The discrepancy between the two data sets at forward angles for the inclusive $\alpha$ yield from the ${ }^{208} \mathrm{~Pb}$ target is more perplexing. The data from this work clearly show a distinct maximum $\sim 10^{\circ}$ inside the grazing angle $\left(\theta_{\text {graz. }} \sim 35^{\circ}\right)$ falling by a factor of 2 another $10^{\circ}$ inside this peak. This is almost exactly the same behaviour reported for the inclusive $\alpha$ yield from $75 \mathrm{MeV}{ }^{6} \mathrm{Li}$ on ${ }^{197} \mathrm{Au}[\mathrm{Ca} 80]$. The data from [Da87] however, have their turn-over at much smaller angles $\left(\sim 10^{\circ}\right)$. No solution has been found for this disparity. The total integrated cross-section for the ${ }^{208} \mathrm{~Pb}$ target will, fortunately, be rather insensitive to this problem due to the $\sin \theta$ term in the integrand.

The total integrated cross-section for each target and outgoing fragment was found by Simpsons rule integration of equation 4.1 with the fitted parameters. The results are listed in table 4.1 .

The uncertainty of the behaviour of the ${ }^{12} \mathrm{C}$ and ${ }^{120} \mathrm{Sn}$ data at extreme forward angles means that these values represented an upper limit to the cross-section and may be over-estimated by up to $\sim 30 \%$. The ${ }^{208} \mathrm{~Pb}$ data are more reliable as the peak was well fitted by the least squares routine. It can be seen that for all three targets there is a marked excess of beam velocity alpha particles over beam 


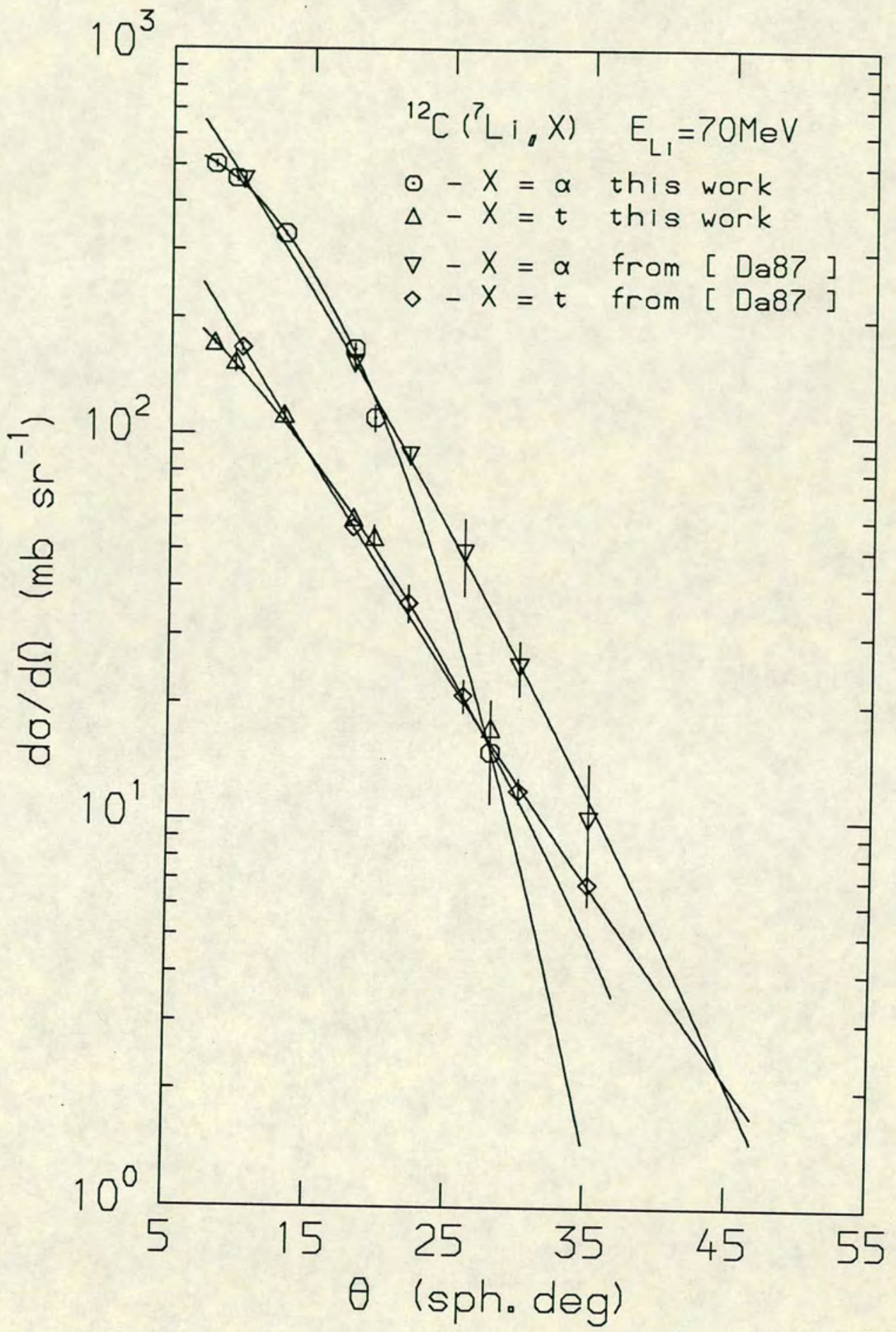

Figure 4.7: Inclusive, energy integrated alpha and triton data for $70 \mathrm{MeV}{ }^{7} \mathrm{Li}$ incident on ${ }^{12} \mathrm{C}$ along with data from [Da87]. Curves are least squares fits and are explained in the text. 


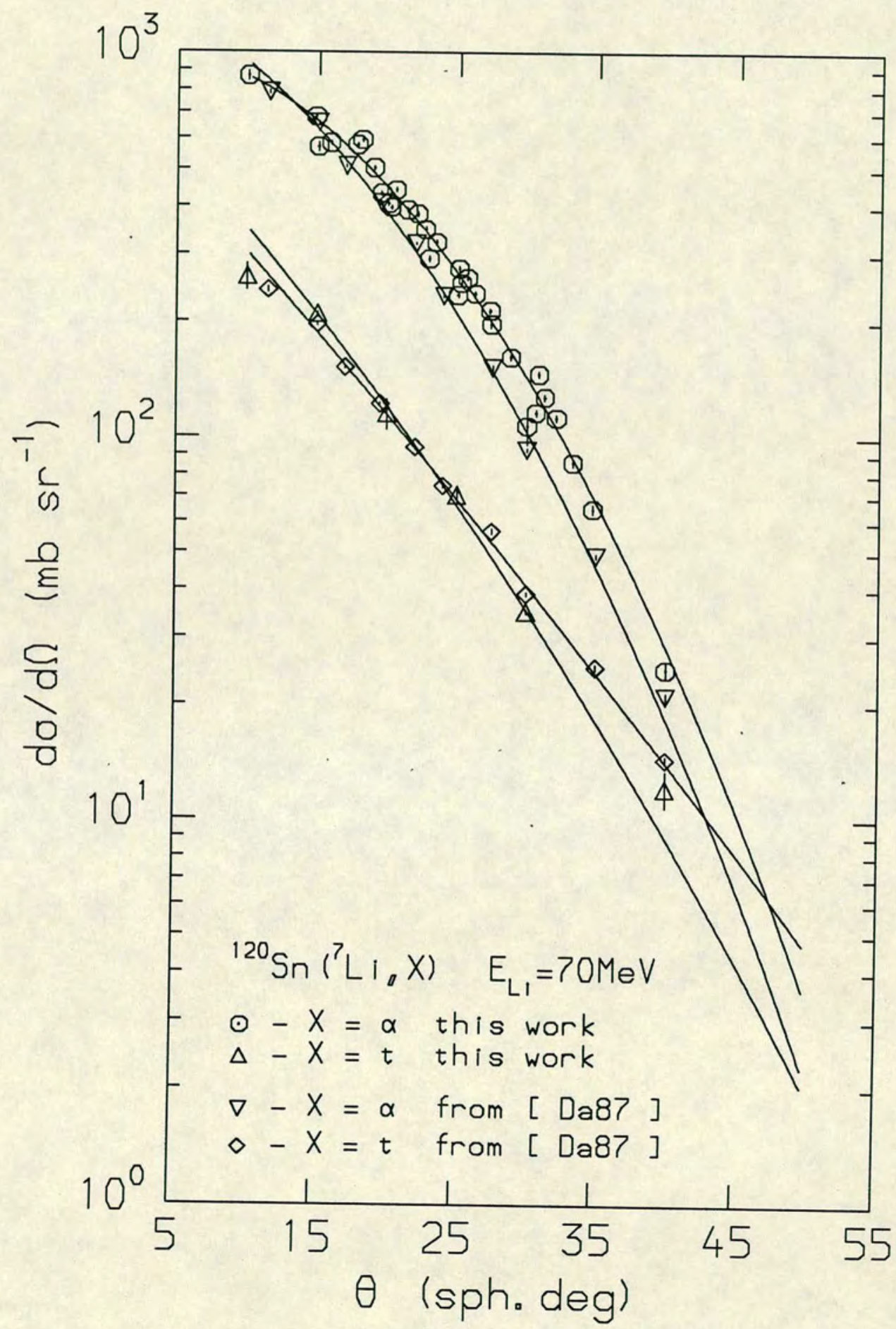

Figure 4.8: Inclusive, energy integrated alpha and triton data for $70 \mathrm{MeV}{ }^{7} \mathrm{Li}$ incident on ${ }^{120} \mathrm{Sn}$ along with data from [Da87]. Curves are least squares fits and are explained in the text. 


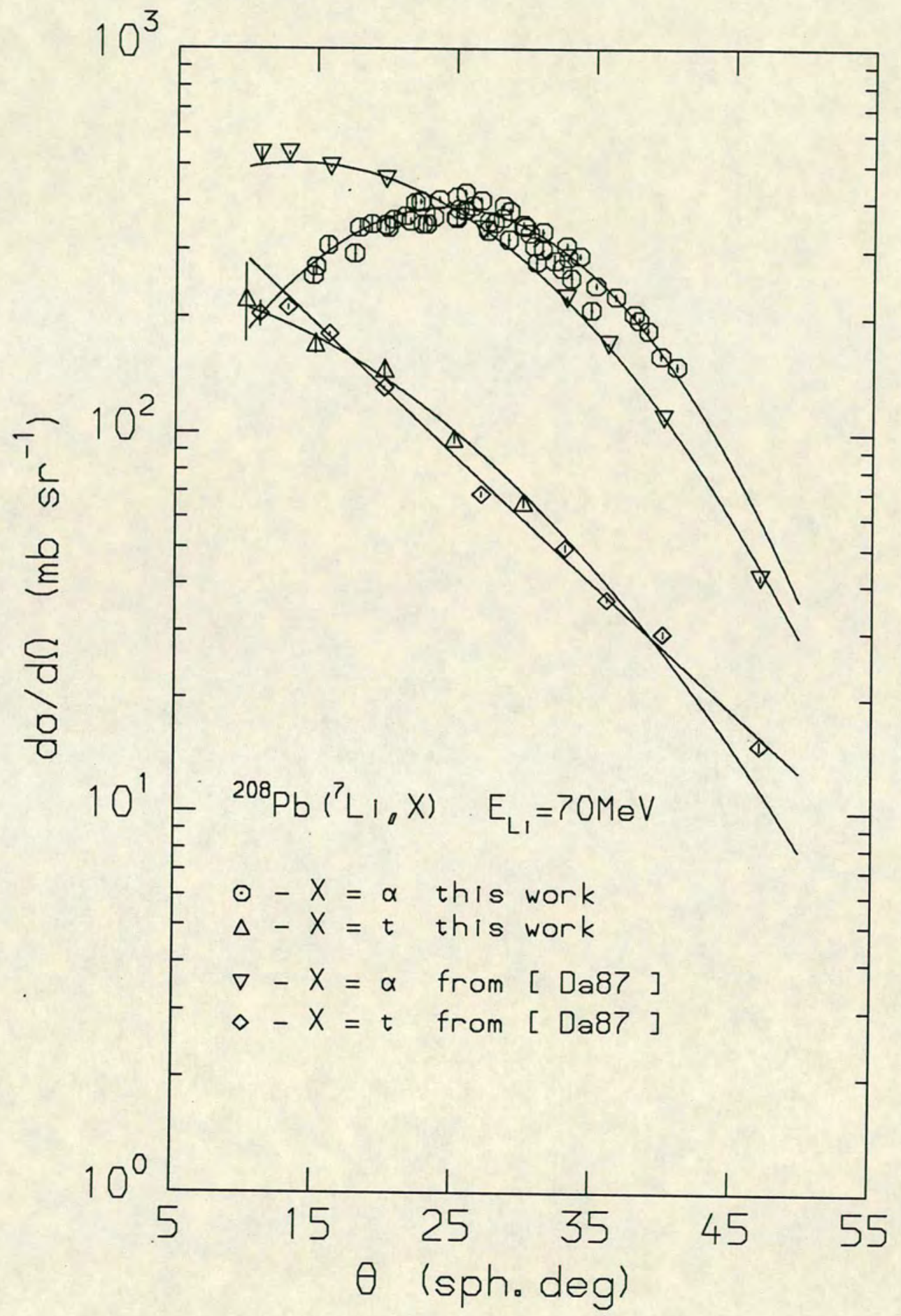

Figure 4.9: Inclusive, energy integrated alpha and triton data for $70 \mathrm{MeV}{ }^{7} \mathrm{Li}$ incident on ${ }^{208} \mathrm{~Pb}$ along with data from [Da87]. Curves are least squares fits and are explained in the text. 


\begin{tabular}{|c|c|c|c|c|c|}
\hline target & \multicolumn{2}{|c|}{$\sigma_{\alpha}^{t o t}(\mathrm{mb})$} & \multicolumn{2}{|c|}{$\sigma_{\text {trit. }}^{\text {tot }}(\mathrm{mb})$} & $\sigma_{\text {reac }}^{\text {tot }}(\mathrm{mb})$ \\
\hline${ }^{12} \mathrm{C}$ & $138 \pm 20$ & $170 \pm 30$ & $58 \pm 8$ & $70 \pm 10$ & 1020 \\
\hline${ }^{120} \mathrm{Sn}$ & $450 \pm 60$ & $420 \pm 60$ & $153 \pm 25$ & $150 \pm 20$ & 1960 \\
\hline${ }^{208} \mathrm{~Pb}$ & $500 \pm 70$ & $530 \pm 80$ & $160 \pm 25$ & $190 \pm 30$ & 2040 \\
\hline
\end{tabular}

Table 4.1: Angle integrated inclusive cross-sections for alphas and tritons from ${ }^{12} \mathrm{C},{ }^{120} \mathrm{Sn}$ and ${ }^{208} \mathrm{~Pb}$. Other data from [Da87].Total reaction cross-sections calculated from equ.4.2. Errors quoted include estimates of systematic and statistical errors.

velocity tritons. This indicates that there are more mechanisms contributing to the inclusive bumps than simple projectile fragmentation and probably reflects the increased probability of survival of an alpha over a triton after an interaction with the target. Table 4.1 also lists the total reaction cross-section calculated from the strong absorption formula [Ho78]:

$$
\sigma_{\text {reac }}^{\text {tot }}=\pi \mathrm{R}_{c}^{2}\left(1-\frac{\mathrm{V}_{c}}{\mathrm{E}}\right)
$$

with $\mathrm{R}_{c}=1.4\left(\mathrm{~A}_{p}^{1 / 3}+\mathrm{A}_{t}^{1 / 3}\right)$ and $\mathrm{V}_{c}=\frac{\mathrm{Z}_{p} \mathrm{Z}_{t} \mathrm{e}^{2}}{\mathrm{R}_{c}}$

It can be seen that the inclusive break-up yield for alphas and tritons takes a substantial fraction of the total reaction cross-section and thus represent an important reaction channel in the interaction of $70 \mathrm{MeV}{ }^{7} \mathrm{Li}$ with the various targets. When these cross-sections are plotted against the mass number of the target the data follow an $\mathrm{A}$ dependance close to $\mathrm{A}^{1 / 3}$ [Da87]. This suggests a surface peaked reaction mechanism rather than one dependant on the target area $\left(\propto \mathrm{A}^{2 / 3}\right)$ or volume $(\propto \mathrm{A})$. It is also shown in [Da87] that the relative particle yields are rather independant of the target mass indicating that the 
particle production is more a reflection of the structure of the projectile than the target.

In summary, the inclusive energy spectrum for alpha particles and triton particles have been found to exhibit the typical features attributed to particles arising from fragmentation of the projectile through a surface peaked interaction with the target nucleus. The total angle integrated inclusive yields of these break-up bumps were calculated and found to be in good agreement with previous data [Da87]. This gave increased confidence in the data collection system and data analysis procedure, used to analyse the coincidence data.

\subsection{Exclusive Data}

The particle-particle coincidence data were collected as outlined in Chapter 3 , with the strip detectors detecting the alpha particle and the SiLi detector telescopes detecting the triton particle. Only alpha-triton coincidences will be presented in this thesis.

Figures 4.10 to 4.19 show the total kinetic energy spectrum $\left(\mathrm{E}_{\alpha}+\mathrm{E}_{t}\right)$ from each of the pixels on the alpha detector for a number of different angle combinations. In this representation, different final states of the target are easily identified by separate peaks. Fig.4.11 clearly shows evidence for excitation of the first $\left(4.439 \mathrm{MeV}, 2^{+}\right)$and third $\left(9.641 \mathrm{MeV}, 3^{-}\right)$excited states of the ${ }^{12} \mathrm{C}$ target along with quasi-elastic events which leave the target in its ground state. In contrast, the ${ }^{120} \mathrm{Sn}$ and ${ }^{208} \mathrm{~Pb}$ targets show little or no evidence for target excitation to their lower levels. This behaviour has been identified in other experiments detecting the alpha and triton at small angular separations [Da87] but it can be seen here that even at large separations the quasi-elastic channel takes a significant proportion of the $\alpha-t$ coincident cross-section.

The enhancement in the cross-section at $\sim 45-65 \mathrm{MeV}$ in fig.4.10 and 4.16 has been attributed to break-up of ${ }^{7} \mathrm{Li}$ on hydrogen present in the targets. The structure seen in these events is due to the projection of the available phase 


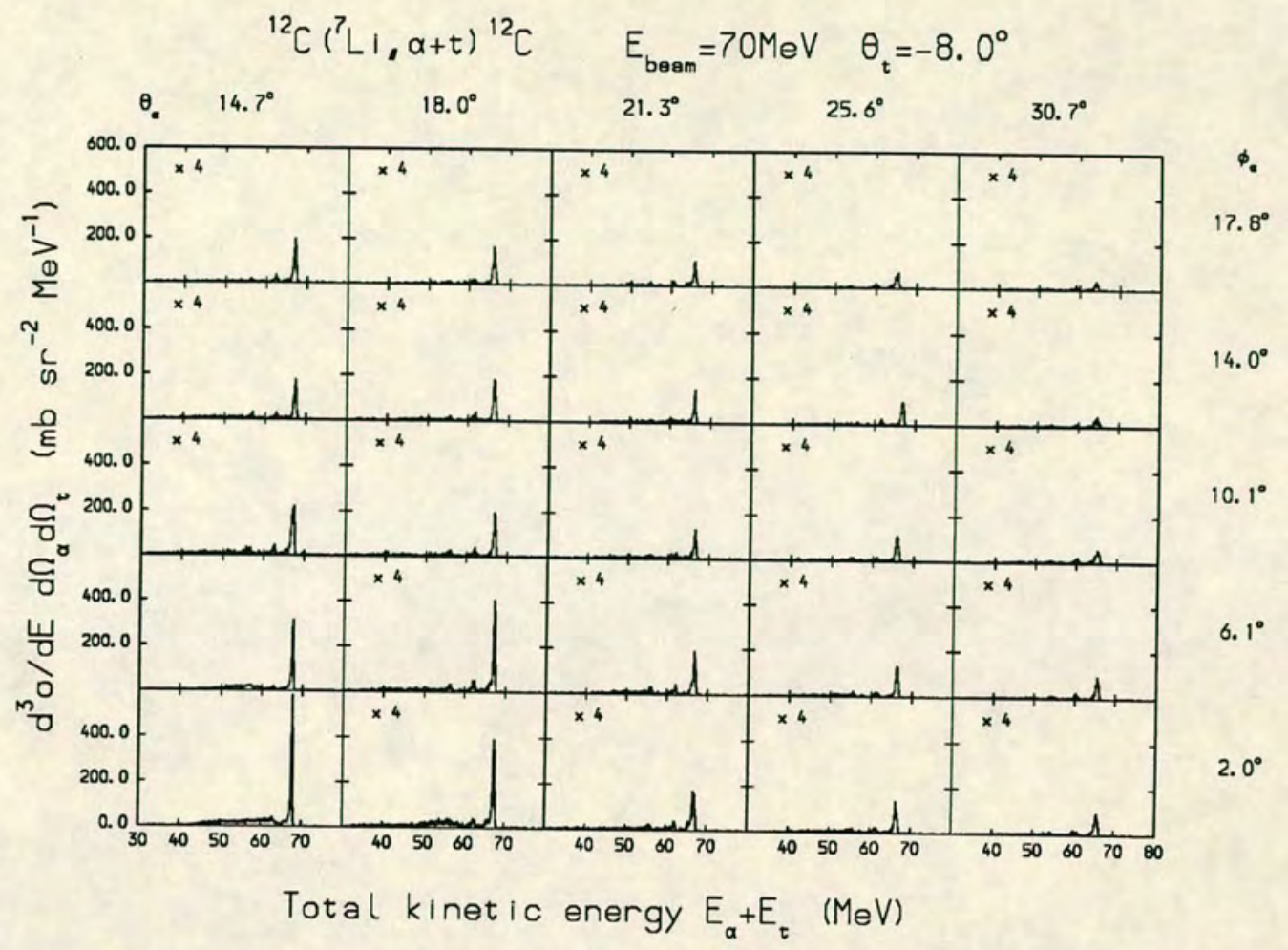

Figure 4.10: Total kinetic energy spectra from the strip detector pixels with ${ }^{12} \mathrm{C}$ target and both particles in the forward direction.

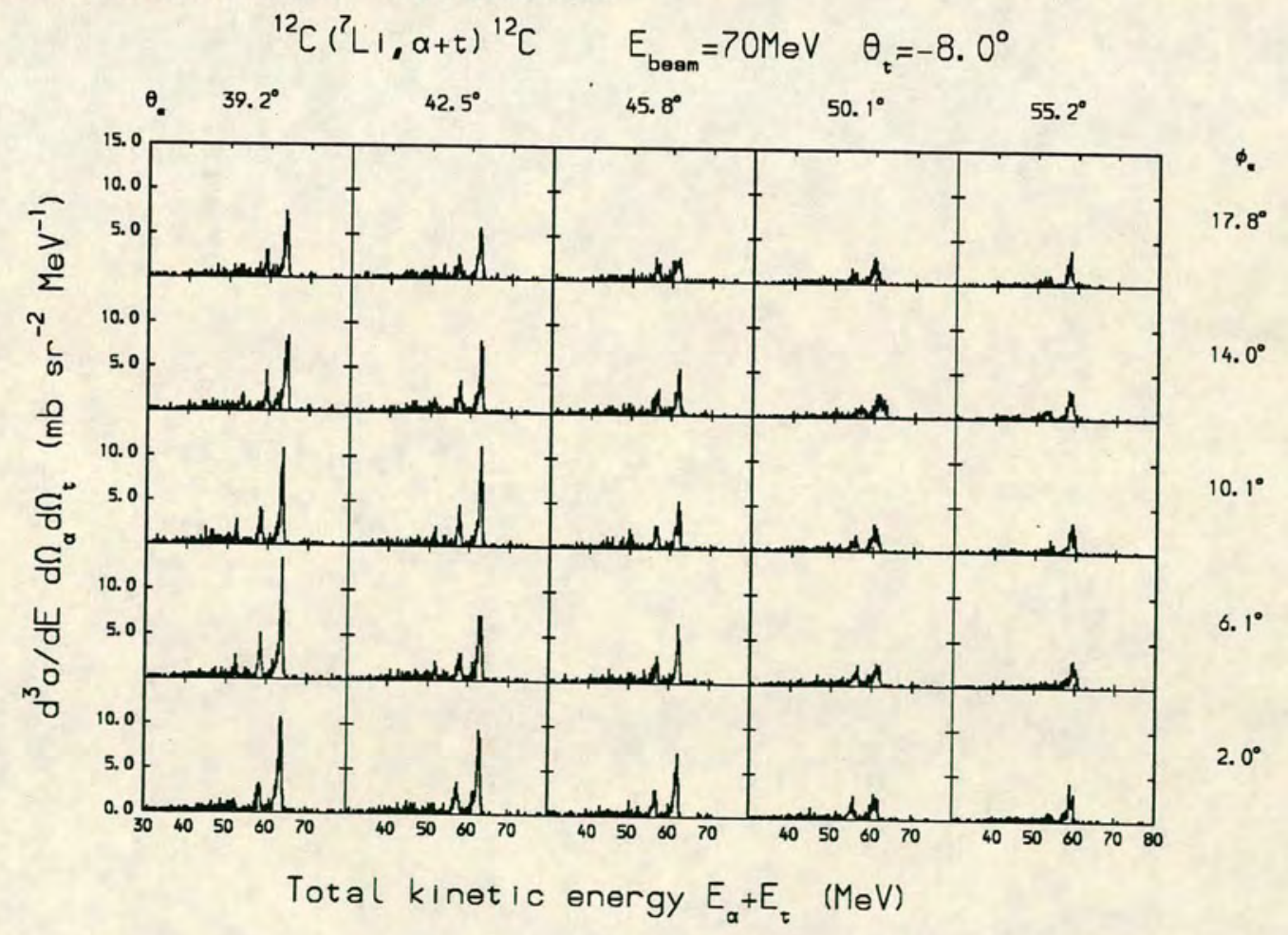

Figure 4.11: Total kinetic energy spectra from the strip detector pixels with ${ }^{12} \mathrm{C}$ target, triton in forward direction, alpha in backward direction. 


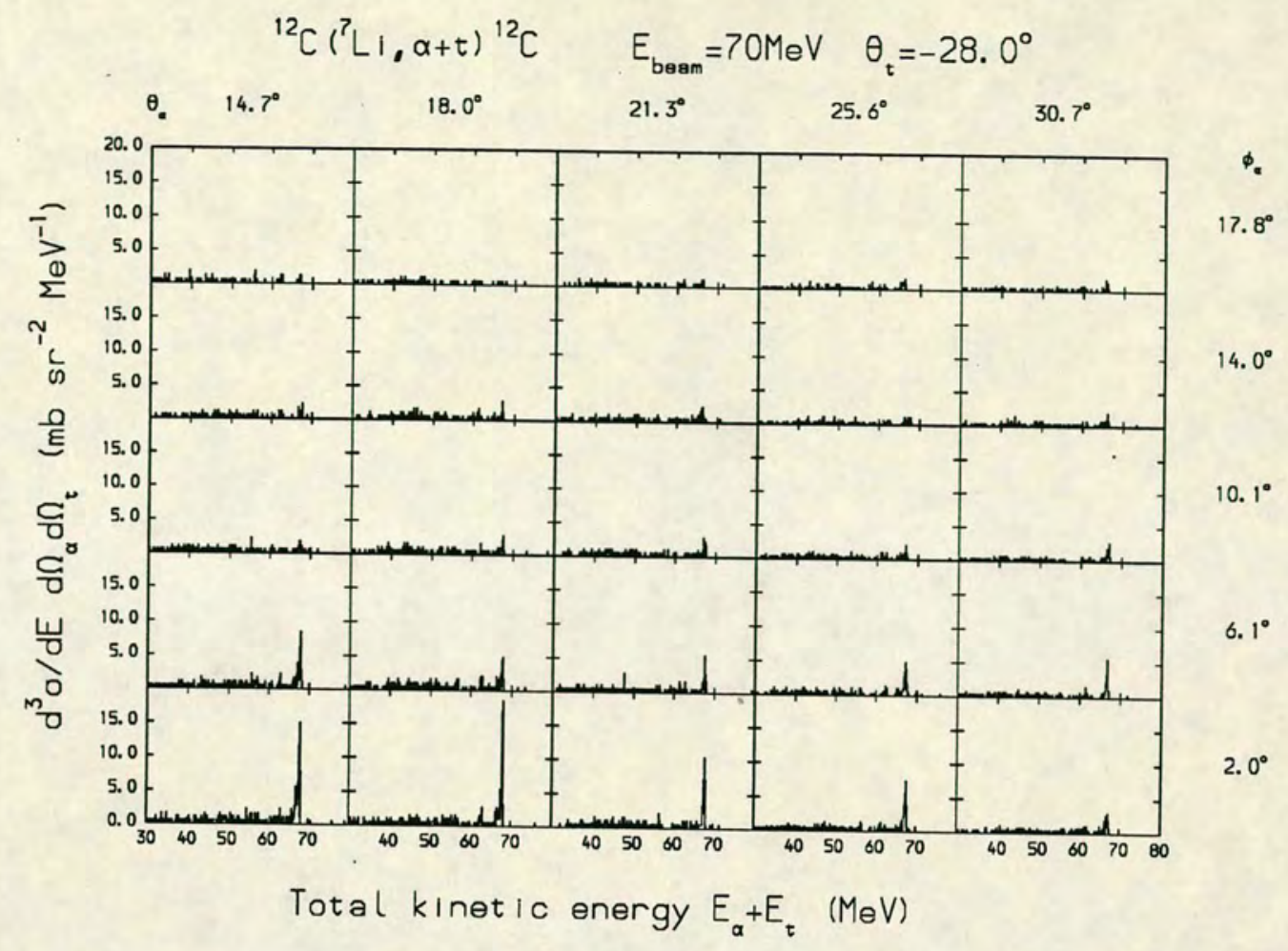

Figure 4.12: Total kinetic energy spectra from the strip detector pixels with ${ }^{12} \mathrm{C}$ target, alpha in forward direction, triton in backward direction.

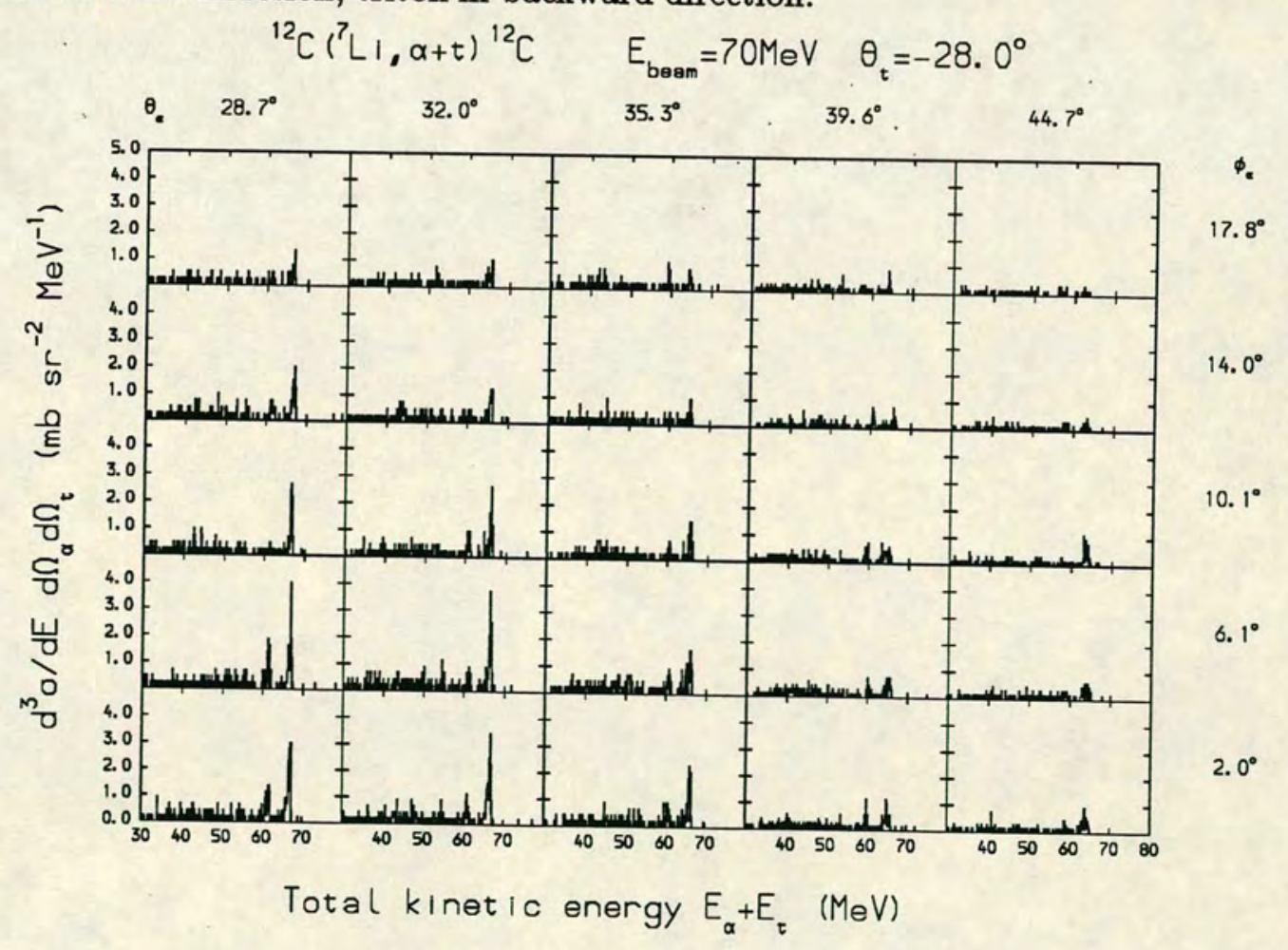

Figure 4.13: Total kinetic energy spectra from the strip detector pixels with ${ }^{12} \mathrm{C}$ target and both particles in the backward direction. 


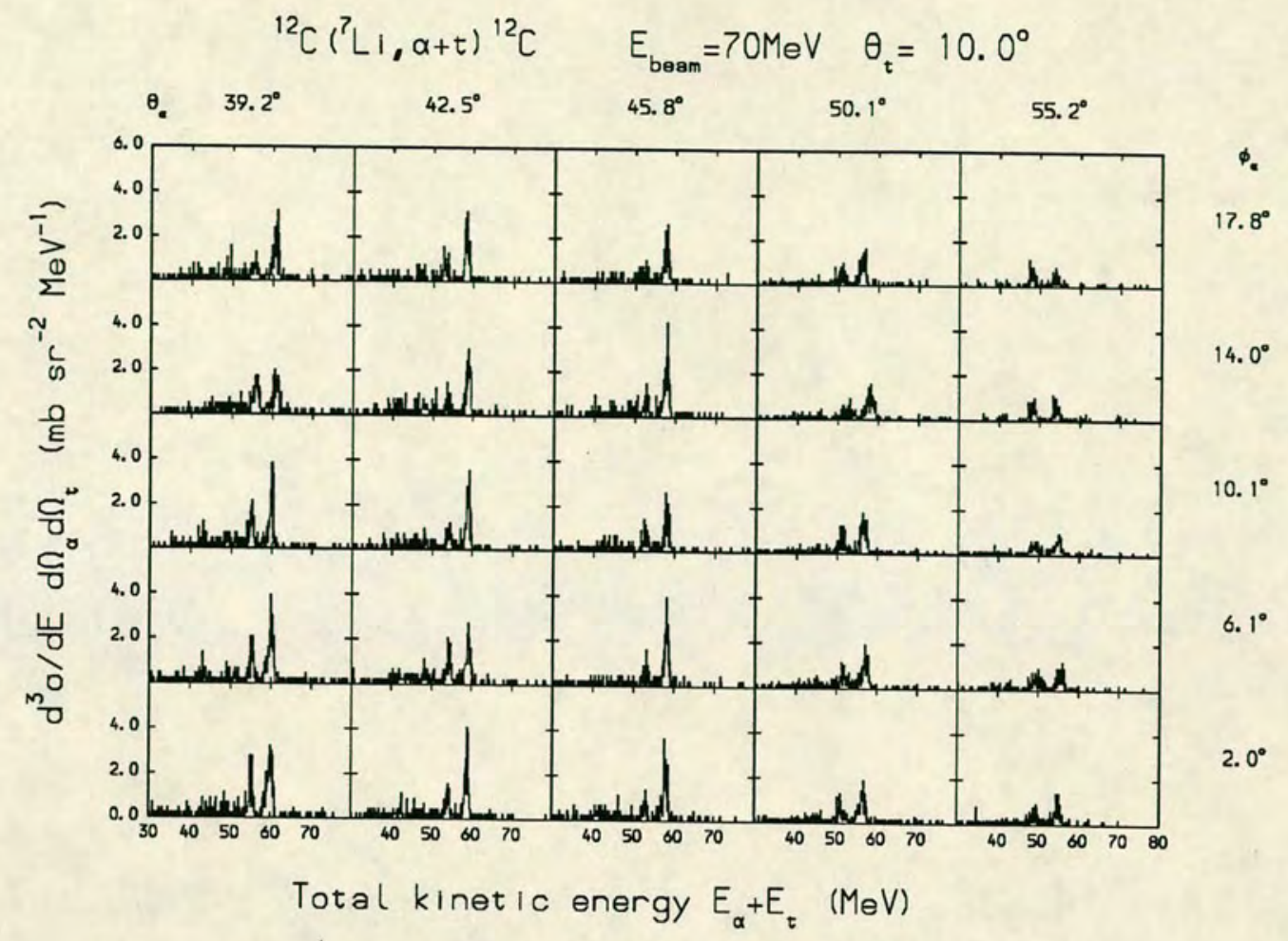

Figure 4.14: Total kinetic energy spectra from the strip detector pixels with ${ }^{12} \mathrm{C}$ target and both detectors on same side of beam.

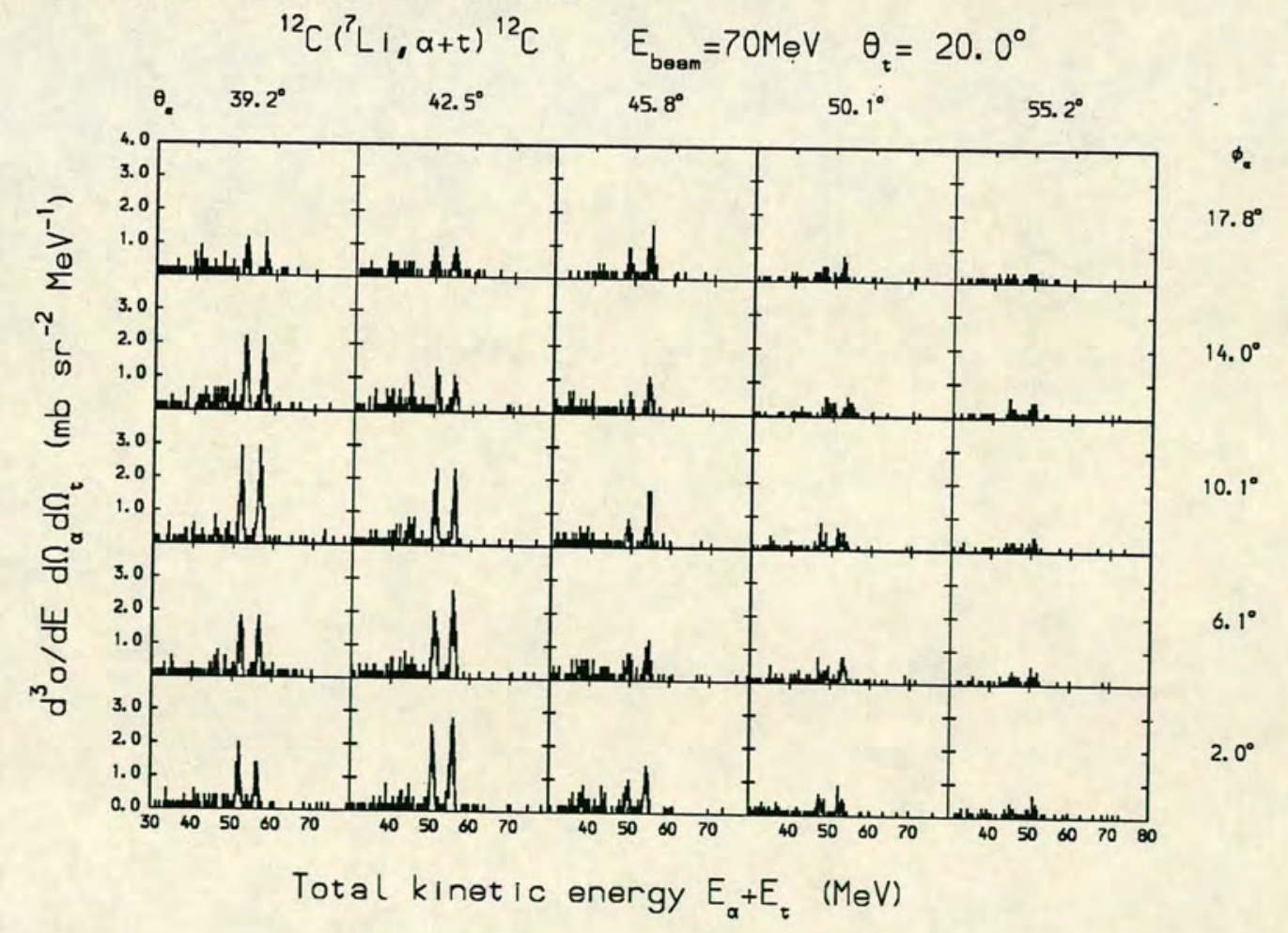

Figure 4.15: Total kinetic energy spectra from the strip detector pixels with ${ }^{12} \mathrm{C}$ target and both detectors on same side of beam. 


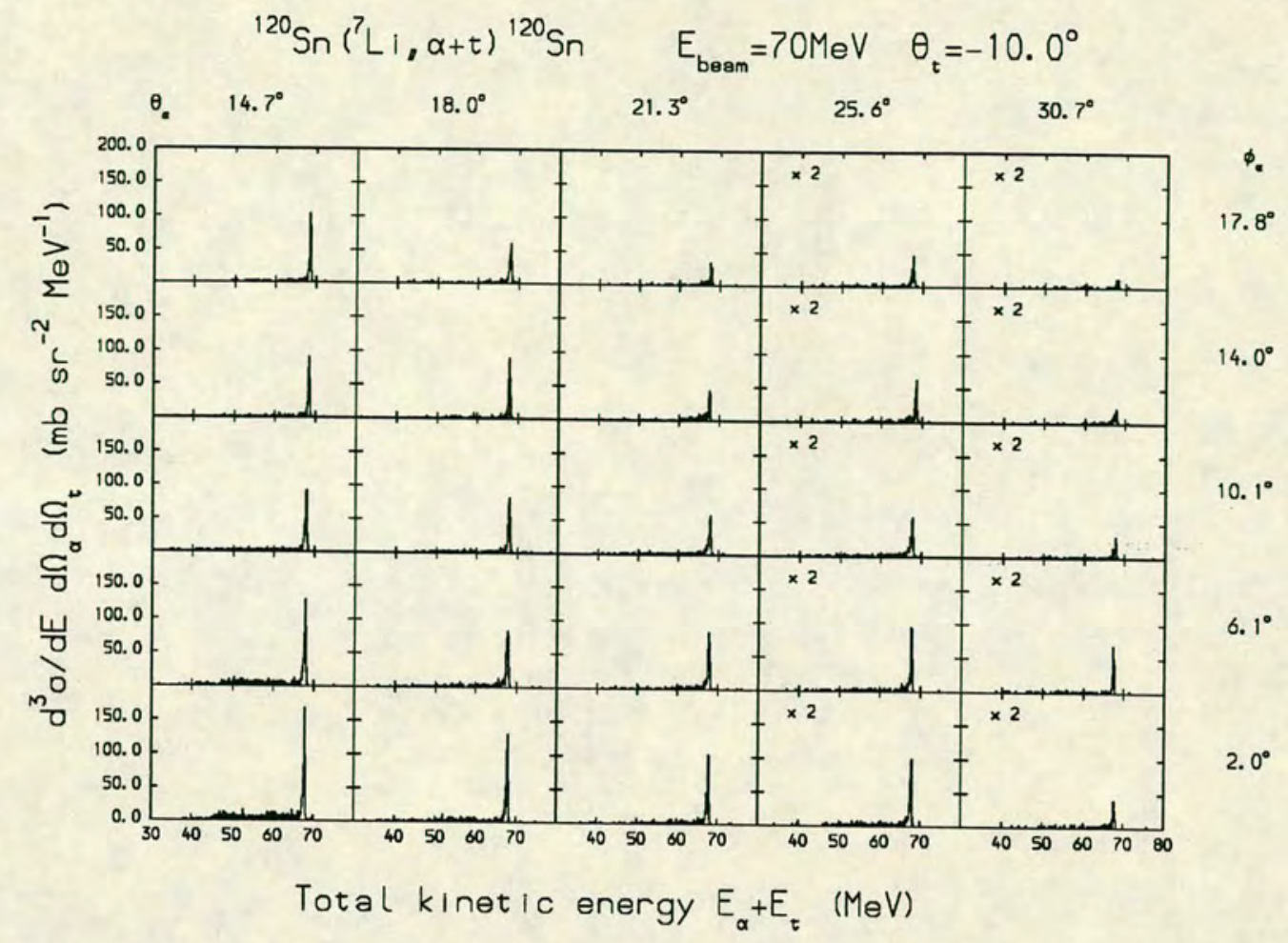

Figure 4.16: Total kinetic energy spectra from the strip detector pixels with ${ }^{120} \mathrm{Sn}$ target and both particles in the forward direction.

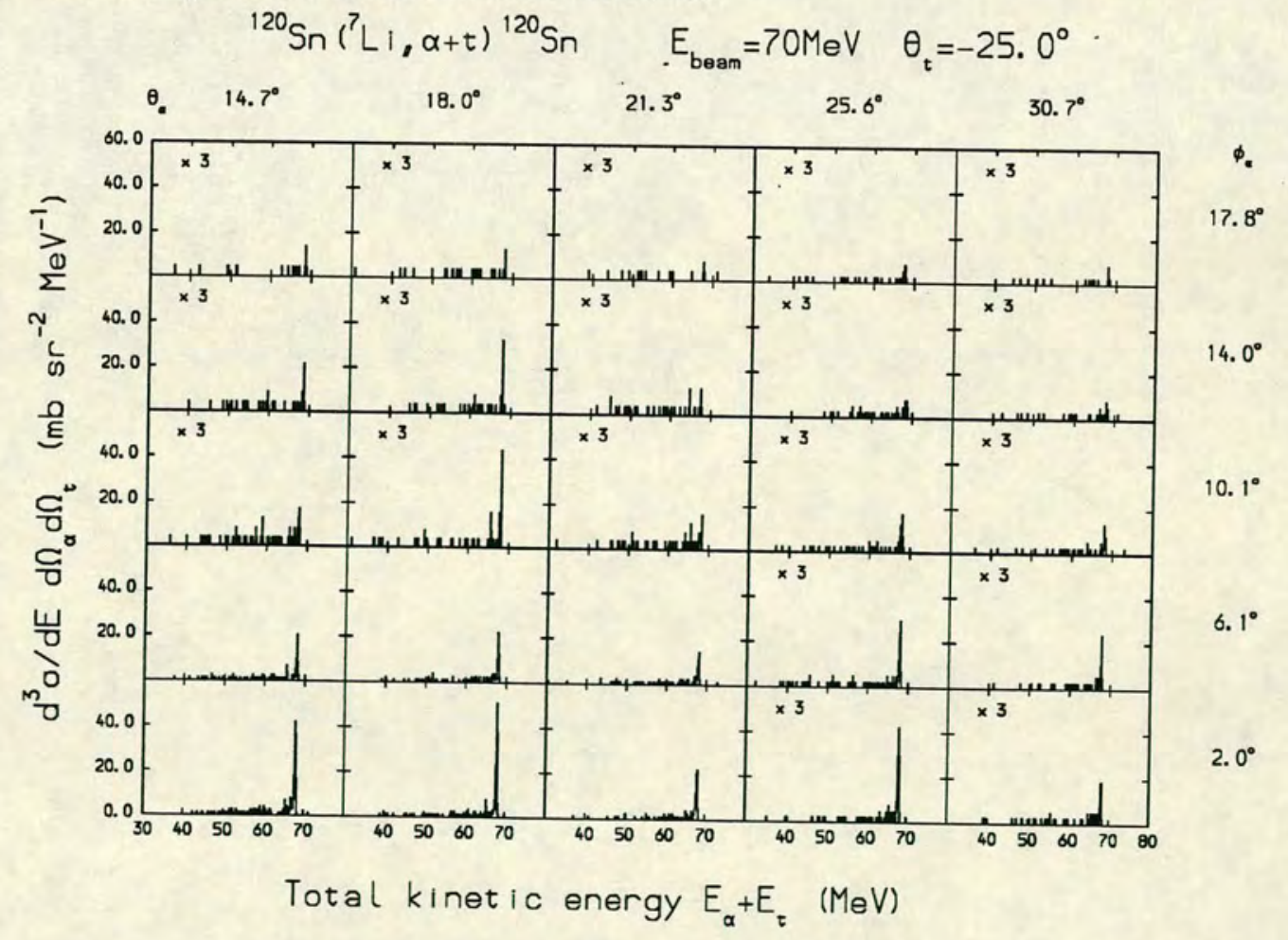

Figure 4.17: Total kinetic energy spectra from the strip detector pixels with ${ }^{120} \mathrm{Sn}$ target, alpha in forward direction, triton in backward direction. 


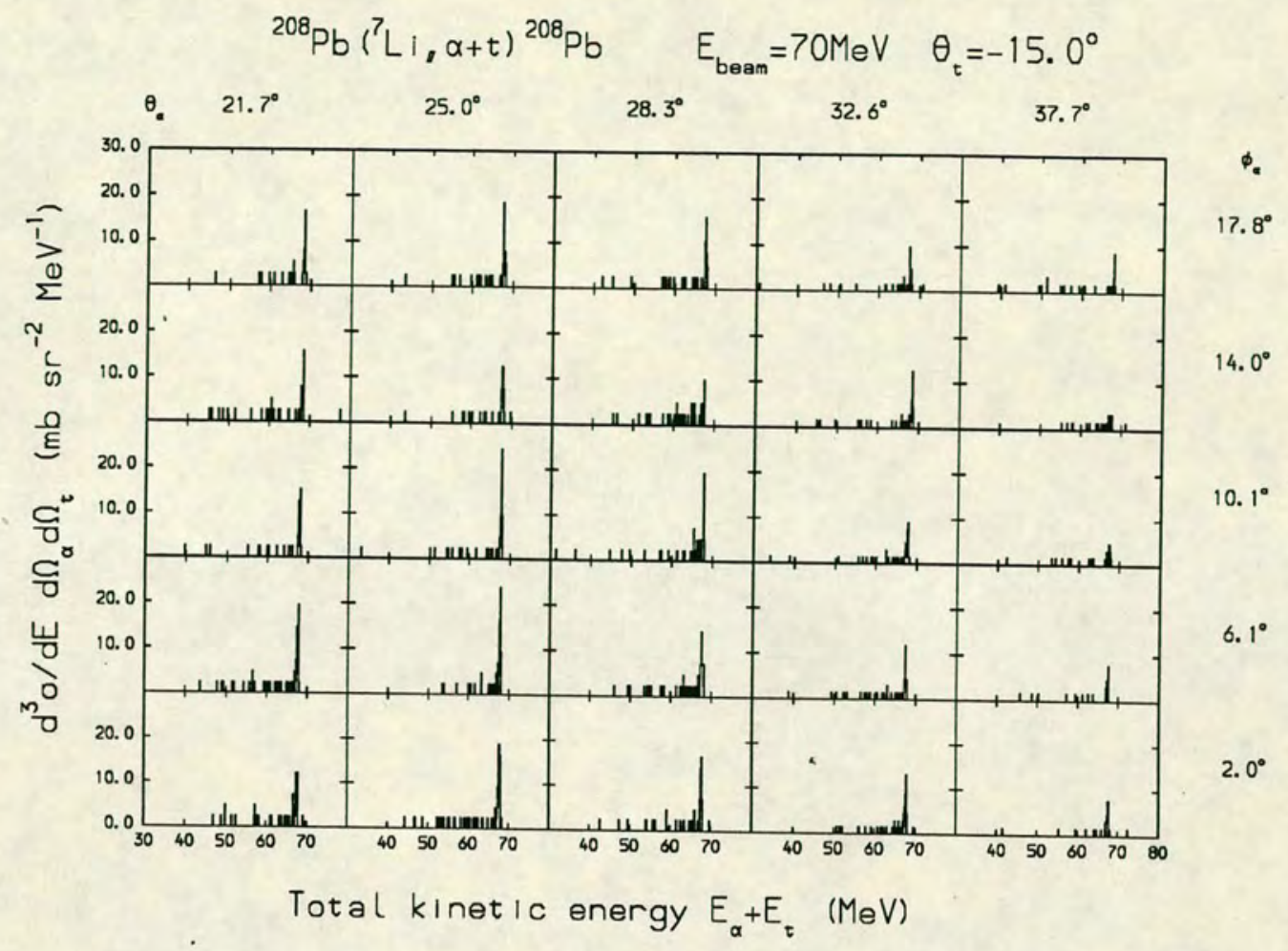

Figure 4.18: Total kinetic energy spectra from the strip detector pixels with ${ }^{208} \mathrm{~Pb}$ target and both particles in the forward direction.

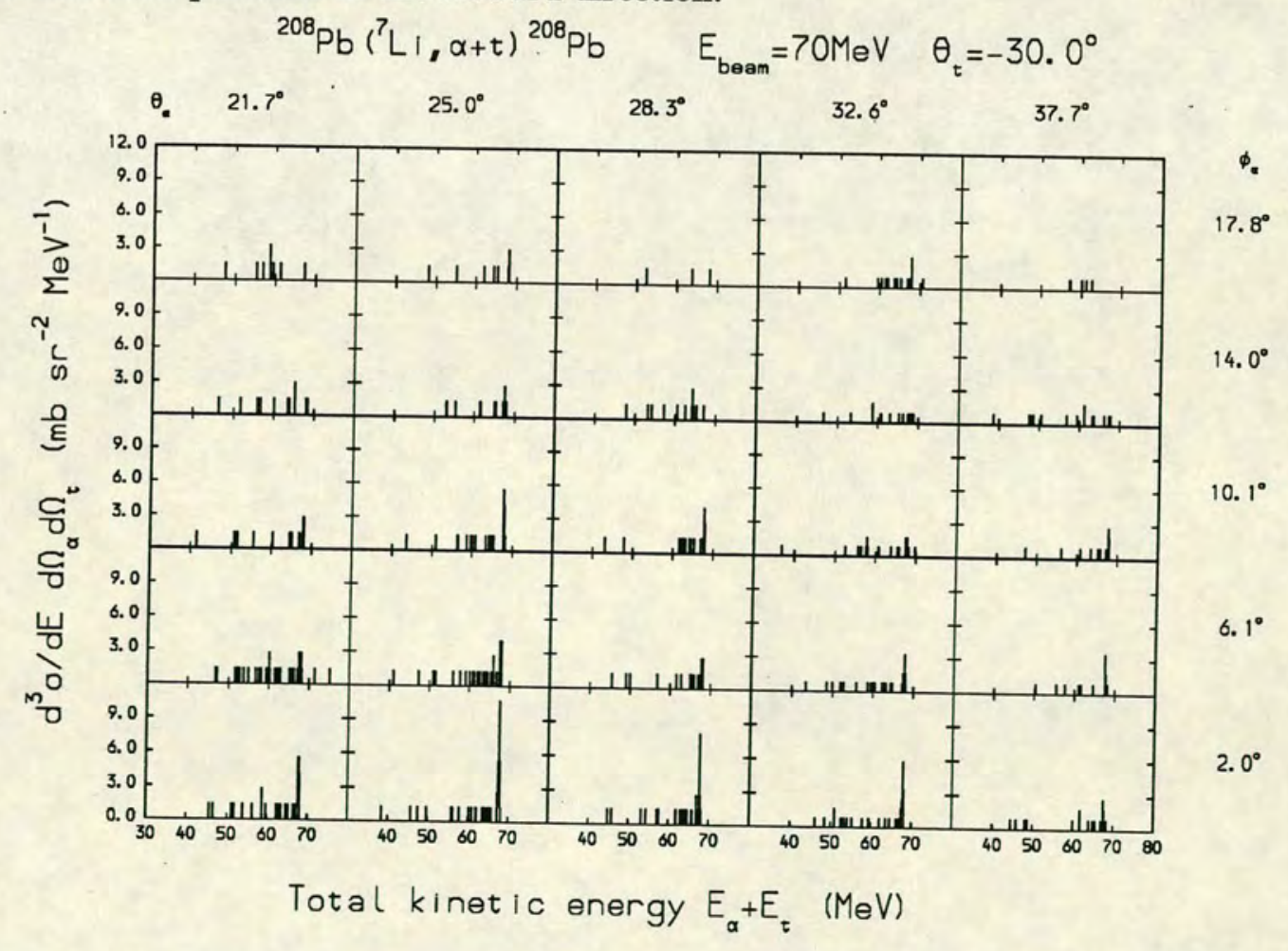

Figure 4.19: Total kinetic energy spectra from the strip detector pixels with ${ }^{208} \mathrm{~Pb}$ target, alpha in forward direction, triton in backward direction. 
space for this type of break-up onto the $\mathrm{E}_{\alpha}+\mathrm{E}_{t}$ axis. It can be seen that this contamination disappears from all but the most forward angled pixels. This is as would be expected from the kinematics of the ${ }^{1} \mathrm{H}\left({ }^{7} \mathrm{Li}, \alpha+\mathrm{t}\right){ }^{1} \mathrm{H}$ reaction. It was possible to discriminate against these events when the quasi-elastic cross-sections were being calculated, but the total energy double differential cross sections will not be presented for those pixels where this hydrogen contamination was present.

One point of interest is the relative intensity of the ground state and the first excited state of the ${ }^{12} \mathrm{C}$ target. When both outgoing fragments are on opposite sides of the beam, the quasi-elastic events were generally much stronger than the events leaving the ${ }^{12} \mathrm{C}$ in its $4.44 \mathrm{MeV}$ state. When both particles are detected on the same side of the beam however, the intensity of these two processes becomes comparable (see fig.4.14). This type of behaviour, with the enhancement of the excited states at smaller angular separations and larger momentum transfers to the target, has also been reported in ${ }^{12} \mathrm{C}\left({ }^{20} \mathrm{Ne},{ }^{16} \mathrm{O}+\alpha\right){ }^{12} \mathrm{C}$ experiments at $7.85 \mathrm{MeV} / \mathrm{A}$ [Si87]. There are a number of possible explanations for this behaviour. The simplest and probably the most reasonable is that mutual excitation of both the ${ }^{12} \mathrm{C}$ and the ${ }^{7} \mathrm{Li}$ becomes more probable with larger momentum transfer to the target. This would suggest that as the angular difference increases between fragments on opposite sides of the beam, target excitation should become more noticeable. In calculating the momentum transferred to the recoiling ${ }^{12} \mathrm{C}$ target for a fixed value of $\theta_{\alpha}$ and $\theta_{t}$ at the minimum detectable relative energy, it becomes clear that the elements corresponding to larger momentum transfers do indeed exhibit more excitation of the target (eg. fig.4.11 element F1M1 $\theta_{\alpha}=39.2^{\circ}, \theta_{t}=-8.0^{\circ}, \Delta \mathrm{p}_{\text {targ }} \sim 290 \mathrm{MeV} / \mathrm{c}$; fig.4.13 element F4M1 $\left.\theta_{\alpha}=39.6^{\circ}, \theta_{t}=-28.0^{\circ}, \Delta \mathrm{p}_{\text {targ }} \sim 340 \mathrm{MeV} / \mathrm{c}\right)$. The elements with smaller momentum transfers display less target excitation (eg. fig.4.12 element F1M1 $\left.\theta_{\alpha}=14.7^{\circ}, \theta_{t}=-28.0^{\circ}, \Delta \mathrm{p} \sim 93 \mathrm{MeV} / \mathrm{c}\right)$. It is also possible that the increased probabilty of excitation of the ${ }^{12} \mathrm{C}$ with larger momentum transfers is due to the increased probability of particle transfer or fragmentation-absorption with reduced impact parameter. The states in ${ }^{16} \mathrm{O}$ and ${ }^{15} \mathrm{~N}$ may then decay by particle re-emission to the ground or first excited state of the ${ }^{12} \mathrm{C}$ nucleus. This mechanism would tend to produce a cross-sectional angular dependance that may be forward focused due to the large width of the excited states populated, but its contribution would also result in a fixed ratio between the intensities of 
the ${ }^{12} \mathrm{C}$ final states.

It is impossible to distinguish between these two processes from the total kinetic energy spectra alone. It is necessary to inspect the projected spectra of events belonging to each separate locus to obtain more information on the mechanisms involved. This is done in more detail in chapter 5 .

It is also interesting to consider why there seems to be no identifiable strength in the mutual excitation of the ${ }^{120} \mathrm{Sn}$ or ${ }^{208} \mathrm{~Pb}$ targets. It is known that mutual excitation of both the target and the projectile is much more probable if they are of similar size [Ka79]. It may also be an indication that the reaction mechanisms involved with these targets and the ${ }^{7} \mathrm{Li}$ projectile are different from those involved with the ${ }^{12} \mathrm{C}$ target. This will also be approached later in chapter 5 where the possible reaction mechanisms will be discussed.

\subsubsection{In-Plane Data}

In this section the differential cross-sections for the elements of the alpha detector which lie in the reaction plane will be presented. Figures 4.20 to 4.23 show the quasi-elastic in-plane differential cross section for varying $\theta_{t}$ and $\theta_{\alpha}$ for the ${ }^{12} \mathrm{C}$ target, fig.4.24 to fig.4.27 show the data for the ${ }^{120} \mathrm{Sn}$ target and fig.4.28 to fig. 4.30 the data for the ${ }^{208} \mathrm{~Pb}$ target. Each figure shows the cross-section for a set of fixed $\theta_{t}$ and varying $\theta_{\alpha}$. Some figures also show the result of a simple PWBA calculation which will be discussed in more detail in chapter 5 .

It can be seen that the data recorded with the ${ }^{12} \mathrm{C}$ target shows a rather structureless decrease with increasing $\theta_{t}$ and $\theta_{\alpha}$. There is no detectable diffraction structure. This is probably a result of the angular resolution of the detector set-up. There is a marked increase for forward angles with the triton and alpha detectors close to the beam direction. (eg. fig.4.20 with $\theta_{t}=-8.0^{\circ}$ and $\theta_{\alpha}=14.7^{\circ}$ ). This has been attributed to the detectors being within the sequential break-up cone for the $4.63 \mathrm{MeV}$ state in ${ }^{7} \mathrm{Li}$. Apart from this enhancement at forward angles, the average slope of the quasi-elastic differential cross-section seems to be rather insensitive to the triton angle. This feature is common to all 


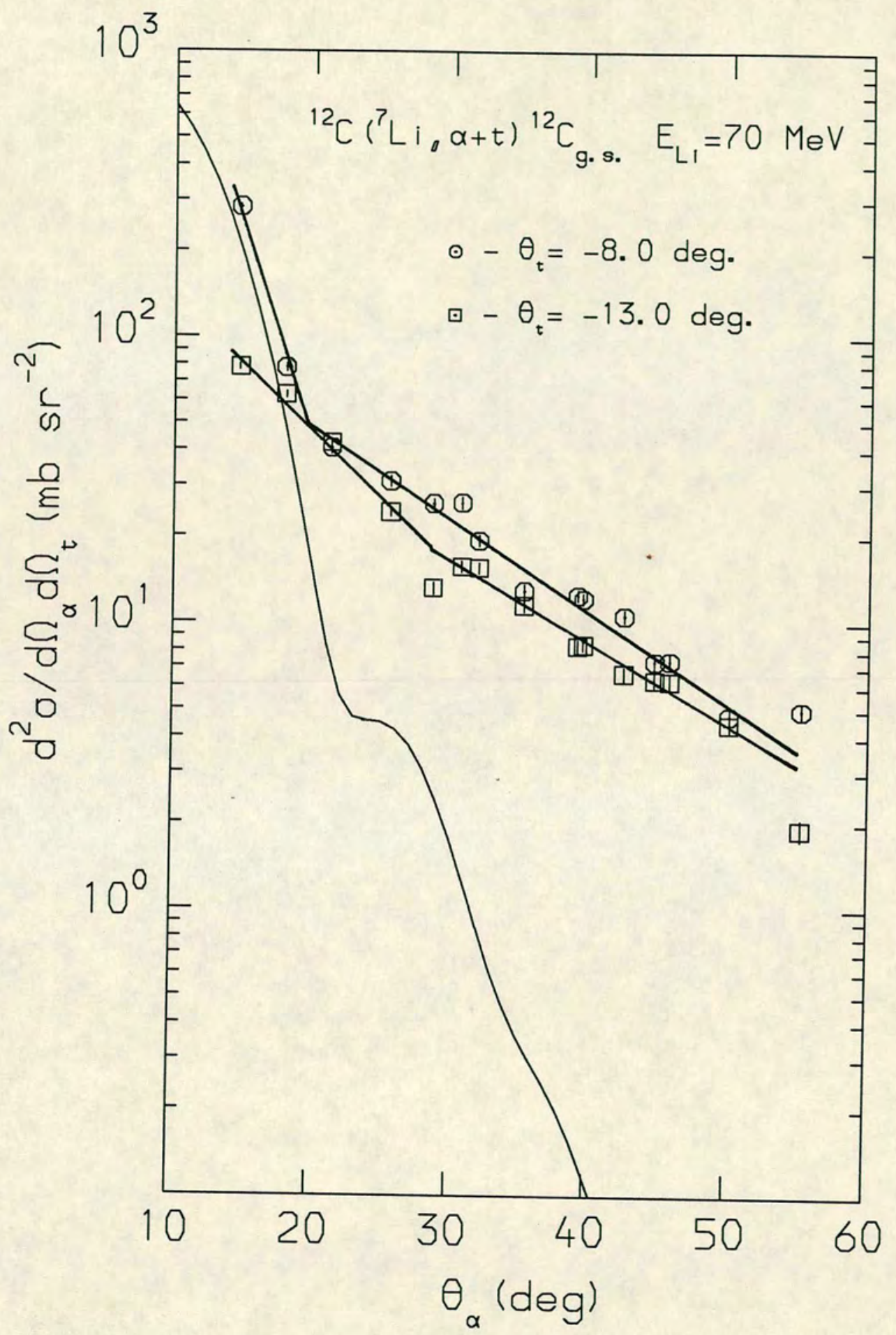

Figure 4.20: Angular distribution for quasi-elastic events from ${ }^{12} \mathrm{C}$ target with $\phi_{\alpha}=2.0^{\circ}$ ( straight lines to guide the eye ). PWBA fit for $\theta_{t}=-8.0^{\circ}$ normalised to forward angled data points. 


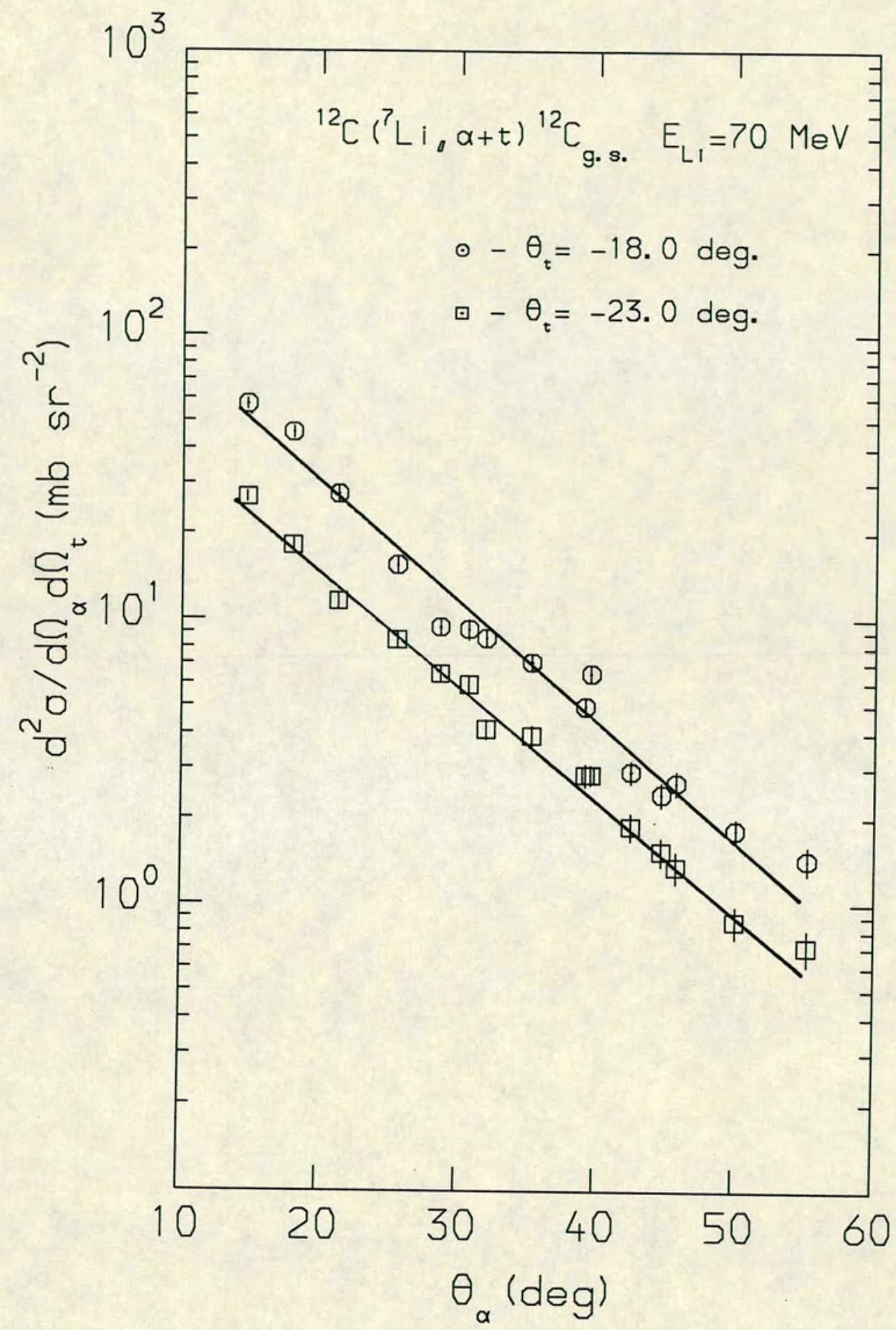

Figure 4.21: Angular distribution for quasi-elastic events from ${ }^{12} \mathrm{C}$ target with $\phi_{\alpha}=2.0^{\circ}$ ( lines to guide the eye). 


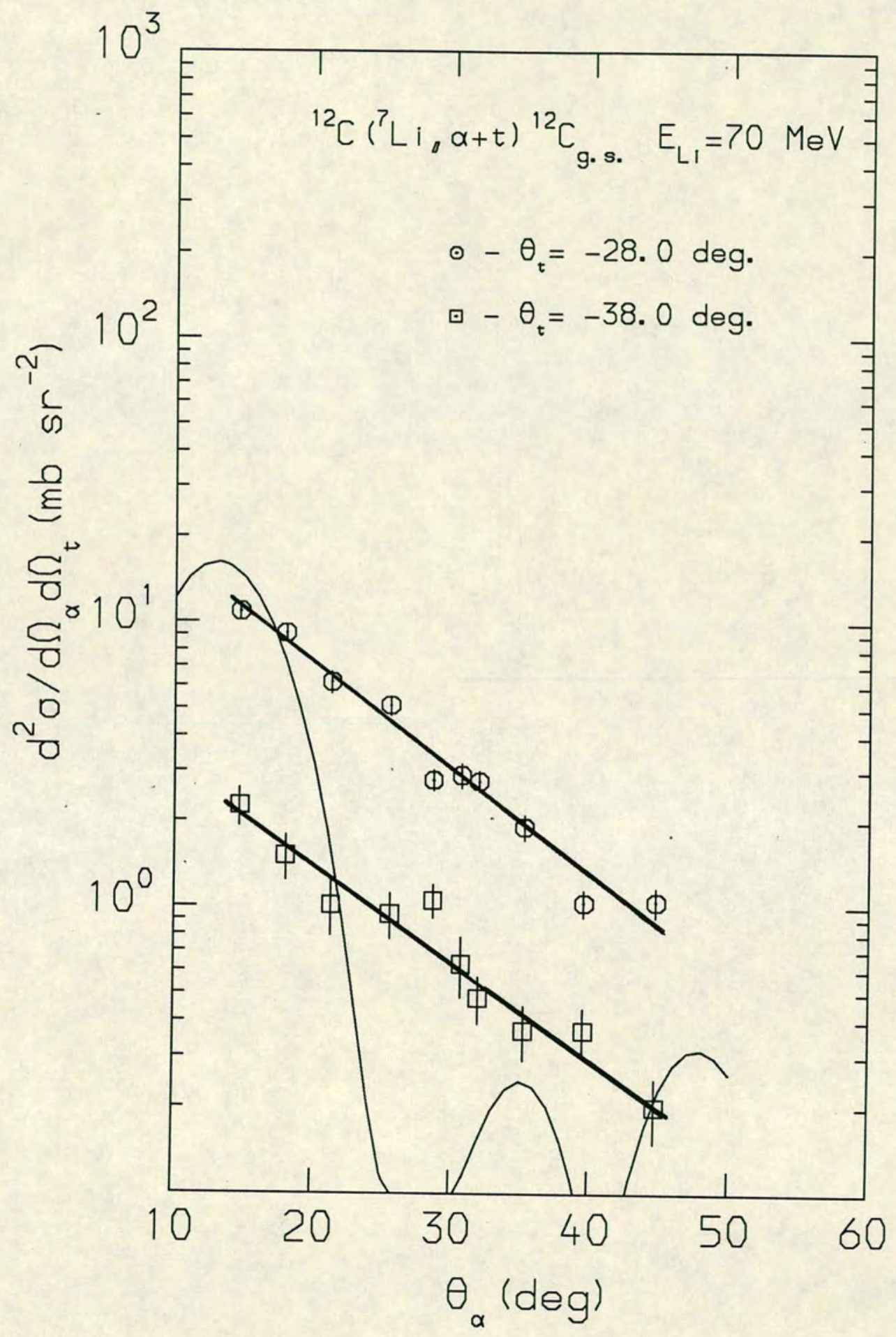

Figure 4.22: Angular distribution for quasi-elastic events from ${ }^{12} \mathrm{C}$ target with $\phi_{\alpha}=2.0^{\circ}$ ( straight lines to guide the eye ). PWBA fit for $\theta_{t}=-28.0^{\circ}$ normalised to forward angled data points. 


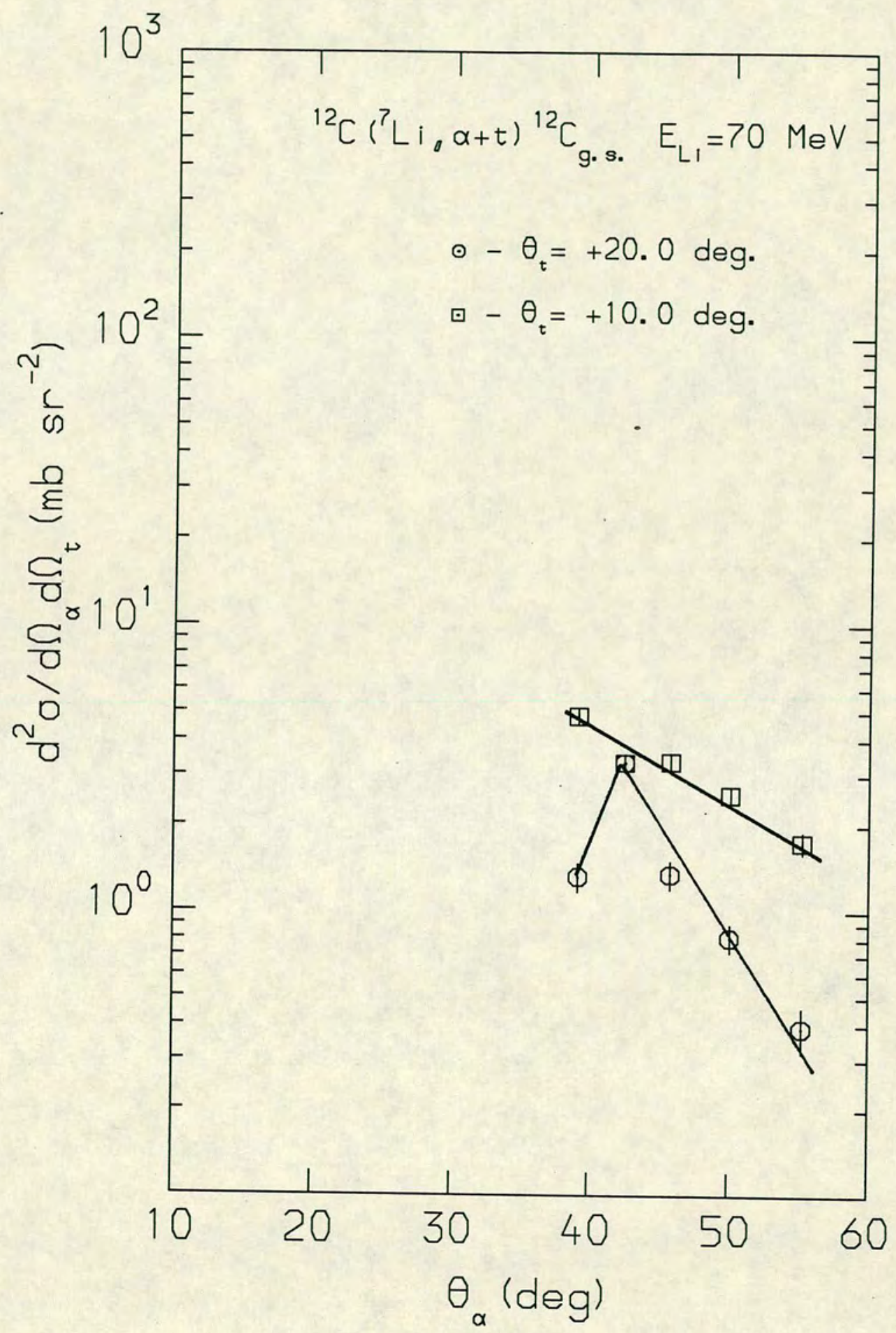

Figure 4.23: Angular distribution for quasi-elastic events from ${ }^{12} \mathrm{C}$ target with $\phi_{\alpha}=2.0^{\circ}$ and detectors on same side of beam (lines to guide the eye ). 


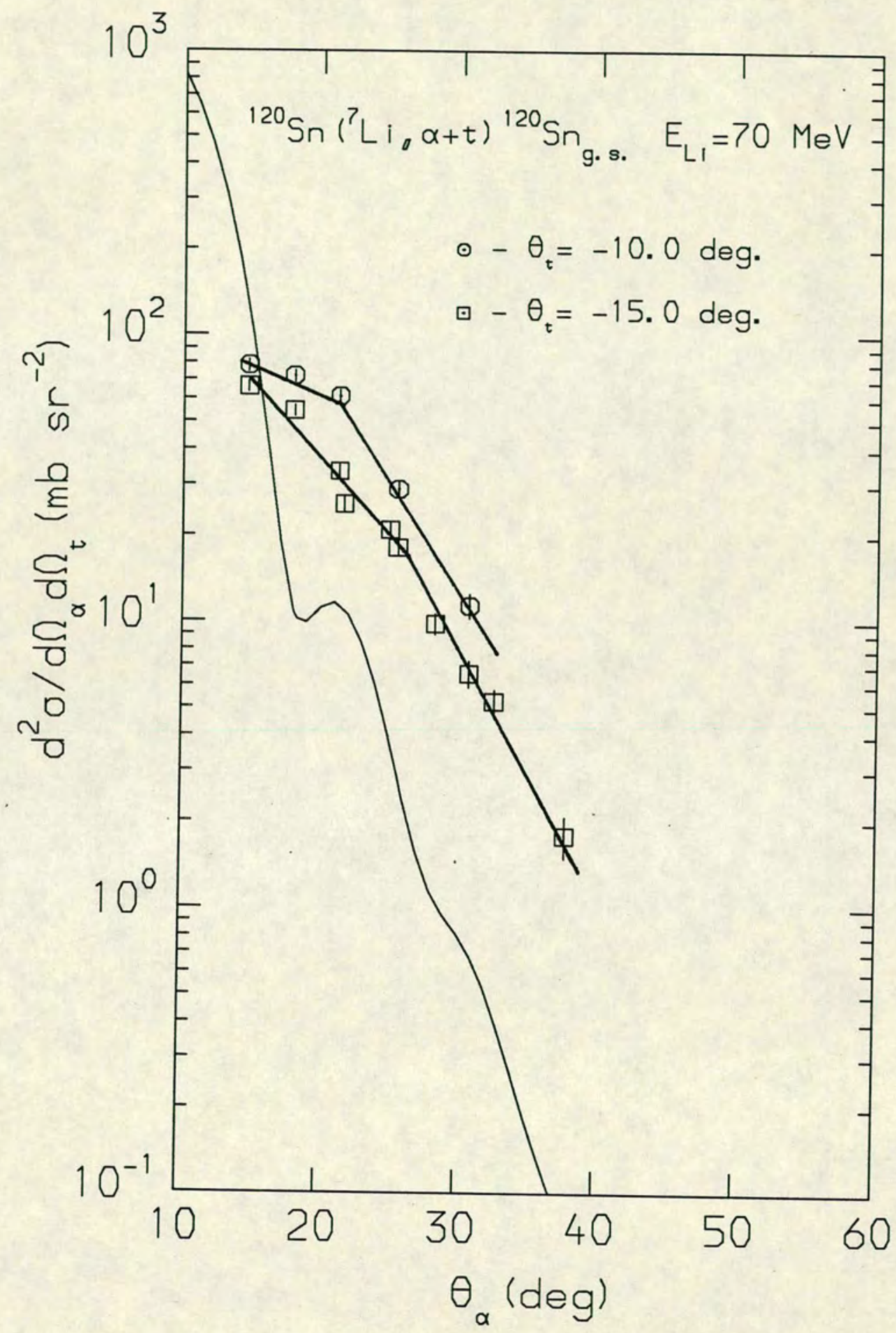

Figure 4.24: Angular distribution for quasi-elastic events from ${ }^{120} \mathrm{Sn}$ target with $\phi_{\alpha}=2.0^{\circ}$ ( straight lines to guide the eye ). PWBA fit for $\theta_{t}=-10.0^{\circ}$ normalised to forward angled data points. 


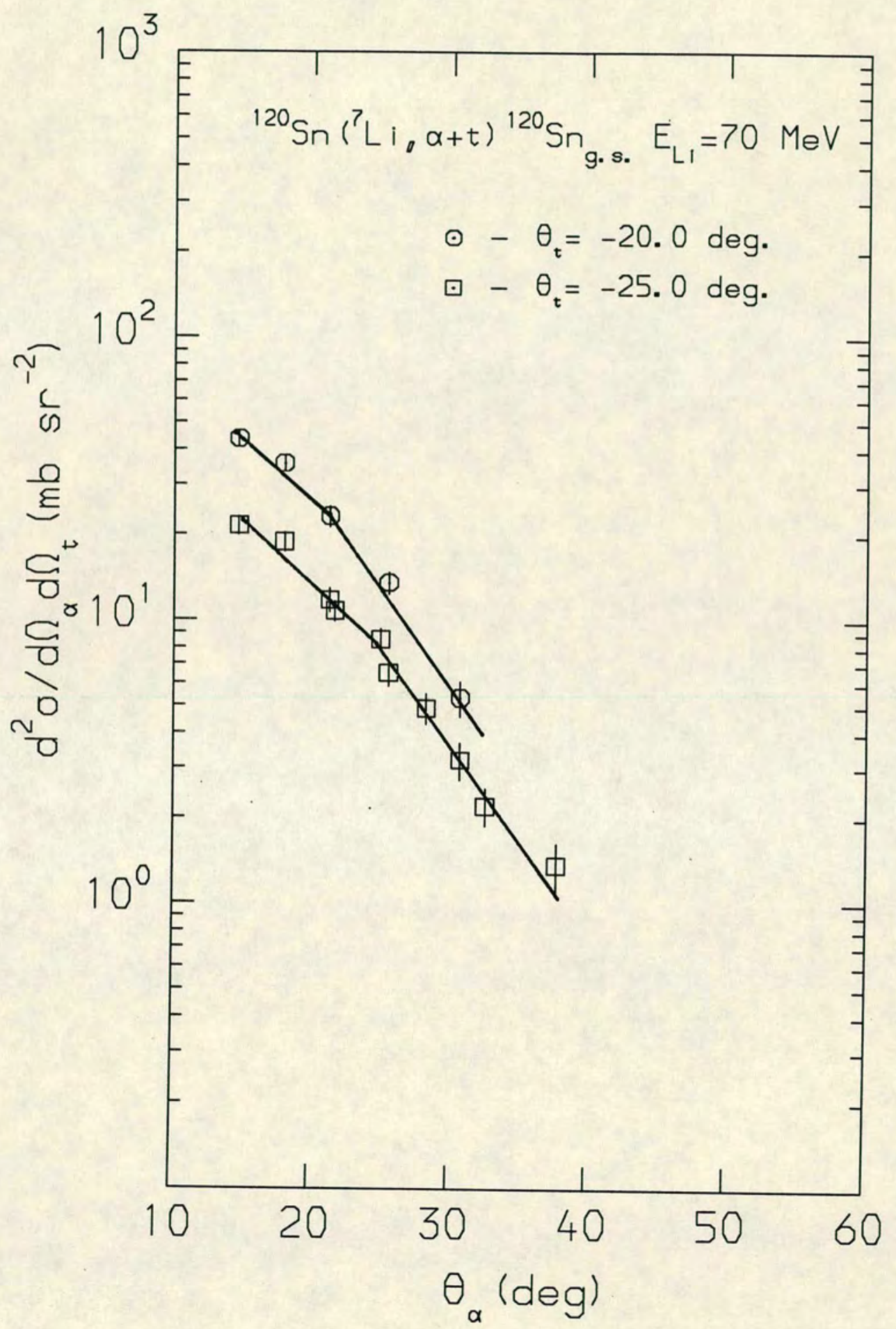

Figure 4.25: Angular distribution for quasi-elastic events from ${ }^{120} \mathrm{Sn}$ target with $\phi_{\alpha}=2.0^{\circ}$ ( lines to guide the eye ). 


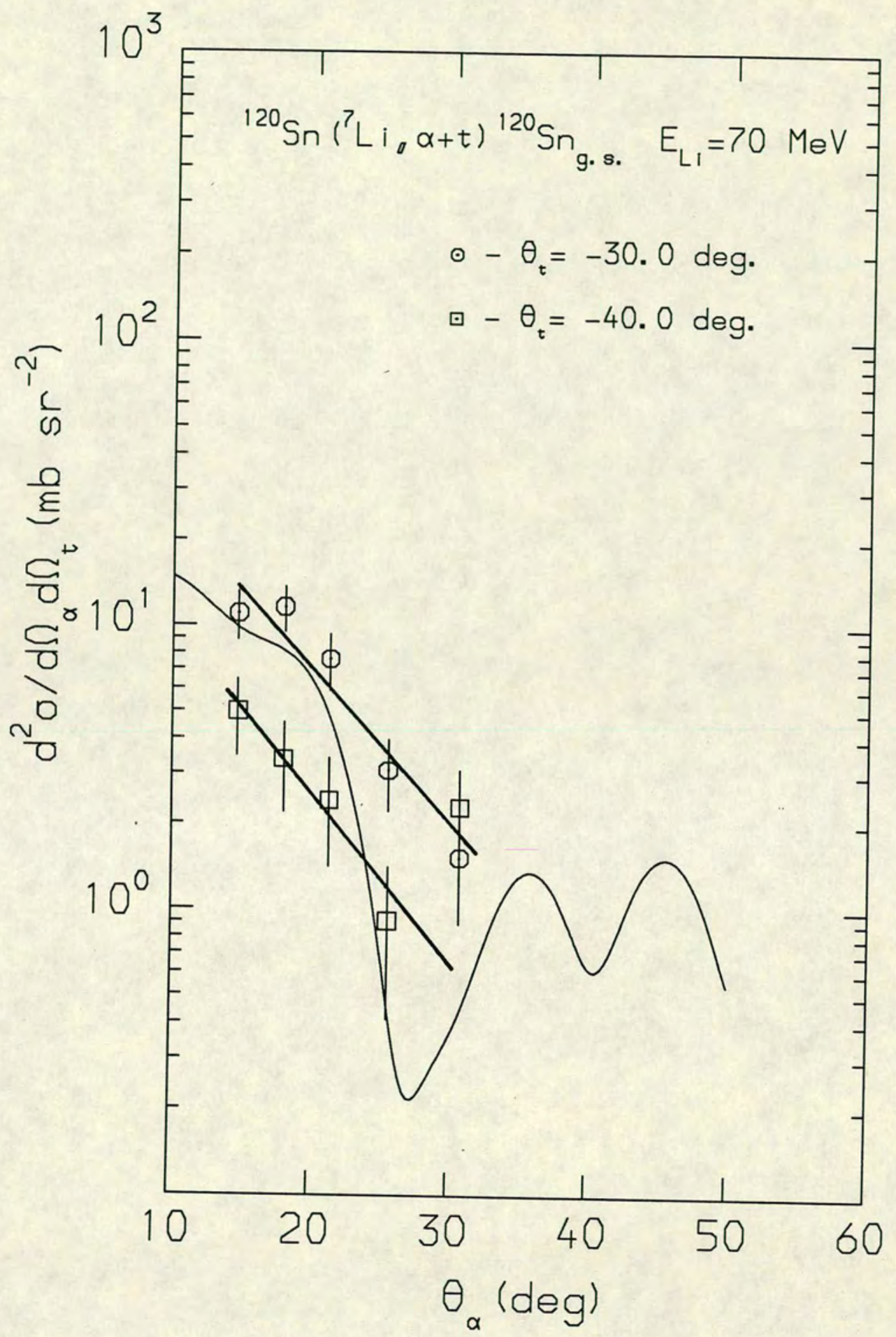

Figure 4.26: Angular distribution for quasi-elastic events from ${ }^{120} \mathrm{Sn}$ target with $\phi_{\alpha}=2.0^{\circ}$ ( straight lines to guide the eye ). PWBA fit for $\theta_{t}=-30.0^{\circ}$ normalised to forward angled data points. 


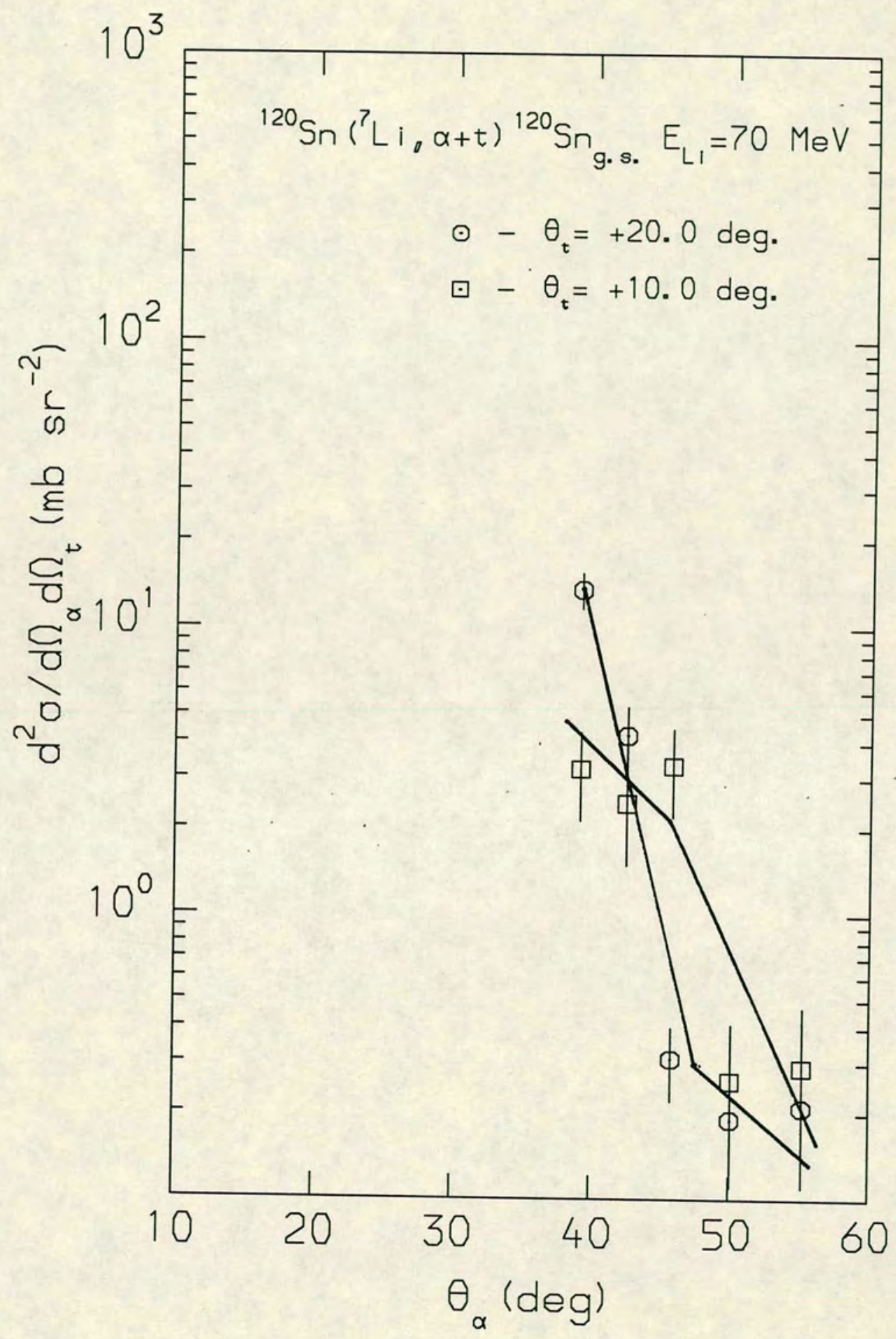

Figure 4.27: Angular distribution for quasi-elastic events from ${ }^{120} \mathrm{Sn}$ target with $\phi_{\alpha}=2.0^{\circ}$ and detectors on same side of beam (lines to guide the eye ). 


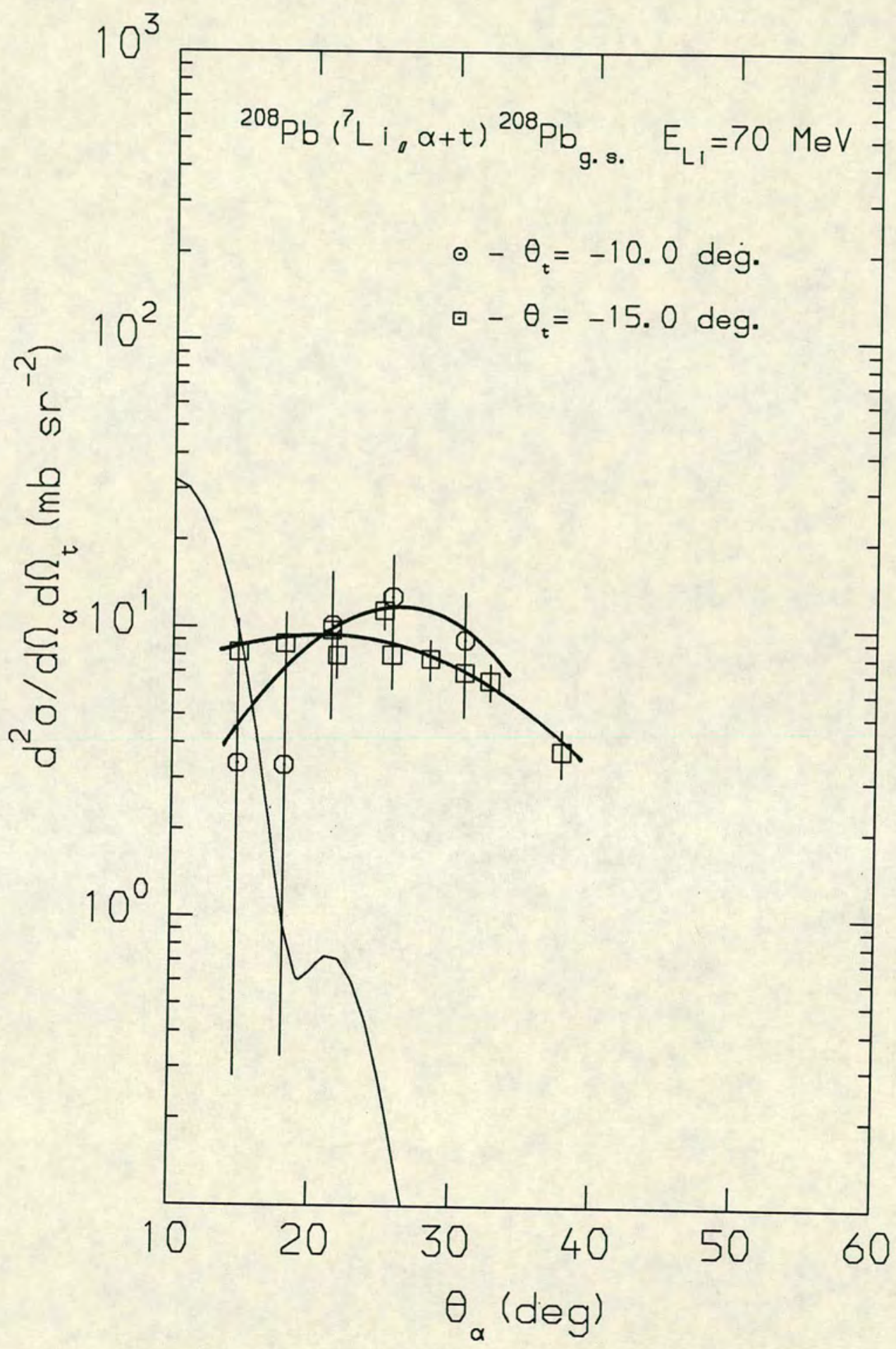

Figure 4.28: Angular distribution for quasi-elastic events from ${ }^{208} \mathrm{~Pb}$ target with $\phi_{\alpha}=2.0^{\circ}$ ( lines to guide the eye ). PWBA fit for $\theta_{t}=-15.0^{\circ}$ normalised to forward angled data points. 


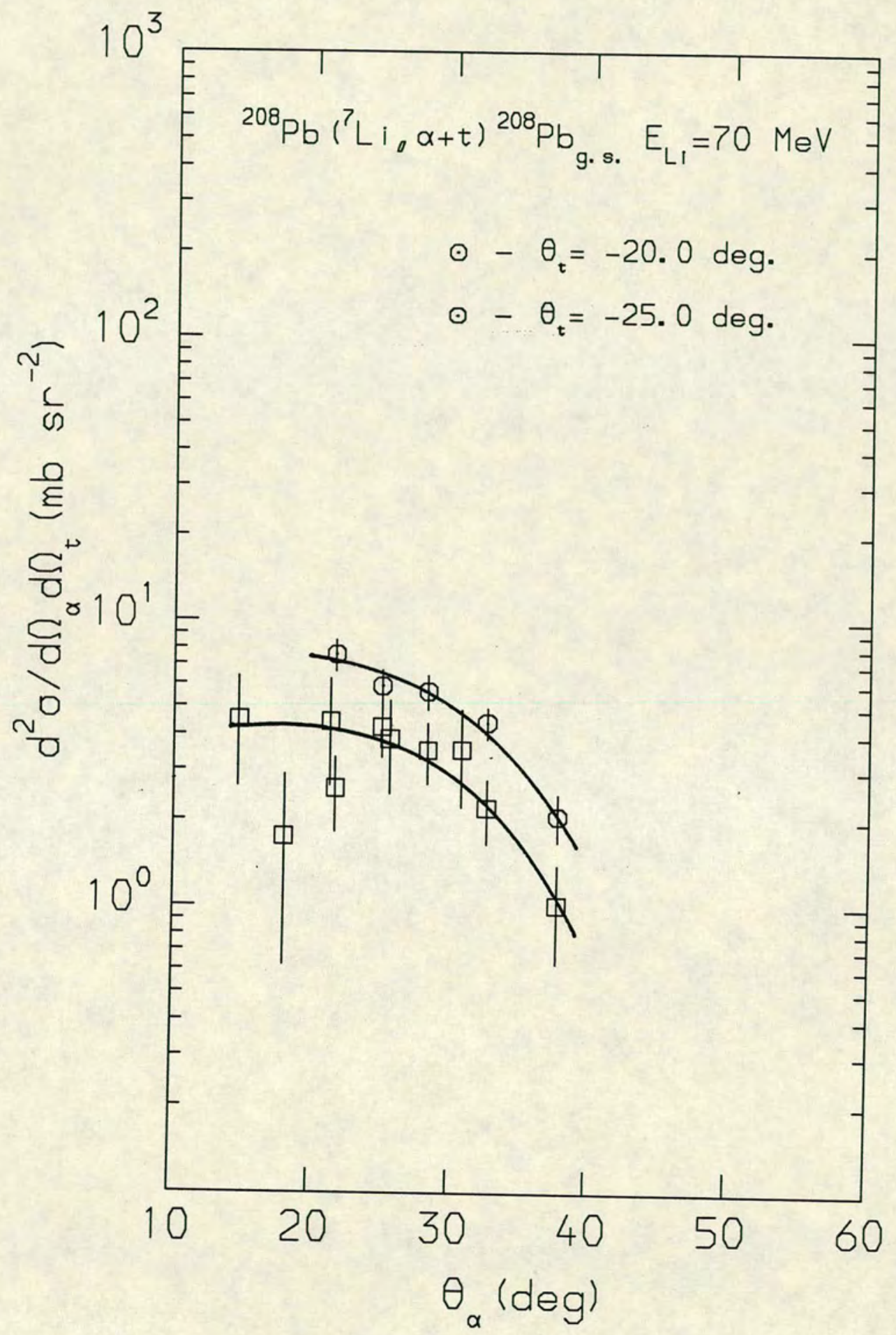

Figure 4.29: Angular distribution for quasi-elastic events from ${ }^{208} \mathrm{~Pb}$ target with $\phi_{\alpha}=2.0^{\circ}$ ( lines to guide the eye ). 


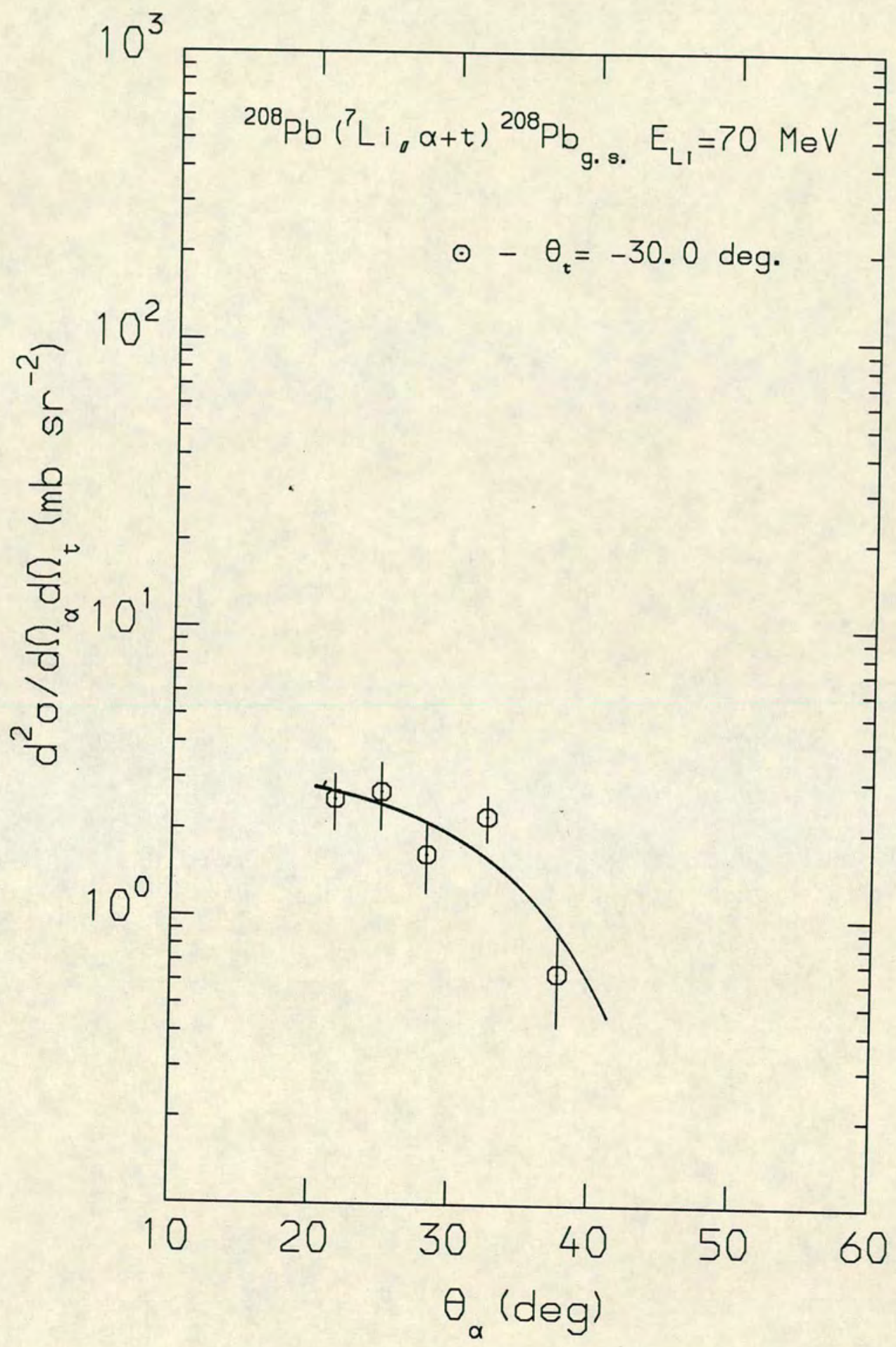

Figure 4.30: Angular distribution for quasi-elastic events from ${ }^{208} \mathrm{~Pb}$ target with $\phi_{\alpha}=2.0^{\circ}$ ( line to guide the eye ). 
the targets. The ${ }^{120} \mathrm{Sn}$ quasi-elastic data seem to exhibit a levelling out of the differential cross-section inside the grazing angle $\left(\theta_{\text {graz. }} \sim 21^{\circ}\right)$ with the fall off outside this value of $\theta_{\alpha}$ again being independant of $\theta_{t}$. The ${ }^{208} \mathrm{~Pb}$ quasi-elastic data follow the shape of the alpha particle singles data presented in fig. 4.9 with the cross-section remaining rather flat inside $\theta_{\alpha} \sim 30^{\circ}$.

The ${ }^{12} \mathrm{C}$ quasi-elastic differential cross-section is similar to the ${ }^{120} \mathrm{Sn}$ differential cross-section at forward angles but falls off more slowly with increasing $\theta_{\alpha}$. The ${ }^{208} \mathrm{~Pb}$ cross-section at forward angles is a factor of 10 smaller and has a fall off, outside $\sim 30^{\circ}$ approximately the same as the ${ }^{120} \mathrm{Sn}$ data. At larger values of $\theta_{t}$ the ${ }^{208} \mathrm{~Pb}$ cross-section tends to remain constant in the $1-10 \mathrm{mb} \mathrm{sr}^{-2}$ region whereas both the ${ }^{12} \mathrm{C}$ and ${ }^{120} \mathrm{Sn}$ data fall by a factor of $\sim \times 10$ from their values at smaller $\theta_{t}$. Fig.4.31 to fig.4.34 show the double differential cross-sections for $\alpha$-t coincident events leaving the ${ }^{12} \mathrm{C}$ target in its $4.44 \mathrm{MeV}$ excited state. These show similar behaviour to the quasi-elastic events with a decreasing magnitude as $\theta_{t}$ and $\theta_{\alpha}$ increase and a slope rather insensitive to the $\theta_{t}$ angle. This slope is shallower than that of the quasi-elastic events and agrees with the earlier statement that as the angular difference between the detectors increases, the excitation of the $4.44 \mathrm{MeV}{ }^{12} \mathrm{C}$ state becomes more important. At these larger angles, with the alpha and triton on opposite sides of the beam, it is still weaker than the quasi-elastic channel.

To complete the in-plane angular distributions, fig. 4.35 to fig. 4.45 show the total double differential cross-sections for all $\alpha$-t coincidence events, irrespective of the excitation in the outgoing channel. It can be seen from these data that the shape of the total double differential cross-sections are essentially the same as the quasi-elastic data showing little or no diffraction structure for any of the targets. The similarity of the fall off is somewhat surprising and indicates that even at large angular separation where both fragments must have undergone a strong interaction with the target, the strength of the quasi-elastic channel is still significant for all three targets. The quasi-elastic contribution to the total in-plane differential cross-section remains at around $50 \sim 70 \%$ for almost all target, angle combinations, only falling to the lower edge of this range for the large angular separations. The smallest contribution is $\sim 15 \%$ for the ${ }^{12} \mathrm{C}$ target and this is only for the extreme limits of the angular separation $\left(\Delta \theta \sim 80^{\circ}\right)$ on 


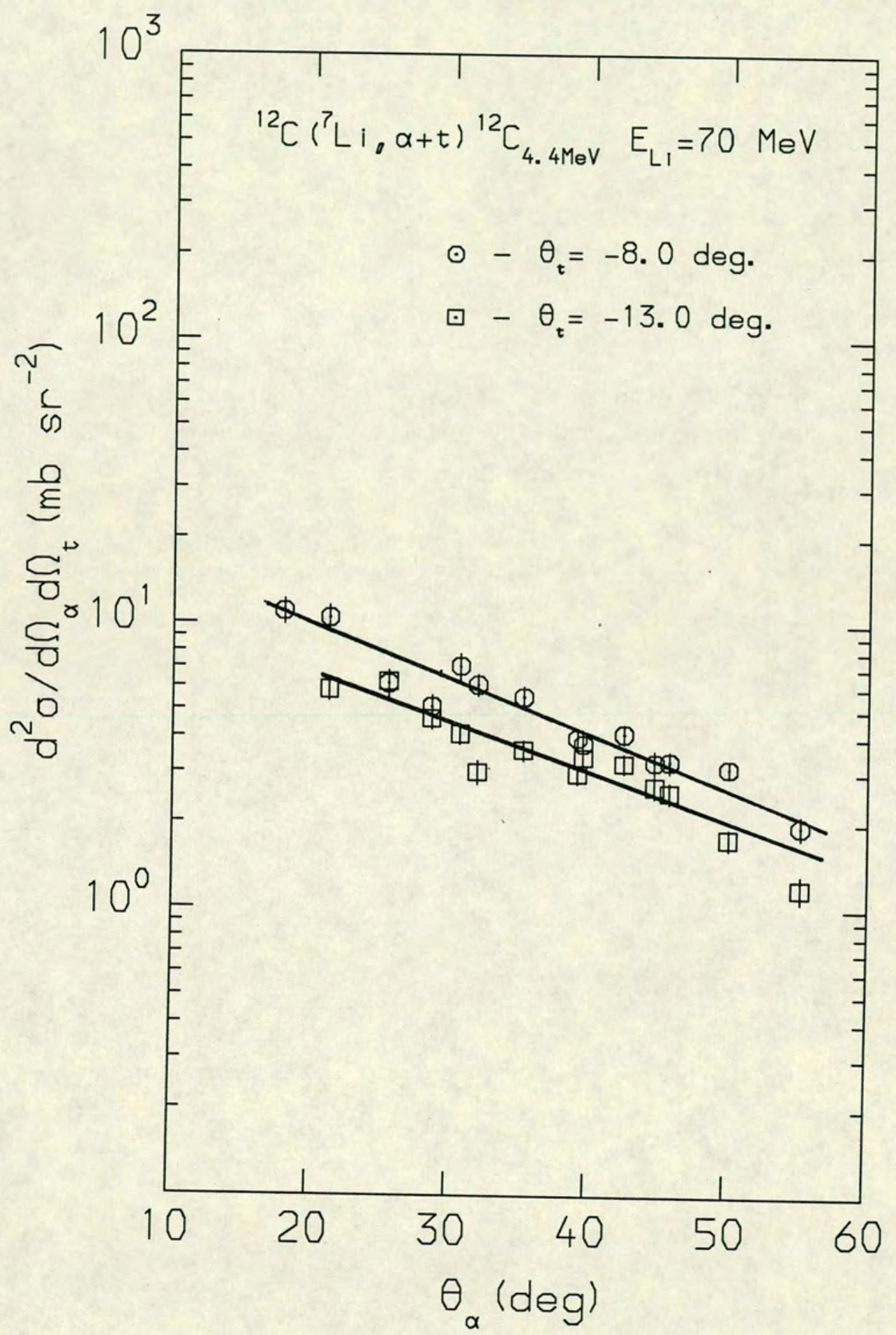

Figure 4.31: Angular distribution for first excited state events from ${ }^{12} \mathrm{C}$ target with $\phi_{\alpha}=2.0^{\circ}$ ( lines to guide the eye ). 


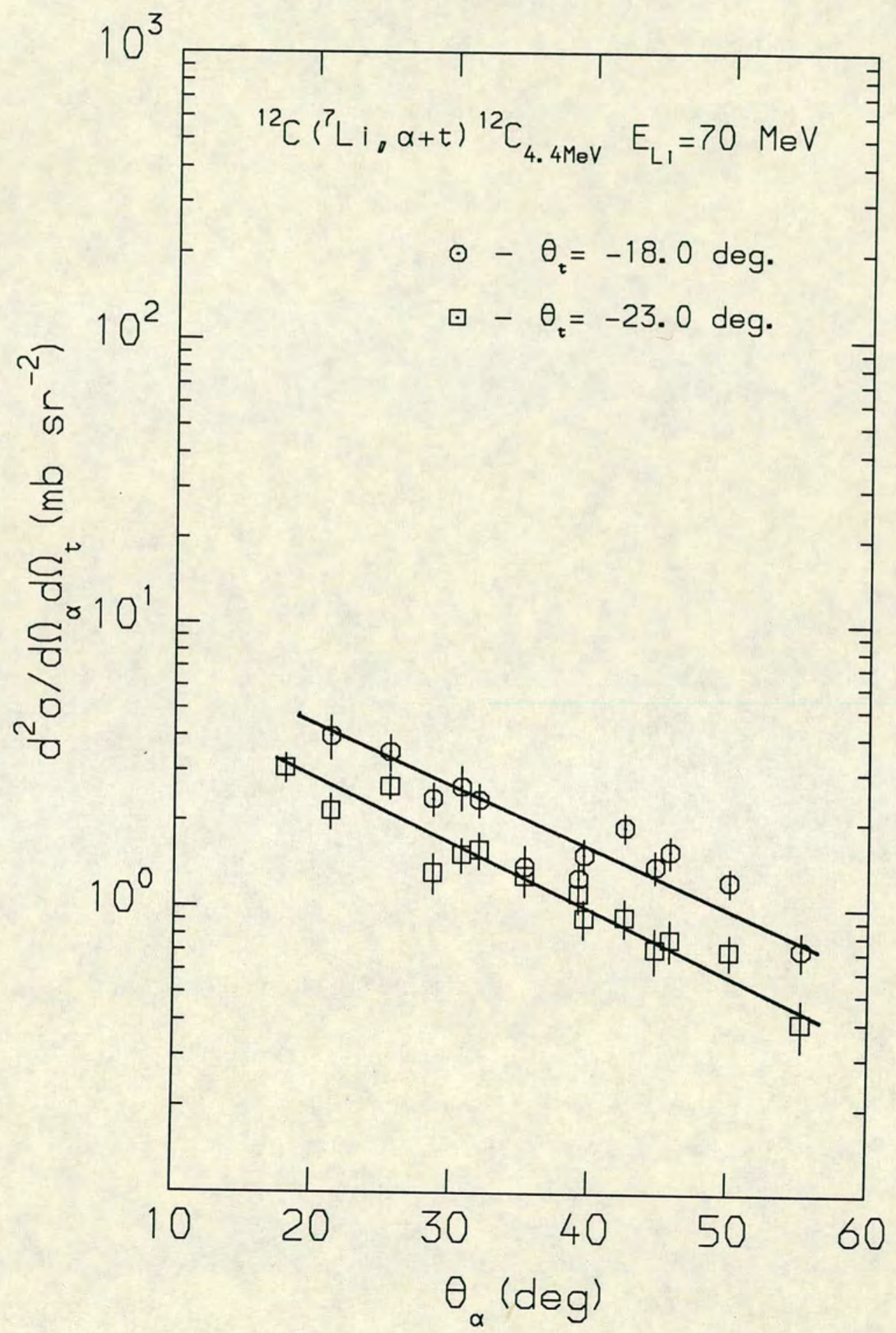

Figure 4.32: Angular distribution for first excited state events from ${ }^{12} \mathrm{C}$ target with $\phi_{\alpha}=2.0^{\circ}$ ( lines to guide the eye ). 


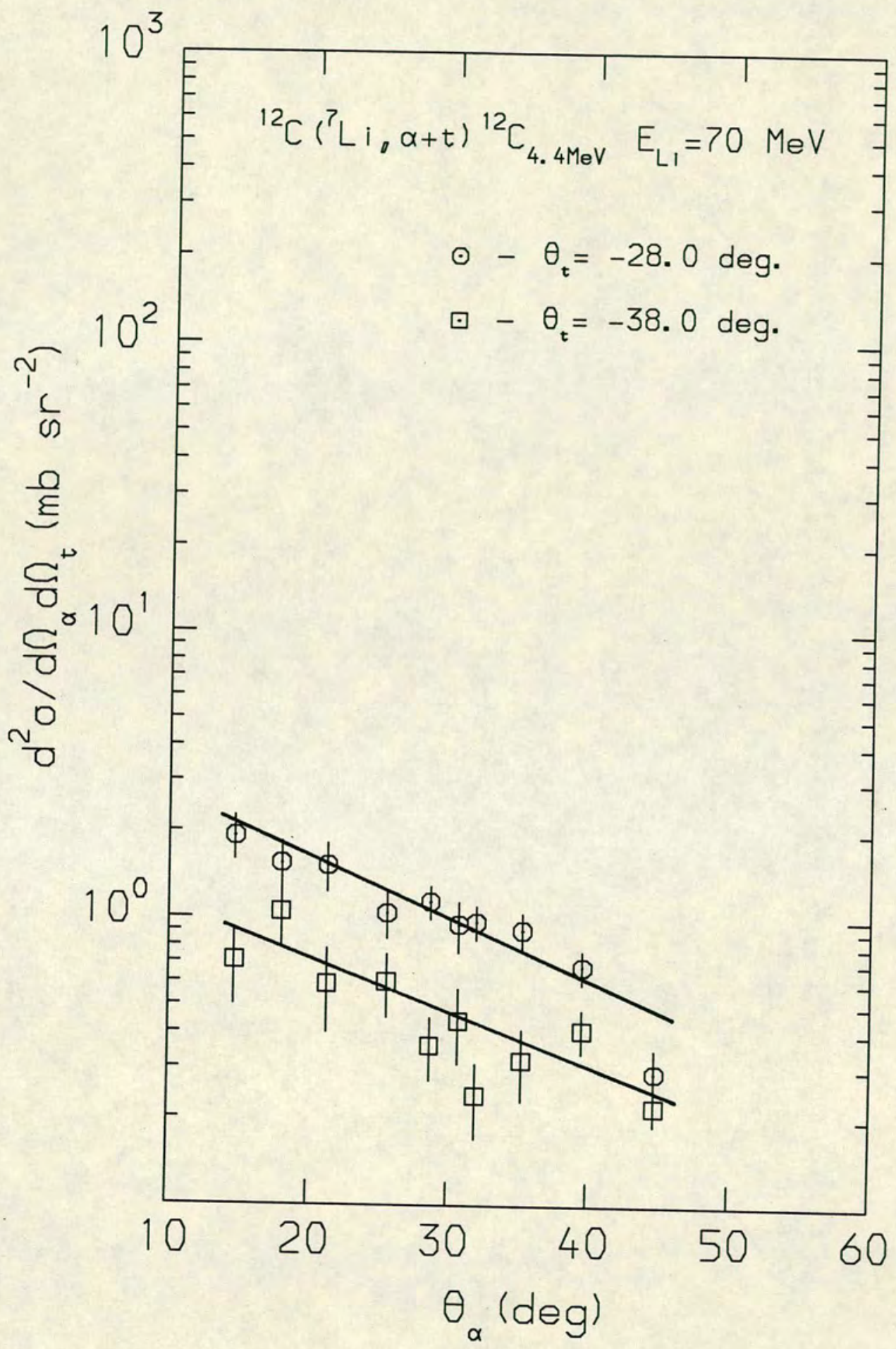

Figure 4.33: Angular distribution for first excited state events from ${ }^{12} \mathrm{C}$ target with $\phi_{\alpha}=2.0^{\circ}$ ( lines to guide the eye ). 


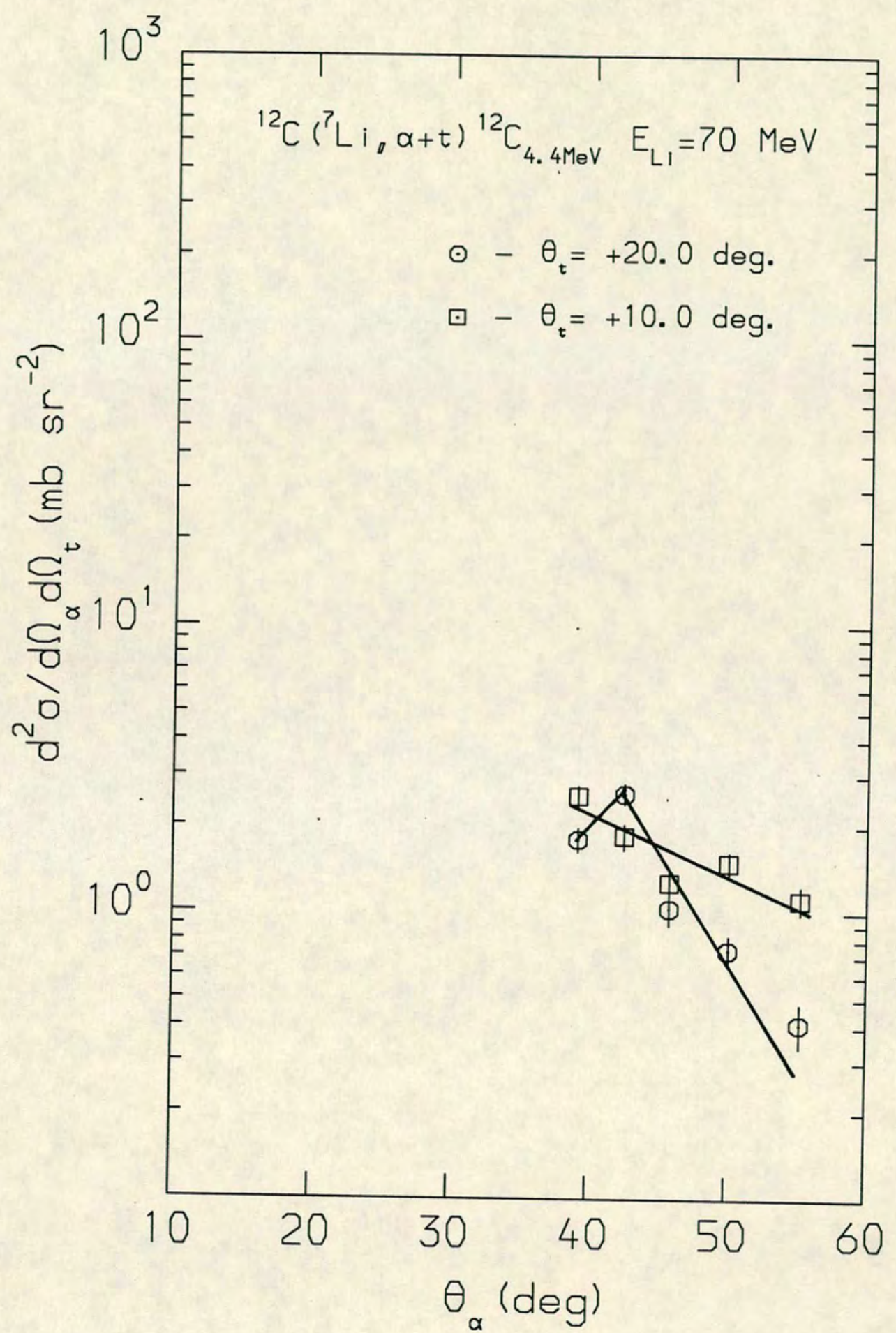

Figure 4.34: Angular distribution for first excited state events from ${ }^{12} \mathrm{C}$ target with $\phi_{\alpha}=2.0^{\circ}$ and detectors on same side of beam (lines to guide the eye ). 


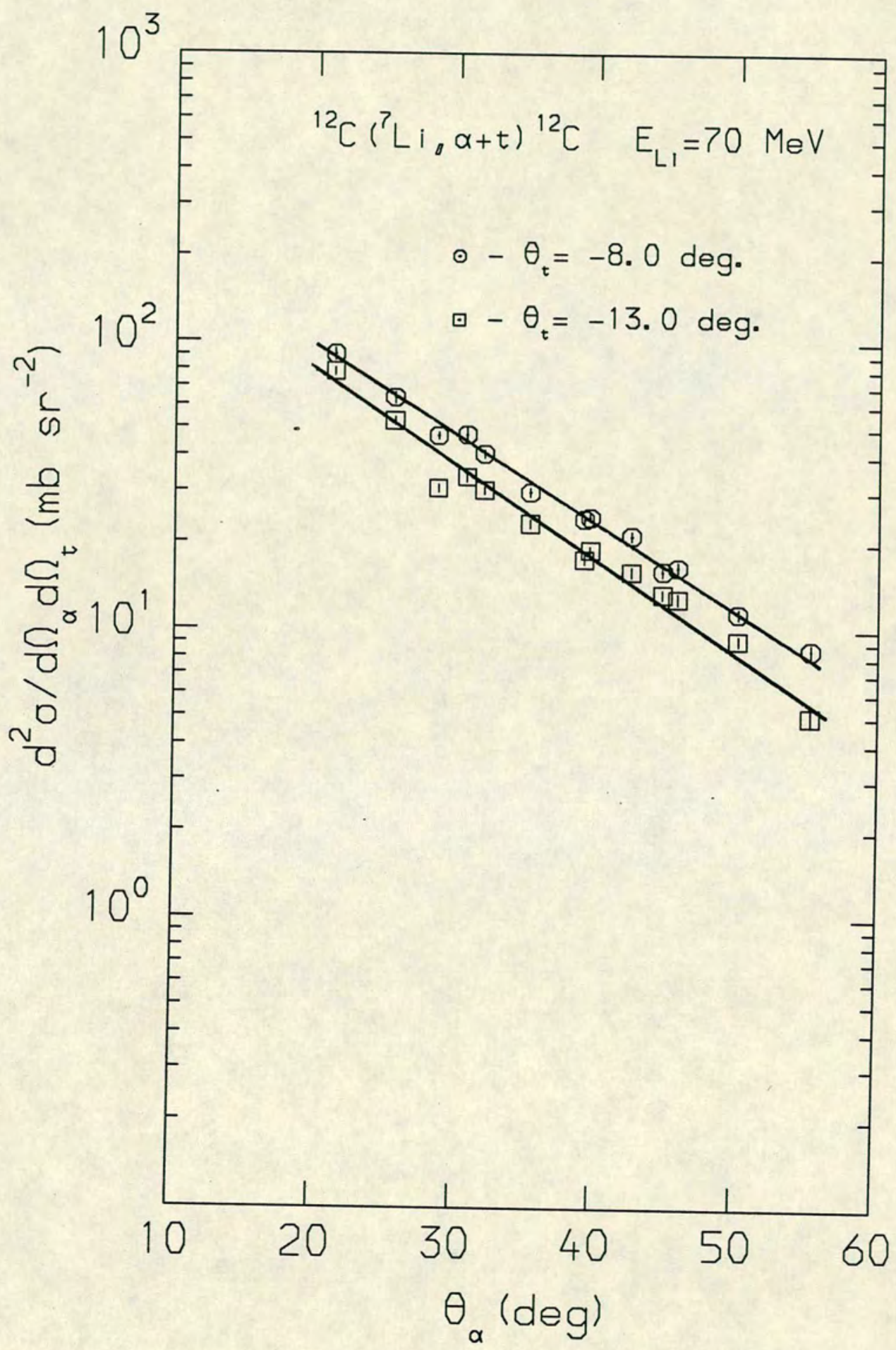

Figure 4.35: Angular distribution for all coincident $\alpha$-t events from ${ }^{12} \mathrm{C}$ target with $\phi_{\alpha}=2.0^{\circ}$ ( lines to guide the eye ). 


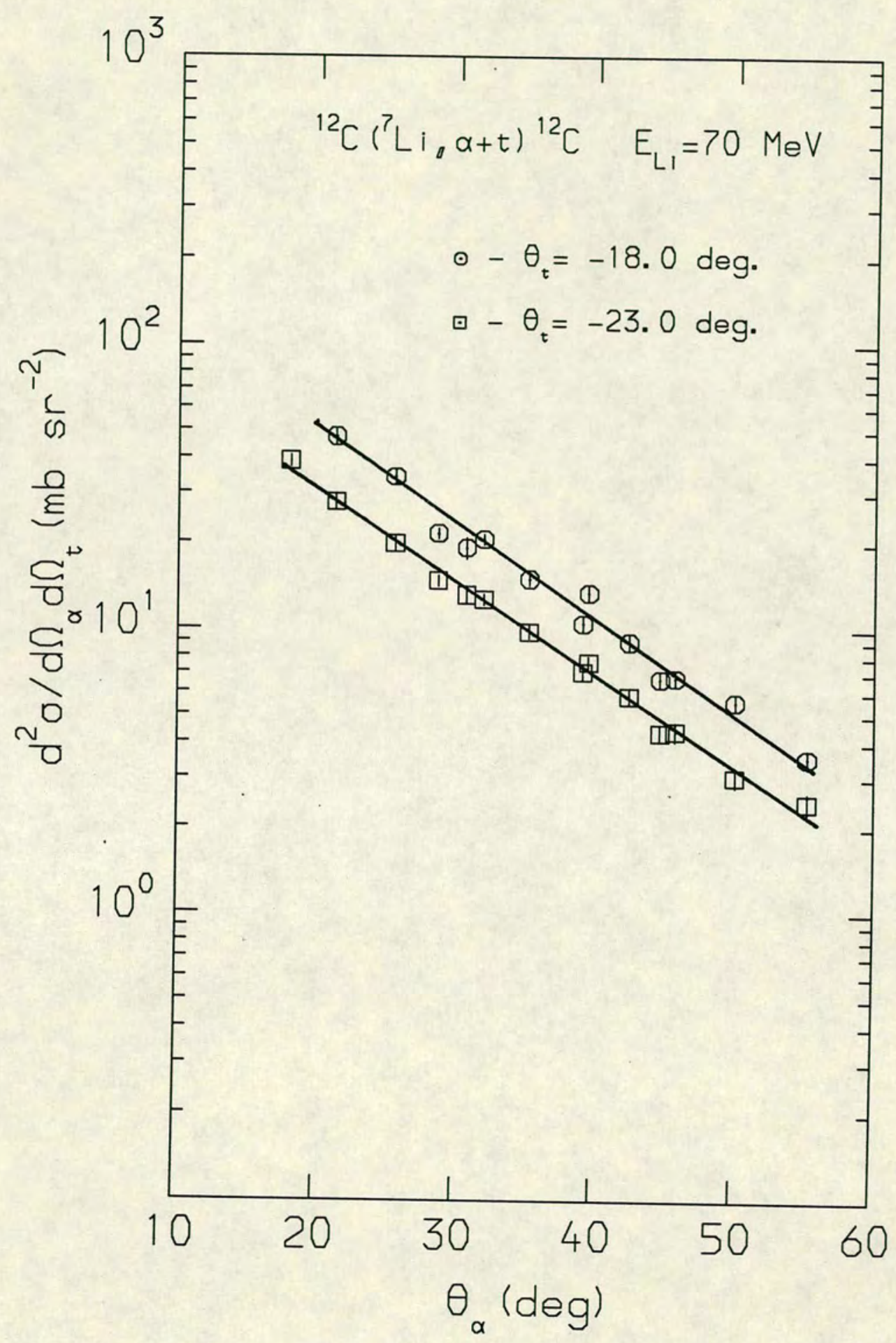

Figure 4.36: Angular distribution for all coincident $\alpha$-t events from ${ }^{12} \mathrm{C}$ target with $\phi_{\alpha}=2.0^{\circ}$ ( lines to guide the eye ). 


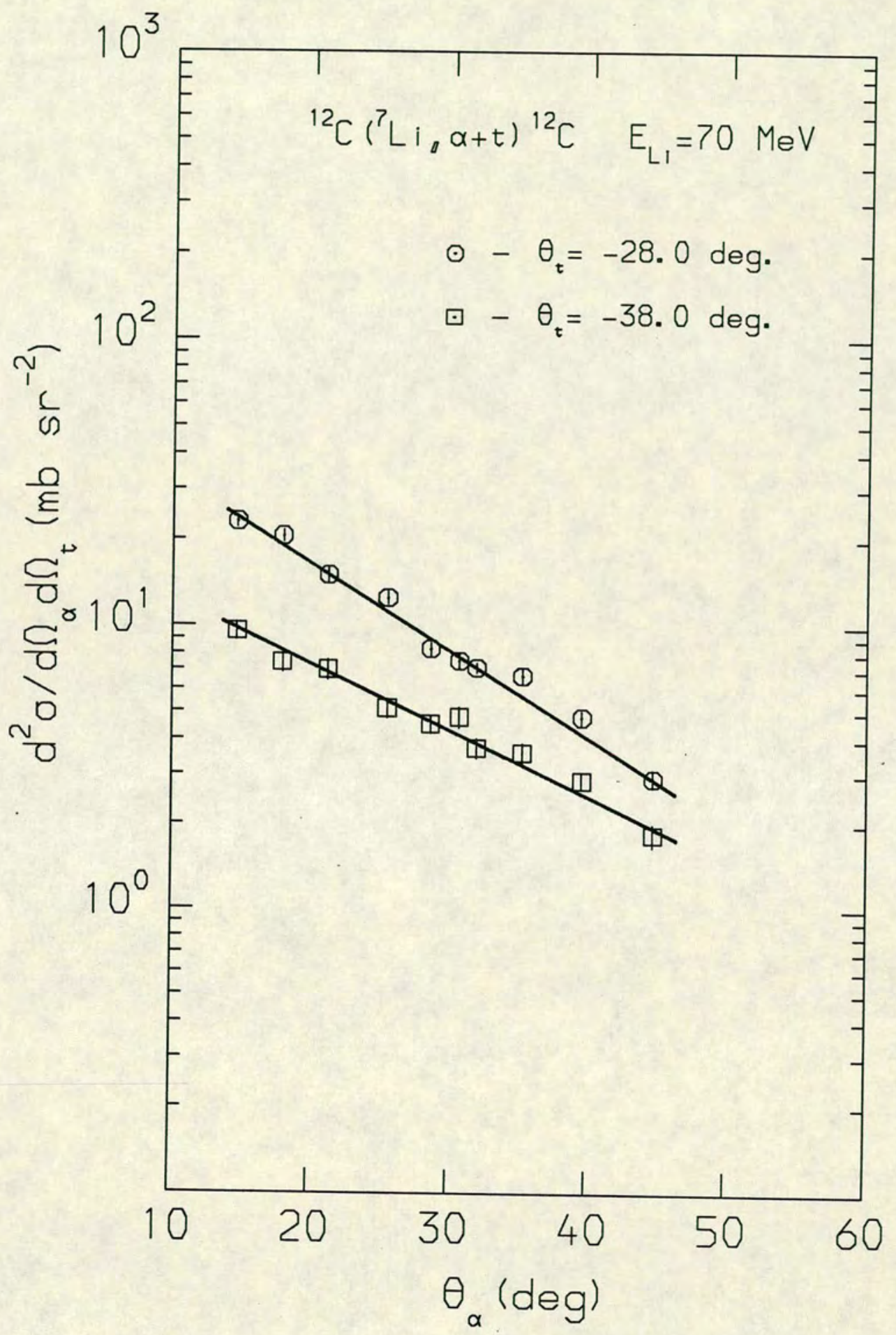

Figure 4.37: Angular distribution for all coincident $\alpha$-t events from ${ }^{12} \mathrm{C}$ target with $\phi_{\alpha}=2.0^{\circ}$ ( lines to guide the eye ). 


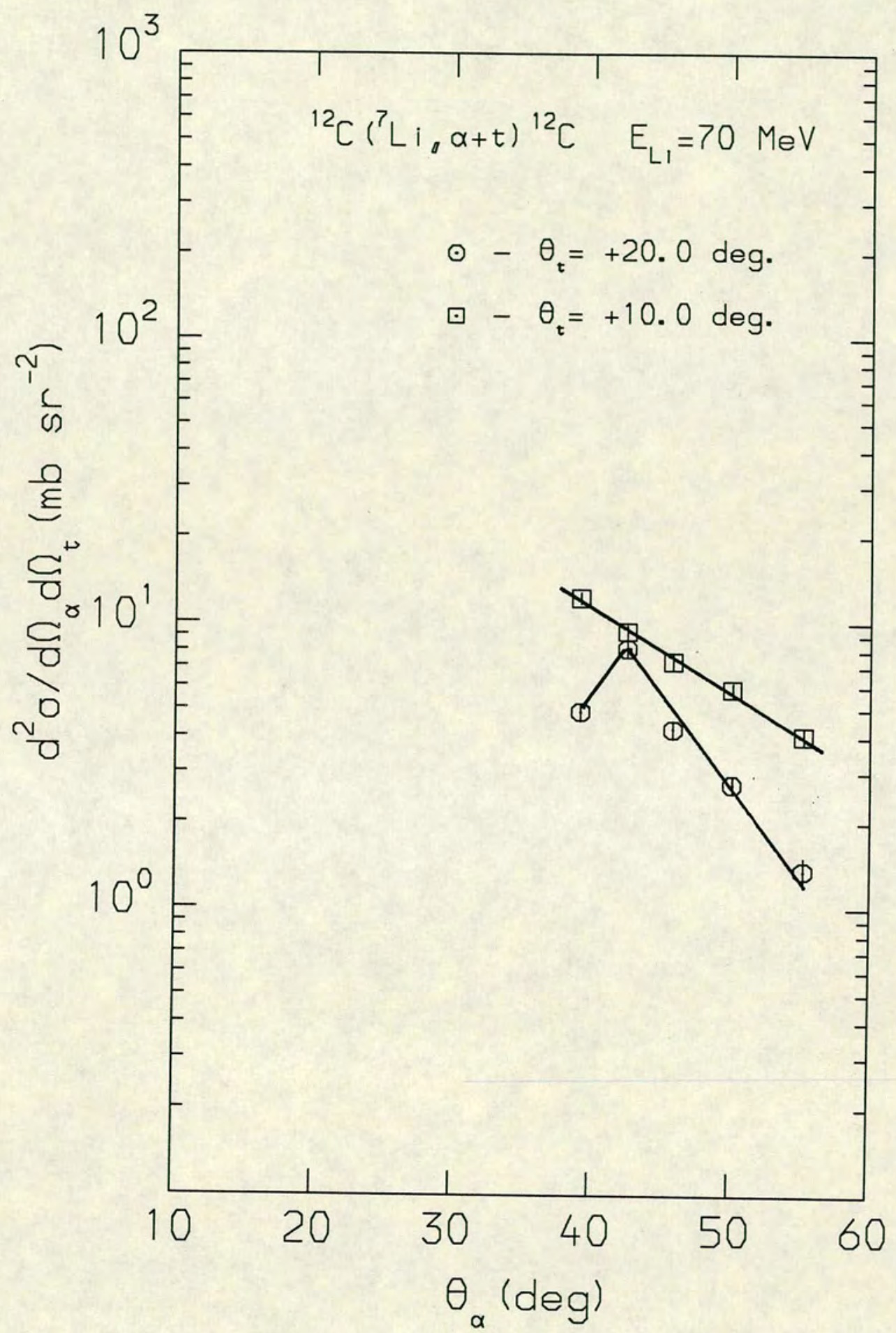

Figure 4.38: Angular distribution for all coincident $\alpha$-t events from ${ }^{12} \mathrm{C}$ target with $\phi_{\alpha}=2.0^{\circ}$ and detectors on same side of beam (lines to guide the eye ). 


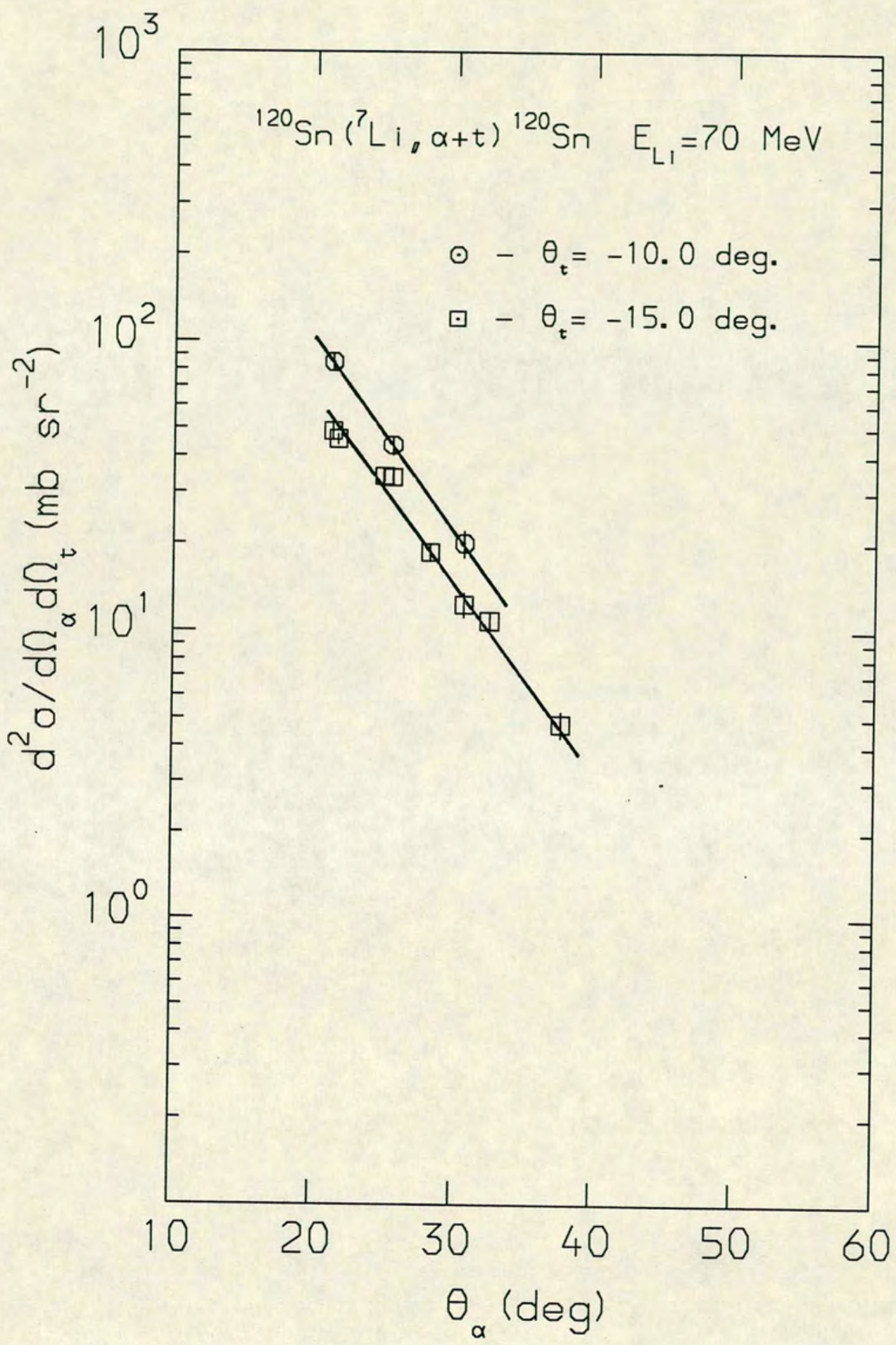

Figure 4.39: Angular distribution for all coincident $\alpha$-t events from ${ }^{120} \mathrm{Sn}$ target with $\phi_{\alpha}=2.0^{\circ}$ ( lines to guide the eye ). 


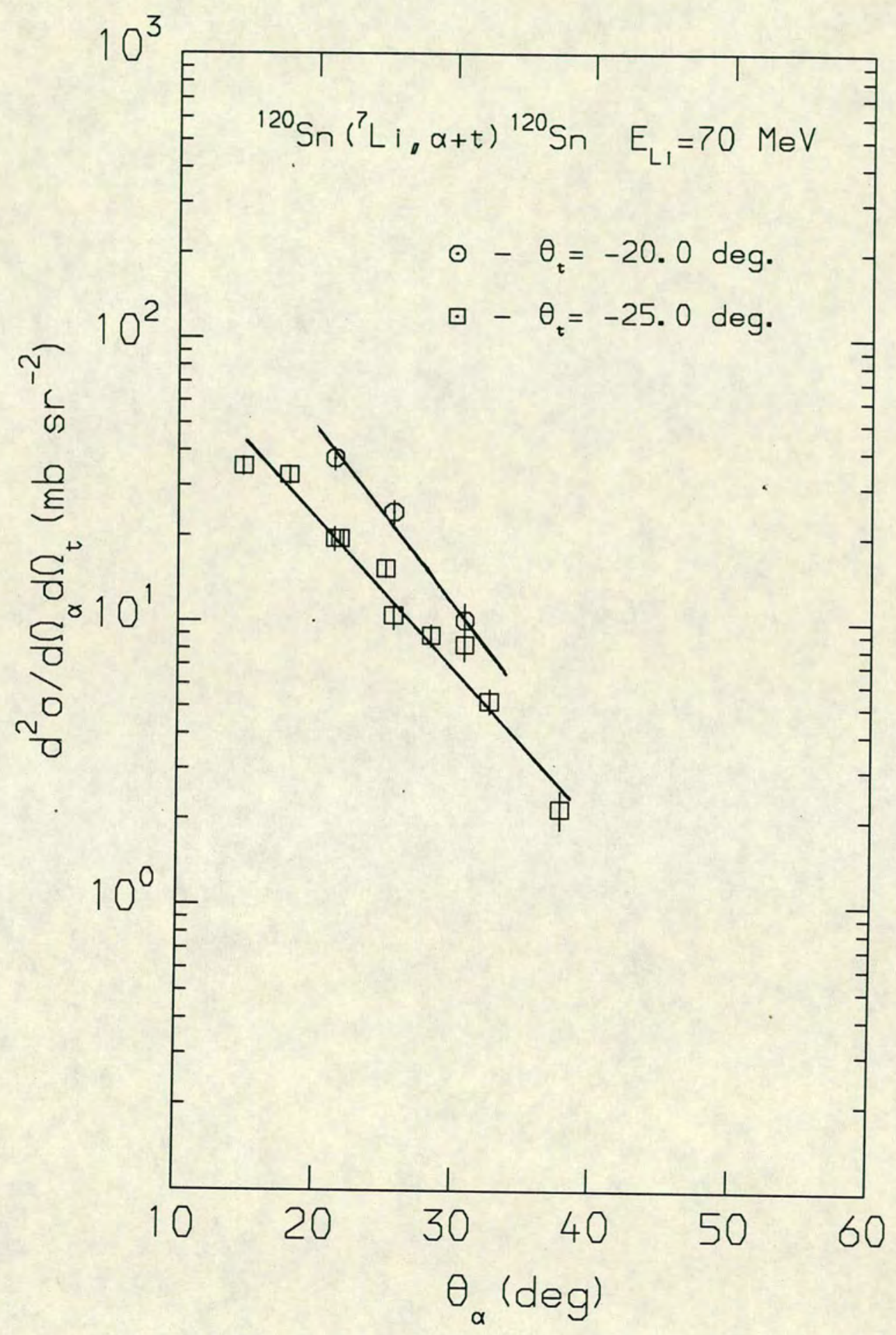

Figure 4.40: Angular distribution for all coincident $\alpha$-t events from ${ }^{120} \mathrm{Sn}$ target with $\phi_{\alpha}=2.0^{\circ}$ ( lines to guide the eye ). 


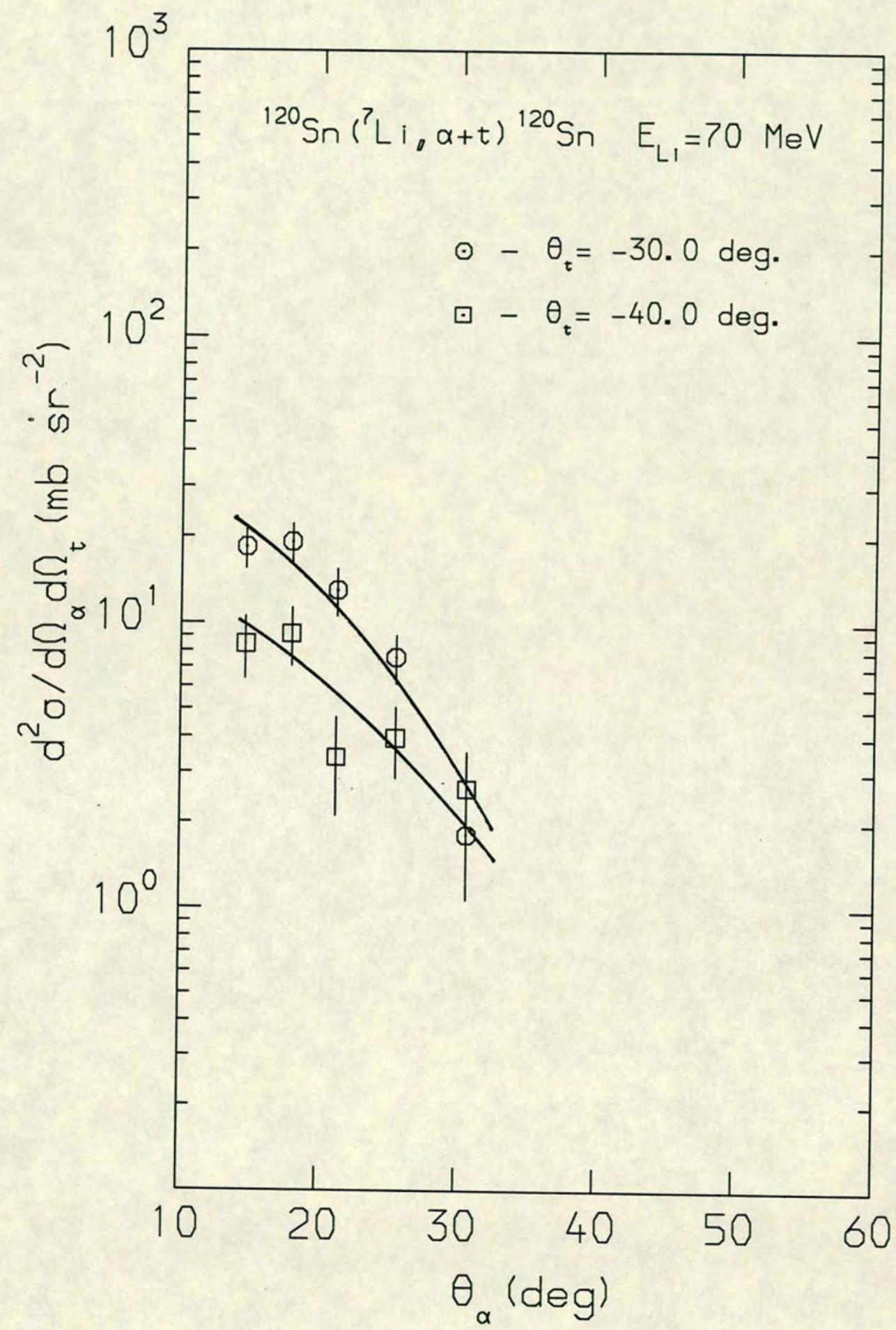

Figure 4.41: Angular distribution for all coincident $\alpha$-t events from ${ }^{120} \mathrm{Sn}$ target with $\phi_{\alpha}=2.0^{\circ}$ ( lines to guide the eye ). 


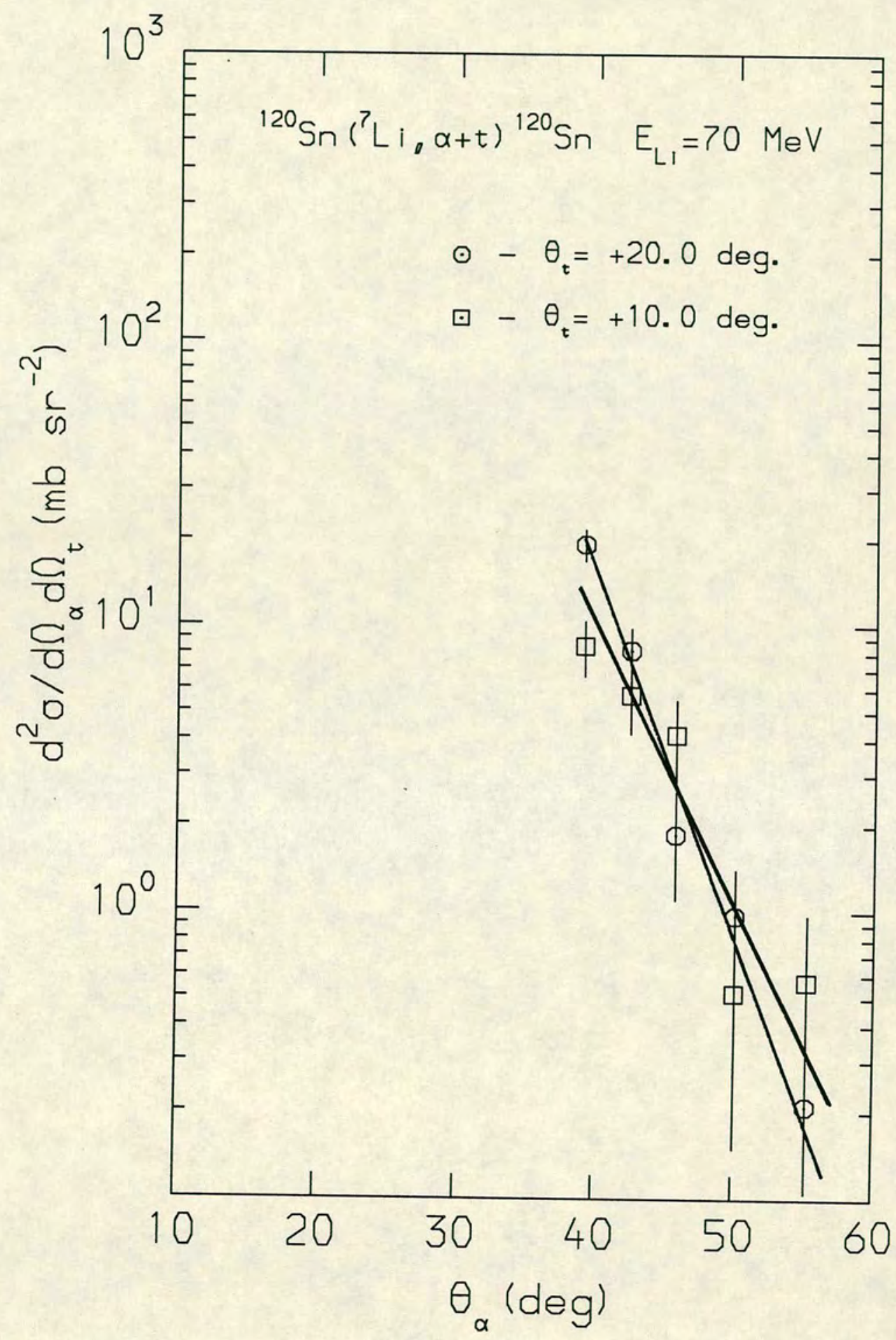

Figure 4.42: Angular distribution for all coincident $\alpha$-t events from ${ }^{120} \mathrm{Sn}$ target with $\phi_{\alpha}=2.0^{\circ}$ and detectors on same side of beam (lines to guide the eye ). 


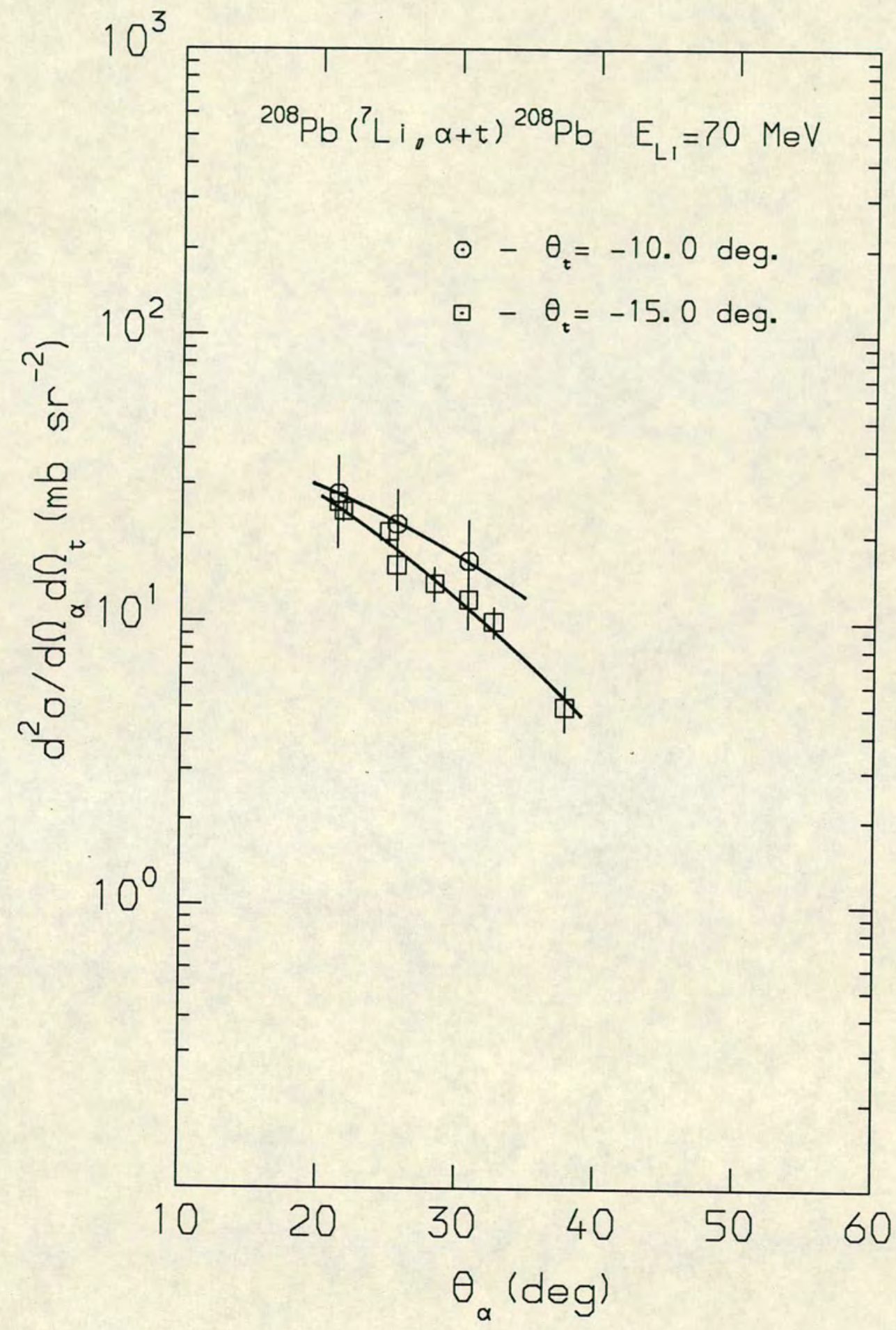

Figure 4.43: Angular distribution for all coincident $\alpha$-t events from ${ }^{208} \mathrm{~Pb}$ target with $\phi_{\alpha}=2.0^{\circ}$ ( lines to guide the eye ). 


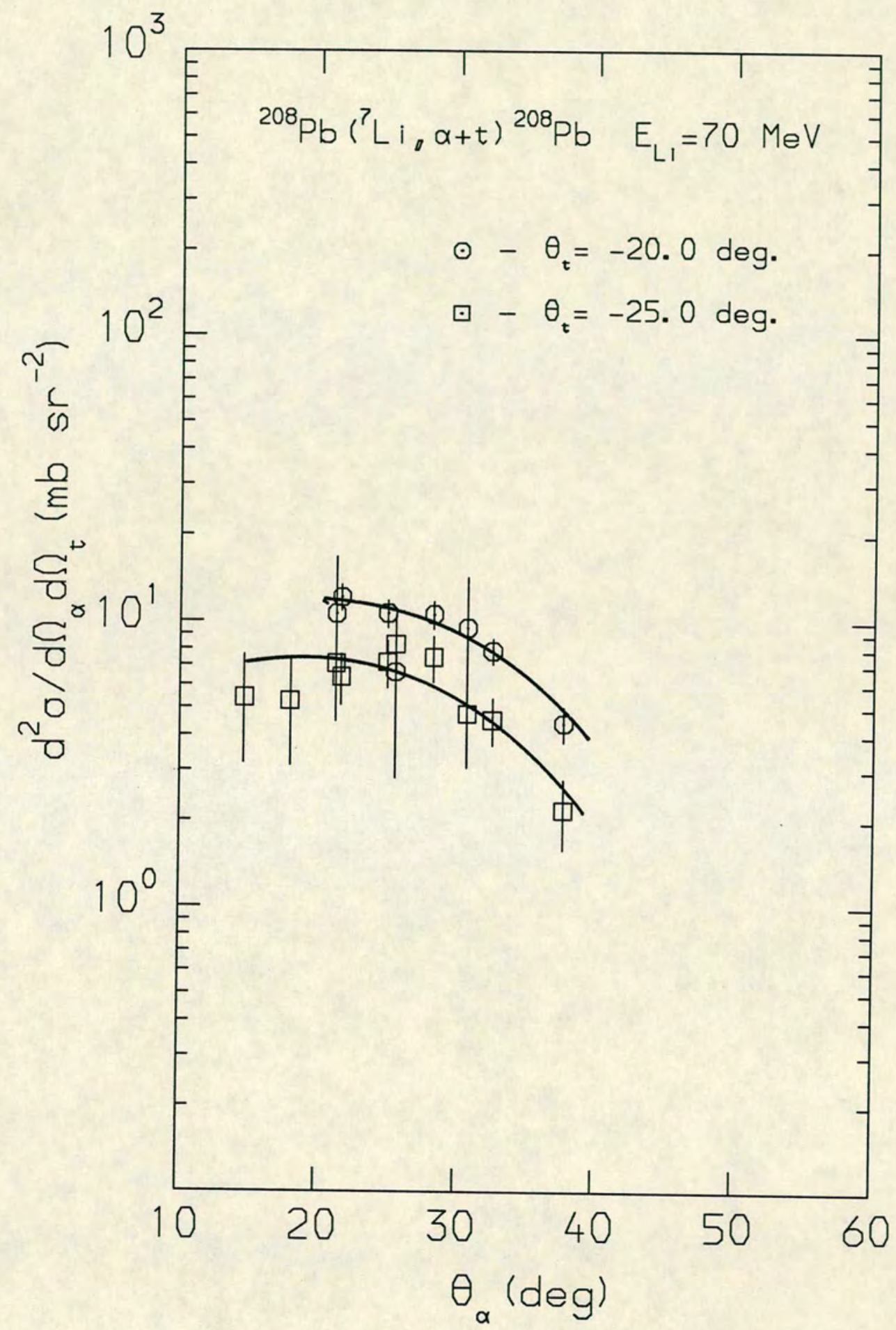

Figure 4.44: Angular distribution for all coincident $\alpha$-t events from ${ }^{208} \mathrm{~Pb}$ target with $\phi_{\alpha}=2.0^{\circ}$ ( lines to guide the eye ). 


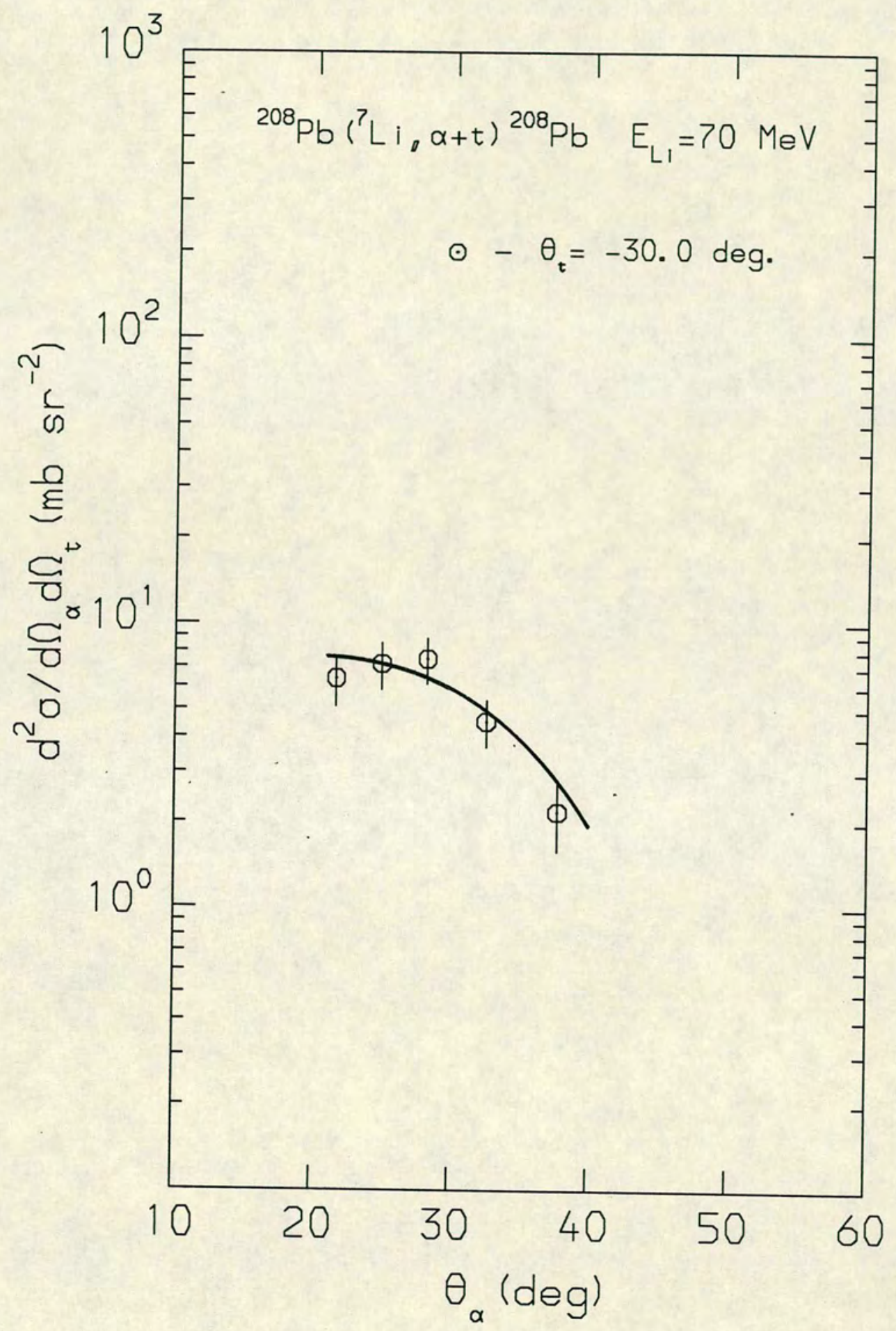

Figure 4.45: Angular distribution for all coincident $\alpha$-t events from ${ }^{208} \mathrm{~Pb}$ target with $\phi_{\alpha}=2.0^{\circ}$ ( line to guide the eye ). 
opposite sides of the beam.

So far only the data with the detectors on opposite sides of the beam have been discussed. Cross-sections were also measured for events with both the alpha and triton on the same side of the beam. These data were only collected for the ${ }^{12} \mathrm{C}$ and ${ }^{120} \mathrm{Sn}$ targets and are presented in figures $4.23,4.27,4.38$ and 4.34.

It can be seen from these figures that the ${ }^{12} \mathrm{C}$ data show a preference for the triton to be emitted in the beam direction whereas the ${ }^{120} \mathrm{Sn}$ data show a preference for emission of the triton closer to the alpha particle direction. This is more clearly indicated in figures 4.46 to 4.49 which present the angular double differential cross-sections for quasi-elastic events with a fixed $\theta_{\alpha}$, and $\phi_{\alpha}=2.0^{\circ}$ but varying $\theta_{t}$ (the solid curves are the result of a PWBA calculation described in section 5.2). Although the ${ }^{120} \mathrm{Sn}$ data were not collected for $\theta_{\alpha}=+39.2^{\circ}$ and $\theta_{t}<0^{\circ}$ the values of these cross-sections were extrapolated from the data with $\theta_{\alpha}$ closer to the beam i.e. figs.4.24 to 4.26. Figures 4.46 to 4.49 show that, for the ${ }^{12} \mathrm{C}$ target, there is an enhanced probability for particle emission to opposite sides of the beam direction. Similar correlations have been reported [Ma80b] for kinematically complete experiments with lighter, more energetic, projectiles where the coincident, quasi-elastic proton and deuteron from the fragmentation of $90 \mathrm{MeV},{ }^{3} \mathrm{He}$ were found to be preferentially emitted on opposite sides of the beam. In contrast to this behaviour, the cross-section for the ${ }^{120} \mathrm{Sn}$ target, with the triton telescope close to the alpha detector $\left(\theta_{t}=+20.0^{\circ}, \theta_{\alpha}=+39.2^{\circ}\right)$ is much larger than the corresponding case for the ${ }^{12} \mathrm{C}$ target. This can be explained by the detection of sequential break-up events from the $4.63 \mathrm{MeV}$ excited state of ${ }^{7} \mathrm{Li}$. The cross-section for this process is a factor of $\sim \times 10$ greater [Da87] for ${ }^{120} \mathrm{Sn}$ than ${ }^{12} \mathrm{C}$ in this angular region. This interpretation is also strengthened by the Monte Carlo simulation shown in section 5.1.2 fig.5.36. Unfortunately the statistics for the ${ }^{120} \mathrm{Sn}$ data were not sufficient to allow extraction of this sequential component. This meant that no investigation of the correlation about the beam direction for the lower intensity components could be made for the ${ }^{120} \mathrm{Sn}$ target. The enhancement in the ${ }^{12} \mathrm{C}$ data at $\theta_{t}=+20^{\circ}, \theta_{\alpha}=+42.5^{\circ}$ (see fig.4.23) has been identified as originating from an increased detection efficiency for fragments from the sequential break-up of the $4.63 \mathrm{MeV}$ state in ${ }^{7} \mathrm{Li}$. In this configuration the detectors are on the edge of the kinematic break-up cone for 


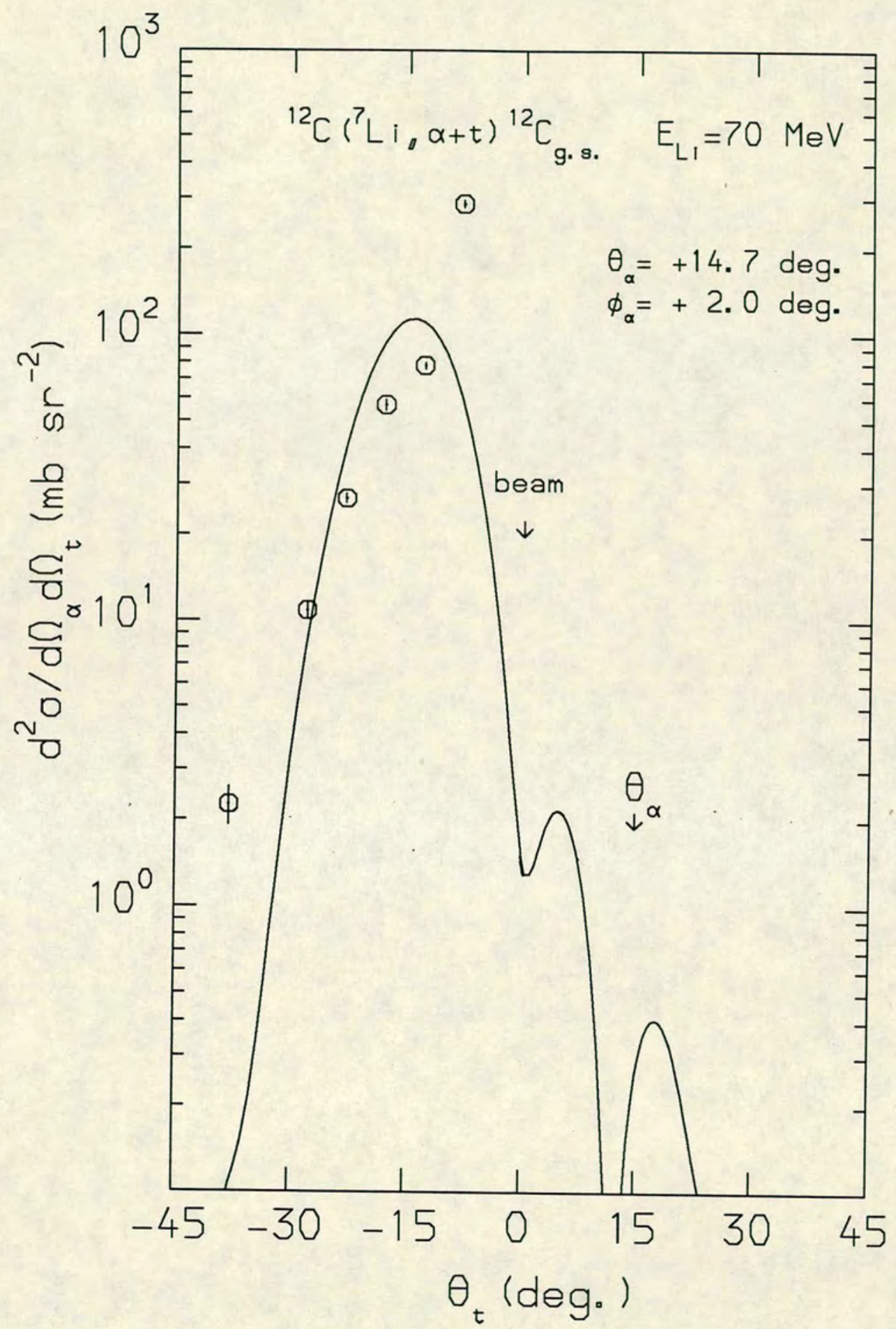

Figure 4.46: Quasi-elastic angular distribution for a fixed $\theta_{\alpha}=+14.7^{\circ}$ in the forward direction and varying $\theta_{t}$. PWBA fit is normalised to the data outside the influence of the sequential break-up of the ${ }^{7} \mathrm{Li}_{4.63 \mathrm{MeV}}$ state. 


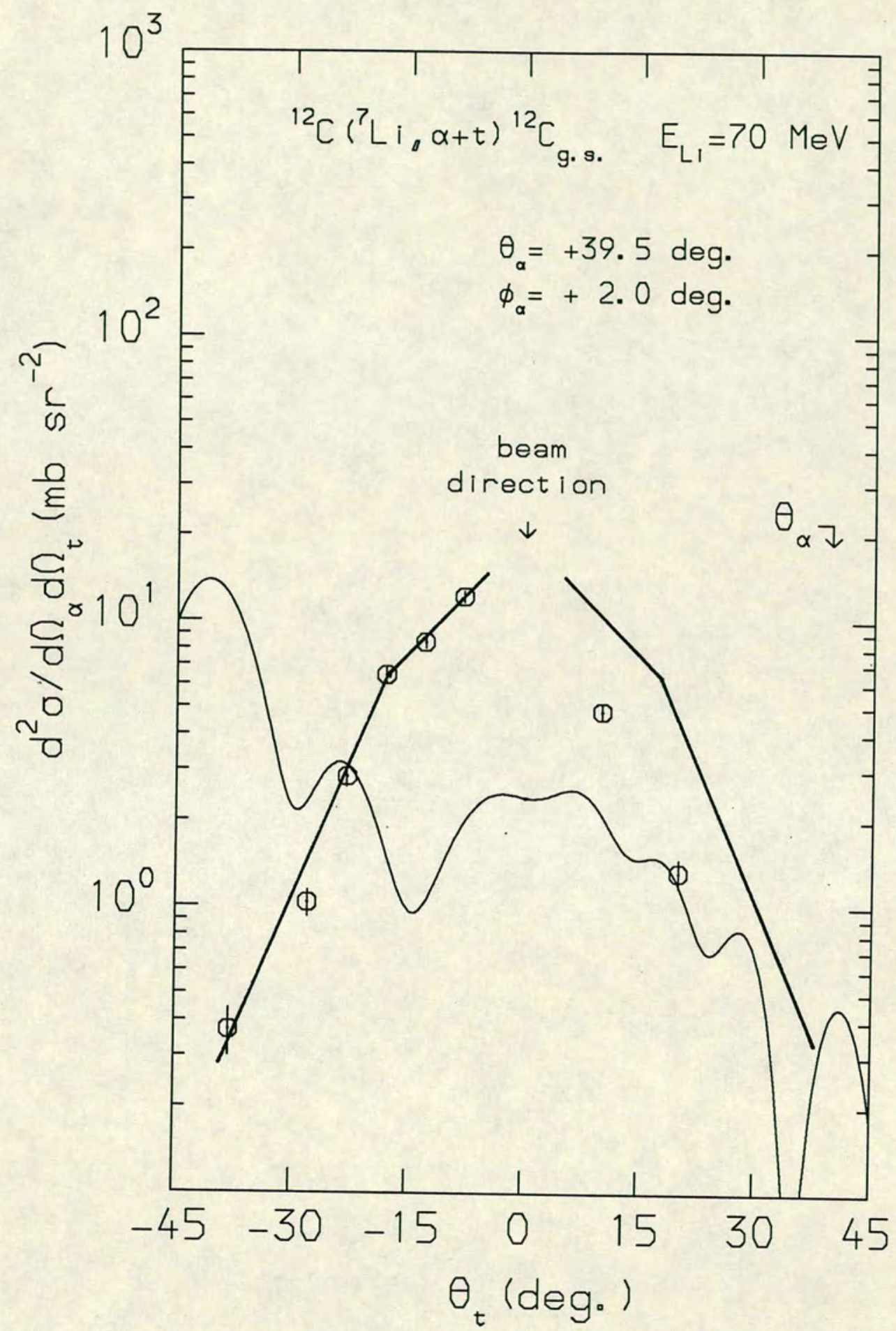

Figure 4.47: Quasi-elastic angular distribution for a fixed $\theta_{\alpha}=+39.2^{\circ}$ in the backward direction and varying $\theta_{t}$. PWBA fit is normalised to the data. The straight lines are symmetric about the beam direction. 


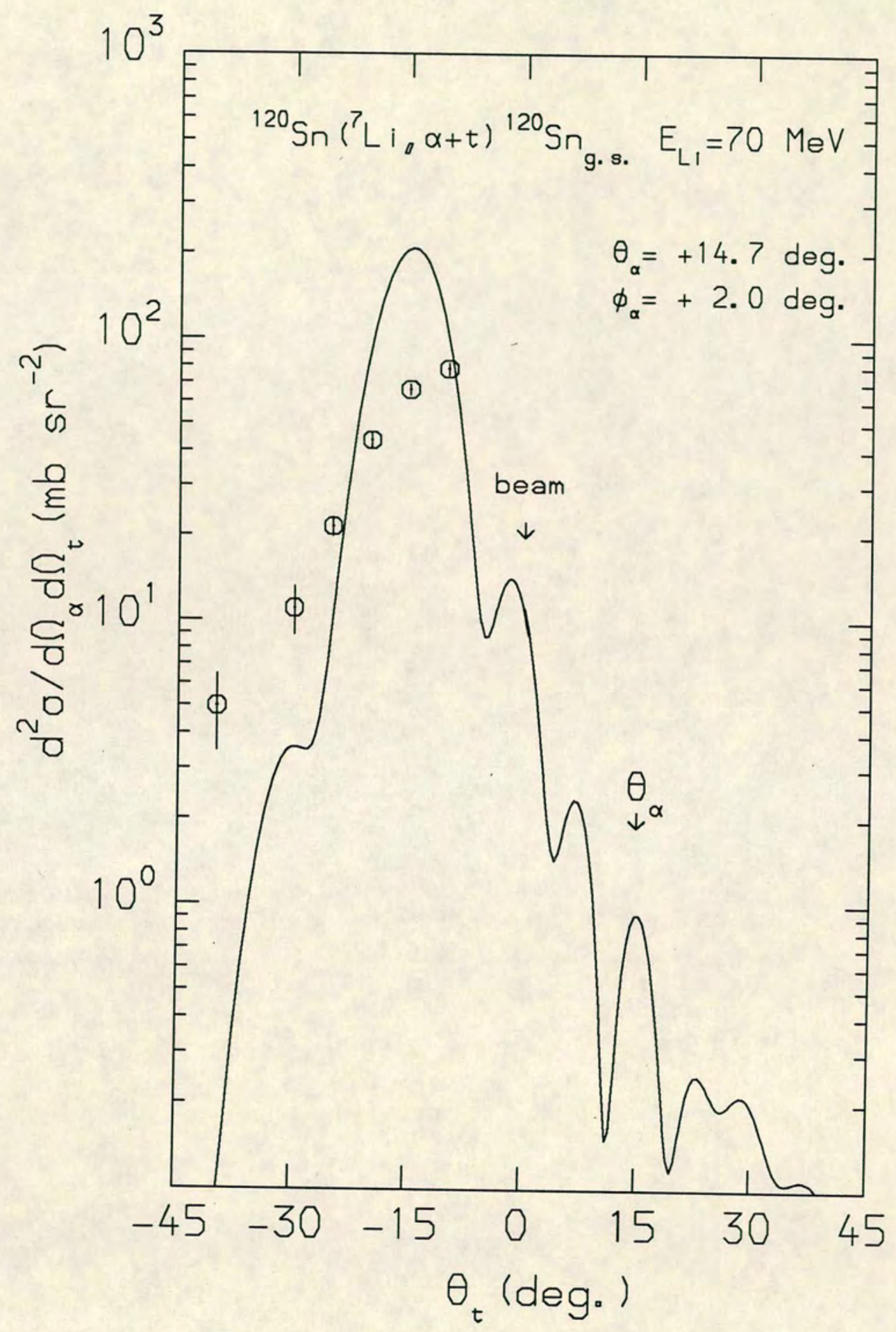

Figure 4.48: Quasi-elastic angular distribution for a fixed $\theta_{\alpha}=+14.7^{\circ}$ in the forward direction and varying $\theta_{t}$. PWBA fit is normalised to the data. 


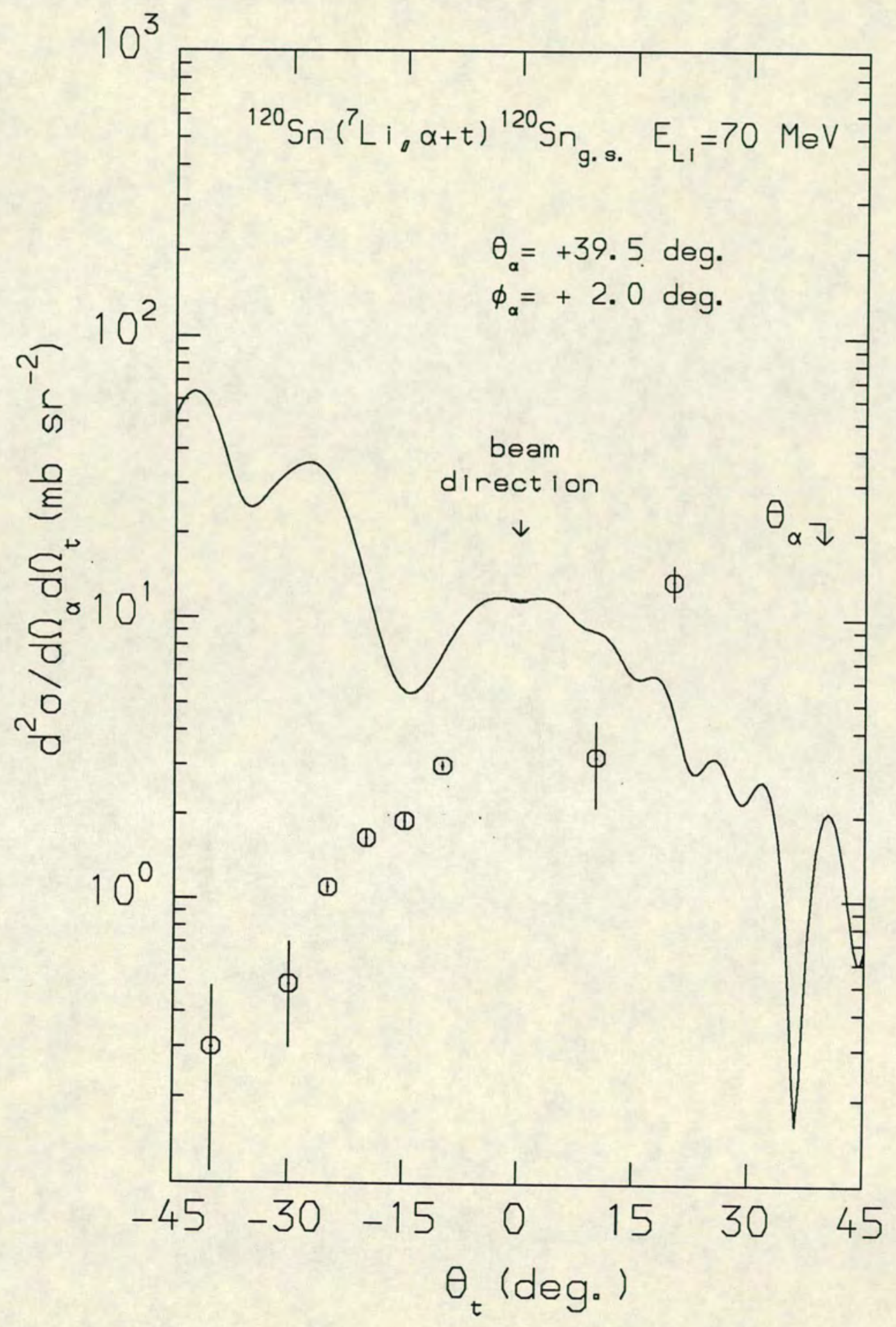

Figure 4.49: Quasi-elastic angular distribution for a fixed $\theta_{\alpha}=+39.2^{\circ}$ in the backward direction and varying $\theta_{t}$. PWBA fit is normalised to the data. 
decay of the $4.63 \mathrm{MeV}$ state. This produces an enhanced efficiency for detection of these events (see fig.5.19 which is a Monte-Carlo simulation of the projected $\mathrm{E}_{\alpha}$ for this case ). The higher recoil energy of the ${ }^{7} \mathrm{Li}_{4.63 \mathrm{MeV}}$ from the ${ }^{120} \mathrm{Sn} \mathrm{nu}$ cleus reduces the width of this cone which explains the absence of an equivalent enhancement for the ${ }^{120} \mathrm{Sn}$ target. (see fig.5.36, Monte-Carlo projected $\mathrm{E}_{\alpha}$ for this case.)

In summary this section has dealt with the $\alpha$-t coincidences recorded in the reaction plane. It has been shown that even at large angular separations between the coincident fragments the quasi-elastic reaction channel remains a significant component of the total $\alpha$-t coincidence cross-section. The ${ }^{12} \mathrm{C}$ data show a clear preference for emission of the fragments to opposite sides of the beam, whilst quasi-elastic events from the ${ }^{120} \mathrm{Sn}$ target, with both fragments on the same side of the beam, are dominated by sequential break-up of the ${ }^{7} \mathrm{Li}$ through its 4.63 $\mathrm{MeV}$ excited state.

\subsubsection{Out-of-plane Data}

In addition to the in-plane data presented in the previous section the positional information combined with the strip detector's large area allowed the out-ofplane intensity distribution to be measured as a function of $\phi_{\alpha}$. Due to the large number of out-of-plane data points collected in this investigation, this section will only present a respresentative sample of the full data set. A complete collection of all the out-of-plane data is given in Appendix B.

Fig.4.50 to 4.56 show the out-of-plane quasi-elastic intensity distributions for a range of $\theta_{\alpha}$ and $\theta_{t}$ from the ${ }^{12} \mathrm{C},{ }^{120} \mathrm{Sn}$ and ${ }^{208} \mathrm{~Pb}$ targets.

It can be seen that in general, the magnitude of the differential cross-section decreases with increasing $\theta_{\alpha}$ and $\theta_{t}$. This is just a reflection of the in-plane behaviour. In most instances it also decreases monotonically out-of-plane with increasing $\phi_{\alpha}$.

The ${ }^{12} \mathrm{C}$ data show that as $\theta_{\alpha}$ increases, for a fixed $\theta_{t}$, the in-plane enhancement 


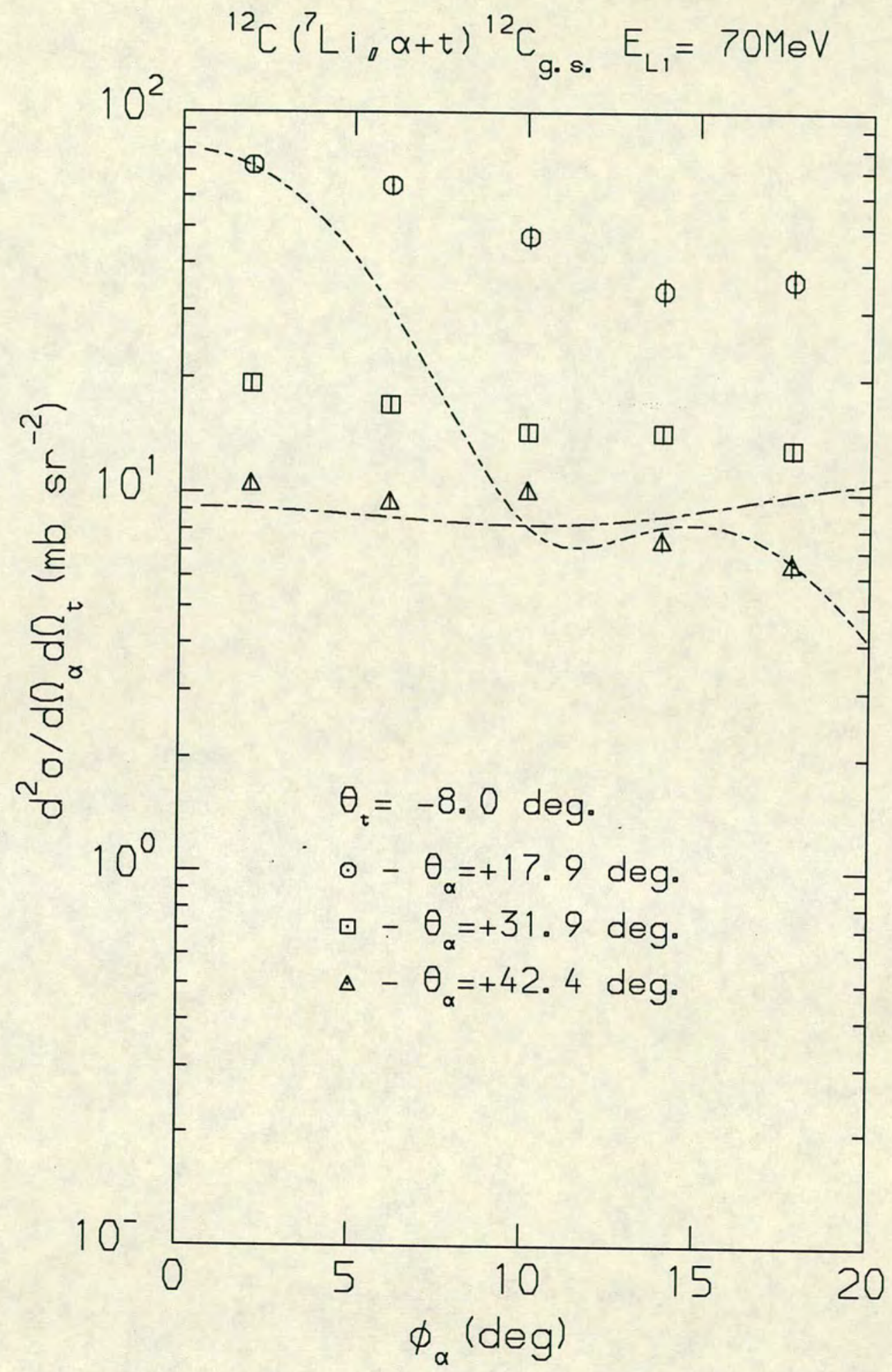

Figure 4.50: Out-of-plane angular distributions for quasi-elastic events from the ${ }^{12} \mathrm{C}$ target with fixed $\theta_{t}=-8.0^{\circ}$, and varying $\theta_{\alpha}=+17.9^{\circ}-+42.4^{\circ}$. Dashed line is PWBA calculation for $\theta_{\alpha}=+17.9^{\circ}$ and dotted dashed line is for $\theta_{\alpha}=+42.4^{\circ}$, both are normalised to the forward angled data points. 


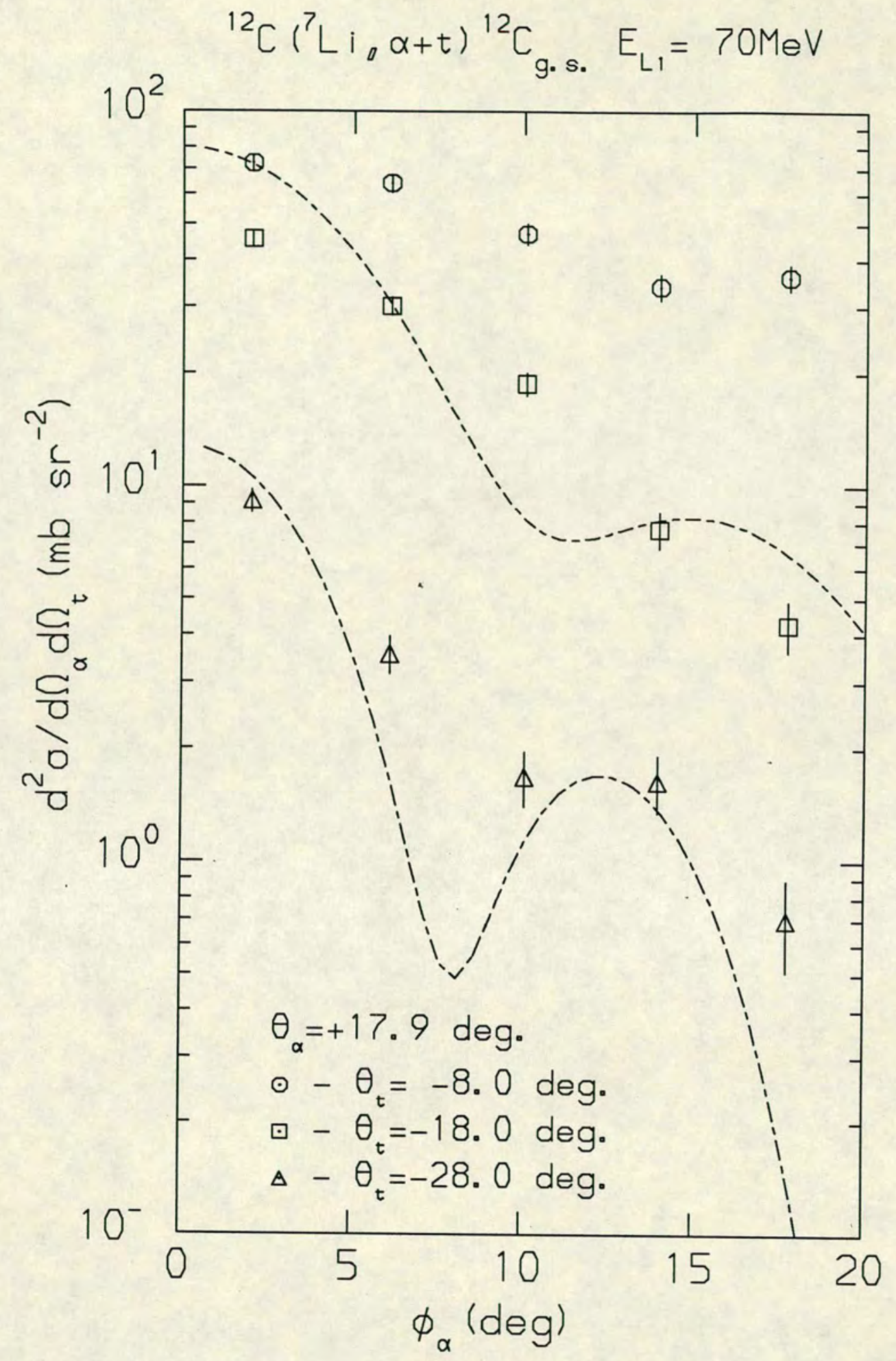

Figure 4.51: Out-of-plane angular distributions for quasi-elastic events from the ${ }^{12} \mathrm{C}$ target with fixed $\theta_{\alpha}=+17.9^{\circ}$, and varying $\theta_{t}=-8.0^{\circ}--28.0^{\circ}$. Dashed line is PWBA calculation for $\theta_{t}=-8.0^{\circ}$ and dotted dashed line is for $\theta_{t}=-28.0^{\circ}$, both are normalised to the forward angled data points. 


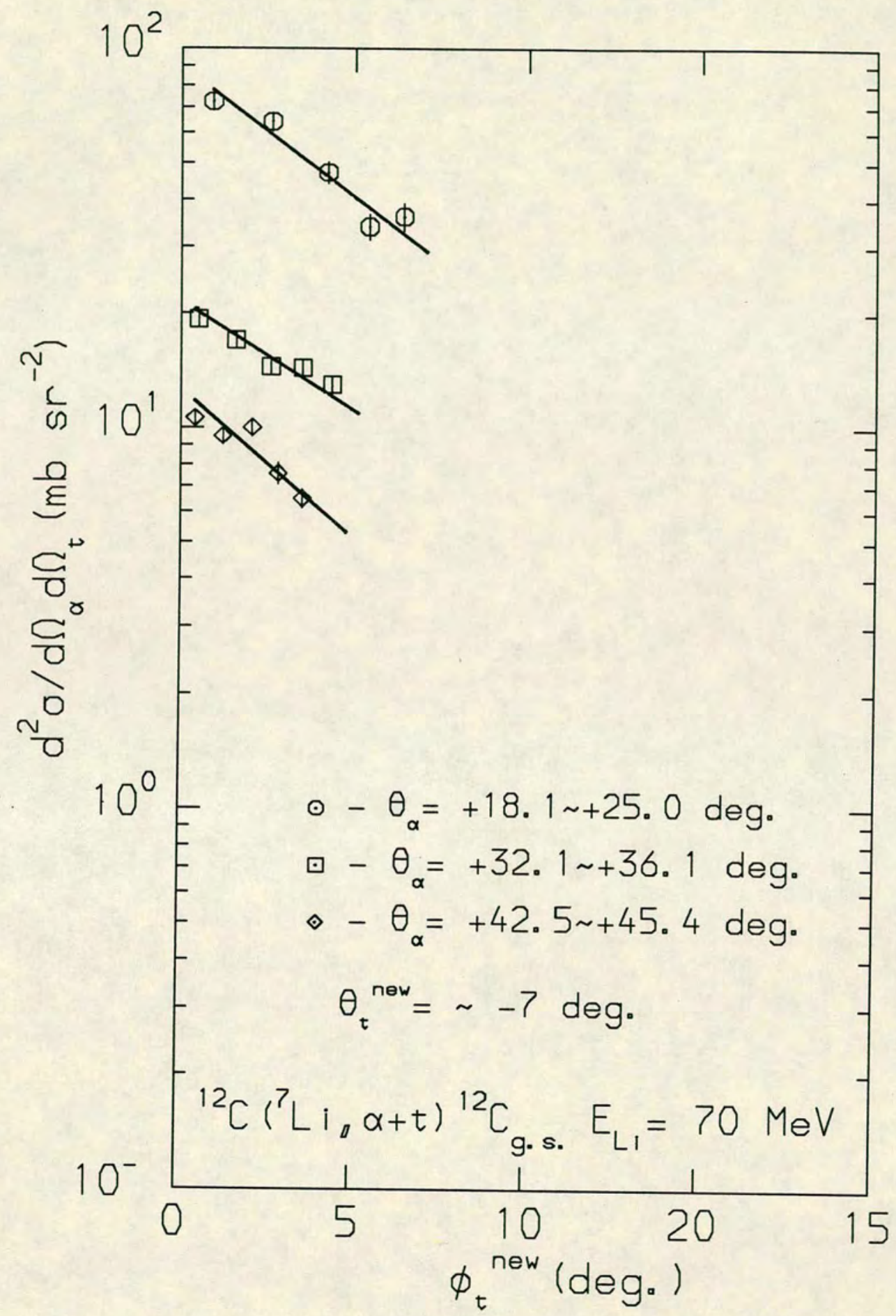

Figure 4.52: Out-of-plane data from fig.4.50 with reaction plane redefined by beam and relevant alpha detector pixel, showing triton out-of-plane behaviour. ( Lines to guide the eye.) 


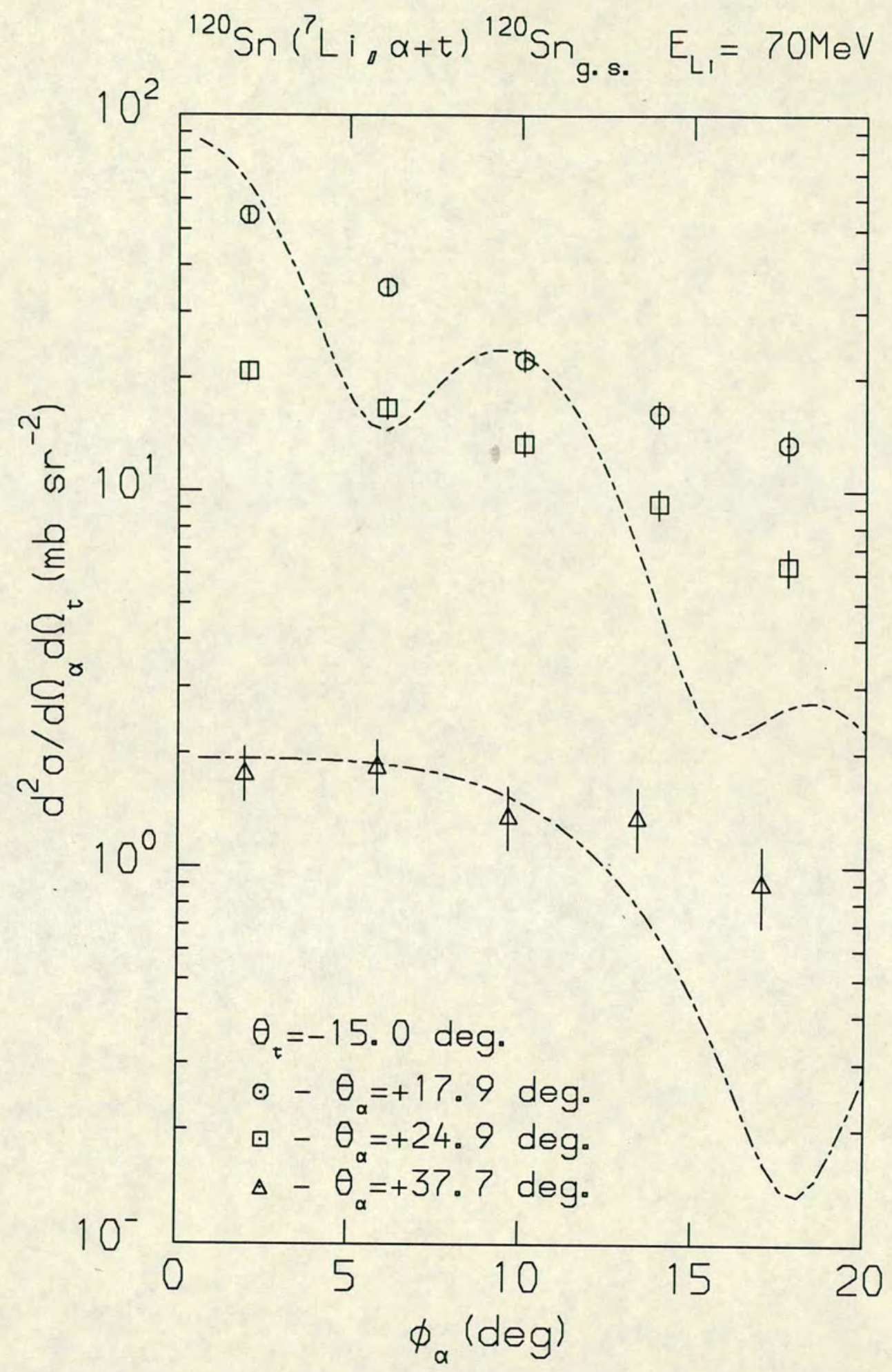

Figure 4.53: Out-of-plane angular distributions for quasi-elastic events from the ${ }^{120} \mathrm{Sn}$ target with fixed $\theta_{t}=-15.0^{\circ}$, and varying $\theta_{\alpha}=+17.9^{\circ}-+37.7^{\circ}$. Dashed line is PWBA calculation for $\theta_{\alpha}=+17.9^{\circ}$ and dotted dashed line is for $\theta_{\alpha}=+37.7^{\circ}$, both are normalised to the forward angled data points. 


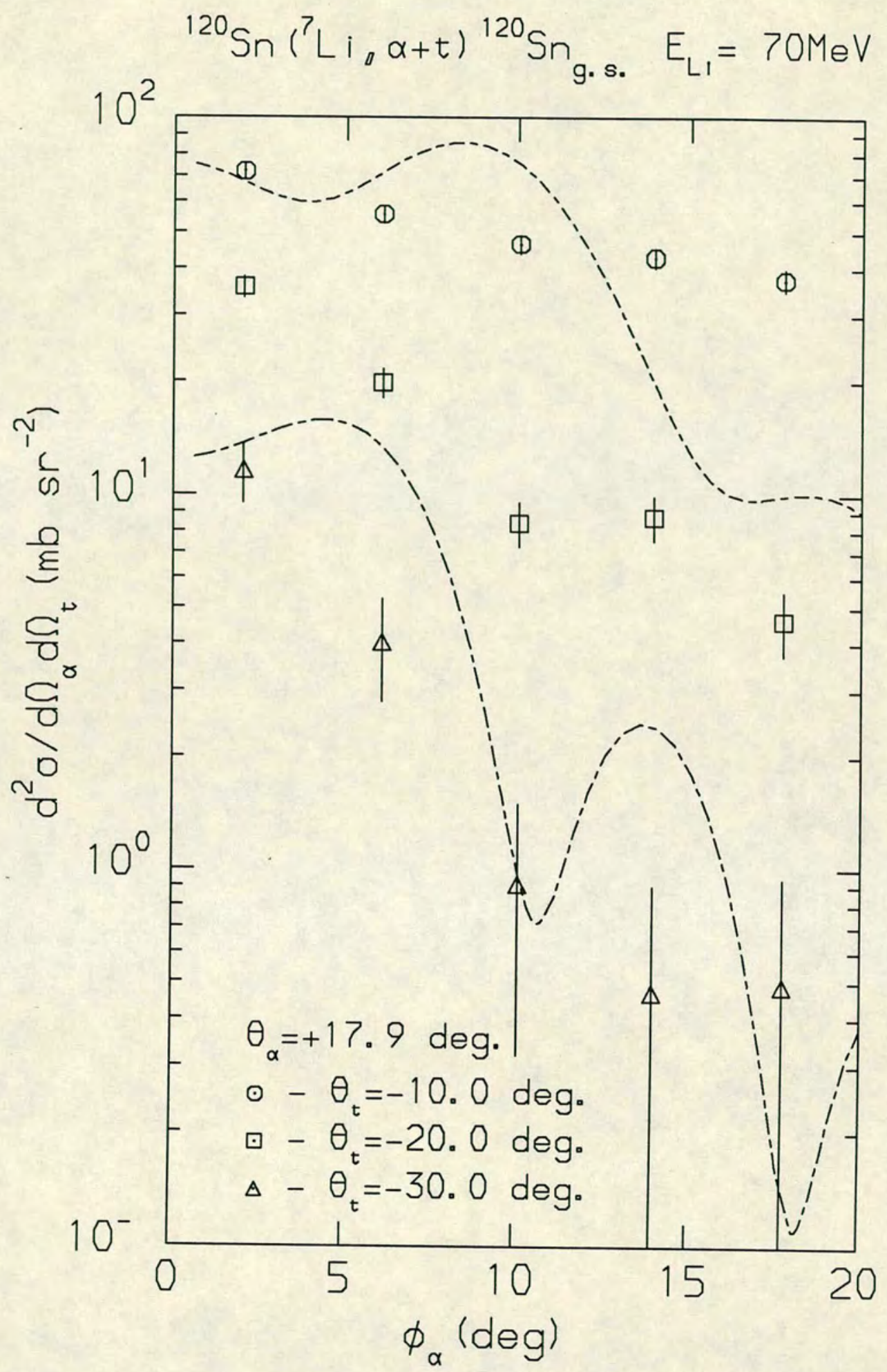

Figure 4.54: Out-of-plane angular distributions for quasi-elastic events from the ${ }^{120} \mathrm{Sn}$ target with fixed $\theta_{\alpha}=+17.9^{\circ}$, and varying $\theta_{t}=-10.0^{\circ}--30.0^{\circ}$. Dashed line is PWBA calculation for $\theta_{t}=-10.0^{\circ}$ and dotted dashed line is for $\theta_{t}=-30.0^{\circ}$, both are normalised to the forward angled data points. 


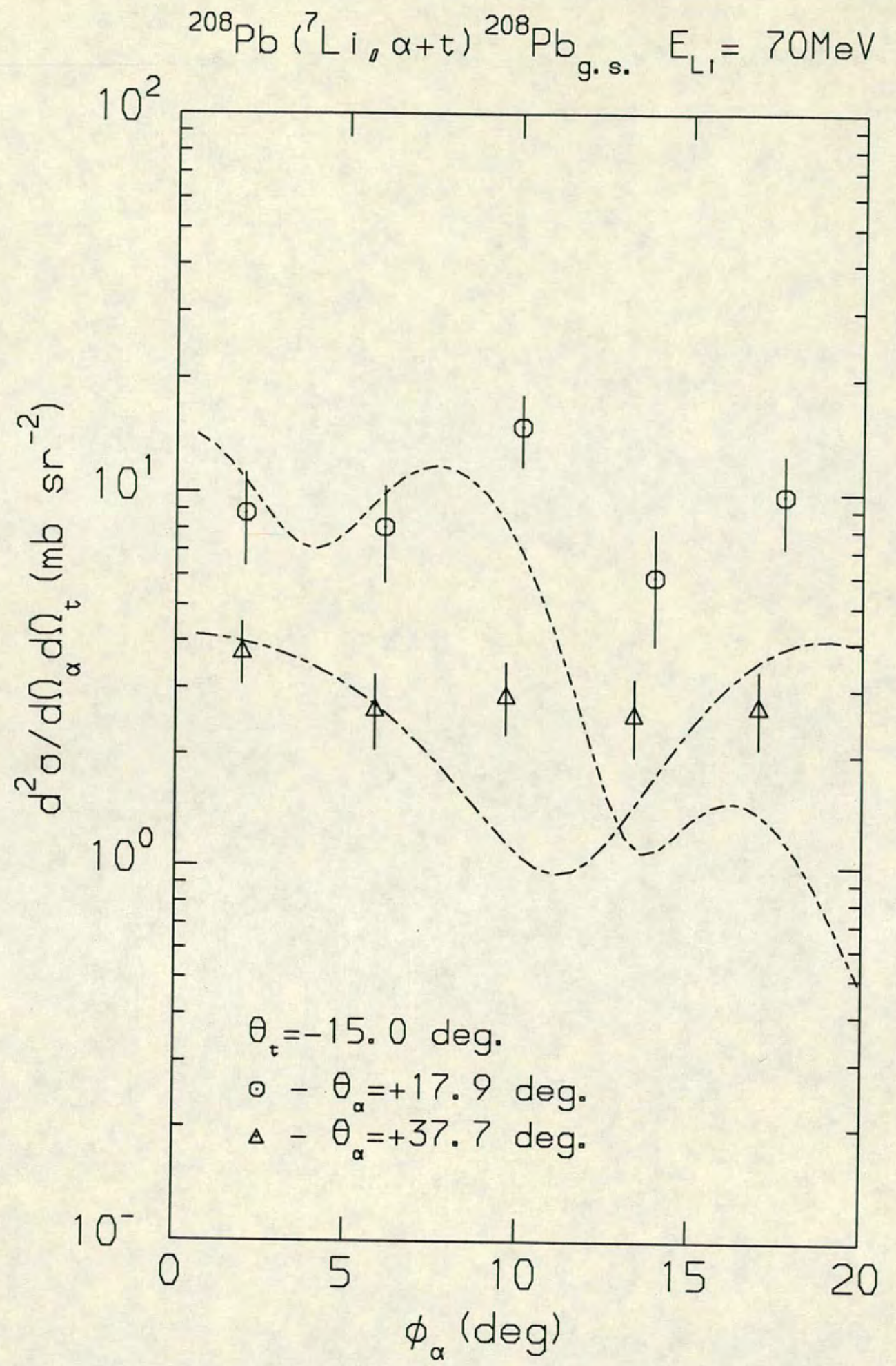

Figure 4.55: Out-of-plane angular distributions for quasi-elastic events from the ${ }^{208} \mathrm{~Pb}$ target with fixed $\theta_{t}=-15.0^{\circ}$, and varying $\theta_{\alpha}=+17.9^{\circ}-+37.7^{\circ}$. Dashed line is PWBA calculation for $\theta_{\alpha}=+17.9^{\circ}$ and dotted dashed line is for $\theta_{\alpha}=+37.7^{\circ}$, both are normalised to the forward angled data points. 


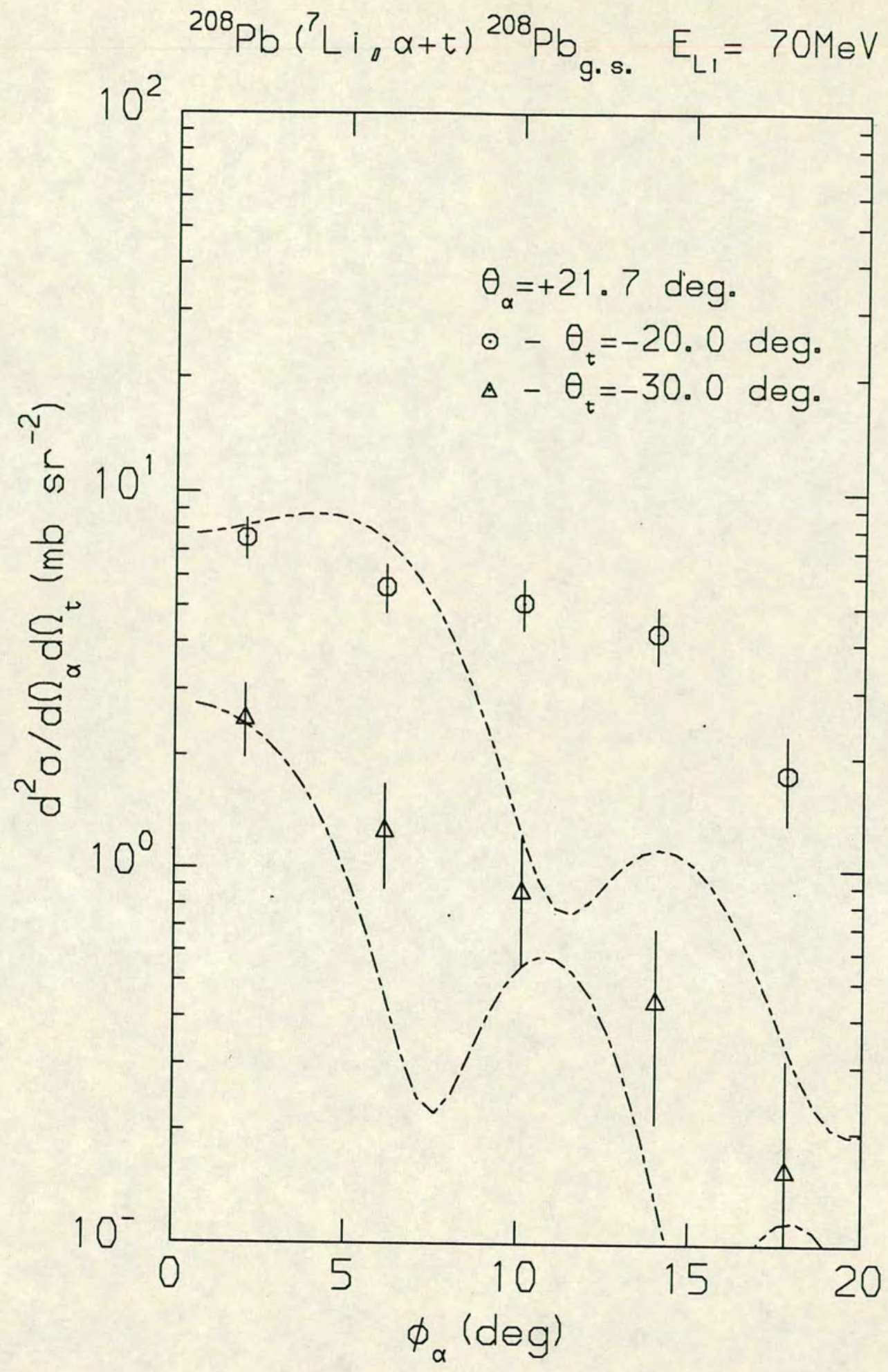

Figure 4.56: Out-of-plane angular distributions for quasi-elastic events from the ${ }^{208} \mathrm{~Pb}$ target with fixed $\theta_{\alpha}=+21.7^{\circ}$, and varying $\theta_{t}=-20.0^{\circ}--30.0^{\circ}$. Dashed line is PWBA calculation for $\theta_{t}=-20.0^{\circ}$ and dotted dashed line is for $\theta_{t}=-30.0^{\circ}$, both are normalised to the forward angled data points. 
tends to become less pronounced. In contrast to this as $\theta_{t}$ increases, for a fixed $\theta_{\alpha}$, the in-plane enhancement becomes stronger. At first sight, this different behaviour with varying triton and alpha angle, seems to be rather confusing. It should be noted however that the two cases mentioned above are not equivalent. In order to find the corresponding triton out-of-plane dependance for a fixed triton in-plane angle with varying alpha in-plane angle, one must re-define the reaction plane. This new reaction plane is defined by the beam direction and the relevant alpha pixel. The correct angle for comparison is then the triton out-of-plane angle with respect to this plane. Fig.4.52 shows the data with such a redefined co-ordinate system for $\theta_{t}^{\text {new }} \sim-7^{\circ}$ and $\theta_{\alpha}$ varying from $+18^{\circ}$ to $+45^{\circ}$. It can be seen that the triton out-of-plane intensity becomes slightly more enhanced in-plane as $\theta_{\alpha}$ increases.

In an attempt to present a more quantitative measure of this in-plane enhancement, the out-of-plane distributions were fitted with a least squares technique similar to that used in section 4.1. The fits were made with the parameter $\mathrm{C}=0$. The value of the out-of-plane angle at which the fitted functions fell to approximately one half of the $\phi=0$ value were then calculated. A small value for this angle meant a strong in-plane enhancement whilst a large value corresponded to a weaker enhancement. Figs.4.57 to 4.62 show this half angle variation for the ${ }^{12} \mathrm{C}$ and ${ }^{120} \mathrm{Sn}$ targets. The rather large error bars on some of the points reflect the statistical quality of the fitted data and the sensitivity of the evaluation of the half angle for slopes with shallower gradient. It can be seen from figs.4.57 to 4.59 that as previously mentioned, the out-of-plane intensity distribution for the ${ }^{12} \mathrm{C}$ target becomes less steep as $\theta_{\alpha}$ increases but steeper as $\theta_{t}$ increases.

The behaviour of the ${ }^{120} \mathrm{Sn}$ out-of-plane distributions are slightly different, with the in-plane enhancement being rather constant or only slightly increasing with increasing $\theta_{\alpha}$ for a fixed triton angle. As the triton angle is increased, the behaviour of the ${ }^{120} \mathrm{Sn}$ distributions is similar to the ${ }^{12} \mathrm{C}$ data with the in-plane enhancement becoming more pronounced. In general the ${ }^{208} \mathrm{~Pb}$ distributions (see appendix B) seem to behave in a similar way to the ${ }^{120} \mathrm{Sn}$ data, but the statistical errors make any accurate assessment rather difficult. 


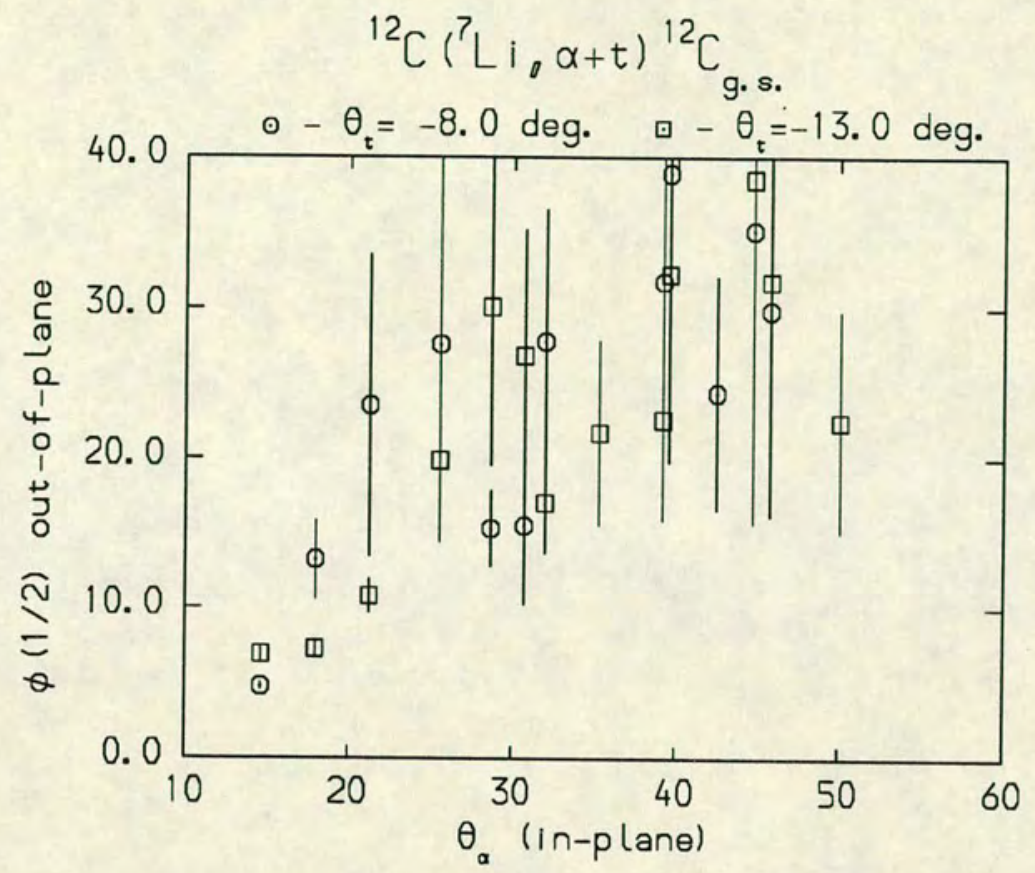

Figure 4.57: Value of the half angle for the quasi-elastic out-of-plane distributions for the ${ }^{12} \mathrm{C}$ target with $\theta_{t}=-8.0^{\circ}$ and $\theta_{t}=-13.0^{\circ}$.

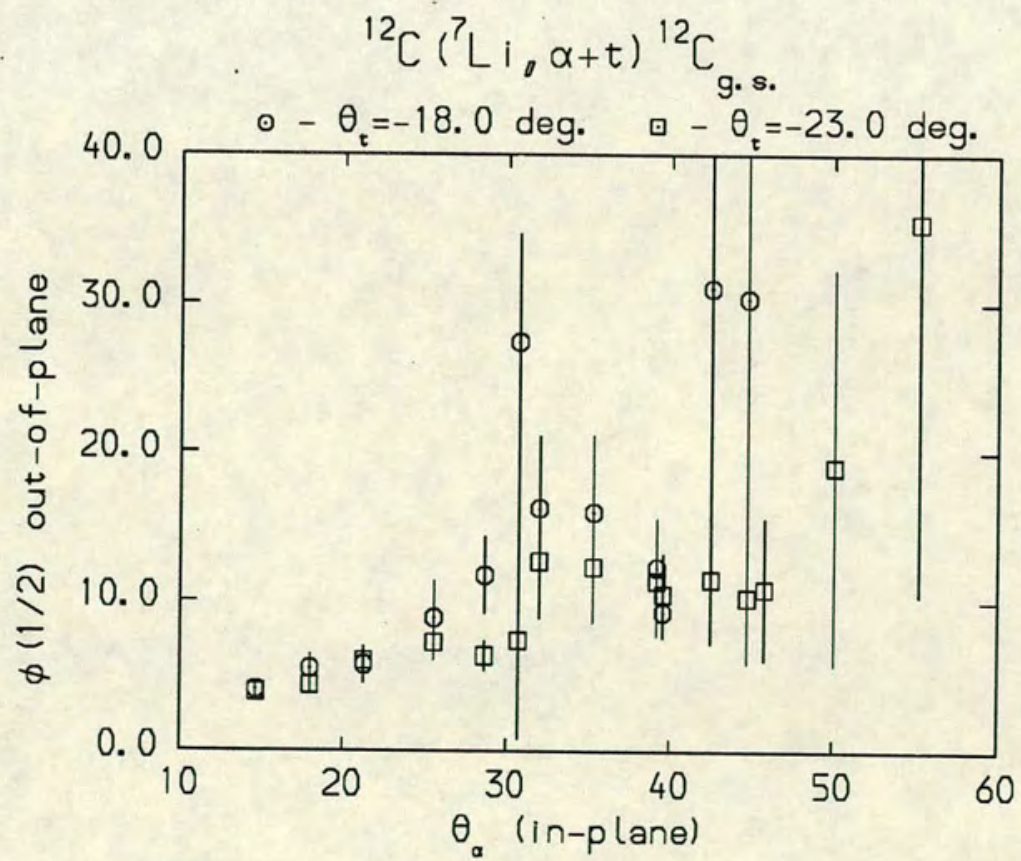

Figure 4.58: Value of the half angle for the quasi-elastic out-of-plane distributions for the ${ }^{12} \mathrm{C}$ target with $\theta_{t}=-18.0^{\circ}$ and $\theta_{t}=-23.0^{\circ}$. 


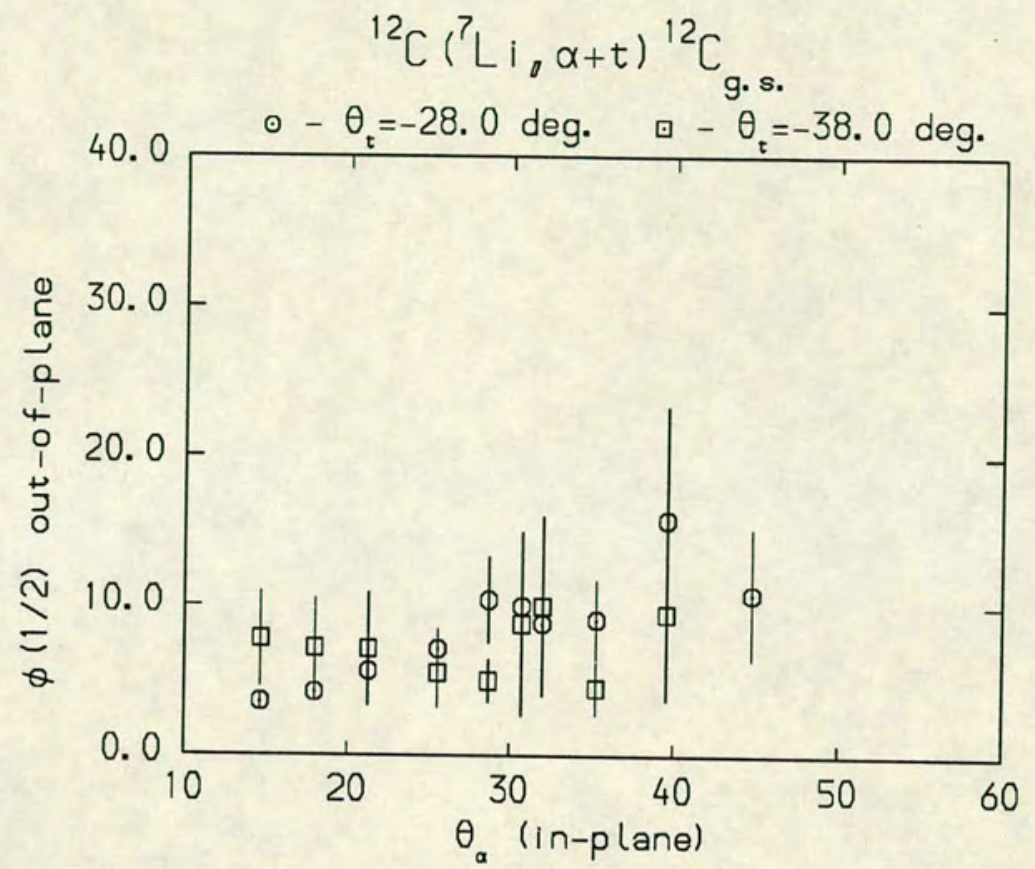

Figure 4.59: Value of the half angle for the quasi-elastic out-of-plane distributions for the ${ }^{12} \mathrm{C}$ target with $\theta_{t}=-28.0^{\circ}$ and $\theta_{t}=-38.0^{\circ}$.

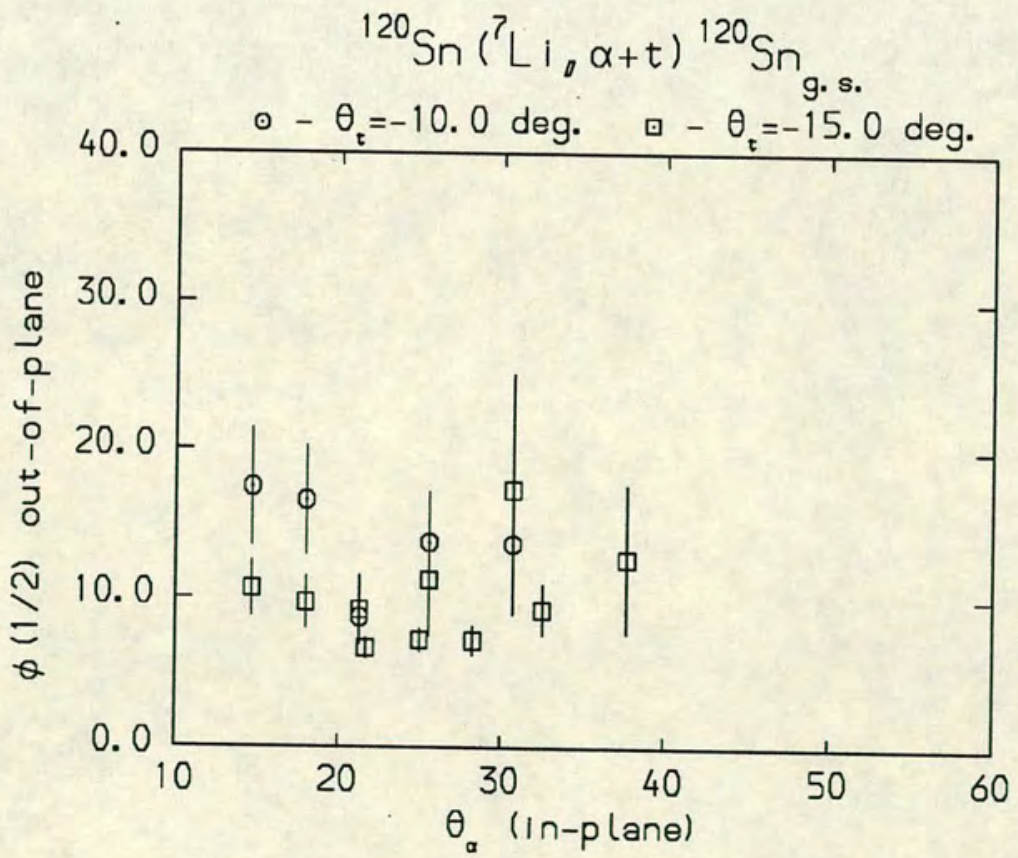

Figure 4.60: Value of the half angle for the quasi-elastic out-of-plane distributions for the ${ }^{120} \mathrm{Sn}$ target with $\theta_{t}=-10.0^{\circ}$ and $\theta_{t}=-15.0^{\circ}$. 


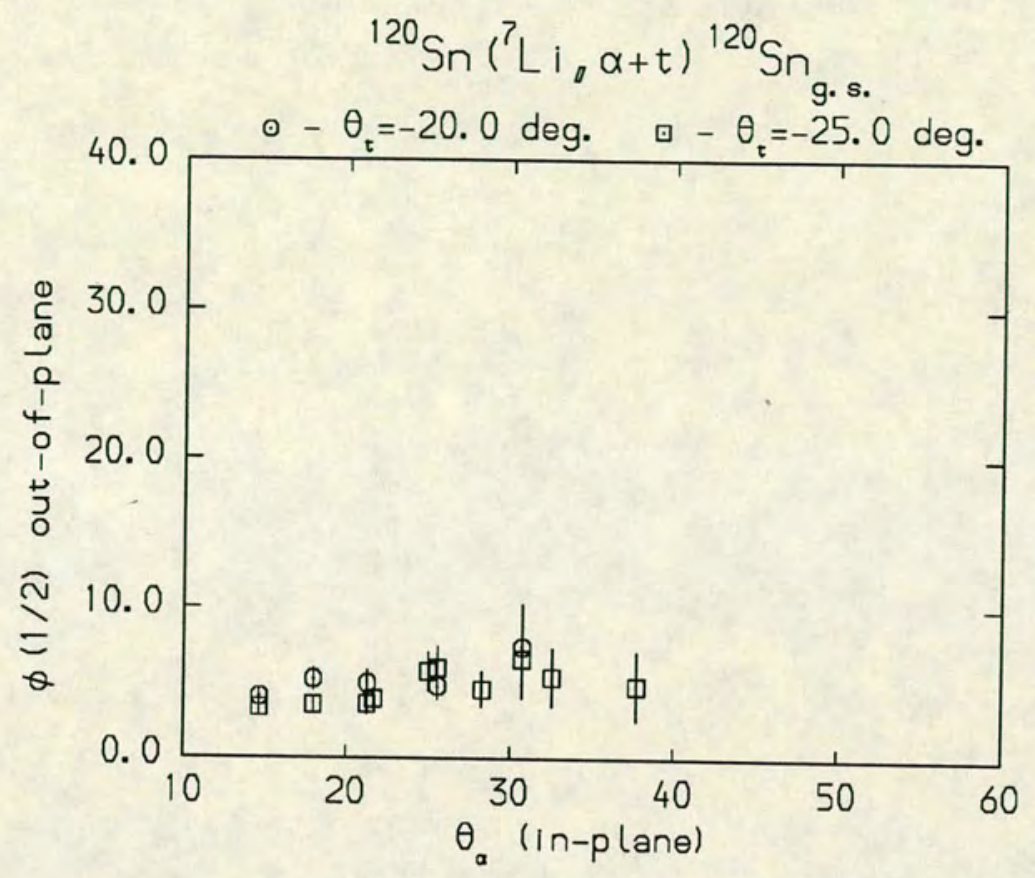

Figure 4.61: Value of the half angle for the quasi-elastic out-of-plane distributions for the ${ }^{120} \mathrm{Sn}$ target with $\theta_{t}=-20.0^{\circ}$ and $\theta_{t}=-25.0^{\circ}$.

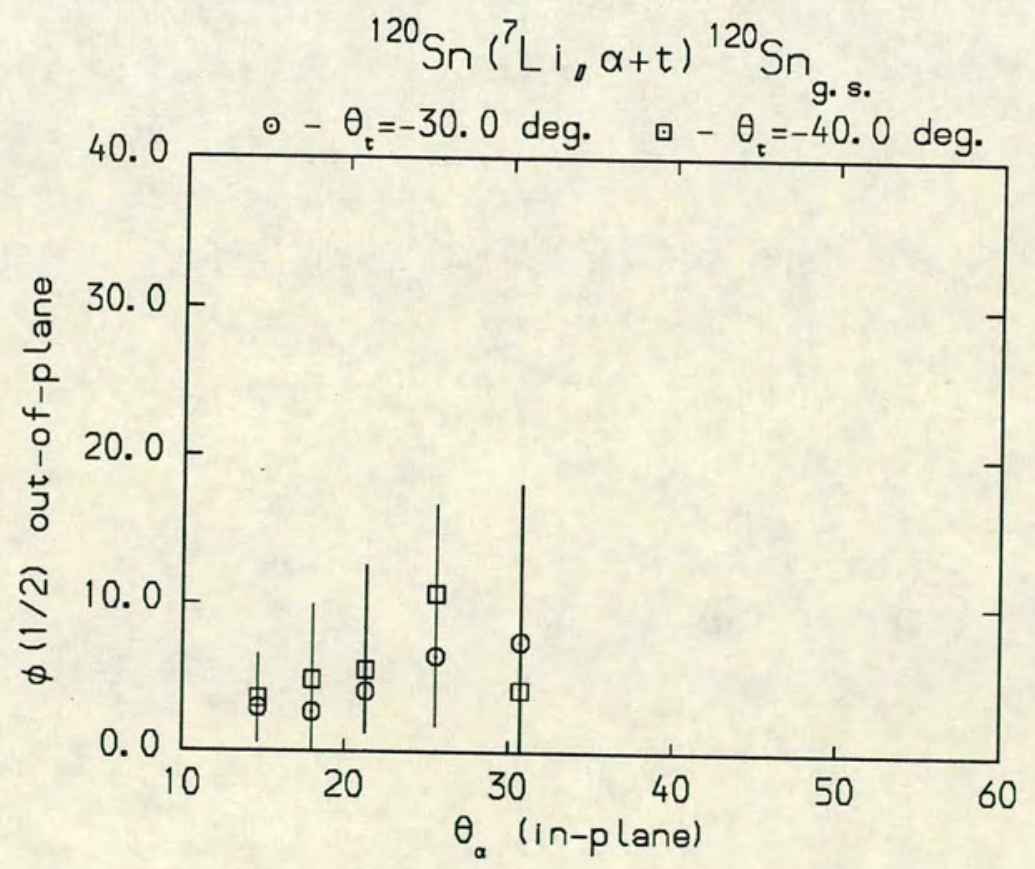

Figure 4.62: Value of the half angle for the quasi-elastic out-of-plane distributions for the ${ }^{120} \mathrm{Sn}$ target with $\theta_{t}=-30.0^{\circ}$ and $\theta_{t}=-40.0^{\circ}$. 
It seemed possible that this increased enhancement with increasing $\theta_{t}$ could be due to the improved definition of the reaction plane with the larger values of $\theta_{t}$. If one assumes that the actual out-of-plane dependance is an exponential fall off, it is possible to investigate this effect. Taking a cross-section of the form:

$$
d \sigma \sim e^{-m \phi}
$$

the experimentally observed cross-section $\overline{d \sigma}$ is an average over the angular resolution $\Delta \phi$ and is given by:

$$
\overline{d \sigma}=\frac{\int_{\Delta \phi} e^{-m \phi} d \phi}{\Delta \phi}
$$

if we let $\Delta \phi=\phi \pm \frac{\Delta \phi}{2}$ then evaluation of equ. 4.3 gives:

$$
\overline{d \sigma}=\frac{e^{m \phi}}{m \Delta \phi}\left(e^{\frac{m \Delta \phi}{2}}-e^{\frac{-m \Delta \phi}{2}}\right)
$$

and expanding the term in the bracket in this expression gives:

$$
\begin{aligned}
\overline{d \sigma} & =e^{-m \phi}\left(1+\frac{(m \Delta \phi)^{2}}{2^{2} 3 !}+\frac{(m \Delta \phi)^{4}}{2^{4} 5 !}+\cdots\right) \\
& =a e^{-m \phi} \quad a \geq 1
\end{aligned}
$$

In the angular distributions the log of this average cross-section is plotted. This gives a angular dependance of shape:

$$
y=\log \overline{d \sigma}=\log a-m \phi
$$

This is a straight line fall off, whose gradient is independent of $\Delta \phi$. It is probable therefore that the fall off enhancement is not due to this simple geometrical effect ${ }^{1}$. The variation of in-plane enhancement must be due to the reaction processes producing the coincident particles. These will be dealt with in the discussion of Chapter 5 .

In summary, this section has presented a representative selection of the quasielastic out-of-plane intensity distributions for all three targets. The full set of data are contained in Appendix B. It was shown that there is a distinct in-plane enhancement for all the targets. With the ${ }^{120} \mathrm{Sn}$ target the fall-off out-of-plane becomes more pronounced for larger detection angles, whereas for the ${ }^{12} \mathrm{C}$ target the enhancement increases with increasing $\theta_{t}$ but decreases for increasing $\theta_{\alpha}$. A simple calculation shows this change is not related to the improved definition of the reaction plane for larger detection angles.

\footnotetext{
${ }^{1}$ It can similarly be shown that a linear out-of-plane fall off has a similar independence.
} 


\subsection{Integrated Cross-Sections}

In his work on the fragmentation of $70 \mathrm{MeV}^{7} \mathrm{Li}$ Davinson [Da87] decomposed the inclusive break-up bump cross-section for beam velocity tritons into a number of components. He successfully accounted for $\sim 50 \%$ of the ${ }^{208} \mathrm{~Pb}$ inclusive triton yield at grazing $\left(\theta_{\text {graz }} \sim 32 \mathrm{degr}\right)$ in terms of sequential decay of the ${ }^{7} \mathrm{Li}$ state, direct break- up of the ${ }^{7} \mathrm{Li}$ and $\left({ }^{7} \mathrm{Li}, \operatorname{txn} \gamma\right)$ partial fusion channels. He also accounted for $\sim 30 \%$ of the ${ }^{120} \mathrm{Sn}$ inclusive triton yield at $17^{\circ}$ in the same reaction channels. The ${ }^{7} \mathrm{Li}_{4.63 \mathrm{MeV}}$ sequential break-up channel only accounted for $7.0 \%$ of the ${ }^{12} \mathrm{C}$ inclusive triton yield at $10^{\circ}$ with there being no indication of any direct break-up component. No measure of the partial fusion cross-section was attempted for this target.

It was proposed that a significant proportion of the missing reaction crosssections may be absorbed by final state interactions between the fragments and the target. These interactions would distort or completely destroy the momentum correlation of the outgoing fragments and may deflect one or both of the fragments to large angles outwith the narrow angular detection range used in the experiment. These events would then be misidentified or lost altogether resulting in an underestimate of the reaction channel strength. It can be seen that this effect will be most significant for those channels where fragmentation takes place close to the target. Consequently the identification of the direct break-up channel will be most affected by this mechanism. To make an estimate of the wide angle contribution to the total inclusive triton yield the experimental data presented in the previous two sections, 4.2.1. and 4.2.2., was integrated over the alpha detector angle. A trapezoidal integration technique was used to determine the cross-section contribution from each of the pixels of the alpha detector. The integration was only performed between the actual detection limits of the alpha detector system and no attempt was made to fit the double differential cross-sections outside this range. It is assumed that the contribution from these external regions is small due to the $\sin \theta$ term in the solid angle integration and to the in-plane enhancement illustrated in section 4.2.2. The calculated contributions therefore represent a lower limit to the full wide angle strength. In the regions where the total cross-sections were contaminated by hydrogen break-up, 
the cross-sections used for integration were estimated from the trends of the neighbouring data points. Again, since this only occured at the most forward angles, the resulting uncertainty was assumed to be a minimal contributor to the overall uncertainty calculated from the extremes of the statistical errors. In the forward angled ${ }^{12} \mathrm{C}$ data where contributions from sequential ${ }^{7} \mathrm{Li}$ break-up have been identified, this component was extracted from the data before the integration was performed. Again the $\sin \theta$ term ensured that errors in this procedure were minimal. Figures 4.63 to 4.65 show the results of this integration for all three targets.

It is immediately obvious from these figures that even for large triton angles the $\alpha$-t coincidence channel has an important quasi-elastic component for the ${ }^{120} \mathrm{Sn}$ and ${ }^{208} \mathrm{~Pb}$ targets but for the ${ }^{12} \mathrm{C}$ target the processes became significantly more inelastic at larger $\theta_{t}$. This point has already been mentioned in Section 4.2.1. These figures may be directly compared to the inclusive triton data presented in figs.4.7,4.8 and 4.9 and show that contrary to what was proposed in [Da87] the wide angle $\alpha$-t channel does not contribute significantly to the inclusive triton yield for any of the targets. For the ${ }^{12} \mathrm{C}$ target at $10^{\circ}$ it is only $6 \%$ of the inclusive yield, for the ${ }^{120} \mathrm{Sn}$ target $1.5 \%$ at $17^{\circ}$ and $0.5 \%$ for the ${ }^{208} \mathrm{~Pb}$ target at $32^{\circ}$. A similar procedure has been followed by Castenada et al [Ca80] to explain the fast particle inclusive cross-section at grazing for $75 \mathrm{MeV},{ }^{6} \mathrm{Li}$ on ${ }^{197} \mathrm{Au}$. There he attributes almost $50 \%$ of the inclusive deuteron yield at $25^{\circ}$ to the sequential and non-sequential $\alpha$ - $d$ coincidence channel. This is a much higher contribution than reported here and in [Da87] for what one would assume to be a very similar system. This disparity may be due to Castenada's assumption of azimuthal symmetry of the coincidence yield around the deuteron direction. The data in Section 4.2.2. and appendix B show that there is an exponential rather than a linear fall off out-of-plane. The effect of this is not clear however, since the two different cases use different angular conventions. Depending on the gradient of the assumed linear fall off, Castenada may over-estimate this outof-plane component. It is possible that for values of $\phi_{\alpha}$ near $180^{\circ}$ there may be a substantial increase in the cross-section as one approaches the reaction plane. This would mean that the calculation in this thesis would underestimate the true value. At present it is not clear if these reasons are sufficient to explain this disparity. 


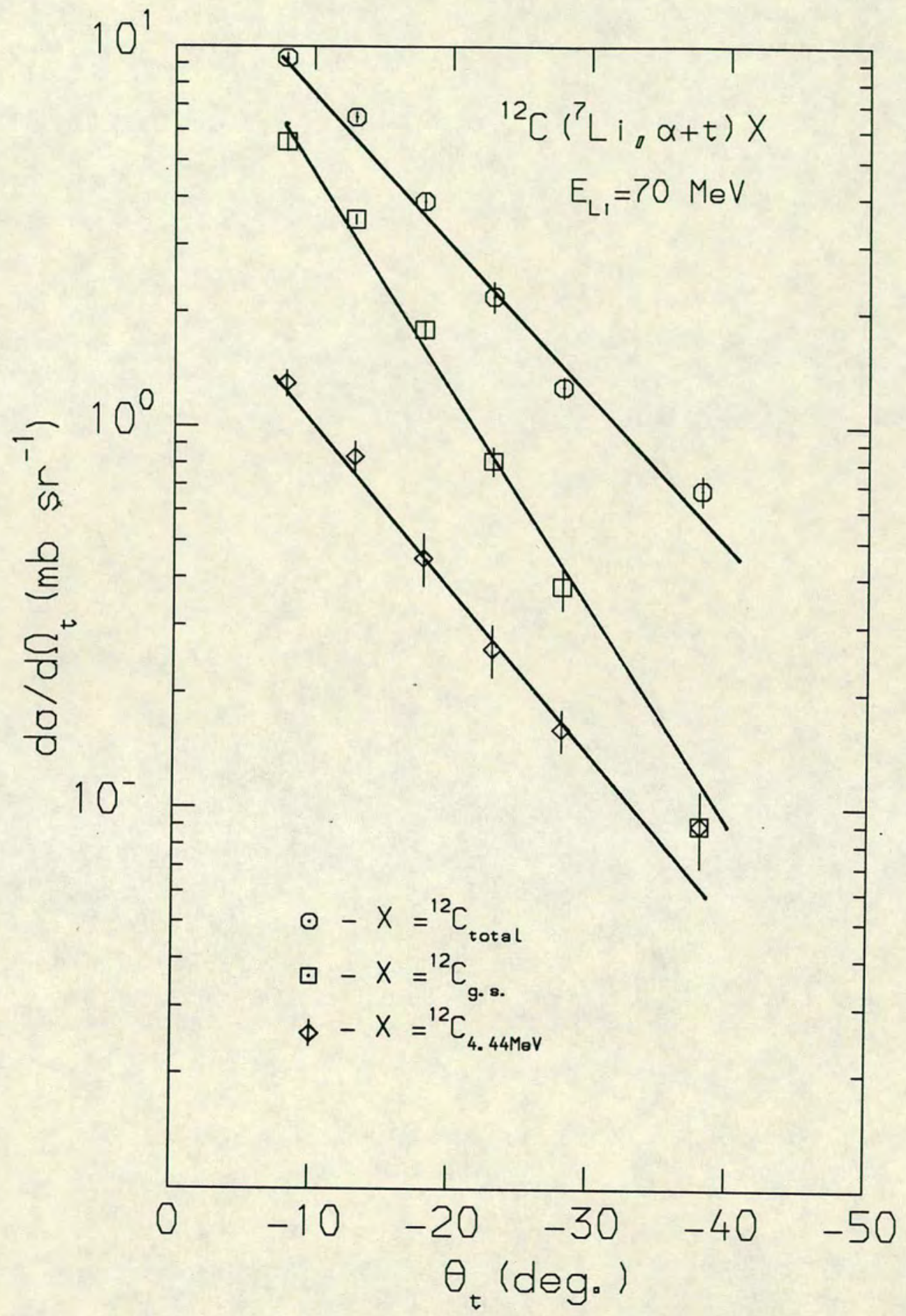

Figure 4.63: Differential cross section for tritons, integrated over all recorded alpha angles. Data is shown for quasi-elastic events, events leaving ${ }^{12} \mathrm{C}$ in its first excited state and for all $\alpha$-t coincidence events. 


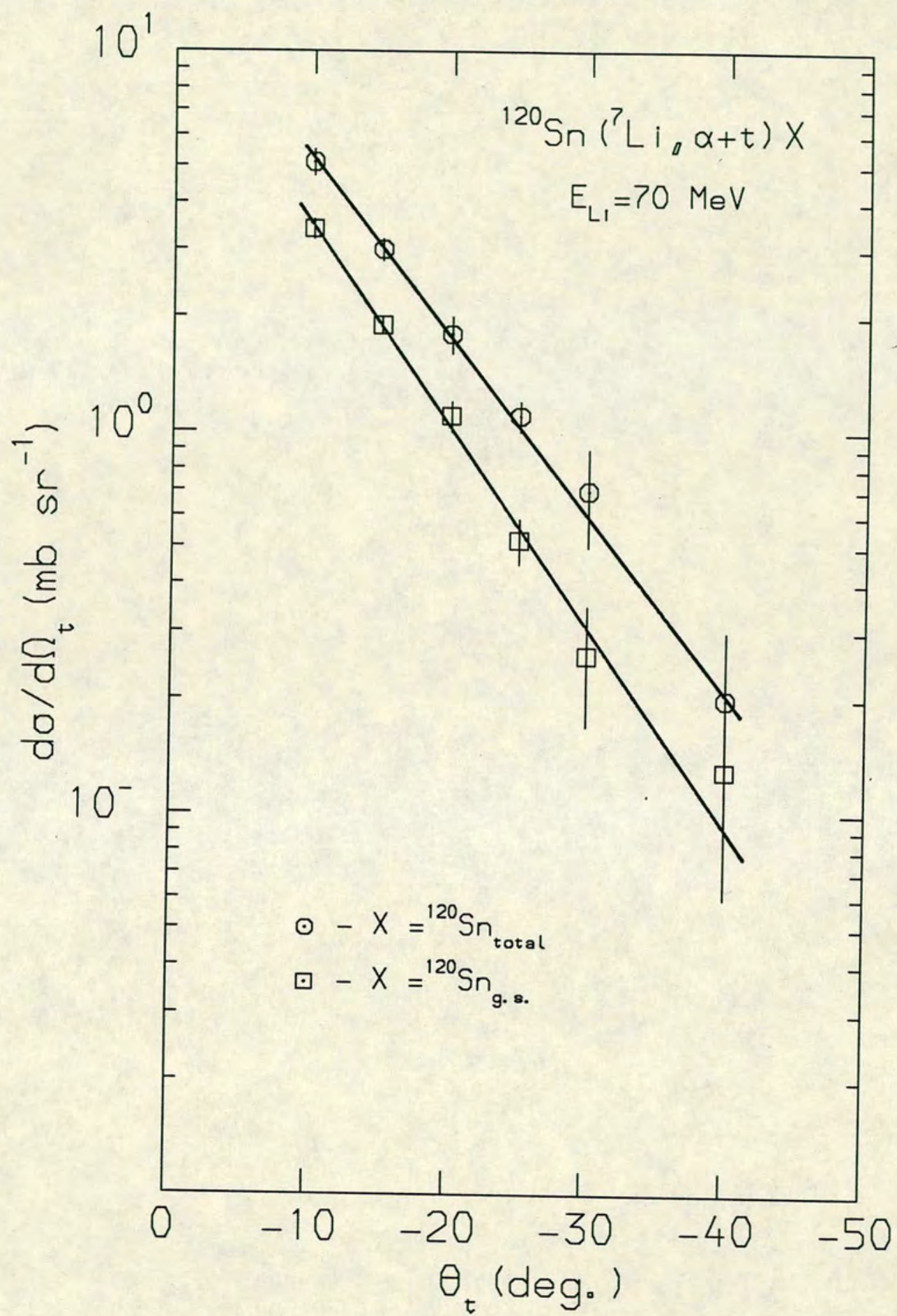

Figure 4.64: Differential cross section for tritons, integrated over all recorded alpha angles. Data is shown for quasi-elastic events and for all $\alpha$-t coincidence events. 


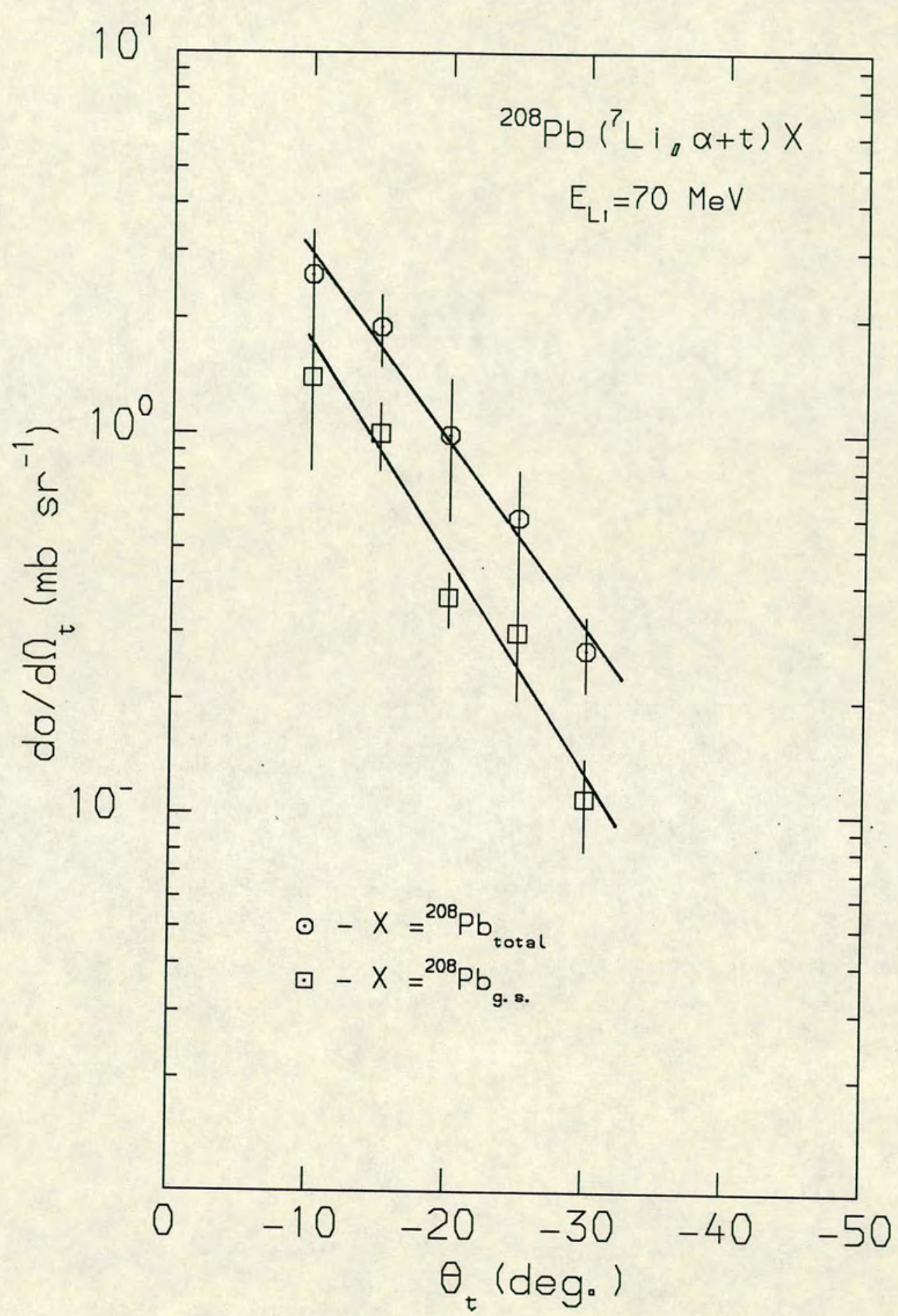

Figure 4.65: Differential cross section for tritons, integrated over all recorded alpha angles. Data is shown for quasi-elastic events and for all $\alpha$-t coincidence events. 
This leaves us with the question of where the rest of the inclusive triton yield comes from. For the ${ }^{12} \mathrm{C}$ target it is possible that the partial fusion channel and the multi-fragmentation channel will be strong contributors but the identification of both these channels is rather difficult. For the heavier targets, fission of the compound nucleus resulting from partial fusion can occur and has not, as yet, been measured. Other charged particle decaying channels will also contribute as will other sequentially decaying particle transfer channels. None of these reactions have been measured in this work or in [Da87] and it seems likely that their identification is necessary to fully account for the inclusive, beam-velocity yields. 


\section{Chapter 5}

\section{Discussion and Conclusions}

In the interpretation of heavy ion reactions the currently available methods for analysing and understanding the data, tend to fall into two general groups; those that utilise sophisticated mathematical and computational techniques in an attempt to incorporate all the important reaction channels within the one approach (eg. C.D.C.C.) and those that rely on simplifying approximations and intuitive reasoning to explain, more qualitatively, the general trends and overall behaviour of certain groups of experimental data (eg. critical angular momentum model).The following discussion will deal exclusively with the latter type of model, giving only passing mention to the former where appropriate.

It is known that the fragments contributing to the inclusive beam velocity bump and the $\alpha-t$ break-up channel at small angular separations [Da87] mainly originate from peripheral collisions. In this region the type of interaction between the target and the projectile will be very sensitive to the projectile's impact parameter. Consequently if one considers the possible reaction mechanisms leading to large angular separations between the outgoing fragments one would expect a number of processes to contribute. The incident ${ }^{7} \mathrm{Li}$ may be excited to a high lying particle unstable state which sequentially decays into an alpha and a triton at large angular separations. This decay would happen at large distances from the target where final state interactions between the fragments and the target are unlikely. The ${ }^{7} \mathrm{Li}$ may also be excited directly into the $\alpha-t$ continuum [Da87][Sh81][Sh84]. This excitation/fragmentation occurs much closer to the tar- 
get where the possibility of fragment-target final state interactions is much more likely. These may deflect one or both of the fragments to large angles where their inferred relative energy is much greater than the initial break-up value. This type of reaction, also known as partial orbitting, was proposed as a possible mechanism for the production of wide angle $\alpha-\mathrm{d}$ coincidences in the fragmentation of $75 \mathrm{MeV}{ }^{6} \mathrm{Li}$ [Ca80]. Shotter et.al. [Sh88] have performed Coulomb excitation calculations for the excitation of the $\alpha-t$ system into the continuum near the break-up threshold for a ${ }^{208} \mathrm{~Pb}$ target at $70 \mathrm{MeV}$. At smaller scattering angles for the effective ${ }^{7} \mathrm{Li}$ centre of mass, the data and the calculation agree well but at angles $\geq \theta_{g r} \sim 35^{\circ}$ the data fall substantially below the calculation. It is exactly in this region that one would expect any final state interactions to become important. It is conceivable that the reason for the disparity is that final state interactions deflect one of the fragments from its trajectory, reducing the recorded coincidence cross-section in the original direction.

If the deflected fragment is to survive to large scattering angles it must have a large mean free path in nuclear matter since it will be in contact with the surface of the target for a substantial fraction of the nuclear circumference. However, the interacting particles normal m.f.p. may be enhanced (with regard to fusion) if its grazing angular momentum is above the critical angular momentum for fusion of the fragment with the target. Table 5.1 lists the grazing angular momenta for alpha and triton fragments in a $70 \mathrm{MeV}{ }^{7} \mathrm{Li}$ incident on the various targets used in this investigation. Also shown is the value of the critical angular momenta for fusion calculated from the parameterisation of Siwek-Wilczynska [Si79]. It can be seen that at grazing the fragment's angular momenta are all much greater than the critical angular momenta for fusion of the fragment. In the absence of any tangential frictional forces the probability for fusion of these peripherally produced fragments should therefore be small. This will not affect the possibility of inelastic interactions between the target and the participant. It has however been shown ( in chapter 4 ) that, at least for the heavier targets, this inelastic reaction channel is weak.

In a situation where both the fragments are scattered to angles on opposite sides of the beam well outside the grazing angle, the concept of a non-interacting spectator becomes rather tenuous. It requires fragments on the very tail of the 


\begin{tabular}{|c|c|c|c|c|c|c|}
\hline & \multicolumn{2}{|c|}{$\alpha$} & \multicolumn{2}{c|}{ triton } & \multicolumn{2}{c|}{${ }^{7} \mathrm{Li}$} \\
\hline & $\ell_{\text {graz. }}$ & $\ell_{\text {crit. }}$ & $\ell_{\text {graz. }}$ & $\ell_{\text {crit. }}$ & $\ell_{\text {graz. }}$ & $\ell_{\text {crit. }}$ \\
${ }^{12} \mathrm{C}$ & 11 & 4 & 9 & 3 & 20 & 5 \\
${ }^{120} \mathrm{Sn}$ & 21 & 8 & 16 & 7 & 37 & 11 \\
${ }^{208} \mathrm{~Pb}$ & 22 & 9 & 16 & 8 & 38 & 13 \\
\hline
\end{tabular}

Table 5.1: Angular momentum (in units of $\hbar$ ) of clusters within a grazing ${ }^{7} \mathrm{Li}$ on various targets at $70 \mathrm{MeV}$. The critical angular momentum for fusion of the fragment at beam velocity is also shown.

${ }^{7} \mathrm{Li}$ internal momentum wave function to produce spectator fragments with large relative velocities scattering to these large angles. The probability of break-up from these states will be rather small. It may be that in this case the impact parameter is smaller than that of the previous case and both fragments undergo final state interactions with the target - one feeling the repulsive core of the interaction potential with the other experiencing a more gentle deflection similar to the orbitting previously mentioned. A limiting case for this model would be the complete fusion of one of the fragments with the target. The compound system formed in a highly excited state, may then re-emit a particle identical to the fused participant. This would tend to be emitted non-isotropically with the degree of focusing and in-plane enhancement dependent on the width and spin of the occupied state.

A similar but distinct process to this break-up transfer is the direct transfer of a set of nucleons to the target. The fragments from such a direct transfer process will exhibit a different energy dependence (via. the appropriate $Q$ value) to those from a break-up transfer mechanism.

In the following sections a number of simple models will be considered in an attempt to identify the contributions from these different mechanisms. The first section (5.1), deals with sequential break-up and direct transfer where the coincident particles have a distinctive energy relationship. The next section (5.2), models the reaction from a simple plane wave approach where the interaction between the fragments and the target is assumed to be small. Such a simple approach may be applicable to fragments travelling in the forward direction where 
such approximations are more valid. Section 5.3 is an attempt to analyse the quasi-elastic coincidence data by factorising it into a product of the singles inclusive yields. This type of approach would be successful for processes where the fragments have completely lost their momentum correlation due to interactions with the target. Finally, the last section contains the conclusions of the thesis.

\subsection{Sequential Decay and Partial Fusion}

One of the reaction channels that has been identified as contributing to the quasielastic events in kinematically complete fragmentation studies is the sequential decay of projectile like fragments that have been excited above their particle threshold. This is seen as a two step process where the projectile is first raised to an excited state via the interaction with the target and then subsequently decays via particle emission, at large distances from the target. This ensures that there is little possibility for final state interactions between the target and either of the fragments. The fragments therefore maintain their momentum correlation making it possible to identify the projectile states involved in the reaction. This has been outlined in chapter 1 and entails the projection of the target ground state locus (in a two dimensional $\mathrm{E}$ (frag 1) vs $\mathrm{E}$ (frag 2) plot) onto one of the energy axes. Different projectile states are then characterised by distinct peaks in this projected energy spectrum. It is possible to enhance the efficiency for detection of sequential events from specific projectile states by careful choice of the detector configuration [Bi82], [Da87]. However, it is not always obvious what the system response for different sequential states will be. For any detailed investigation into these sequential break-up events, this response must be determined in order to correctly interpret the experimental data. One method that has been used to good effect [Bi82], [Da87], [Ma88] is to simulate the reaction kinematics by a Monte Carlo technique. This will be described in more detail in the next section. Section 5.1.2 will then present a comparison of the experimental and Monte Carlo results. 


\subsubsection{Monte Carlo Simulations}

The Monte Carlo code used to simulate the present detector system was based on the programme Montegen, written by MacDonald [Ma 88]. The programme was modified to determine the response of a coincidence set-up incorporating two circular telescopes and a detector system constructed from crossed strip detectors. The actual detection geometry was specified by:

- radii and distances of the two circular telescopes from the target.

- the strip detector distances and orientation.

- the number and width of strips on each strip detector.

- and the angular position of the detectors.

Once these parameters are fixed the properties of the reaction itself were specified. These were:

- the incident beam energy.

- the initial target and projectile masses.

- the final outgoing masses of the target-like (TLF) and projectile-like (PLF) fragments.

- the $\mathrm{Q}$ value for this first reaction.

- the sequential state of the PLF being simulated (ie. its energy and width).

- the final sequential break-up channel and the $Q$ value for this fragmentation.

The structure of the programme was of a sufficiently general nature that any type of break-up reaction with three particles in the outgoing channel could be simulated by changing these input parameters. The first step in the calculation was to determine the excitation of the outgoing PLF. The state being simulated 
was taken to have a Lorentzian line shape and was randomly populated by the Monte Carlo technique. The initial scattering angle of the PLF* system was then randomly chosen, again by the Monte Carlo technique, to give an isotropic distribution in the centre-of-mass frame.

Fixed angular limits on this initial scattering direction were used to improve the efficiency of the calculation. These relied on the fact that for both fragments to be recorded in the detectors, the centre-of-mass of the fragment + fragment system (ie. the direction of the excited $\mathrm{PLF}^{*}$ system) had to lie within a certain range of angles determined by the detector geometry. Once this direction was chosen the kinematics of the initial scattering process were calculated giving the velocity of the $\mathrm{PLF}^{*}$ system. The $\mathrm{PLF}^{*}$ system was then used as the centre-ofmass reference frame for the second stage of the simulation. The two fragments were emitted isotropically in this frame with the direction of fragmentation relative to the $\mathrm{PLF}^{*}$ direction again chosen by the Monte Carlo method. Once this final direction was chosen, the paths of the fragments were transformed into the lab reference frame and checked to determine if they hit the correct detectors. If either of the fragments missed the detectors, a new event was started. If they were both recorded, their lab energies were calculated and checked against the experimental energy thresholds before the correct pixel energy spectra were incremented.

The final energy spectra gave the actual response of the detector set-up to the sequential decay of a specific state of the $\mathrm{PLF}^{*}$ system. It was also possible to calculate the effective solid angle and direction of maximum efficiency for each pixel-telescope combination. The Monte Carlo spectra thus take into account the effects of all the energy thresholds and finite angular resolutions that influence the experimentally observed data. By this method it was possible to compare the experimental projected energy spectra with the corresponding Monte Carlo spectra for various states of the $\mathrm{PLF}^{*}$ system and to remove the effect of geometrically enhanced efficiencies from subsequent cross-section calculations. The results of these simulations were therefore a valuable aid in the interpretation of the experimental data. 


\begin{tabular}{|c|c|c|c|}
\hline $\mathbf{E}_{x}(\mathrm{MeV} \pm \mathrm{keV})$ & width $(\mathrm{keV})$ & spin & fragmentation dist. (fermis) \\
\hline $4.630 \pm 9$ & $93 \pm 8$ & $\frac{7}{2}-$ & 311 \\
$6.68 \pm 50$ & $875 \pm 200$ & $\frac{5}{2}-$ & 33 \\
$7.459 \pm 1.2$ & $89 \pm 7$ & $\frac{5}{2}-$ & 325 \\
$9.67 \pm 100$ & $\sim 400$ & $\frac{7}{2}-$ & 72 \\
\hline
\end{tabular}

Table 5.2: Excited states of ${ }^{7} \mathrm{Li}$ used in simulations ( taken from [Aj84]) along with distance of fragmentation calculated from $\Delta \mathrm{E} \times \Delta \mathrm{t} \sim \hbar$.

\subsubsection{Sequential Decay}

The Monte Carlo procedure outlined in the previous section was used to simulate the detector system response to the sequential break-up of the ${ }^{7} \mathrm{Li}$ projectile via the known states of ${ }^{7} \mathrm{Li}$ shown in table 5.2 below [Aj84]. These are all known to decay into the $\alpha+\mathrm{t}$ channel. The sequential break-up of the $4.63 \mathrm{MeV}$ state has already been extensively investigated by Davinson et al [Da87] for the same target projectile systems as used in this work. That investigation involved the use of a detector system specifically designed to enhance the detection efficiency for this channel. Clear evidence for the sequential decay path was obtained from the projected spectra for quasi-elastic events. Sequential events via the $4.63 \mathrm{MeV}$ state in ${ }^{7} \mathrm{Li}$ leaving the ${ }^{12} \mathrm{C}$ in its $4.44 \mathrm{MeV}$ first excited state were also recorded. This process had a differential cross-section similar in magnitude to the quasi-elastic process.

For any given detector angular separation set-up there will be a minimum relative energy between the outgoing fragments that will be detectable. The value of this minimum energy is dependant on the velocity of the centre-of-mass of the fragment-fragment system (ie. the recoil energy of the excited ${ }^{7} \mathrm{Li}$ in this case). This ${ }^{7} \mathrm{Li}$ recoil velocity will change with target mass and with the ${ }^{7} \mathrm{Li}$ direction. As a result, a fixed angular separation between detectors will be sensitive to different ${ }^{7} \mathrm{Li}$ excitation ranges as the detectors are moved relative to the beam. For a fixed $\theta_{t}$ direction, as one moves the value of $\theta_{\alpha}$, eg. along the in-plane M1 pixels, the detector system will also be most efficient at detecting break-up events from different directions of the outgoing ${ }^{7} \mathrm{Li}$. The cross-section for the excitation 
of the excited states of ${ }^{7} \mathrm{Li}$ are known to vary with the outgoing angle of the ${ }^{7} \mathrm{Li}$ system [Da87], [Sa86]. Consequently, it is difficult, a priori, to determine the exact relative intensities one would expect for the same sequential state in different detector pixels and for different sequential states in the same pixel. This can only be done if information is known about the angular distributions of the ${ }^{7} \mathrm{Li}$ excited states being modelled. This information is available for the $4.63 \mathrm{MeV}$ state (from [Da87]) but this state is kinematically suppressed from most of the detector pixels at the angular ranges covered.

The large solid angle subtended by both the alpha detector pixels and the triton detectors tend to average out the effect of the variation of excitation cross-section with ${ }^{7} \mathrm{Li}$ angle. This allows at least a nominal comparison between the intensities of the same state in neighbouring pixels. This is not true for comparison between different sequential states. These may have distinctly different angular distributions to the $4.63 \mathrm{MeV}$ state and have not as yet, been individually investigated. One final approximation made in the Monte Carlo simulations is the assumption of isotropic emission in the centre-of-mass frame of the excited projectile. All the simulated excited states have non-zero spin and if the initial interaction causes any alignment of this angular momentum, the ejectile will decay non-isotropically in its rest frame [Sc77], [Os74], [Ut83], [Ma88], [Da87]. This will have little effect on the energy distributions obtained but may distort the inferred out-of-plane intensity dependance. Any alignment would tend to increase the in-plane enhancement. Fig.5.1 shows the result of a simulation of break-up from the $6.68 \mathrm{MeV}$ state in ${ }^{7} \mathrm{Li}$ on ${ }^{12} \mathrm{C}$. It exhibits the features one can use to distinguish sequential break-up peaks from other processes. The main characteristic is the two distinct peaks which merge together into one as the angular separation between the fragments increases. The intensity of this combined peak increases just as the detectors reach the edge of the break-up cone and then decreases rapidly as the detectors move outside the kinematically allowed region.

With these properties in mind, figs.5.2 to 5.21 show the experimental $\mathrm{E}_{\alpha}$ projected spectra from the ${ }^{12} \mathrm{C}$ target for various angles of the alpha and triton detectors. Where it was instructive to do so, the corresponding Monte Carlo simulations are also shown. All the kinematically observable sequential states 


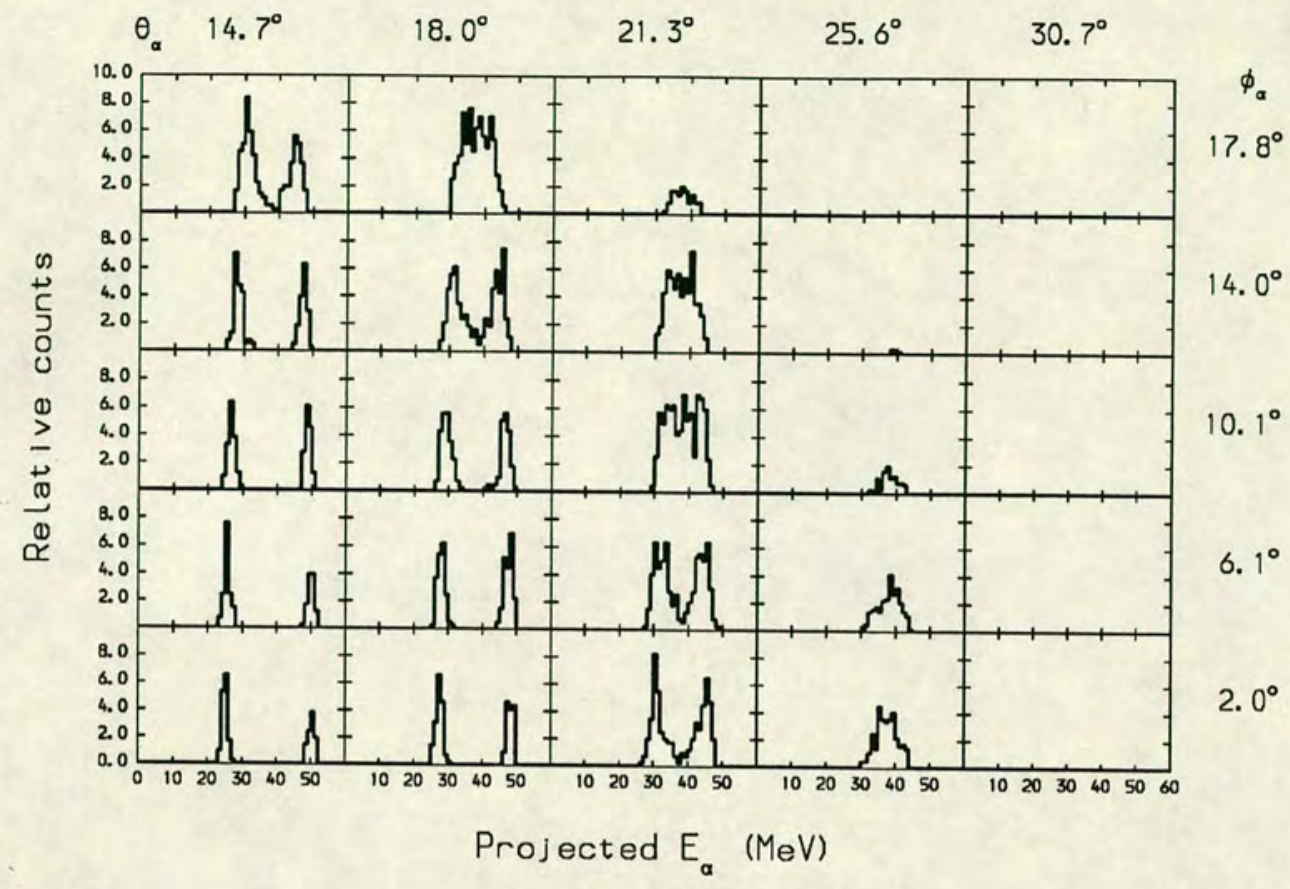

Figure 5.1: Example of the Monte Carlo simulations for sequential break-up from the ${ }^{7} \mathrm{Li}_{7.47 \mathrm{MeV}}$ state with the ${ }^{12} \mathrm{C}$ target and $\theta_{t}=-8.0^{\circ}$.

were simulated by the same number of random events and the resulting spectra plotted together to form the final spectra. The states simulated in the Monte Carlo calculations are given in the caption of each figure. It can be seen that for angular settings where the alpha and triton detectors are within the $4.63 \mathrm{MeV}$ state break-up cone (eg. fig.5.2 pixels F1M1 and F1M2, fig.5.19 pixel F1M1 etc.) there is clear evidence for break-up via this state. When the detectors are on the edge of the break-up cone as is the case for these elements, the effective solid angle is extremely sensitive to the angular separation. A small change of $\sim 0.5^{\circ}$ can alter the effective solid angle of the system by a factor of $\sim \times 20$. This meant it was rather meaningless to calculate the efficiency corrected cross-section for excitation of the $4.63 \mathrm{MeV}$ state at the corresponding ${ }^{7} \mathrm{Li}$ scattered angle. 


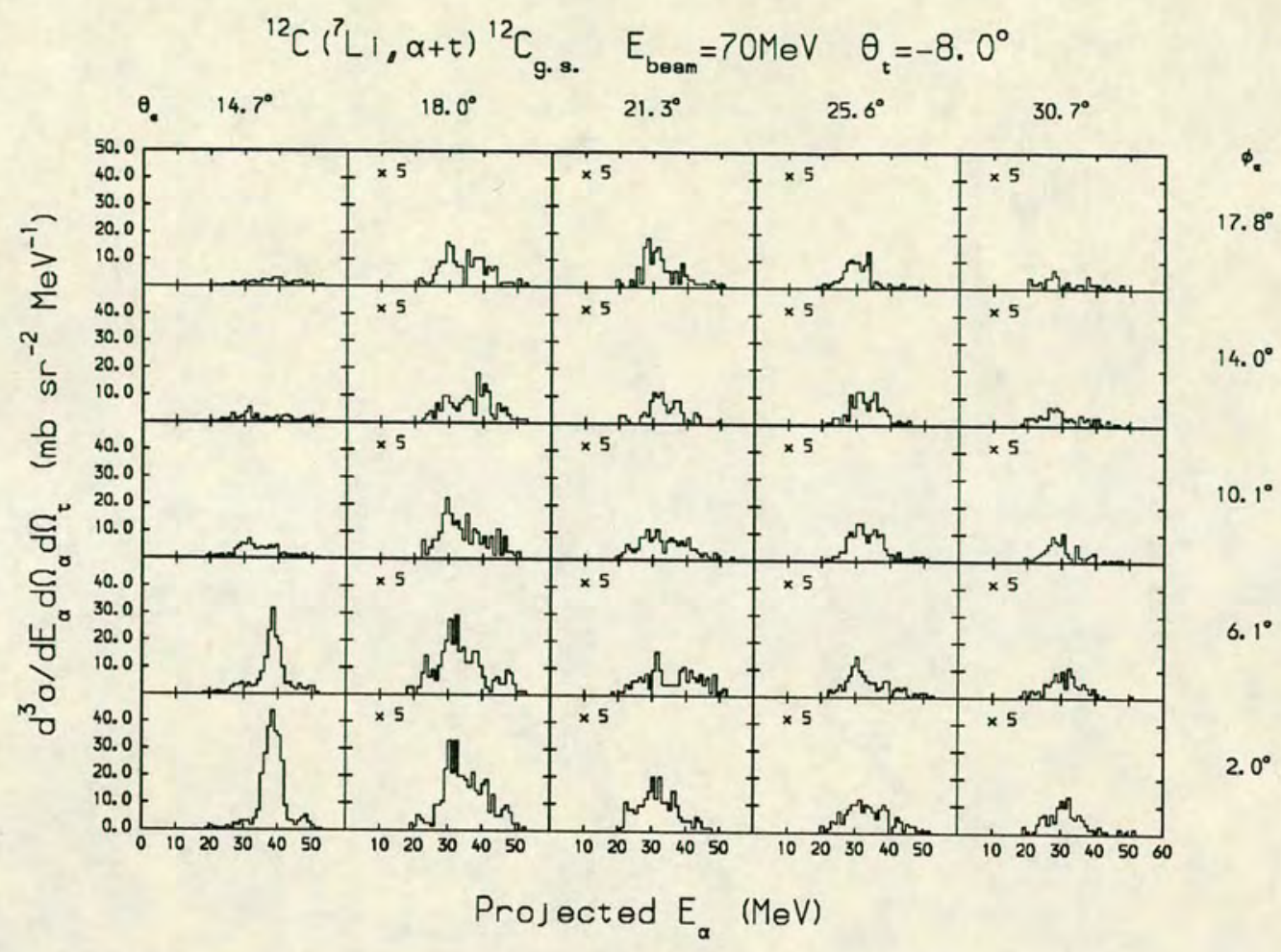

Monte-Car lo Simulation

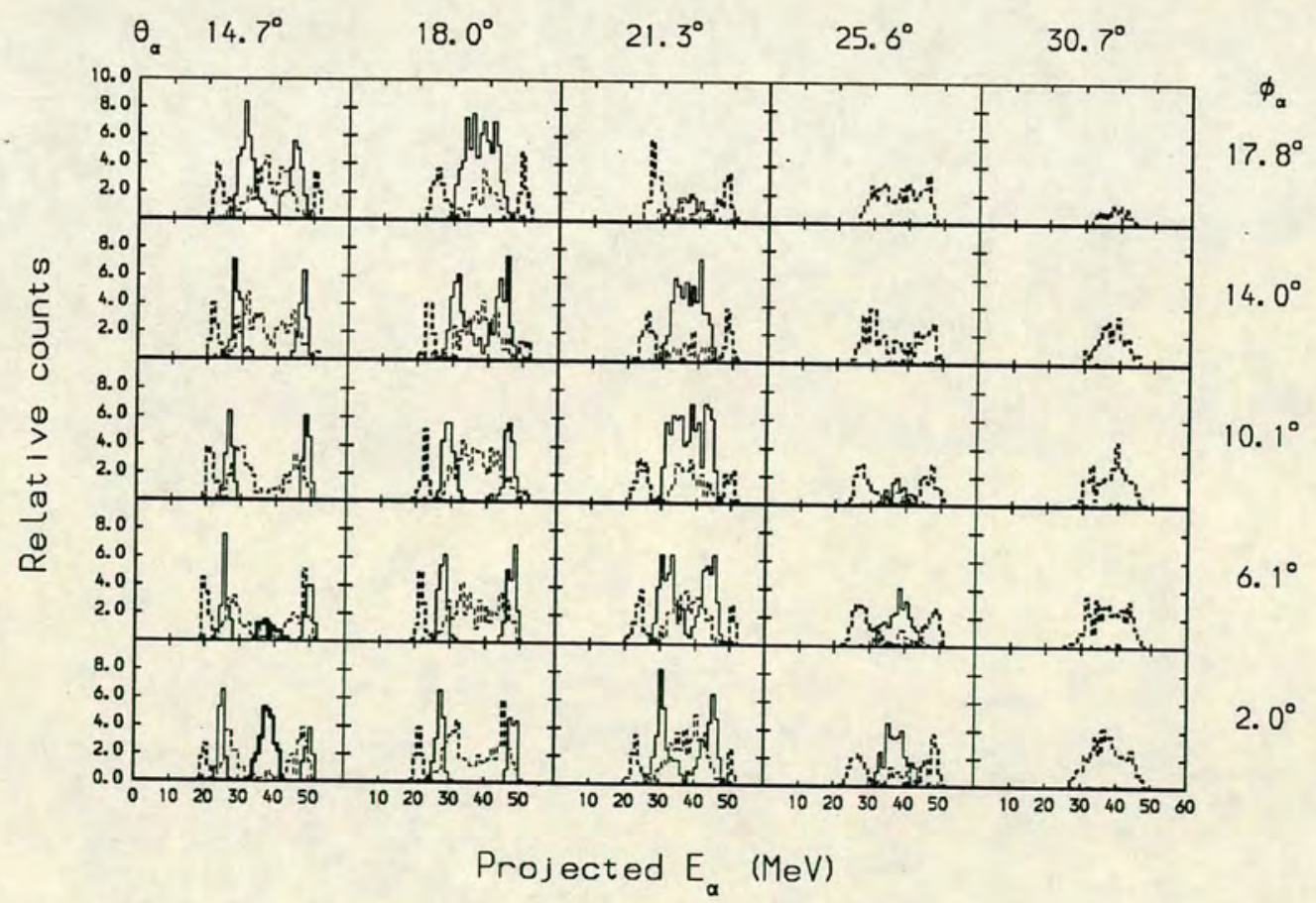

Figure 5.2: Comparison of data and Monte Carlo simulations for $\theta_{t}=-8.0^{\circ}$. States simulated were ${ }^{7} \mathrm{Li}_{4.63 \mathrm{MeV}}$ (thick line), ${ }^{7} \mathrm{Li}_{6.68 \mathrm{MeV}}$ (dotted line), ${ }^{7} \mathrm{Li}_{7.47 \mathrm{MeV}}$ (thin line), ${ }^{7} \mathrm{Li}_{9.96 \mathrm{MeV}}$ (thick dotted line). 


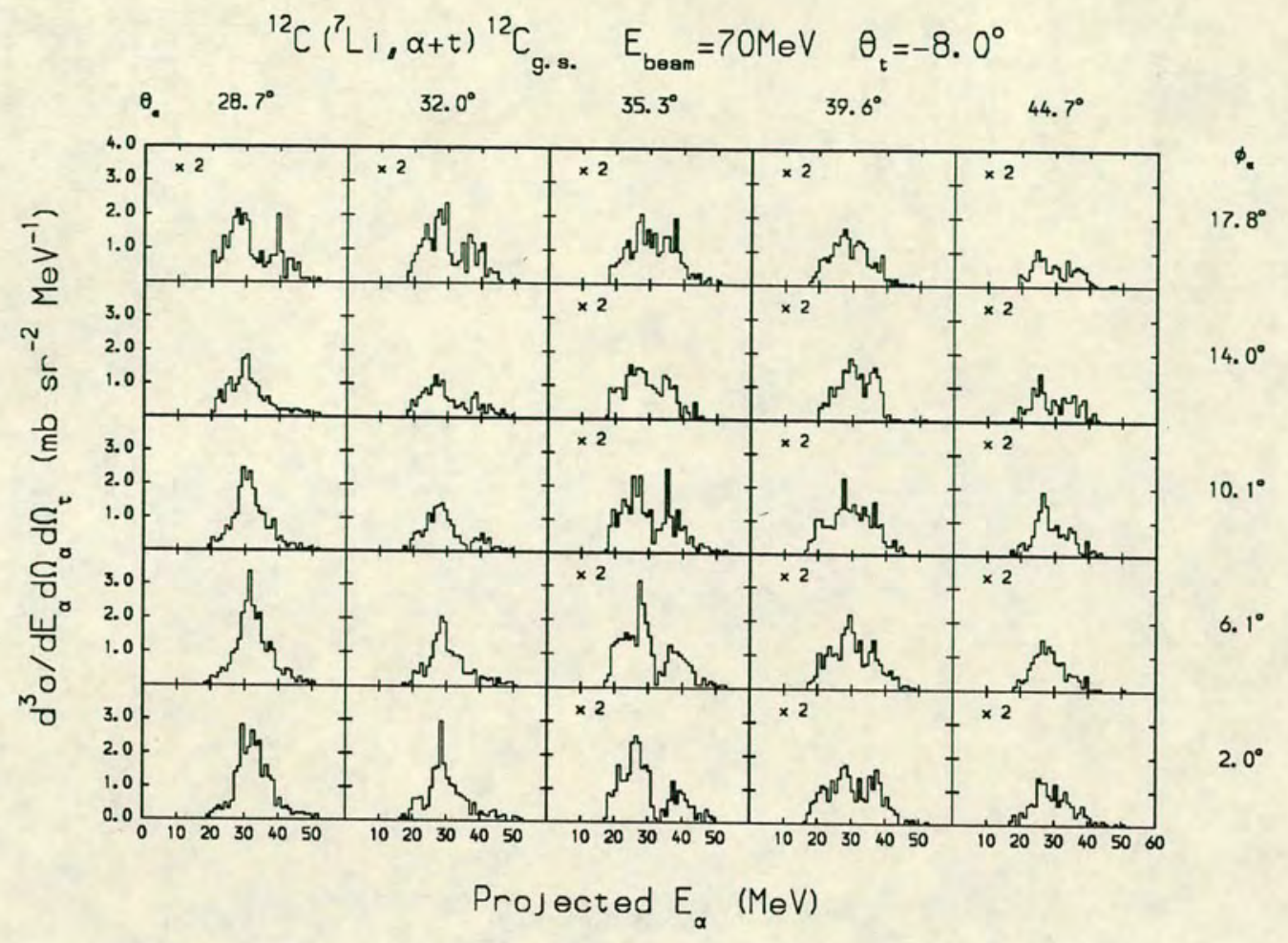

Monte-Car lo Simulat ion

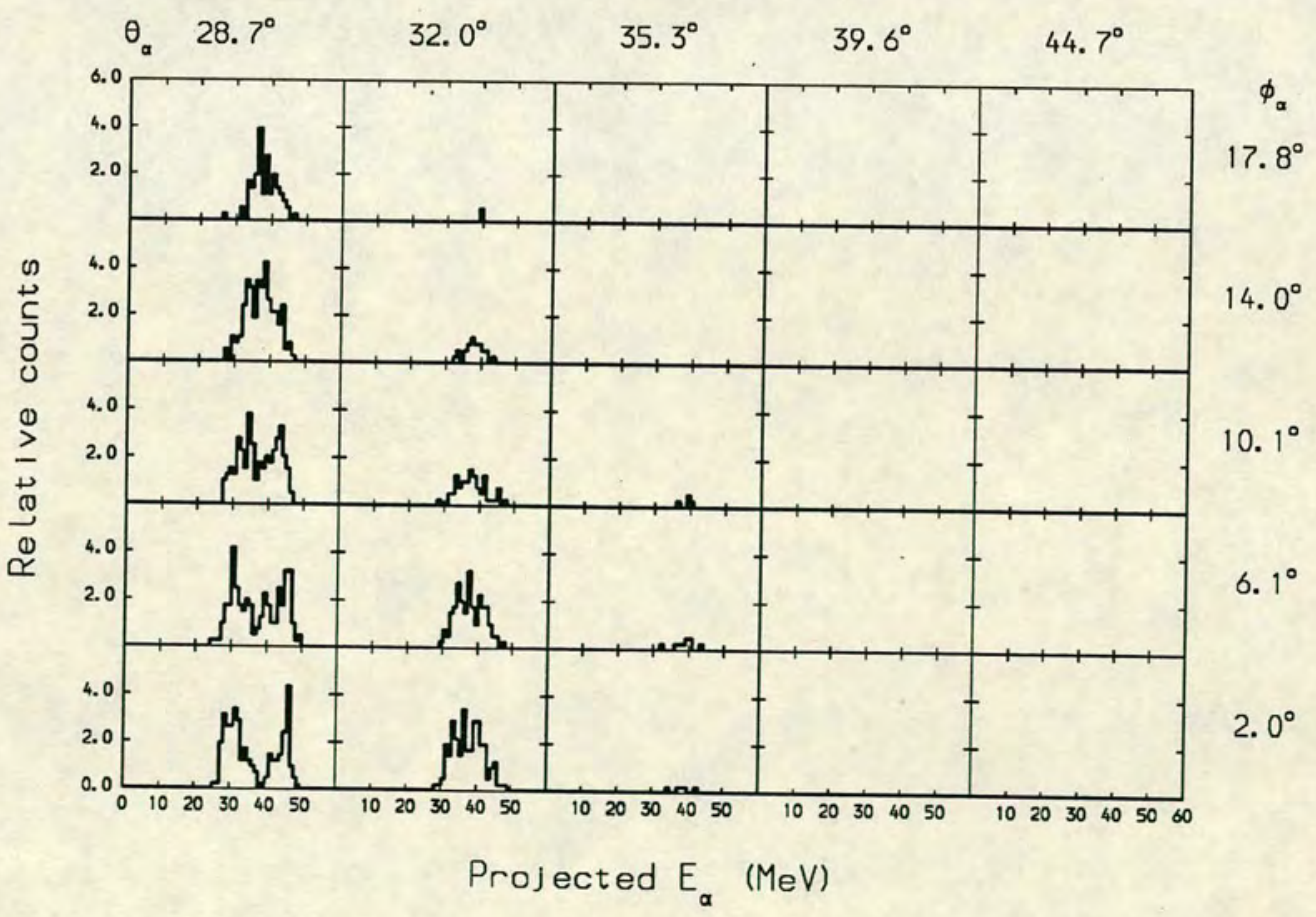

Figure 5.3: Comparison of data and Monte Carlo simulations for $\theta_{t}=-8.0^{\circ}$. State simulated was ${ }^{7} \mathrm{Li}_{9.96 \mathrm{MeV}}$ (thick line). 


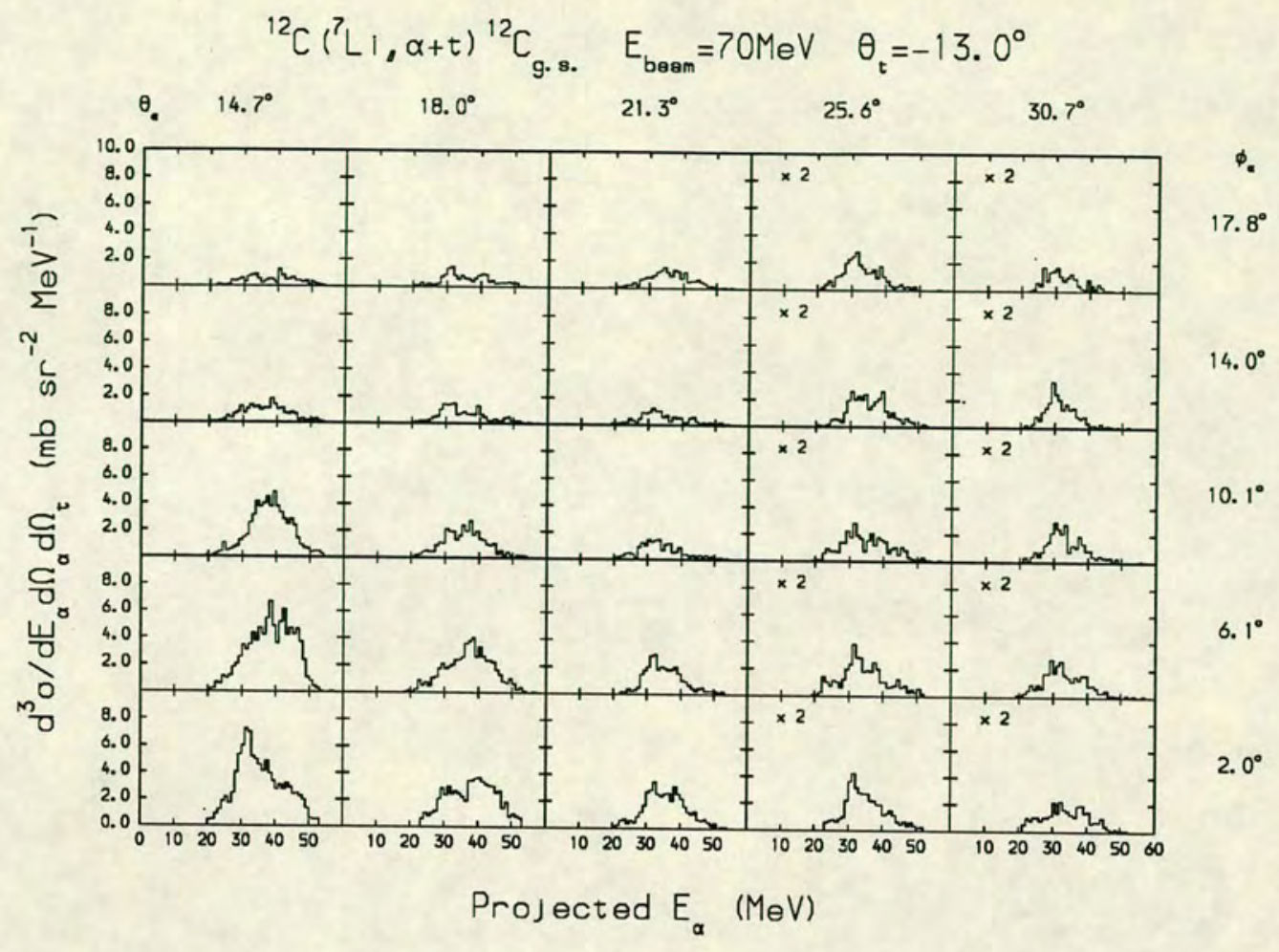

Monte-Car lo Simulat ion

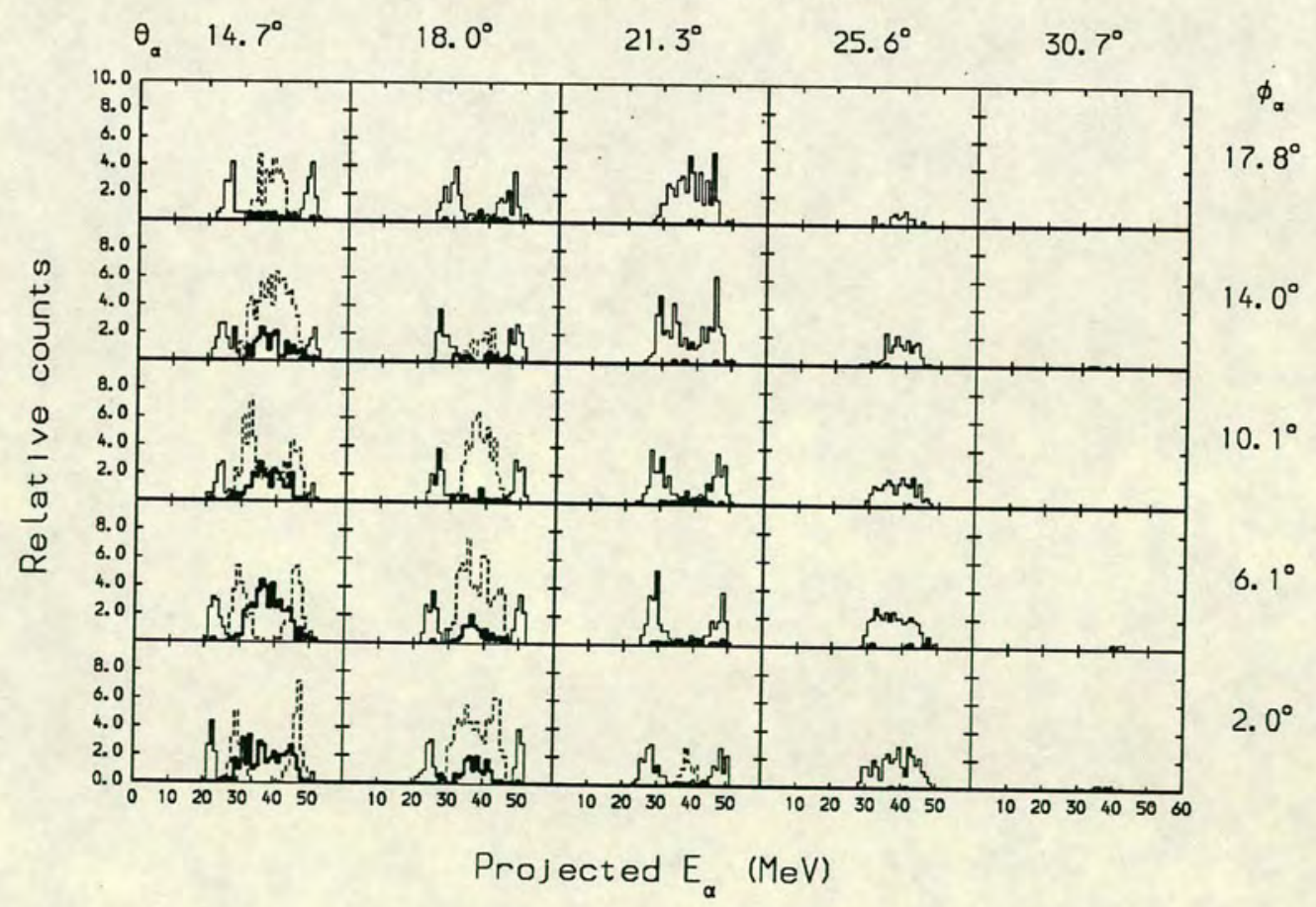

Figure 5.4: Comparison of data and Monte Carlo simulations for $\theta_{t}=-13.0^{\circ}$. States simulated were ${ }^{7} \mathrm{Li}_{6.68 \mathrm{MeV}}$ (thick line), ${ }^{7} \mathrm{Li}_{7.47 \mathrm{MeV}}$ (dotted line), ${ }^{7} \mathrm{Li}_{9.96 \mathrm{MeV}}$ (thin line). 


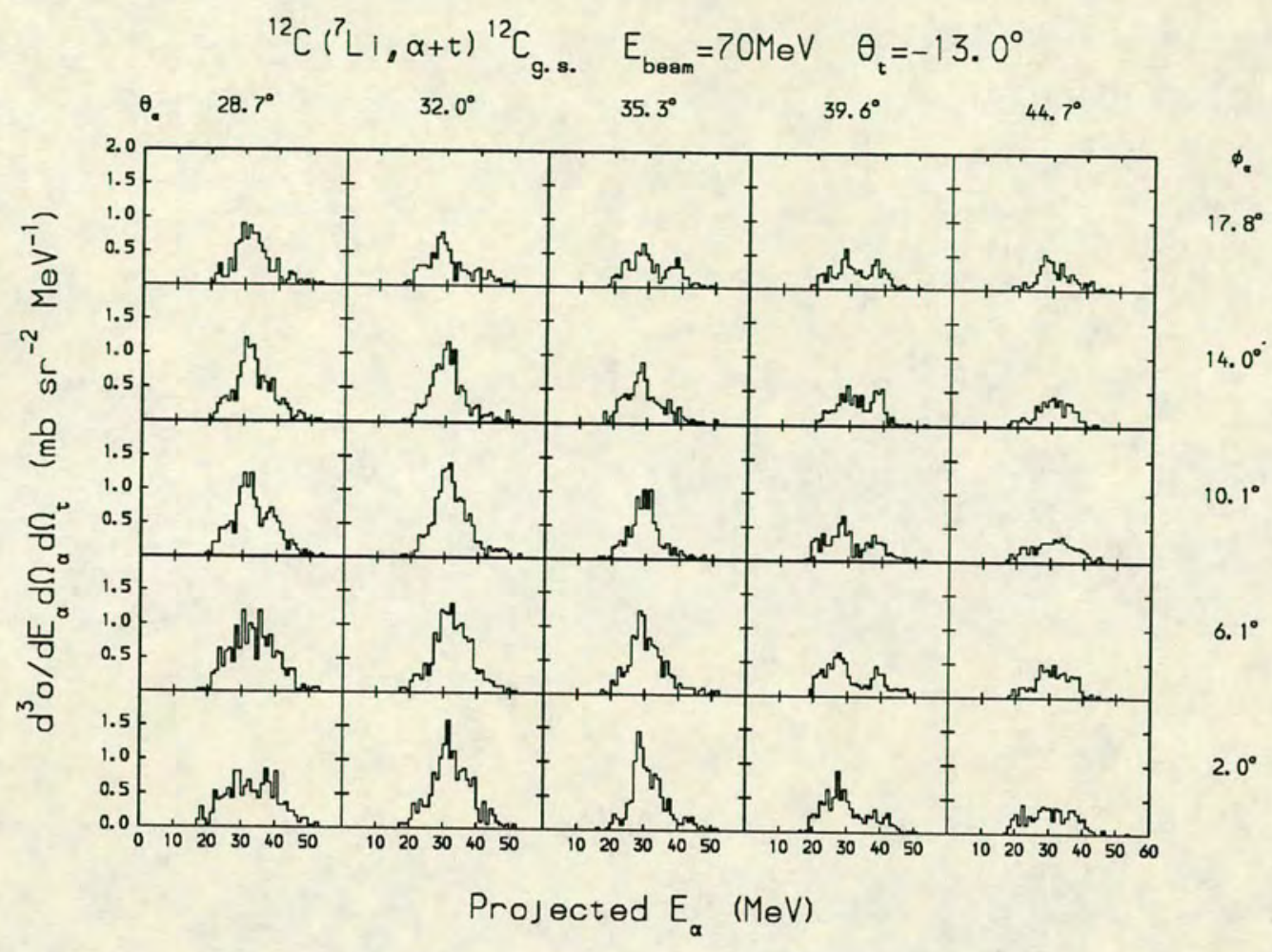

Monte-Car lo Simulation

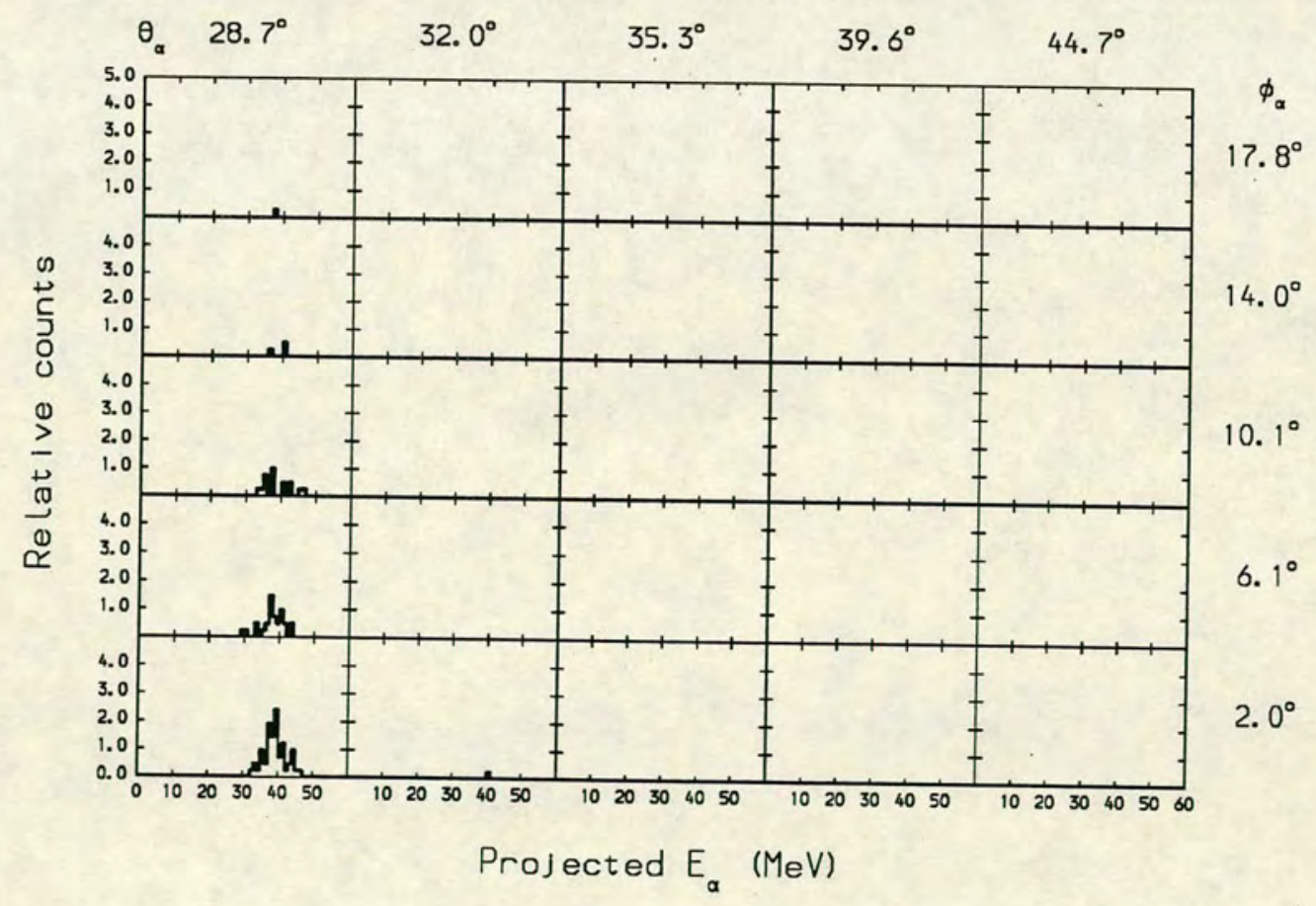

Figure 5.5: Comparison of data and Monte Carlo simulations for $\theta_{t}=-13.0^{\circ}$. State simulated was ${ }^{7} \mathrm{Li}_{9.96 \mathrm{MeV}}$ (thick line). 


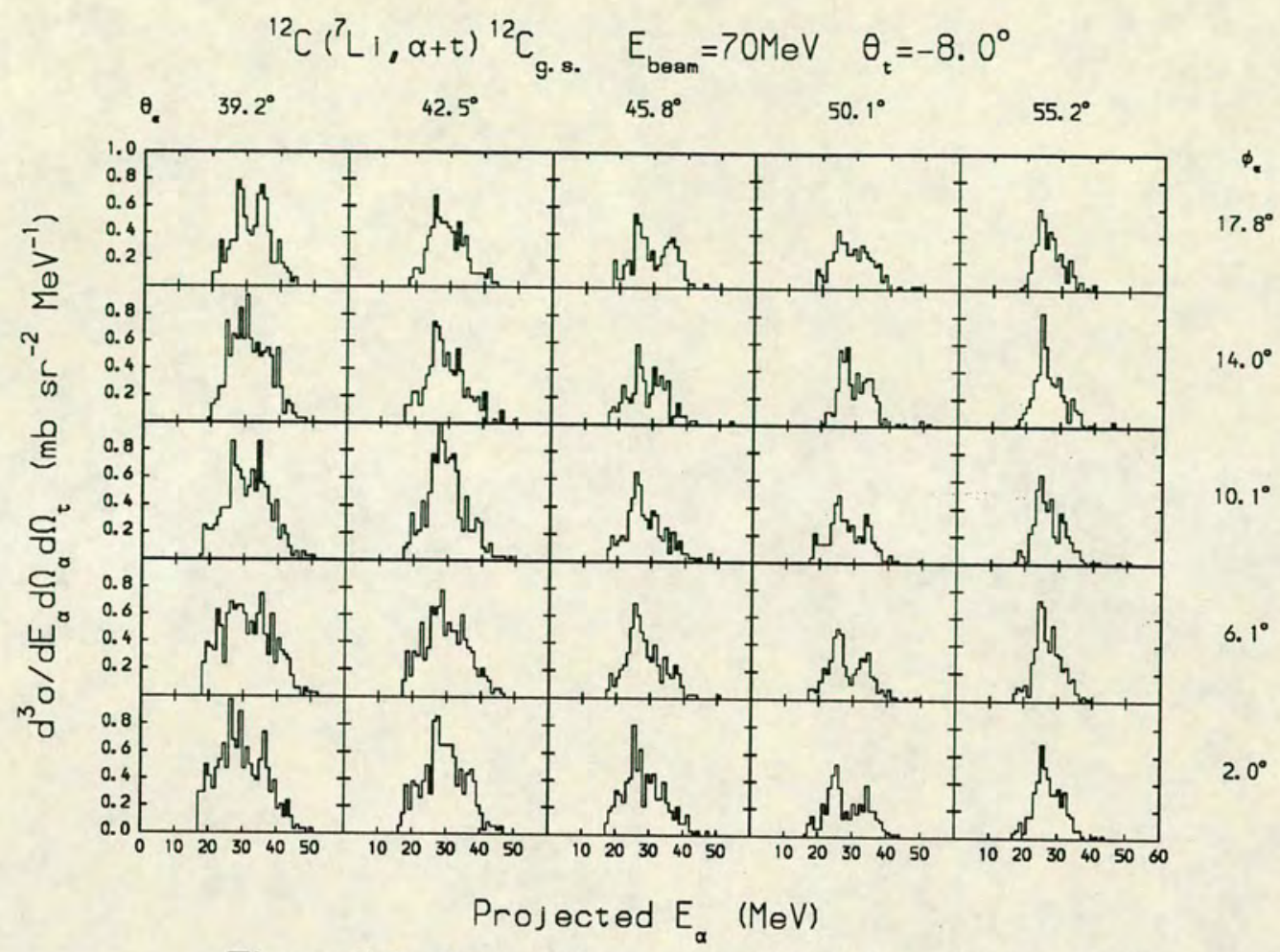

Figure 5.6: Projected quasi-elastic $\mathrm{E}_{\alpha}$ data for $\theta_{t}=-8.0^{\circ}$.

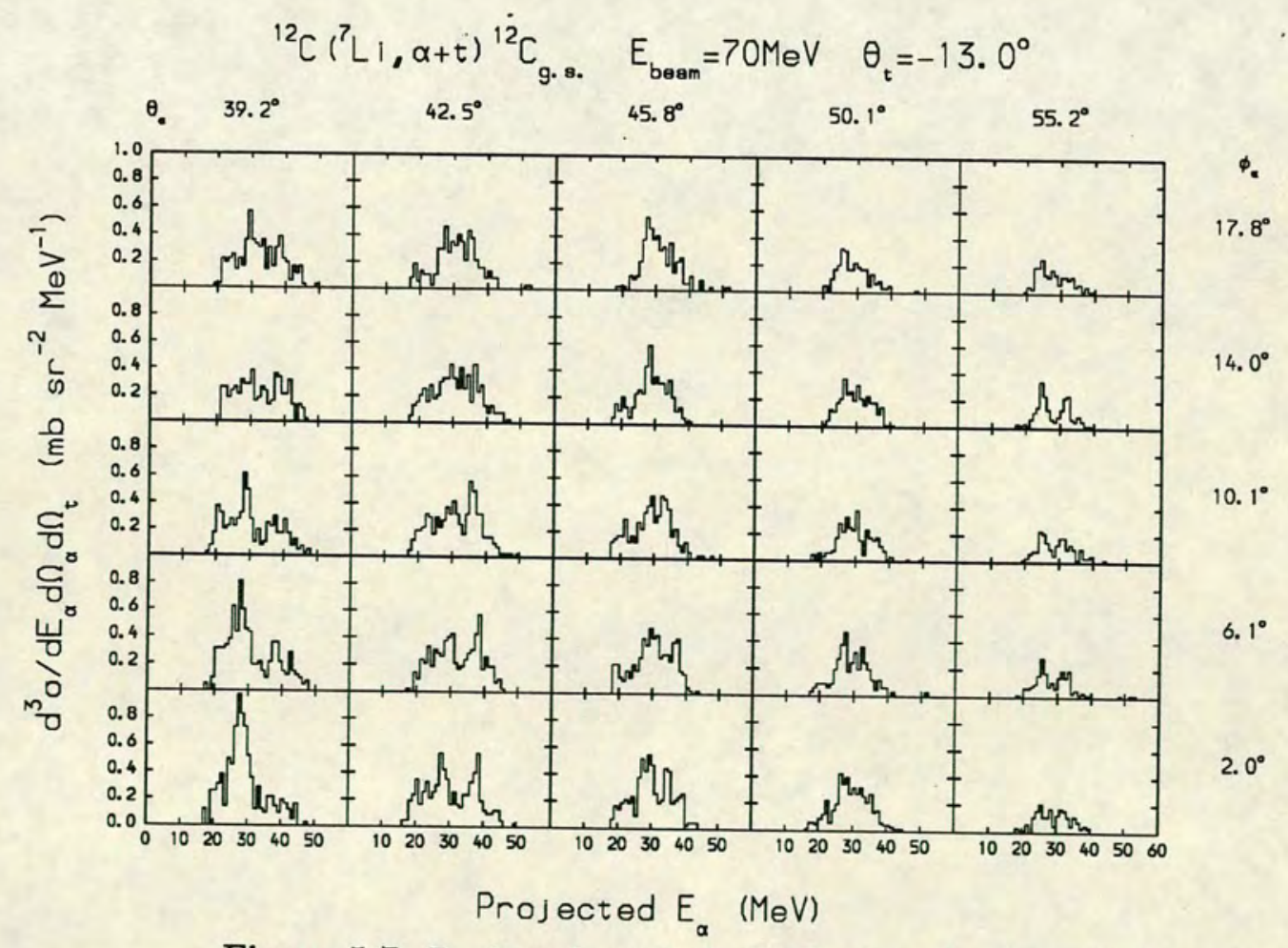

Figure 5.7: Projected quasi-elastic $\mathrm{E}_{\alpha}$ data for $\theta_{t}=-13.0^{\circ}$. 


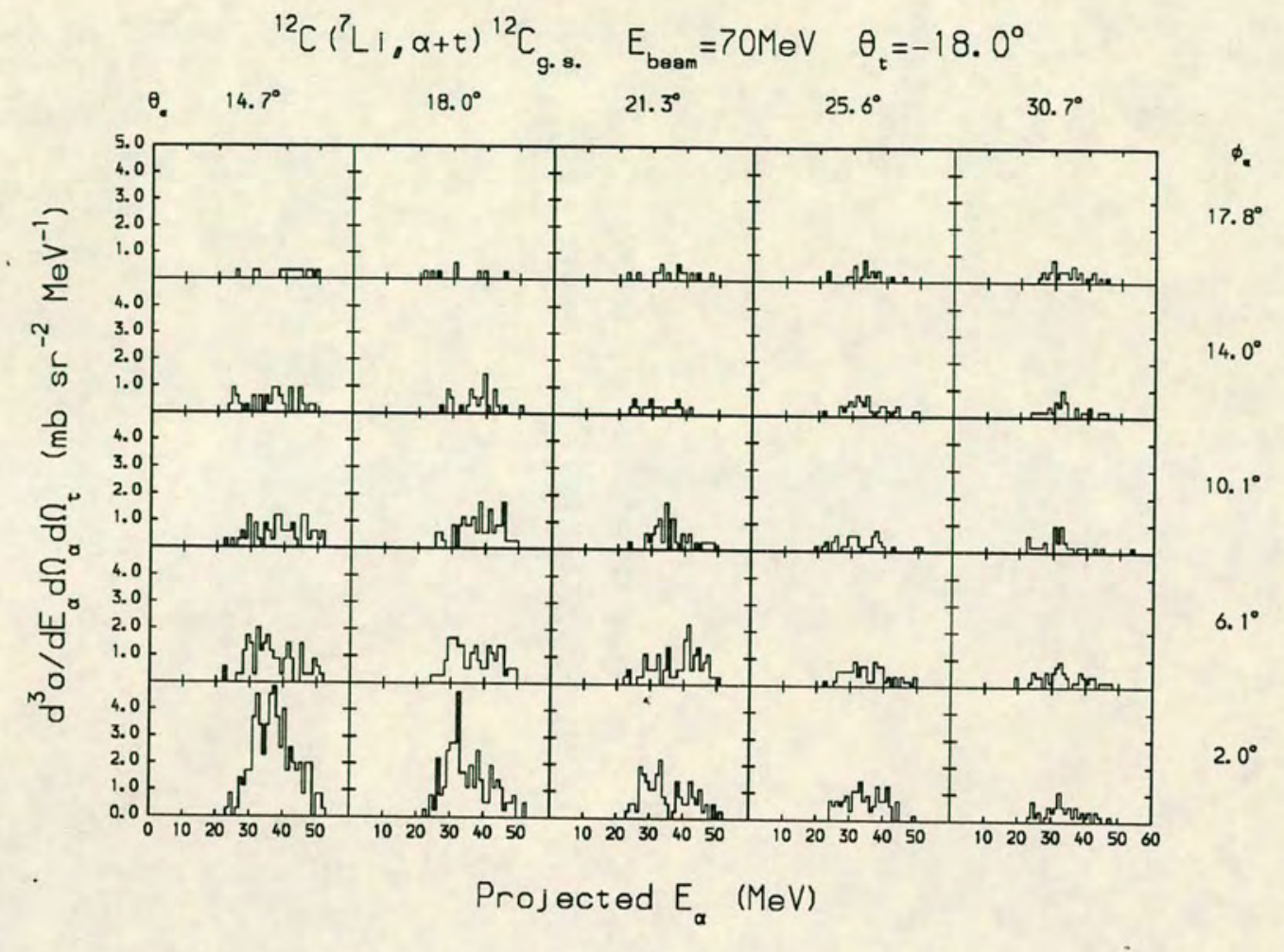

Monte-Car lo Simulation

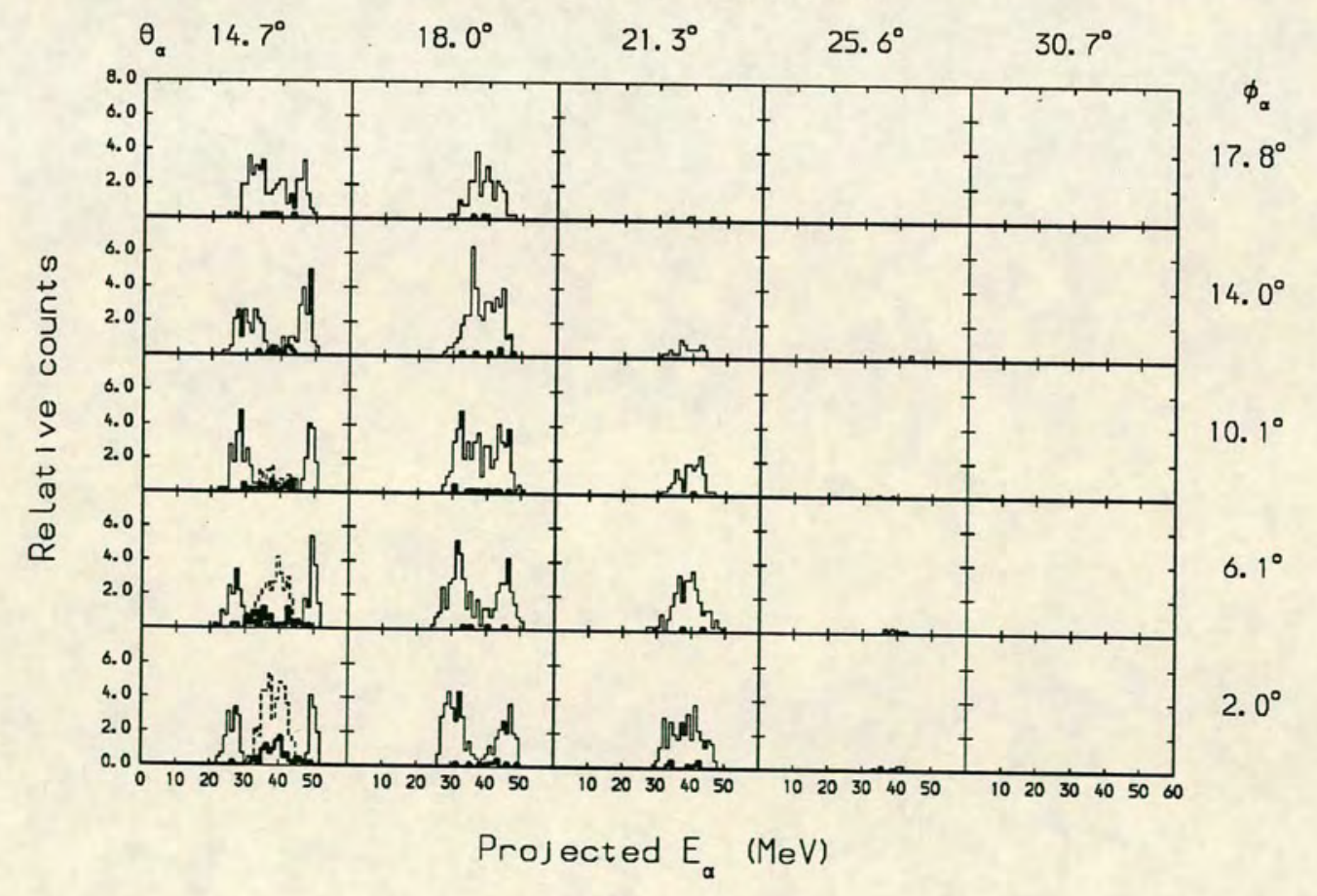

Figure 5.8: Comparison of data and Monte Carlo simulations for $\theta_{t}=-18.0^{\circ}$. States simulated were ${ }^{7} \mathrm{Li}_{6.68 \mathrm{MeV}}$ (thick line), ${ }^{7} \mathrm{Li}_{7.47 \mathrm{MeV}}$ (dotted line), ${ }^{7} \mathrm{Li}_{9.96 \mathrm{MeV}}$ (thin line). 


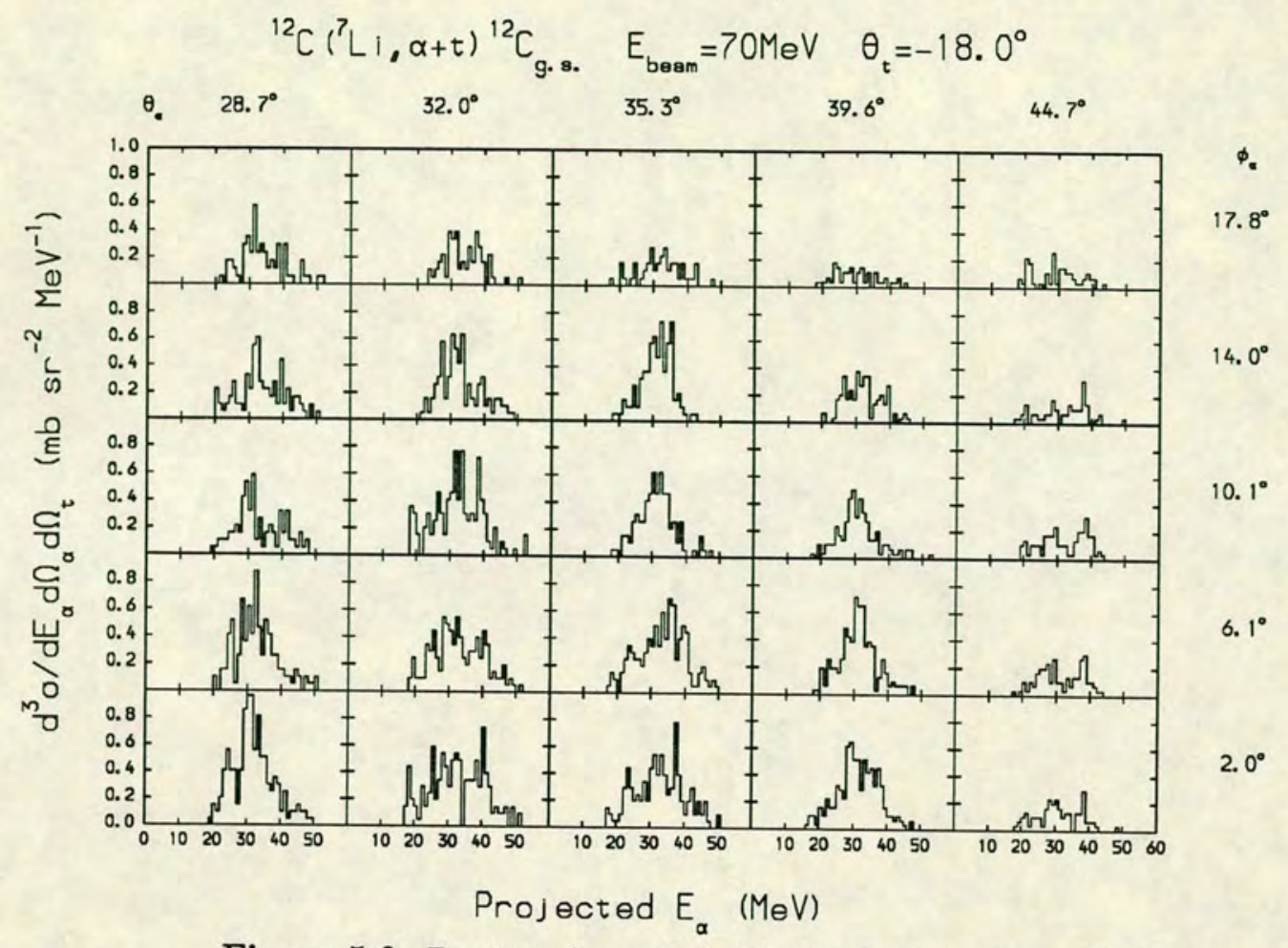

Figure 5.9: Projected quasi-elastic $\mathrm{E}_{\alpha}$ data for $\theta_{t}=-18.0^{\circ}$.

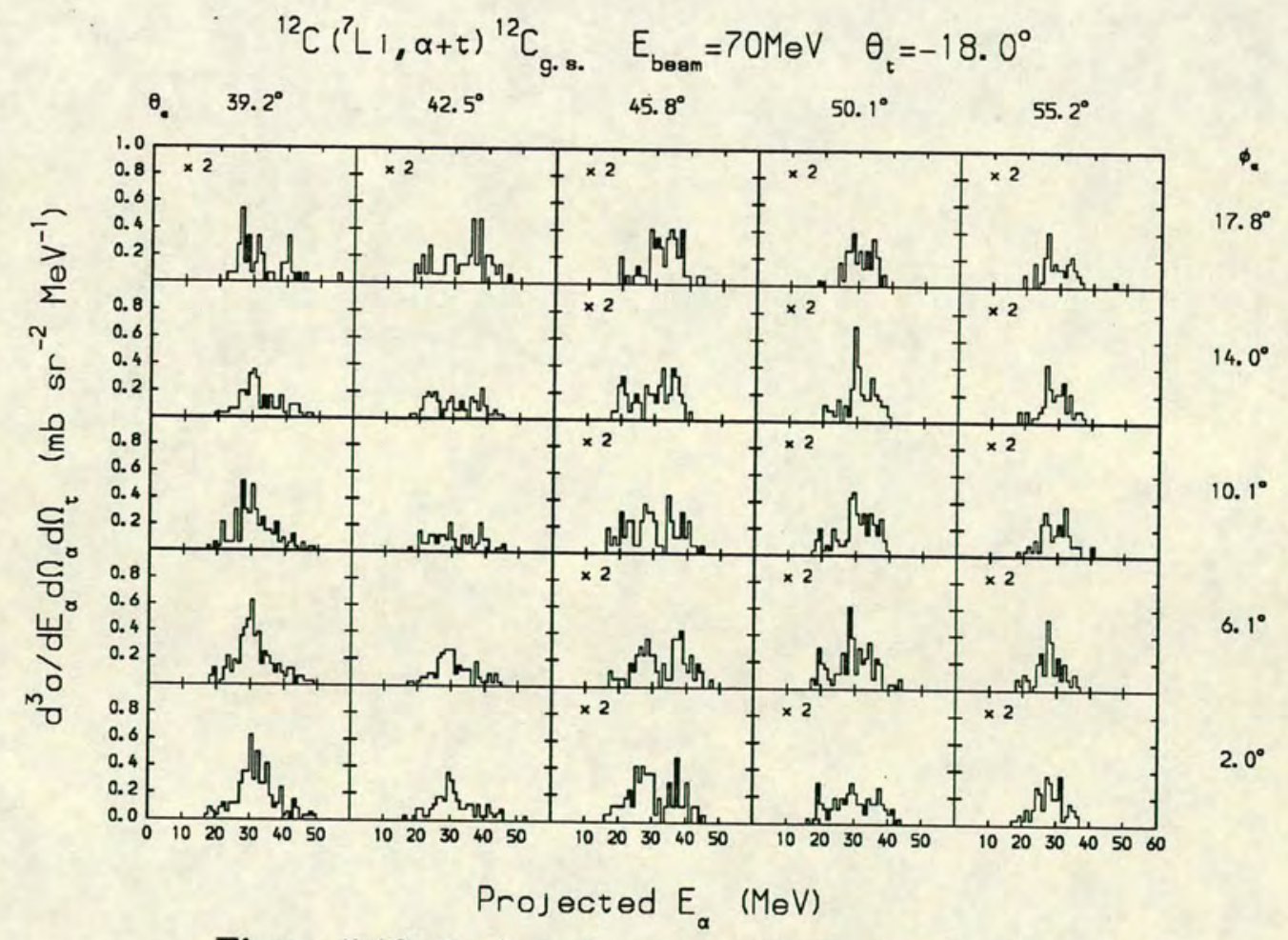

Figure 5.10: Projected quasi-elastic $\mathrm{E}_{\alpha}$ data for $\theta_{t}=-18.0^{\circ}$. 


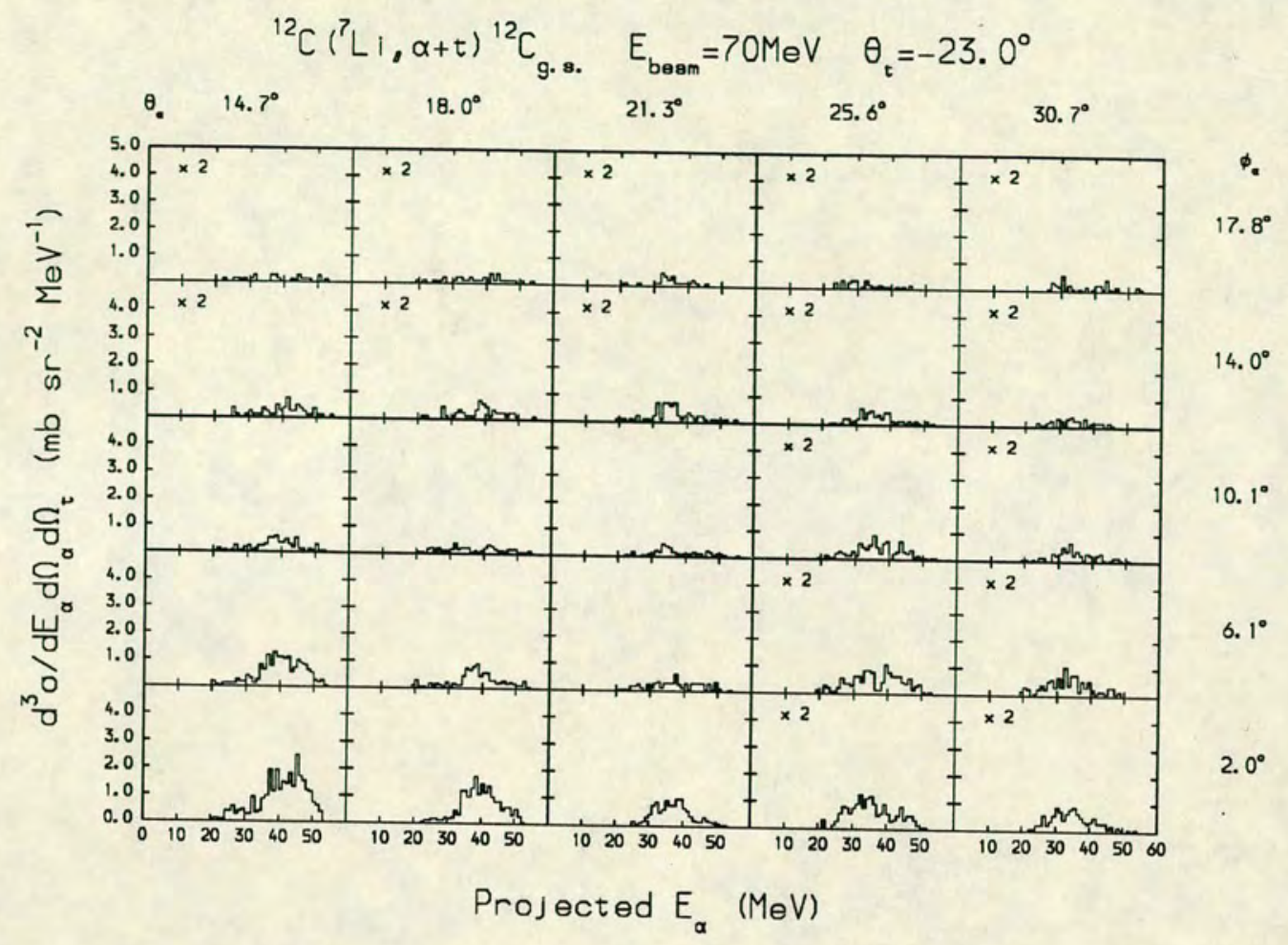

Monte-Car lo Simulat ion

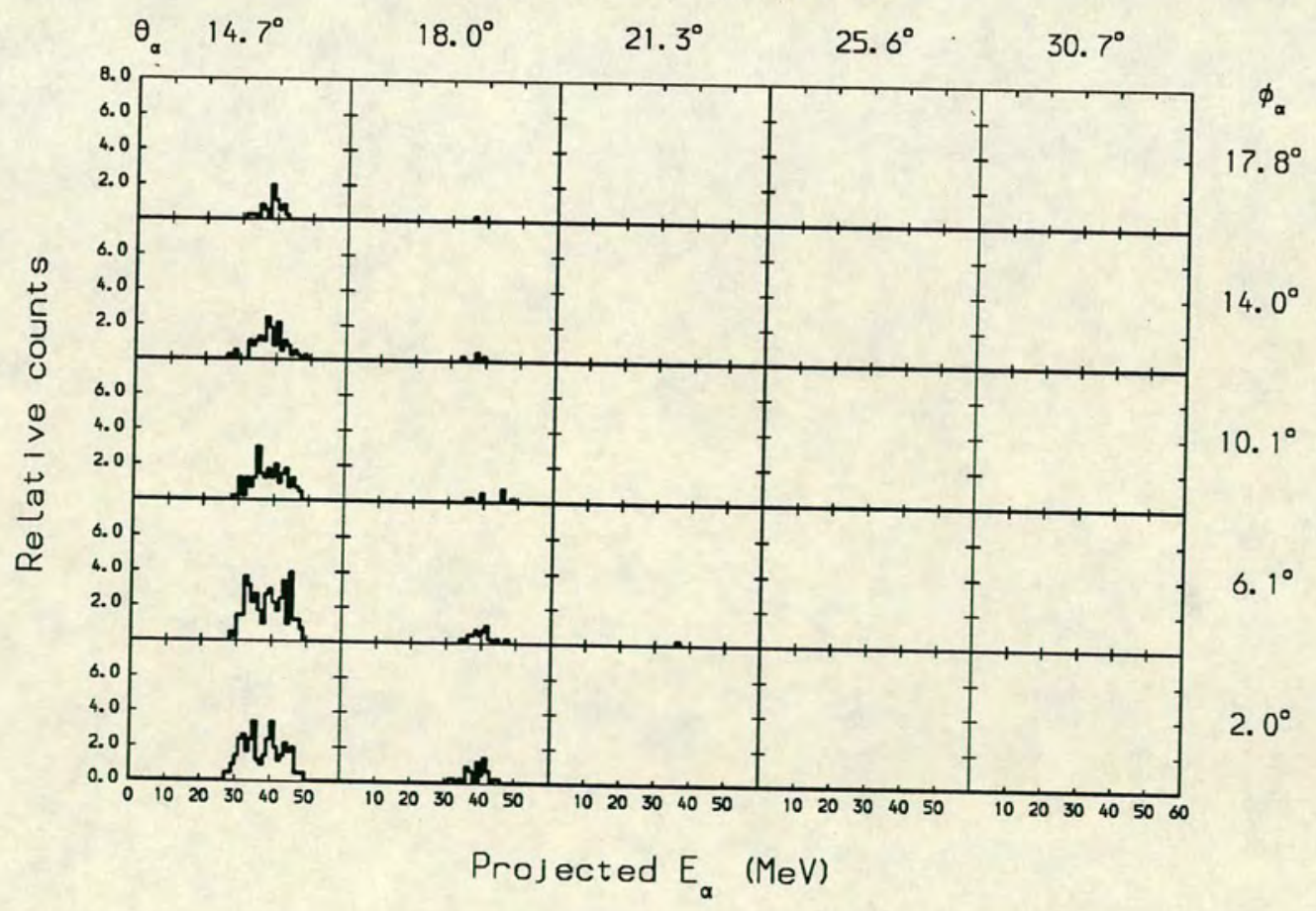

Figure 5.11: Comparison of data and Monte Carlo simulations for $\theta_{t}=-23.0^{\circ}$. State simulated was ${ }^{7} \mathrm{Li}_{9.96 \mathrm{MeV}}$ (thick line). 


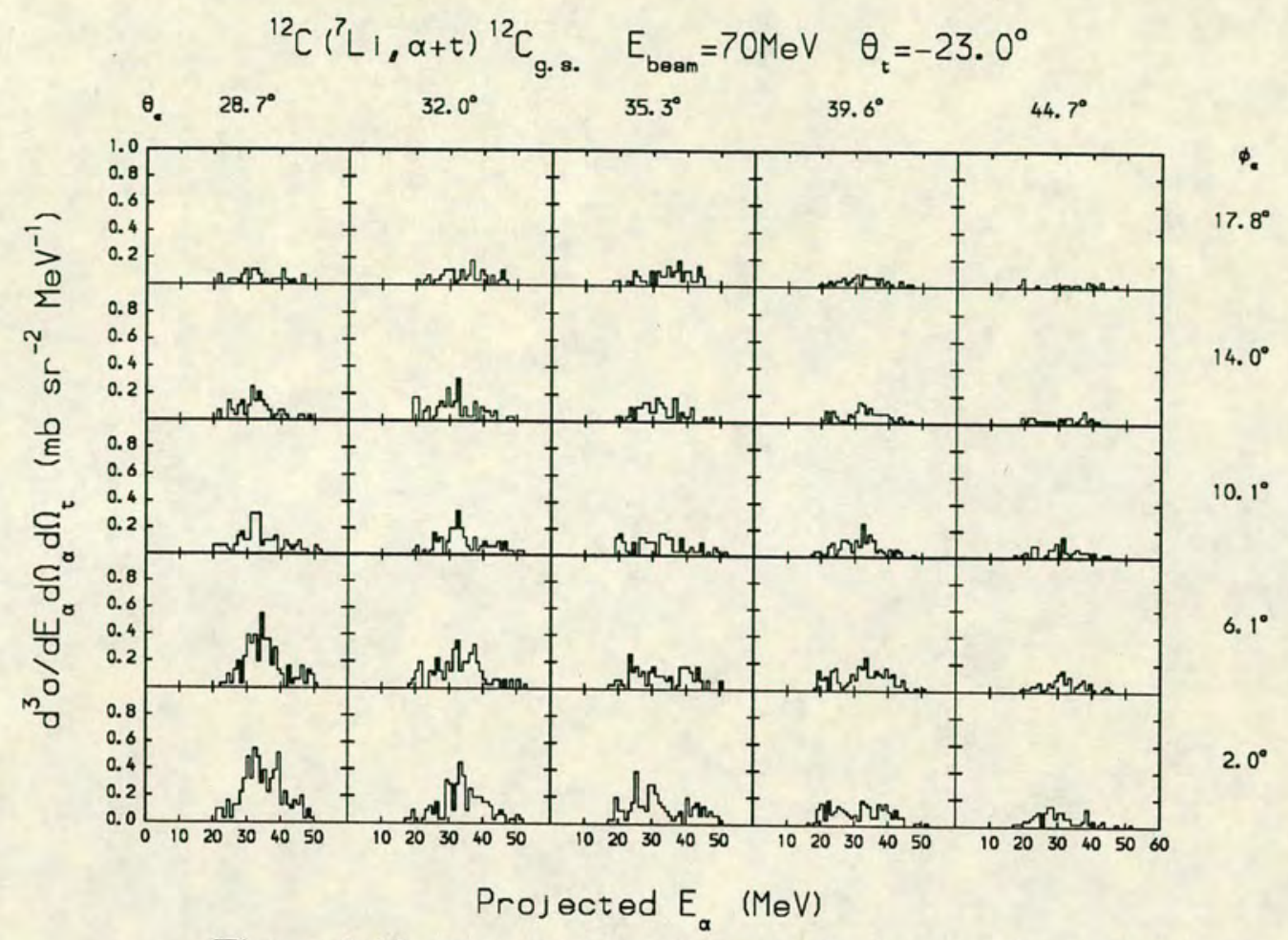

Figure 5.12: Projected quasi-elastic $\mathrm{E}_{\alpha}$ data for $\theta_{t}=-23.0^{\circ}$.

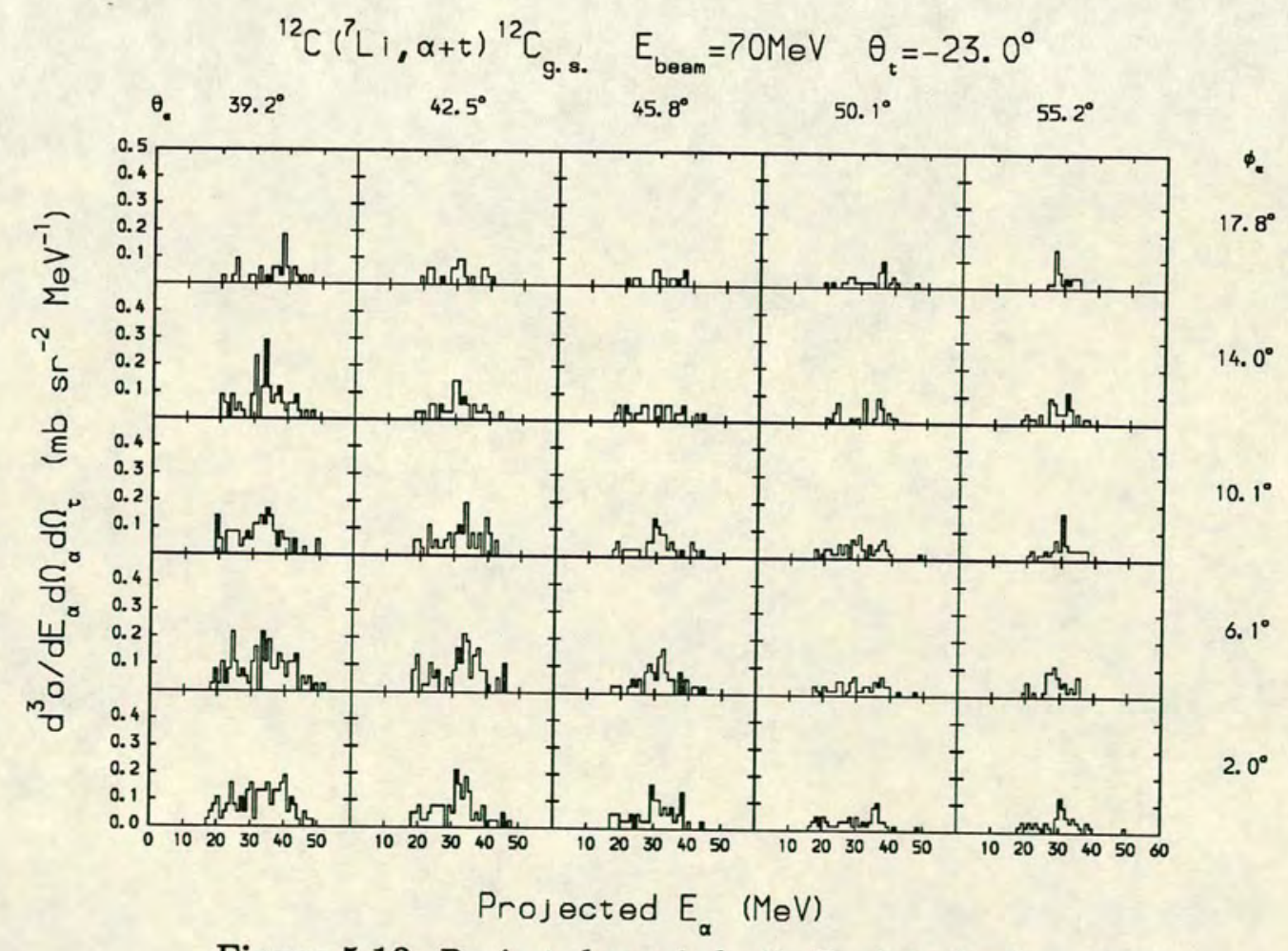

Figure 5.13: Projected quasi-elastic $\mathrm{E}_{\alpha}$ data for $\theta_{t}=-23.0^{\circ}$. 


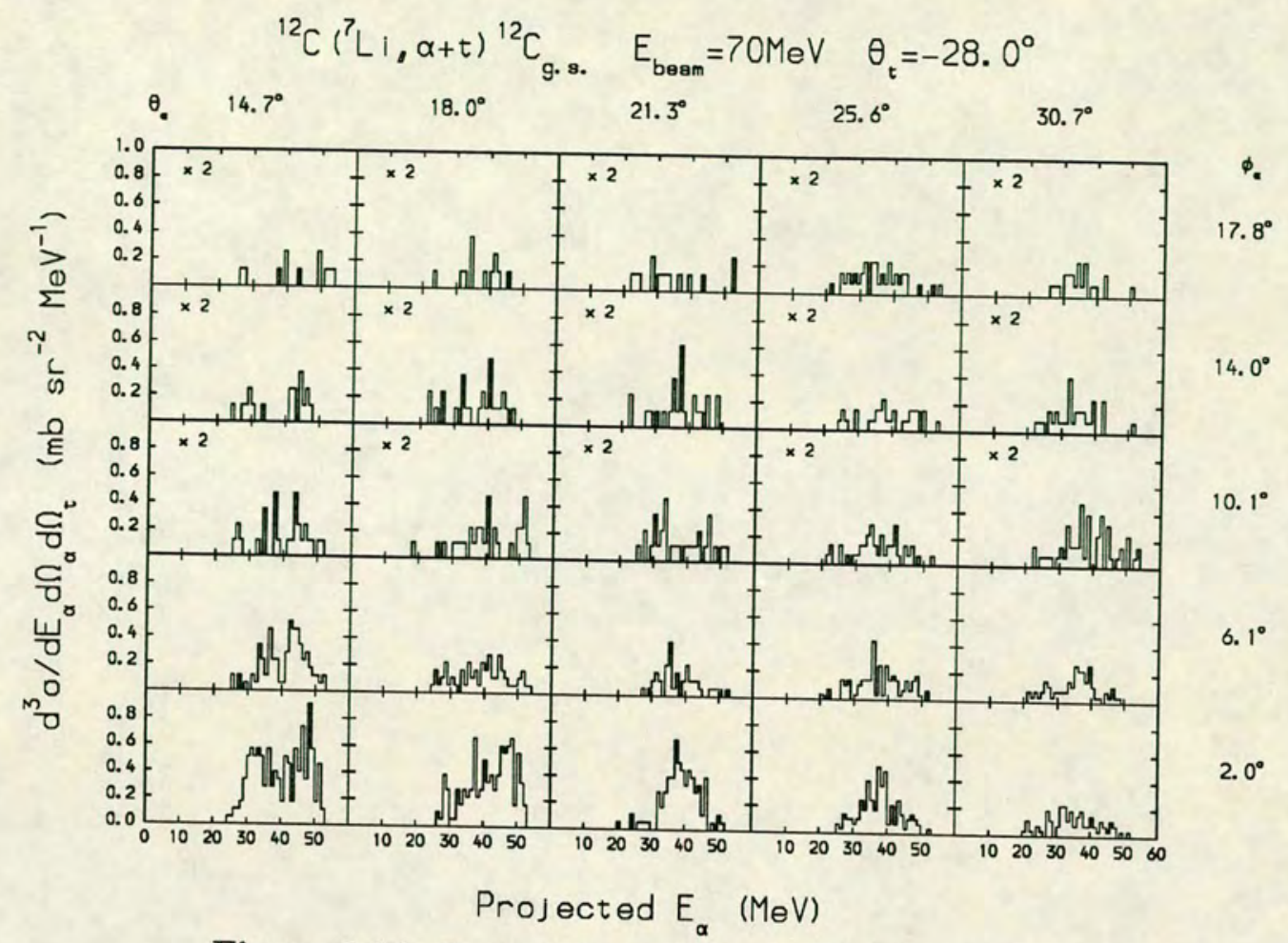

Figure 5.14: Projected quasi-elastic $\mathrm{E}_{\alpha}$ data for $\theta_{t}=-28.0^{\circ}$.

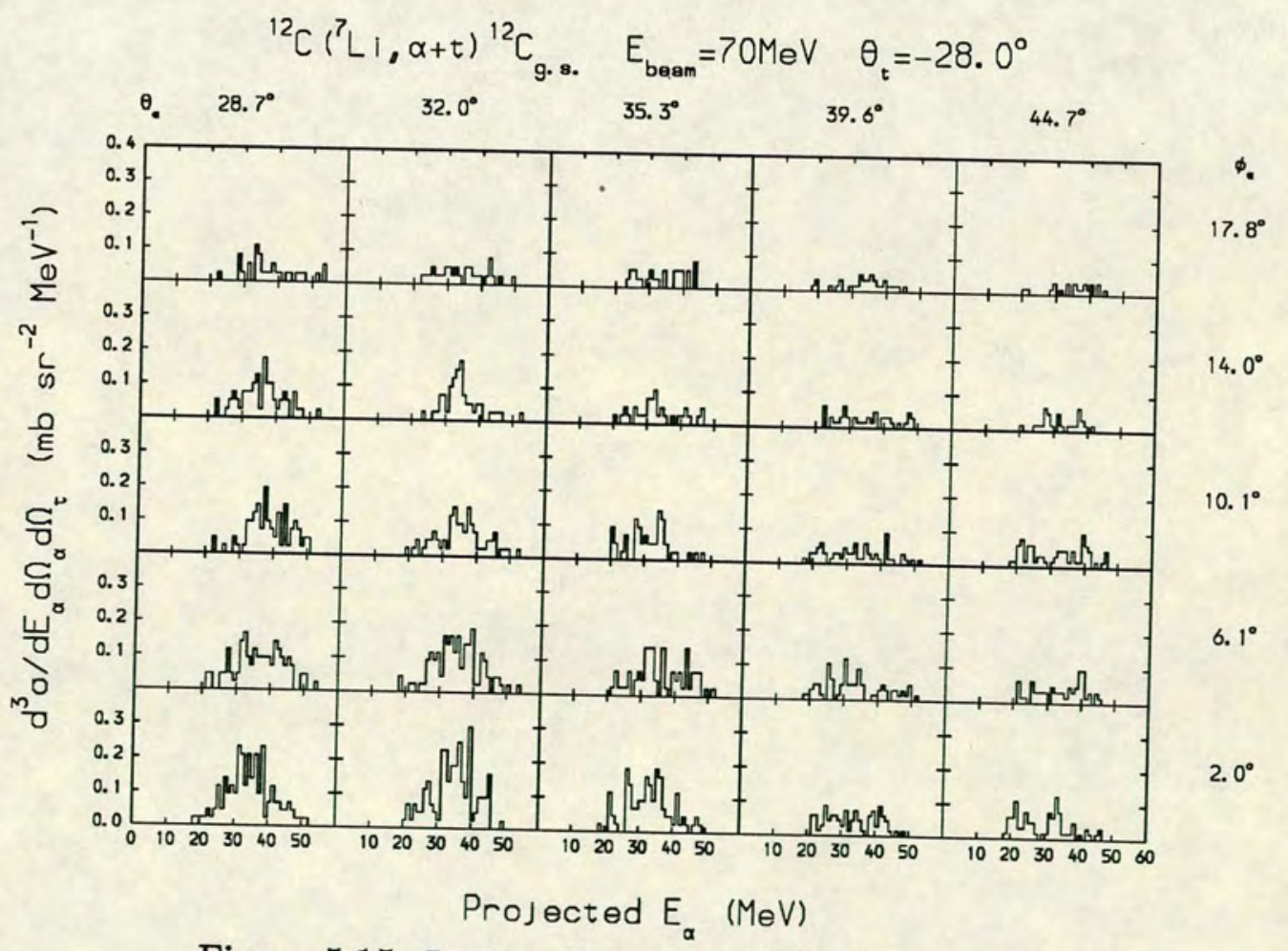

Figure 5.15: Projected quasi-elastic $\mathrm{E}_{\alpha}$ data for $\theta_{t}=-28.0^{\circ}$. 


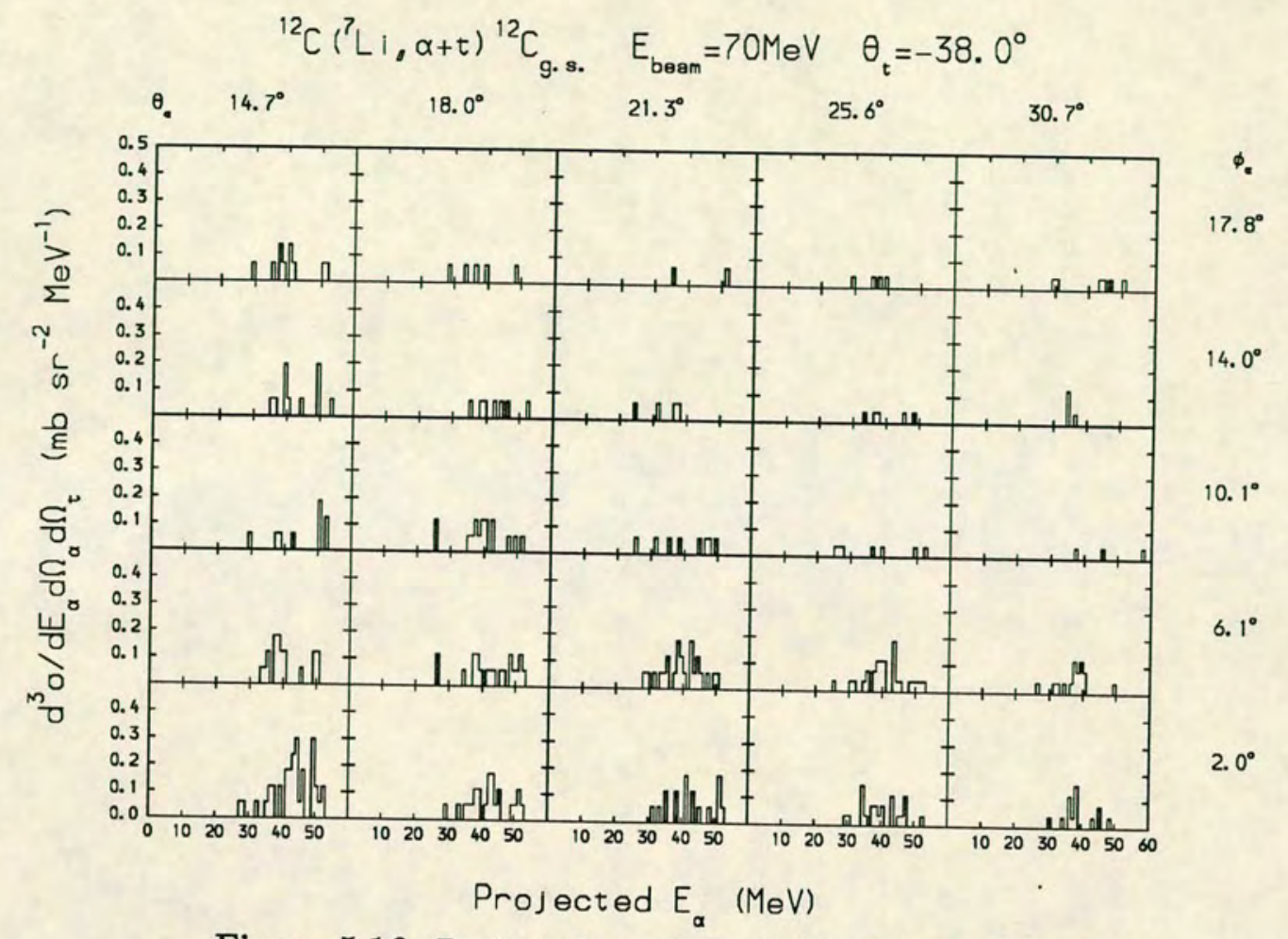

Figure 5.16: Projected quasi-elastic $\mathrm{E}_{\alpha}$ data for $\theta_{t}=-38.0^{\circ}$.

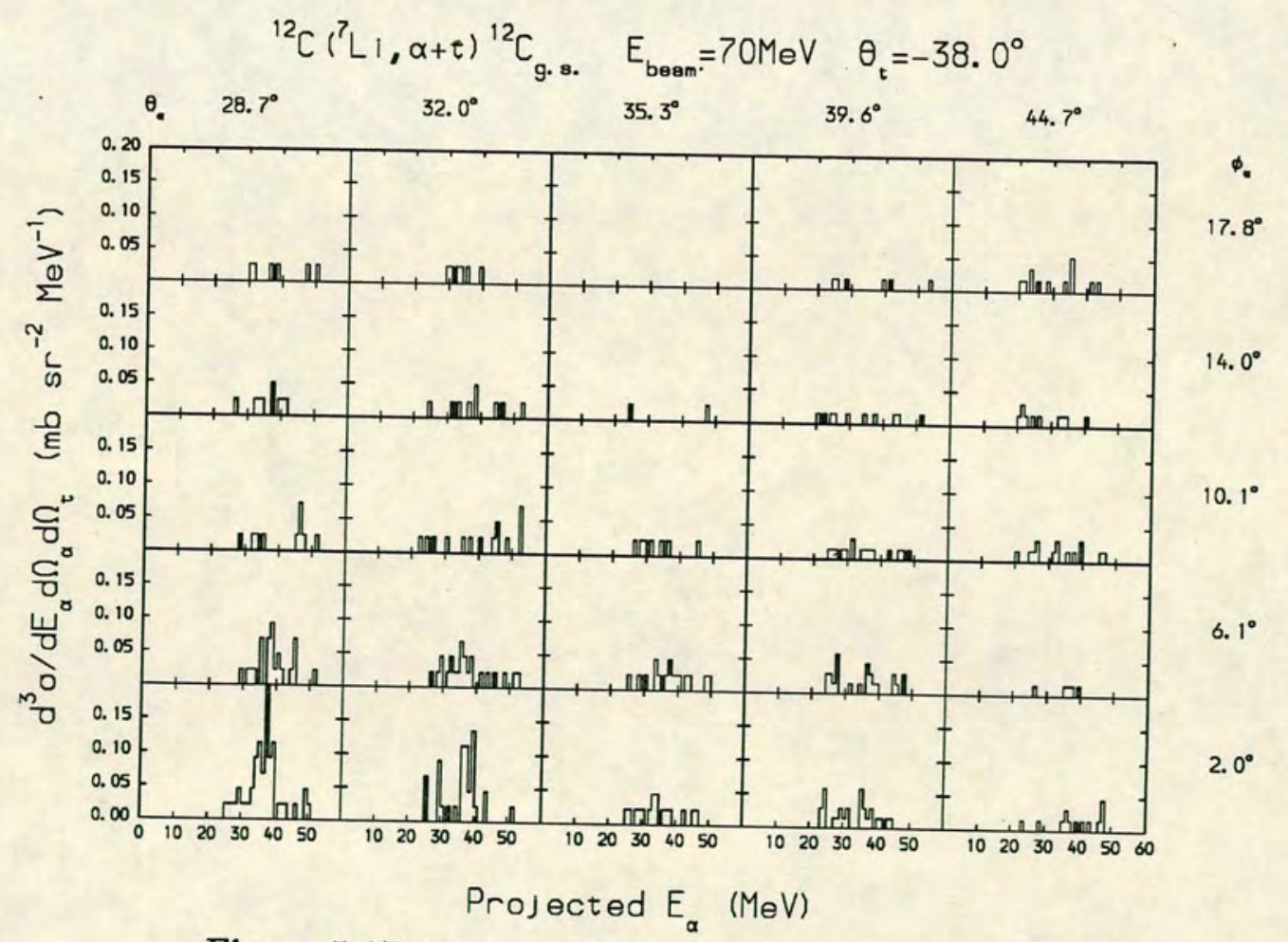

Figure 5.17: Projected quasi-elastic $\mathrm{E}_{\alpha}$ data for $\theta_{t}=-38.0^{\circ}$. 


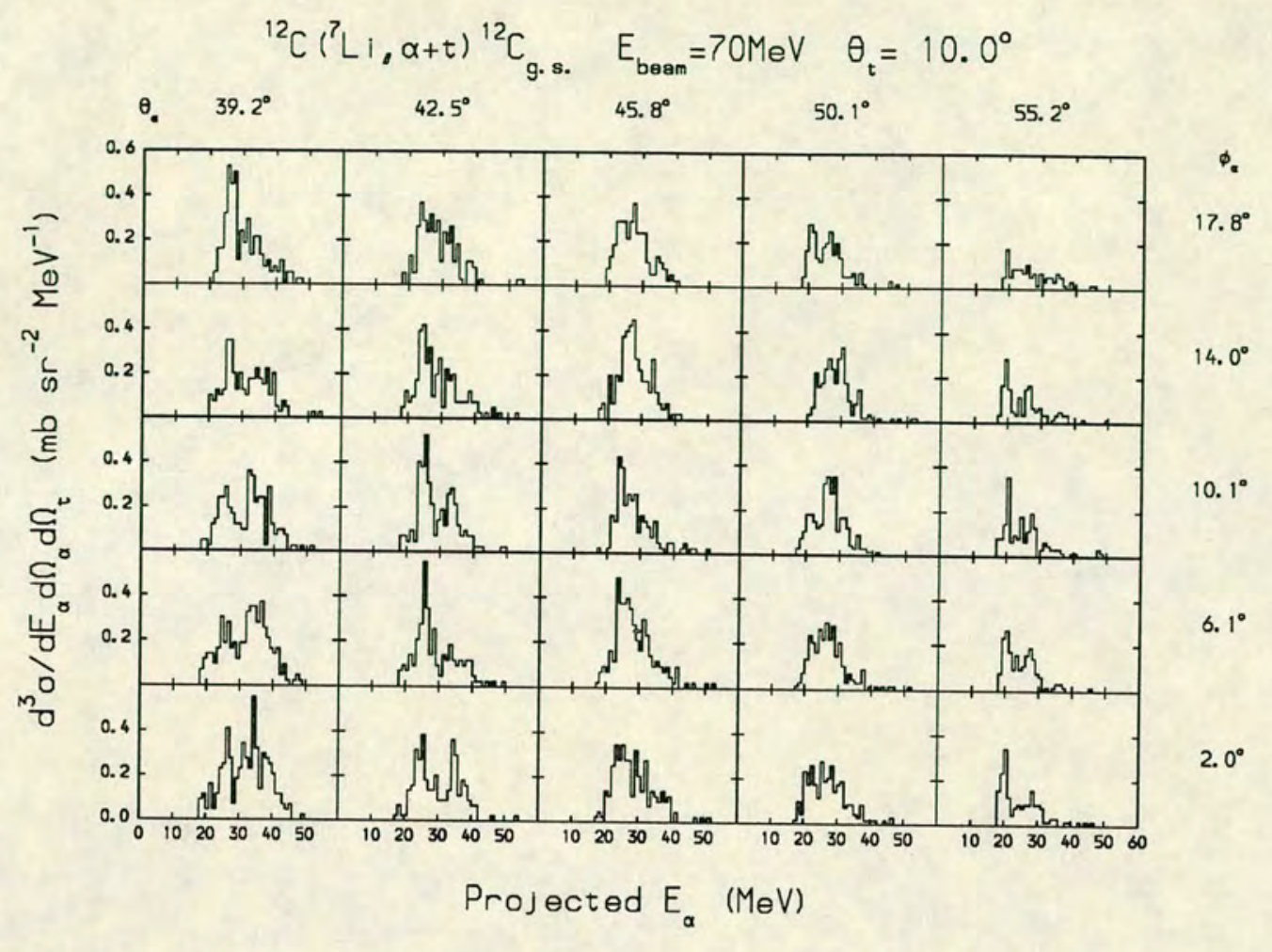

Monte-Car lo Simulation
$\begin{array}{ll}\theta_{\alpha} & 39.2^{\circ}\end{array}$
42. $5^{\circ}$
45. $8^{\circ}$
50. $1^{\circ}$
$55.2^{\circ}$

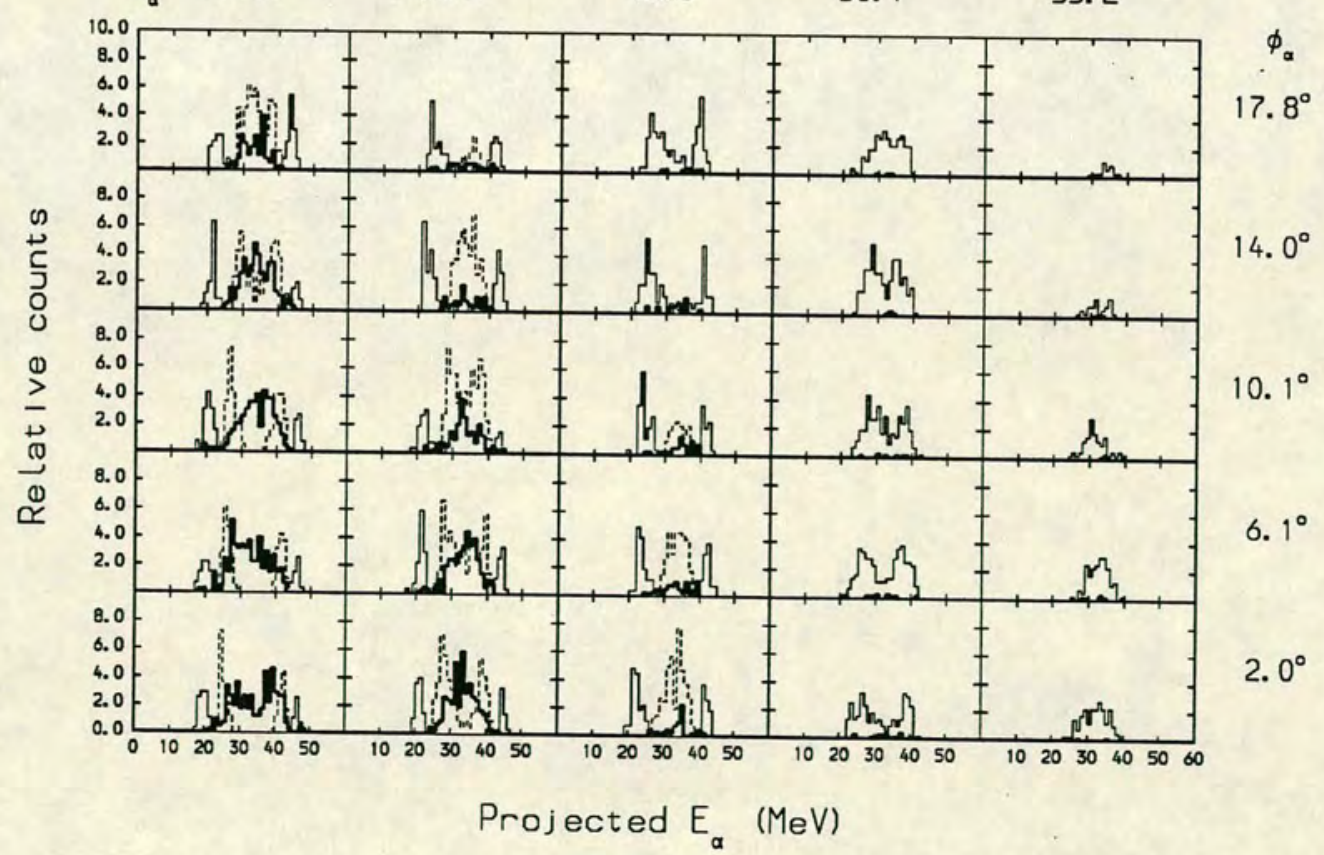

Figure 5.18: Comparison of data and Monte Carlo simulations for $\theta_{t}=+10.0^{\circ}$, and both detectors on same side of beam. States simulated were ${ }^{7} \mathrm{Li}_{6.68 \mathrm{MeV}}$ (thick line), ${ }^{7} \mathrm{Li}_{\text {7.47MeV }}$ (dotted line), ${ }^{7} \mathrm{Li}_{9.96 \mathrm{MeV}}$ (thin line). 


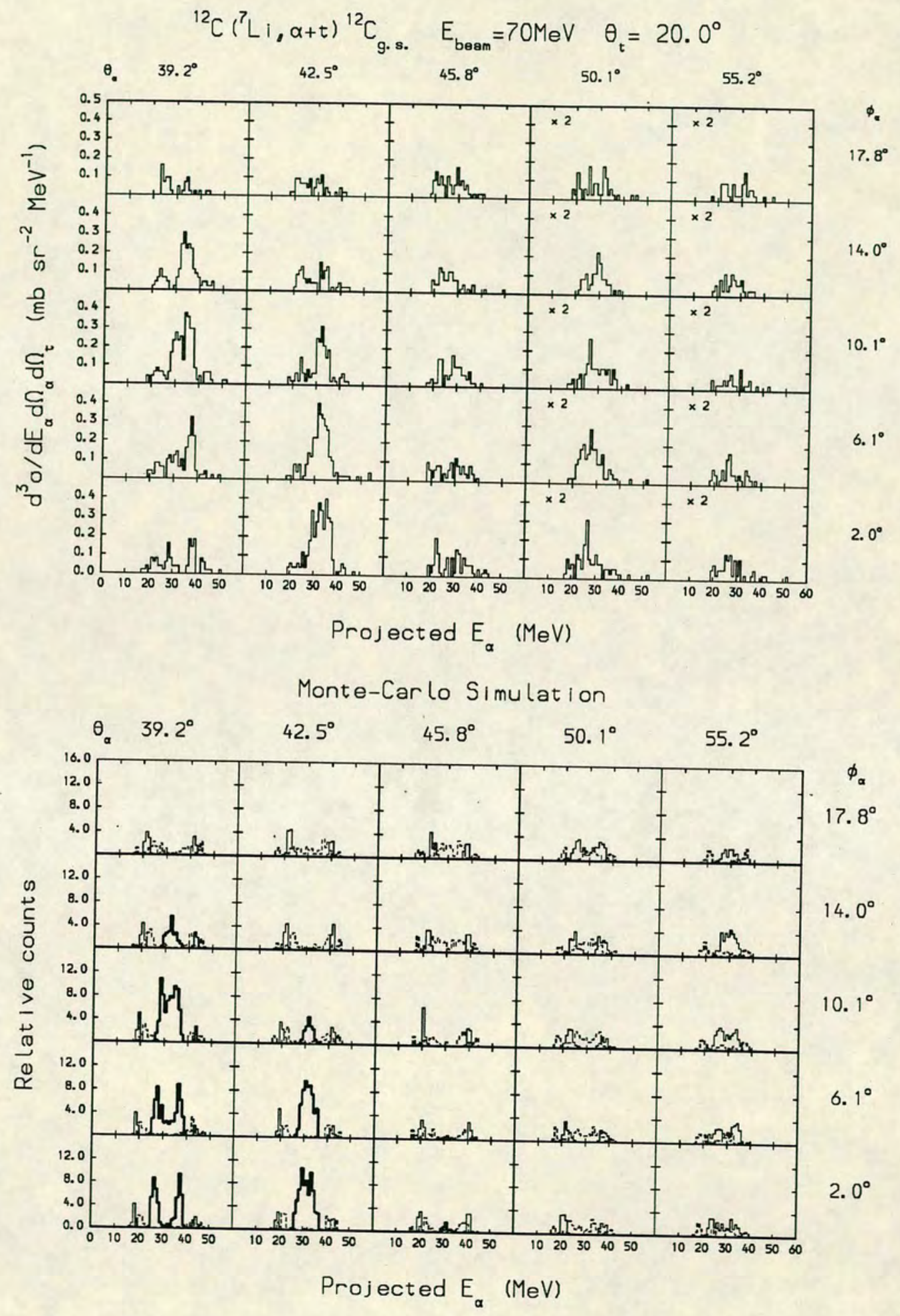

Figure 5.19: Comparison of data and Monte Carlo simulations for $\theta_{t}=+20.0^{\circ}$, and both detectors on same side of beam. States simulated were ${ }^{7} \mathrm{Li}_{4.63 \mathrm{MeV}}$ (thick line), ${ }^{7} \mathrm{Li}_{6.68 \mathrm{MeV}}$ (dotted line), ${ }^{7} \mathrm{Li}_{7.47 \mathrm{MeV}}$ (thin line), ${ }^{7} \mathrm{Li}_{9.96 \mathrm{MeV}}$ (thick dotted line). 


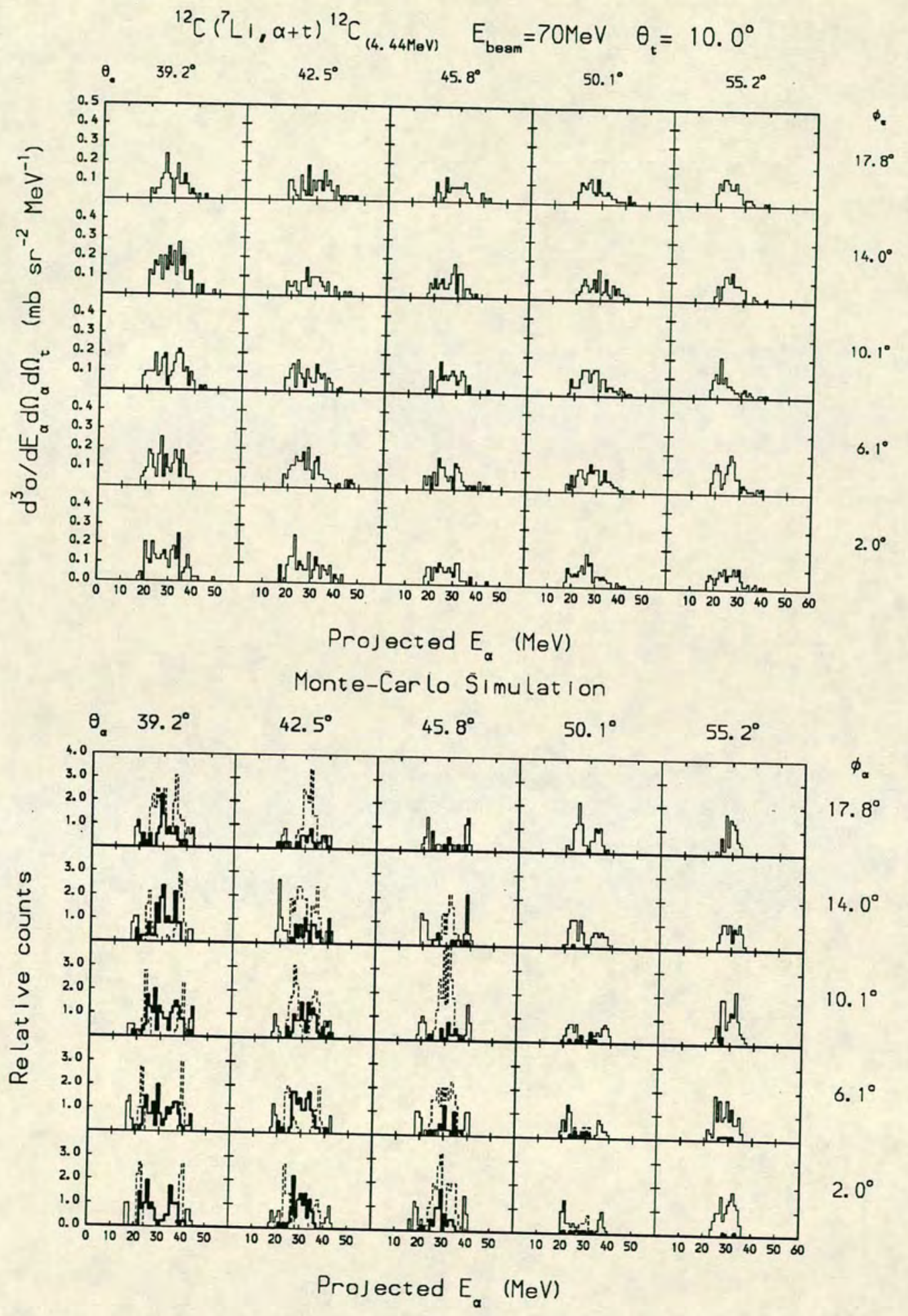

Figure 5.20: Comparison of data and Monte Carlo simulations for $\theta_{t}=+10.0^{\circ}$, and both detectors on same side of beam. Data and simulations are for events leaving ${ }^{12} \mathrm{C}$ in its first excited state. States simulated were ${ }^{7} \mathrm{Li}_{6.68 \mathrm{MeV}}$ (thick line), ${ }^{7} \mathrm{Li}_{7.47 \mathrm{MeV}}$ (dotted line), ${ }^{7} \mathrm{Li}_{9.96 \mathrm{MeV}}$ (thin line). 


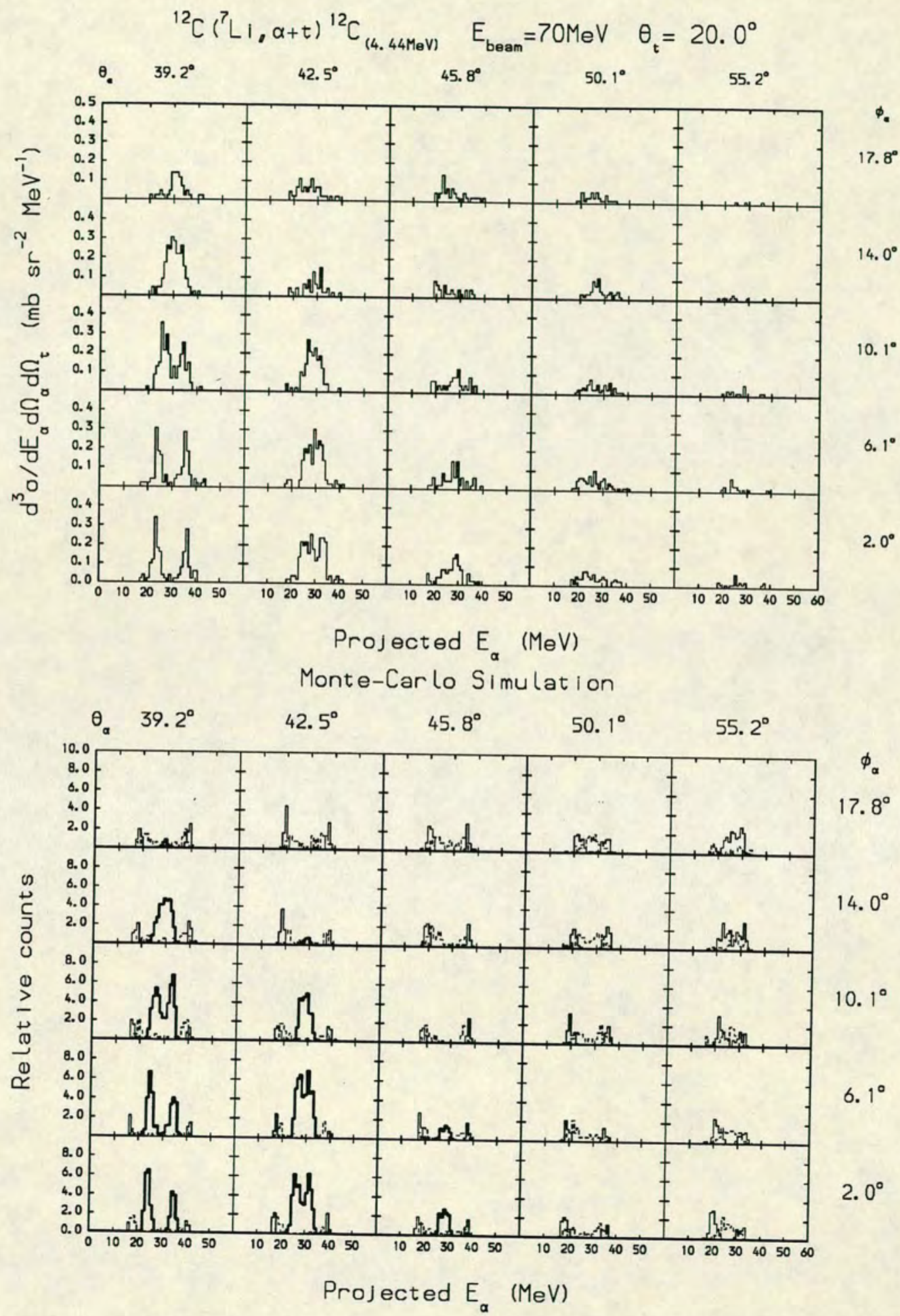

Figure 5.21: Comparison of data and Monte Carlo simulations for $\theta_{t}=+20.0^{\circ}$, and both detectors on same side of beam. Data and simulations are for events leaving ${ }^{12} \mathrm{C}$ in its first excited state. States simulated were ${ }^{7} \mathrm{Li}_{4.63 \mathrm{MeV}}$ (thick line), ${ }^{7} \mathrm{Li}_{6.68 \mathrm{MeV}}$ (dotted line), ${ }^{7} \mathrm{Li}_{7.47 \mathrm{MeV}}$ (thin line), ${ }^{7} \mathrm{Li}_{9.96 \mathrm{MeV}}$ (thick dotted line). 
This sequential decay from ${ }^{7} \mathrm{Li}$ is responsible for the enhancement in the angular distributions at forward angles seen in fig.4.20. Removing these events from the double differential cross-sections brings these forward angled points down into line with the trend of the rest of the data points at these angular settings. Sequential decay is also responsible for the peak in the quasi-elastic angular distribution with both the alpha and the triton on the same side of the beam (ie. fig.4.23).

It has been shown [Ra84] that the most important factor in resolving sequential states via the projected energy spectra is the angular resolution of the detector set-up. Even more than the energy resolution of the system, the angular resolution determines the ability of the detector set-up to discriminate between different sequential states. It can be seen from the Monte Carlo simulations that the angular resolution of the detector system used makes it difficult to unambiguously identify states other than the $4.63 \mathrm{MeV}$ state already mentioned. The simulations were useful however, in determining whether peaks seen in the projected spectra did not arise from sequential decay of the ${ }^{7} \mathrm{Li}$. They show how the sequential peaks should change in energy with varying $\theta_{t}$ and $\theta_{\alpha}$ and any peak not obeying this behaviour can confidently be attributed to processes not involving ${ }^{7} \mathrm{Li}$ sequential decay. The simulations also give the limiting angles where one would expect to detect sequential events and any events outside these limits can again be attributed to alternative processes.

From a comparison of the experimental and Monte Carlo projected spectra at the larger angular separation eg. fig.5.3 it is clear there is a substantial amount of cross-section outside the kinematic limits for sequential decay from the highest excited ${ }^{7} \mathrm{Li}$ states.

Within the kinematic limits of the highest sequential state the interpretation of the data is rather complicated. The width of the states and the angular resolution of the system combine to make identification of the individual components extremely difficult. In certain pixels where the simulations predict two separate energy solutions for break-up from the higher ${ }^{7} \mathrm{Li}$ states there does seem to be experimental evidence for the lower energy peak (eg. fig. $5.4 \theta_{t}=-13.0^{\circ}, \theta_{\alpha}=14.7^{\circ}$ peak at $30 \mathrm{MeV}$ is at position of lower energy solution for sequential decay from 


\begin{tabular}{|c|c|}
\hline${ }^{7} \mathrm{Li}$ state $(\mathrm{MeV})$ & Max. angle \\
\hline 6.88 & $\sim 22^{\circ}$ \\
7.46 & $\sim 27^{\circ}$ \\
9.67 & $\sim 34^{\circ}$ \\
\hline
\end{tabular}

Table 5.3: Kinematical limits for $\theta_{\alpha}$ with $\theta_{t}=-8.0^{\circ}$ to detect events from given sequential state.

${ }^{7} \mathrm{Li}_{\text {7.47MeV }}$ state). In most of these spectra there is no real evidence for the accompanying upper energy peak which should be visible. This seems to indicate that another process is responsible for these peaks in the experimental spectra. As one moves from one pixel to the next (in the region within the kinematic limits) there does seem to be a concentration of events in the allowed energy range which broadly agree with the behaviour of the simulations. It is tempting to associate these events with sequential decay but it will require another measurement with better angular resolution or a detailed study of the angular distribution of these higher lying states, (allowing accurate intensity contributions to be calculated) before a more accurate assessment of the decomposition of these events can be made. One would expect however, that if sequential decay from any of the higher lying states (ie. not the $4.63 \mathrm{MeV}$ state) was a significant contributor to the quasi-elastic cross-section then there would be a distinct change in the shape of the quasi-elastic angular distribution at the limit of the corresponding break-up cone. This is seen for the $4.63 \mathrm{MeV}$ state in fig.4.20 $\theta_{\alpha} \sim+18.0^{\circ}$ where the slope of the angular distribution changes sharply. The corresponding values of $\theta_{\alpha}$ for $\theta_{t}=-8.0^{\circ}$ (taken from fig.5.2 to fig.5.3) are given in table 5.3: It can be seen that there is no significant change in the slope of the quasi-elastic angular distribution at these values of $\theta_{\alpha}$. The average slope seems to be fairly constant out to much larger values of $\theta_{\alpha}$ than the kinematical limit for even the most energetic levels of ${ }^{7} \mathrm{Li}$. This is true for all the values of $\theta_{t}$ on the opposite side of the beam from the alpha particle. Although this behaviour does not necessarily imply the absence of sequential decay from these higher excited states it does indicate that no single state makes a significantly dominant contribution in any angular range (except of course for the $4.63 \mathrm{MeV}$ state which dominates the projected spectra in the relevant pixels). 
The projected spectrum of $\mathrm{E}_{\alpha}$ for the events leaving the ${ }^{12} \mathrm{C}$ in its $4.44 \mathrm{Mev}$ excited state at $\theta_{t}=+20^{\circ}, \theta_{\alpha}=+39.2^{\circ}$ (ie. fig.5.21) clearly show the double peaked structure for break-up from the $4.63 \mathrm{Mev}$ state in ${ }^{7} \mathrm{Li}$. Most of the cross-section is contained in these peaks with only a small amount outside the kinematically allowed energies. This remaining cross-section is possibly due to the sequential break-up of any combination of the other three states, the angular resolution making it impossible to extract the separate components.

\section{Partial and Complete Fusion}

There are a number of peaks in the projected quasi-elastic alpha energy spectra from the ${ }^{12} \mathrm{C}$ target which are not associated with sequential decay (eg. peak at $\sim 29 \mathrm{MeV}$ in element F3M1 fig.5.5 $\theta_{t}=-13.0^{\circ}, \theta_{\alpha}=35.3^{\circ}$ ). It is possible for such a peak to originate from absorption re-emission processes. This mechanism has been identified in another light ion fragmentation experiment performed by Siwek-Wilczynska et al [Si87]. In the bombardment of ${ }^{12} \mathrm{C}$ with $157 \mathrm{MeV}{ }^{20} \mathrm{Ne}$ unambiguous evidence for population of a $20.8 \mathrm{MeV}$ state, $2.5 \mathrm{MeV}$ wide in ${ }^{16} \mathrm{O}$ has been obtained where the outgoing fragments $\left({ }^{12} \mathrm{C}\right.$ and $\left.\alpha\right)$ were detected on opposite sides of the beam. The events analysed corresponded to all three outgoing fragments in their ground state. The authors performed a HauserFeshbach calculation to verify that the partial width for $\alpha$ decay of these high lying states to the ground state of ${ }^{12} \mathrm{C}$ was reasonably large. These calculations showed that even for excitations up to $\sim 25 \mathrm{MeV}$ in the excited ${ }^{16} \mathrm{O}$ nucleus the width of the states were almost solely due to alpha emission to the ground state of ${ }^{12} \mathrm{C}$.

Peaks in the projected alpha energy spectra of the present data may result from a similar transfer re-emission process with either an alpha or triton fusing with the ${ }^{12} \mathrm{C}$ to form ${ }^{16} \mathrm{O}$ or ${ }^{15} \mathrm{~N}$ respectively. This process will be characterised by the behaviour of the energy peaks with angle of the triton and alpha, depending on which is transferred and which is the spectator.

One clear candidate for such a reaction is the distinct peak at $\mathrm{E}_{\alpha}=20 \mathrm{MeV}$ with $\theta_{t}=+10^{\circ}$ and $\theta_{\alpha}=55.2^{\circ}$, (ie. fig.5.18 pixel F5M1 with both alpha and triton on 
the same side of the beam). This peak is consistent with a triton transfer reaction to a state in ${ }^{15} \mathrm{~N}$ with excitation energy of $39.4 \mathrm{MeV}$. When the two body kinematics are calculated for such a process with the alpha at angles corresponding to pixels F1M1 to F4M1, the predicted alpha energies agree well with peaks seen in these spectra (ie. F1M1 $\mathrm{E}_{\alpha} \sim 26.8 \mathrm{MeV}, \mathrm{F} 2 \mathrm{M} 1 \mathrm{E}_{\alpha} \sim 25.4 \mathrm{MeV}$, F3M1 $\mathrm{E}_{\alpha} \sim 23.8 \mathrm{MeV}, \mathrm{F} 4 \mathrm{M} 1 \mathrm{E}_{\alpha} \sim 22.2 \mathrm{MeV}$ ). At first sight it would seem that this process would give an isotropic angular distribution more characteristic of compound reaction mechanisms with the peak intensity independant of the triton detector angle. It is seen however, that there are very few other pixels which exhibit a peak in the correct energy region. The lack of strength for this peak may be due to the width of the state in ${ }^{15} \mathrm{~N}$. Unfortunately levels of this excitation have not been studied to date, but a simple calculation assuming a width of $4 \mathrm{MeV}$ (this being the width of the alpha peak) gives a lifetime of $1.6 \times 10^{-22}$ secs. In this time a beam velocity triton will travel $\sim 7$ fermis round the surface of a ${ }^{12} \mathrm{C}$ nucleus. This means that a resonance in the ${ }^{12} \mathrm{C}+\mathrm{t}$ wave function of width $\sim 4 \mathrm{MeV}$ will not produce an isotropic distribution but will re-emit the triton more in the forward direction.

If the ${ }^{15} \mathrm{~N}$ system is to re-emit a triton leaving the ${ }^{12} \mathrm{C}$ in its ground state the wave-function of the excited level must have a strong $t+{ }^{12} \mathrm{C}_{g . s}$. cluster configuration. It is also possible in such a cluster system for the ${ }^{12} \mathrm{C}$ to be in its first excited state. Many of the known ${ }^{12} \mathrm{C}+\mathrm{t}$ resonances show such a mixture of wavefunctions [Aj86b] If one inspects the projected energy spectra for $\alpha+t$ coincidences leaving the ${ }^{12} \mathrm{C}$ in its $4.44 \mathrm{MeV}$ state, at the same angles where the quasi-elastic events show the previously mentioned peak (ie. fig.5.20 $\theta_{t}=+10^{\circ}$ ) then one does indeed find evidence for a peak at $\mathrm{E}_{\alpha} \sim 20 \mathrm{MeV}$. This is further evidence for the process contributing to this peak being one where the alpha particle is the spectator. It is also known that the angular distributions of transfer reactions at such high energies exhibit diffraction structure and with such a light target and high beam energy it is possible that this accounts for the observed variation of the peak intensity with alpha angle. With the detectors on opposite sides of the beam a ${ }^{15} \mathrm{~N}$ excitation of $\sim 27.5 \mathrm{MeV}$ explains the $35 \mathrm{MeV}$ peak with $\theta_{t}=-8.0^{\circ}$ and $\theta_{\alpha}=50.1^{\circ}, \phi_{\alpha}=6.1^{\circ}$ (fig.5.6), the $37 \mathrm{MeV}$ peak with $\theta_{t}=-13.0^{\circ}$ and $\theta_{\alpha}=42.5^{\circ}, \phi_{\alpha}=10.1^{\circ}$ (fig.5.7) and the other less distinct peaks at similar energies in the neighbouring pixels. This is close to the $26.8 \mathrm{MeV}$ resonance in 
${ }^{12} \mathrm{C}+\mathrm{t}$ which decays by triton emission to the ground state of ${ }^{12} \mathrm{C}$ [Aj86b].

Clear identification of alpha transfer states in ${ }^{16} \mathrm{O}$ is more difficult. Artemov et.al. [Ar82] have identified $\alpha+{ }^{12} \mathrm{C}$ resonance states in ${ }^{16} \mathrm{O}^{*}$ at $\sim 29 \mathrm{MeV}$ and $\sim 34 \mathrm{MeV}$ excitation in a ${ }^{12} \mathrm{C}\left({ }^{6} \mathrm{Li}, \mathrm{d}+\alpha\right)$ reaction at $60 \mathrm{MeV}$. Similar excitation states have been observed independantly by Becchetti et.al. [Be88], [Ja88] in a ${ }^{12} \mathrm{C}\left({ }^{7} \mathrm{Li}, \alpha+\mathrm{t}\right)$ experiment at $101 \mathrm{MeV}$ and by Rae et.al. [Ra85] in a ${ }^{12} \mathrm{C}\left({ }^{12} \mathrm{C},{ }^{16} \mathrm{O}^{*}\right){ }^{8} \mathrm{Be}$ reaction at $120 \mathrm{MeV}$. These states are all rather broad $(\Gamma \sim 6 \mathrm{MeV})$ and their identification consequently quite difficult. There is however, some evidence for excitation of states in this region in a number of the detector pixels eg. fig.5.3 $\theta_{t}=-8.0^{\circ}, \theta_{\alpha}=35.3^{\circ}$, peak at $\mathrm{E}_{\alpha} \sim 27 \mathrm{MeV}$ corresponds to $\mathrm{E}_{16} \mathrm{O}^{*} \sim 28 \mathrm{MeV}$, fig.5.4 $\theta_{t}=-13.0^{\circ}, \theta_{\alpha}=14.7^{\circ}$, peak at $\mathrm{E}_{\alpha} \sim 30 \mathrm{MeV}$ corresponds to $\mathrm{E}_{18} \mathrm{O}^{*} \sim 28 \mathrm{MeV}$, and fig.5.11 $\theta_{t}=-23.0^{\circ}, \theta_{\alpha}=18.0^{\circ}$, peak at $\mathrm{E}_{\alpha} \sim 40 \mathrm{MeV}$ corresponds to $\mathrm{E}_{16} \mathrm{O}^{*} \sim 34 \mathrm{MeV}$. Most of the peaks seen in the projected spectra from the ${ }^{12} \mathrm{C}$ target in the $20 \sim 30 \mathrm{MeV}$ range can be explained by an excitation of $\sim 28 \mathrm{MeV}$ in ${ }^{16} \mathrm{O}$.

It has been found from particle- $\gamma$ coincidence experiments on heavier targets [Da87], [Ut83] that triton fusion is more probable than alpha particle fusion. This has been attributed both to the $Q$ value dependance of the compound nucleus excitation energy and level density and an orientation effect of the incoming ${ }^{7} \mathrm{Li}$ by the long range Coulomb field. The alpha, having a larger charge to mass ratio than the triton, is orientated preferentially on the outside of the ${ }^{7} \mathrm{Li}$ as it approaches the target. This results in an enhanced probability of triton fusion. With a ${ }^{12} \mathrm{C}+{ }^{7} \mathrm{Li}$ system, where the Coulomb field effects are significantly less than with heavier targets, the applicability of such an argument is rather questionable.

The partial fusion model of limiting angular momentum [Wi73] gives a crosssection for triton absorption approximately equal to that for alpha absorption $\left(\ell_{\text {crit. }}(\alpha)=4 \hbar, \ell_{\text {crit. }}(\mathrm{t})=3 \hbar\right)$ if one assumes that both may occur with equal probability within their limiting angular momentum values $l_{c r}$. Once more, the quantal nature of this system probably reduces the applicability of such semi-classical concepts. 
Combining the concept of the critical angular momentum for complete fusion of the ${ }^{7} \mathrm{Li}+{ }^{12} \mathrm{C}$ and the strong absorption model which gives the total cross-section for such a process as:

$$
\begin{array}{rlrl}
\sigma_{\text {fusion }} \sim \pi \hbar^{2} & \sum_{\ell=0}^{\ell_{\text {crit. }}}(2 \ell+1) T_{\ell} & & T_{\ell}=1 \ell \leq \ell_{\text {crit. }} \\
=\pi \hbar^{2} \ell_{\text {crit. }}^{2} & & T_{\ell}=0 \ell>\ell_{\text {crit. }}
\end{array}
$$

allows an estimate for the strength of the complete fusion channel. If one then assumes that every ${ }^{19} \mathrm{~F}$ nucleus formed decays via triton and alpha emission to the ground state of ${ }^{12} \mathrm{C}$ it is possible to calculate an upper limit for the contribution of this complete fusion channel to the quasi-elastic events.

This simple calculation results in an upper limit of $\sim 0.5 \mathrm{mbsr}^{-2}$ for the double differential cross-section which is an order of magnitude too small to be seen in the projected spectra. This limit could probably be reduced by another factor of 10 or 100 due to the assumption of the decay channels populated. It seems unlikely therefore that the events not explained by sequential break-up can be accounted for by this process.

\section{Kinematical Model}

In an attempt to explain the wide angle cross-section another rather simple model was considered. This followed the idea of an optimum $Q$ value for transfer [Br72], [Wi73b], [Oe85] and incorporated in an elementary way the idea of a critical angular momentum for fusion. The ${ }^{7} \mathrm{Li}$ was viewed as approaching the target up to a point of closest approach where it would be possible for the participant to fuse with the target. The velocity of the ${ }^{7} \mathrm{Li}$ at this point was then found, after correction for the energy contained in the Coulomb field and the $Q$ value of the proposed reaction. The main assumption of the model was that the centre of mass velocity of the spectator fragment was conserved and made equal to the velocity of the ${ }^{7} \mathrm{Li}$ at this point. The lab velocity of the spectator fragment corrected for Coulomb effects in the outgoing channel could then be calculated for any lab detection angle. The direction and velocity of the recoiling compound nucleus were found from momentum conservation in the centre of mass and the energy necessary for conservation was attributed to excitation of the compound 
nucleus. Unlike the models of Brink and others the angular momentum was not explicitly calculated and it was assumed to be conserved by an arbitary choice of the angular momentum state of the recoiling excited nucleus. If the particle whose projected energy was being investigated was assumed to be the spectator this was the end of the calculation and gave a predicted optimum value for the projected energy. If the particle was assumed to be the transferred fragment (ie. the participant) the kinematics for the re-emission stage were again worked out assuming a two body interaction, the inital conditions of which were specified by the first interaction. It was possible using this approach to model two types of interaction which are distinctly different in visualisation. If one uses the $Q$ value of simple fragmentation of the ${ }^{7} \mathrm{Li}$ into the alpha and the triton (ie. $-2.47 \mathrm{MeV}$ ) then this represents a direct fragmentation-absorption type of process where the spectator knows little about the interaction of the participant with the target. The two events, the fragmentation and the absorption are viewed as being separate occurences. If, on the other hand one uses the complete intermediate channel ground state $\mathrm{Q}$ value $\left(\right.$ eg. $\left.{ }^{12} \mathrm{C}\left({ }^{7} \mathrm{Li}, \mathrm{t}\right){ }^{16} \mathrm{O}, \mathrm{Q}_{\text {g.s. }}=+4.96 \mathrm{MeV}\right)$ to calculate the ${ }^{7} \mathrm{Li}$ centre-of-mass velocity, the reaction being modelled is more a one step transfer process where the spectator is more intimately involved in the reaction.

For the projected quasi-elastic alpha energy this results in four situations; the alpha is either a spectator or a participant and the process is either a transfer or a direct fragmentation reaction.

Figs.5.22 to 5.27 show the results of these calculations for varying in-plane alpha and triton angles along with the experimentally observed average alpha energy $\left\langle\mathrm{E}_{\alpha}\right\rangle$ calculated from the projected spectra. The calculations tend to be rather insensitive to changes in the transfer radius affecting the inital and final channel Coulomb energies. It can be seen from these figures that the data is clearly inconsistent with any major contribution from a process where the mechanism is a transfer reaction with the alpha as the outgoing spectator. It is not so easy to discriminate against the other three processes. It is quite possible that each of these may contribute varying amounts at different detection angles. 


$$
{ }^{12} \mathrm{C}\left({ }^{7} L i, \alpha+t\right){ }^{12} \mathrm{C}_{\text {g.s. }} .
$$

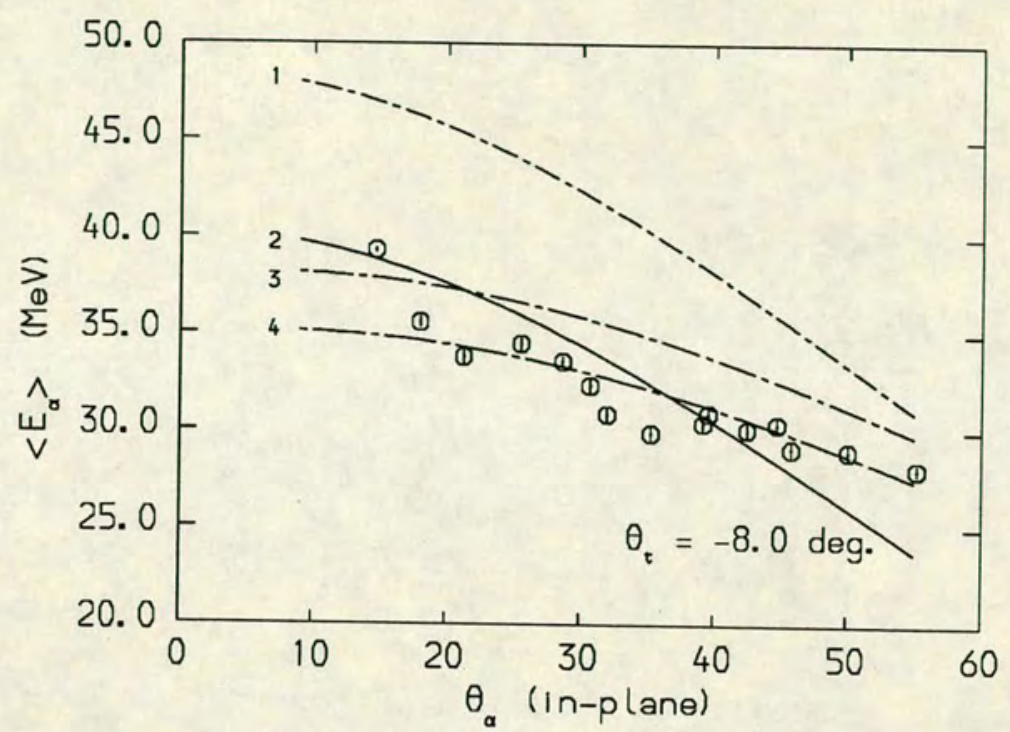

Figure 5.22: Experimental average energies of projected $E_{\alpha}$ spectra with $\theta_{t}=-8.0^{\circ}$. Curves are results of kinematical model explained in text for 1 . transfer spectator, 2. direct spectator, 3. direct participant, 4. transfer participant model.

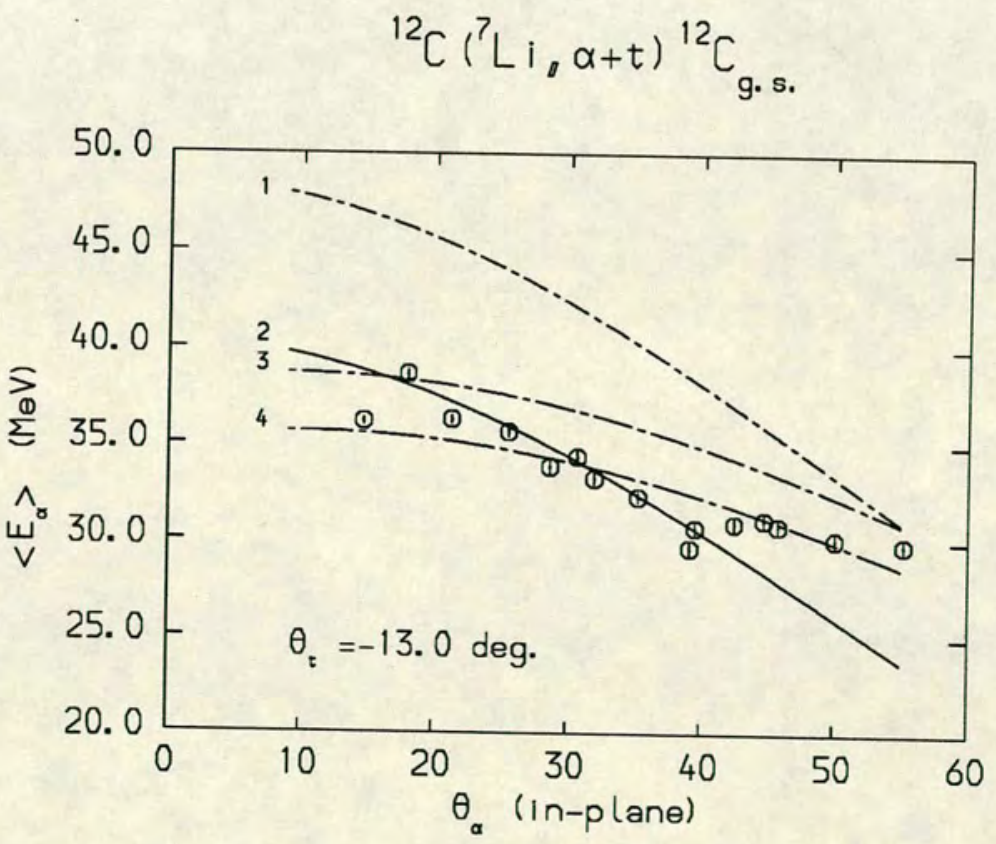

Figure 5.23: Experimental average energies of projected $\mathrm{E}_{\alpha}$ spectra with $\theta_{t}=-13.0^{\circ}$. Curves are results of kinematical model explained in text and given in fig.5.22. 


$$
{ }^{12} \mathrm{C}\left({ }^{7} L i, \alpha+t\right){ }^{12} \mathrm{C}_{\text {g.s. }}
$$

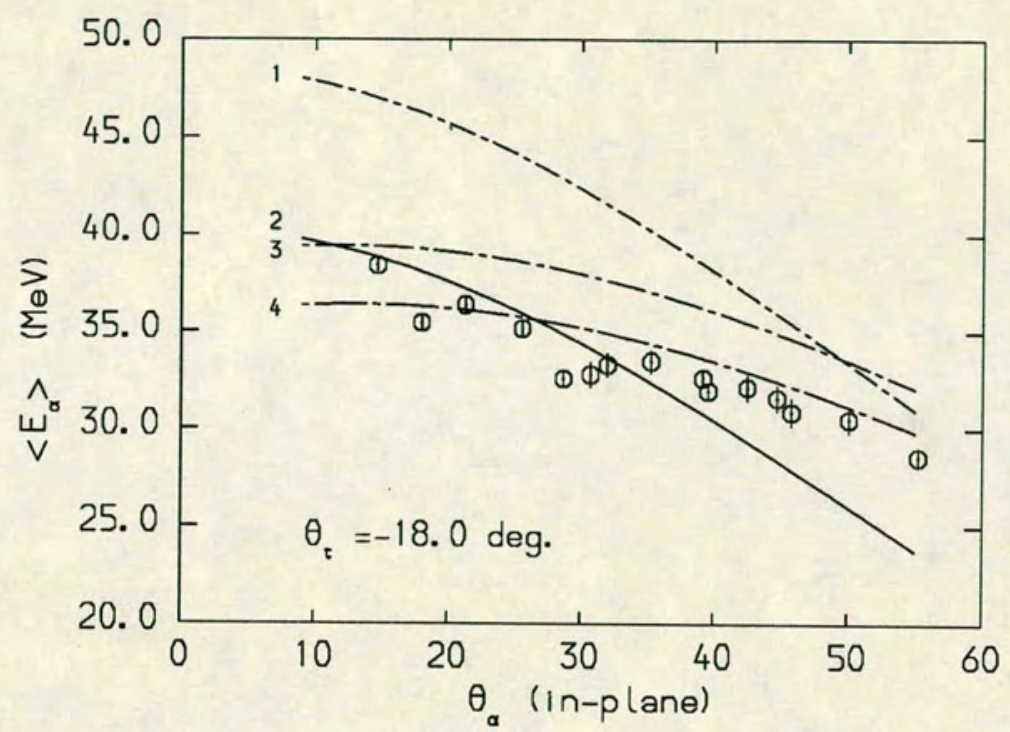

Figure 5.24: Experimental average energies of projected $\mathrm{E}_{\alpha}$ spectra with $\theta_{t}=-18.0^{\circ}$. Curves are results of kinematical model explained in text and given in fig.5.22.

$$
{ }^{12} \mathrm{C}\left({ }^{7} \mathrm{Li}, \alpha+t\right){ }^{12} \mathrm{C}_{\text {g.s. }}
$$

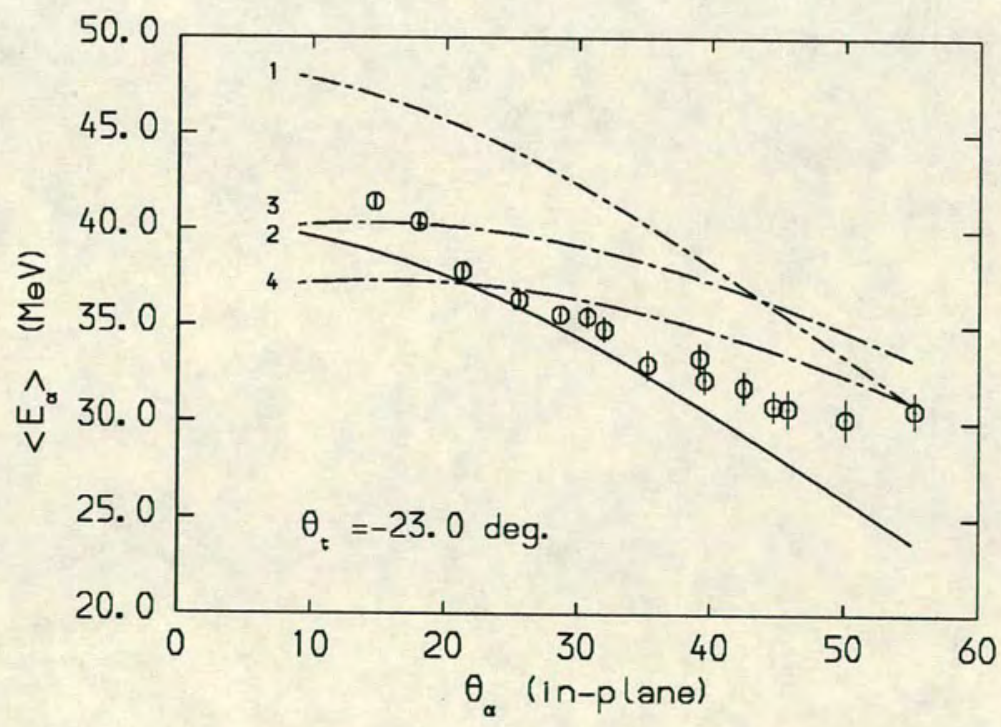

Figure 5.25: Experimental average energies of projected $\mathrm{E}_{\alpha}$ spectra with $\theta_{t}=-23.0^{\circ}$. Curves are results of kinematical model explained in text and given in fig.5.22. 


$$
{ }^{12} C\left({ }^{7} L i, \alpha+t\right){ }^{12} C_{\text {g.s. }} .
$$

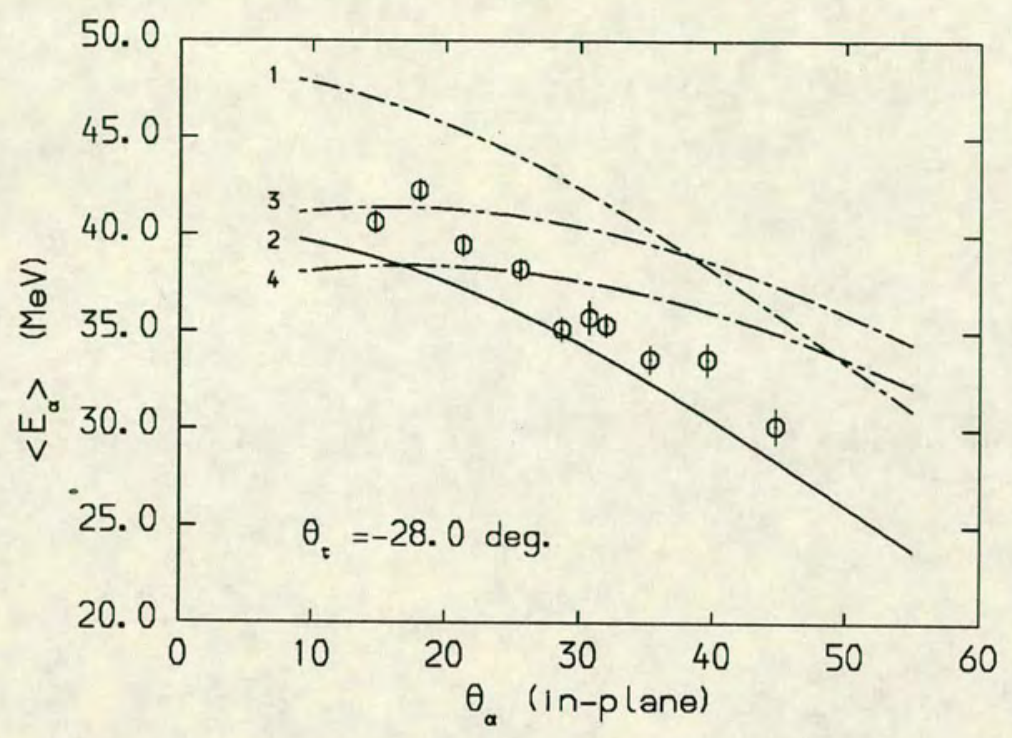

Figure 5.26: Experimental average energies of projected $\mathrm{E}_{\alpha}$ spectra with $\theta_{t}=-28.0^{\circ}$. Curves are results of kinematical model explained in text and given in fig.5.22.

$$
{ }^{12} \mathrm{C}\left({ }^{7} L i, \alpha+t\right){ }^{12} \mathrm{C}_{\text {g.s. }}
$$

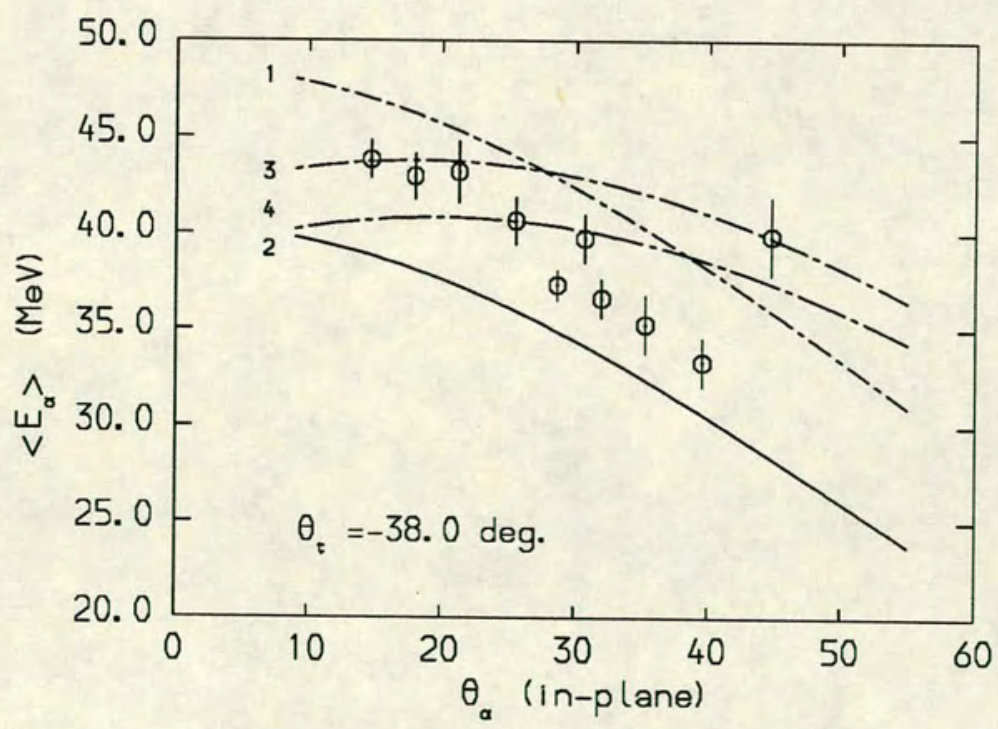

Figure 5.27: Experimental average energies of projected $E_{\alpha}$ spectra with $\theta_{t}=-38.0^{\circ}$. Curves are results of kinematical model explained in text and given in fig.5.22. 


\begin{tabular}{|c|c|c|c|}
\hline Mechanism & Interaction & Compound Nucleus & Excitation \\
\hline Direct & $\alpha$ spectator & ${ }^{15} \mathrm{~N}$ & $36.1 \mathrm{MeV}$ \\
Direct & $\alpha$ participant & ${ }^{16} \mathrm{O}$ & $34.9 \mathrm{MeV}$ \\
Transfer & $\alpha$ spectator & ${ }^{15} \mathrm{~N}$ & $29.3 \mathrm{MeV}$ \\
Transfer & $\alpha$ participant & ${ }^{16} \mathrm{O}$ & $32.6 \mathrm{MeV}$ \\
\hline
\end{tabular}

Table 5.4: Optimum excitation energies for compound nucleus predicted from a simple kinematic break-up model.

However, from a close inspection of the behaviour of the experimental average energies with varying $\theta_{\alpha}$ then for $\theta_{t}$ up to approximately $-23.0^{\circ}$ and at backward $\theta_{\alpha}$ angles the data seems to be consistent with a mechanism where the alpha is the participant in a transfer like process to states in ${ }^{16} \mathrm{O}$. For values of $\theta_{t}$ at more backward angles the data is more consistent with the slope of a direct spectator mechanism where the alpha is emitted more independantly of the participanttarget interaction. Clearly this interpretation is rather speculative since the actual energy distributions are rather broad. This method of analysis predicts an optimum excitation energy for the recoiling compound system and these are given in table 5.4 for the corresponding states in ${ }^{16} \mathrm{O}$ and ${ }^{15} \mathrm{~N}$. It can be seen from this table that the predicted excitation energies for a transfer mechanism to states in ${ }^{15} \mathrm{~N}$ and ${ }^{16} \mathrm{O}$ are quite close to the states proposed to explain the peaks in the projected $\mathrm{E}-\alpha$ spectra implying that transfer to these states will not be kinematically suppressed.

In the analysis of the ${ }^{120} \mathrm{Sn}$ data, one important kinematical effect is the smaller recoil of the target. This means that the break-up cones for detecting events from the sequential break-up of the projectile are more forward focused. Consequently, only the angular settings with the detectors on the same side of the beam are within the ${ }^{7} \mathrm{Li}_{4.63 \mathrm{MeV}}$ break-up cone. This causes the enhancement around the alpha direction seen in fig.4.49.

For the angular settings with the detectors on the opposite side of the beam, the quasi-elastic energy distributions tend to be broad featureless bumps. These alpha energy distributions are shown in fig.5.28 to 5.35 along with the Monte Carlo simulations performed for the relevant ${ }^{7} \mathrm{Li}$ states. The experimental spectra do 
not show any obvious peaks that can confidently be attributed to sequential break-up from any of these states. As with the ${ }^{12} \mathrm{C}$ target there is a concentration of events around the beam velocity in most pixels. These could be attributed to sequential break-up but the distributions do not show any structure whose behaviour with changing $\theta_{t}$ and $\theta_{\alpha}$ is indicative of this. The behaviour of the energy distributions do not show any effects which can be correlated with the position of the break-up cone edges such as intensity enhancement or energy shift. Again this could be reflecting the fact that no single sequential state is making significantly dominant contribution to the quasi-elastic cross-section at the angles investigated.

One point worthy of mention is the lower energy limit in all the projected spectra from the ${ }^{120} \mathrm{Sn}$ target. These are all $\sim 30 \mathrm{MeV}$ which is much higher than the experimental threshold of $\sim 18 \mathrm{MeV}$. Any sequential state which has events lower than this energy cannot be contributing to the projected spectra to any significant degree. Inspecting the F1M1 element in fig.5.28, it can be seen that this rules out break-up from the ${ }^{7} \mathrm{Li}_{7.47 \mathrm{MeV}}$ and ${ }^{7} \mathrm{Li}_{9.96 \mathrm{MeV}}$ states (at least in this pixel). If the sequential break-up from these states is absent from this pixel then it is most probably absent from the neighbouring pixels. Again, from inspection of this same pixel, the energy distribution seems to be completely different to that expected from break-up of the ${ }^{7} \mathrm{Li}_{6.68 \mathrm{MeV}}$ state shown in the Monte Carlo data. The simulation has a distinct dip in intensity at $\sim 40 \mathrm{MeV}$ whereas the experimental data in this region are seen to peak. This seems to indicate that these events are arising from some process other than sequential break-up. Figs.5.36 and 5.37 show the projected spectra for both detectors on the same side of the beam. Although these have relatively poor statistics it is clear that the spectrum with $\theta_{t}=+20.0^{\circ}$ is dominated by ${ }^{7} \mathrm{Li}_{4.63 \mathrm{MeV}}$ sequential break-up. 


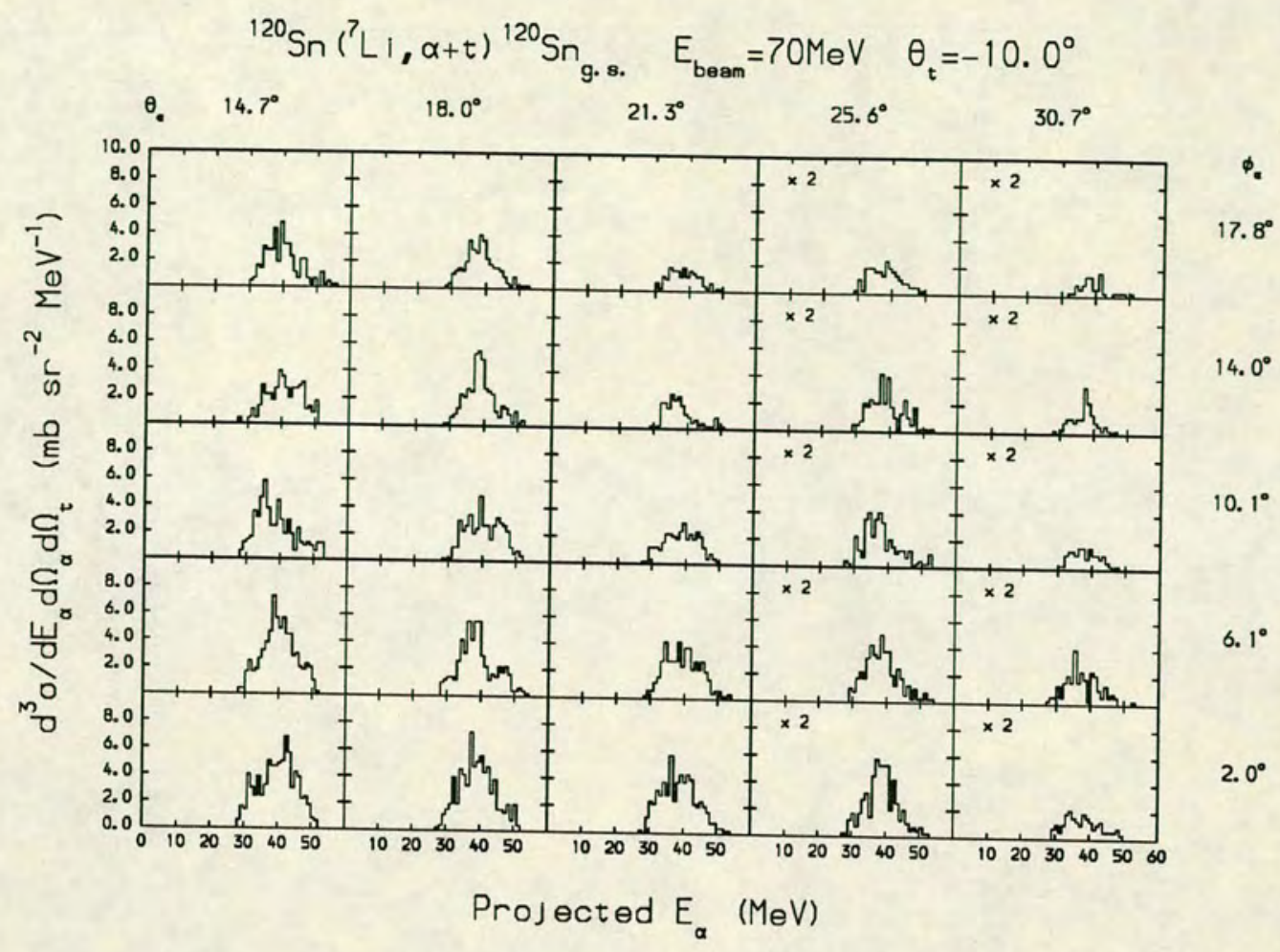

Monte-Car lo Simulat ion

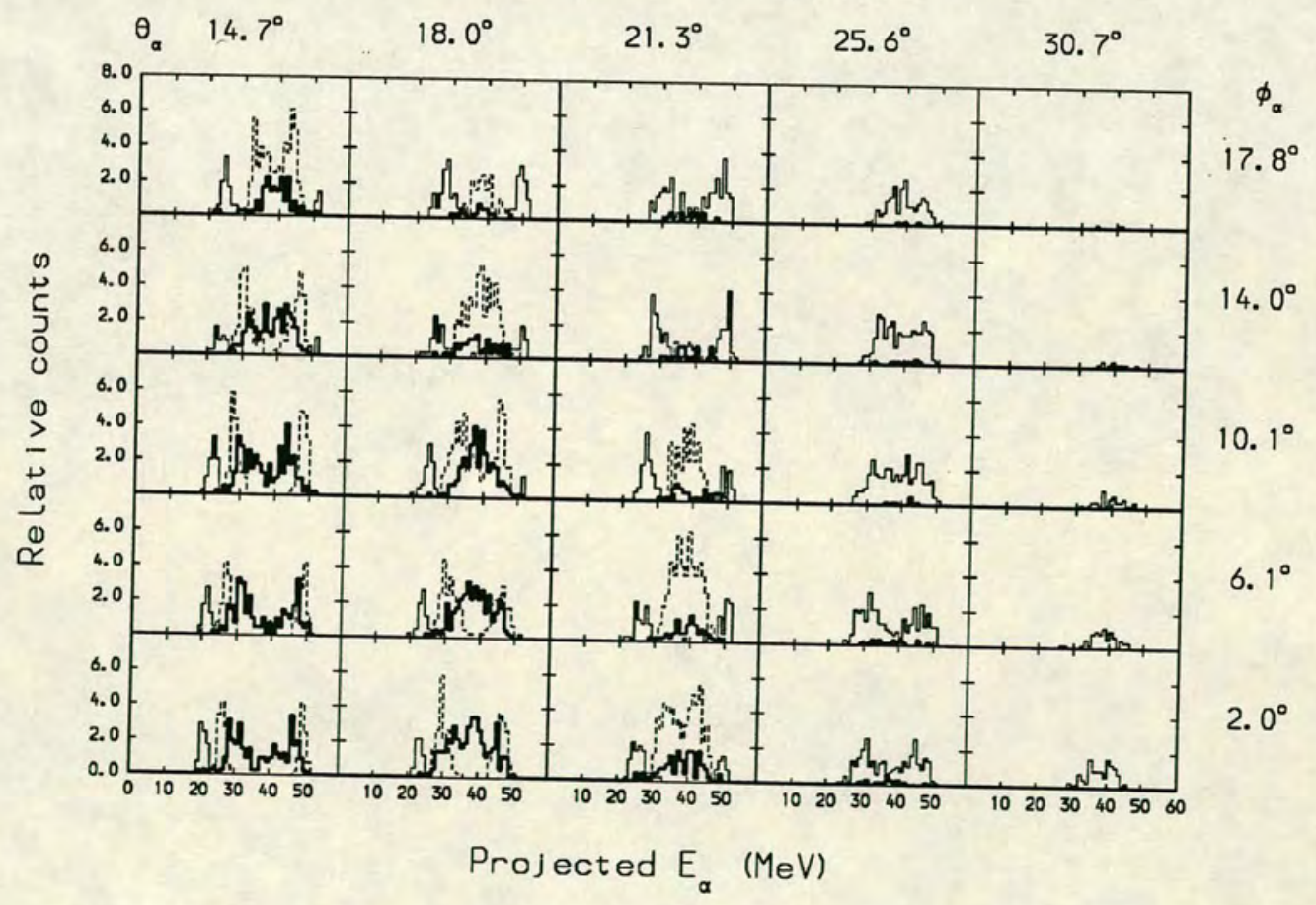

Figure 5.28: Comparison of data and Monte Carlo simulations for $\theta_{t}=-10.0^{\circ}$. States simulated were ${ }^{7} \mathrm{Li}_{6.68 \mathrm{MeV}}$ (thick line), ${ }^{7} \mathrm{Li}_{7.47 \mathrm{MeV}}$ (dotted line), ${ }^{7} \mathrm{Li}_{9.96 \mathrm{MeV}}$ (thin line). 

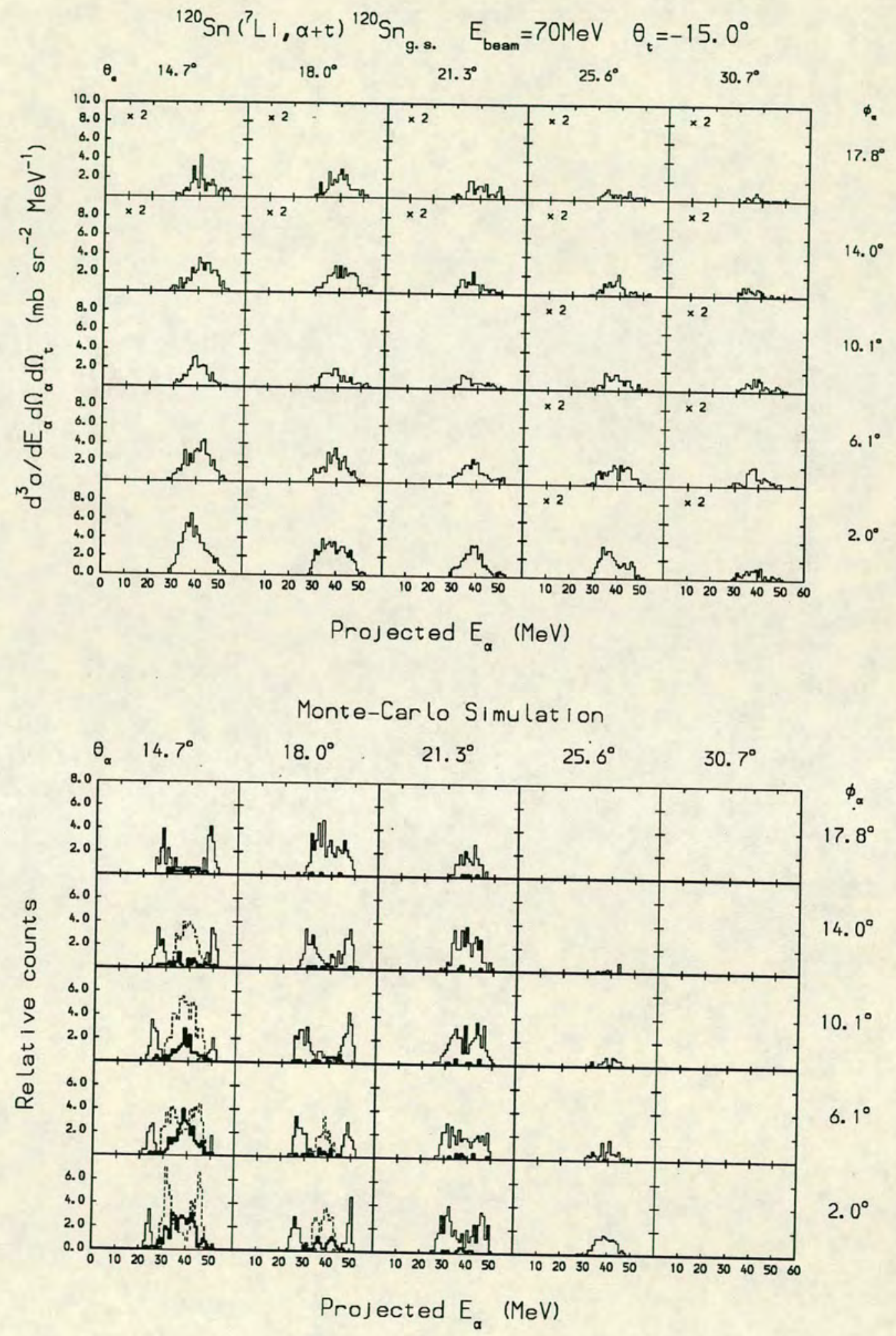

Figure 5.29: Comparison of data and Monte Carlo simulations for $\theta_{t}=-15.0^{\circ}$. States simulated were ${ }^{7} \mathrm{Li}_{6.68 \mathrm{MeV}}$ (thick line), ${ }^{7} \mathrm{Li}_{7.47 \mathrm{MeV}}$ (dotted line), ${ }^{7} \mathrm{Li}_{9.96 \mathrm{MeV}}$ (thin line). 


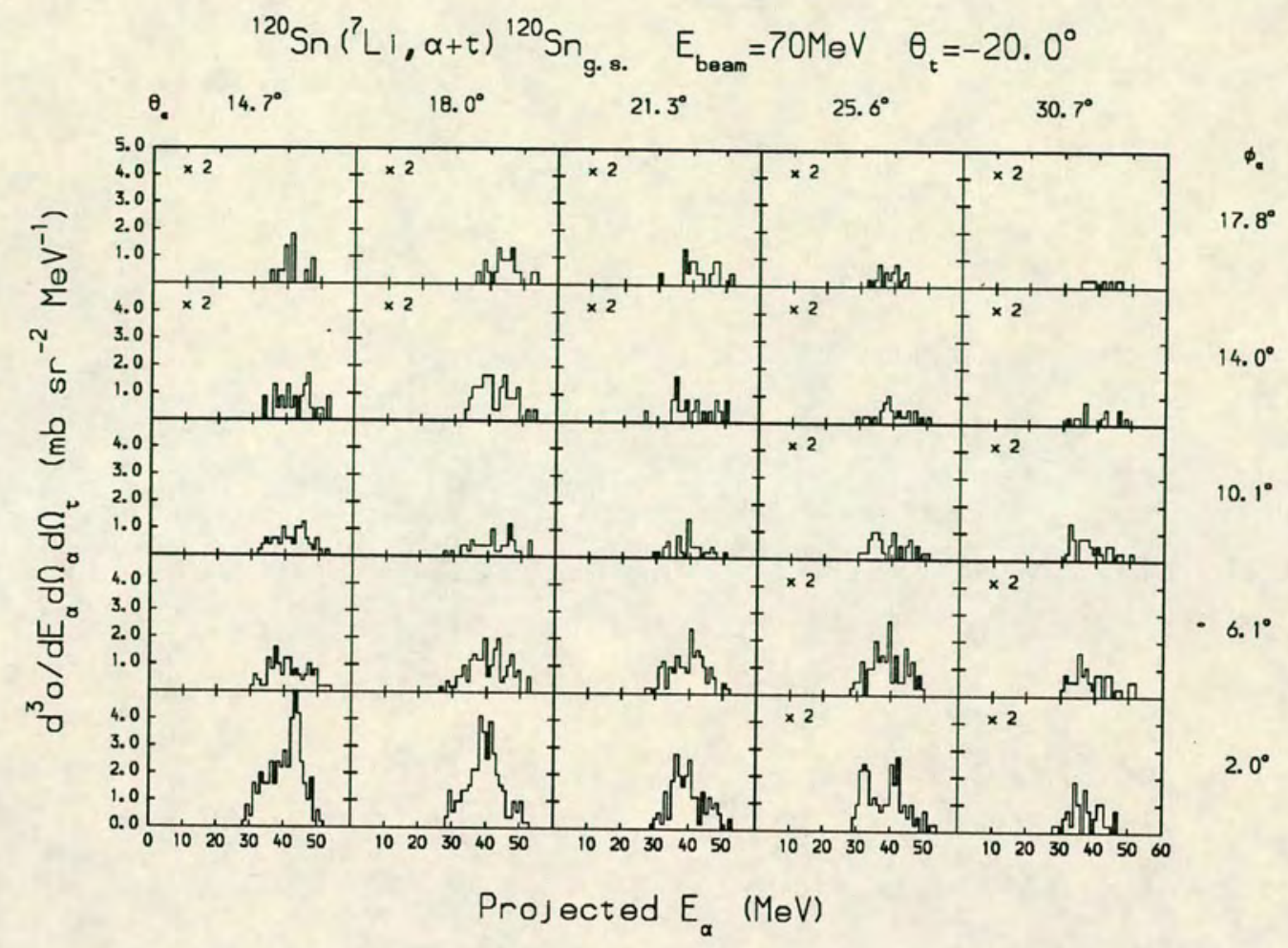

Monte-Car lo Simulat ion

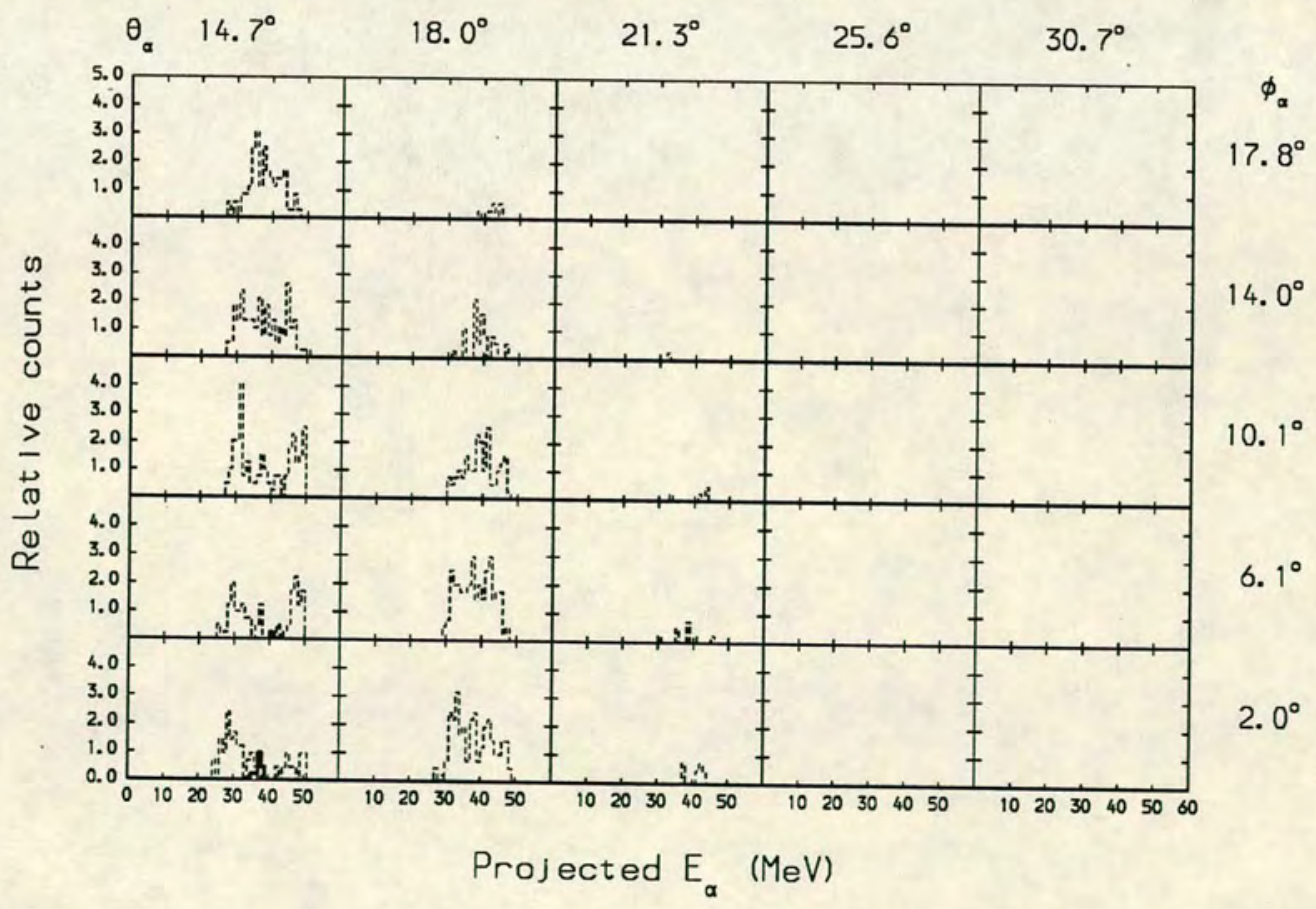

Figure 5.30: Comparison of data and Monte Carlo simulations for $\theta_{t}=-20.0^{\circ}$. States simulated were ${ }^{7} \mathrm{Li}_{7.47 \mathrm{MeV}}$ (thick line), ${ }^{7} \mathrm{Li}_{9.96 \mathrm{MeV}}$ (dotted line). 


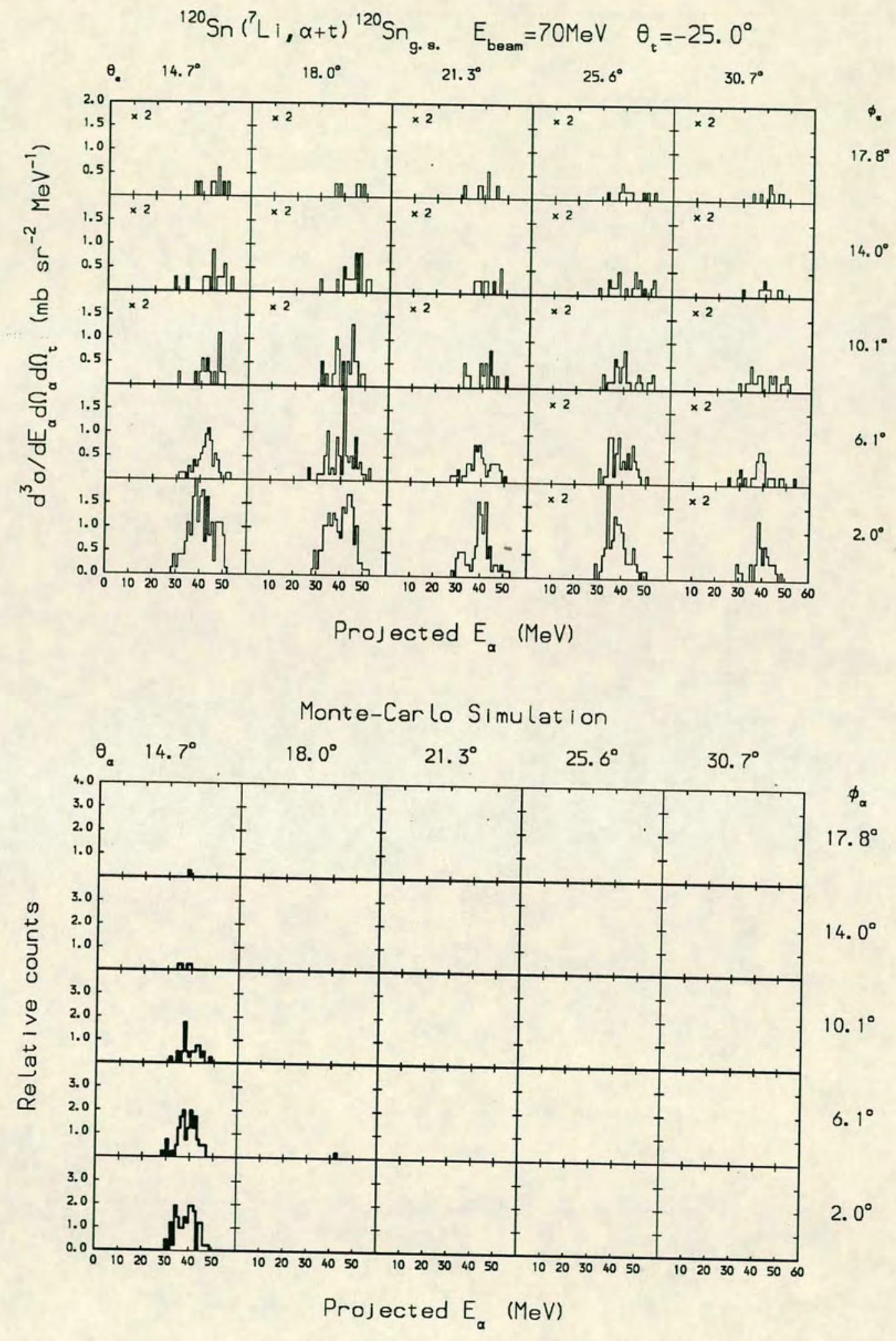

Figure 5.31: Comparison of data and Monte Carlo simulations for $\theta_{t}=-25.0^{\circ}$. State simulated was ${ }^{7} \mathrm{Li}_{9.96 \mathrm{MeV}}$ (thick line). 


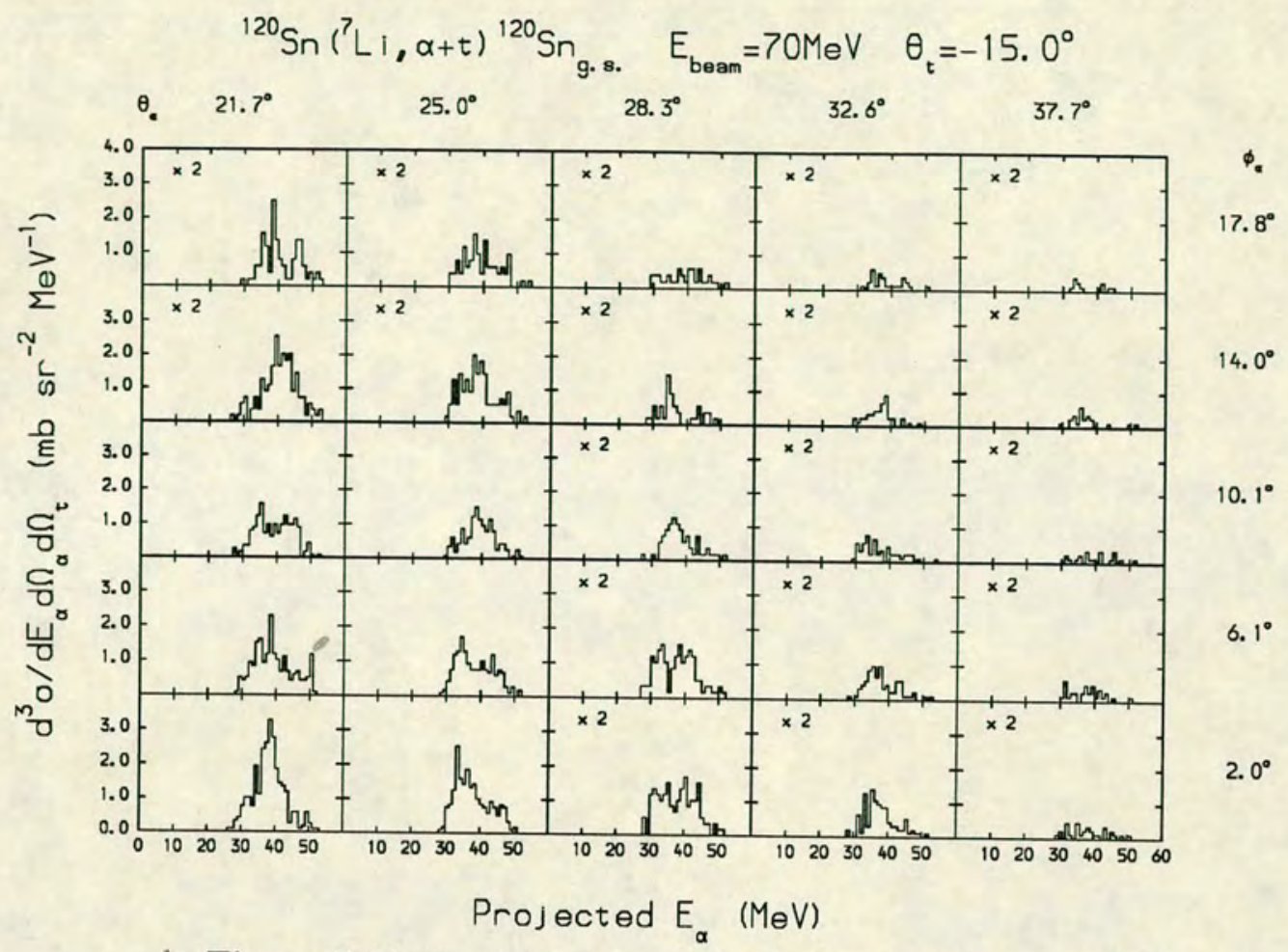

Figure 5.32: Projected quasi-elastic $\mathrm{E}_{\alpha}$ data for $\theta_{t}=-15.0^{\circ}$.

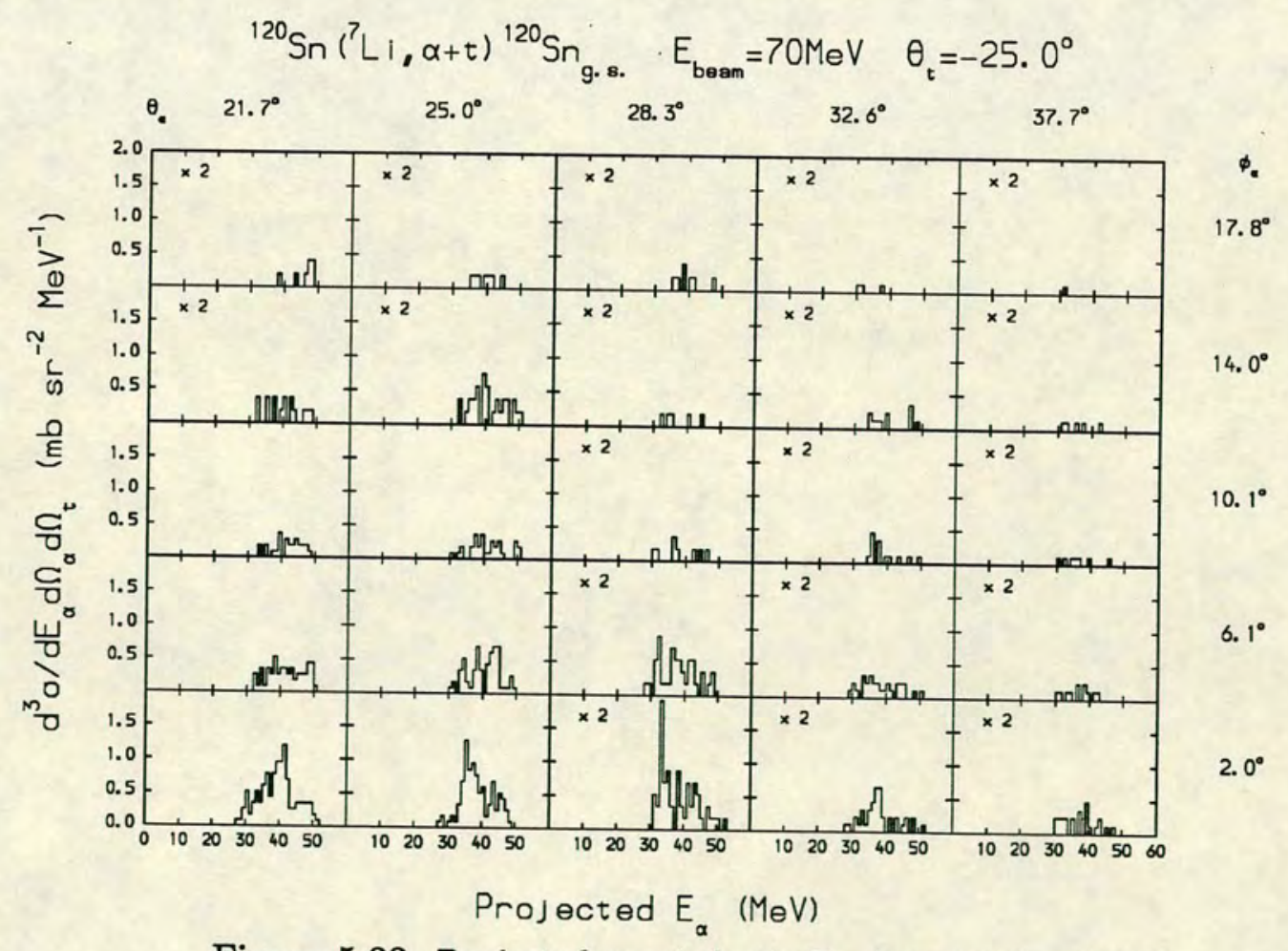

Figure 5.33: Projected quasi-elastic $\mathrm{E}_{\alpha}$ data for $\theta_{t}=-25.0^{\circ}$. 


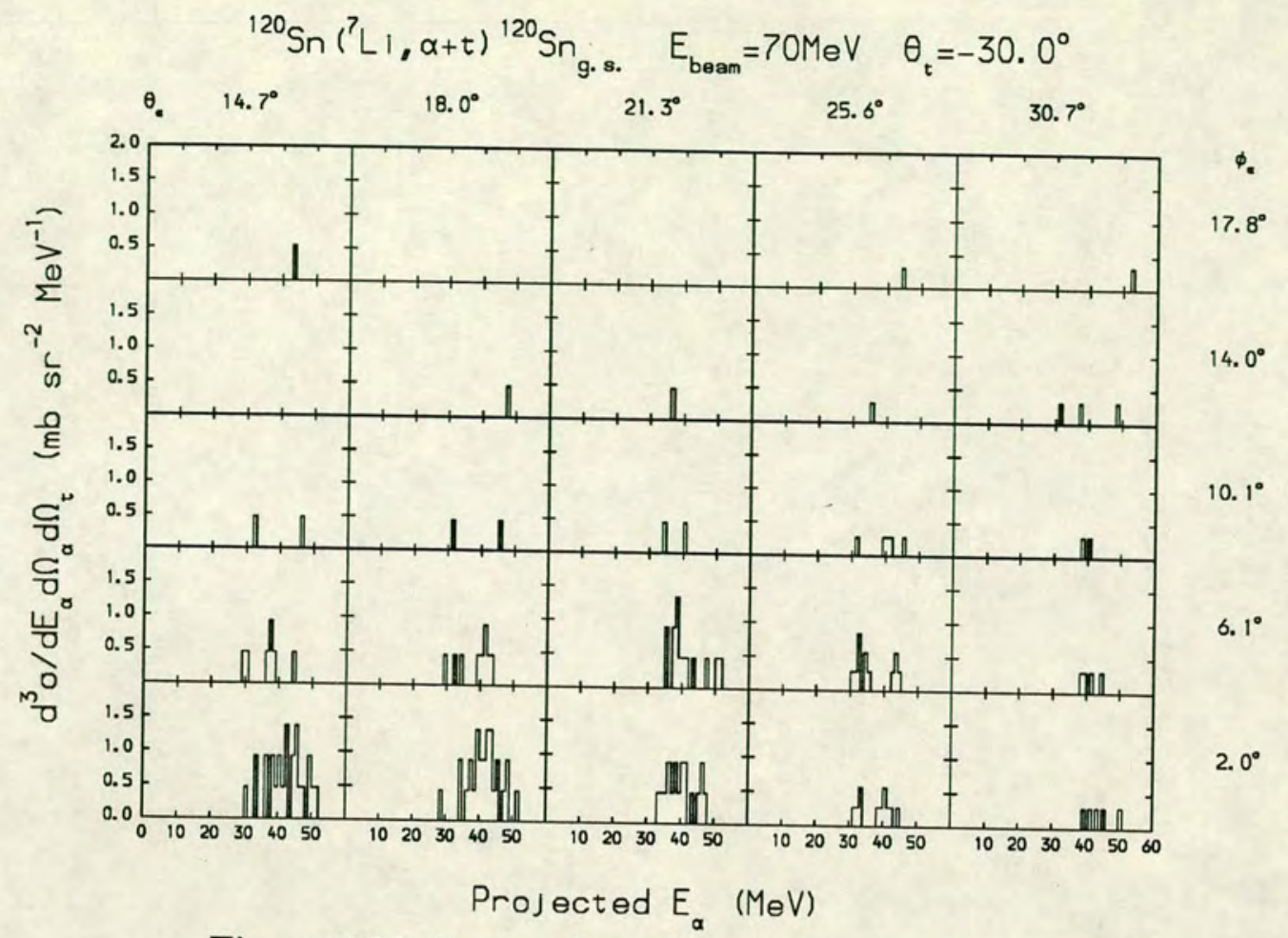

Figure 5.34: Projected quasi-elastic $\mathrm{E}_{\alpha}$ data for $\theta_{t}=-30.0^{\circ}$.

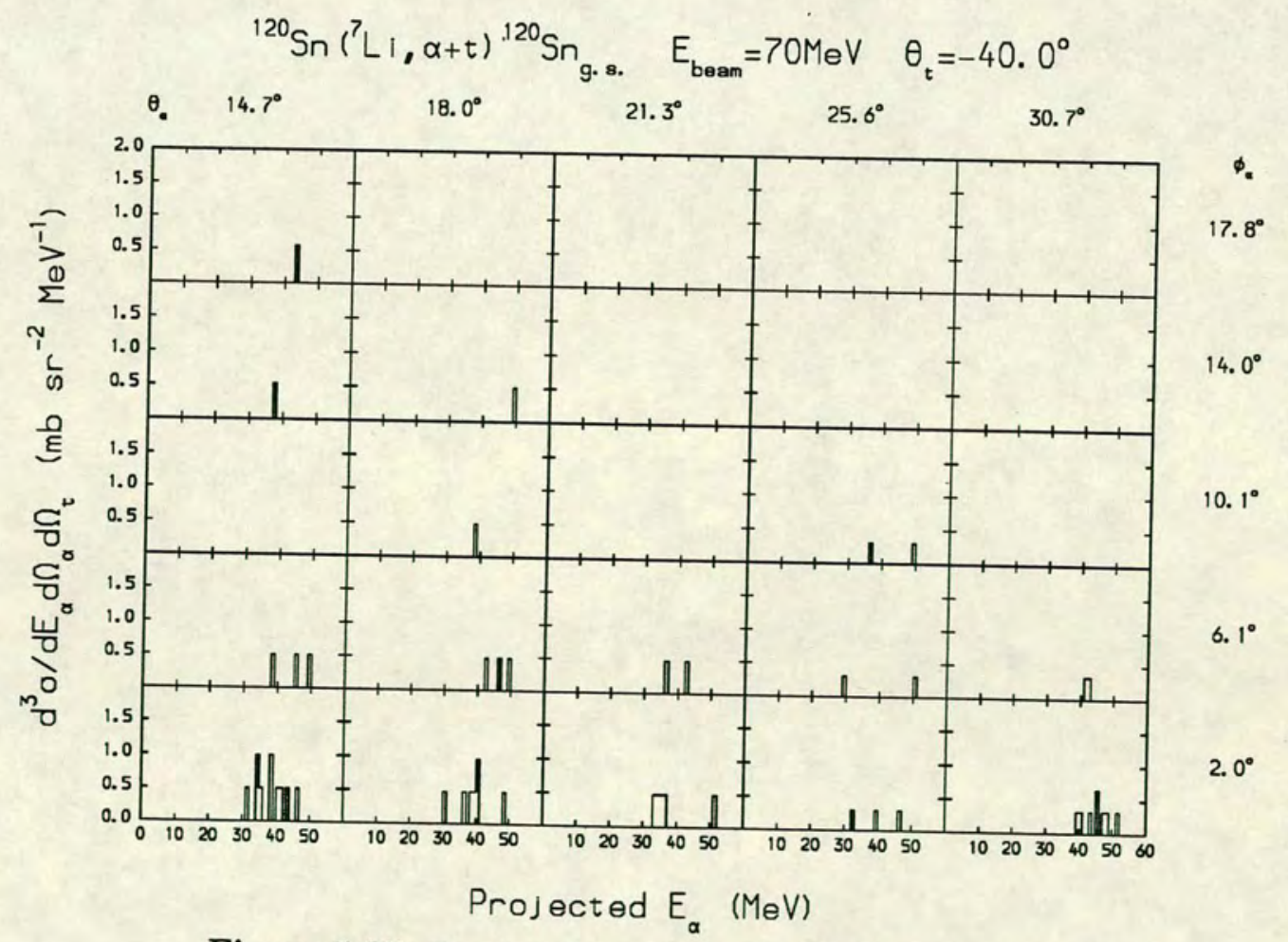

Figure 5.35: Projected quasi-elastic $\mathrm{E}_{\alpha}$ data for $\theta_{t}=-40.0^{\circ}$. 

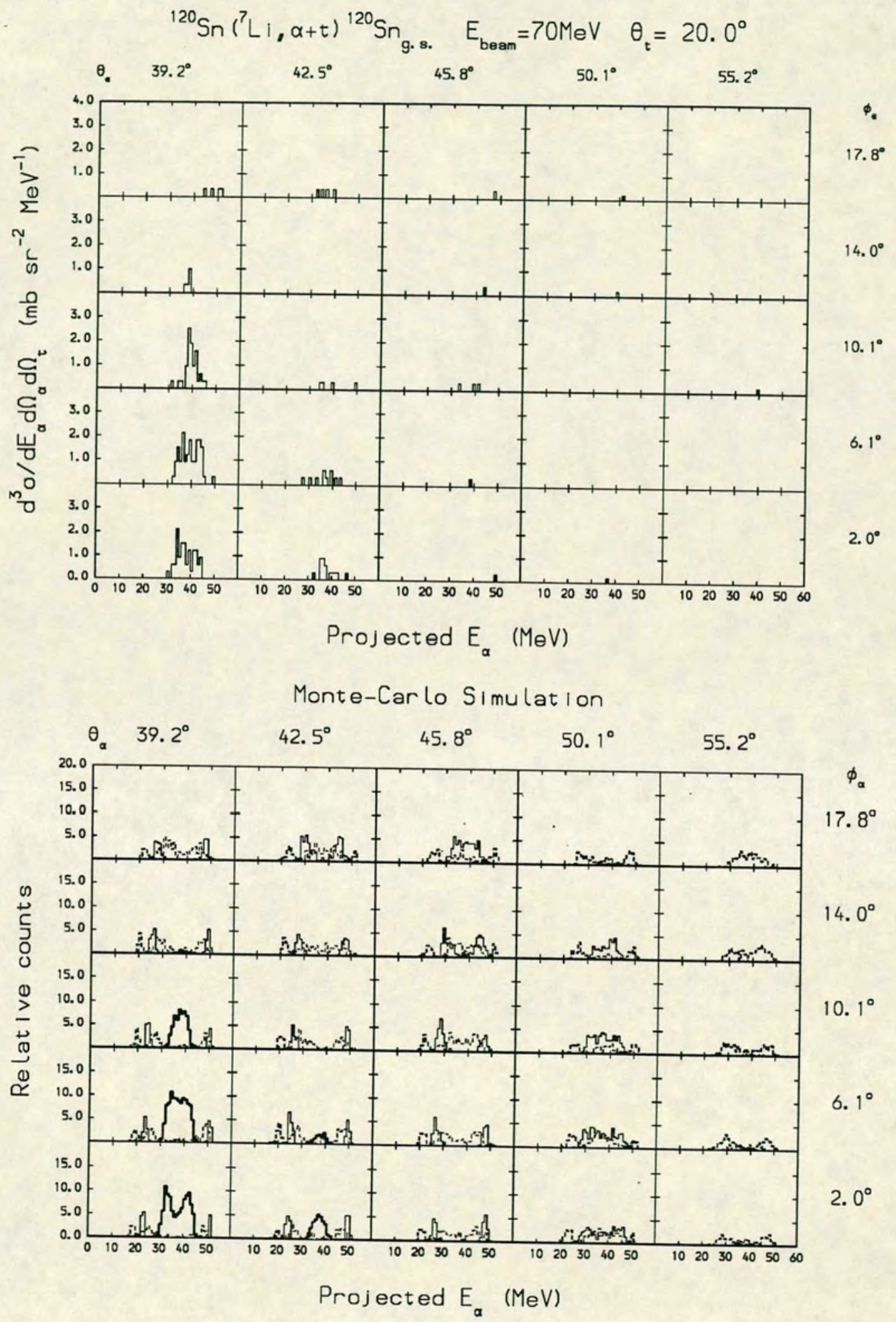

Figure 5.36: Comparison of data and Monte Carlo simulations for $\theta_{t}=+20.0^{\circ}$ (both detectors on same side of beam). States simulated were ${ }^{7} \mathrm{Li}_{4.63 \mathrm{MeV}}$ (thick line), ${ }^{7} \mathrm{Li}_{6.68 \mathrm{MeV}}$ (dotted line), ${ }^{7} \mathrm{Li}_{7.47 \mathrm{MeV}}$ (thin line), ${ }^{7} \mathrm{Li}_{9.96 \mathrm{MeV}}$ (thick dotted line). 

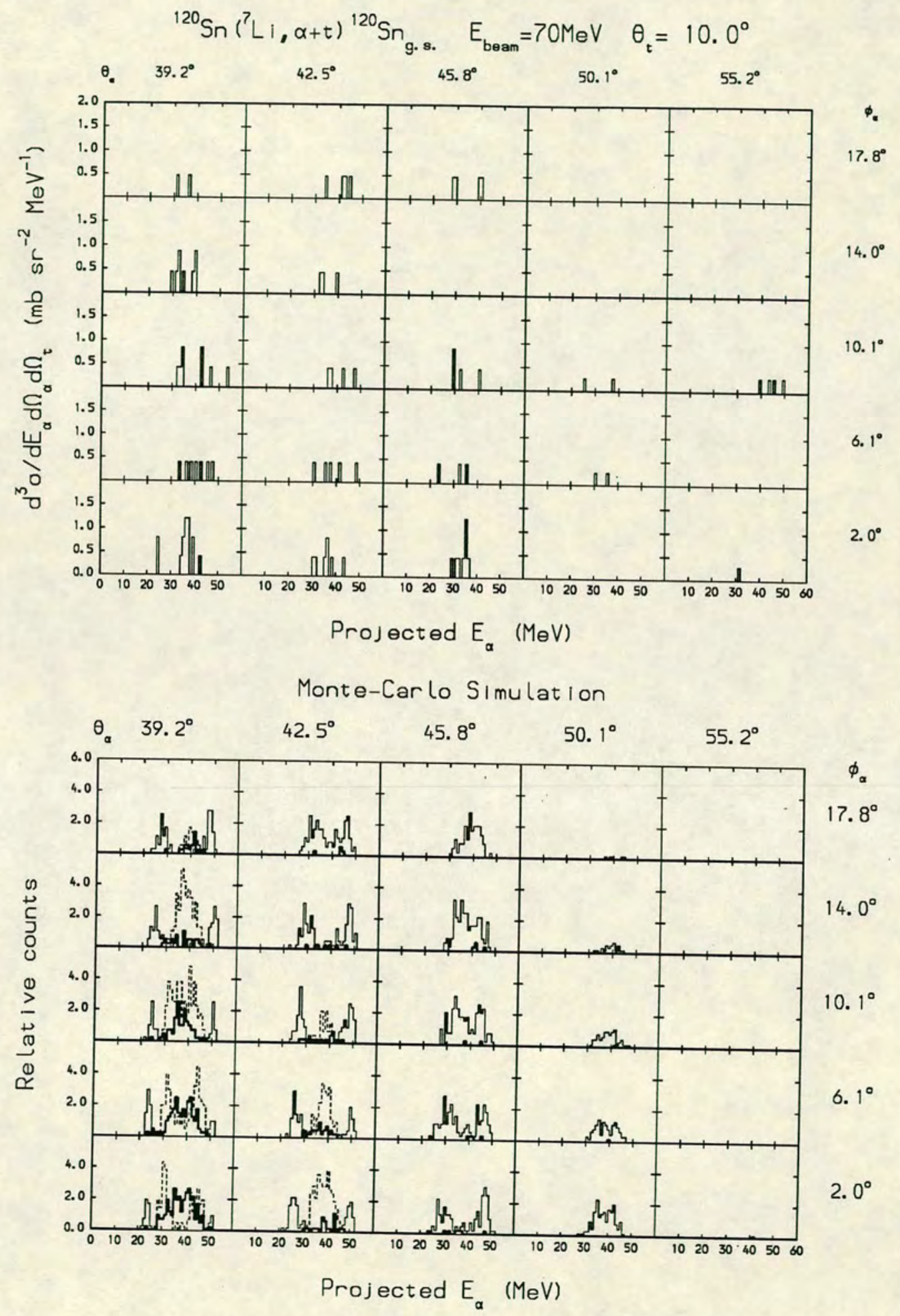

Figure 5.37: Comparison of data and Monte Carlo simulations for $\theta_{t}=+10.0^{\circ}$ ( both detectors on same side of beam). States simulated were ${ }^{7} \mathrm{Li}_{6.68 \mathrm{MeV}}$ (thick line), ${ }^{7} \mathrm{Li}_{7.47 \mathrm{MeV}}$ (dotted line), ${ }^{7} \mathrm{Li}_{9.96 \mathrm{MeV}}$ (thin line). 
Figs.5.38 to 5.43 show the average energies of the projected energy distribution along with the results of the kinematic model previously described. For this target the data is more consistent with a model where the alpha is the participant. Again however, this simple approach must be viewed with caution not least because the actual energy distributions are quite broad. Table 5.5 gives the predicted compound nucleus excitations for the four processes modelled. These are much higher than any measured states in the compound nuclei. However, a heavy compound system such as ${ }^{123} \mathrm{Sb}$ or ${ }^{124} \mathrm{Te}$ will probably have a rather small spectroscopic factor for existence as an $\alpha+{ }^{120} \mathrm{Sn}$, or $\mathrm{t}+{ }^{120} \mathrm{Sn}$ and re-emission of a charged complex nucleus will be strongly Coulomb suppressed [Ba83]. this would imply that re-emission to the ground state of ${ }^{120} \mathrm{Sn}$ would be a rather weak de-excitation process. From the data the quasi-elastic channel is seen to dominate the $\alpha+\mathrm{t}$ channel again suggesting that these events are arising from a process other than fusion.

It has been proposed [ $\mathrm{Ca} 80]$, [Sh85] that a possible mechanism for producing wide angle $\alpha$-t coincidence events is a direct excitation of the ${ }^{7} \mathrm{Li}$ into the continuum with a final state interaction between one of the fragments and the target. Such direct break-up has been identified for both the ${ }^{120} \mathrm{Sn}$ and ${ }^{208} \mathrm{~Pb}$ targets [Da87], and is presumed to arise from the variation of the Coulomb and/or nuclear forces acting on the clusters within the ${ }^{7} \mathrm{Li}$. In this type of process the alpha and triton will be liberated sufficiently quickly for there to be a distinct possibility of further interactions with the target. Measurements made by Shotter et al. [Sh84] with $70 \mathrm{MeV}{ }^{7} \mathrm{Li}$ on ${ }^{120} \mathrm{Sn}$ show that as one approaches the grazing angle the observed projected energy distribution attributed to direct break-up does become distorted from that observed at forward angles. This may be interpreted as originating from the increased nuclear influence on the outgoing fragments and indicates the possibility of a process where one of the fragments is deflected to large angles. Without any theoretical predictions for this interaction it is difficult to make any more quantitative statements about this process. One model which implicitly includes these final state interactions is the C.D.C.C. calculations outlined in chapter1. As yet no calculations appropriate to this experimental configuration have been performed due to the large amount of computing time required to incorporate all the relevant angular momentum channels. The direct spectator and direct participant versions of 


\begin{tabular}{|c|c|c|c|}
\hline Mechanism & Interaction & Compound Nucleus & Excitation \\
\hline Direct & $\alpha$ spectator & ${ }^{123} \mathrm{Sb}$ & $37.5 \mathrm{MeV}$ \\
Direct & $\alpha$ participant & ${ }^{124} \mathrm{Te}$ & $40.6 \mathrm{MeV}$ \\
Transfer & $\alpha$ spectator & ${ }^{123} \mathrm{Sb}$ & $30.5 \mathrm{MeV}$ \\
Transfer & $\alpha$ participant & ${ }^{124} \mathrm{Te}$ & $39.8 \mathrm{MeV}$ \\
\hline
\end{tabular}

Table 5.5: Optimum excitation energies for compound nucleus predicted from a simple kinematic break-up model.

the simple kinematic model previously outlined resembles a direct quasi-elastic type of final state interaction where the target is left in its ground state. This predicts the expected energy of the outgoing fragment but gives no indication of the relative strengths of the different processes and hence is of limited use in separating out the different components.

Direct break-up followed by an interaction of one of the fragments with the nuclear field of the target may also explain some of the ${ }^{12} \mathrm{C}$ wide-angle cross-section. A theoretical calculation modelling the direct break-up as a Coulomb excitation process has produced rather good quantitative fits to the close geometry ${ }^{120} \mathrm{Sn}$ direct break-up component at forward angles [Sh88] and a similar calculation predicts a large component for this process at grazing on ${ }^{12} \mathrm{C}$.

In conclusion, this section has presented the results of a Monte Carlo simulation modelling sequential break-up from all the known states in ${ }^{7} \mathrm{Li}$. These were compared with the experimentally observed quasi-elastic projected alpha energy and identified break-up from the ${ }^{7} \mathrm{Li}_{4.63 \mathrm{MeV}}$ state of the projectile. No other sequentially decaying states were clearly identified for either the ${ }^{12} \mathrm{C}$ target or the ${ }^{120} \mathrm{Sn}$ target. In retrospect it is perhaps not too surprising that evidence for sequential break-up from the higher lying states of ${ }^{7} \mathrm{Li}$ was not found with the ${ }^{120} \mathrm{Sn}$ target. The detector system, with both fragments being detected on opposite sides of the beam was most efficient at detecting sequentially decaying ${ }^{7} \mathrm{Li}$ travelling in the forward direction. In this region the excitation of the ${ }^{7} \mathrm{Li}$ is proposed to originate mainly from the Coulomb force [Sr88], [Sh88]. The strength of this decreases exponentially as the energy of the excited state increases and is rather inefficient for excitations larger than $\sim 4 \mathrm{MeV}$. 


$$
{ }^{120} \operatorname{Sn}\left(^{7} L_{i}, \alpha+t\right){ }^{120} \operatorname{Sn}_{\text {g.s. }}
$$

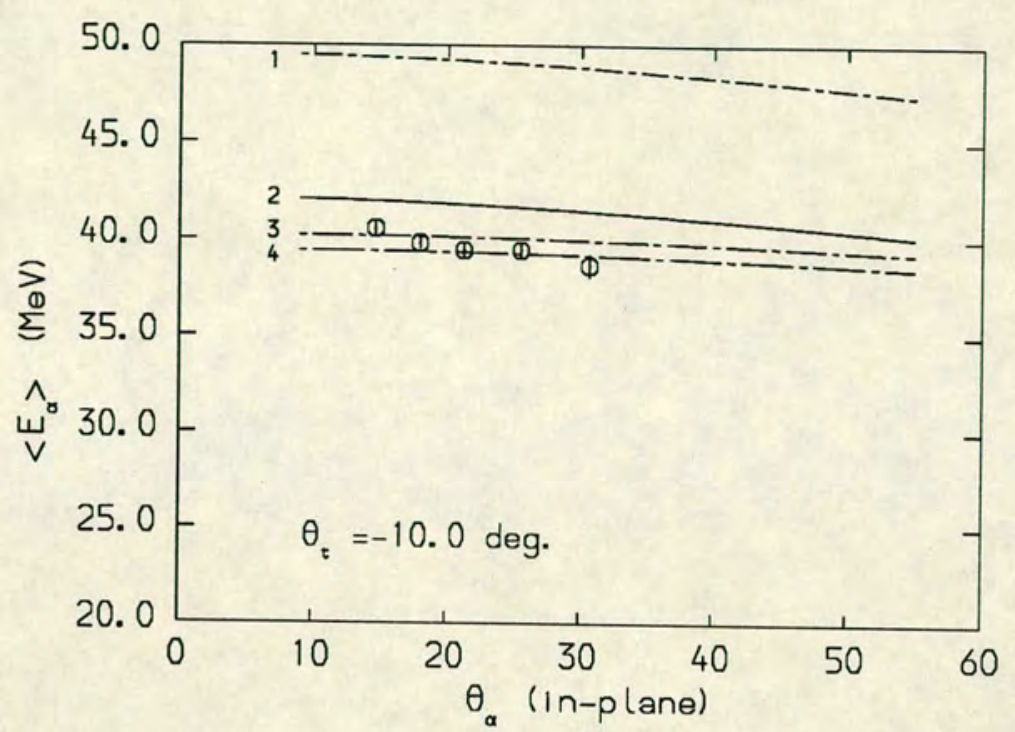

Figure 5.38: Experimental average energies of projected $E_{\alpha}$ spectra with $\theta_{t}=-10.0^{\circ}$. Curves are results of kinematical model explained in text for 1 . transfer spectator, 2. direct spectator, 3. direct participant, 4. transfer participant model.

$$
{ }^{120} \mathrm{Sn}\left({ }^{7} \mathrm{~L} i, \alpha+t\right){ }^{120} \mathrm{Sn} \text { g.s. }
$$

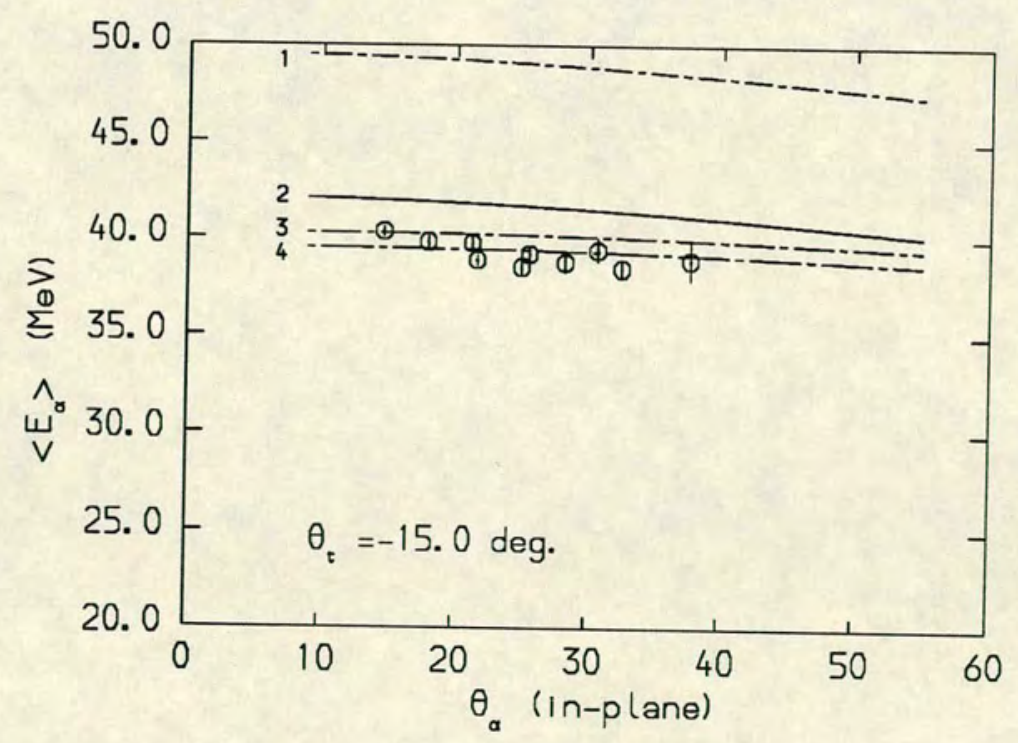

Figure 5.39: Experimental average energies of projected $E_{\alpha}$ spectra with $\theta_{t}=-15.0^{\circ}$. Curves are results of kinematical model explained in text and given in fig.5.38. 


$$
{ }^{120} \mathrm{Sn}\left({ }^{7} L i, \alpha+t\right){ }^{120} \mathrm{Sn} \text { g.s. }
$$

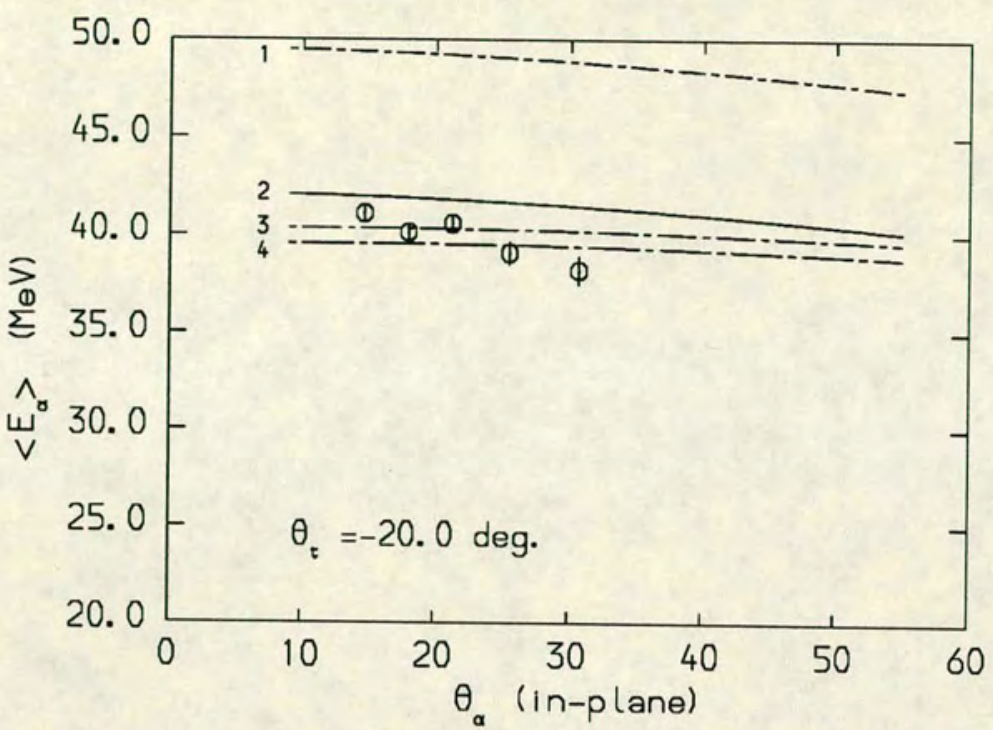

Figure 5.40: Experimental average energies of projected $\mathrm{E}_{\alpha}$ spectra with $\theta_{t}=-20.0^{\circ}$. Curves are results of kinematical model explained in text and given in fig.5.38.

$$
{ }^{120} \mathrm{Sn}\left(^{7} \mathrm{~L}, \alpha+t\right){ }^{120} \mathrm{Sn} \text { g.s. }
$$

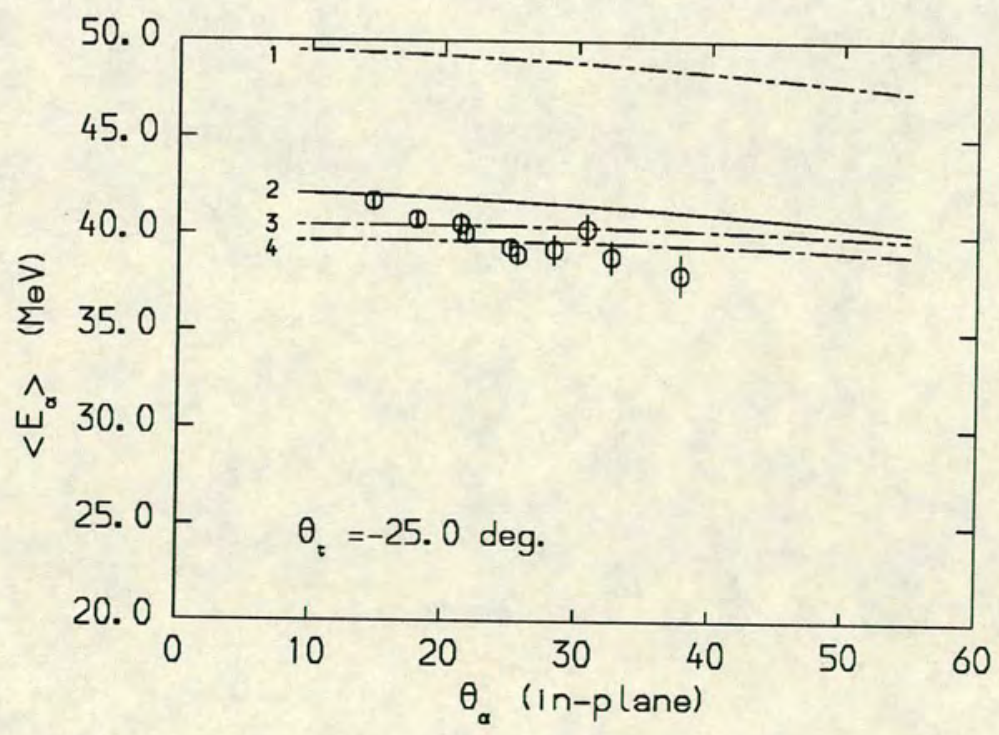

Figure 5.41: Experimental average energies of projected $E_{\alpha}$ spectra with $\theta_{t}=-25.0^{\circ}$. Curves are results of kinematical model explained in text and given in fig.5.38. 


$$
{ }^{120} \operatorname{Sn}\left({ }^{7} L i, \alpha+t\right){ }^{120} \mathrm{Sn}_{\text {g.s. }}
$$

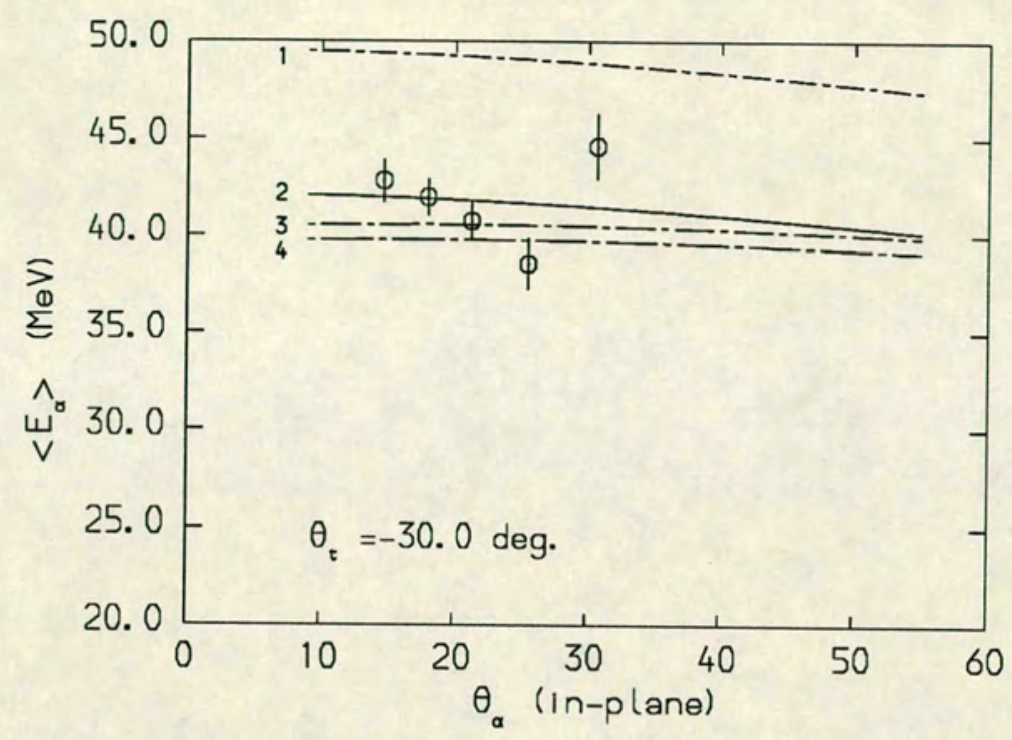

Figure 5.42: Experimental average energies of projected $\mathrm{E}_{\alpha}$ spectra with $\theta_{t}=-30.0^{\circ}$. Curves are results of kinematical model explained in text and given in fig.5.38.

$$
{ }^{120} \operatorname{Sn}\left({ }^{7} L i, \alpha+t\right){ }^{120} \mathrm{Sn}_{g . s .}
$$

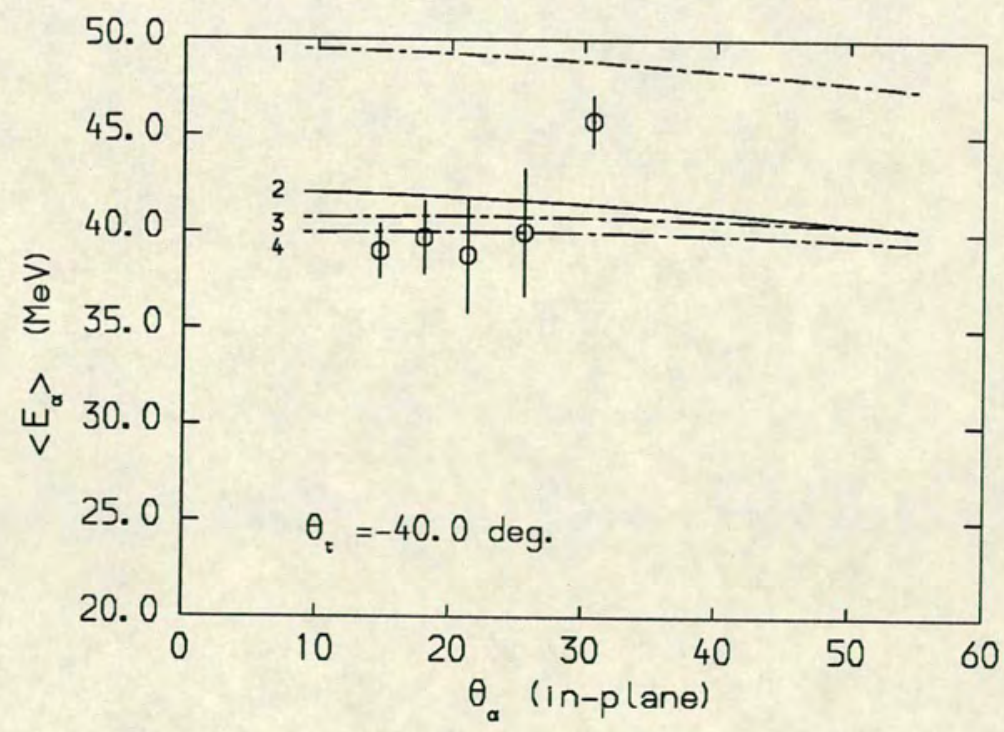

Figure 5.43: Experimental average energies of projected $E_{\alpha}$ spectra with $\theta_{t}=-40.0^{\circ}$. Curves are results of kinematical model explained in text and given in fig.5.38. 
Components due to partial fusion of the triton and alpha fragments with the ${ }^{12} \mathrm{C}$ were proposed to explain certain peaks in the projected spectra. For both targets the bulk of the cross-section remains unidentified except for the conclusion that most of the ${ }^{120} \mathrm{Sn}$ data probably arises from processes other than sequential decay and partial fusion. A direct break-up process via the Coulomb and/or nuclear potential with final state interactions between the fragments and the target is proposed as a possible mechanism producing wide-angle correlations. One final comment at this point is the danger of interpreting the data in too definitive a manner. This was graphically illustrated by the work of Bhowmik et.al. [Bh81] and Goldhoorn et.al. [Go84]. The former interpreted similar projected energy spectra as uncorrelated events. Later Goldhoorn showed this to be a misinterpretation due to the poor angular resolution used in the experiment and on the contrary, a large amount of the cross-section was from sequential states unresolved in the previous experiment. This highlights the pitfalls present in analysis of experiments with large angular resolutions.

\subsection{P.W.B.A Calculations}

It has been proposed [Ca80], [Sh85] that the wide angle correlations observed in this experiment may arise from break-up events where one or other of the fragments have undergone a final state interaction with the target. Any fragment undergoing a final state interaction must be produced close to the target and would thus tend to originate from a direct break-up mechanism rather than a sequential decay process. The strength of the quasi-elastic peak indicates a strong probability for any $\alpha$-t pair surviving this interaction, remaining in the elastic channel. If this is the case it is interesting to discover how well this interaction is described by a simple first order elastic scattering calculation where the interaction between the fragments and the target is assumed to be small. The Plane Wave Born Approximation is a technique for performing such a calculation. Using a P.W.B.A. approach, Matsuoka et al [Ma80b] managed to reproduce the wide angle diffraction structure seen in the quasi-elastic angular distributions for $90 \mathrm{MeV}^{3} \mathrm{He}$ incident on a number of targets. In their approach they assume that the projectile ' $a$ ' consists of the spectator ' $b$ ' and participant 
' $x$ ' and that the reaction can be written as:

$$
a(b+x)+A \longrightarrow b+(x+A) \longrightarrow b+y+B
$$

The T-matrix element of this reaction in the POST form representation is given by:

$$
T_{\text {post }}=<\chi_{f}^{-}\left|W_{f}\right| \Psi_{i}^{+}>
$$

where the incoming solution $\Psi_{i}^{+}$is an eigenfunction of the full hamiltonian $\mathrm{H}$ where:

$$
H=\underbrace{T_{k i n}+V_{b x}+V_{a A}}_{H_{i}}+\underbrace{V_{x A}+V_{b A}-V_{a A}}_{V_{i}}
$$

and the outgoing wavefunction $\chi_{f}^{-}$is an eigenfunction of the final channel hamiltonian $\mathrm{H}_{f}$ where:

$$
H=\underbrace{T_{k i n}+V_{b B}+V_{y B}}_{H_{f}}+\underbrace{V_{b y}}_{V_{f}}
$$

and the residual interaction potential $\mathrm{W}_{f}$ is equal to $\mathrm{V}_{b y}$. In the P.W. approximation both the $\Psi_{i}^{+}$and the $\chi_{f}^{-}$are approximated by plane waves giving:

$$
\begin{gathered}
\left|\Psi_{i}^{+}>\sim\right| e^{i\left(\overrightarrow{\mathbf{k}}_{a} \cdot \overrightarrow{\mathbf{R}}_{a}\right)} \Psi_{a}\left(\overrightarrow{\mathbf{r}}_{b x}, \xi_{b}, \xi_{x}\right) \Psi_{A}\left(\xi_{A}\right)> \\
<\chi_{f}^{-}\left|\sim<e^{-i\left(\overrightarrow{\mathbf{k}}_{b} \cdot \overrightarrow{\mathbf{R}}_{b}\right)} e^{-i\left(\overrightarrow{\mathbf{k}}_{y} \cdot \overrightarrow{\mathbf{R}}_{y}\right)} \Psi_{b}\left(\xi_{b}\right) \Psi_{y}\left(\xi_{y}\right) \Psi_{B}\left(\xi_{B}\right)\right|
\end{gathered}
$$

where the $\Psi$ now represent the intrinsic wave functions of the relevant particles and $\xi$ denotes their internal co-ordinates. The other co-ordinates are defined in fig. 5.44 (from De Meyer). It can be seen from eq. 5.1 and eq. 5.2 that these approximations are equivalent to neglecting $\mathrm{V}_{x A}$ and $\mathrm{V}_{b A}$ in the incoming channel and $\mathrm{V}_{b B}$ and $\mathrm{V}_{y B}$ in the final channel. Clearly these approximations are only really valid when the distortions due to these potentials are small.

For elastic break-up where $\mathrm{y}=\mathrm{x}$ and $\mathrm{B}=\mathrm{A}, \mathrm{W}_{f}$ is just the potential between the components of the projectile $\mathrm{V}_{b x}$. In this case the $\mathrm{T}$ matrix may be written as:

$$
\begin{aligned}
T_{\text {post }}^{P W}= & \iint d \overrightarrow{\mathbf{R}}_{a} d \overrightarrow{\mathbf{r}}_{b x} e^{-i\left(\overrightarrow{\mathbf{k}}_{b} \cdot \overrightarrow{\mathbf{R}}_{b}\right)} e^{-i\left(\overrightarrow{\mathbf{k}}_{x} \cdot \overrightarrow{\mathbf{R}}_{x}\right)} \\
& \times<\psi_{b}\left(\xi_{b}\right) \psi_{x}\left(\xi_{x}\right)\left|V_{b x}\left(\overrightarrow{\mathbf{r}}_{b x}\right)\right| \psi_{a}\left(\overrightarrow{\mathbf{r}}_{b x}, \xi_{b}, \xi_{x}\right)>e^{i\left(\overrightarrow{\mathbf{k}}_{a} \cdot \overrightarrow{\mathbf{R}}_{a}\right)}
\end{aligned}
$$

To reduce this integral to a more manageable form Matsuoka et al. introduce a zero range approximation with a finite range correction term ie.

$$
<\psi_{b}\left(\xi_{b}\right) \psi_{x}\left(\xi_{x}\right)\left|V_{b x}\left(\overrightarrow{\mathbf{r}}_{b x}\right)\right| \psi_{a}\left(\overrightarrow{\mathbf{r}}_{b x}, \xi_{b}, \xi_{x}\right)>\sim D_{0} f\left(r_{b x}\right)
$$




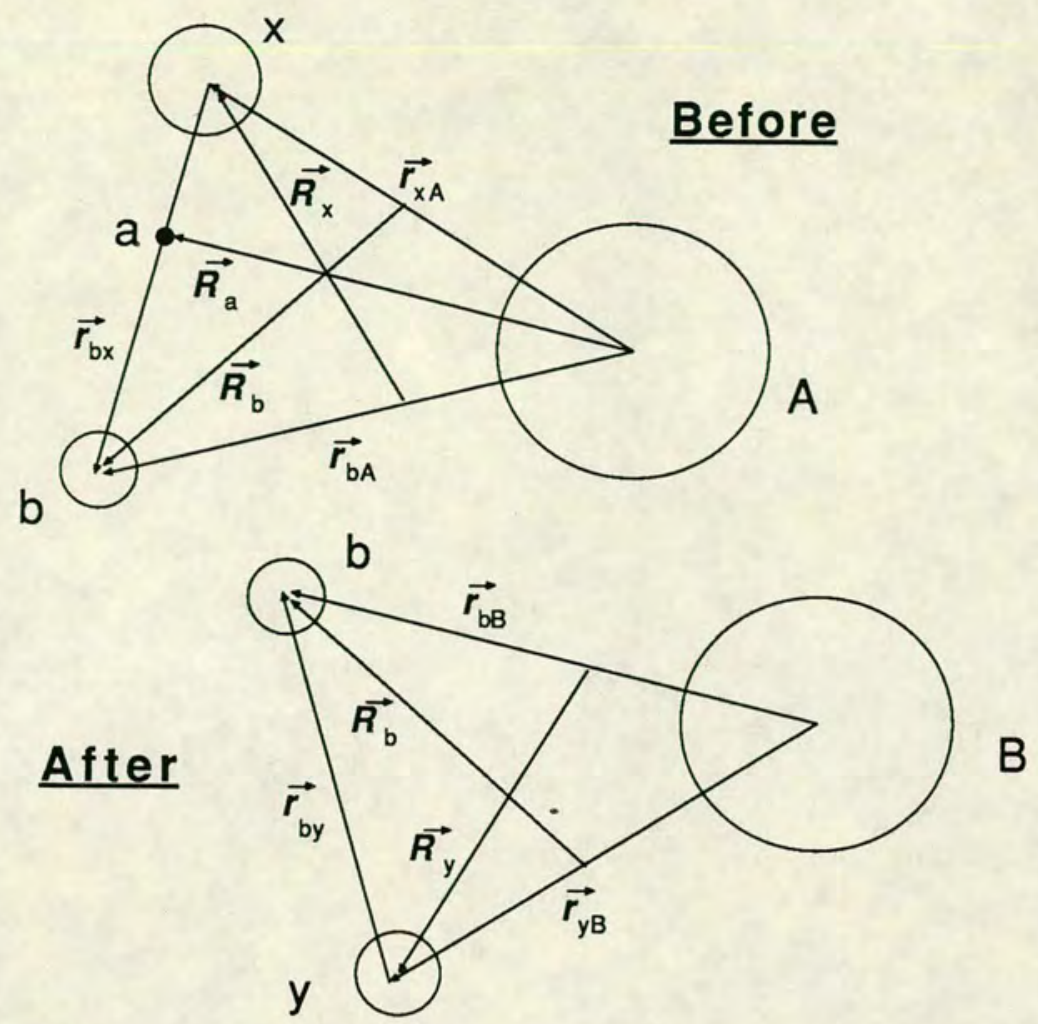

Figure 5.44: Coordinate system for P.W.B.A calculations

with $f\left(r_{b x}\right)$ a Yukawa-type range function of the form:

$$
f\left(r_{b x}\right)=\frac{\beta^{2} e^{-\beta r_{b x}}}{4 \pi r_{b x}} \quad \text { with } \beta \equiv \text { range parameter of interaction }
$$

This allows the transition matrix to be rewritten in the form:

$$
T_{\text {post }}^{P W}=\int d \overrightarrow{\mathbf{R}}_{a} e^{i\left(\overrightarrow{\mathbf{Q}} \cdot \overrightarrow{\mathbf{R}}_{a}\right)} \int d \overrightarrow{\mathbf{r}}_{b x} e^{-i\left(\overrightarrow{\mathbf{q}}^{\prime} \cdot \overrightarrow{\mathbf{r}}_{b x}\right)} f\left(r_{b x}\right)
$$

where $\overrightarrow{\mathbf{Q}}$ is the momentum transfer to the target and $\overrightarrow{\mathbf{q}}$ is the relative momentum of the particles $\mathrm{b}$ and $\mathrm{x}$ within $\mathrm{a}$ and are given by:

$$
\overrightarrow{\mathbf{Q}}=\overrightarrow{\mathbf{k}}_{a}-\overrightarrow{\mathbf{k}}_{b}-\overrightarrow{\mathbf{k}}_{x} \quad \overrightarrow{\mathbf{q}}=\frac{m_{x}}{m_{a}} \overrightarrow{\mathbf{k}}_{b}-\frac{m_{b}}{m_{a}} \overrightarrow{\mathbf{k}}_{x}
$$

The integral over $\overrightarrow{\mathbf{R}}_{a}$ in eq. 5.3 is a delta function for limits 0 to $\infty$ and Matsuoka et al. envoke the strong absorption concept to introduce a radial cut-off distance $\mathrm{R}_{c}$, within which all particles are absorbed. This allows an explicit expression for the integral to be found:

$$
\int_{R_{a} \geq R_{c}}^{\infty} d \overrightarrow{\mathbf{R}}_{a} e^{i\left(\vec{Q} \cdot \overrightarrow{\mathbf{R}}_{a}\right)}=-4 \pi R_{c}^{2} \frac{j_{1}\left(Q R_{c}\right)}{Q}
$$

where $j_{1}\left(Q R_{c}\right)$ is the first spherical Bessel function. Using this explicit expression and the Yukawa type range functions, they produce satisfactory fits for small 
momentum transfers. They also achieved a fit to the wide angle diffraction structure by adjusting the value of the $R_{c}$ parameter.

If direct break-up of the ${ }^{7} \mathrm{Li}$ projectile does occur in the Coulomb field of the nucleus it may be possible to view any final state interactions as simple elastic scattering of the participant from the target. The small $Q$ value $(-2.47 \mathrm{MeV})$ for break-up of the ${ }^{7} \mathrm{Li}$ into an alpha and triton suggests that only a soft interaction is required for excitation of this channel. The presence of beam velocity bumps in the projected $\mathrm{E}_{\alpha}$ spectrum for the $\alpha$-t quasi-elastic events (eg. see fig.5.28) and the lack of target excitation in the collisions with the heavier targets may be indicative of rather soft collisions between the projectile and targets, especially at forward angles. These ideas suggest that a simple P.W.B.A. treatment of the data along the lines outlined by Matsuoka is worth pursuing as a first step in modelling these final state interactions. The P.W.B.A. calculation performed in this work followed the treatment of [Ma80] but with a different form of the internal wavefunction for the projectile $\psi_{a}\left(\overrightarrow{\mathbf{r}}_{b x}\right)$. It is known that for heavier projectiles a full finite range calculation is necessary to produce any realistically accurate fits to the experimental data [Ba84]. Expanding $\Psi_{a}\left(\overrightarrow{\mathbf{r}}_{b x}, \xi_{b}, \xi_{x}\right)$ in eq. 5.3 into $\psi\left(\overrightarrow{\mathbf{r}}_{b x}\right) \psi_{b}\left(\xi_{b}\right), \psi_{x}\left(\xi_{x}\right)$, (assuming a spectroscopic factor of 1 for representing ${ }^{7} \mathrm{Li}$ as an alpha and a triton) the integration over the internal co-ordinates can be performed to give:

$$
T_{\text {post }}^{P W}=\int d \overrightarrow{\mathbf{R}}_{a} e^{i\left(\overrightarrow{\mathbf{Q}} \cdot \overrightarrow{\mathbf{R}}_{a}\right)} \int d \overrightarrow{\mathbf{r}}_{b x} V_{b x}\left(\overrightarrow{\mathbf{r}}_{b x}\right) \psi_{a}\left(\overrightarrow{\mathbf{r}}_{b x}\right) e^{-i\left(\mathbf{q} \cdot \overrightarrow{\mathbf{r}}_{b x}\right)}
$$

The integral over $\overrightarrow{\mathbf{r}}_{b x}$ in equ 5.6 was performed numerically by expanding the ground state wave function $\psi_{a}\left(\overrightarrow{\mathbf{r}}_{b x}\right)$ of the clusters $\mathrm{b}$ and $\mathrm{x}$ in the projectile, in terms of spherical harmonics. The plane wave $e^{-i\left(\vec{q} \cdot \vec{r}_{b z}\right)}$ is also expanded in terms of its angular momentum components. In the work by El Lithi [E187] it is shown that this results in the following expression for the integral of interest:

$$
\int d \overrightarrow{\mathbf{r}}_{b x} V_{b x}\left(\overrightarrow{\mathbf{r}}_{b x}\right) \psi_{a}\left(\overrightarrow{\mathbf{r}}_{b x}\right) e^{-i\left(\overrightarrow{\mathbf{q}} \cdot \vec{r}_{b x}\right)}=\frac{4 \pi}{\sqrt{3}} \int d r_{b x} j_{1}\left(q r_{b x}\right) R\left(r_{b x}\right) V_{b x}\left(r_{b x}\right) r_{b x}^{2}
$$

This was then evaluated numerically using Simpsons rule integration to a radius of $\mathrm{r}_{b x}=15 \mathrm{fm}$. The potential $\mathrm{V}_{b x}$ was separated into its nuclear and Coulomb components. The nuclear part was modelled with a Woods Saxon potential of the form:

$$
V_{b x}^{n u c l}\left(r_{b x}\right)=V_{0} \frac{1}{\left(e^{x}+1\right)} \text { and } x=\left(\frac{r_{b x}-R_{0}}{a_{0}}\right)
$$




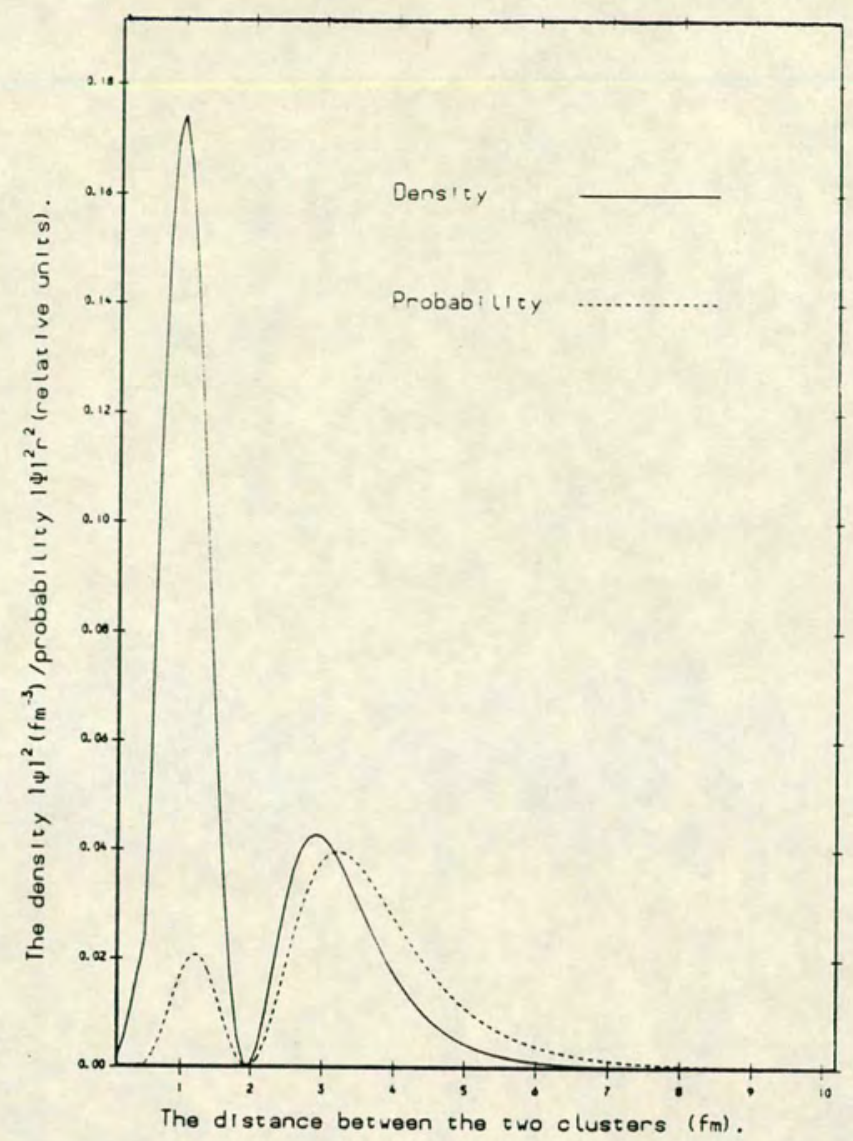

Figure 5.45: Radial wave function of bound $\alpha$-t system in ${ }^{7} \mathrm{Li}$.

with the parameters $\mathrm{V}_{0}=-91.2 \mathrm{MeV}, \mathrm{R}_{0}=2.05 \mathrm{fm}$ and $\mathrm{a}_{0}=0.7 \mathrm{fm}$, taken from [Ku72]. The Coulomb component was taken to be:

$$
V_{b x}^{\text {Coul }}\left(r_{b x}\right)= \begin{cases}\frac{Z_{1} Z_{2} e^{2}}{2 R}\left(3-\frac{r^{2}}{R^{2}}\right) & r \leq R \\ \frac{Z_{1} Z_{2} e^{2}}{r} & r>R\end{cases}
$$

The results of the calculation were rather insensitive to the value used for $R$. The final value was chosen as $R=3.6 \mathrm{fm}$, calculated from:

$$
\mathrm{R}=1.2\left(\mathrm{~A}_{1}^{\frac{1}{3}}+\mathrm{A}_{2}^{\frac{1}{3}}\right)
$$

The $R\left(r_{b x}\right)$ was the radial wave function of the $\alpha$-t system with $\ell=1$ in this potential and is shown in fig. 5.45. This was calculated [El87] using the code BOUND [Sm69] with binding energy $2.47 \mathrm{MeV}$ and relative angular momentum of 1 unit of $\hbar$. As with [Ma80] the integral over $\overrightarrow{\mathbf{R}}_{a}$ in eq. 5.6 was performed by introduction of a strong absorption cut-off radius $\mathrm{R}_{c}$ calculated from [Ba80b]

$$
R_{c}=R_{\text {proj }}+R_{\text {targ }}+3 \text { fm with } R_{i}=1.12 A^{1 / 3}-0.94 A^{-1 / 3}
$$

The triple differential cross-section may then be calculated from [Ma80]:

$$
\frac{d^{3} \sigma}{d \Omega_{b} d \Omega_{x} d E_{x}}=\frac{2 \pi}{\hbar^{2}} \frac{m_{a}}{k_{a}}\left|T_{f i}\right|^{2} \rho\left(E_{x}\right)
$$


where $T_{f i}$ is the transition matrix previously calculated and $\rho\left(E_{x}\right)$ is the density of states factor given by [Oh65]:

$$
\rho\left(E_{x}\right)=\frac{h^{-6} m_{b} m_{x} m_{A} p_{b} p_{x}}{\left(m_{b}+m_{A}\right)+m_{b} \frac{\left(\overrightarrow{\mathbf{p}}_{x}-\overrightarrow{\mathbf{P}}\right) \cdot \overrightarrow{\mathbf{p}}_{b}}{p_{b}^{2}}}
$$

The double differential cross-section was then found by integration of eq. 5.8 over the experimentally allowed values of $E_{x}$.

Examples of the resulting cross-sections are shown in figures 4.20, 4.22 4.24, $4.26,4.28,4.46$ to 4.49 and 4.50 to 4.56 . These show the quasi-elastic data for forward and backward angles of the alpha particle for varying $\theta_{t}, \theta_{\alpha}$ and $\phi_{\alpha}$. The fits have been individually normalised to the data points. At the smaller angular separations the calculations produce fits which are not too disimilar to the data. At larger angular separations however, (figs.4.47 and 4.49) the P.W.B.A. calculations completely mispredict the measured distributions, being peaked at much larger values of $\theta_{t}$ than the data. This is hardly surprising since the assumptions made in the P.W.B.A. calculations, of small distortions, are not valid for such large momentum transfers. The calculations peak at values for $\theta_{t}$ reflecting the value of $\theta_{\alpha}$. This is an understandable consequence of the plane wave treatment where an outgoing alpha plane wave, at a specific angle, requires the outgoing triton to peak at a position on the opposite side of the beam determined from conservation of momentum. Although the calculations do not reproduce the shape of the data for fixed $\theta_{t}$ and varying $\theta_{\alpha}$, it is interesting to note that the PWBA calculations reproduce the decrease in the out-of-plane intensity variation for increasing $\theta_{\alpha}$ and the increase for increasing $\theta_{t}$ (see figs.4.50 to 4.56). This may indicate that this behaviour arises from phase space constraints rather than any effects due to the properties of the mechanism involved.

The choice of the post form P.W.B.A. may not be the best way of approaching these final state interactions because they are explicitly ignored in the approximations to the outgoing wave functions. In this representation the final $\mathrm{T}$ matrix separates into a part dependant on the momentum transfer to the target and another part dependant on the internal momentum of the projectile (see equ. 5.6). No mention is made of any participant-target interactions $\mathrm{V}_{x A}$ in the outgoing channel. In the prior form representation however, this interaction is explicitly included in the calculation instead of $\mathrm{V}_{b x}$. The final $\mathrm{T}$ matrix then takes the 
form:

$$
T_{p r i o r}^{P W}=\int d \overrightarrow{\mathbf{r}}_{x A} e^{i\left(\vec{Q}^{\prime} \cdot \overrightarrow{\mathbf{r}}_{x A}\right)} V_{x A}\left(\overrightarrow{\mathbf{r}}_{x A}\right) \int d \overrightarrow{\mathbf{r}}_{b x} \psi_{a}\left(\overrightarrow{\mathbf{r}}_{b x}\right)
$$

which is a product of the internal momentum distribution of the projectile and the $\mathrm{T}$ matrix for the interaction of the participant with the target. It seems likely that this P.W.B.A. representation should be more successful in describing any final state interactions between the participant and target but the integral over $\overrightarrow{\mathbf{r}}_{x A}$ would prove to be more difficult than in the POST case.

In summary, post form P.W.B.A. calculations similar to those of [Ma80] have been performed. These included explicitly the internal momentum distribution of the fragments within the projectile by numerical integration over the relative radial wave function of the $\alpha$-t confined within a Woods Saxon potential. The calculations produce a reasonable fit to the data for small momentum transfers to the target but fail to reproduce the observed experimental distributions for large angular separations between the outgoing fragments.

\subsection{Factorisation and Uncorrelated Processes}

In their work on the fragmentation of the $10-12 \mathrm{MeV} / \mathrm{A}{ }^{15} \mathrm{~N}$ on ${ }^{159} \mathrm{~Tb}$ Bhowmik et.al. [Bh82] identified two processes contributing to the $\alpha$-heavy ion coincidence cross-section. These were:

- sequential alpha decay of excited projectile like fragments (PLF).

- and uncorrelated emission of fast light particles at an early stage of the reaction.

Fukuda et al [Fu83] in experiments with a similar system $\left(\sim 15 \mathrm{MeV} / \mathrm{A}{ }^{15} \mathrm{~N}\right.$ on ${ }^{93} \mathrm{Nb}$ ) have also identified a third component; sequential alpha emission from target like fragments (TLF). This is also implied by Bhowmik et.al. [Bh81] for $\alpha$-HI $(\mathrm{Z}>5)$ correlations for $10 \mathrm{MeV} / \mathrm{A}{ }^{15} \mathrm{~N}$ on ${ }^{58} \mathrm{Ni}$, these being peaked with the alpha around the recoiling target direction. This experiment recorded no evidence for sequential decay of the PLF. Goldhoorn et.al. [Go84] have since 
shown that the lack of distinction of sequential PLF decay in this latter experiment was due to the poor angular resolution between the detected fragments and that the assumed uncorrelated events in this experiment were infact mainly from this sequential PLF decay process. They did however accept that when the fragments were detected on opposite sides of the beam, the high level density in the $(\alpha+\mathrm{HI})$ systems being studied, made it impossible to rule out an uncorrelated contribution to the coincidence cross- section. They concluded that this uncorrelated component, if present would be significant only at larger angular seperations between the fragments [Go84b].

For the region with the alpha and H.I. on opposite sides of the beam Bhowmik et al [Bh82] were able to decompose the coincidence cross-section into a product of the alpha inclusive and H.I. inclusive cross-sections:

$$
\frac{d^{4} \sigma}{d \Omega_{\alpha} d E_{\alpha} d \Omega_{H I} d E_{H I}}=K\left(\frac{d^{2} \sigma}{d \Omega_{\alpha} d E_{\alpha}}\right)_{i n c l}\left(\frac{d^{2} \sigma}{d \Omega_{H I} d E_{H I}}\right)_{i n c l}
$$

The factor $\mathrm{K}$ was found to be rather independant of ejectile type. This factorisation was taken as an indication of a two step mechanism in which the alpha particle was first emitted in the early stages of the reaction via a projectile fragmentation process with the remaining fragment undergoing a further (deep inelastic) interaction with the target. (i.e. the alpha is a spectator and the $\left({ }^{15} \mathrm{~N}-\right.$ $\alpha$ ) fragment is a participant). The outcome of this secondary interaction would then be independant of the alpha particle. In their analysis they assume that the quasi-elastic events (eg. as observed for the ${ }^{11} \mathrm{~B}+\alpha$ ) arise mainly from PLF sequential decay and contribute little to the wide angle uncorrelated events. Indeed the alpha angular distribution for the ${ }^{11} \mathrm{~B}+\alpha$ quasi-elastic channel is strongly focused around the ${ }^{11} \mathrm{~B}$ direction with little contribution to the events with the alpha on the opposite side of the beam. In this region the quasi-elastic alpha distribution falls much faster than the inclusive alpha angular distribution used for the factorisation. In the experiment outlined in this thesis the quasi-elastic channel plays an important role in the total $\alpha$ - $t$ coincidence crosssection, even at large angular separations between the fragments. In a similar vein to the analysis of Bhowmik et al it is interesting to determine the degree of correlation of these $\alpha$-t events recorded in the present experiment. One could propose that if the alpha and/or triton were undergoing deflections via final state target-fragment interactions then these may destroy any correlation be- 
tween the outgoing alpha and triton. If this is the case the double differential cross-section should be factorisable as in equation 5.9. Bhowmik et.al. [Bh82] point out that if the triton and alpha formation processes are truely uncorrelated then the angular distributions should show no out of plane dependance. In this experiment the out-of-plane behaviour was directly measured and so the data provides a strict test of the correlation of the outgoing alpha and triton over a wide angular range.

To perform this analysis the data points were plotted according to their spherical polar angle from the beam axis. A weak out-of-plane correlation will mean that this should be the only angle of importance with respect to the reaction plane defined by the triton detector and the beam direction. Fig. 5.46 to 5.53 show the data plotted in this manner for a few selected values of the triton angle for all three targets. These illustrate the general trend of the data. Symbols of the same type correspond to the data from pixels of the same F strip (ie. to data moving out of the reaction plane) and are connected together by straight lines. The dashed lines are the functions

$$
K \times\left(\frac{d \sigma\left(\theta_{t}\right)}{d \Omega_{t}}\right)_{i n c l} \times\left(\frac{d \sigma}{d \Omega_{\alpha}}\right)_{i n c l}
$$

which are the inclusive alpha yields normalised by the value of the corresponding inclusive triton yield at the specific $\theta_{t}$ (sph deg.). The inclusive alpha yields are the least squares fits shown in fig.4.7, 4.8 and 4.9. The values of $\mathrm{K}$ are as shown in the figures. They are much smaller than the corresponding values from the ${ }^{15} \mathrm{~N}$ work where $\mathrm{K} \sim 0.2-0.5$. This reflects the fact that in this case the quasielastic channel is only a small contributor to the inclusive cross-sections. It can be seen from these spectra that as $\theta_{t}$ increases, all three targets exhibit a strong dependance on the out-of-plane angle whether it be in azimuthal or spherical co-ordinates [Bh81]. This would seem to preclude the factorisation of the data into the product of the inclusive yields. It is interesting to note however that the shape of the coincident in-plane data for the ${ }^{120} \mathrm{Sn}$ target is very similar to the inclusive alpha distribution ${ }^{1}$ (see fig.5.49 to fig.5.51). This is not the case for the ${ }^{12} \mathrm{C}$ target, with the inclusive alpha yield falling off more rapidly than the quasi-elastic in-plane coincidence distribution. Although it seems from

\footnotetext{
${ }^{1}$ The in-plane data corresponds to moving from the first data point on one $\mathrm{F}$ strip identified
} by its symbol type, to the first data point on the next $\mathrm{F}$ strip and so on. 


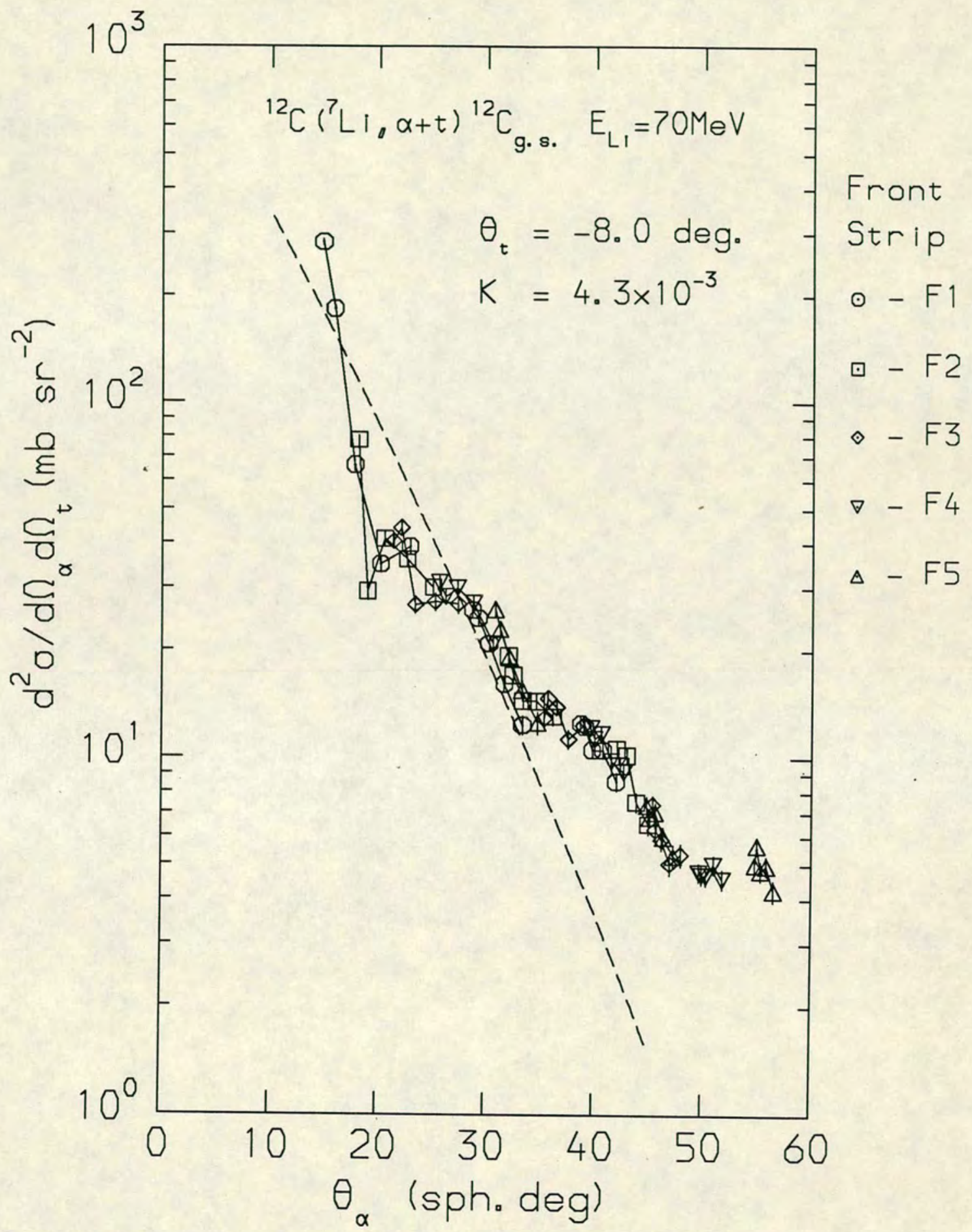

Figure 5.46: ${ }^{12} \mathrm{C}$ quasi-elastic $\alpha$-t cross section plotted as a function of the alpha's spherical polar angle. Dotted line is the normalised alpha inclusive yield. Data from the same F strip are plotted with the same symbol. 


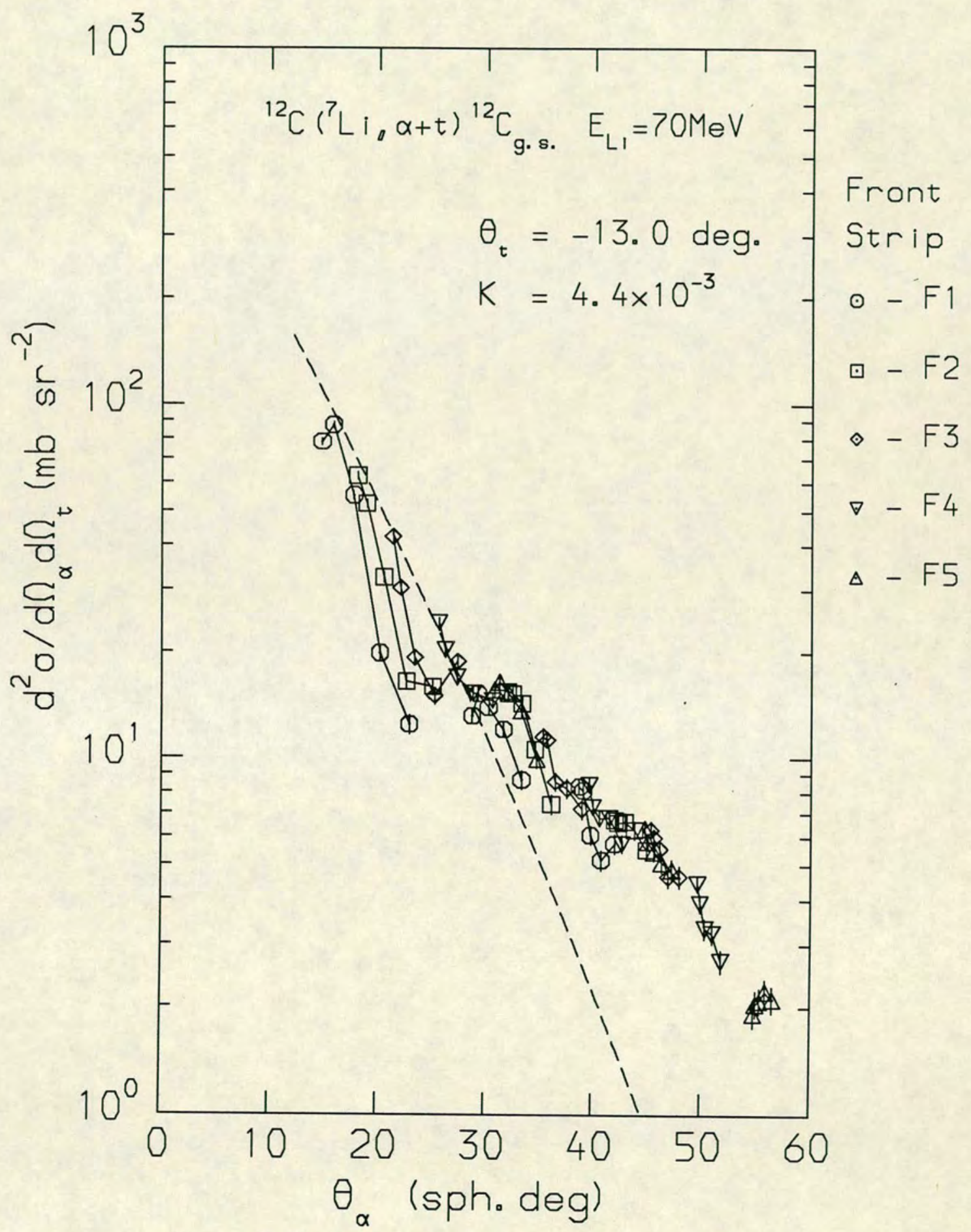

Figure 5.47: Same as fig. 5.46 but with $\theta_{t}=-13.0^{\circ}$ 


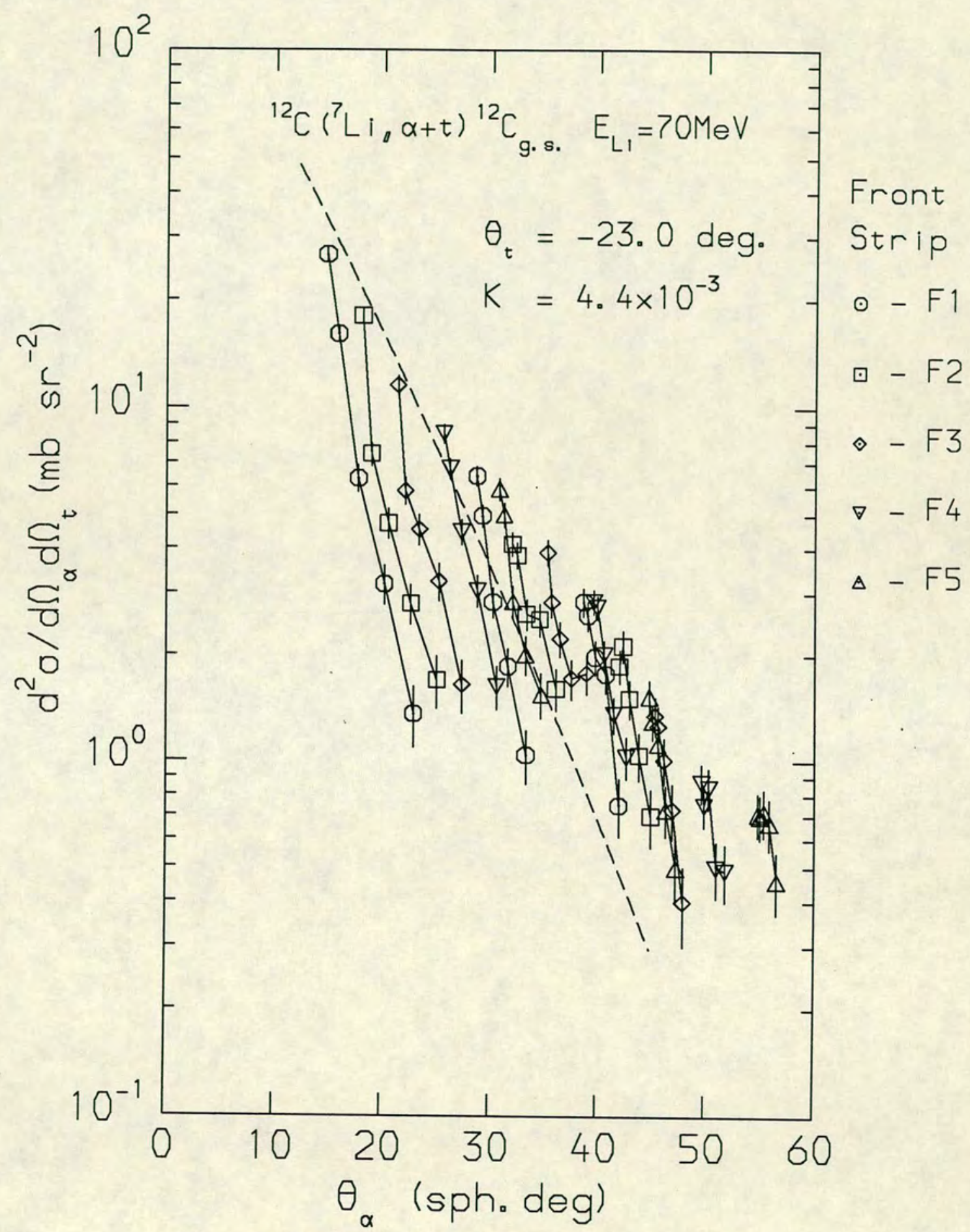

Figure 5.48: Same as fig. 5.46 but with $\theta_{t}=-23.0^{\circ}$ 


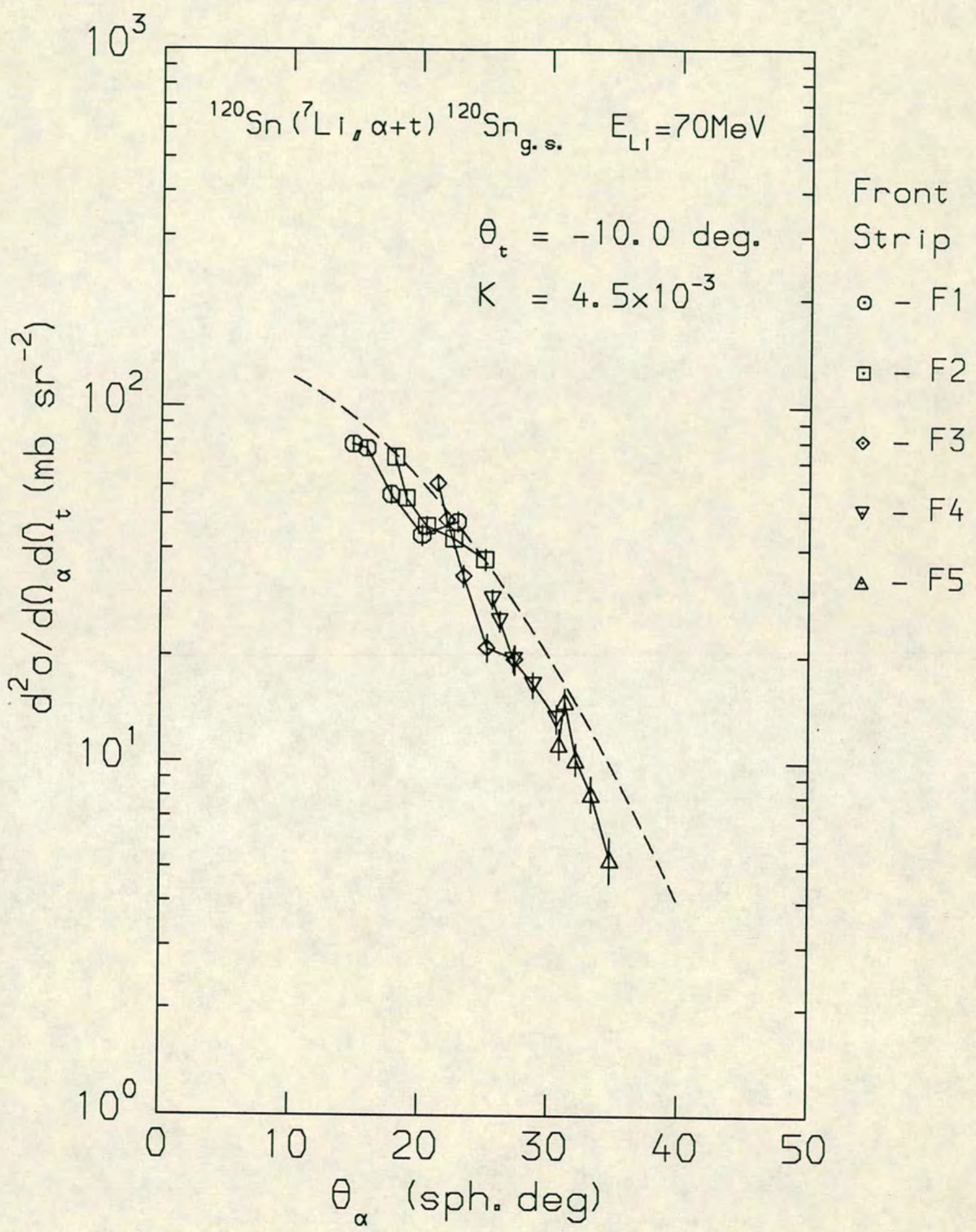

Figure 5.49: ${ }^{120} \mathrm{Sn}$ quasi-elastic $\alpha$-t cross section plotted as a function of the alpha's spherical polar angle. Dotted line is the normalised alpha inclusive yield. 


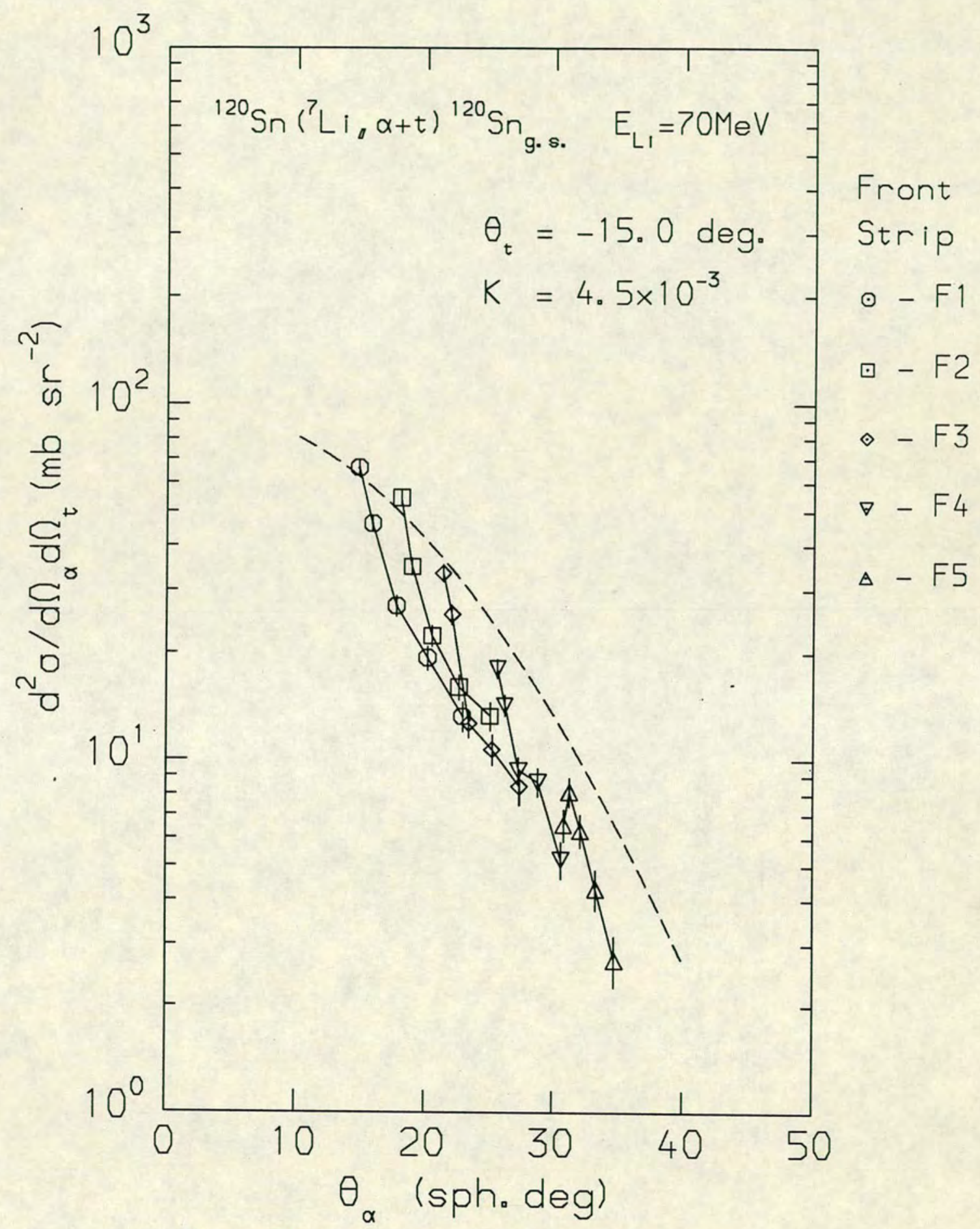

Figure 5.50: Same as fig. 5.50 but with $\theta_{t}=-15.0^{\circ}$ 


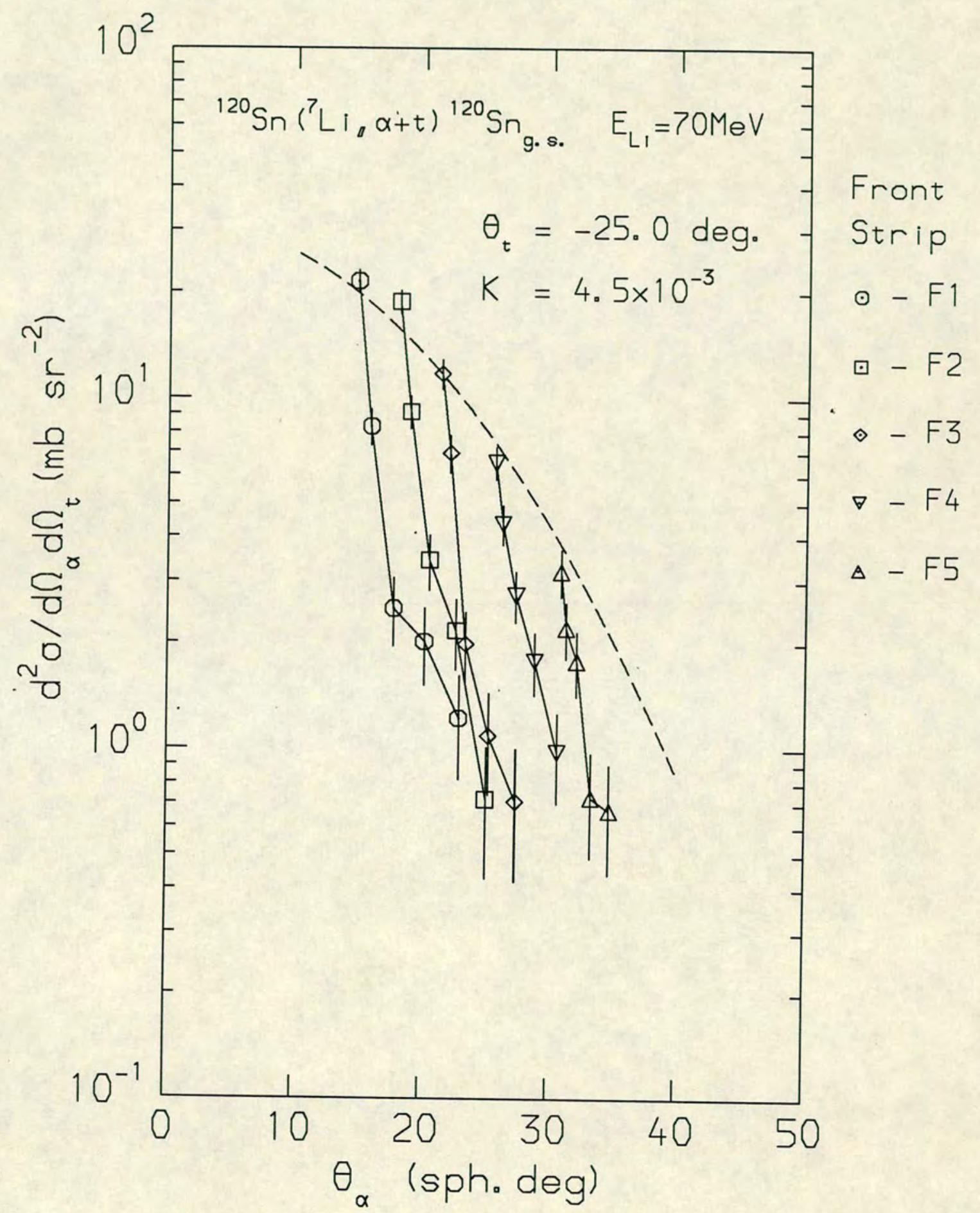

Figure 5.51: Same as fig. 5.50 but with $\theta_{t}=-25.0^{\circ}$ 


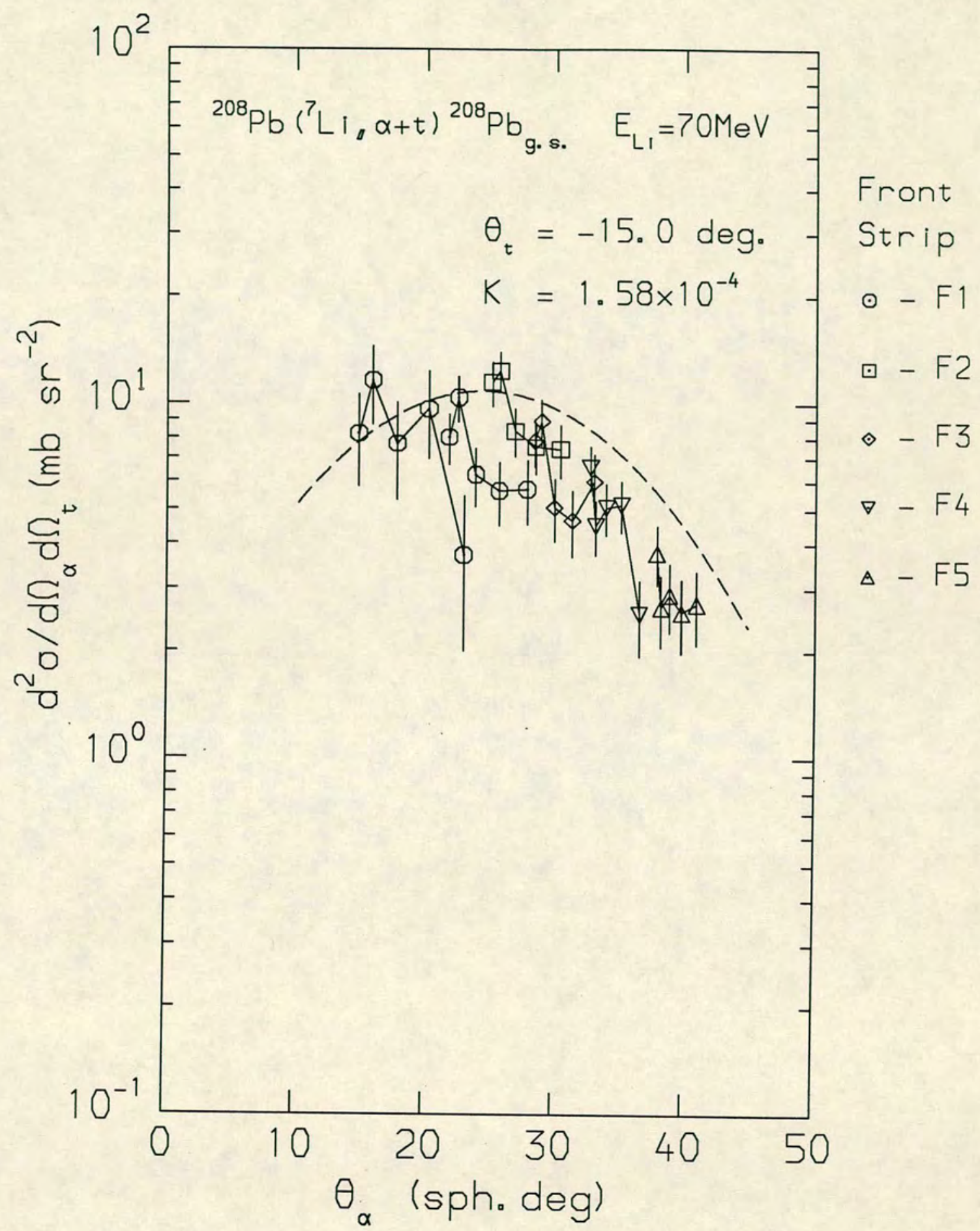

Figure 5.52: ${ }^{208} \mathrm{~Pb}$ quasi-elastic $\alpha$-t cross section plotted as a function of the alpha's spherical polar angle. Dotted line is the normalised alpha inclusive yield. 


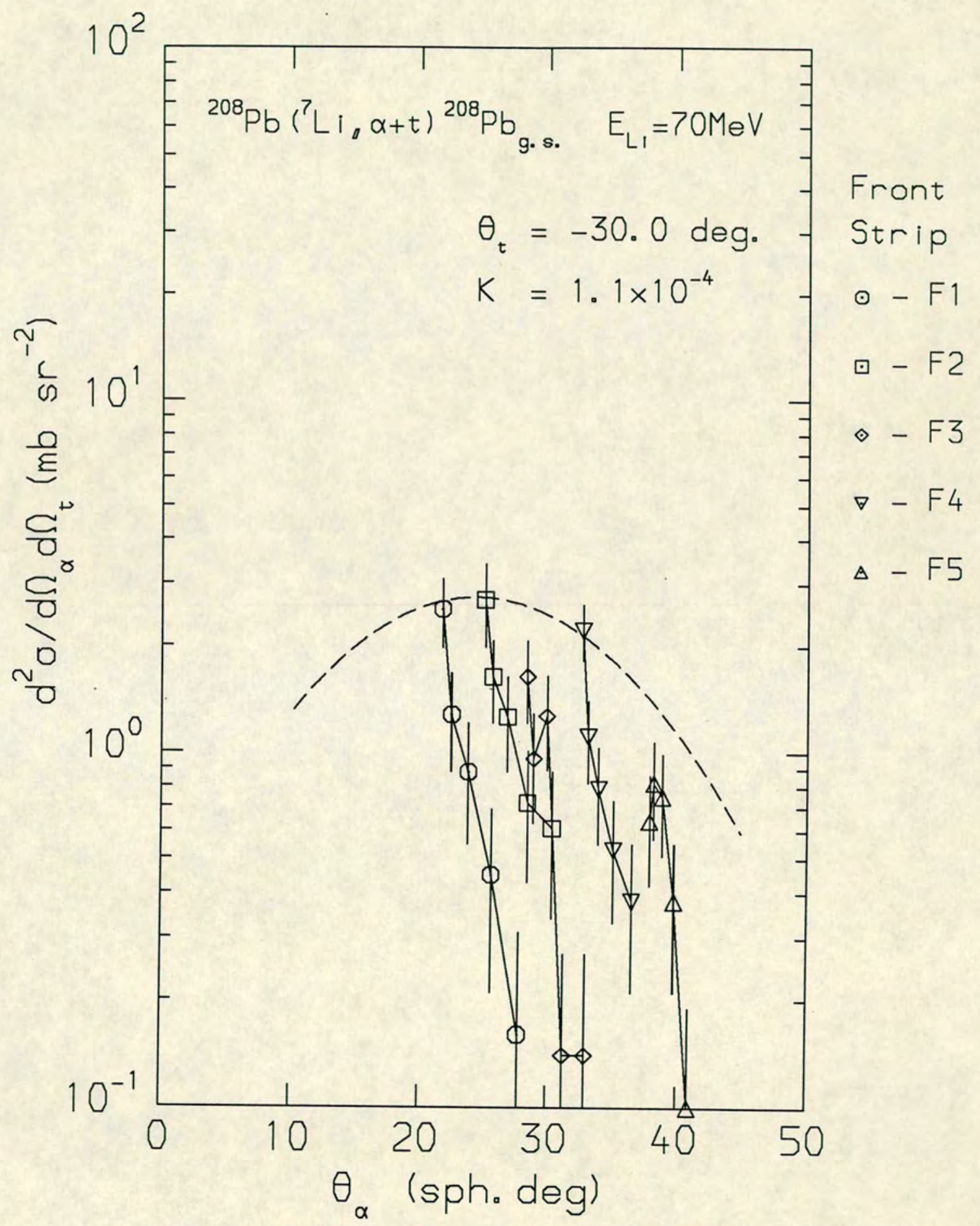

Figure 5.53: Same as fig. 5.53 but with $\theta_{t}=-30.0^{\circ}$ 
this, that the ${ }^{12} \mathrm{C}$ data cannot be factorised, the insensitivity of the fall off in the in-plane distribution to the triton angle may indicate that an uncorrelated process is occuring. If this process did not contribute significantly to the inclusive yield then one would not expect a factorisation in terms of the triton and alpha inclusive yields to be possible. The statistical quality of the ${ }^{208} \mathrm{~Pb}$ data makes it difficult to make any statement about the factorisation procedure except that the in-plane enhancement seen with the other targets is also present with the ${ }^{208} \mathrm{~Pb}$.

If the alpha and triton were emitted totally uncorrelated the dashed lines plotted on the figures would exactly reproduce the data with one fixed value of $\mathrm{K}$. As mentioned, this is only the case for the in-plane ${ }^{120} \mathrm{Sn}$ data where the experimental results are well reproduced for all $\theta_{t}$ by the factorisation technique with $\mathrm{K}=4.5 \times 10^{-3}$. It is possible that this factorisation in the reaction plane but strong correlation out-of-plane arises from a reaction mechanism strongly dependant on the angular momentum involved in the reaction. One possibility is the transfer of one of the fragments to a high spin, highly excited state in the $\left({ }^{120} \mathrm{Sn}+\right.$ frag. $)$ compound system with a rapid re-emission of the fragment. This re-emission would be from a system with its total spin aligned perpendicular to the reaction plane giving a strong dependance out of this plane. In $\left({ }^{7} \mathrm{Li} \alpha, \mathrm{xn} \gamma\right)$ experiments Davinson [Da87] and Utsunomiya et.al. [Ut83] have measured a distinct in to out-of-plane anisotropy for the coincident $\gamma$ ray intensity. This indicates that an alignment of the emitting compound system in a direction perpendicular to the reaction plane is indeed possible. A similar absorption reemission process has been identified in the lighter system of ${ }^{12} \mathrm{C}+{ }^{20} \mathrm{Ne}$ at 7.85 $\mathrm{MeV} / \mathrm{A}$ [Si87]. One would expect however, that the re-emission of a complex nucleus from such a heavy target would be strongly Coulomb suppressed, with the excited compound nucleus decaying mainly by neutron emission. The high density of states at these large excitations precludes the identification of such a reaction mechanism through the projected energy spectra of the spectator or participant, but the branching ratio for alpha or triton decay to the ground state of ${ }^{120} \mathrm{Sn}$ may be calculated using a Hauser-Feshbach approach. This would give an idea of the expected strength in this channel which could be compared to the experimentally observed cross-section. It does however seem strange that this type of process should leave the residual ${ }^{120} \mathrm{Sn}$ in its ground state with no 
evidence for any population of of the low excited states of ${ }^{120} \mathrm{Sn}$.

A reaction mechanism whereby one of the fragments experiences an interaction with the target and is deflected to the opposite side of the beam may also produce a similar out-of-plane correlation. This has been shown in semi-classical trajectory calculations performed by El-Lithi [El87]. In these calculations the full three body forces between all three particles in the final channel were explicitly calculated for each step along a classical trajectory for the $\alpha+t+{ }^{208} \mathrm{~Pb}$ system. No target or fragment excitation was allowed but partial fusion and orbiting were a natural consequence of the method used. The nuclear field was modelled by a Woods Saxon potential with real and imaginary parts, the former being used in the trajectory calculations and the latter in the calculations of the absorption of particles. The Coulomb forces between all the particles were also taken into account. The Sommerfeld parameter for this system at $70 \mathrm{MeV}$ bombarding energy is $\sim 12$ which makes such a semi-classical picture reasonably valid. These calculations have had remarkable success (considering their basic simplicity) in reproducing the angular dependance and absolute magnitude of the experimental cross-section for direct break-up. They also predict that as the alpha and the triton are detected at larger angles, there will be a strong probability that they are deflected to opposite sides of the beam with an increasing enhancement in-plane as the detection angles increase. The experimental data for this system do indeed show an enhanced in-plane behaviour as the detection angles increase and it is conceivable that the experimental data agrees with this prediction. However, the poor statistical quality of the ${ }^{208} \mathrm{~Pb}$ data make a definite confirmation impossible. Unfortunately these calculations have only been performed for this one system and the question of the in and out-of-plane dependance was not fully investigated. It would be extremely interesting to extend this type of calculation to the ${ }^{120} \mathrm{Sn}$ data where the Sommerfeld parameter is still relatively large $(\sim 7.5)$ and more reliable experimental data are available. It would also be interesting to investigate more fully the out-of-plane dependance with one particle direction fixed and the other varying to allow a more direct comparison with experiment. Extension of these semi-classical calculations to the ${ }^{7} \mathrm{Li}+{ }^{12} \mathrm{C}$ system would be rather unrealistic due to the small Sommerfeld parameter (in this case 0.9 ). 
One final interesting point concerning these semi-classical calculations is that they predict a distinct peak in the contribution of the partial fusion channel to the inclusive alpha particle spectrum at $\theta_{\alpha}=25^{\circ}$. In other experiments this channel is known to be one of the strongest contributors to the inclusive beam velocity fragments spectra eg.[Wi79], [Ne79], [Ut83]. The experimental inclusive alpha yield measured in this work peaks exactly in this angular range. This gives increased confidence in the philosophy behind these semi-classical trajectory calculations.

In summary this section has attempted to answer the question of whether or not the alpha and triton particles were correlated with one another when they were detected at large angular separations. This question was addressed only for the quasi-elastic component of the $\alpha$-t coincidence cross-section. This puts very stringent kinematic constraints on the processes being investigated. Not surprisingly therefore it was found that the quasi-elastic events from the ${ }^{12} \mathrm{C}$ target could not be factorised into the product of the individual particle inclusive cross-sections in either the out-of-plane or in-plane directions. In contrast to this behaviour it was found that the quasi-elastic events from the ${ }^{120} \mathrm{Sn}$ target could be approximately factorised into the inclusive yields in the in-plane direction but still maintained a correlation in the out-of-plane direction. This behaviour was predicted from a semi-classical trajectory calculation taking into account the full three body forces involved in the interaction and is not inconsistent with final state interations between the fragments and the target.

\subsection{Conclusion}

This section will present a final, quick review of the conclusions reached in each chapter of this thesis.

The experiment involved the use of a new type of solid state particle detector to record coincident fragments from the break-up of $70 \mathrm{MeV}{ }^{7} \mathrm{Li}$ on ${ }^{12} \mathrm{C}{ }^{120} \mathrm{Sn}$ and ${ }^{208} \mathrm{~Pb}$. These were known as strip detectors and their properties and general behaviour were investigated before they were used for the collection of experi- 
mental data. Their leakage current, energy resolution and detection efficiency were measured and found to be acceptable for use in the experiment. On certain detectors there was a time dependant limit on the breakdown bias which increased with time under applied bias. This was important when full depletion of the detectors was required since it limited the thickness of silicon that could be depleted. Little evidence for 'normal' charge sharing was found for adjacent strips but an anomalous polarity charge sharing effect between strips was identified for particles entering the detector in the volume between the implantation regions of neighbouring strips. This was attributed to charge build up on the $\mathrm{SiO}_{2}$ passivation layer on the detector surface leading to a local field reversal in the interstrip region. A computer simulation of this effect produced excellent agreement with the proposed model.

At the present time the behaviour of these strip detectors tends to be more characteristic of the individual detector rather than the different manufacturers. It is presumed that the reproducibility of strip detector manufacture will improve as more experience is gained with them. This was identified as one of the areas for future development to ensure that strip detectors achieve their full potential in future nuclear physics experiments. Other areas identified for development were the associated electronics and the philosophy behind the the experimental design. All these are currently being investigated and it seems reasonable to predict that silicon strip detectors will become a common tool in nuclear physics experiments in the near future.

The experiment performed with these detectors was the measurement of the wide-angle and out-of-plane coincidence cross-section for the $\alpha$-t break-up channel of $70 \mathrm{MeV}^{7} \mathrm{Li}$ on various targets. The experimental set-up incorporated the strip detectors in an alpha particle telescope with the coincident tritons being detected by a standard $\triangle \mathrm{E}-\mathrm{E} \mathrm{SiLi}$ telescope. The electronic set-up used conventional NIM electronic units to record simultaneously the inclusive particle cross-sections and the exclusive coincidence cross-section.

The inclusive data for beam velocity tritons and alphas agreed well with previous inclusive data for the same systems. All the inclusive energy spectra exhibited the characteristic beam velocity bumps at forward angles and the in- 
clusive angular distributions from the ${ }^{12} \mathrm{C}$ and ${ }^{120} \mathrm{Sn}$ targets were the same as previously measured. The ${ }^{7} \mathrm{Li}+{ }^{208} \mathrm{~Pb}$ alpha inclusive cross-section however, was slightly different from the previous inclusive angular distributions, with a more pronounced peak just inside the grazing angle. The position of this peak was as predicted from a semi-classical trajectory calculation. The inclusive beam velocity cross-sections were found to contain a significant fraction of the total reaction cross-section so the identification of the processes leading to beam velocity fragments is important to the understanding of the reaction mechanisms at these energies.

The exclusive $\alpha-\mathrm{t}$ coincidence cross-section was measured at the same time as the inclusive cross-section. The angular resolution of the detector system meant that any underlying diffraction structure in the $\alpha-\mathrm{t}$ angular distributions was not detectable. A steady decrease of the coincidence cross-section with increasing angles was seen for all the targets used, although this was less steep inside grazing angle for the ${ }^{120} \mathrm{Sn}$ and ${ }^{208} \mathrm{~Pb}$ targets. A distinct in-plane enhancement was observed for all three targets and this became more pronounced as the angular separation between the outgoing fragments increased. For larger momentum transfers to the ${ }^{12} \mathrm{C}$ target, excitation of its excited states became apparent. For the ${ }^{120} \mathrm{Sn}$ and ${ }^{208} \mathrm{~Pb}$ targets the quasi-elastic component dominated the coincidence cross-sections, and even at large angular separations no evidence for target excitation was seen. The total integrated wide-angle yield of coincident $\alpha-t$ events was found to be a small contribution to the forward angled inclusive triton yield. It was proposed that the remaining cross-section originated from partial fusion of the alpha followed by proton emission or fission. Neither of these channels would have been detected in the particle- $\gamma$ ray coincidence experiments performed by other authors [Da87], [Ut85] to try and account for the missing triton inclusive yield. It is also possible that fragmentation with more than three particles in the outgoing channel contribute to the triton inclusive yield at these relatively high energies.

In an attempt to identify the underlying mechanisms producing the wide angle $\alpha-\mathrm{t}$ coincidence cross-section a number of approaches were considered. A Monte Carlo simulation programme was used to predict the effects of geometry enhanced detection efficiency on the projected energy spectra. This modelled 
break-up from all the known sequentially decaying states of ${ }^{7} \mathrm{Li}$ and produced projected energy spectra that were compared with the experimental spectra. Although the angular resolution of the detection system and the width of the excited states combined to make unambiguous identification of sequentially decaying higher excitation states rather difficult, it was generally seen that the experimental projected spectra did not exhibit any behaviour consistent with sequential decay. The shape and intensity of the experimental spectra showed no dramatic change in going from angular separations within the relevant kinematically allowed region to outside the allowed region for sequential decay. One exception to this was the sequential decay from the ${ }^{7} \mathrm{Li}_{4.63 \mathrm{MeV}}$ state. Although detection of this sequential channel was kinematically impossible for most of the angular settings of the detector system, it was clearly identified for those settings within the corresponding break-up cone. In general however, the behaviour of the projected energy spectra seemed to be incompatible with dominant contributions from any of the higher excited states of ${ }^{7} \mathrm{Li}$. Particle re-emission from excited states in ${ }^{15} \mathrm{~N}$ and ${ }^{16} \mathrm{O}$ was proposed as a possible mechanism producing the distinct peaks seen in the projected quasi-elastic alpha energy spectra with the ${ }^{12} \mathrm{C}$ target. The corresponding energies of the excitation levels in ${ }^{16} \mathrm{O}$ correlated with high excitation alpha cluster states recorded by other authors. Similar particle re-emission was assumed to be unlikely from the heavier targets due to the larger Coulomb barrier.

To try and model a reaction mechanism where free particles are scattered from the target after direct break-up of the ${ }^{7} \mathrm{Li}$ a simple plane wave calculation was performed. This generally followed a prescription applied successfully to lighter projectiles at higher energies. Although the absolute magnitude of the quasielastic coincidence cross-section was not correctly predicted, the P.W.B.A. calculation gave reasonable fits to the in-plane angular distributions for small momentum transfers to the target. Not surprisingly though, the calculations failed to fit the distributions at larger momentum transfers. The P.W.B.A. approach did manage to fit the observed enhancement in the quasi-elastic out-of-plane distributions for varying $\theta_{t}$ and $\theta_{\alpha}$. This probably shows the effect of phase space constraints on the coincidence cross-sections.

In an attempt to investigate if the outgoing alpha and triton directions were at 
all correlated with one another, the quasi-elastic coincidence yield was factorised into the product of the inclusive yields. This produced the surprising result that although the data showed some degree of in-plane correlation, there remained a strong out-of-plane correlation between the outgoing fragments. This outof-plane correlation increased as the angular separation between the outgoing fragments increased. This behaviour was predicted by a semi-classical trajectory calculation but the precise meaning of this dependance is not yet clear.

One of the objectives of this thesis was to try and identify the different processes leading to large angle scattering of the outgoing fragments. It was proposed that this may be due to a two step process where the projectile breaks-up in a direct interaction with the Coulomb and/or nuclear field of the target, and one (or both) of the fragments then undergo further interactions with the target. Possibly the only way to identify this type of process is the subtraction of the cross-section contributing from other processes. This may be difficult for lighter targets, but for ${ }^{120} \mathrm{Sn}$ and ${ }^{208} \mathrm{~Pb}$ where fragment re-emission should be negligible, this is a feasible approach. To convincingly account for the possible contributions from sequential decay from the higher lying states of ${ }^{7} \mathrm{Li}$, it seems necessary to perform an experiment to specifically measure the angular distributions of these states. Once this is done it would be a simple matter to fold these distributions into a Monte Carlo type calculation (similar to the one performed here) and subtract the resulting cross-sections from those presented in this thesis. Only in this way can one be sure that the wide angle cross-section is not contaminated by sequential decay. For the heavier targets it is difficult to propose any other mechanisms that could realistically contribute to the wide angle data other than fragmentation close to the target followed by further final state interactions.

It is clear that further work, both theoretical and experimental, will be necessary before a fuller understanding of the mechanisms contributing to the wide-angle cross-section can be achieved. 


\section{Appendix A}

\section{Strip Detector Solid Angles}

This appendix presents the solid angles of each pixel on the strip detector telescope. The vertical and horizontal angular width of each pixel is also given.

\begin{tabular}{||c|c||c|c||c|c||c|c||c|c||}
\hline \hline F1M5 & 3.58 & F2M5 & 3.55 & F3M5 & 3.49 & F4M5 & 5.59 & F5M5 & 5.23 \\
\hline 3.33 & 3.71 & 3.31 & 3.69 & 3.27 & 3.68 & 5.29 & 3.63 & 5.04 & 3.56 \\
\hline \hline F1M4 & 3.79 & F2M4 & 3.76 & F3M4 & 3.69 & F4M4 & 5.90 & F5M4 & 5.51 \\
\hline 3.33 & 3.85 & 3.31 & 3.84 & 3.27 & 3.82 & 5.29 & 3.77 & 5.04 & 3.69 \\
\hline \hline F1M3 & 3.96 & F2M3 & 3.92 & F3M3 & 3.85 & F4M3 & 6.15 & F5M3 & 5.73 \\
\hline 3.33 & 3.96 & 3.31 & 3.95 & 3.27 & 3.93 & 5.29 & 3.88 & 5.04 & 3.79 \\
\hline \hline F1M2 & 4.08 & F2M2 & 4.04 & F3M2 & 3.96 & F4M2 & 6.33 & F5M2 & 5.89 \\
\hline 3.33 & 4.04 & 3.31 & 4.03 & 3.27 & 4.00 & 5.29 & 3.95 & 5.04 & 3.86 \\
\hline \hline F1M1 & 4.14 & F2M1 & 4.10 & F3M1 & 4.02 & F4M1 & 6.43 & F5M1 & 5.97 \\
\hline 3.33 & 4.08 & 3.31 & 4.07 & 3.27 & 4.04 & 5.29 & 3.99 & 5.04 & 3.89 \\
\hline \hline
\end{tabular}

\begin{tabular}{||c|c||}
\hline \hline element. & solid ang. \\
\hline $\begin{array}{c}\text { in-plane } \\
\text { width }\end{array}$ & $\begin{array}{c}\text { out-of-plane } \\
\text { width }\end{array}$ \\
\hline \hline
\end{tabular}

Table A.1: Solid angles (in milli-steradians), in-plane and out-of-plane angular widths (in degrees) of the different strip detector elements. 


\section{Appendix B}

\section{Out-of-Plane Data Presentation.}

This appendix presents the out-of-plane data recorded from each pixel on the strip detector set-up. The data recorded from the pixels moving out-of-plane along a fixed $\mathrm{F}$ strip are shown by the same symbol. The alpha angle quoted in each figure is for the edge of the strip detector set-up. To obtain the value of the in-plane angle to the centre of each $\mathrm{F}$ group, $\theta_{\alpha}$, one must add the values given below to this quoted angle. The lines on these graphs are the least squares fits outlined in section 4.2.2. The values of the out-of-plane half angle for each of the distributions is also given at the end of the appendix.

$\begin{array}{cc}\text { F strip. } & \text { additional angle. } \\ \text { F1 } & 1.7^{\circ} \\ \text { F2 } & 4.9^{\circ} \\ \text { F3 } & 8.3^{\circ} \\ \text { F4 } & 12.5^{\circ} \\ \text { F5 } & 17.7^{\circ}\end{array}$

Table B.1: Angles to be added to values quoted in the figures for calculation of $\theta_{\alpha}$ for different front $\mathrm{F}$ strips. 
Front Strip Detector Groups

$$
\odot-F 1_{0} \bullet-F 2_{0} \diamond-F 3, \nabla-F 4, \Delta-F 5
$$
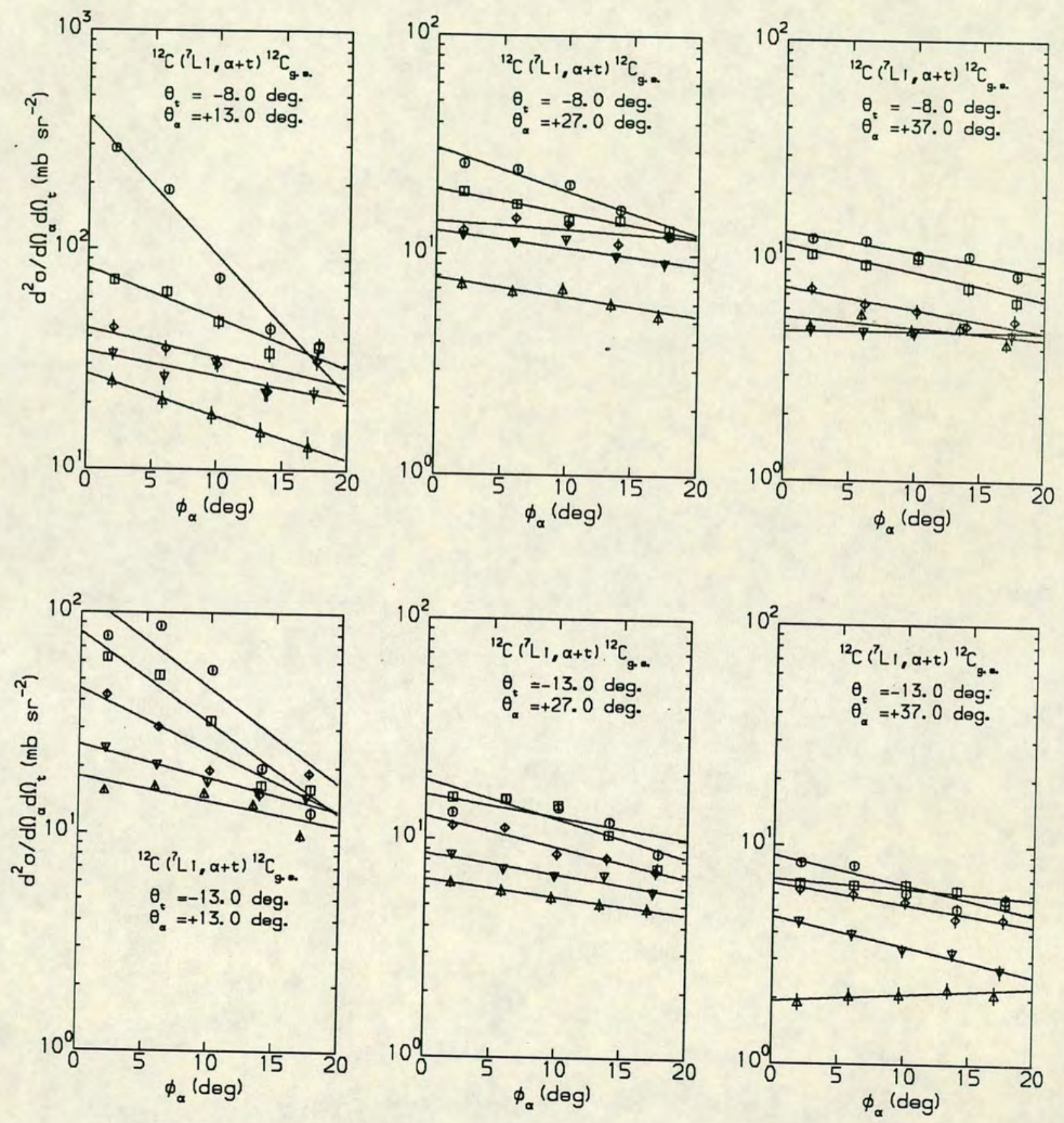

Figure B.1: Out-of-plane angular distributions for quasi-elastic ${ }^{12} \mathrm{C}$ coincidence events for $\theta_{t}=-8.0^{\circ}$ and $\theta_{t}=-13.0^{\circ}$ 
Front Strip Detector Groups

$$
\odot-F 1_{0} \bullet-F 2_{0} \diamond-F 3, \nabla-F 4 \Delta \Delta-F 5
$$
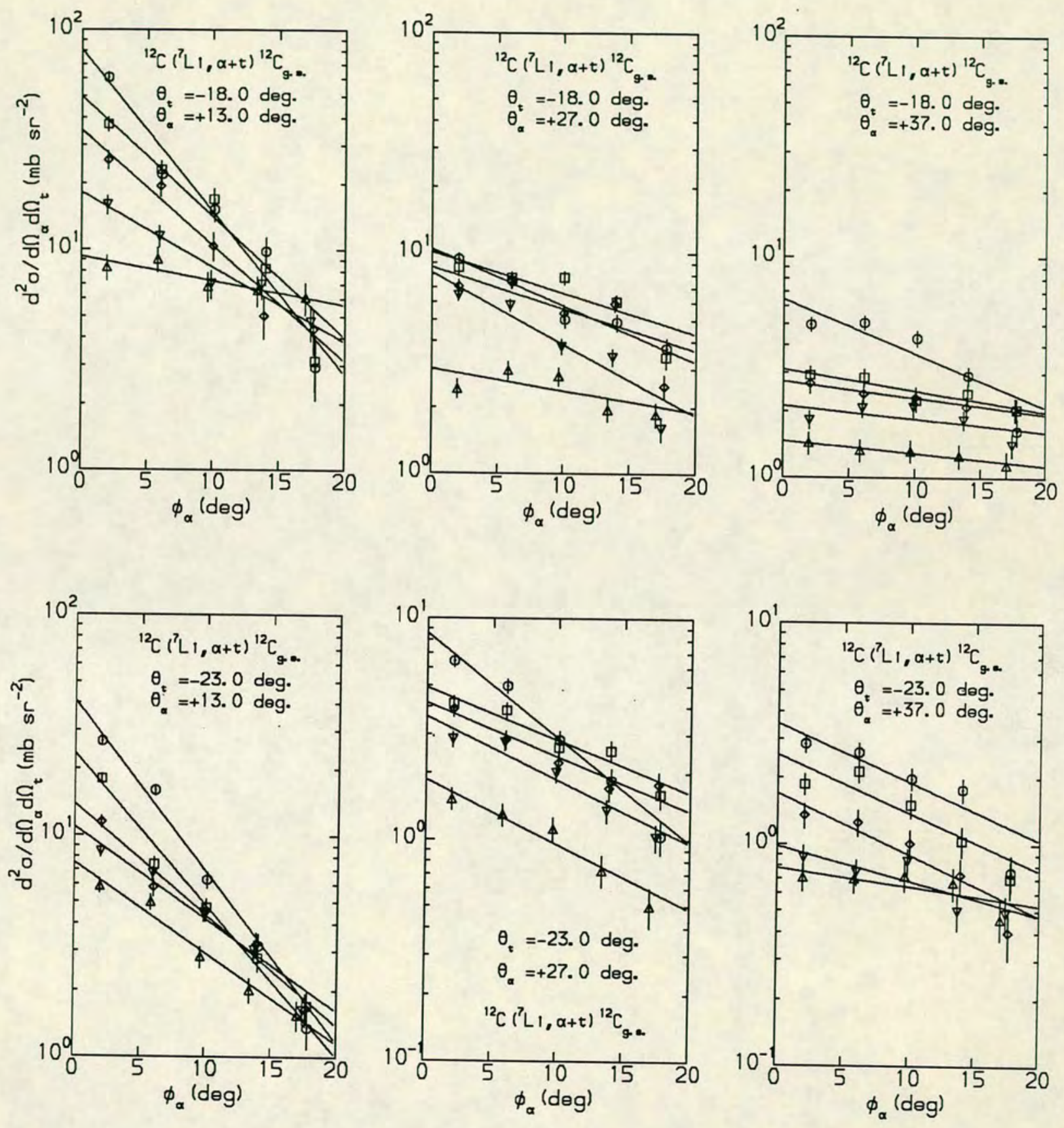

Figure B.2: Out-of-plane angular distributions for quasi-elastic ${ }^{12} \mathrm{C}$ coincidence events for $\theta_{t}=-18.0^{\circ}$ and $\theta_{t}=-23.0^{\circ}$ 
Front Strip Detector Groups

$\odot-F 1_{0} \square-F 2_{0} \diamond-F 3, \nabla-F 4_{\theta} \Delta-F 5$
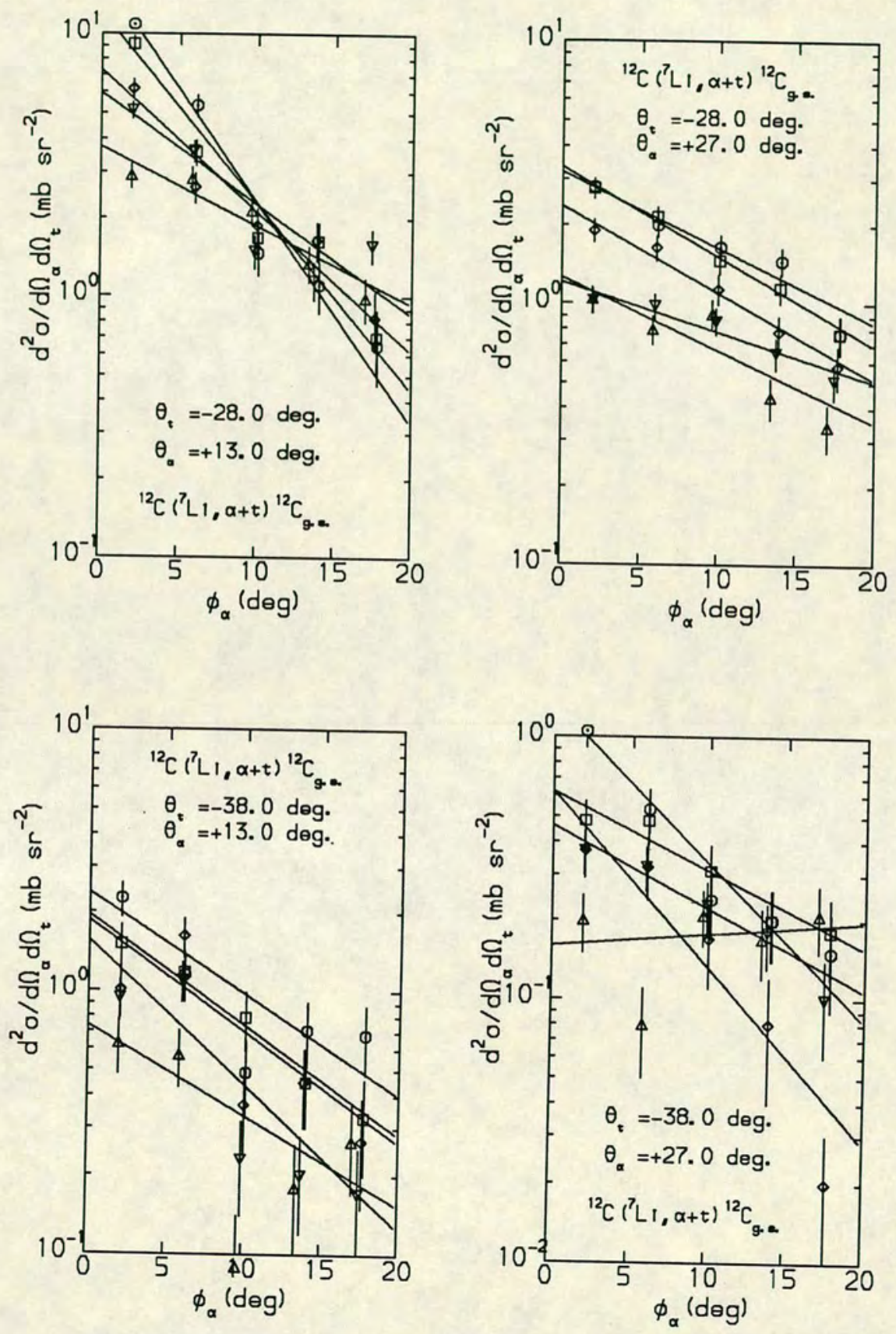

Figure B.3: Out-of-plane angular distributions for quasi-elastic ${ }^{12} \mathrm{C}$ coincidence events for $\theta_{t}=-28.0^{\circ}$ and $\theta_{t}=-38.0^{\circ}$ 


\section{Front Strip Detector Groups}

$$
\odot-F 1_{\theta} \bullet-F 2_{\theta} \diamond-F 3, \nabla-F 4, \Delta-F 5
$$
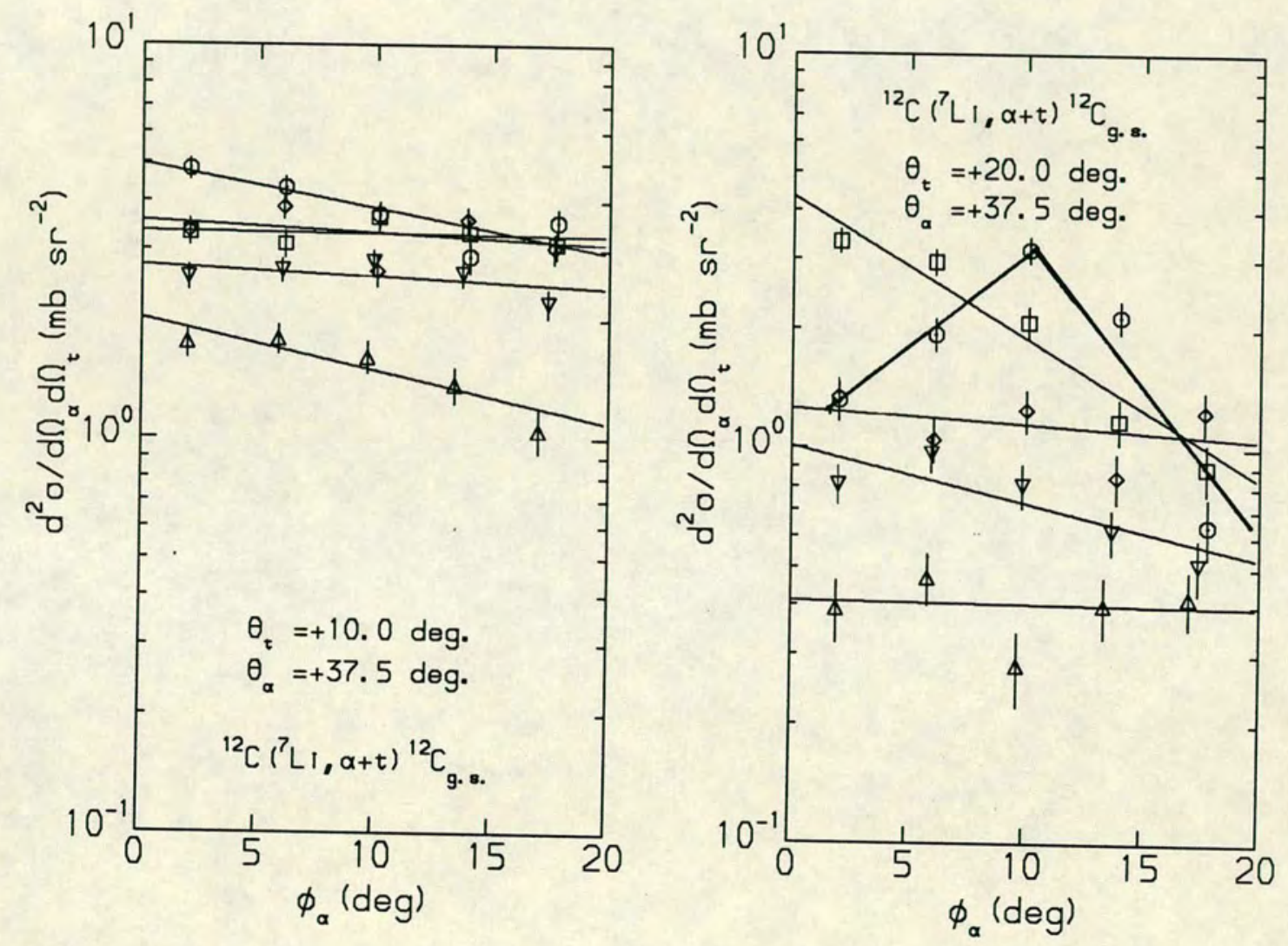

Figure B.4: Out-of-plane angular distributions for quasi-elastic ${ }^{12} \mathrm{C}$ coincidence events with alpha and triton on same side of beam for $\theta_{t}=+10.0^{\circ}$ and $\theta_{t}=+20.0^{\circ}$ 
Front Strip Detector Groups

$$
\odot-F 1_{\theta} \square-F 2_{\theta} \diamond-F 3, \nabla-F 4 \Delta \Delta-F 5
$$
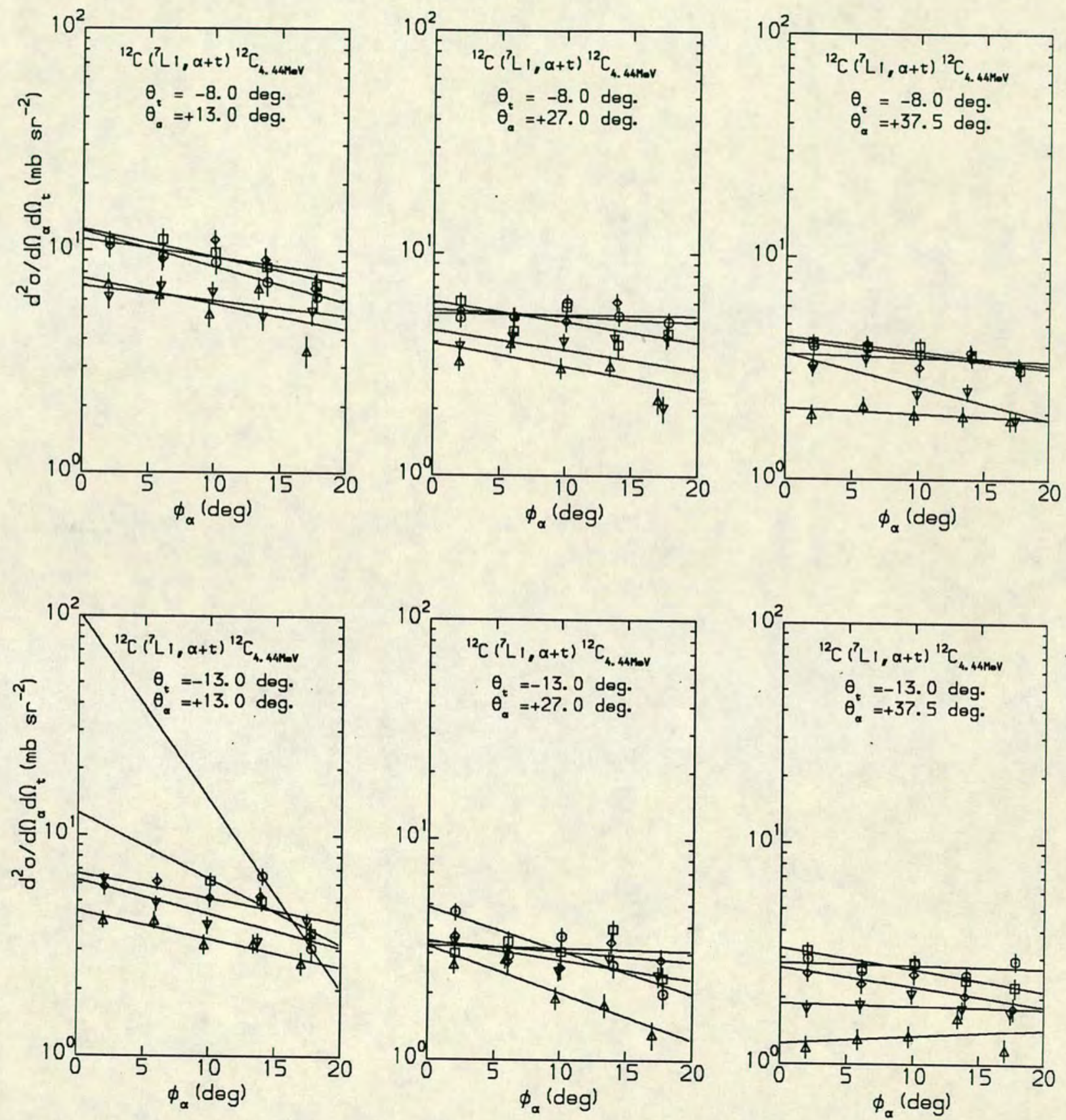

Figure B.5: Out-of-plane angular distributions for events leaving ${ }^{12} \mathrm{C}$ in its first excited state for $\theta_{t}=-8.0^{\circ}$ and $\theta_{t}=-13.0^{\circ}$ 
Front Strip Detector Groups

$$
\odot-F 1_{0} \square-F 2_{0} \diamond-F 3, \nabla-F 4, \Delta-F 5
$$
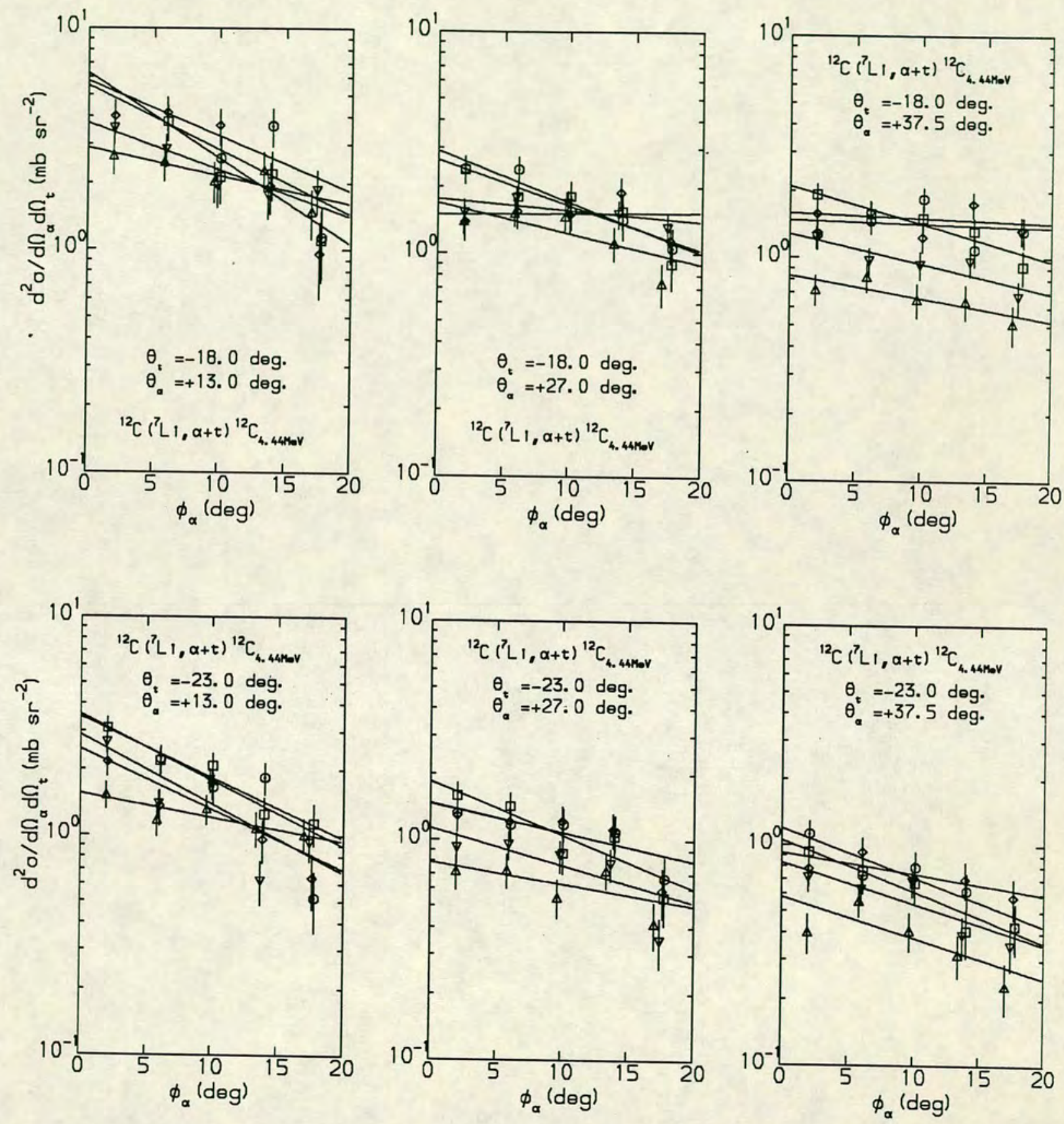

Figure B.6: Out-of-plane angular distributions for events leaving ${ }^{12} \mathrm{C}$ in its first excited state for $\theta_{t}=-18.0^{\circ}$ and $\theta_{t}=-23.0^{\circ}$ 
Front Strip Detector Groups

$\odot-F 1_{\theta} \bullet-F 2, \diamond-F 3, \nabla-F 4, \Delta-F 5$
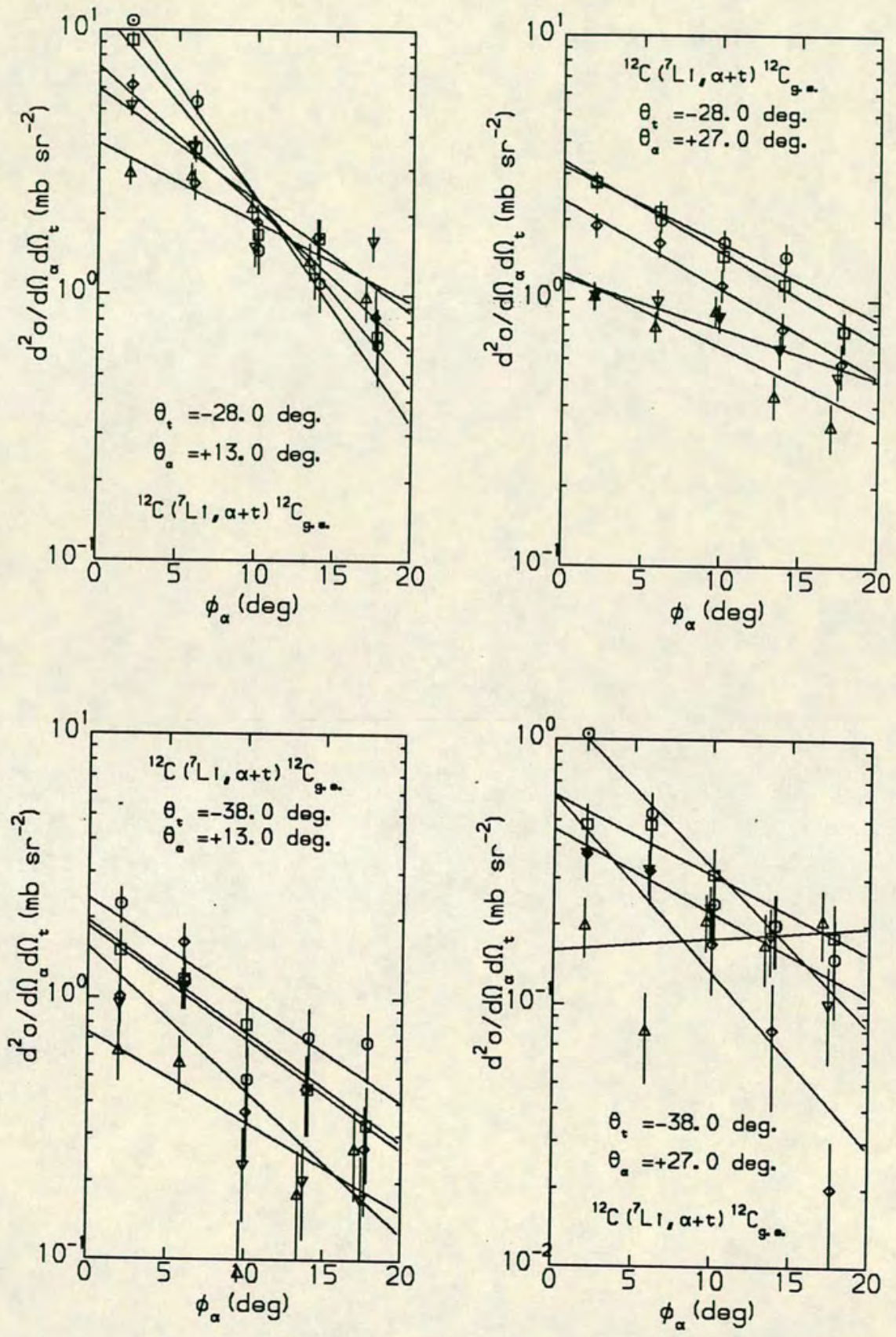

Figure B.7: Out-of-plane angular distributions for events leaving ${ }^{12} \mathrm{C}$ in its first excited state for $\theta_{t}=-28.0^{\circ}$ and $\theta_{t}=-38.0^{\circ}$ 
Front Strip Detector Groups
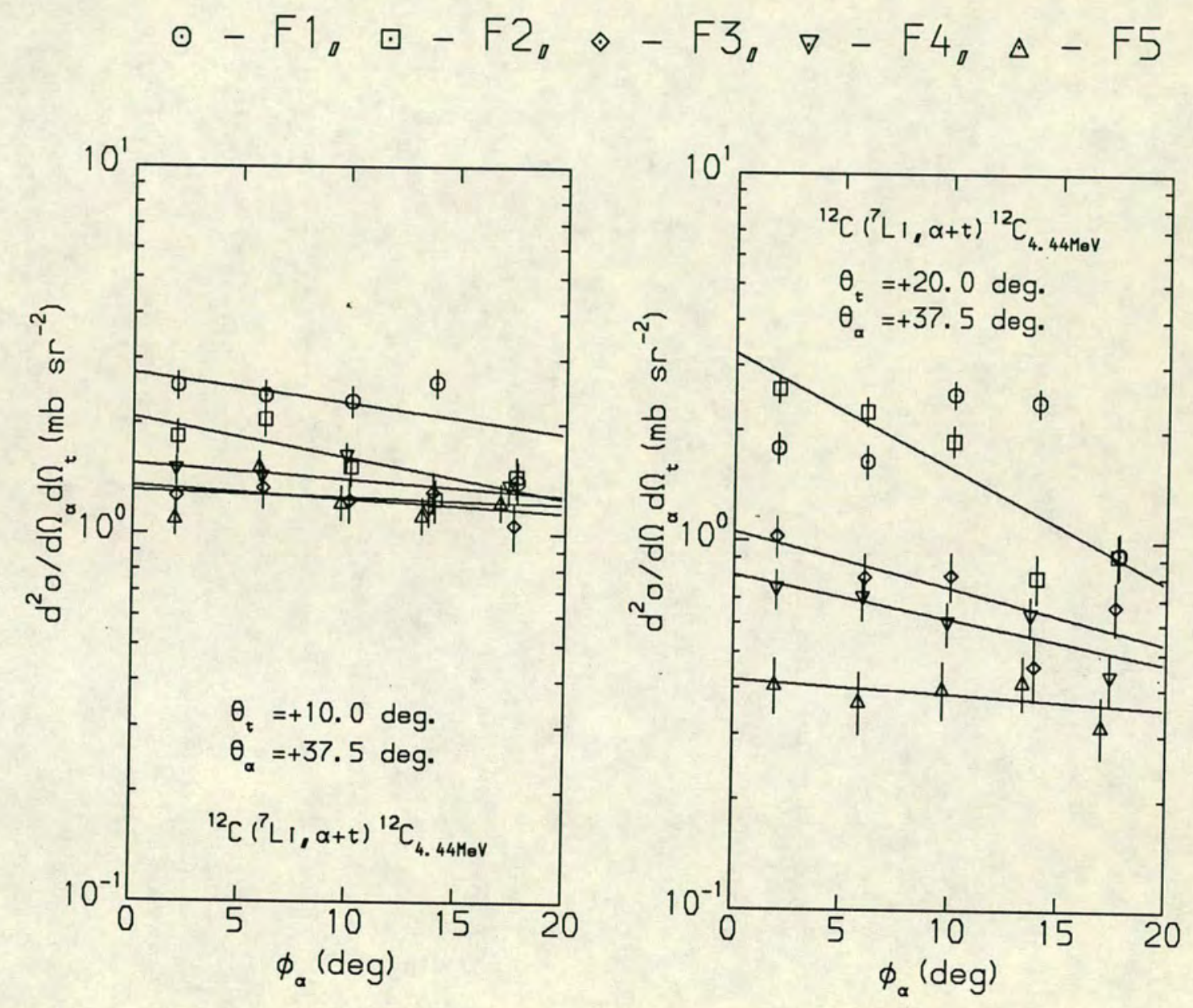

Figure B.8: Out-of-plane angular distributions for events leaving ${ }^{12} \mathrm{C}$ in its first excited state with alpha and triton on the same side of the beam for $\theta_{t}=+10.0^{\circ}$ and $\theta_{t}=+20.0^{\circ}$ 
Front Strip Detector Groups
$\odot-F 1$ 。
$\square-F 2, \diamond-F 3$,
$\nabla-F 4$
$\Delta-F 5$
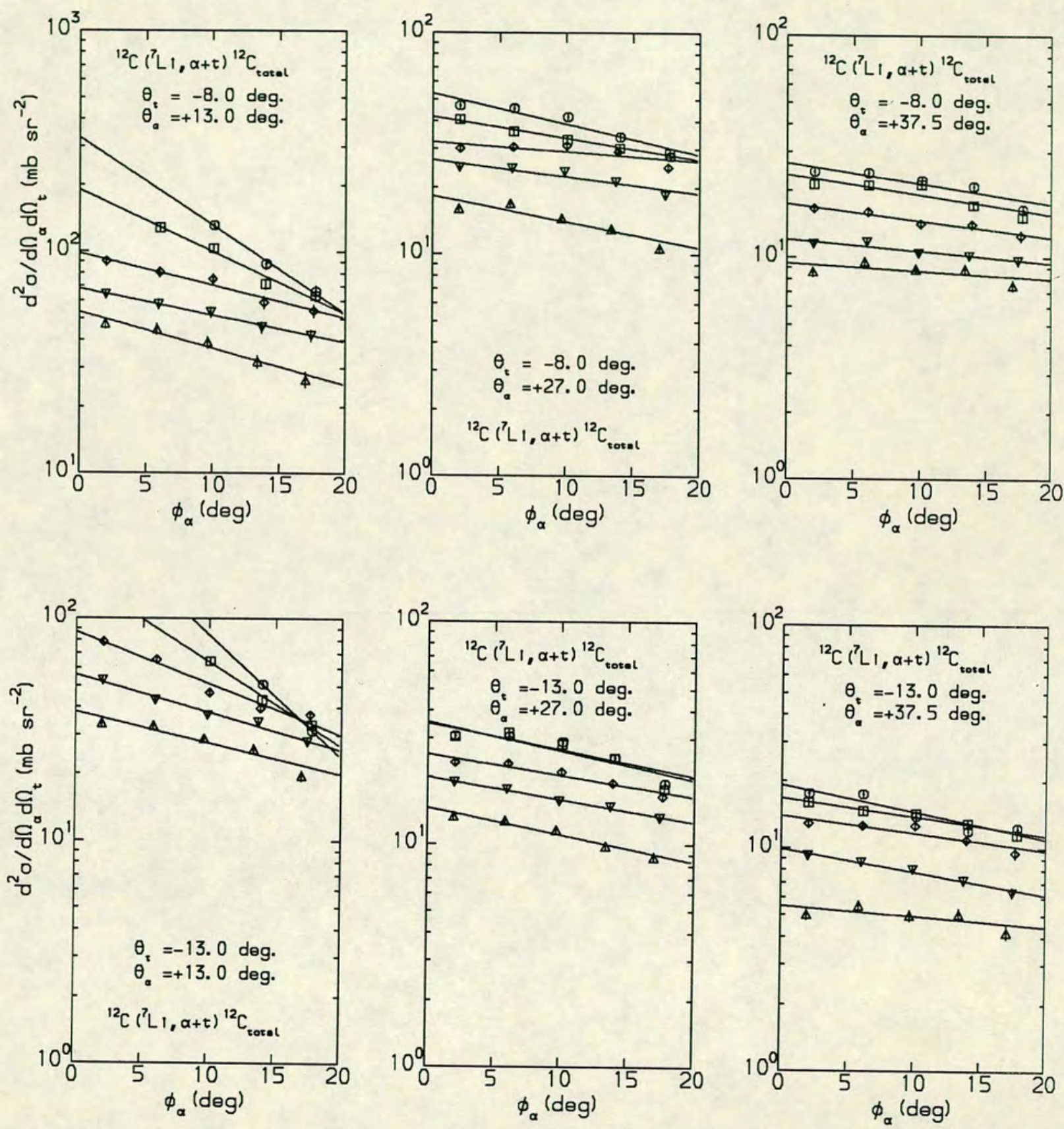

Figure B.9: Out-of-plane angular distributions for all ${ }^{12} \mathrm{C}$ coincidence events for $\theta_{t}=-8.0^{\circ}$ and $\theta_{t}=-13.0^{\circ}$ 
Front Strip Detector Groups

$$
\odot-F 1_{0} \bullet-F 2_{0} \diamond-F 3_{0} \nabla-F 4_{\theta} \Delta-F 5
$$
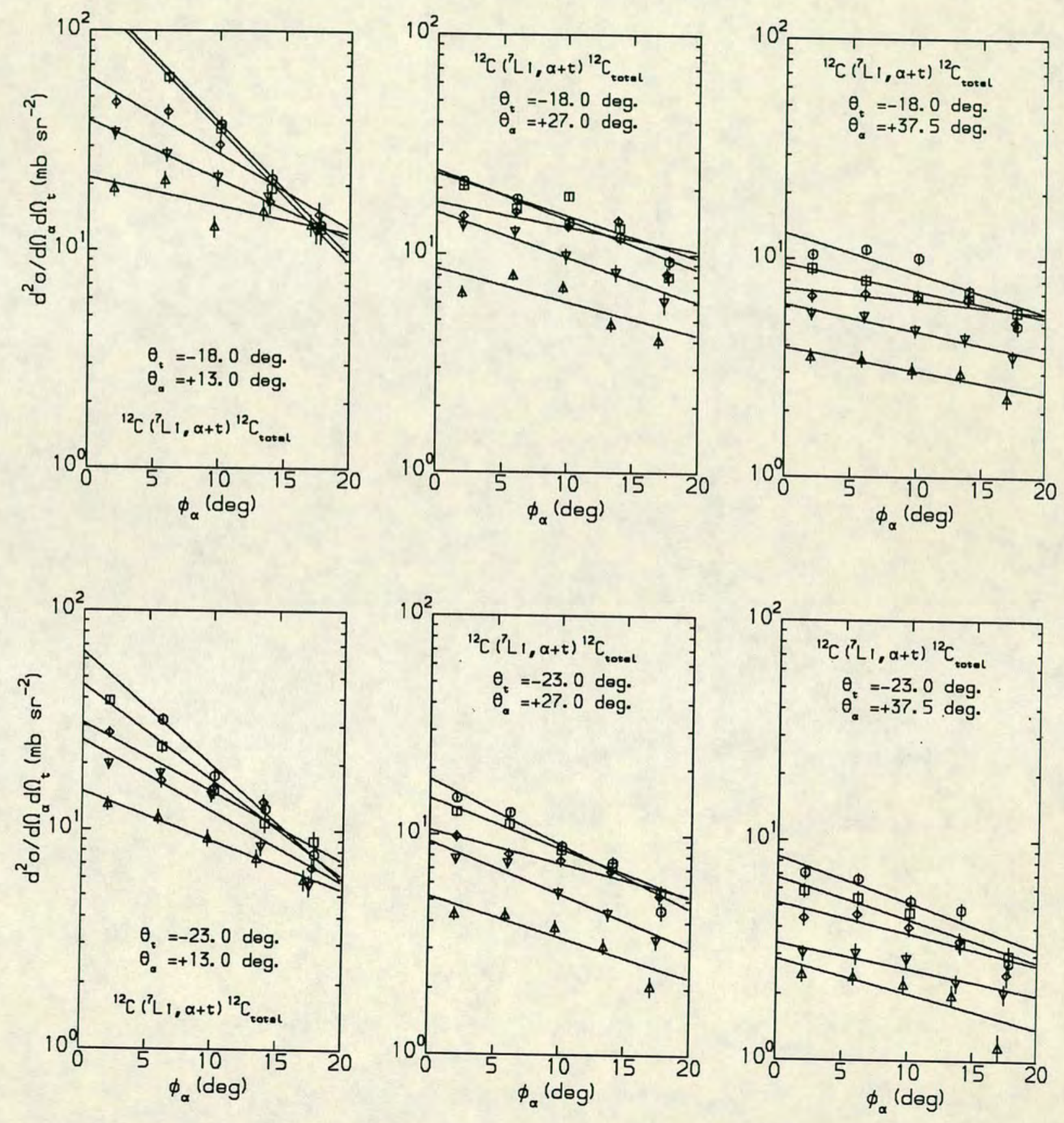

Figure B.10: Out-of-plane angular distributions for all ${ }^{12} \mathrm{C}$ coincidence events for $\theta_{t}=-18.0^{\circ}$ and $\theta_{t}=-23.0^{\circ}$ 
Front Strip Detector Groups

$\odot-F 1_{0} \bullet-F 2, \diamond-F 3, \nabla-F 4, \Delta-F 5$
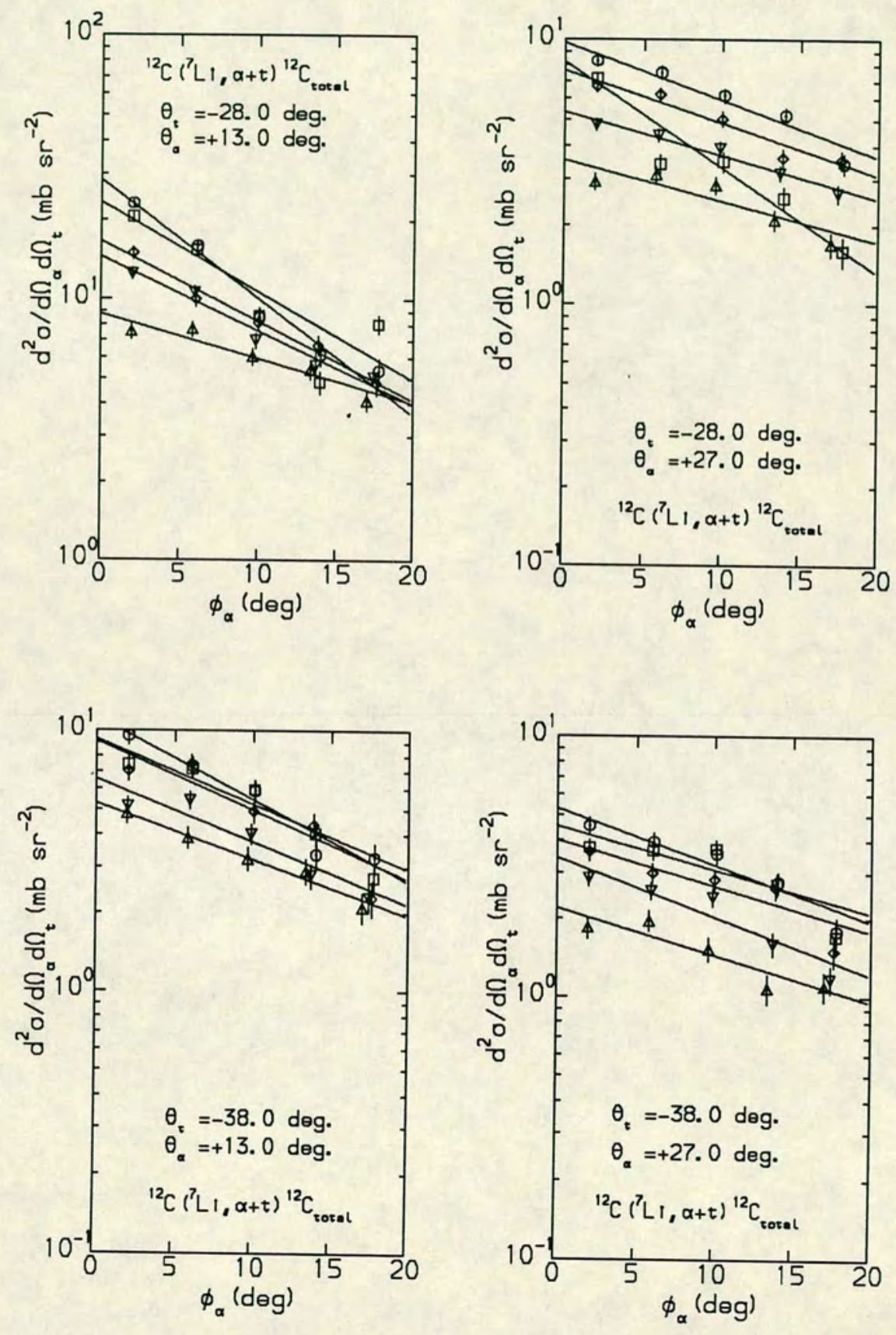

Figure B.11: Out-of-plane angular distributions for all ${ }^{12} \mathrm{C}$ coincidence events for $\theta_{t}=-28.0^{\circ}$ and $\theta_{t}=-38.0^{\circ}$ 
Front Strip Detector Groups

$$
\odot-F 1_{\theta} \bullet-F 2, \diamond-F 3, \nabla-F 4, \Delta-F 5
$$
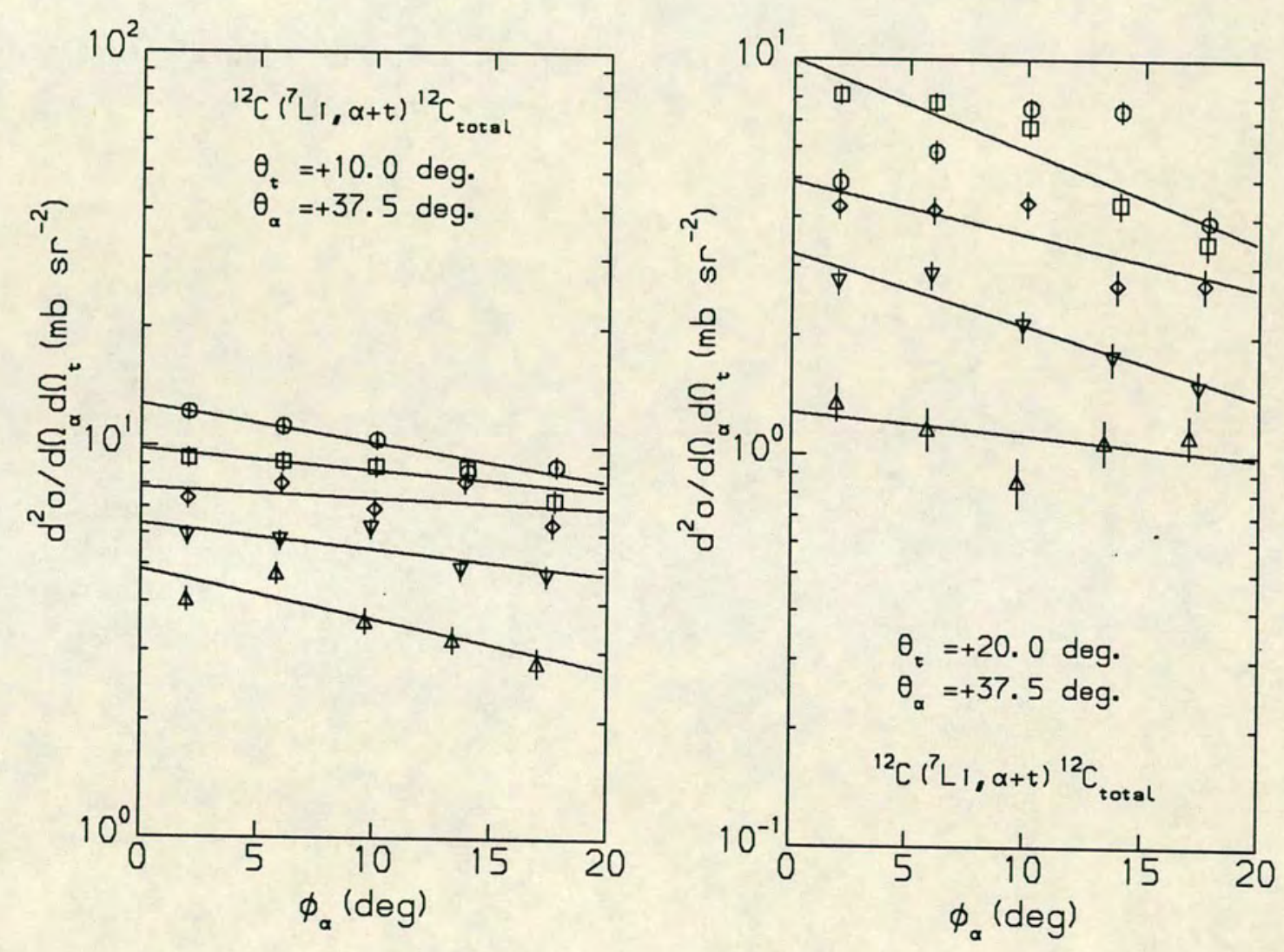

Figure B.12: Out-of-plane angular distributions for all ${ }^{12} \mathrm{C}$ coincidence events with alpha and triton on same side of beam for $\theta_{t}=+10.0^{\circ}$ and $\theta_{t}=+20.0^{\circ}$ 
Front Strip Detector Groups

$$
\odot-F 1_{0} \bullet-F 2_{0} \diamond-F 3, \nabla-F 4 \Delta \Delta-F 5
$$
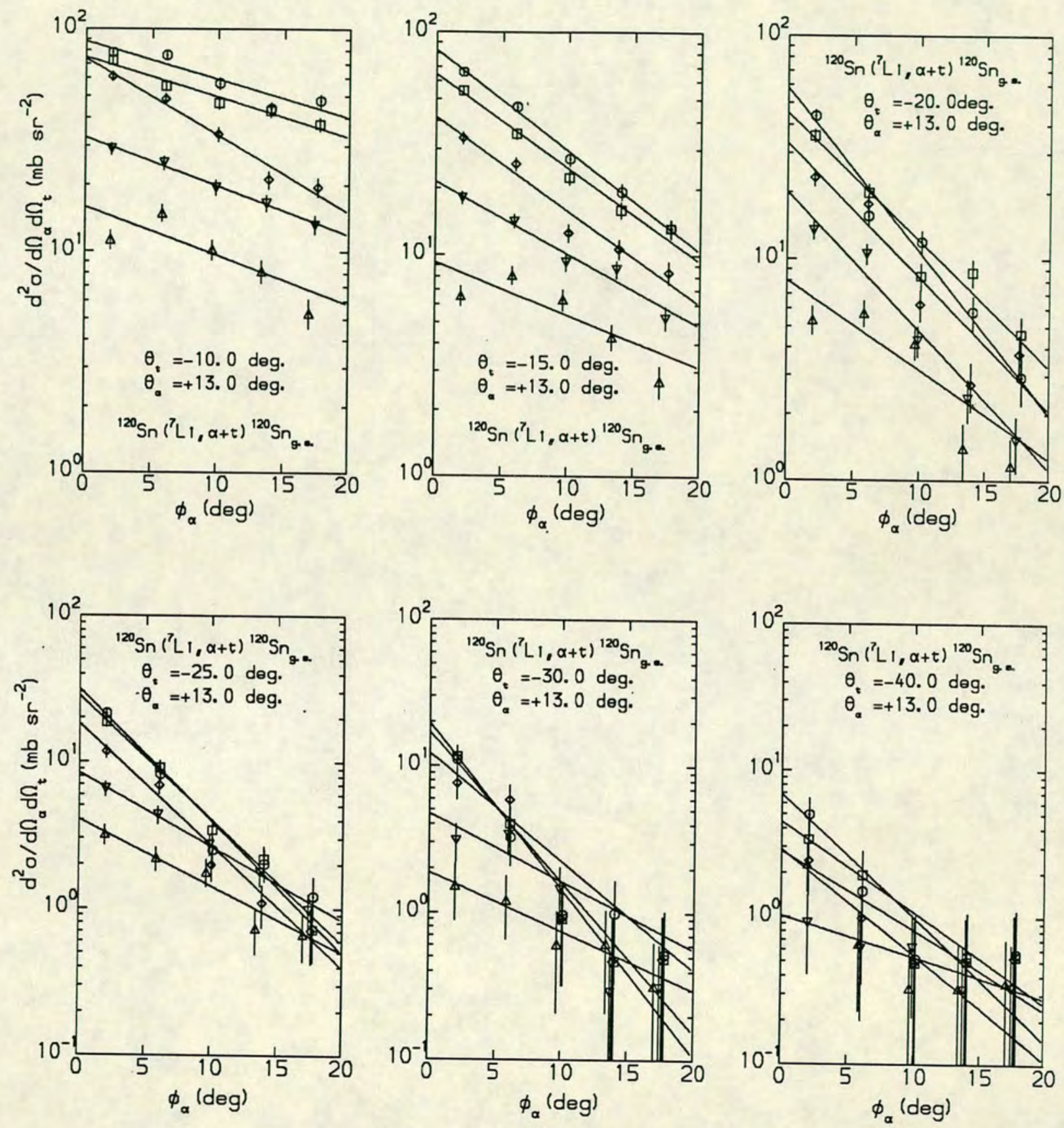

Figure B.13: Out-of-plane angular distributions for quasi-elastic ${ }^{120} \mathrm{Sn}$ coincidence events. 
Front Strip Detector Groups

$\odot-F 1_{\theta} \bullet-F 2, \diamond-F 3, \nabla-F 4 \Delta \Delta-F 5$
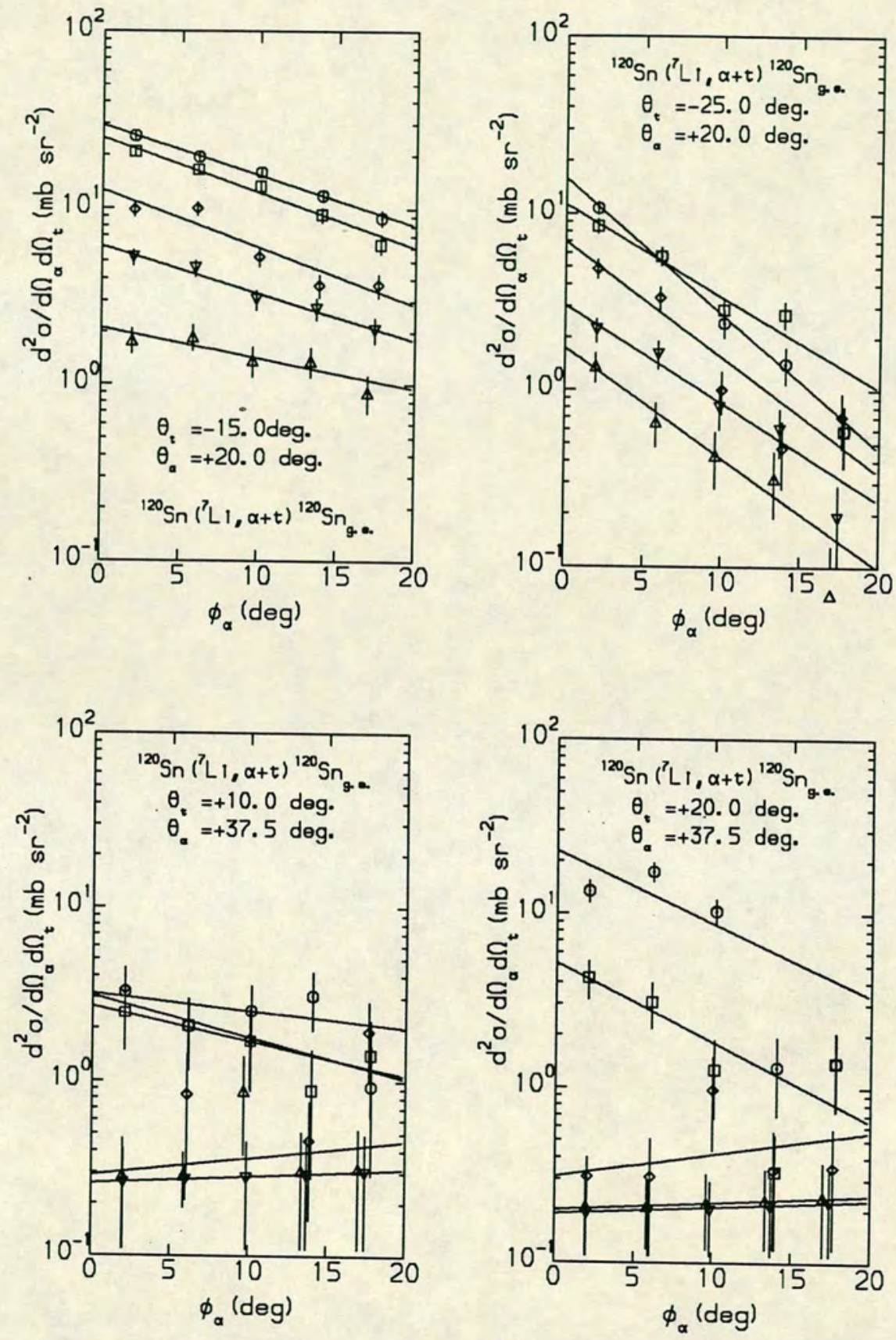

Figure B.14: Out-of-plane angular distributions for quasi-elastic ${ }^{120} \mathrm{Sn}$ coincidence events. 
Front Strip Detector Groups $\odot-F 1_{0} \bullet-F 2_{0} \diamond-F 3, \nabla-F 4, \Delta-F 5$
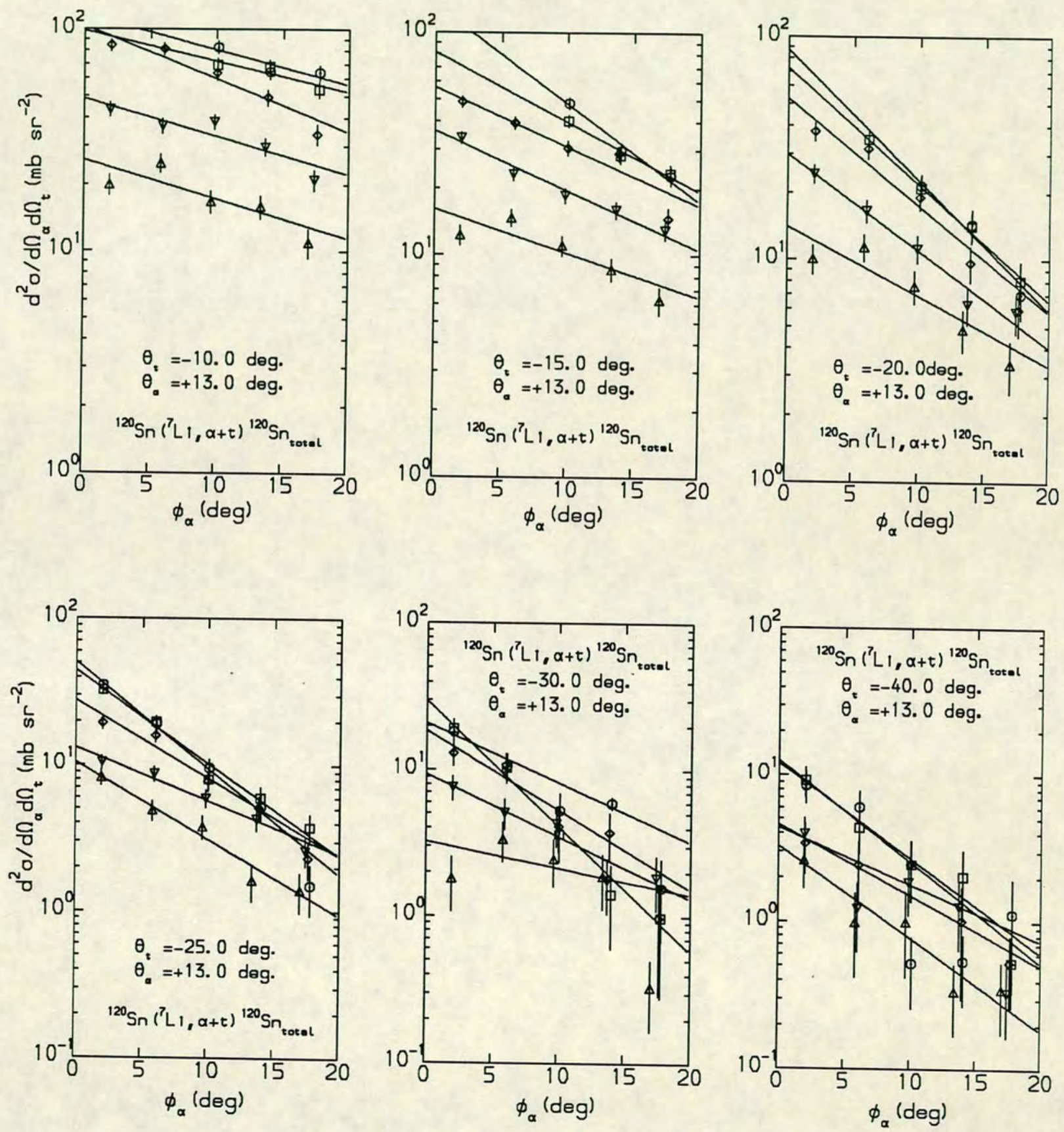

Figure B.15: Out-of-plane angular distributions for all ${ }^{120} \mathrm{Sn}$ coincidence events. 

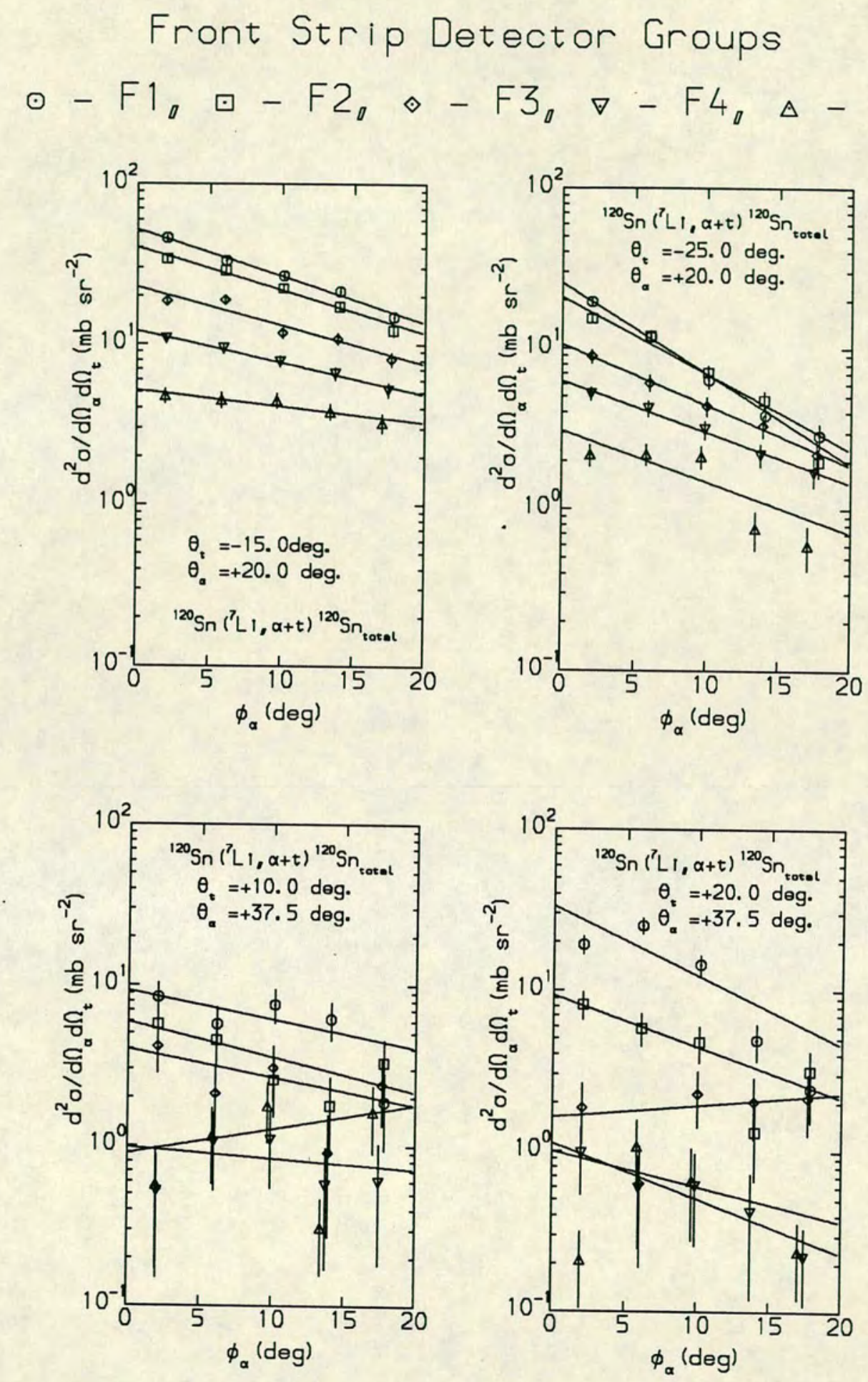

Figure B.16: Out-of-plane angular distributions for all ${ }^{120} \mathrm{Sn}$ coincidence events. 
Front Strip Detector Groups

$\odot-F 1_{\theta} \bullet-F 2_{0} \diamond-F 3, \nabla-F 4, \Delta-F 5$
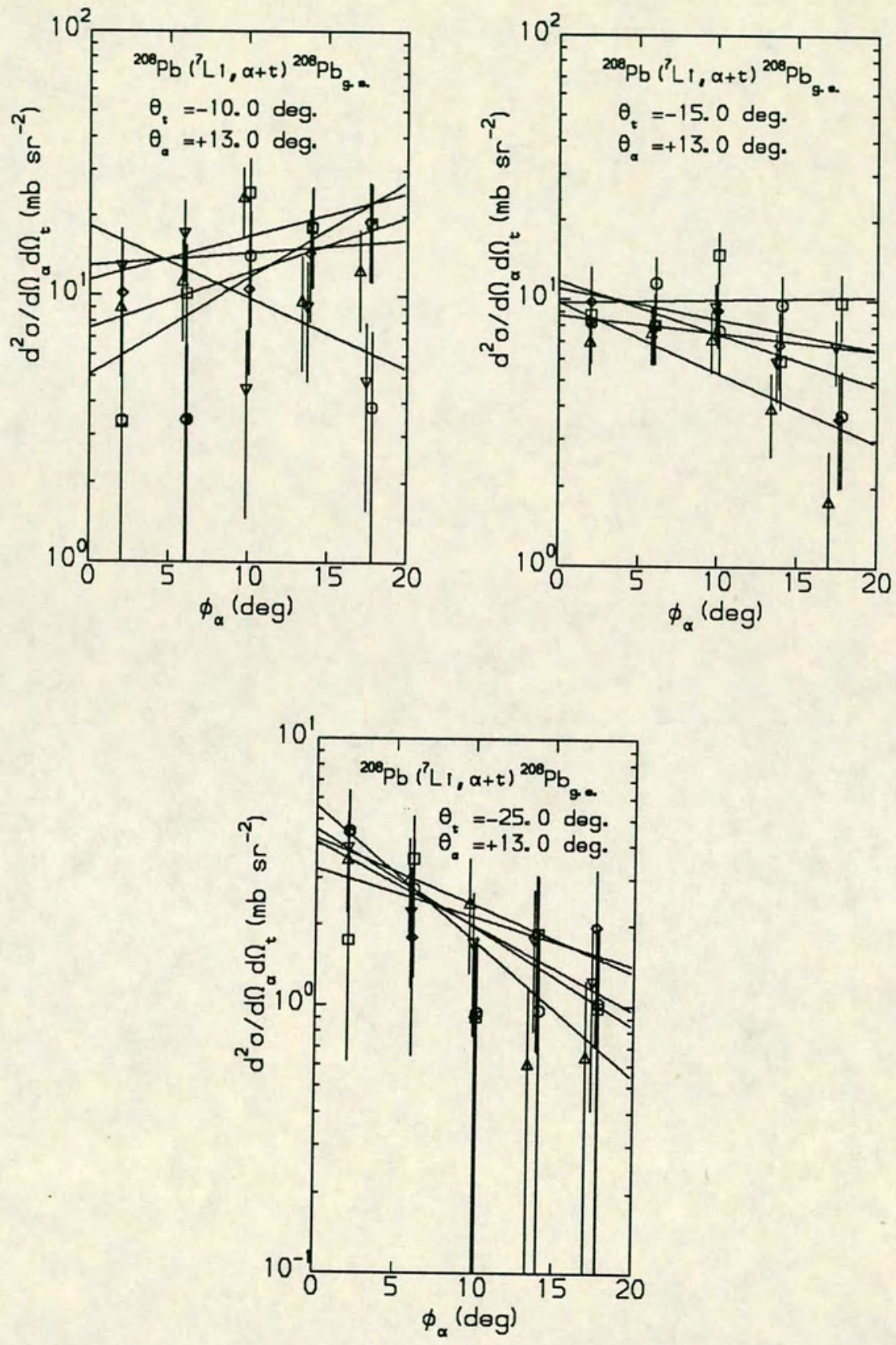

Figure B.17: Out-of-plane angular distributions for quasi-elastic ${ }^{208} \mathrm{~Pb}$ coincidence events. 
Front Strip Detector Groups

$\odot-F 1, \nabla-F 2, \diamond-F 3, \nabla-F 4, \Delta-F 5$
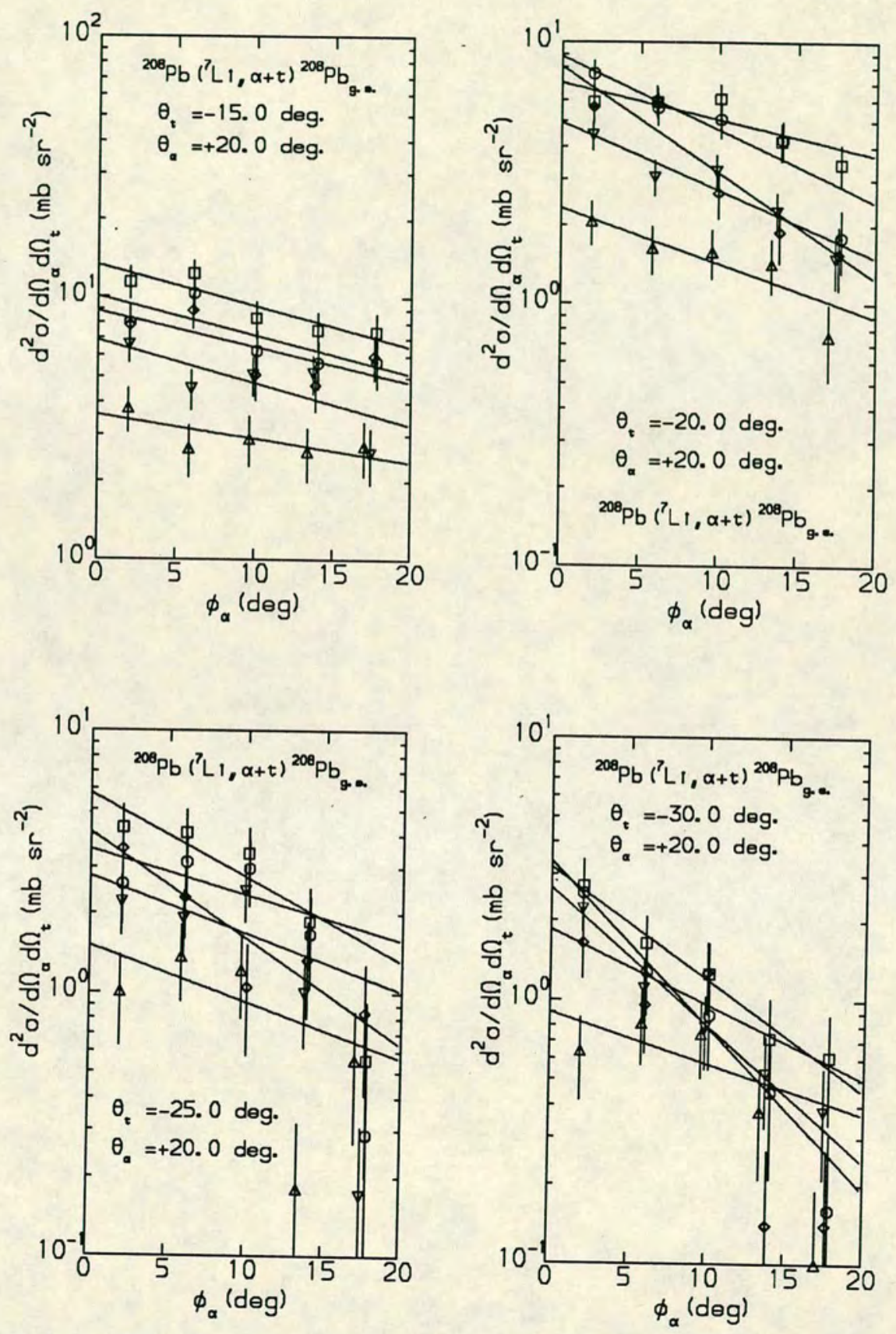

Figure B.18: Out-of-plane angular distributions for quasi-elastic ${ }^{208} \mathrm{~Pb}$ coincidence events. 
Front Strip Detector Groups

$\odot-F 1_{0} \bullet-F 2_{0} \otimes-F 3, \nabla-F 4_{0} \Delta-F 5$
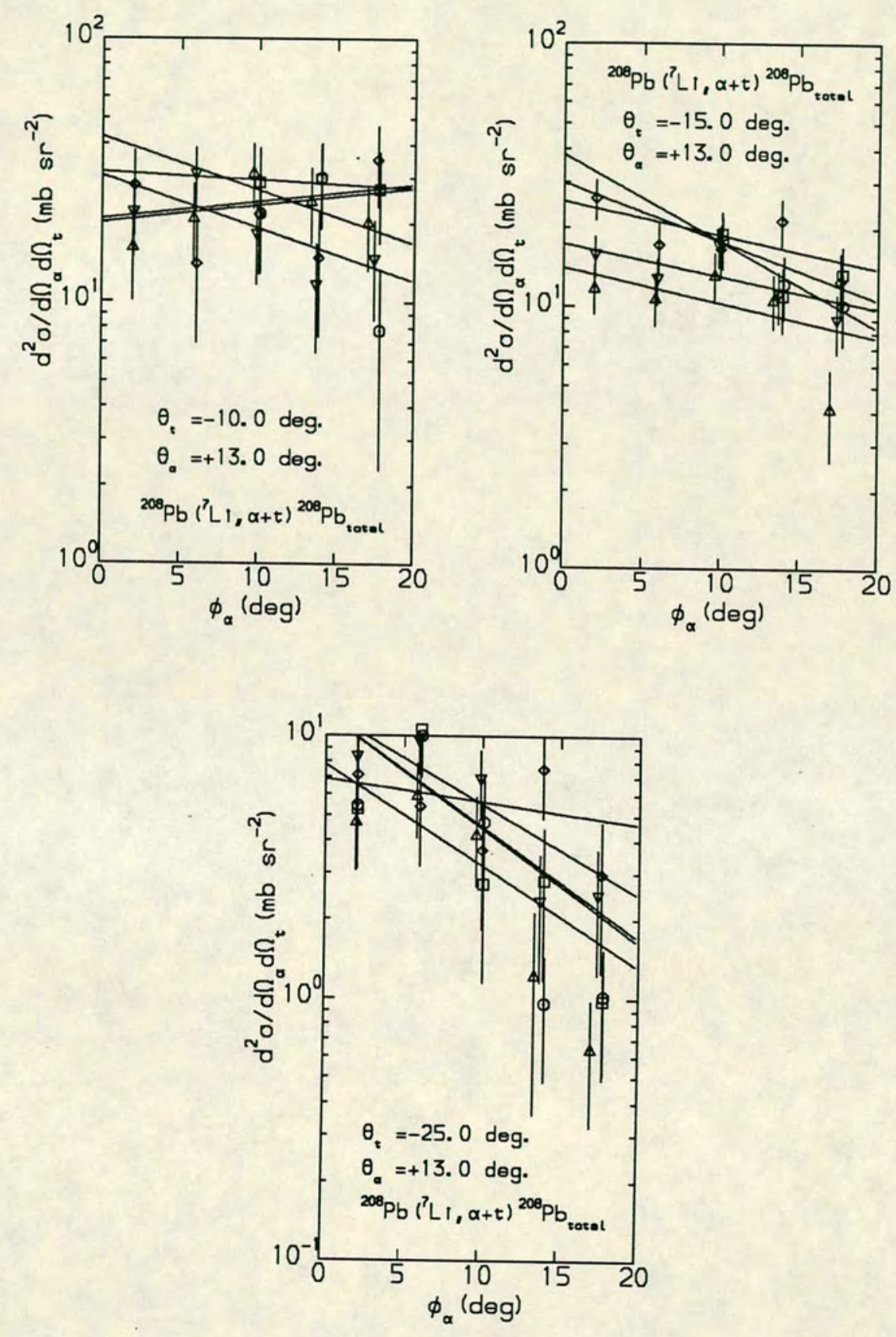

Figure B.19: Out-of-plane angular distributions for all ${ }^{208} \mathrm{~Pb}$ coincidence events. 
Front Strip Detector Groups $\odot-F 1_{0} \bullet-F 2_{0} \diamond-F 3, \nabla-F 4_{0} \Delta-F 5$
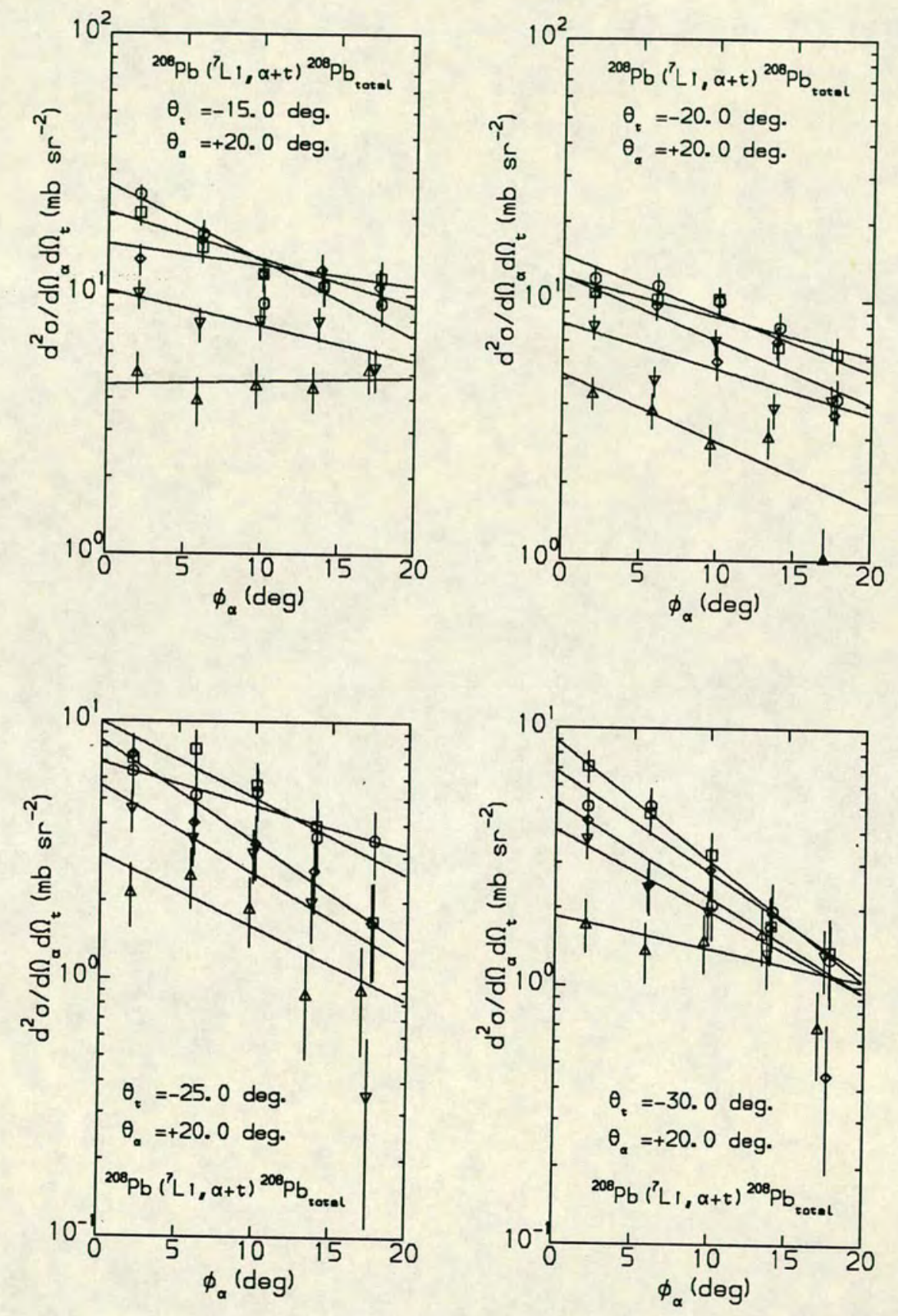

Figure B.20: Out-of-plane angular distributions for all ${ }^{208} \mathrm{~Pb}$ coincidence events. 


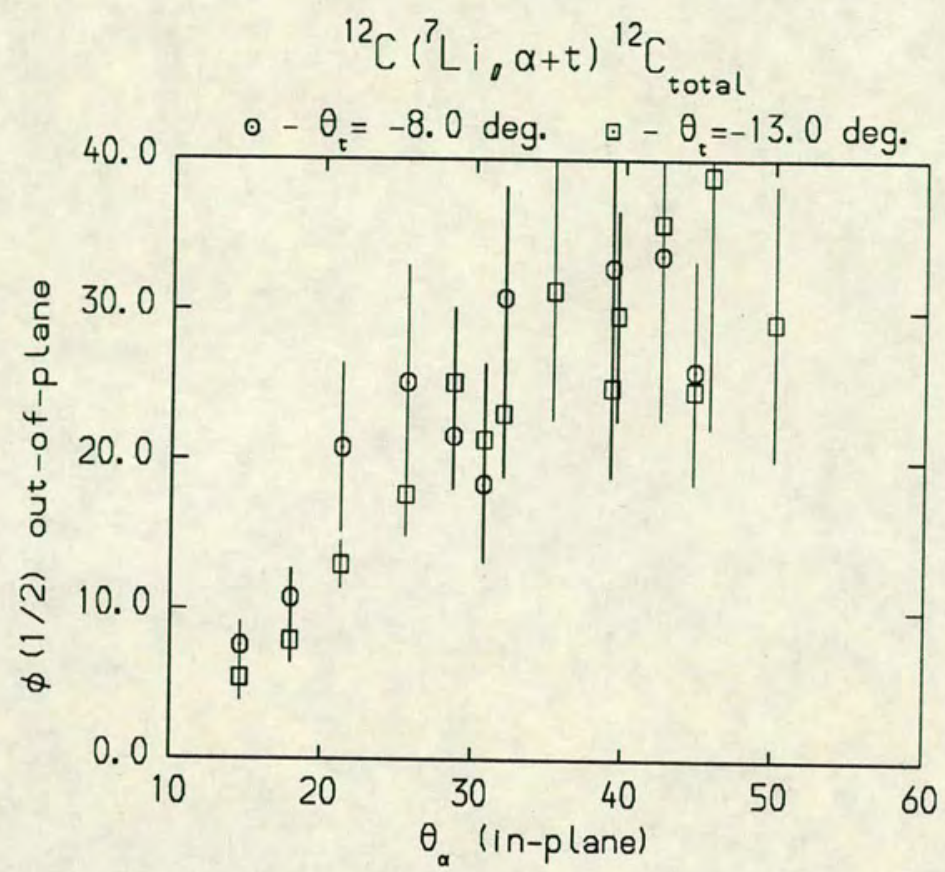

Figure B.21: Value of the half angle for the full out-of-plane distributions for the ${ }^{12} \mathrm{C}$ target with $\theta_{t}=-8.0^{\circ}$ and $\theta_{t}=-13.0^{\circ}$.

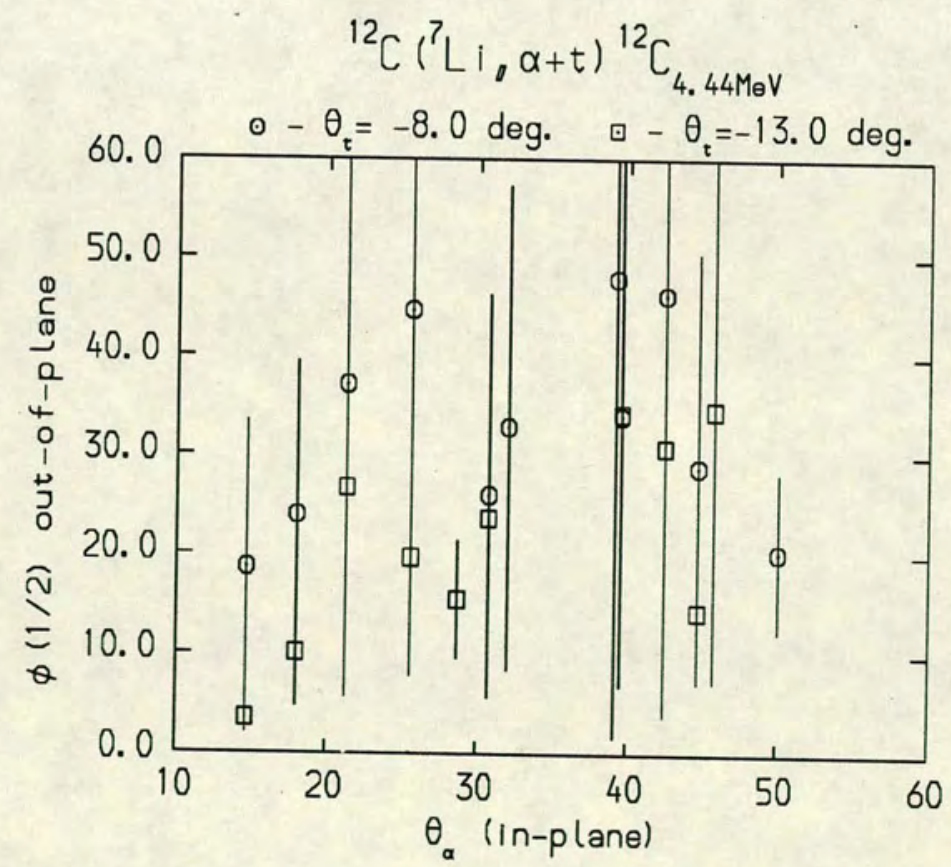

Figure B.22: Value of the half angle for the out-of-plane distributions for events leaving the ${ }^{12} \mathrm{C}$ target in its $4.44 \mathrm{MeV}$ state, with $\theta_{t}=-8.0^{\circ}$ and $\theta_{t}=-13.0^{\circ}$. 


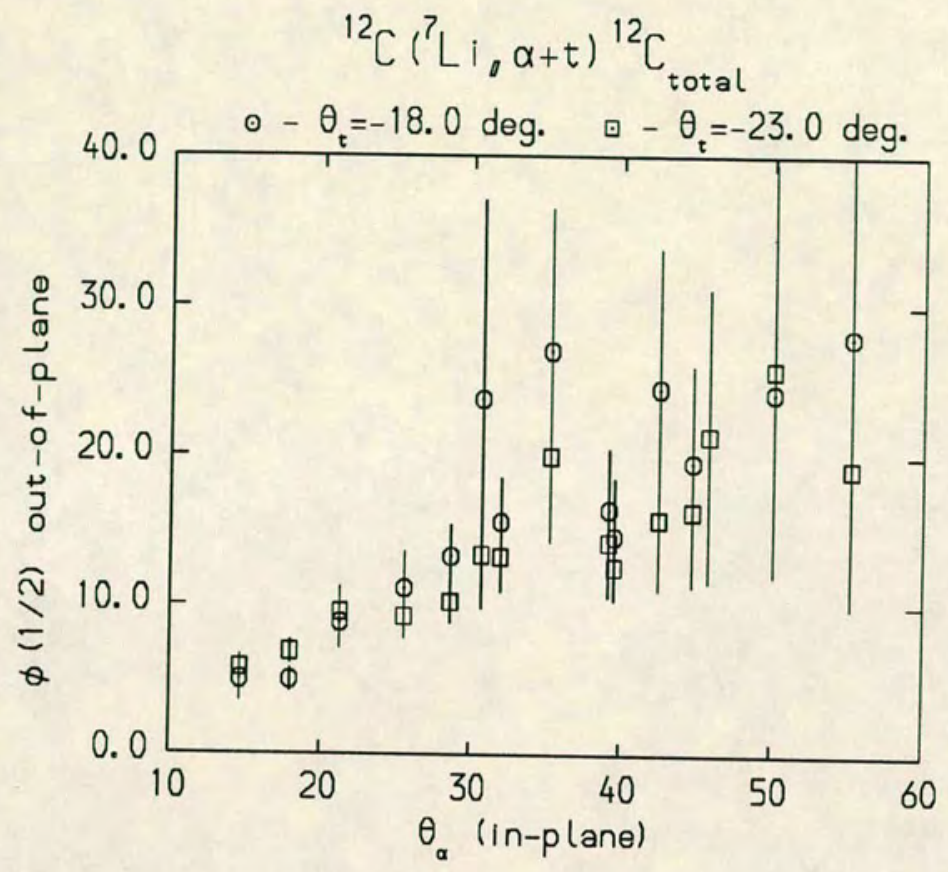

Figure B.23: Value of the half angle for the full out-of-plane distributions for the ${ }^{12} \mathrm{C}$ target with $\theta_{t}=-18.0^{\circ}$ and $\theta_{t}=-23.0^{\circ}$.

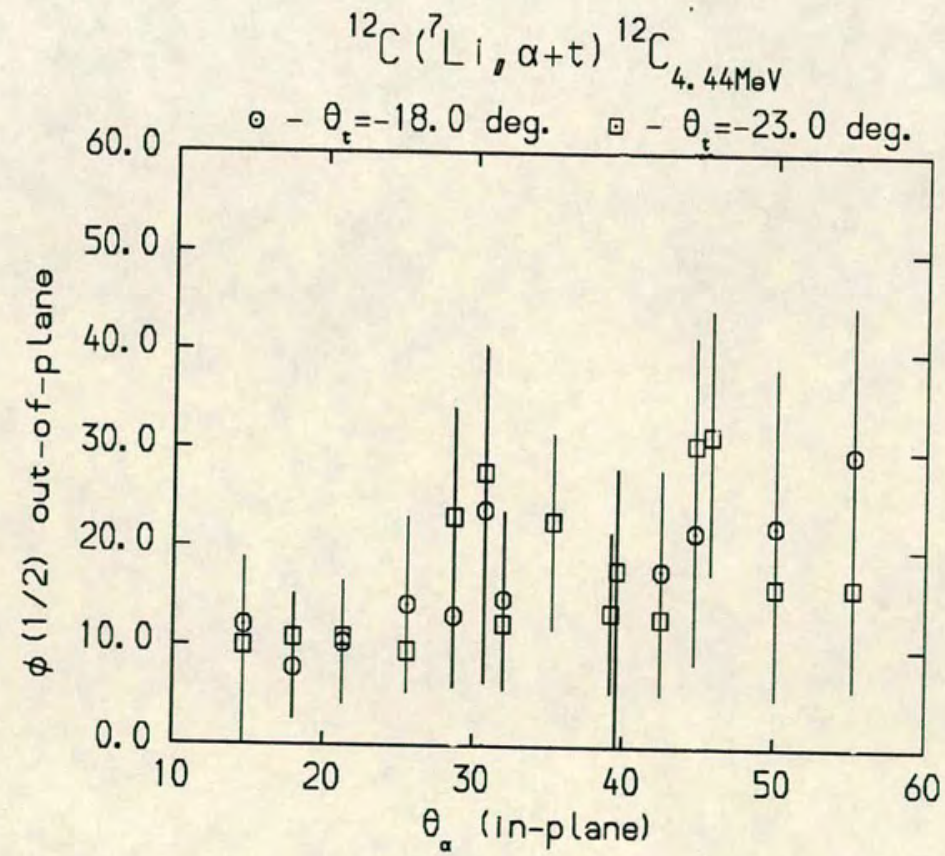

Figure B.24: Value of the half angle for the out-of-plane distributions for events leaving the ${ }^{12} \mathrm{C}$ target in its $4.44 \mathrm{MeV}$ state, with $\theta_{t}=-18.0^{\circ}$ and $\theta_{t}=-23.0^{\circ}$. 


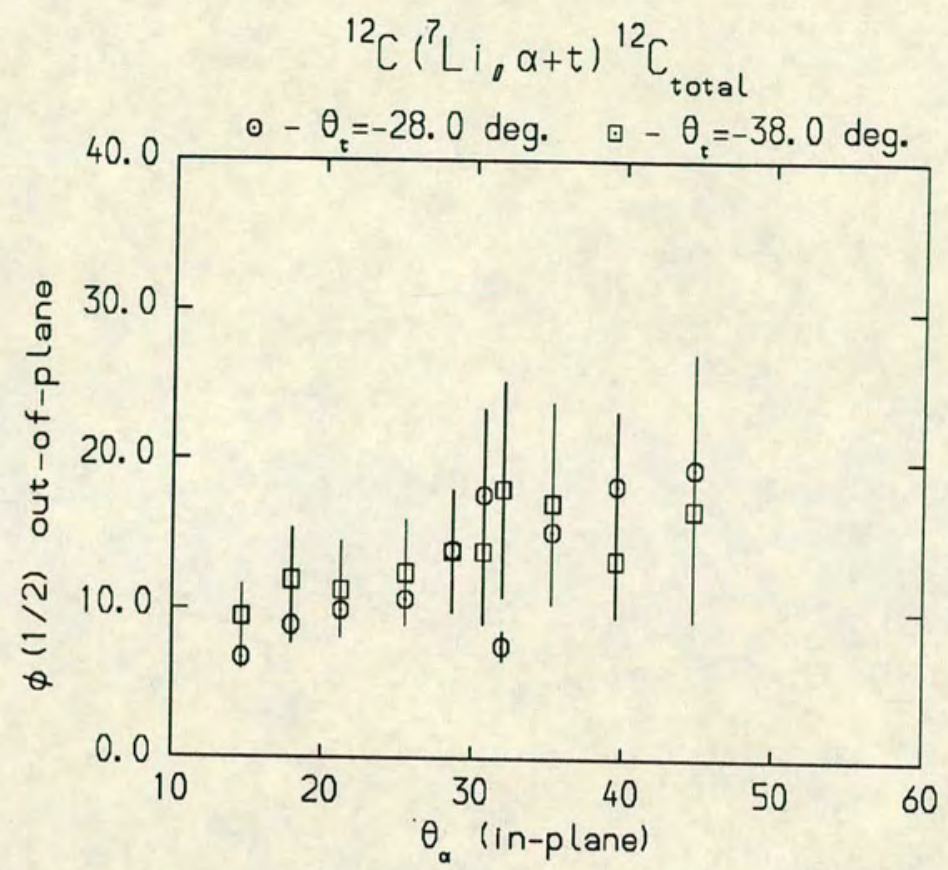

Figure B.25: Value of the half angle for the full out-of-plane distributions for the ${ }^{12} \mathrm{C}$ target with $\theta_{t}=-28.0^{\circ}$ and $\theta_{t}=-38.0^{\circ}$.

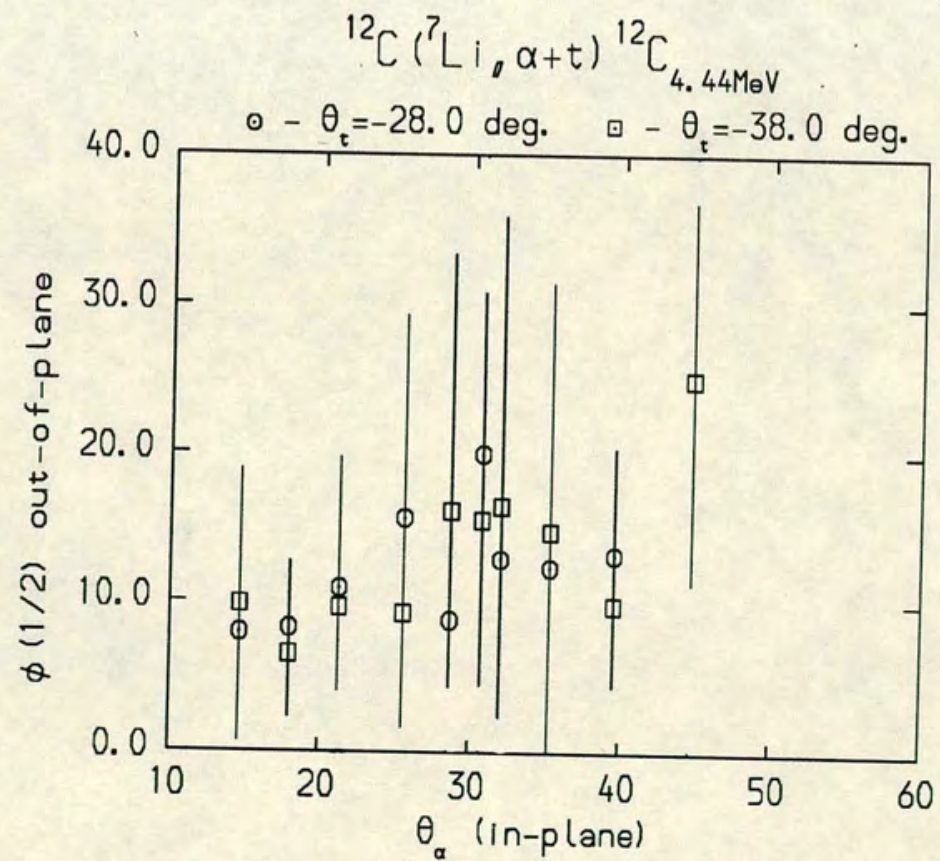

Figure B.26: Value of the half angle for the out-of-plane distributions for events leaving the ${ }^{12} \mathrm{C}$ target in its $4.44 \mathrm{MeV}$ state, with $\theta_{t}=-28.0^{\circ}$ and $\theta_{t}=-38.0^{\circ}$. 


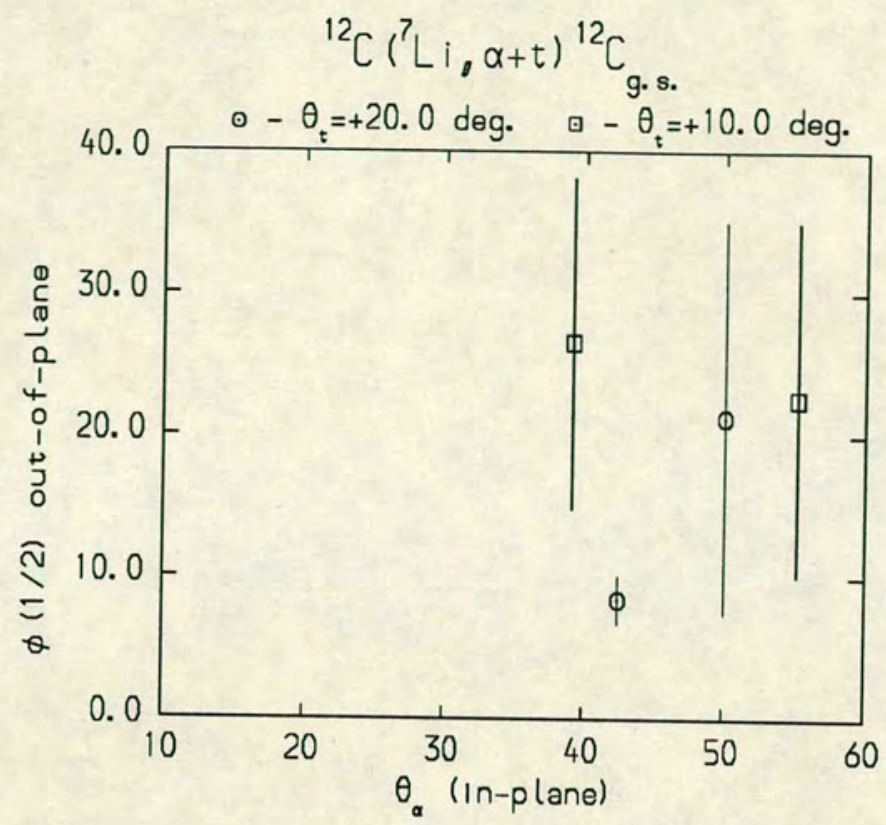

Figure B.27: Value of the half angle for the quasi-elastic out-of-plane distributions for the ${ }^{12} \mathrm{C}$ target with detectors on the same side of the beam, $\theta_{t}=+10.0^{\circ}$ and $\theta_{t}=+20.0^{\circ}$.

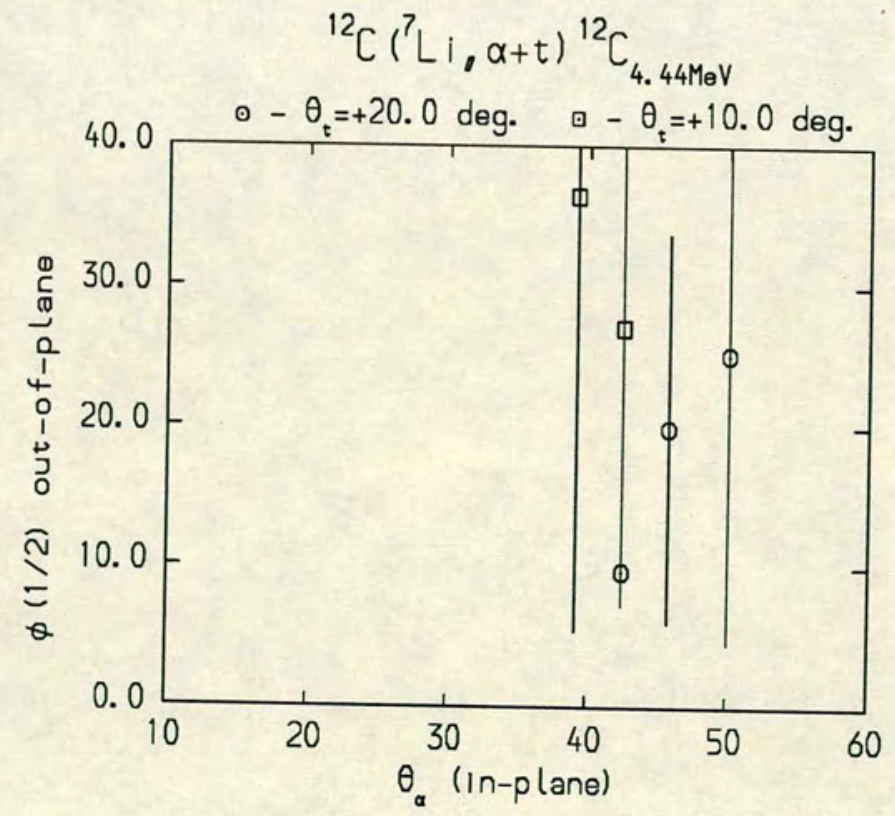

Figure B.28: Value of the half angle for the out-of-plane distributions for events leaving the ${ }^{12} \mathrm{C}$ target in its $4.44 \mathrm{MeV}$ state, and both detectors on the same side of the beam, $\theta_{t}=+10.0^{\circ}$ and $\theta_{t}=+20.0^{\circ}$. 


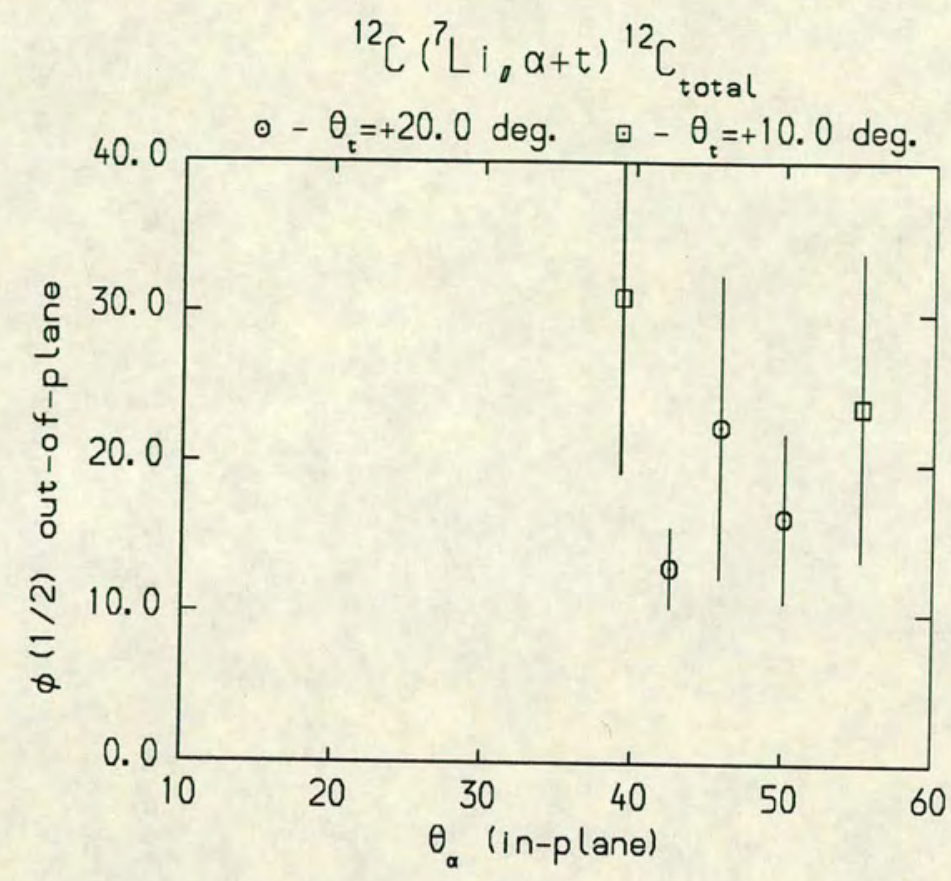

Figure B.29: Value of the half angle for the full out-of-plane distributions for the ${ }^{12} \mathrm{C}$ target with both detectors on the same side of the beam, $\theta_{t}=+10.0^{\circ}$ and $\theta_{t}=+20.0^{\circ}$.

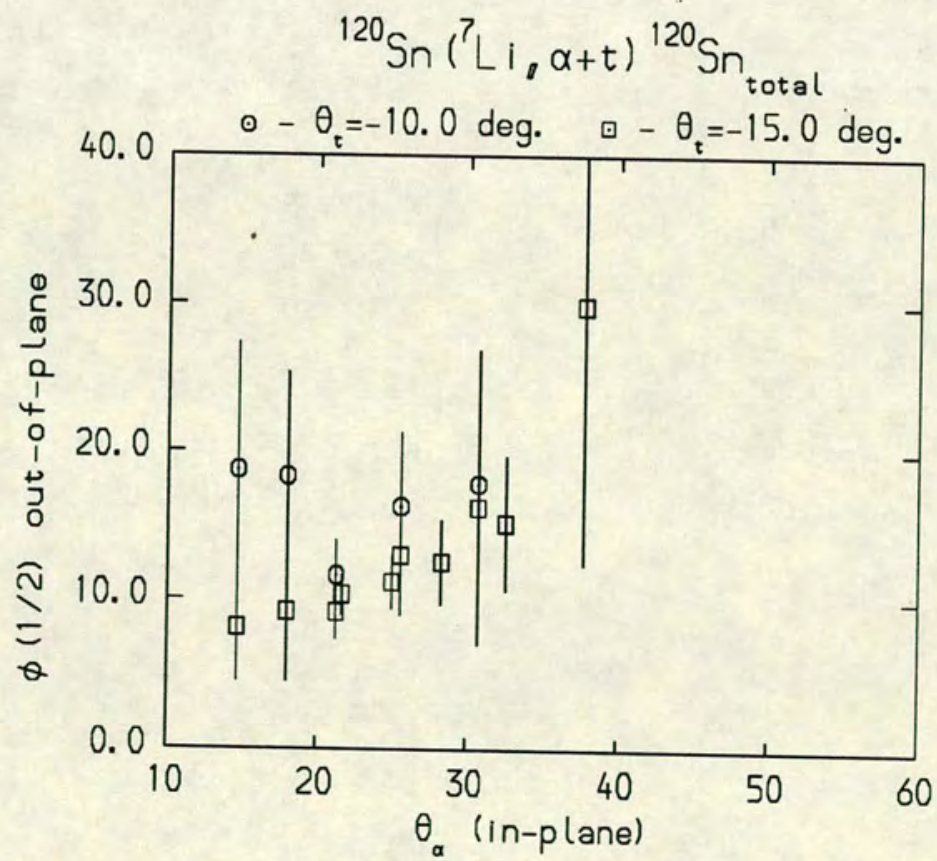

Figure B.30: Value of the half angle for the full out-of-plane distributions for the ${ }^{120} \mathrm{Sn}$ target with $\theta_{t}=-10.0^{\circ}$ and $\theta_{t}=-15.0^{\circ}$. 


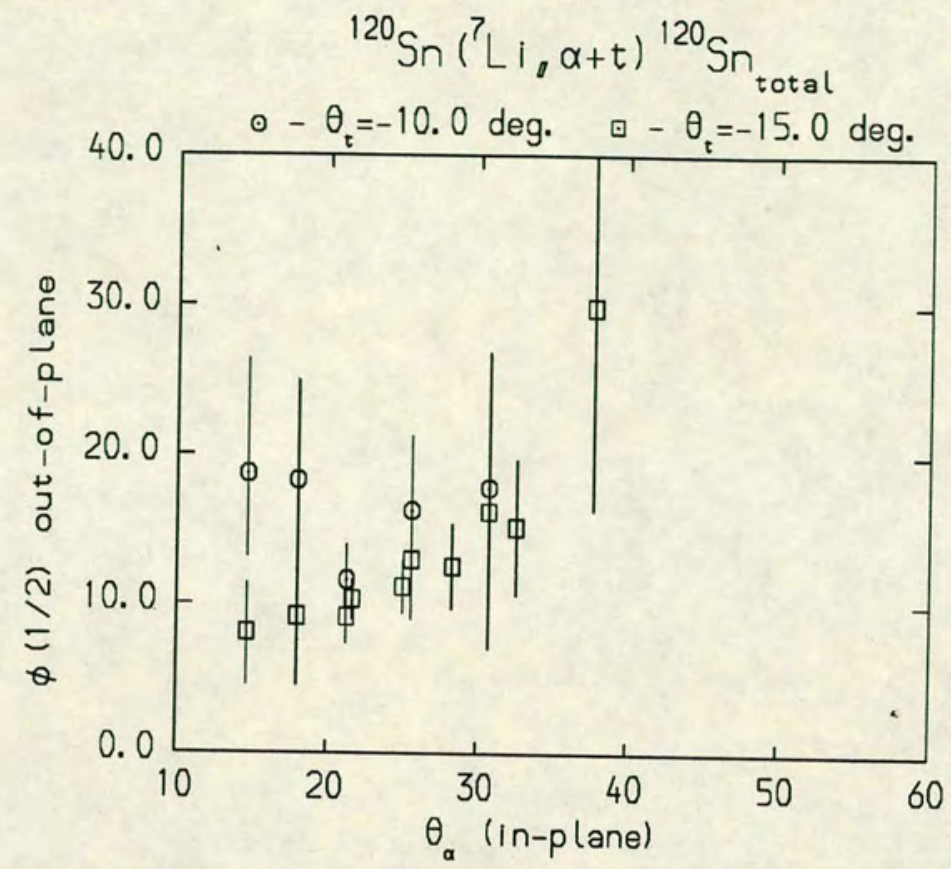

Figure B.31: Value of the half angle for the full out-of-plane distributions for the ${ }^{120} \mathrm{Sn}$ target with $\theta_{t}=-20.0^{\circ}$ and $\theta_{t}=-25.0^{\circ}$.

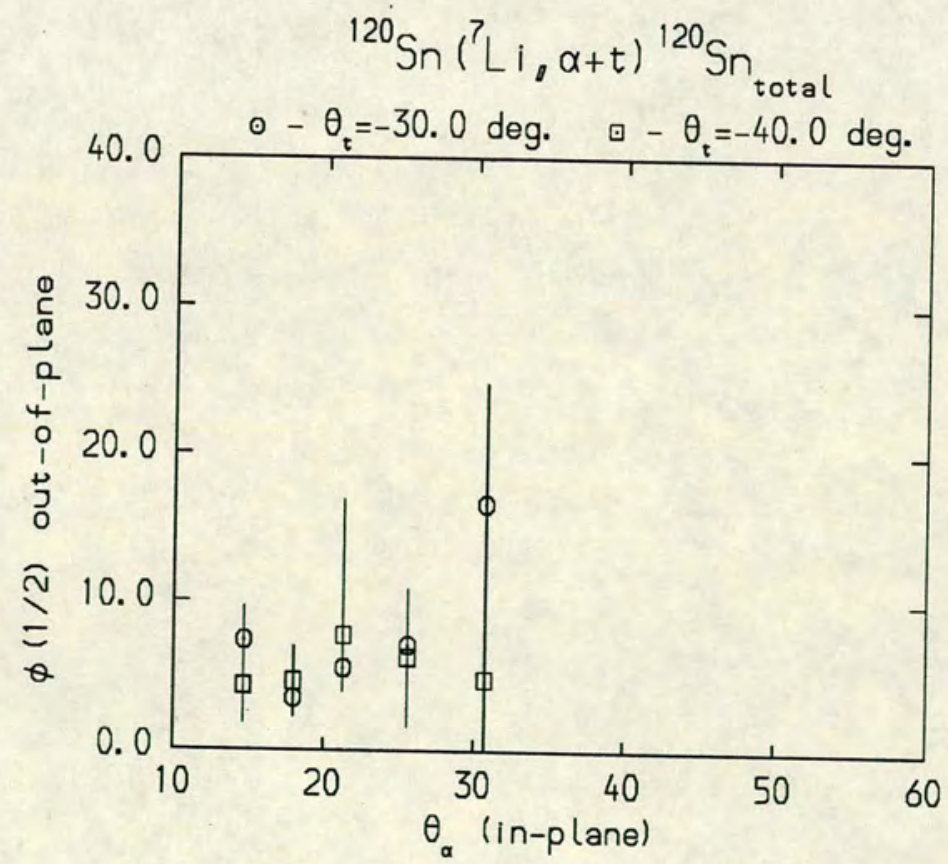

Figure B.32: Value of the half angle for the full out-of-plane distributions for the ${ }^{120} \mathrm{Sn}$ target with $\theta_{t}=-30.0^{\circ}$ and $\theta_{t}=-40.0^{\circ}$. 


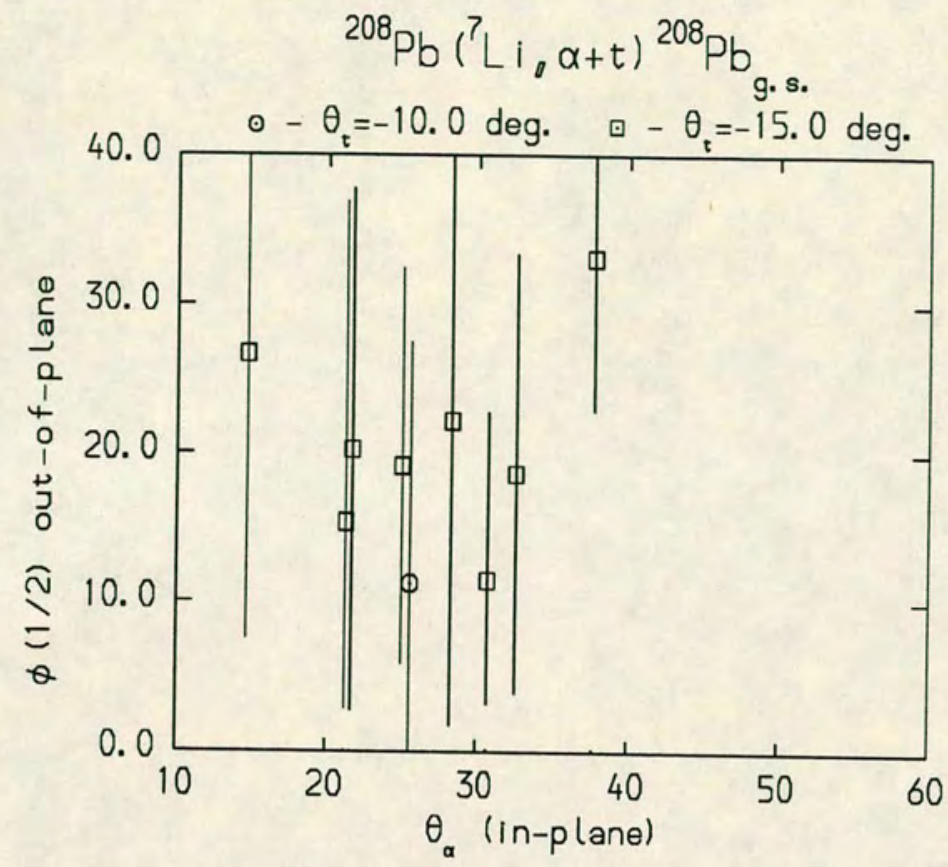

Figure B.33: Value of the half angle for the quasi-elastic distributions for the ${ }^{208} \mathrm{~Pb}$ target with $\theta_{t}=-10.0^{\circ}$ and $\theta_{t}=-15.0^{\circ}$.

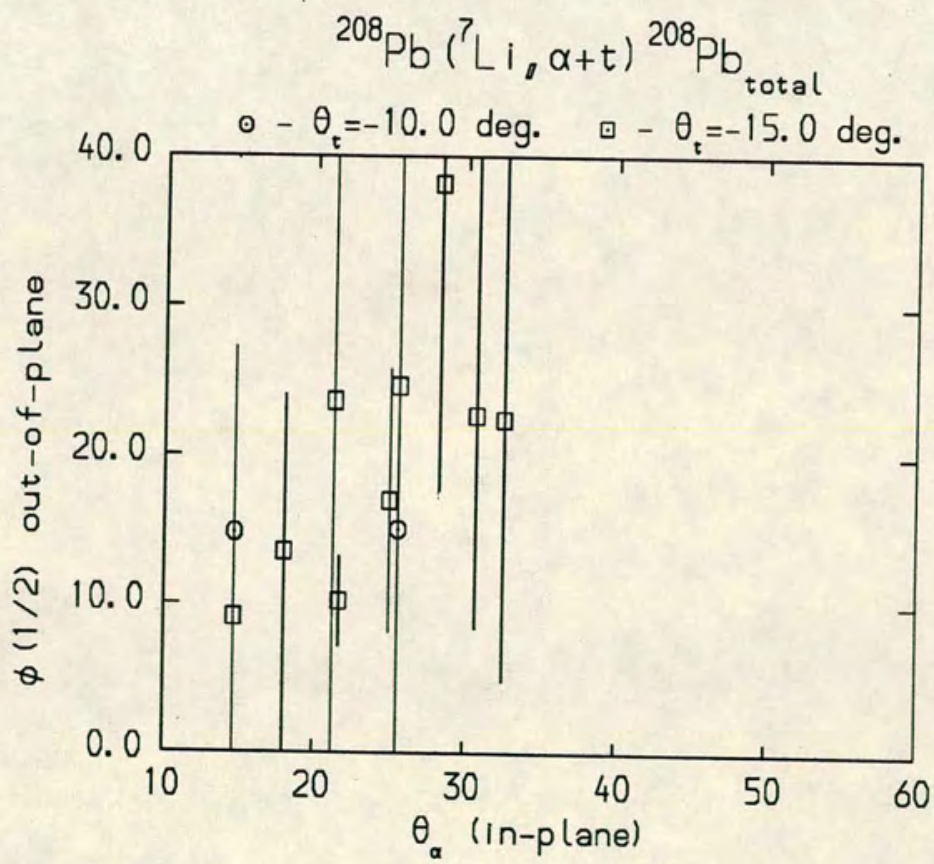

Figure B.34: Value of the half angle for the full out-of-plane distributions for the ${ }^{208} \mathrm{~Pb}$ target with $\theta_{t}=-10.0^{\circ}$ and $\theta_{t}=-15.0^{\circ}$. 


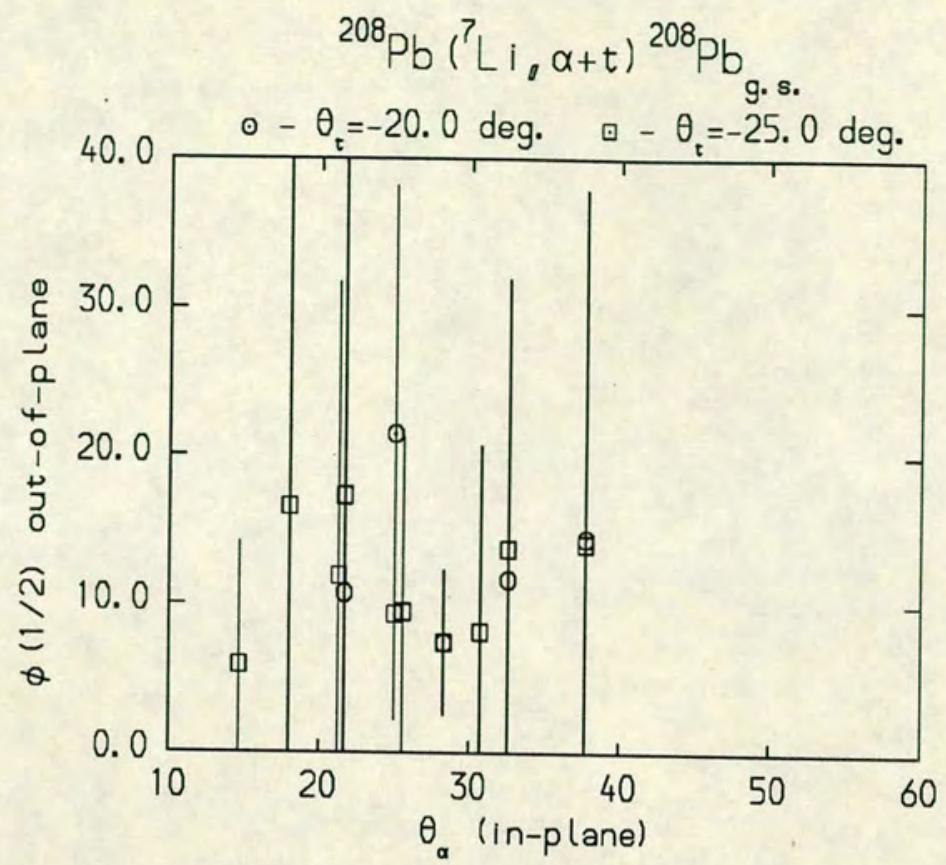

Figure B.35: Value of the half angle for the quasi-elastic distributions for the ${ }^{208} \mathrm{~Pb}$ target with $\theta_{t}=-20.0^{\circ}$ and $\theta_{t}=-25.0^{\circ}$.

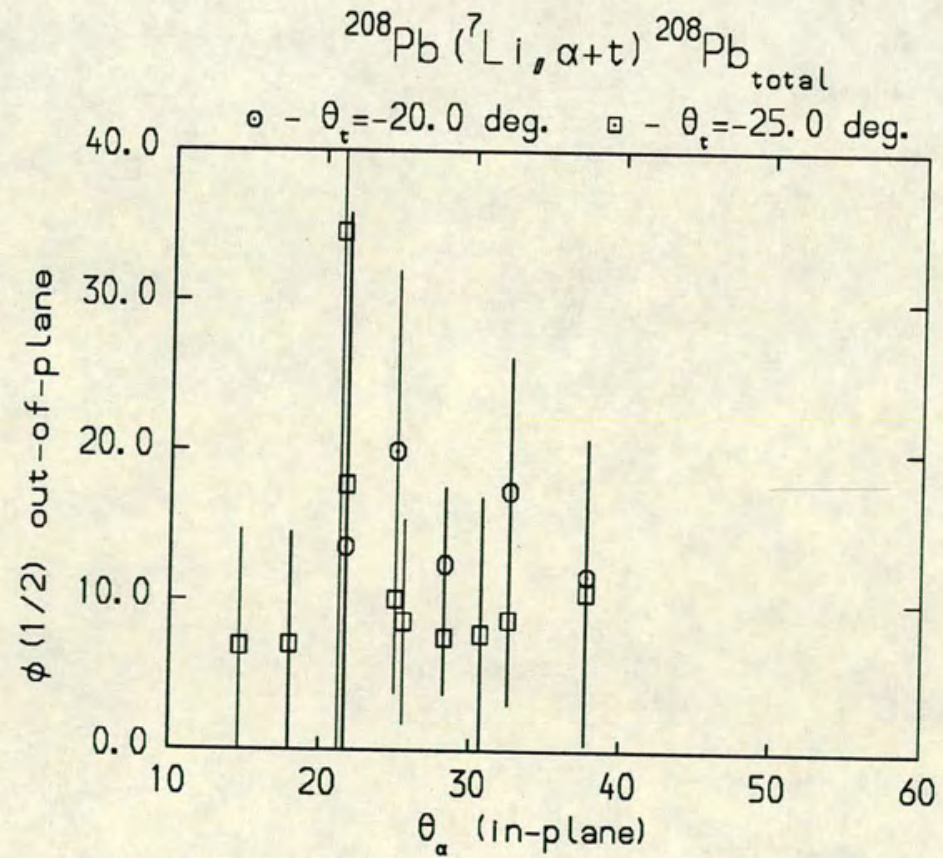

Figure B.36: Value of the half angle for the full out-of-plane distributions for the ${ }^{208} \mathrm{~Pb}$ target with $\theta_{t}=-20.0^{\circ}$ and $\theta_{t}=-25.0^{\circ}$. 


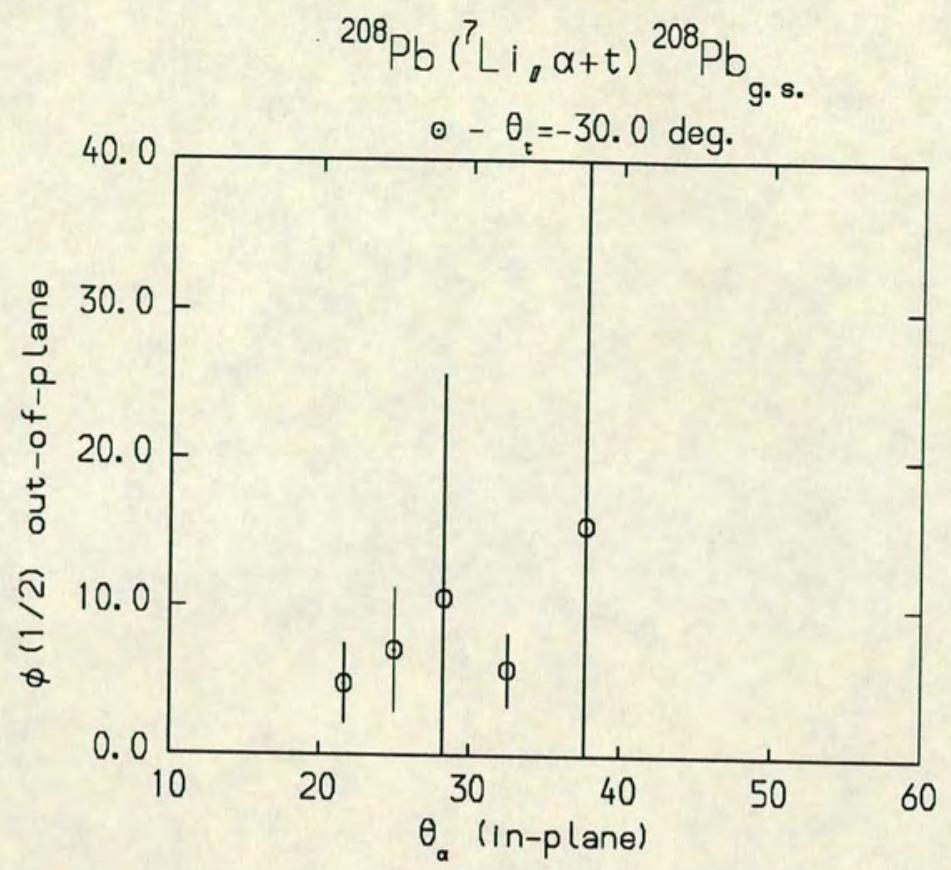

Figure B.37: Value of the half angle for the quasi-elastic distributions for the ${ }^{208} \mathrm{~Pb}$ target with $\theta_{t}=-30.0^{\circ}$.

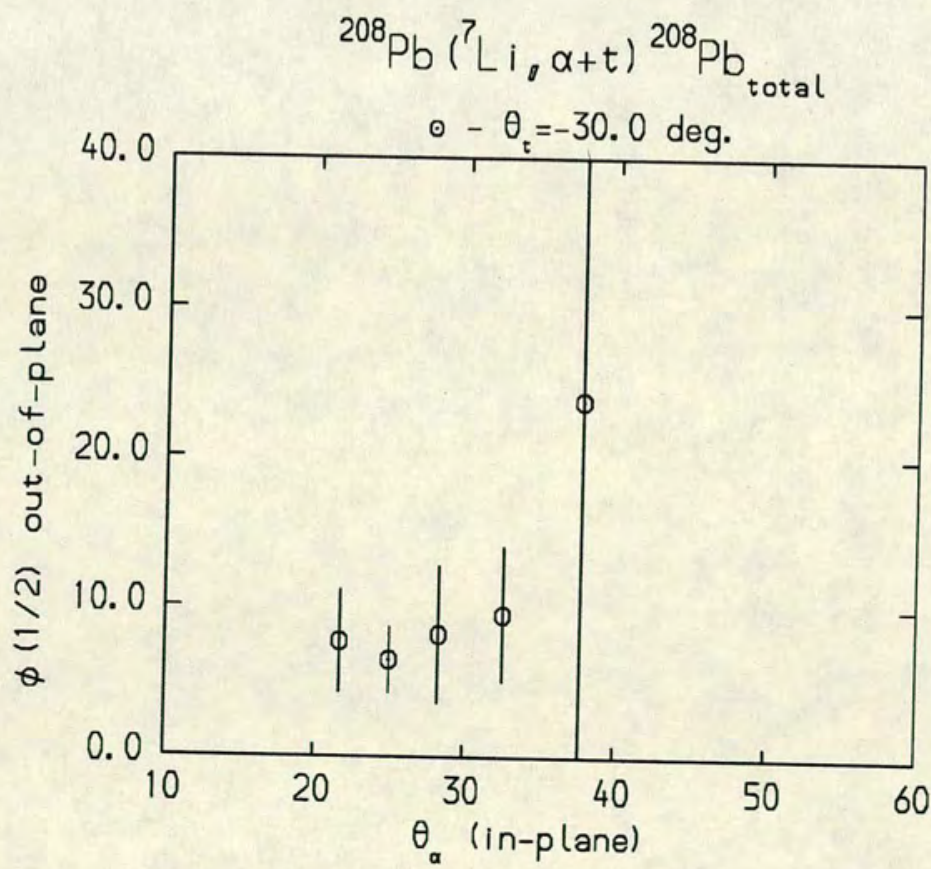

Figure B.38: Value of the half angle for the full out-of-plane distributions for the ${ }^{208} \mathrm{~Pb}$ target with $\theta_{t}=-30.0^{\circ}$. 


\section{Appendix C}

\section{Strip Detector Paper}

This appendix contains the paper on the anomalous interstrip surface effects published in Nuclear Instruments and Methods 262 (1987) 353. 


\title{
INTERSTRIP SURFACE EFFECTS IN OXIDE PASSIVATED ION-IMPLANTED SILICON STRIP DETECTORS
}

\author{
J. YORKSTON and A.C. SHOTTER \\ Department of Physics, Edinburgh University, The King's Buildings, Edinburgh, EH9 $3 J Z$ UK
}

\author{
D.B. SYME and G. HUXTABLE \\ Nuclear Physics Division, AERE, Harwell, Oxfordshire, UK
}

Received 6 July 1987

An investigation is reported into charge sharing effects for highly ionising particles penetrating the interstrip gap for ion-implanted silicon strip detectors. It is found that under certain conditions anomalous polarity pulses are induced on adjacent strips. This effect is analysed with a model that takes account of the surface charge trapped on the $\mathrm{Si}-\mathrm{SiO}{ }_{2}$ interface between the adjacent strips.

\section{Introduction}

Silicon strip detectors have, for a number of years, been used as active targets in high energy physics [1] where the excellent spatial resolution attainable by modern semiconductor fabrication techniques makes them particularly suitable for accurate vertex determination and flight path direction measurements.

In contrast to the minimum ionising particles detected in high energy applications, heavy ions of energy $\sim 10 \mathrm{MeV} / A$ will deposit large amounts of energy within the detector. The resulting higher ionisation density will generate intense internal fields which may give rise to charge sharing effects from the region between strips, although no such problems have been reported in previous work using minimum ionising particles [2]. This paper reports some results of an investigation to specifically determine charge sharing characteristics for highly ionising particles.

\section{Experimental procedure}

The detector used for this investigation was an oxide passivated, ion-implanted, silicon strip detector supplied by Enertec (Strasbourg). It consisted of 25 aluminium strips of $1.8 \mathrm{~mm}$ width and $200 \mu \mathrm{m}$ separation evaporated on a $42 \mathrm{~mm} \times 50 \mathrm{~mm}$ slice of $5 \mathrm{k} \Omega$ $\mathrm{cm}$ resistivity, n-type silicon and was manufactured using the planar process [3]. A schematic cross section of the construction between two adjacent strips is shown in fig. 1 .
The performance of the detector was evaluated using the IBIS (intense bunched ion source) accelerator at AERE, Harwell. The machine was used in its microbeam mode [4] with $3 \mathrm{MeV}$ alpha particles. Under normal operating conditions it can produce a beam of cross sectional dimensions better than $10 \mu \mathrm{m} \times 10 \mu \mathrm{m}$ which is ideal for probing this interstrip structure. However, due to the extremely small beam current required to allow exposure directly onto the detector surface $\left(\sim 10^{-16} \mathrm{~A}\right)$ the beam profile did not approach this value. From experimental results it was concluded that the beam had an intense spot with one sharply defined edge of $\sim 5 \mu \mathrm{m}$ but an extended tail on its opposite edge. This tail was wider than the interstrip gap but narrower than the individual strip width. The detector was mounted on a mechanical scanning mount allowing it to be positioned relative to the microbeam with an accuracy of $1 \mu \mathrm{m}$.

For this investigation four adjacent strips were biased to their full depletion voltage of $-40 \mathrm{~V}$ with reference to the earthed rear connection. The microbeam was then scanned across the front surface of the detector, between the two centre strips, sharp edge first, by moving the detector mount assembly in steps of $25 \mu \mathrm{m}$. Energy spectra from singles events from each strip were recorded for each position, along with the spectra of any pulses which occurred in coincidence in both strips.

\section{Results}

Fig. 2a shows a typical energy spectrum of the alpha particles when the beam illuminated only one strip, i.e., 


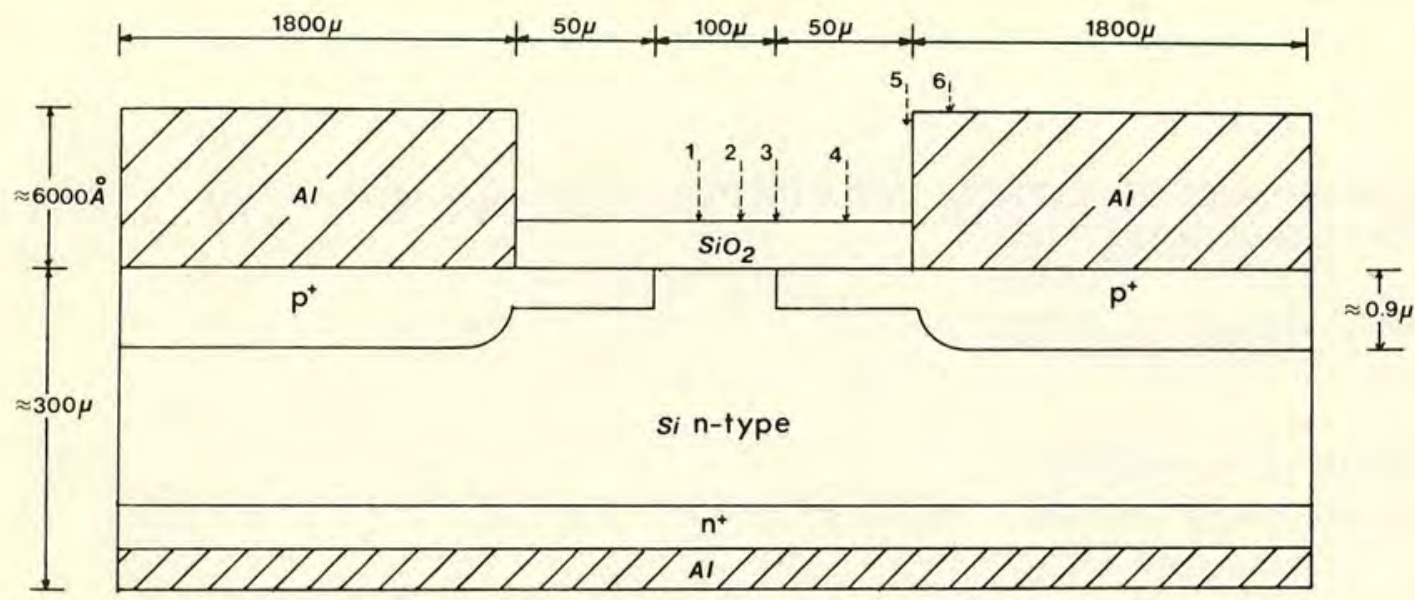

Fig. 1. Cross-sectional details of detector between two adjacent strips. The numbered arrows refer to fig. 3 .

no interstrip spacing was illuminated. The energy resolution was beam-limited at $40 \mathrm{keV}$. Fig. $2 \mathrm{~b}$ shows a typical spectrum when the whole interstrip gap was illuminated. Obviously the latter spectrum has a number of anomalous features compared to fig. 2a. By a comparison of figs. $2 \mathrm{a}$ and $\mathrm{b}$ it is clear that the most intense peak in fig. $2 \mathrm{~b}$ is due to alphas entering the active region through the aluminium strip. The upper energy peak in fig. $2 \mathrm{~b}$ is produced by alpha particles

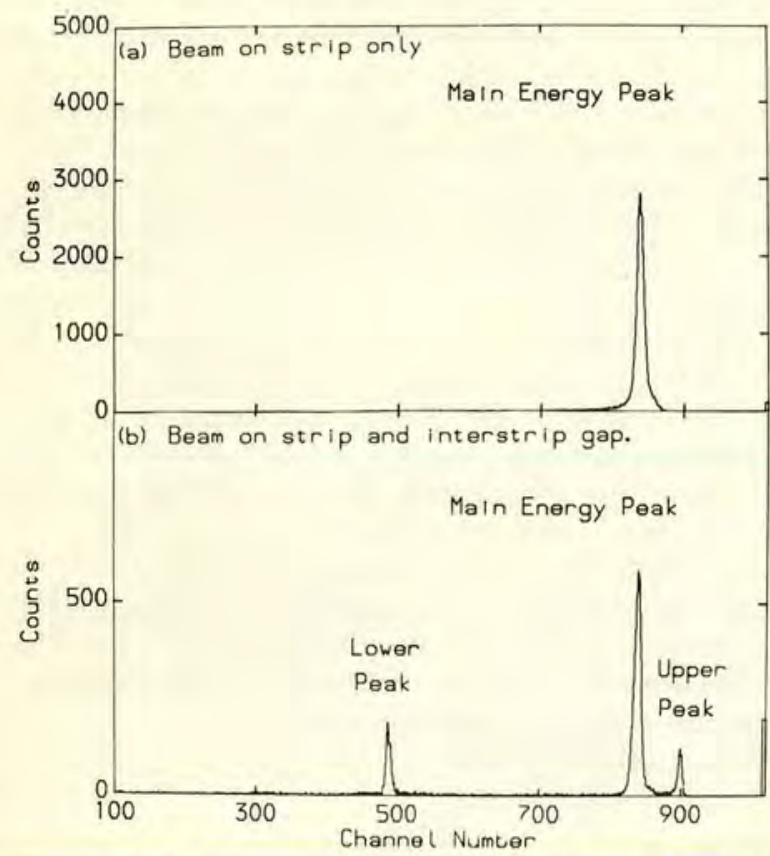

Fig. 2. Typical energy spectrum of $3 \mathrm{MeV}$ alpha particles incident on (a) the strip only and (b) the strip and interstrip gap. entering the detector via the thinner $\mathrm{SiO}_{2}$ layer between the $6000 \AA$ thick $\mathrm{Al}$ strips. The $200 \mathrm{keV}$ energy difference between the main peak and this upper peak is consistent with this interpretation. The origin of the lower peak will be discussed later.

The response of the detector was further tested by scanning the beam between two neighbouring strips. It was found experimentally that there were no events generated in the right-hand strip due to particles being detected at points further away than the midpoint of the interstrip gap on the left. The evolution of the right-hand strip energy spectrum, for a scan starting midway between the two adjacent strips, is shown in fig. 3 . The approximate position of the sharp edge of the beam for each spectrum is as shown in fig. 1. It can be seen that, as the beam is scanned across the interstrip gap, the different features begin to become apparent at distinctly different positions of the scan. This suggests that an explanation of their existence may be obtained from a consideration of the geometrical details of the detector.

In previous tests [2] the absence of any charge sharing between adjacent strips in passivated ion-implanted strip detectors has been an important advantage of this design over other types, e.g. surface barrier detectors. These tests were reported on detectors being used in a transmission mode and determined coincidences between pulses of normal polarity from adjacent strips. No such coincidences were reported for ion-implanted detectors. However, for surface barrier detectors, coincident pulses of opposite polarity have been observed from adjacent strips [5]. In the present test with ion-implanted detectors it was noted that with the beam in the interstrip position both adjacent strips exhibited noncoincident pulses of the opposite polarity to that expected from a normal energy signal but again there were never coincident normal-polarity pulses from adjacent strips. Fig. 4 shows a typical anomalous-polarity pulse 


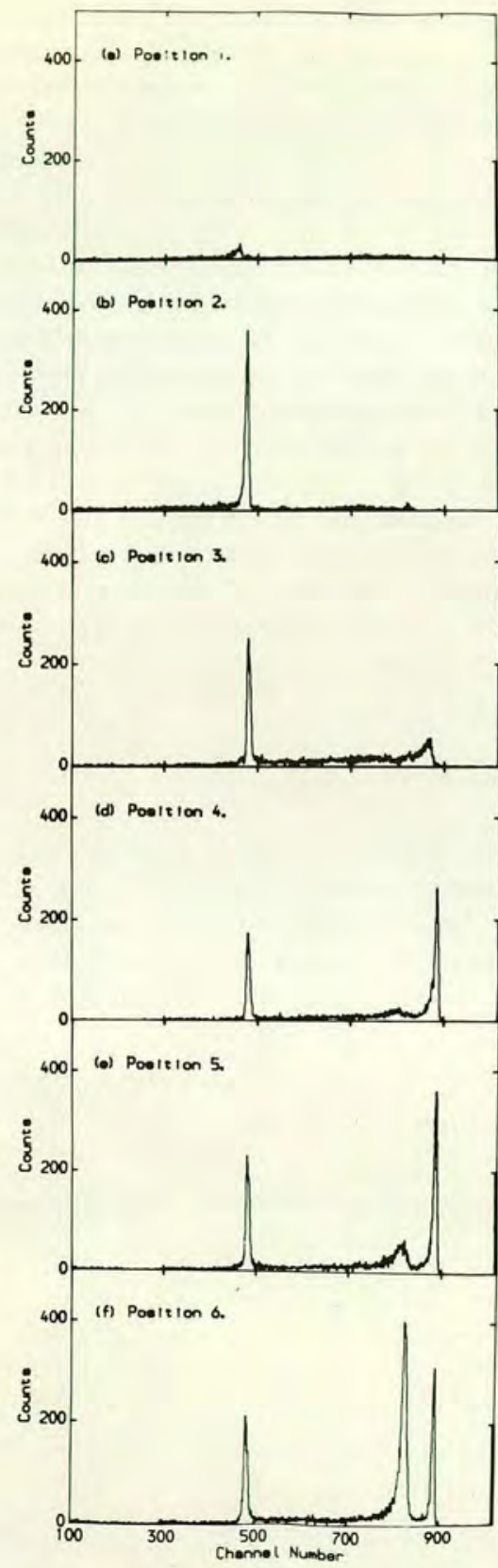

Fig. 3. Energy spectrum from one strip as the beam is moved towards it, sharp edge first, from the centre of the interstrip gap. The approximate location of the edge for each position is shown in fig. 1.

height spectrum from one strip. On further investigation it was found that these anomalous-polarity pulses always occurred in coincidence with a normal-polarity pulse in the adjacent strip. Furthermore, it was observed that these coincident normal-polarity pulses were smaller than a full energy pulse and contributed to the

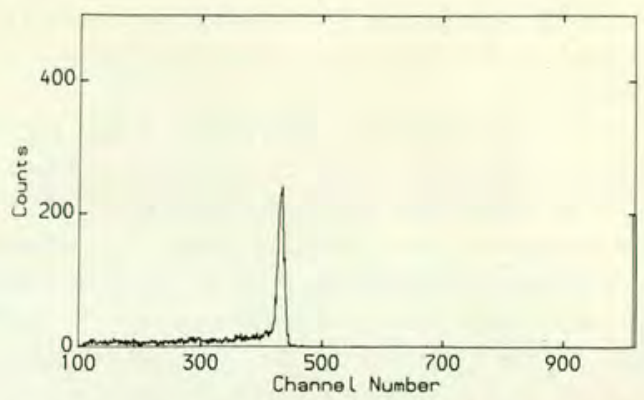

Fig. 4. A typical anomalous negative polarity pulse height spectrum.

lower energy satellite peak in fig. $2 \mathrm{~b}$. In order to explain this lower peak consider the spectrum taken at the centre of the interstrip gap (i.e. fig. $3 b$ ) which exhibits a strong lower energy peak. It is the pulses contributing to this peak which are in coincidence with the anomalous-polarity pulses from the adjacent strip. This leads to the conclusion that these coincident pulses of opposite polarity originate from the region between the strips and more specifically from the volume below the central region where there is no $\mathrm{p}^{+}$implantation.

When one looks at a two-dimensional plot of the anomalous-polarity pulse amplitude signal from one strip against the coincident normal-polarity pulse amplitude from its neighbour as shown in fig. 5 , it can be seen that the reduction below the full-energy charge for a normal-polarity pulse is about equal to the charge of the coincident anomalous-polarity pulse. This is not then charge sharing but could be due to the imposition of equal anomalous-polarity charges on both strips simultaneously, added to the normal collected charge. Prompted by these results a number of other simple

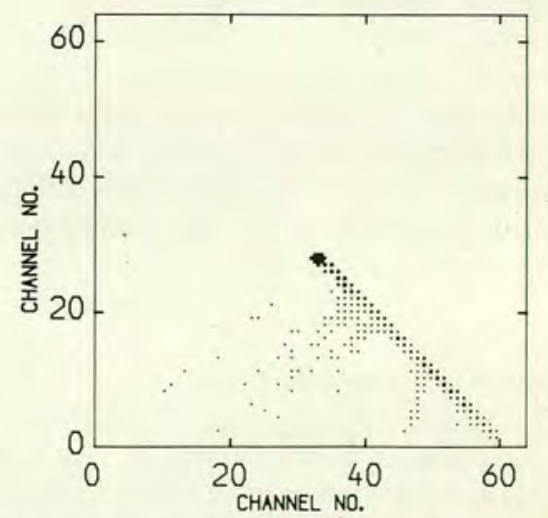

Fig. 5. A two-dimensional plot of coincident normal-polarity ( $x$-axis) vs anomalous-polarity ( $y$-axis) pulse heights. This was taken using an ${ }^{241} \mathrm{Am}$ alpha source rather than the IBIS beam, so is not expected to correspond in all details with figs. 2-4. 
tests were performed on the detector to try and shed more light on the mechanism producing these coincident pulses.

It was found that they disappeared when the bias was removed from one of the central strips and that the number of consecutive negative pulses from one strip, before a negative pulse from the other strip occurred, did not follow any identifiable pattern. To check whether these pulses were due to a difference in the voltage applied to the central strips the voltage on one was lowered by $10 \mathrm{~V}$ (a quarter of the full depletion voltage) to try and enhance the rate of negative pulses in either strip. This had no effect. It was also noticed that there was a threshold bias voltage below which the negative pulses disappeared. This may indicate that the growth of the depletion layers from the $\mathrm{p}^{+}$regions could be an important factor in their production. Using a fast-timing coincidence circuit the time difference between the coincident positive and negative pulses was found to be less than resolution of the setup ( $\sim 2-3 \mathrm{~ns})$.

The anomalous-polarity effect was also present when the detector was illuminated with a ${ }^{252} \mathrm{Cf}$ source whose fission fragments have a range comparable to that of the alpha particles used, but which deposit $\sim 90 \mathrm{MeV}$.

When the detector was illuminated with electrons from a ${ }^{207} \mathrm{Bi}$ source no anomalous-polarity pulses were observed. These electrons have a range of approximately $1 \mathrm{~mm}$ in $\mathrm{Si}$ and the full energy peak observed in the spectra was presumably due to the increased likelihood of large angle scattering within the detector. This lack of anomalous pulses seems to agree with the recent tests at CERN [2] where no anomalous effects were seen or reported when the detector was being used as a transmission detector. With $5.5 \mathrm{MeV}$ alpha particles from an ${ }^{241} \mathrm{Am}$ source illuminating the rear of the detector the anomalous effect was also absent suggesting that it arises from the front surface only. There was, however, a normal-normal coincidence rate of $<1 \%$ of the individual strip singles rate which was attributed to charge sharing following the diffusion of the hole cloud as it travelled through the detector towards the strips.

This absence of negative pulses with transmitted ionising particles may be due to their ionisation density in the volume between the adjacent $\mathrm{p}^{+}$regions being much smaller than that of the highly ionising particles stopping near the front surface.

\section{Analysis of observations}

The behaviour of $\mathrm{p}-\mathrm{n}$ junctions of this type under charged-particle irradiation is a topic of current interest in electron device design and is receiving some attention from a number of authors [6]. In his paper on surface barrier detectors Kraner [5] identified similar anomalous-polarity pulses with charge injection from the aluminium strip contact due to the perturbation of the local field by the ionising particle. A crude calculation shows that the maximum instantaneous concentration of electron-hole pairs resulting from a $5.5 \mathrm{MeV}$ alpha particle is of the same order as the fixed space charge in the $\mathrm{p}^{+}$region of full depletion $\left(\sim 5 \times 10^{18} \mathrm{~cm}^{-3}\right)$ so that the field would indeed be perturbed. However, there is no known mechanism to instantaneously separate these associated concentrations of holes and electrons in order to generate the perturbing field necessary to cause charge injection. An alternative explanation is required. The computer simulations of Hsieh et al. [6] predict that an anomalous charge collection funnelling effect will be of increasing importance with higher resistivity substrate and be a strong function of junction bias. However, we have developed a rather different model from the viewpoint of charge induction rather than charge injection which seems to explain many of the observed effects.

\section{Proposed model}

Between the strips is a highly insulating surface layer of $\mathrm{SiO}_{2}$, and the depleted junction extends underneath this layer. Leakage (and also ionisation) causes current to flow across the junction, and a steady flow of holes arrives at the strip side, to be collected by the strips.

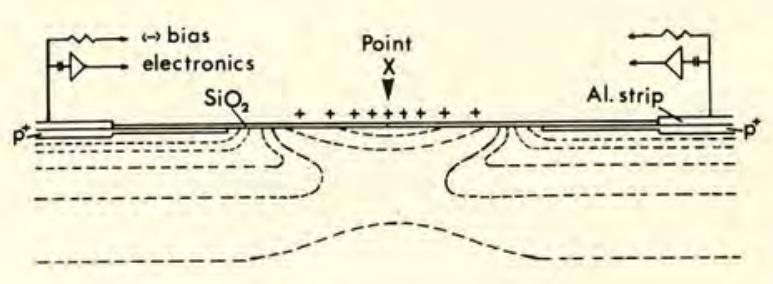

n-type Si

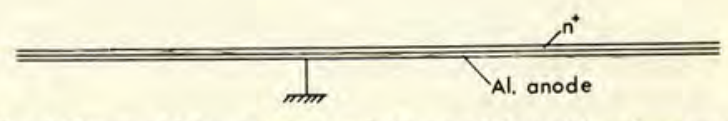

Fig. 6. Presumed form of potential map with positive charge on insulating surface, showing reversed-field region. 
Because the interstrip gap is highly insulating, there can be no net flow of current to this area, so a positive charge must be built up at the surface of the insulating layer, sufficient (in the equilibrium state) to repel further holes from arriving in the interstrip gap. This charge is distributed symmetrically about the midpoint of the gap (point $\mathrm{X}$ in fig. 6). The assumption is that this positive charge is sufficient to cause a local field reversal so that the potential, measured across the junction from X, falls locally to a minimum value between the strips, close below the surface, before the normal potential rise across the junction then commences. Between the strips there will be a saddle point in the potential map, as sketched in fig. 6 .

\section{Hole sharing}

One effect of the hump in the potential between the strips is that when hole-electron pairs are produced by an ionising particle in the interstrip gap, the holes are driven towards the nearest strip by the field gradient, depending on which side of the saddle they were created. Therefore, there will be a strong tendency for the holes to be collected on one or other of the strips, rather than divided between them. Only from a very restricted band of positions, right on the interstrip centre, will holes

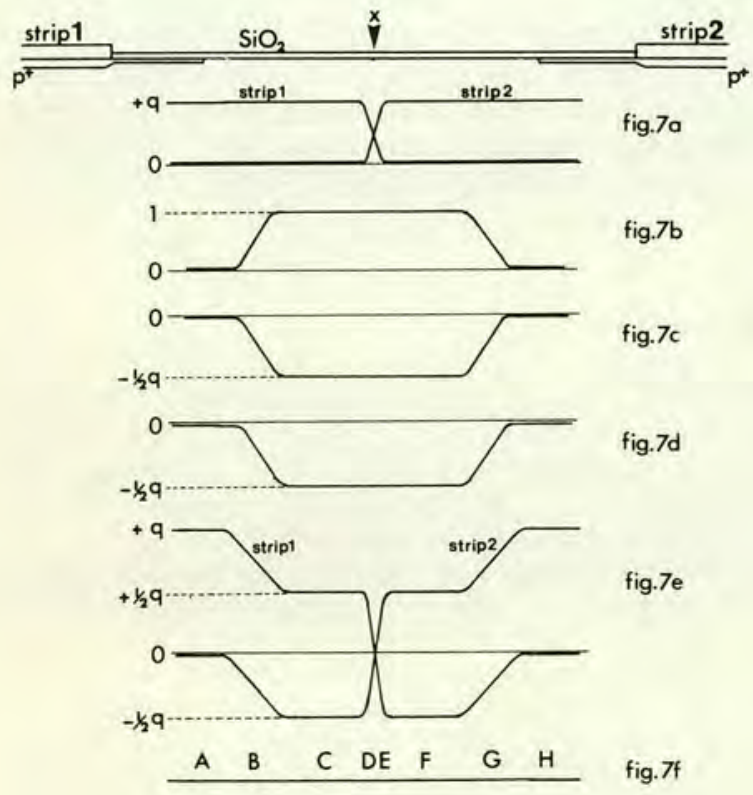

Fig. 7. Charge collection from tracks at different position within the interstrip gap. (a) Charge due to holes collected. (b) Fraction of electrons trapped near X. (c) Charge induced on strip 1 due to electrons trapped near X. (d) Charge induced on strip 2 due to electrons trapped near X. (e) Net charge collected on strips. (f) Position of track across interstrip gap. from a track be divided between the strips, as suggested in fig. 7a. A sharp division of charge between strips is observed experimentally. This would give ideal characteristics for the detector if all the electrons from all tracks were to travel to the anode for collection. However, this does not happen as we will see.

\section{Electron trapping}

When a particle track occurs near the centre of the interstrip gap, the electrons will be pulled towards the potential hump at X, rather than towards the anode, if they were created on the reversed-field side of the potential saddle point. This will be particularly likely for electrons in the tracks of short range highly ionising particles such as $3 \mathrm{MeV}$ alphas (range approximately 10 $\mu \mathrm{m})$. For more penetrating particles, or for tracks which occur near one strip or the other, some of the electrons will be pulled towards the anode. But the model implies that over a finite area around X, all the electrons created by an alpha track lie in this anomalous reversefield region and all move to $\mathrm{X}$, as shown in fig. $7 \mathrm{~b}$. There is no electrode here to conduct them away; if they remain trapped at this point for a time long compared with the pulse-shaping times of the electronics, or if they immediately recombine at this point with a trapped positive charge, the effect of the net signals at the detector electrodes will be exactly the same, a charge deficit will arise in the signal which depends on the charge which has been induced on the corresponding electrode by these electrons. Consider first a "normal" full-energy signal produced by a track passing through, or close to the edge of a strip. The track produces a charge $+q$ of holes $-q$ of electrons. All the holes are collected on a strip and all the electrons travel away from the influence of the strip to the anode, resulting in a net charge $q$ flowing between the strip and the anode and zero charge on adjacent strips. There may be a transient pulse electrostatically induced on the adjacent strip but its net charge will integrate to zero.

However, if the track is between the strips, and the electrons become trapped at $\mathrm{X}$, the result is very different. The charge $-q$ carried by the electrons is electrostatically coupled entirely to the two strips, and hardly at all to the anode, because of the geometry (the anode is much further away). The induced charge flow from each strip is $-\frac{1}{2} q$ if we suppose that the charge has ended up symmetrically between the strips at $\mathrm{X}$ (figs. 7c and d). As all the holes will usually be collected by one strip, the resulting net charge flow from that strip is $+q-\frac{1}{2} q$, of $+\frac{1}{2} q$, i.e. a pulse or normal polarity but halved amplitude. As there is no hole collection on the adjacent strip, the net charge flow from it is $-\frac{1}{2} q$, i.e. an anomalous-polarity pulse of half amplitude. Many such signal pairs were observed in this experiment. 


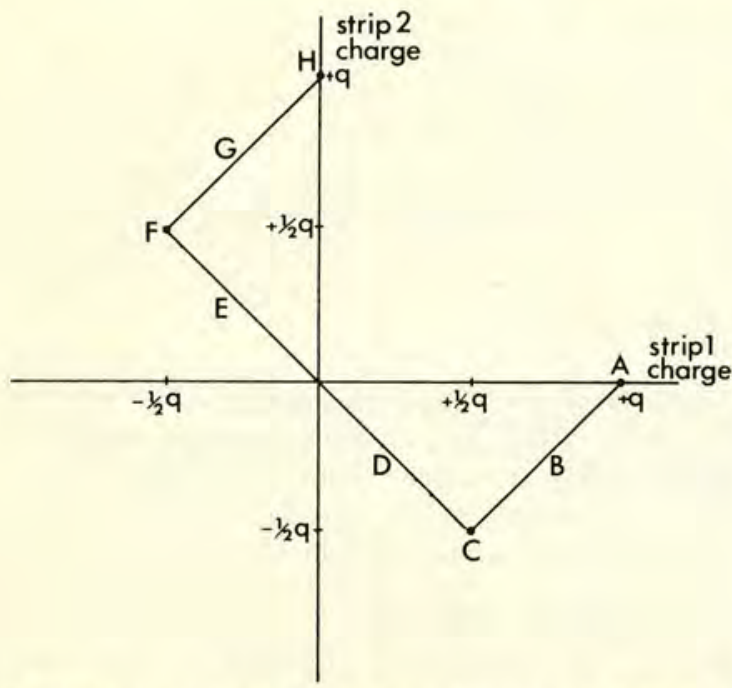

Fig. 8. Correlation diagram for charge collected on strip 1 and strip 2 for different positions of track within the interstrip gap. The letters A to $\mathrm{H}$ correspond to the regions shown in fig. $7 \mathrm{e}$.

It is worth emphasising that the anomalous pulse is due entirely to induced charge: no electrons or holes are collected by that strip, but the incompletely collected ionisation from the particle track results in a charge $-q$ ending up near the strip and inducing a charge $-\frac{1}{2} q$ to flow out from it and into the external circuit (see fig. 7e, regions $\mathrm{C}$ and $\mathrm{F}$ ).

It appears from the measurements that trapping of all electrons occurs for tracks over a significant fraction of the interstrip gap, near its centre (i.e. regions $\mathrm{CDEF}$ in fig. 7). Outside these regions the nearer to a strip a track occurs the smaller is the fraction of the electrons that are trapped. The trapped charge $-\Delta q$ then becomes progressively smaller than $q$. Assuming still that all the trapped electrons migrate to the midpoint $X$, the net signal on the nearer strip would be $q-\frac{1}{2} \Delta q$, and on the further strip $-\frac{1}{2} \Delta q$. This is exactly the correlation that has been observed experimentally and corresponds to regions $B$ and $G$ of fig. 7e. For tracks near the edge of the strip, and for all tracks that pass through the strip, complete collection is expected, and $\Delta q=0$. This corresponds to regions $\mathrm{A}$ and $\mathrm{H}$ of fig. 7e.

A correlation diagram (fig. 8) has been developed from fig. $7 \mathrm{e}$ and shows how the simultaneous pulses from adjacent strips correlate for tracks in various regions of the interstrip gap. This shows remarkable agreement with the observed correlation reported in this paper.

To summarise, the following effects are well explained by the model.
(1) The sharp splitting of signals between the strips.

(2) The peaks at $\frac{1}{2}$ and $-\frac{1}{2}$ of normal pulse height.

(3) The fact that simultaneous normal-polarity pulses are never seen: one is always reversed.

(4) The detailed form of the correlation function between simultaneous pulses from adjacent strips.

In addition it seems likely that some of the "peculiar effects" of reversed-polarity pulses described in ref. [5] might be caused by a similar charge-trapping mechanism, though many details of that measurement differ from the present one, and a weaker effect of the same variety would be anticipated.

\section{Conclusion}

The existence of interstrip surface effects in oxide passivated ion-implanted silicon strip detectors has been identified. They seem to be important only for highly ionising nontransmitting particles and arise from a volume beneath the $\mathrm{SiO}_{2}$ passivation layer between the main $\mathrm{p}^{+}$implantation regions under each strip. The effect gives rise to anomalous-polarity pulses and anomalous peaks in the energy spectrum of the ionising particle. They have been observed in a number of different strip detectors of different design and from different manufacturers but all of which have been produced by the planar technique incorporating ion implantation. It is clear from these results that although strip detectors have a number of advantages over more conventional detectors for a range of applications, care must be taken when incorporating them into certain experiments, especially those involving fission fragments and other highly ionising radiation.

\section{References}

[1] E.H.M. Heijne et al., Nucl. Instr. and Meth. 178 (1980) 331 ;

E.H.M. Heijne and P. Jarron, IEEE Trans. Nucl. Sci. NS-29 (82);

J. Kemmer et al., IEEE Trans. Nucl. Sci. NS-29 (82) 733; and other references in Nucl. Instr. and Meth. 226 (1984).

[2] P. Bonamy et al., EP Internal Report CERN 81-06 (23 Sept. 1981).

[3] J. Kemmer, Nucl. Instr. and Meth. 169 (1980) 499.

[4] J.A. Cookson, Nucl. Instr. and Meth. 165 (1979) 477.

[5] H.W. Kraner et al., IEEE Trans. Nucl. Sci. NS-30 (83) 405.

[6] C.M. Hsieh et al., IEEE Electron Dev. Lett. EDL-2 (81) 103;

C. Hu, IEEE Electron Dev. Lett. EDL-3 (82) 31; J.S. Chern, IEEE Trans. Electron Dev. ED-33 (86) 822. 


\section{Bibliography}

[Aa81] E.H.L.Aarts et.al. Physics Letters 102B (1981) 307.

[Aa84] E.H.L.Aarts et.al. Nuclear Physics $\underline{\mathrm{A} 425}$ (1984) 23.

[Aj84] F.Ajzenberg-Selove Nuclear Physics $\underline{\text { A413 (1984) } 1 .}$

[Aj86a] F.Ajzenberg-Selove Nuclear Physics $\underline{460}$ (1986) 1.

[Aj86b] F.Ajzenberg-Selove Nuclear Physics $\underline{449}$ (1986) 1.

[Al56] K.Alder,A.Bohr et.al. Rev. of Mod. Physics $\underline{28}$ (1956) 432.

[Ar82] K.P.Artemov et.al. Sov.J.Nucl.Phys.36 (1982) 779.

[Ba72] G.Baur and D.Trautman Nuclear Physics A191 (1972) 321.

[Ba72b] G.Baur and D.Trautman Physics Letters 42B (1972) 31.

[Ba73] G.Baur et.al. Nuclear Physics A208 (1973) 261.

[Ba76] G.Baur and D.Trautman Physics Reports 25C (1976) 293.

[Ba80] J.H.Barker et.al. Physics Review Lett. $\underline{45}$ (1980) 474.

[Ba80b] R.Bass, Nuclear Reactions with Heavy Ions. Published by SpringerVerlag. (1980) 89.

[Ba83] S.Bayegan Ph.D thesis Edinburgh University (1983) unpublished.

[Ba84] G.Baur, F.Rosel et.al. Physics Reports $\underline{111}$ (1984) 333.

[Ba84b] G.J.Balster, R.K.Bhowmik et.al. Physics Letters 143B (1984) 79. 
[Be84] G.Bellini et.al. Nucl. Instr.\& Meth. $\underline{225}$ (1984) 619.

[Be88] F.D.Becchetti, M.Dowell et.al. Invited paper at $5^{\text {th }}$ Int. Conference on Clustering in Nuclei. Koyoto Japan, July 1988.

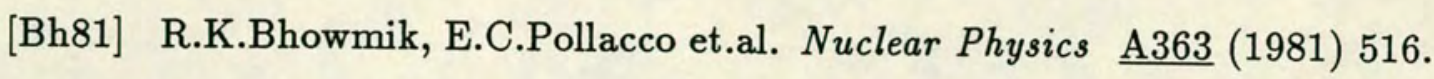

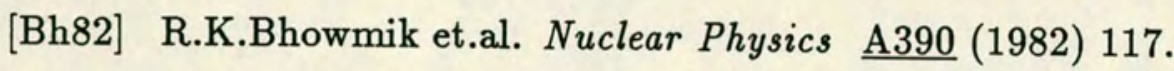

[Bi81] A.N.Bice, A.C Shotter et.al. Physics Letters 101B (1981) 27.

[Bi82] A.N.Bice, A.C.Shotter et.al. Nuclear Physics $\underline{\text { A390 }}$ (1982) 161.

[Bo81] P.Bonamy, P.Borgeaud et.al. E.P. Internal repört 81-06 (23 ${ }^{\text {rd }}$ Sept. 1981)

[Br61] H.C.Britt and A.R.Quinton Physics Review $\underline{124}$ (1961) 877.

[Br72] D.M.Brink Physics Letters $\underline{40 B}$ (1972) 37.

[Bu78] A.Budzanowski et.al. Physics Review Lett. $\underline{41}$ (1978) 635.

[Bu88] P.Burger Private communication.

[Ca80] C.M.Castenada et.al. Physics Review C $\underline{21}$ (1980) 179.

[Ce66] J.Cerny, S.W.Cosper et.al. Nucl. Instr. \& Meth. $4 \underline{45}$ (1966) 337.

[Co79] J.A.Cookson Nucl. Instr.\& Meth. $\underline{165}$ (1979) 477.

[Cu78] A.Cunsolo, A.Foti et.al. Physics Review C $\underline{18}$ (1978) 856.

[Cu80] A.Cunsolo, A.Foti et.al. Physics Review C $\underline{21}$ (1980) 2345.

[Da84] C.J.S. Damerell. Developments in solid state vertex detectors. Lectures given at SLAC Summer Inst. 1984. Ral-84-123

[Da85] T.Davinson Private communication.

[Da87] T.Davinson. Ph.D. thesis Edinburgh University (1987) unpublished.

[Da88] T.Davinson Private communication. 
[Dr80] J.van.Driel. Ph.D. thesis University of Groningen (1980) unpublished.

[En81] J.B.A.England, B.D.Hyams et.al. Nucl. Instr.\& Meth. $\underline{185}$ (1981) 43.

[En82] J.B.A.England, B.D.Hyams et.al. Nucl. Instr.\& Meth. 196 (1982) 149.

$[\mathrm{EG}+\mathrm{G}] \mathrm{EG}+\mathrm{G}$ Ortec, Silicon Charged Particle Radiation Detector Instruction Manual.

[E187] A.El-Lithi Ph.D thesis Edinburgh University (1987) unpublished.

[Fu83] T.Fukuda, M.Ishihara et.al. Physics Review C $\underline{27}$ (1983) 2029.

[Ge78] H.Gemmeke, B.Delingi et.al. Zeit. Physik $\underline{\mathrm{A} 266}$ (1978) 73.

[Ge79] K.A.Geoffroy, D.G.Sarantites et.al. Physics Review Lett. $\underline{43}$ (1979) 1303.

[Go86] C.Geossling, E.H.M.Heijne et.al. IEEE.Trans. on Nucl.Sci. NS-33 (1986) 272.

[Go84] P.B.Goldhoorn, G.J.Balster et.al. Physics Letters $\underline{142}$ (1984) 14.

[Go84b] P.B.Goldhoorn in Proceedings of the Workshop on Coincident Particle Emission from Continuum States in Nuclei. Edited by H.Machner and P.Jahn. Published by World Scientific (1984).

[Ha72] O.Hausser, A.B.M ${ }^{c}$ Donald et.al. Physics Letters $\underline{38 \mathrm{~B}}$ (1972) 75.

[He47] A.C.Helmholtz et.al. Physics Review $\underline{72}$ (1947) 1003.

[He80] E.H.M.Heijne, L.Hubbeling et.al. Nucl. Instr.\& Meth. $\underline{178}$ (1980) 331.

[Ho78] P.E.Hodgson in Nuclear Heavy Ion Reactions. Published by Oxford University Press (1978) 33.

[Ho84] R.Hofmann, R.Klanner et.al. Nucl. Instr.\& Meth. $\underline{225}$ (1984) 601.

[Ho84b] R.Hofmann et.al. Nucl. Instr.\& Meth. $\underline{226}$ (1984) 200.

[Hs83] C.Hsieh et.al. IEEE.Trans. on Elect.Dev. ED-30 (1983) 686. 
[Hy83] B.Hyams, V.Koetz et.al. Nucl. Instr.\& Meth. $\underline{205}$ (1983) 99.

[Hy84] B.Hyams Nucl. Instr.\& Meth. $\underline{225}$ (1984) 606.

[In77] T.Inamura, M.Ishihara et.al. Physics Letters $\underline{68 \mathrm{~B}}$ (1977) 51.

[Ja70] D.F.Jackson Nuclear Reactions. Published by Methuen (1970) 60.

[Ja73] L.Jarczyk, J.Lang et.al. Physics Review C $\underline{8}$ (1973) 68.

[Ja88] J.W.Janecke Private communication.

[Jo70] R.C.Johnson and P.R.Soper Physics Review C $\underline{1}$ (1970) 976.

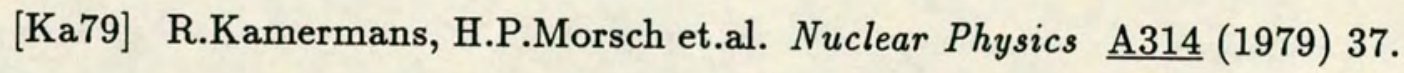

[Ke80] J.Kemmer Nucl. Instr.\& Meth. $\underline{169}$ (1980) 499.

[Ke82] J.Kemmer, P.Burger et.al. IEEE.Trans. on Nucl.Sci. NS-29 (1982) 733.

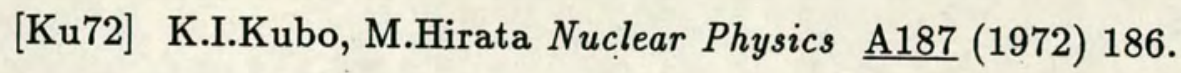

[La35] E.O.Lawrence, E.M ${ }^{\mathrm{c}}$ Millan et.al. Physics Review $\underline{48}$ (1935) 500.

[Ma78] N.Matsuoka et.al. Nuclear Physics A311 (1978) 173.

[Ma80] N.Matsuoka et.al. Nuclear Physics $\underline{\text { A345 }}$ (1980) 1.

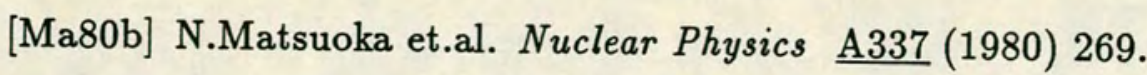

[Ma82] N.Matsuoka et.al. Nuclear Physics $\underline{\text { A391 (1982) } 357 .}$

[Ma88] E.W.Macdonald Ph.D. thesis Edinburgh University (1988) unpublished.

[Me83] R.J.de Meijer et.al. Nuclear Physics $\underline{\mathrm{A} 402}$ (1983) 15.

[Me85] R.J.de Meijer et.al. Rev. of Mod. Physics $\underline{57}$ (1985) 147. 
[Me84] R.J.de Meijer and P.D.Bond in Proceedings of the Workshop on Coincident Particle Emission from Continuum States in Nuclei. Edited by H.Machner and P.Jahn. Published by World Scientific (1984) 71.

[Mc80] K.Mc Voy and M.C.Nemes Zeit. Physik $\underline{\mathrm{A} 295}$ (1980) 177.

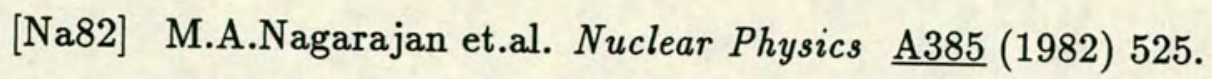

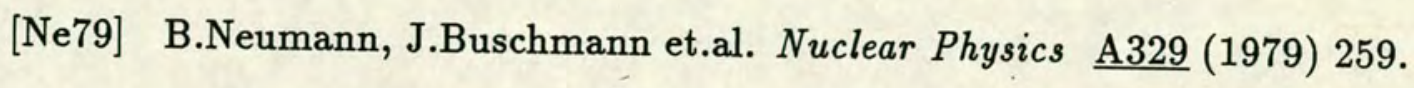

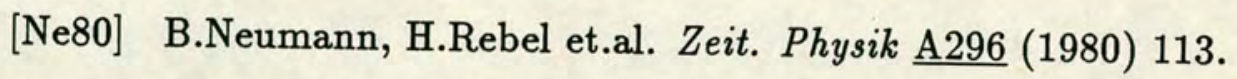

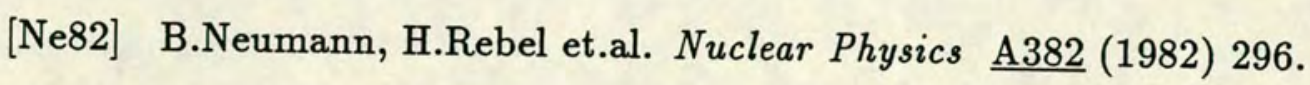

[Oe85] W.von Oertzen Physics Letters 151B (1985) 95.

[Oh65] G.G.Ohlsen Nucl. Instr.\& Meth. $\underline{37}$ (1965) 240.

[Op35] J.R.Oppenheimer Physics Review $\underline{47}$ (1935) 845.

[Op35b] J.R.Oppenheimer and M.Phillips Physics Review $\underline{48}$ (1935) 500.

[Os74] R.Ost, K.Bethge et.al. Zeit. Physik $\underline{\text { A266 }}$ (1974) 369.

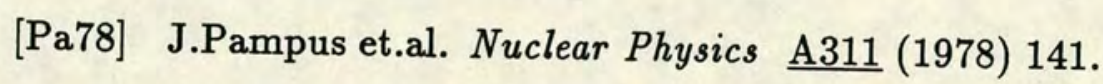

[Pa84] D.J.Parker, J.Asher et.al. U.K.A.E.A. report no.AERE-R 11217 (1984).

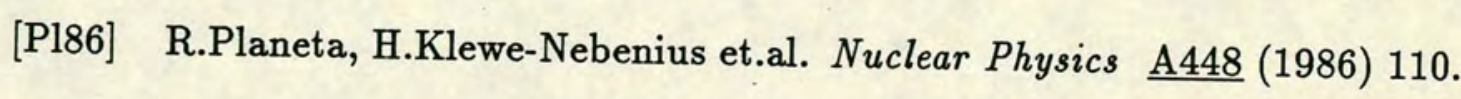

[Qu74] J.L.Quebert, B.Frois te.al. Physics Review Lett. $\underline{32}$ (1974) 1136.

[Ra75] G.H.Rawitscher Physics Review C 11 (1975) 1152.

[Ra82] V.Rapp Edinburgh Nuclear Physics Group Annual Report (1982) 30.

[Ra84] W.D.M.Rae et.al. Physics Review C $\underline{30}$ (1984) 158. 


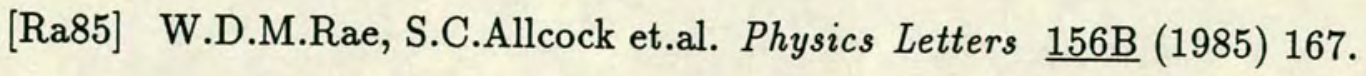

[Sa86] Y.Sakuragi, M.Yahiro et.al. Institute for Nuclear Study, University of Tokyo, Japan, INS-Report-600 Aug.1986.

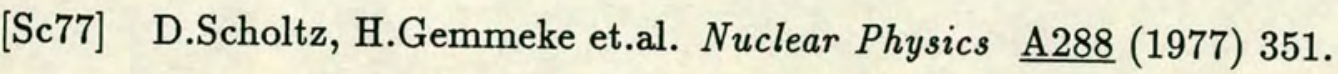

[Se47] R.Serber Physics Review $\underline{72}$ (1947) 1008.

[Sh81] A.C.Shotter, A.N.Bice et.al. Physics Review Lett. $\underline{46}$ (1981) 12.

[Sh84] A.C.Shotter, V.Rapp et.al. Physics Review Lett. $\underline{53}$ (1984) 1539.

[Sh85] A.C.Shotter in Proceedings of $4^{\text {th }}$ Int. Conf. on Clustering Aspects of Nuclear Structure and Nuclear Reactions. Edited by J.Lilley and M.A.Nagarajan. Published by Reidel (1985).

[Sh85b] A.C.Shotter from lectures presented at National Postgraduate School on Nuclear Physics 1985.

[Sh88] A.C.Shotter and M.A.Nagarajan Jour. of Phys. G 14 (1988) L109.

[Sh88b] A.C.Shotter Private communication.

[Sh88c] A.C.Shotter et.al. Jour. of Phys. G $\underline{14}$ (1988) L169.

[Si79] K.Siwek-Wilczynska et.al. Physics Review Lett. $\underline{42}$ (1979) 1599.

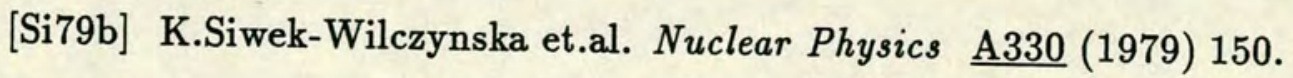

[Si87] K.Siwek-Wilczynska, J.Wilczynski et.al. Physics Review C $\underline{35}$ (1987) 1316 .

[Sm69] W.R.Smith Computer Physics Comm. 1 (1969) 55.

[Sr88] D.K.Srivastava et.al. Physics Letters 206B (1988) 391.

[Th83] I.Thompson and M.A.Nagarajan Physics Letters 123B (1983) 379.

[Ut83] H.Utsunomiya, S.Kubono et.al. Physics Review C $\underline{28}$ (1983) 1975. 
[Ut88] H.Utsunomiya, R.P.Schmidt et.al. Physics Letters 211B (1988) 24.

[Wa84] J.T.Walker et.al. Nucl. Instr.\& Meth. $\underline{226}$ (1984) 200.

[Wo72] G.J.Wozniak, H.L.Harney et.al. Physics Review Lett. $\underline{28}$ (1972) 1278.

[Wi73] J.Wilczynski Nuclear Physics A216 (1973) 386.

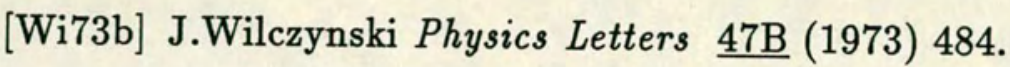

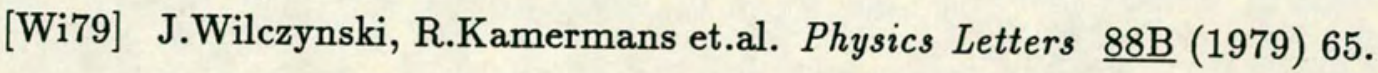

[Wi83] H.W.Wilschut, R.K.Bhowmik et.al. Physics Letters 123B (1983) 173.

[Yo87] J.Yorkston, A.C.Shotter et.al. Nucl. Instr.\& Meth. $2 \underline{262}$ (1987) 353.

[Zo78] D.R.Zolnowski, H.Yamada et.al. Physics Review Lett. $\underline{41}$ (1978) 93. 